\title{
Migrationsbedingte Heterogenität im Sportunterricht
}

\author{
Eine Repertory Grid-Studie zu schüler- und fachbezogenen Überzeugungen \\ Sport unterrichtender Lehrpersonen
}

\author{
Dissertation zur Erlangung der Würde eines Doktors der Philosophie \\ vorgelegt dem Institut für Bildungswissenschaften der Universität Basel \\ von \\ Jonas Steiger
}




\section{Danksagung}

An dieser Stelle danke ich allen, die mich in irgendeiner Weise bei der Anfertigung dieser Arbeit unterstützt und begleitet haben.

Allen voran danke ich den Lehrpersonen, die sich als Teilnehmende dieser Untersuchung zur Verfügung gestellt haben. Ihre Bereitschaft, Offenheit und insbesondere ihr zeitliches Engagement erlaubten mir einen tiefen Einblick in ihre Berufsrealität.

Ein besonderer Dank gilt Prof. Dr. André Gogoll für die stets konstruktiv-motivierende fachliche und nicht-fachliche Beratung während allen Phasen und Schwierigkeiten des Projekts. Dankbar bin ich ihm zudem für das grosse mir entgegengebrachte Vertrauen und die mir gewährten Freiheiten während der Zeit als Doktorand in seinem Team.

Ein überaus grosser Dank gebührt auch Prof. Dr. Roland Messmer für die wertvollen fachlichen und forschungsmethodischen Hinweise und Anregungen sowie seine wohlwollende, unterstützende Art der Zusammenarbeit.

Ein herzliches Dankeschön geht auch an Prof. Dr. Uwe Pühse für seine Bereitschaft, sich als weiterer Gutachter zur Verfügung zu stellen. Dass mit ihm eine Person gewonnen werden konnte, die durch eigene Forschungsprojekte und Publikationen mit dem Thema Migration bestens vertraut ist, freut mich speziell.

Dr. Harald Seelig und Dr. Gregory Hadley danke ich für ihre unentbehrliche Beratung im Bereich der in dieser Arbeit angewandten Repertory Grid-Technik. Ihre eigenen, mir vermittelten Erfahrungen mit Repertory Grid-Studien haben wesentlich zu meinem Verständnis (einer erfolgreichen Anwendung) dieser Methode beigetragen.

Für das kritische Mitdenken, das hohe Interesse und die unzähligen, in vielerlei Hinsicht hilfreichen Diskussionen und Gespräche danke ich allen Kolleginnen und Kollegen, die mich im Promotionsprozess begleiteten. Besonders bedanken möchte ich mich bei Dr. Matthias Baumgartner, Dr. Eric Jeisy, Dr. Dominique Fankhauser, Mischa Stillhart, Matthias Grabherr, Dr. Elke Gramespacher, Jolanda Vogler, Katja Schönfeld und Dr. Sonja Büchel.

Schliesslich danke ich meiner Familie, die für all meine Hochs und Tiefs stets ein offenes Ohr hatte, bei Zweifel an mich glaubte und mir half, immer wieder mal Distanz zur Arbeit zu gewinnen. 


\section{Inhalt}

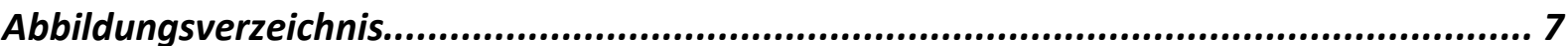

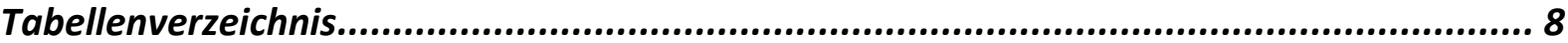

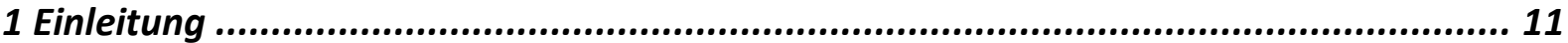

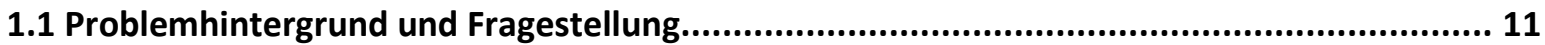

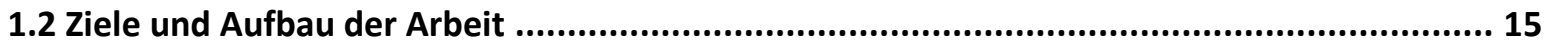

2 Lehrerüberzeugungen (teachers' beliefs) ............................................................ 17

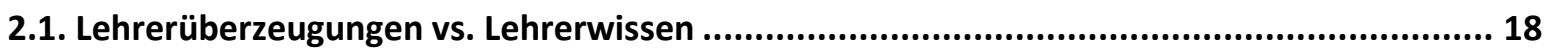

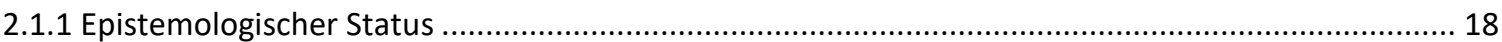

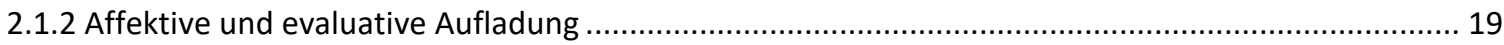

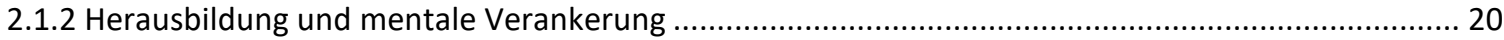

2.2 Gegenstandsbereiche von Lehrerüberzeugungen ......................................................... 22

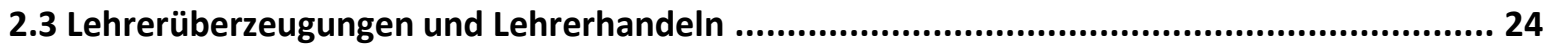

2.3.1 Lehrerüberzeugungen als Aspekt professioneller Kompetenz.................................................... 24

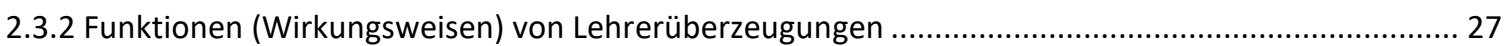

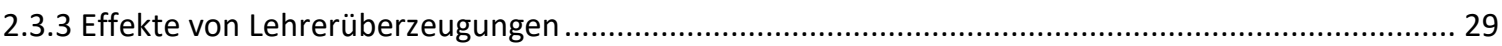

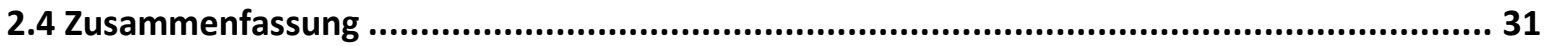

3 Lehrerüberzeugungen angesichts migrationsbedingter Heterogenität ...................... 33

3.1 Empirische Ergebnisse ausserhalb der Sportlehrerforschung ....................................... 34

3.1.1 Akkulturationseinstellungen und Ideologien im Umgang mit migrationsbedingter Heterogenität ... 34

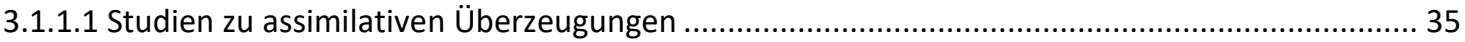

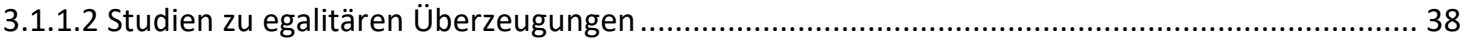

3.1.1.3 Studien zu integrativ-multikulturellen Überzeugungen.......................................................... 40

3.1.2 Überzeugungen über Schülerinnen und Schüler mit Migrationshintergrund .................................. 43

3.1.3 Vorstellungen zur unterrichtlichen Umsetzung und Umsetzbarkeit interkultureller Erziehung......... 50

3.2 Empirische Ergebnisse aus der Sportlehrerforschung..................................................... 54

3.3 Zusammenfassung der Forschungsergebnisse und Identifikation von Forschungslücken...... 58

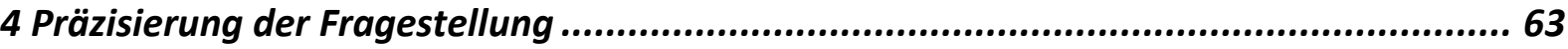

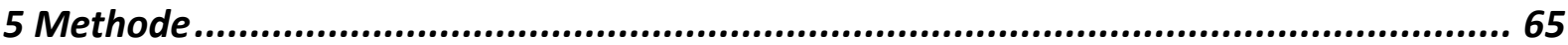

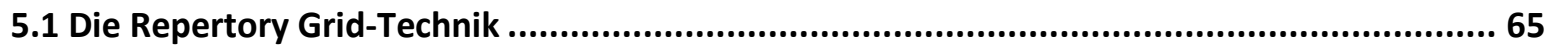




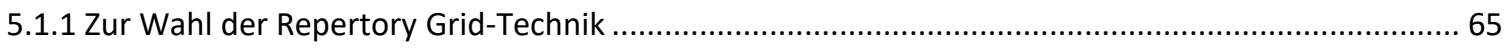

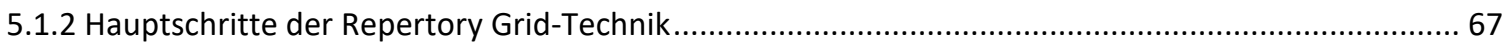

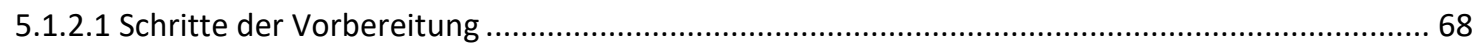

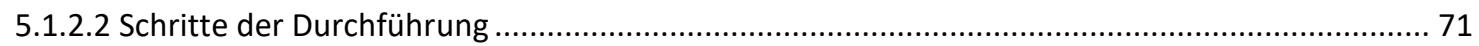

5.2 Untersuchungsteilnehmende....................................................................... 75

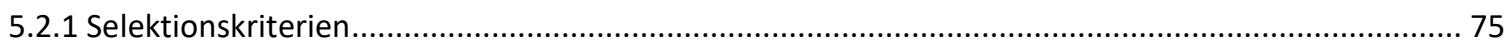

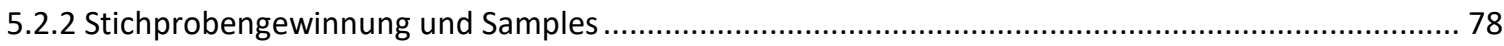

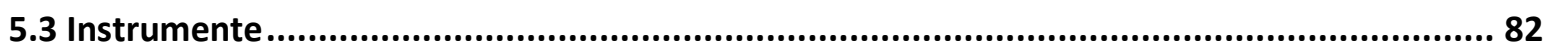

5.3.1 Überzeugungen über Schülerinnen und Schüler mit Migrationshintergrund im Sportunterricht ...... 82

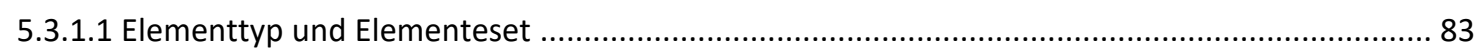

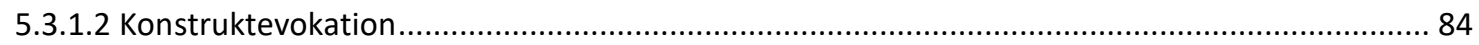

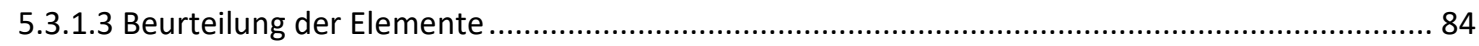

5.3.2 Vorstellungen zur unterrichtlichen Umsetzung und Umsetzbarkeit interkultureller Erziehung im

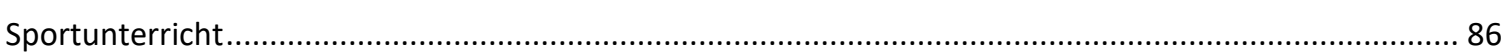

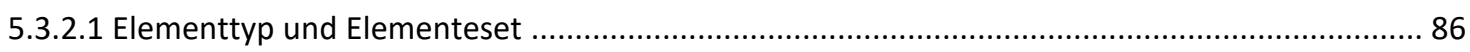

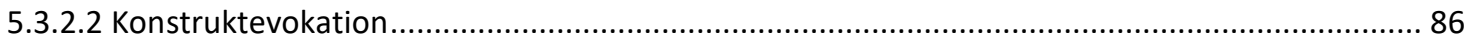

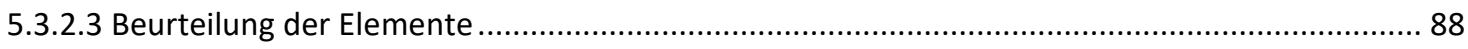

5.4 Durchführung der Interviews ..................................................................... 89

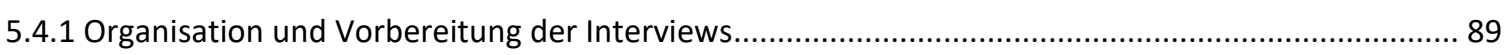

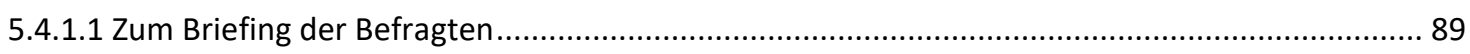

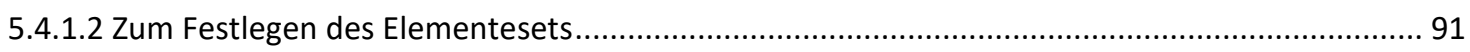

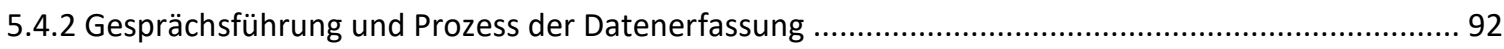

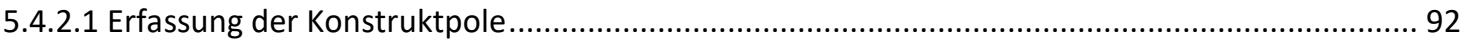

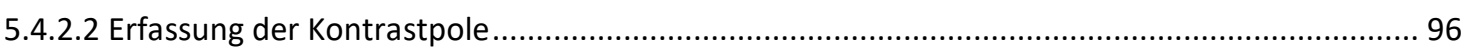

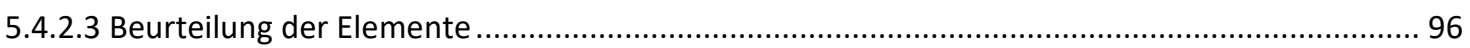

5.5 Auswertungsstrategie und Analyseverfahren ................................................97

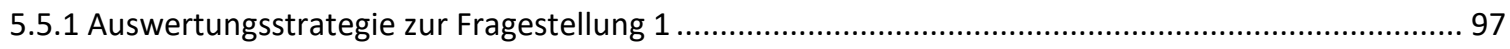

5.5.2 Auswertungsstrategie zur Fragestellung 2 ....................................................................... 101

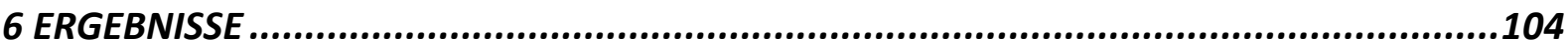

6.1 Schülerinnen und Schüler mit Migrationshintergrund im Sportunterricht aus Sicht der

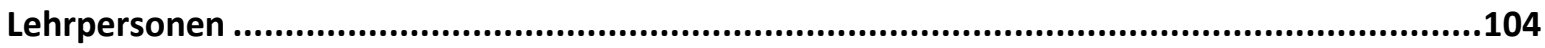

6.1.1 Unterschied zwischen fremdsprachigen und nicht-fremdsprachigen Schülerinnen und Schülern... 106

6.1.2 Kategorienspezifische Unterschiede zwischen fremdsprachigen und nicht-fremdsprachigen

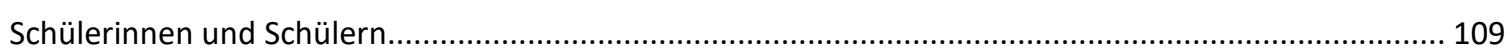

6.1.2.1 Körperlichkeit/Sportliche Leistungsfähigkeit ...................................................................... 111

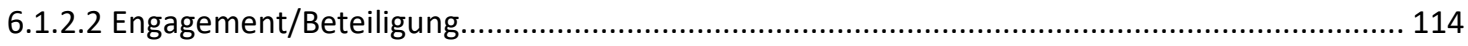




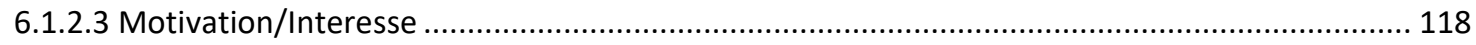

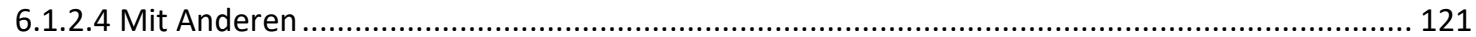

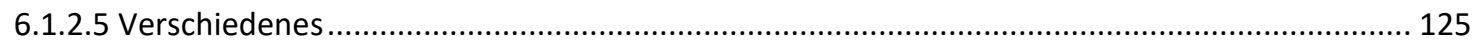

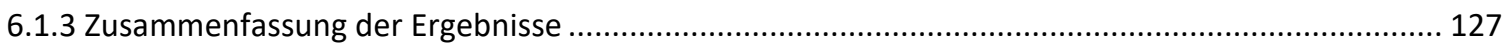

6.2 Sportunterricht und interkulturelles Lernen aus Sicht der Lehrpersonen..........................130

6.2.1 Allgemeine Chancen interkulturellen Lernens aus Sicht der Lehrpersonen ................................ 130

Exkurs: Interkulturelles Lernen aus Sicht der Lehrpersonen - eine Heuristik .................................... 145

6.2.2 Chancen interkulturellen Lernens im Sportunterricht aus Sicht der Lehrpersonen ........................ 152

6.2.2.1 Fachimmanente Chancen des Sportunterrichts ............................................................... 155

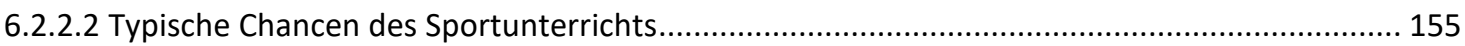

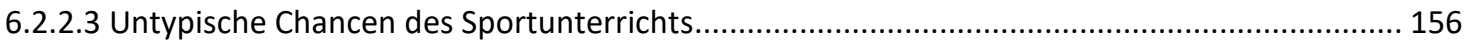

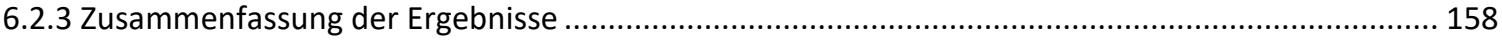

7 ZUSAMMENFASSUNG UND DISKUSSION DER ERGEBNISSE .....................................160

7.1 Zu den Lehrerüberzeugungen über Schülerinnen und Schüler mit Migrationshintergrund im Sportunterricht. .161

7.2 Zu den Lehrervorstellungen zur unterrichtlichen Umsetzung und Umsetzbarkeit interkultureller Erziehung im Sportunterricht ............................................................163

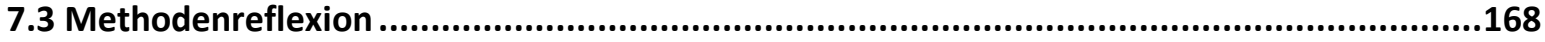

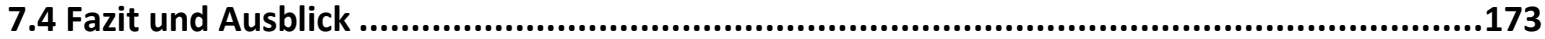

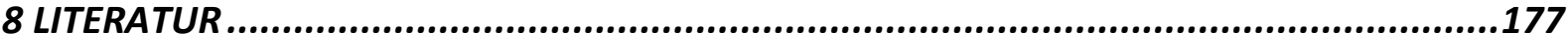

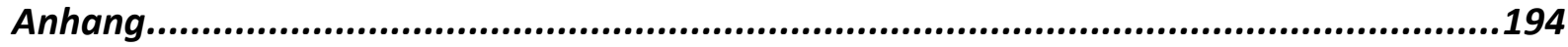

A Anfrage Schulen ........................................................................................194

B Kurzfragebogen ............................................................................................195

C Lehrberechtigung/Unterrichtserfahrung und Elementeset pro Lehrperson....................196

D Rohgrids Fragestellung 1....................................................................197

E Konstrukt-Retrievals Kategorien Fragestellung 1 ...............................................223

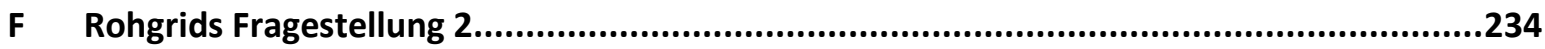

G Konstrukt-Retrievals Kategorien Fragestellung 2 ...............................................258 


\section{Abbildungsverzeichnis}

Abbildung 1. Entwicklung des Anteils ausländischer und fremdsprachiger Schülerinnen und Schüler in der obligatorischen Schule der Schweiz. Quelle: BFS (2016). Eigene Darstellung.

Abbildung 2. Aspekte professioneller Kompetenz von Lehrpersonen. Quelle: Verändert nach Baumert und Kunter (2011)

Abbildung 3. Professionelle Kompetenz als Kontinuum zwischen Disposition und Handeln. Quelle: Blömeke et al. $(2015$, S. 7).

Abbildung 4. Funktionen von Lehrerüberzeugungen. Quelle: Fives und Buehl (2012, S. 478)...

Abbildung 5. Schematische Darstellung der Hauptschritte einer Repertory Grid-Untersuchung. Quelle: Verändert nach Fromm (2010, S. 529); Rosenberger und Freitag (2009, S. 481) 68

Abbildung 6. Protokollblatt einer (fiktiven) Repertory Grid-Untersuchung in der Phase der Konstruktevokation 72

Abbildung 7. Protokollblatt einer (fiktiven) Repertory Grid-Untersuchung nach Abschluss der Datenerfassung 75

Abbildung 8. Verkleinerte Darstellung des Protokollblatts zum Interview über Schülerinnen und Schüler 85

Abbildung 9. Beispiel eines komplettierten Protokollblatts zum Interview über Schülerinnen und Schüler

Abbildung 10. Verkleinerte Darstellung des Protokollblatts zum Interview über Fächer und interkulturelles Lernen.

Abbildung 11. Beispiel eines komplettierten Protokollblatts zum Interview über Fächer und interkulturelles Lernen.

Abbildung 12. Beispiel einer Elementewahl (angekreuzte Fächer) und einer ersten Grobeinschätzung des Potenzials für interkulturelles Lernen.

Abbildung 13. Anteil Schülerinnen und Schüler, die den Idealvorstellungen der Lehrpersonen entsprechen.

Abbildung 14. Inhaltliche Verknüpfungen der Chancen-Kategorien mit gemeinsamem übergeordnetem Konstrukt. 146

Abbildung 15. Einschätzung des Fachs Sport hinsichtlich genannter Chancen interkulturellen Lernens 153

Abbildung 16. Einschätzung des Fachs Sport im Vergleich zum Fach Mathematik hinsichtlich genannter Chancen interkulturellen Lernens..... 154 


\section{Tabellenverzeichnis}

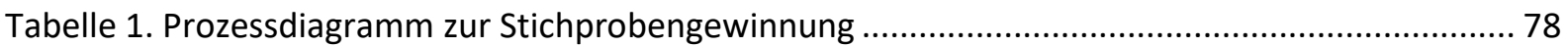

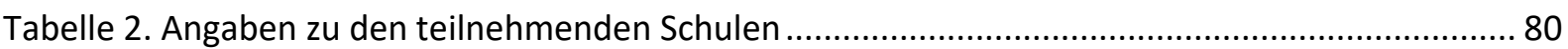

Tabelle 3. Alter, Sportunterrichtserfahrung (SU-Erfahrung), Ausbildung (Lehrerpatent, Lehrdiplom SI, Lehrdiplom SII) und Unterrichtspensum der befragten Lehrpersonen differenziert nach Stichprobe 1 $\left(n_{1}\right)$ und Stichprobe $2\left(n_{2}\right)$

Tabelle 4. Anteil fremdsprachiger SuS pro Klasse und pro Grid differenziert nach Niveaustufe der Klasse

Tabelle 5. Muttersprachen der beurteilten Schülerinnen und Schüler 106

Tabelle 6. Mittelwerte der mittleren matching scores [\%] fremdsprachiger und nicht-fremdsprachiger Schülerinnen und Schüler mit dem „Ideal“-Schüler. 107

Tabelle 7. Mittlere matching scores [\%] fremdsprachiger und nicht-fremdsprachiger SuS mit dem „Ideal"-S differenziert nach Lehrperson. 108

Tabelle 8. Kategorien und Subkategorien genannter Konstrukte. 110

Tabelle 9. Beispiele genannter Konstrukte der Kategorie „Körperlichkeit/Sportliche Leistungsfähigkeit“

Tabelle 10. Mittelwerte, Standardabweichungen und range der mittleren Anteile [\%] fremdsprachiger und nicht-fremdsprachiger Schülerinnen und Schüler, die dem präferierten Pol in der Kategorie „Körperlichkeit/Sportliche Leistungsfähigkeit“ entsprechen

Tabelle 11. Beispiele genannter Konstrukte der Kategorie „Engagement / Beteiligung“..... 115 Tabelle 12. Mittelwerte, Standardabweichungen und range der mittleren Anteile [\%] fremdsprachiger und nicht-fremdsprachiger Schülerinnen und Schüler, die dem präferierten Pol in der Kategorie „Engagement/Beteiligung“ entsprechen

Tabelle 13. Beispiele genannter Konstrukte der Kategorie „Motivation / Interesse“ 118 Tabelle 14. Mittelwerte, Standardabweichungen und range der mittleren Anteile [\%] fremdsprachiger und nicht-fremdsprachiger Schülerinnen und Schüler, die dem präferierten Pol in der Kategorie „Motivation/Interesse“ entsprechen 121

Tabelle 15. Beispiel genannter Konstrukte der Kategorie „Mit Anderen“ 122 Tabelle 16. Mittelwerte, Standardabweichungen und range der mittleren Anteile [\%] fremdsprachiger und nicht-fremdsprachiger Schülerinnen und Schüler, die dem präferierten Pol in der Kategorie „Mit Anderen" entsprechen 124

Tabelle 17. Beispiele genannter Konstrukte in der Kategorie „Verschiedenes“ 125 
Tabelle 18. Mittelwerte, Standardabweichungen und range der mittleren Anteile [\%] fremdsprachiger und nicht-fremdsprachiger Schülerinnen und Schüler, die dem präferierten Pol in den Subkategorien

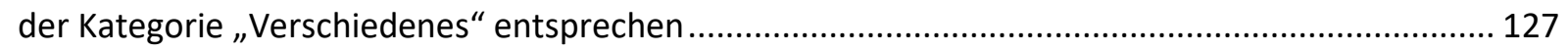

Tabelle 19. Induktive Kategorien an Chancen für interkulturelles Lernen ........................................ 131

Tabelle 20. Beispiele genannter Konstrukte der Chancen-Kategorien (1) - (4) ................................. 135

Tabelle 21. Beispiele genannter Konstrukte der Chancen-Kategorien (5) - (8) ................................ 138

Tabelle 22. Beispiel genannter Konstrukte der Chancen-Kategorien (9) - (12) ................................ 141

Tabelle 23. Beispiele genannter Konstrukte der Chancen-Kategorien (13) - (17) ............................ 144

Tabelle 24. Einschlägige Konstruktpol-Begründungen der übergeordneten Chancen (3), (1) und (12)

Tabelle 25. Einschlägige Konstruktpol-Begründungen der übergeordneten Chancen (9) und (6)

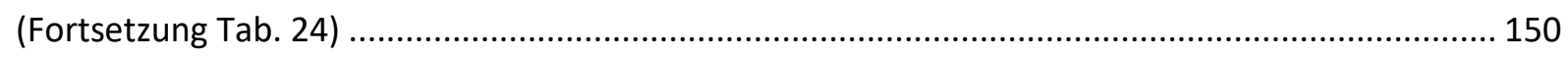

Tabelle 26. Einschlägige Chancen bzw. Konstruktpol-Begründungen der übergeordneten

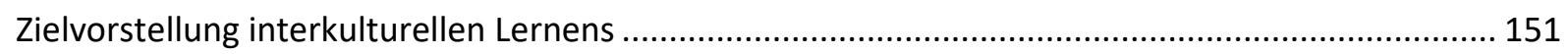




\section{Einleitung}

\subsection{Problemhintergrund und Fragestellung}

Seit der Immigration von Arbeitsmigrantinnen und -migranten während des Wirtschaftsbooms in der Nachkriegszeit gehört die Schweiz zu den modernen Einwanderungsländern. Nachdem sich zunächst insbesondere italienische und spanische Arbeitskräfte in der Schweiz niederliessen, führten vereinfachte Aufenthaltsbedingungen sowie die allgemeine Zunahme der Mobilität und des internationalen Austauschs zu einem stetigen Wachstum der Ausländerzahlen, einer geographischen Erweiterung der Herkunftsländer sowie einer Diversifizierung der Migrationsgründe (vgl. Piguet, 2006). Heute sind rund ein Viertel (24.6\%) der ständig in der Schweiz wohnhaften Bevölkerung ausländische Staatsangehörige (vgl. Bundesamt für Statistik (BFS), 2017a). ${ }^{1}$ Ein weitaus grösserer Anteil von 36\% verfügt über einen Migrationshintergrund (vgl. BFS, 2017b). ${ }^{2}$ Zu den grössten Ausländergruppen gehören Personen mit italienischer (15.2\%), deutscher (14.7\%), portugiesischer (13.1\%), französischer (6.0\%), kosovarischer (5.2\%), spanischer (4.0\%), serbischer (3.5\%), türkischer (3.4\%) und mazedonischer (2.6\%) Staatsbürgerschaft. Der Anteil ausländischer Personen, die dem Asyl- und Flüchtlingsbereich zugerechnet werden, betrug Ende 2016 2.5\% (vgl. Staatssekretariat für Migration (SEM), 2017).

Analog zum Trend einer ständig wachsenden ausländischen Wohnbevölkerung ist der durchschnittliche Anteil ausländischer Schülerinnen und Schüler in den obligatorischen Schulen der Schweiz im Zeitraum der Schuljahre $1980 / 81$ bis $2014 / 15$ kontinuierlich von $12.4 \%$ auf $26.3 \%$ angestiegen. Parallel dazu bzw. als Folge davon hat sich auch der durchschnittliche Anteil fremdsprachiger Schülerinnen und Schüler, d.h. solcher, deren Muttersprache nicht der Unterrichtssprache entspricht, von $13.9 \%$ um mehr als das Doppelte auf 30.0\% vergrössert (vgl. Abb. 1).

\footnotetext{
${ }^{1}$ Das Bundesamt für Statistik bemisst den Ausländeranteil in der Schweizer Bevölkerung anhand der ständigen ausländischen Wohnbevölkerung. Diese umfasst alle Personen mit ausländischer Staatsbürgerschaft mit einer Gesamtaufenthaltsdauer oder einer Anwesenheitsbewilligung von mindestens zwölf Monaten (vgl. BFS, 2017a). ${ }^{2}$ Nach Definition des Bundesamts für Statistik gehören zu den Personen mit Migrationshintergrund „Personen ausländischer Staatsangehörigkeit und eingebürgerte Schweizerinnen und Schweizer - mit Ausnahme der in der Schweiz Geborenen mit Eltern, die beide in der Schweiz geboren wurden - sowie die gebürtigen Schweizerinnen und Schweizer mit Eltern, die beide im Ausland geboren wurden“ (BFS, 2017b).
} 


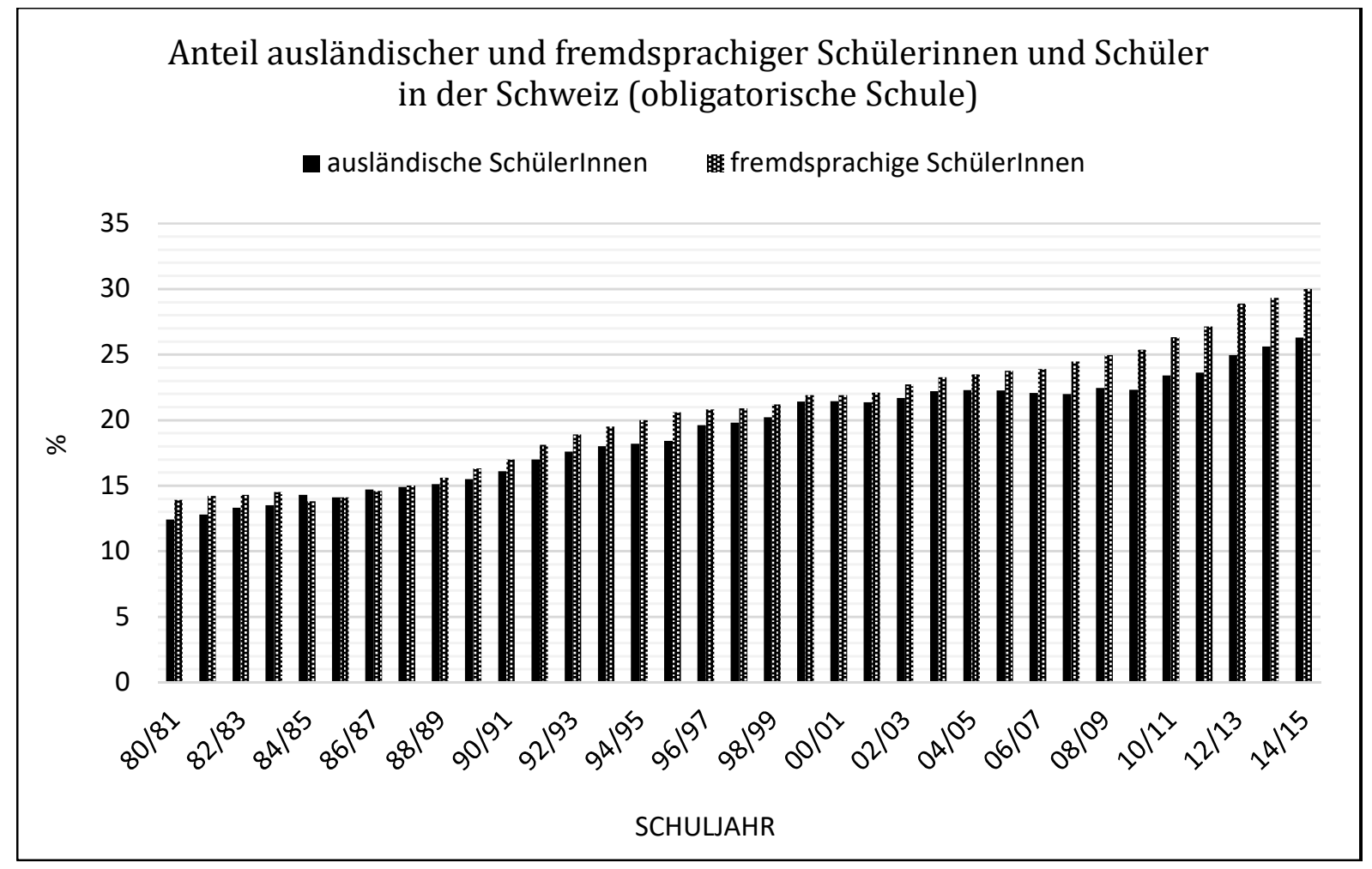

Abbildung 1. Entwicklung des Anteils ausländischer und fremdsprachiger Schülerinnen und Schüler in der obligatorischen Schule der Schweiz. Quelle: BFS (2016). Eigene Darstellung.

Die schweizerische Bildungspolitik beschäftigt sich seit Beginn dieser Entwicklung, d.h. seit den 1960er Jahre mit den Folgen von Migration für die Schulen. Unter Einfluss des erziehungswissenschaftlichen Diskurses der „Ausländerpädagogik“ wurden zunächst „Grundsätze zur Schulung der Gastarbeiterkinder" (Schweizerische Konferenz der kantonalen Erziehungsdirektoren (EDK), 1995, S. 11), später bzw. den Positionen und Praktiken der Interkulturellen Pädagogik folgend „Empfehlungen zur Schulung der fremdsprachigen Kinder" (ebd., S. 161) formuliert. Seit 1992 umfasst das Volksschulgesetz (VSG) des Kantons Bern - in dem die vorliegende Untersuchung durchgeführt wurde - zudem den sog. Integrationsartikel, der vorsieht, „Schülerinnen und Schüler, deren schulische Ausbildung (...) durch Probleme bei der sprachlichen und kulturellen Integration erschwert wird (...)“(Art. 17, VSG, BSG 432.210), wenn möglich in Regelklassen zu integrieren und zu fördern.

Ins Zentrum der bildungspolitischen und schulpädagogischen Aufmerksamkeit rückte das Thema Zuwanderung jedoch erst ab 2000. Als wesentlicher Grund dafür werden insbesondere die Ergebnisse der PISA-Studien von 2000 gesehen (z.B. Budde, 2013, S. 12), die für die Schweiz (und auch Deutschland) überdurchschnittlich hohe Zusammenhänge zwischen den Testleistungen und dem familiären Hintergrund der Schülerinnen und Schüler auswiesen (z.B. Artelt et al., 2001). Dieser gemeinhin als Defizit im Umgang mit sprachlich-kultureller Heterogenität interpretierte Befund führte in der Folge zur Forderung (seitens EDK), interkulturelle Pädagogik verbindlich in die Lehrerbildung aufzunehmen und die „Lehrpläne im Lichte der sprachlichen und kulturellen Vielfalt" zu überarbeiten (vgl. Lanfranchi, 
Perregaux \& Thommen, 2001, S. 42). Zudem wurde der schon länger formulierte Anspruch einer chancengerechten Schule, d.h. einer Schule jenseits von Stereotypen, verzerrten Erwartungen oder Diskriminierungen, wieder laut (vgl. Coradi Vellacott \& Wolter, 2005, S. 16).

Mit Blick auf die heutige Situation zeigt sich, dass Curriculuminhalte Interkultureller Pädagogik zwar zunehmend zu einem verpflichtenden Bestandteil der Lehrerausbildung in der Schweiz geworden sind, in der Konzeption und damit in der inhaltlichen Schwerpunktsetzung, aber auch in der Art der Implementierung in das Curriculum allerdings grosse Unterschiede bestehen (vgl. Buholzer, Zulliger \& Zutavern, 2015; Sieber, 2009). Unabhängig davon umfasst der jüngst erarbeitete (und für die Kantone zur Einführung freigegebene) Lehrplan 21 den Kompetenzbereich „Umgang mit Vielfalt“. Als wesentliche Neuerung gegenüber dem bis anhin im Kanton Bern gültigen Lehrplan ist Interkulturelle Erziehung nicht nur mehr als „zusätzliche Aufgabe“ (Erziehungsdirektion des Kantons (ERZ), 1995), sondern im Bereich der für alle Lehrpersonen und auf allen Stufen verbindlich aufzubauenden und zu fördernden „überfachlichen Kompetenzen“ verankert. Zu den zu erarbeitenden Schülerkompetenzen gehören dabei u.a. das Wahrnehmen- und Verstehenkönnen von „Menschen in ihren Gemeinsamkeiten und Differenzen“ oder der „respektvolle Umgang mit Menschen (...), die sich in Geschlecht, Hautfarbe, Sprache, sozialer Herkunft, Religion oder Lebensform unterscheiden“ (ERZ, 2016b, S. 16). Schliesslich entspricht die im Kanton Bern 2008 in Kraft getretene Verordnung über die besonderen Massnahmen in der Volksschule (BMV) der Umsetzung des über zehn Jahre zuvor verfassten Integrationsartikels. Darin ist festgehalten, dass „Schülerinnen und Schüler, die besonderer Massnahmen bedürfen, in der Regel die Regelklasse [besuchen]“ sollten (ERZ, 2016a, S. 26).

Auf Unterrichtsebene - und damit zum Fokus dieser Arbeit - entsprechen die beschriebenen, sich verändernden sozialen Realitäten und die damit einhergehenden bildungspolitischen Entwicklungen vor allem einem gestiegenen Anspruch an das Lehrerhandeln. Zwar sind die Forderungen nach einem chancengerechten und interkulturell erziehenden Unterricht im Grundsatz nicht neu, ihre Realisierung ist jedoch angesichts der (aufgrund kontinuierlicher Zuwanderung und der Umsetzung integrativer Schulmodelle) auf Klassenebene stark gewachsenen sprachlich-kulturellen Heterogenität deutlich schwieriger geworden. Erschwerend kommt ausserdem hinzu, dass mangels konsensfähiger didaktischer Konzeptionen und empirisch überprüfter Modelle zu den erforderlichen Kompetenzen für einen angemessenen Umgang mit heterogenen Lernvoraussetzungen der Schülerinnen und Schüler (vgl. Allemann-Ghionda, 2006; Buholzer et al., 2015) bis heute von einer unzureichenden Professionalisierung der Lehrpersonen auszugehen ist.

Tatsächlich fühlt sich denn auch die Mehrheit der Schweizer Lehrpersonen unzureichend auf den Umgang mit Heterogenität vorbereitet (vgl. Oser et al., 2010). Weitere Untersuchungen stellen ausserdem 
fest, dass viele Lehrpersonen im Umgang mit heterogenen Klassen verunsichert oder gar überfordert sind (z.B. Edelmann, 2006; Fischer, Veber, Fischer-Ontrup \& Buschmann, 2015; Hallitzky \& Schiessleder, 2008). In ihrer Studie mit Zürcher Lehrpersonen kommt Edelmann (2008, S. 60, 191) denn auch zu dem Schluss, dass eine „Dominanz der persönlichen Einstellungen“ vorherrscht und „die subjektive Interpretation“ (...) sowie das persönliche Interesse [der Lehrperson] ihren Umgang mit der migrationsbedingten Vielfalt massgeblich beeinflussen".

Da Einstellungen und Haltungen (im Folgenden: Überzeugungen) in der aktuellen Lehrer(kompetenz)forschung (z.B. Baumert \& Kunter, 2006) - und speziell in der schulpädagogischen Literatur zum Thema Heterogenität (z.B. Wischer, 2007) - eine zentrale Rolle hinsichtlich des Unterrichtshandelns zugeschrieben wird, erscheint ihre Erforschung mit Blick auf die offenkundig gewordene Diskrepanz zwischen (bildungspolitischen) Erwartungen und dem tatsächlich von Lehrpersonen realisierten Handeln bzw. auf einen professionellen Umgang mit migrationsbedingter Heterogenität von hoher Bedeutung. So stellen Lehrerüberzeugungen im Zusammenhang mit migrationsbedingter Heterogenität einen wichtigen Zugang dar, um Aufschlüsse über Herausforderungen und Chancen von Lehrpersonen im Umgang mit migrationsbedingter Heterogenität zu gewinnen.

Trotz dieser hohen Relevanz sind Forschungsbemühungen zu diesem Gegenstandsbereich jedoch bislang vergleichsweise überschaubar geblieben. Fast gänzlich unerforscht blieben dabei Lehrerüberzeugungen im Zusammenhang mit migrationsbedingter Heterogenität in einem bestimmten Fach. Dass auch das Fach Sport - mit seinen vergleichsweise fachspezifischen Bedingungen und Praktiken, aber auch aufgrund seiner hohen öffentlich-politischen Aufmerksamkeit im Zusammenhang mit migrationsbedingter Heterogenität (z.B. Jurinak, 2017) - diesbezüglich kaum in den Blick genommen wurde, erstaunt dabei umso mehr.

Vor dem Hintergrund der beschriebenen Problemlage und dem bestehenden Forschungsdesiderat beschäftigt sich die vorliegende Arbeit somit mit den Überzeugungen Sport unterrichtender Lehrpersonen angesichts migrationsbedingter Heterogenität. Folgende Fragestellung ist dabei forschungsleitend:

Welche Überzeugungen haben Sport unterrichtende Lehrpersonen angesichts der migrationsbedingten Heterogenität im Sportunterricht? 


\subsection{Ziele und Aufbau der Arbeit}

Ziel dieser Arbeit ist es, (erstes) Wissen über den Umgang Sport unterrichtender Lehrpersonen mit migrationsbedingter Heterogenität zu gewinnen. Ausgehend von der Prämisse, dass das Unterrichtshandeln von (Sport unterrichtenden) Lehrpersonen im Kontext migrationsbedingter Heterogenität zu grossen Teilen durch deren subjektive Überzeugungen geleitet ist, sollen insbesondere Erkenntnisse über diesbezügliche Denkstrukturen und -prozesse der Lehrpersonen gewonnen werden. Unter diesem - einem explorativen Forschungsansatz folgenden - Erkenntnisinteresse generiert die vorliegende Untersuchung Orientierungswissen, das etwa für die (Konzeption der) Aus- und Weiterbildung von Sport unterrichtenden Lehrpersonen genutzt werden kann. Darüber hinaus soll sie aber auch Impulse für die hinsichtlich Interkulturalität noch sehr überschaubare, sportdidaktische Theoriebildung liefern und die Thematik damit letztlich für hypothesengeleitete Untersuchungen zugänglich machen. Schliesslich soll die Arbeit methodisches Innovationspotenzial im Bereich der Lehrer(bildungs)forschung aufzeigen.

Kapitel 2 beleuchtet hierfür zunächst das Leitkonstrukt dieser Untersuchung, die Lehrerüberzeugungen (teachers' beliefs). Neben einer Auseinandersetzung mit den Kernaspekten dieses Konstrukts (in Abgrenzung zum Konstrukt des Lehrerwissens) (vgl. Kap. 2.1) und den mannigfaltigen Gegenstandsbereichen, auf die sich Lehrerüberzeugungen beziehen können (vgl. Kap. 2.2), wird insbesondere auf das Verhältnis von Lehrerüberzeugungen und Lehrerhandeln (im Diskurs um professionelle Kompetenz von Lehrpersonen) eingegangen (vgl. Kap. 2.3).

Kapitel 3 widmet sich dem aktuellen Stand der empirischen Forschung zu Lehrerüberzeugungen angesichts migrationsbedingter Heterogenität. Da bislang kaum Studien aus der (deutschsprachigen) Sportlehrerforschung vorliegen (vgl. Kap. 3.2), wurden insbesondere Arbeiten mit anderen bzw. keinen spezifischen Fachperspektiven und hierin wiederum auch Befunde aus dem nord- bzw. US-amerikanischen Raum berücksichtigt (vgl. Kap. 3.1). Diese sollen einerseits der Identifikation von Forschungslücken innerhalb der Sportlehrerforschung bzw. der Konkretisierung der untersuchungsleitenden Fragestellung, andererseits der Diskussion der Ergebnisse dienen.

Vor dem Hintergrund der erarbeiteten Forschungslücken werden in Kapitel 4 die konkreten Fragestellungen formuliert.

Kapitel 5 beschreibt die methodologischen und methodischen Aspekte dieser Arbeit. Aufgrund der geringen Standardisierung und in der Lehrerforschung noch schwachen Verbreitung des Verfahrens sowie der Tatsache, dass zwei eigens für die vorliegende Untersuchung entwickelte Instrumente verwendet wurden, fallen die Ausführungen dazu etwas ausführlicher aus. 
Das sechste Kapitel stellt die Ergebnisse der empirischen Untersuchung dar. Es gliedert sich in je ein Kapitel zu den beiden konkreten Fragestellungen und deren jeweiligen Teilfragestellungen (vgl. Kap. 6.1; Kap. 6.2).

Schliesslich werden in Kapitel 7 die konkreten Fragestellungen unter Berücksichtigung der datenbasierten Ergebnisse beantwortet und vor dem Hintergrund vorliegender Forschungsbefunde diskutiert (vgl. Kap. 7.1; Kap. 7.2). Nach einer Reflexion methodischer Aspekte (vgl. Kap. 7.3) schliesst das Kapitel mit einem übergreifenden Fazit und einem Ausblick (vgl. Kap. 7.4). 


\section{Lehrerüberzeugungen (teachers'beliefs)}

Der Beginn der Forschung zu Lehrerüberzeugungen (teachers' beliefs) lässt sich auf über 60 Jahre zurückdatieren (vgl. Ashton, 2015; Fives \& Buehl, 2012). Trotz (oder gerade aufgrund) der unzähligen Arbeiten, die in diesem Zeitraum entstanden sind, wurde jedoch bald einmal festgestellt, dass es an Konsens hinsichtlich einer expliziten Definition mangelt. So betitelte Pajares (1992) seinen Übersichtsartikel zu Teachers' Beliefs and Educational Research mit Cleaning Up a Messy Construct und bemerkte, eine Vielzahl an Alias-Konstrukten ${ }^{3}$ aufzählend: „It will not be possible for researchers to come to grips with teachers' beliefs, however, without first deciding what they wish belief to mean and how this meaning will differ from that of similar constructs" (S. 308, Hervorhebung i. O.).

Obwohl die wissenschaftliche Auseinandersetzung mit Lehrerkognitionen nach der Überwindung der behavioristischen Ansätze und unter Einfluss der kognitiven Wende ${ }^{4}$ an Intensität gewonnen hatte, scheint diese Forderung auch im deutschsprachigen Raum bis heute nicht erfüllt worden zu sein. So erklären Reusser und Pauli (2014, S. 642) in ihrem aktuellen Beitrag zu berufsbezogenen Überzeugungen im Handbuch der Forschung zum Lehrerberuf die unternommenen Klärungsversuche als gescheitert. Schliesslich identifiziert auch Skott (2015, S. 17f.) im ein Jahr später erschienen International Handbook of Research on Teachers' Beliefs den fehlenden Konsens über eine Definition des Konstrukts als eines der Hauptprobleme der (anglo-amerikanischen) Forschung zu Lehrerüberzeugungen.

Als Ursachen dieser Schwierigkeit einer konsistenten Begriffsbestimmung lassen sich im Wesentlichen unterschiedliche Forschungsfelder und -traditionen, verschiedene beteiligte Disziplinen - Richardson (1996, S. 103) zählt neben der pädagogischen Psychologie die Anthropologie, die Sozialpsychologie und die Philosophie auf - aber auch die uneinheitlichen Übersetzungen des Begriffs belief anführen (vgl. Reusser \& Pauli, 2014; Seifried, 2009). So existieren im deutschsprachigen Raum neben dem Begriff der Überzeugungen weitere Begriffe wie Subjektive Theorien (z.B. Groeben \& Scheele, 2010; Groeben, Wahl, Schlee \& Scheele, 1988), Vorstellungen (z.B. Hartinger, Kleickmann \& Hawelka, 2006; Kirchner, 2016), Sichtweisen (z.B. Büker \& Rendtorff, 2015; Heintze, Helbig, Jungbluth, Kienast \& Marburger, 1997; Seifried, 2009), Konzeptionen (z.B. Sembill \& Seifried, 2009; Trautwein, 2013), Werthaltungen (z.B. Baumert \& Kunter, 2006; Baumert \& Kunter, 2013), Haltungen (z.B.Fend, 2008;

\footnotetext{
${ }^{3}$ Pajares (1992, S. 309) zählt u.a. folgende, mit dem Begriff belief verwandte Konstrukte auf: attitudes, values, judgments, axioms, opinions, ideology, perceptions, conceptions, conceptual systems, preconceptions, dispositions, implicit theories, explicit theories, personal theories, internal mental processes, action strategies, rules of practice, practical principles, perspectives, repertories of understanding, and social strategy.

${ }^{4}$ Als kognitive Wende wird gemeinhin der Paradigmenwechsel vom Behaviorismus zum Kognitivismus in der Psychologie verstanden. In der Forschung zu Lehrpersonen findet der Begriff insbesondere in Zusammenhang mit der ab ca. 1985 intensiveren wissenschaftlichen Auseinandersetzung mit den Lehrerkognitionen Verwendung (vgl. Bromme, 1992, S. 4; Krauss \& Bruckmaier, 2014; vgl. auch Kap. 2.3).
} 
Kratzmann \& Pohlmann-Rother, 2012), Einstellungen (z.B. Sievers, 2009; Zick \& Six, 1997) oder Orientierungen (z.B. Marburger, Helbig \& Kienast, 1997) (vgl. auch Reusser \& Pauli, 2014).

Insbesondere aufgrund der erstarkenden empirischen Lehrerbildungsforschung, in der Überzeugungen als Aspekt der professionellen Kompetenz mitberücksichtigt werden (vgl. Kap. 2.3.1), scheint sich der Begriff in den letzten Jahren als allgemeiner Leitbegriff jedoch durchgesetzt zu haben (vgl. Reusser \& Pauli, 2014). Studien wie MT21 (vgl. Blömeke, Kaiser \& Lehmann, 2008), TEDS-M (z.B. Blömeke, 2012) oder COACTIV (vgl. Baumert \& Kunter, 2011) bestimmen den Begriff dabei insbesondere in Abgrenzung zu Wissen. Damit schliessen sie an die zentralen, in der pädagogisch-psychologischen Literatur identifizierbaren Diskussionslinien zur Charakterisierung von Lehrerüberzeugungen an, indem der epistemologische Status, die affektive und evaluative Aufladung sowie die Herausbildung und mentale Verankerung von Überzeugungen in den Mittelpunkt der Betrachtung gestellt werden.

Mangels einer allgemein anerkannten Definition von Lehrerüberzeugungen wird im Folgenden anhand dieser Diskussionslinien versucht, die Kernaspekte von Lehrerüberzeugungen (in Abgrenzung zu Lehrerwissen) herauszuschälen. Eine Zusammenfassung davon findet sich schliesslich in Kapitel 2.4.

\subsection{Lehrerüberzeugungen vs. Lehrerwissen}

\subsubsection{Epistemologischer Status}

Die Diskussion um den epistemologischen Status betrifft insbesondere die Frage nach den Bedingungen einer Rechtfertigung, die die Legitimation dafür liefern, eine Aussage als Überzeugung oder aber als Wissen zu taxieren.

Während für Epistemologen Wissen nur dann Wissen ist, wenn es evident bzw. bewiesen ist (vgl. Chisholm, 1989, S. 16), scheint in der Forschung zum Lehrerwissen eher ein moderaterer Standard zu bestehen. Green (1971, S. 99f.) etwa spricht bereits von (Lehrer-)Wissen, wenn eine (Lehrer-) Überzeugung intersubjektiv bzw. objektiv für wahr gehalten wird. Ähnlich äussert sich auch Fenstermacher (1994): „A proposition is known by its holder if the holder believes the proposition and has evidence to establish its reasonableness in relation to other, competing claims" (S. 24).

Für Überzeugungen hingegen gelten niedrigere Rechtfertigungsansprüche (vgl. Calderhead, 1996; Richardson, 1996). So reicht nach Baumert und Kunter (2006, S. 497) der „individuelle Richtigkeitsglaube“, wobei Überzeugungen im Unterschied zu Wissen „weder den Kriterien der Widerspruchsfreiheit noch den Anforderungen der argumentativen Rechtfertigung und der diskursiven Validierung zu genügen" haben. 
Abgesehen von den unterschiedlichen Rechtfertigungsansprüchen zwischen Wissen und Überzeugungen existiert bezüglich des epistemologischen Status der beiden Konstrukte jedoch kein exakteres Kriterium, das eine trennscharfe Unterscheidung zulassen würde. ${ }^{5}$ In Beiträgen und Übersichtsartikeln zu Lehrerwissen und -überzeugungen wird denn auch von „generally overlapping constructs“ (Woolfolk Hoy, Davis \& Pape, 2006, S. 716) oder von sich zwei gegenseitig umfassenden Konstrukten gesprochen (vgl. auch Reusser \& Pauli, 2014, S. 643). Mit der Begründung, dass Wissen als nichts anderes als eine objektiv für wahr gehaltene Überzeugung verstanden werden kann, meint Kagan (1992, S. 73) etwa: "Most of a teacher's professional knowledge can be regarded more accurately as belief". Überzeugungen hingegen dem Wissen subsumierend, äussern sich Alexander, Schallert und Hare (1991, S. 317): „Knowledge encompasses all that a person knows or believes to be true, whether or not it is verified as true in some sort of objective or external way".

Ob eine Überzeugung als Wissen oder Wissen nur in Form einer Überzeugung existieren kann, ist, wie Philipp (2007) treffend schildert, letzten Endes eine philosophische Frage: „If one takes the ontological view that truth exists and people have access to it, the knowledge might be viewed as true belief. If one takes a view that truth, though it may exist, is not accessible to humans and instead the best one can hope for is viability, then knowledge is belief with certainty" (S. 268).

Sich der Schwierigkeit einer kategorialen Grenzziehung zwischen Überzeugungen und Wissen bewusst, distanzieren sich Reusser und Pauli (2014) in ihrem Beitrag zu berufsbezogenen Überzeugungen von Lehrpersonen denn auch von einer Kontrastierung mit Wissen auf epistemologischer Ebene.

\subsubsection{Affektive und evaluative Aufladung}

Eine weitere Diskussionslinie mit Fokus auf der Unterscheidung zwischen Wissen und Überzeugungen beruht auf der Meinung, dass Überzeugungen mentale Konstrukte darstellen, die im Gegensatz zu Wissen (stärker) affektiv aufgeladen und wertgebundener sind.

So stellt etwa Abelson (1979), Unterschiede zwischen Wissens- und Überzeugungssystemen identifizierend, fest: „Beliefs systems rely heavily on evaluative and affective components“ (S. 358). Während in Wissenssystemen enthaltene Wissensbestände gewissermassen eine emotional neutrale Funktion im System einnehmen, würden Überzeugungssysteme hingegen eine Vielzahl an Vorstellungen aufweisen, die subjektiv als „gut“ oder "schlecht“ bewertet sind. Ähnlich argumentiert auch Pajares (1992, S. 310), der Überzeugungen im Gegensatz zu Wissen als weniger „pure“ bzw. emotional stärker aufge-

\footnotetext{
${ }^{5}$ Dies ist nicht zuletzt darauf zurück zu führen, dass auch über den Wissensbegriff bzw. den epistemologischen Status der verschiedenen Wissenstypen keine Einigung besteht (vgl. auch Baumert \& Kunter, 2011, S. 33; Trautmann \& Wischer, 2011, S. 39).
} 
laden und wertend und daher näher bei der Wahr- oder Falschheit einer Gegebenheit liegend betrachtet. Aufgrund dieser Charakterisierung von Überzeugungen als evaluativ-normativ streicht (Abelson, 1979) auch den motivationalen Aspekt von Überzeugungen hervor (vgl. auch Kap. 2.3.2).

Weitere Autoren verwenden den Affektgehalt von Lehrerüberzeugungen als Kriterium zur feineren Abgrenzung zu Lehrerhaltungen und -emotionen. So ordnet McLoed (1992, S. 576f.) Haltungen und Überzeugungen von (Mathematik-)Lehrpersonen zwar beide dem „affektiven Bereich“ der Lehrerkognitionen zu, begreift Überzeugungen gegenüber Haltungen jedoch als das schwächer durch affektive Komponenten gefärbte Konstrukt. Haltungen charakterisiert er als weniger kognitiv, dafür stärker „gefühlt" und dadurch weniger stabil und sich schneller verändernd. Ähnlich beurteilt auch Goldin (2002, S. 64) Überzeugungen, indem er sie zwar als „cognitive/affective configurations“ bezeichnet, jedoch mit einem - im Vergleich zu Emotionen und Haltungen - höheren Anteil an „cognitive elements“ konzeptualisert.

In welchem Verhältnis die kognitiven und affektiven Komponenten bzw. Elemente in Lehrerüberzeugungen (und anderen mentalen Konstrukten bzw. Wissensformen von Lehrpersonen) letztlich vorkommen, darüber fehlt bis heute ein Konsens (vgl. Reusser \& Pauli, 2014). Als unbestritten gilt hingegen, dass es weder kognitive noch affektive Konstrukte in absoluter Form gibt. Denn nach Hascher und Krapp (2014, S. 681) ist „die in alltagssprachlichen Interpretationen häufig anzutreffende Gegenüberstellung Emotion versus Kognition (...) wissenschaftlich nicht gerechtfertigt. Vielmehr ist von engen wechselseitigen Abhängigkeiten auszugehen“. Dementsprechend stellt auch Pajares (1992, S. 310) die Frage: „What truth, what knowledge, can exist in the absence of judgement or evaluation?”.

Mit Blick in die Forschungspraxis bleibt festzuhalten, dass in Arbeiten, in denen der Aspekt der affektiven Aufladung und Wertgebundenheit von Überzeugungen besonderes gewichtet wird, anstelle von Überzeugung die Begriffe der Einstellung oder (Wert-)Haltung verwendet werden (z.B. Kratzmann \& Pohlmann-Rother, 2012; Sievers, 2009).

\subsubsection{Herausbildung und mentale Verankerung}

Die Diskussionslinie zur Aneignung und mentalen Verankerung von Wissen und Überzeugungen folgt den Fragen nach dem Zustandekommen und der Art der Repräsentation von Überzeugungen und Wissen.

Die Ursprünge von Lehrerüberzeugungen werden nach allgemeiner Auffassung zwar auch in der formalen Ausbildung und beruflichen Erfahrungen, vielmehr jedoch in der persönlichen Lebensgeschichte und in den eigenen Schulerfahrungen gesehen (vgl. Kunter \& PohImann, 2015; Richardson, 1996; Skott, 2015). Insbesondere letzteren wird dabei eine zentrale Bedeutung zugesprochen. Die tausenden von Schulstunden, die eine Lehrperson selber erlebt hat, so Lortie (1975) oder auch (Dirks, 2000), würden 
Überzeugungen von Lehrpersonen deutlich stärker prägen als die viel später in der Biographie einer Lehrperson stattfindende formale Lehrerausbildungsphase. Zum Zeitpunkt des Studienbeginns, so die Meinung, hätten angehende Lehrpersonen, durch das was Lortie $(1975$, S. 61) „apprenticeship of observation“ nennt, somit bereits fest etablierte professionsbezogene Überzeugungen ${ }^{6}$ (vgl. Abelson, 1979; Nespor, 1987; Pajares, 1992).

Entsprechend dieser Vorstellung gelten Lehrerüberzeugungen als stark erfahrungsbasierte Kognitionen. ${ }^{7}$ In Bezug auf die mentale Verankerung unterscheidet Nespor $(1987$, S. 320) Überzeugungen und Wissen folglich dadurch, dass Wissen vorwiegend in semantischen Netzwerken (,semantic networks"), Überzeugungen hingegen vielmehr entlang bestimmter Episoden („episodic storage“) memorisiert sind . Gleichzeitig wird aber auch davon ausgegangen, dass Überzeugungen mehr oder weniger theorieförmig vorliegen können. Insbesondere unter Verwendung des Begriffs der Subjektiven Theorien (z.B. Groeben et al., 1988) werden Überzeugungen in Analogie zu wissenschaftlichen Theorien deshalb auch als Kognitionen mit „quasi-logischer Struktur" aufgefasst (vgl. Reusser \& Pauli, 2014, S. 644).

Vor diesem Hintergrund werden Überzeugungen denn auch nicht in isolierter, sondern in mental verwobener Konfiguration bzw. als in Überzeugungssysteme eingebunden verstanden: „It is important to think in terms of connections among beliefs instead of in terms of beliefs as independent subsystems" (Pajares, 1992, S. 327). Dabei wird davon ausgegangen, dass gewisse Überzeugungen zentraler im System liegen als andere. Während Rokeach (1989) die Zentralität durch die Stärke der funktionalen Beziehung zu anderen Überzeugungen definiert, gelten für Green (1971) diejenigen Überzeugungen konzentrisch betrachtet als primary beliefs, die nicht von anderen Überzeugungen abgeleitet werden können und in ihrer Genese meist weit zurückliegen. Oser und Blömeke (2012) unterscheiden ausserdem zwischen tiefer verankerten „normativ getränkten beliefs, die auf ein moralisches oder funktionales Muss gerichtet sind“ und weniger tief verankerten „deskriptiven beliefs“ bzw. „deskriptiven Analysen (z.B. Mathematik ist ein Fach, in dem angeborene Fähigkeiten viel wichtiger sind als Anstrengungen)“ (S. 417). Dabei gilt: Je zentraler, vernetzter oder tiefer die Stellung der Überzeugung im System, desto resistenter ist sie gegenüber Veränderung (vgl. Calderhead, 1996; Pajares, 1992; Reusser \& Pauli, 2014; Rokeach, 1989; Wischmeier, 2012). ${ }^{8}$

\footnotetext{
${ }^{6}$ Haben verschiedene Lehrpersonen ähnliche Erfahrungen gemacht und überschneiden sich deshalb manche ihrer Überzeugungen, so spricht man auch von shared beliefs (vgl. Steinmann \& Oser, 2012).

${ }^{7}$ Die Grenzen zu ebenfalls erfahrungsbasierten, in der Literatur zum Lehrerwissen erwähnten Wissensformen wie practical knowledge, case knowledge, strategic knowledge oder craft knowledge (vgl. Calderhead, 1996; Fenstermacher, 1994; Elbaz, 1983; zit. n. Munby, Russell \& Martin, 2001, S. 885; Neuweg, 2014; Shulman, 1986) bleiben hier unscharf.

${ }^{8}$ Da Überzeugungen charakteristischerweise subjektive Bewertungen beinhalten und daher potenziell auf falschen Annahmen beruhen können (vgl. Kap. 2.1.2), gilt die formelle Veränderung bzw. die Ausbildung wissenschaftlich abgestützter Überzeugungen deshalb als zentrale Herausforderung der Lehrerbildung (vgl. Kunter \& Pohlmann, 2015). Um Lehrpersonen sich ihrer Überzeugungen bewusst zu machen, wird dabei insbesondere auf den conceptual change-Ansatz zurückgegriffen (vgl. Kunter \& Pohlmann, 2015; Reusser \& Pauli, 2014).
} 
Schliesslich haben Überzeugungen nach allgemeiner Auffassung einen hohen impliziten Anteil und sind deshalb für ihre Trägerinnen und Träger nur beschränkt bewusstheitsfähig (vgl. Bromme, 1992; Hillier, 1998; Kane, Sandretto \& Heath, 2002; Pajares, 1992; Reusser \& Pauli, 2014; Richardson, 1996). Eine Erklärung dafür bietet Neuweg (2014), der die Dimension der Repräsentation von Wissen mit der Dimension des Lernwegs in Verbindung bringt. Demnach sind Kognitionen insbesondere dann implizit repräsentiert, wenn sie entweder bereits implizit gelernt wurden oder wenn sie zwar explizit gelernt wurden, danach jedoch „nicht mehr oder nur mehr eingeschränkt verbalisierbar“ sind (z.B. gut trainierte Fertigkeiten oder verinnerlichte Theorien) (ebd., S. 601). Damit sind nicht nur Verbindungen zur oben angeführten, lebensgeschichtlichen und damit informellen Aneignung, sondern auch zur vielfach postulierten - und im Vergleich zu formal gelerntem Wissen grossen - Handlungsnähe von Überzeugungen, etwa als impliziten theories-in-use (vgl. Argyris \& Schön, 1974) aufgezeigt (vgl. auch Kap. 2.3).

\subsection{Gegenstandsbereiche von Lehrerüberzeugungen}

Lehrerüberzeugungen weisen stets einen Gegenstandsbezug (Fachinhalt, Schülerinnen und Schüler, bildungspolitische Themen usw.) auf (vgl. Reusser \& Pauli, 2014, S. 644). Dieser wird in aktuellen Überblicksartikeln und -beiträgen oft als Klassifikationskriterium der vielseitigen, bislang unternommenen Forschung zu Lehrerüberzeugungen herangezogen. So unterscheiden Reusser und Pauli (2014) zwischen epistemologischen, personenbezogenen und kontextbezogenen Überzeugungen, Kunter und Pohlmann (2015) in Anlehnung an Woolfolk Hoy et al. (2006) zwischen Überzeugungen, die das Selbst, den Lehr-Lern-Kontext, das Bildungssystem oder die Gesellschaft betreffen. ${ }^{9}$ Obwohl sich die beiden Klassifikationsvorschläge terminologisch offensichtlich nicht überschneiden, lassen sie sich auf inhaltlicher Ebene dennoch zusammenführen. Dies gilt insofern, als sich epistemologische Überzeugungen gemäss Reusser und Pauli (2014) auf „Inhalte und Prozesse des Wissens, Erkennens, Lehrens und Lernens in einem disziplinär-fachlichen oder fachübergreifenden Sinne“ (S. 650) und damit auf den LehrLern-Kontext, personenbezogene Überzeugungen auf die „professionsbezogene Selbstwahrnehmung von Lehrkräften (...), aber auch deren schülerbezogene (...) Überzeugungen“ (ebd.) und damit sowohl auf die Kategorien Selbst und den (die Schülerinnen und Schüler umfassenden) Lehr-Lern-Kontext und kontextbezogene Überzeugungen schliesslich auf Merkmale des „engeren (einzelschulischen) und weiteren (gesellschaftlichen) Kontextes" (ebd., S. 651) der Tätigkeit der Lehrpersonen und damit auf die Kategorien Bildungssystem und Gesellschaft beziehen.

Die folgenden Ausführungen entsprechen somit einer integrativen Zusammenfassung der von Reusser und Pauli (2014) sowie Kunter und Pohlmann (2015) beschriebenen Gegenstandsbereiche von

\footnotetext{
${ }^{9}$ Weitere Klassifikationen von Überzeugungen stammen u.a. von Calderhead (1996), Fives und Buehl (2012) sowie von Blömeke, Kaiser, et al. (2008). Letztere wurde spezifisch mit Bezug zum Fach Mathematik entwickelt. 
Lehrerüberzeugungen. Da die Klassifikationsversion von Reusser und Pauli (2014) m. E. zumindest terminologisch weniger eindeutig ist, erfolgt die Beschreibung entlang der Klassifikation von Kunter und Pohlmann (2015). Dabei werden die von den beiden Autorengruppen exemplarisch erwähnten, untersuchten Gegenstände durch weitere einschlägige Themen und Inhalte, auf die sich untersuchte Überzeugungen bislang bezogen haben, ergänzt.

Überzeugungen mit Bezug zum Selbst betreffen Vorstellungen zur eigenen Identität oder über die eigenen Fähigkeiten. Untersucht wurden etwa die Wahrnehmung der Lehrerrolle (z.B. Müller, Felbrich \& Blömeke, 2008) oder Selbstwirksamkeitsüberzeugungen der Lehrpersonen (z.B. Tschannen-Moran \& Hoy, 2001).

Überzeugungen mit Bezug zum Lehr-Lern-Kontext betreffen den „unmittelbaren Wirkungskontext“ der Lehrpersonen (Kunter \& PohImann, 2015, S. 267). Dazu gehören Überzeugungen über das Lehren und Lernen, über das Fach sowie über Schülerinnen und Schüler.

Überzeugungen über das Lehren und Lernen wurden sowohl fachspezifisch (z.B. Aguirre \& Speer, 2000; Staub \& Stern, 2002; Voss, Kleickmann, Kunter \& Hachfeld, 2011) als auch fachübergreifend (z.B. Kember, 1997; Schlichter, 2012) untersucht. Wiederholt unter die Lupe genommen wurde dabei insbesondere der Unterschied zwischen konstruktivistischen und rezeptiv-transmissiven lerntheoretischen Überzeugungen oder auch deren Zusammenhang mit epistemologischen Überzeugungen, das heisst solchen Überzeugungen, die sich auf die Struktur und Genese von Wissen beziehen (z.B. Voss et al., 2011). Darüber hinaus finden sich auch Untersuchungen zu Überzeugungen über die Verwendung bestimmter Methoden (z.B. Ruys, Van Keer \& Aeltermann, 2014; zit. n. Wilde \& Kunter, 2016) oder zu Fachüberzeugungen im Sinne von Bromme's (1992) Philosophie des Schulfachs, d.h. zu „Auffassungen darüber, wofür der Fachinhalt nützlich ist und in welcher Beziehung (...) [er] zu anderen Bereichen des menschlichen Lebens und Wissens steht" (S. 97; z.B. Seifried, 2009).

Überzeugungen über Schülerinnen und Schüler sind schliesslich insbesondere unter den Stichworten der Erwartungseffekte (z.B. Pygmalion-Effekt) und der impliziten Persönlichkeitstheorien thematisiert worden (vgl. Kunter \& Pohlmann, 2015, S. 269; Reusser \& Pauli, 2014, S. 651). Einschlägige Arbeiten beschäftigten sich dabei mit Schülerwahrnehmungen und -kategorisierungen sowie mit Attributionen für Schülerinnen- und Schülerleistungen (z.B. Dresel, Steuer \& Berner, 2010; Seifried, 2009; Ziegler, Kuhn \& Heller, 1998).

Überzeugungen, die sich auf das Bildungssystem beziehen, haben bildungspolitische Themen, Standards oder Reformen zum Gegenstand (vgl. Kunter \& Pohlmann, 2015, S. 267). So untersuchten etwa Müller et al. (2008) schul- und professionstheoretische Überzeugungen zu den Aufgaben von Lehrpersonen und den Inhalten der Lehrerbildung. Woolfolk Hoy et al. (2006) erwähnen ausserdem Studien zu teachers' beliefs hinsichtlich der Heterogenität von Kindern und Jugendlichen bzw. wie Schulen damit umgehen sollen. 
Dem Gegenstandsbereich Gesellschaft subsumieren Kunter und Pohlmann (2015) schliesslich von kulturspezifischen Normen und Werten geprägte Überzeugungen, welche Bildung und Schule betreffen. So wurden in der oben erwähnten Studie von Müller et al. (2008) etwa auch die Überzeugungen zur (gesellschaftlichen) Funktion der Schule (Sozialisations- vs. Selektionsfunktion) untersucht.

\subsection{Lehrerüberzeugungen und Lehrerhandeln}

\subsubsection{Lehrerüberzeugungen als Aspekt professioneller Kompetenz}

Das vorangegangene Kapitel hat gezeigt, dass die Erforschung von Lehrerüberzeugungen ein sehr breites Spektrum an Inhalten, auf die sich die Überzeugungen beziehen, abdeckt. Dies hat nicht zuletzt damit zu tun, dass Lehrerüberzeugungen seit jeher als wichtiges Antezedens für das Berufshandeln von Lehrpersonen betrachtet werden. Seitdem man vor über 70 Jahren damit begonnen hat, sich mit der Frage nach dem „guten Lehrer" bzw. dem „guten Unterricht“ auseinanderzusetzen, beschäftigen deshalb auch Fragen zu Überzeugungen von Lehrpersonen. ${ }^{10}$

Der Beginn einer intensiveren, wissenschaftlichen Auseinandersetzung mit Lehrerüberzeugungen wird insbesondere mit der dritten Auflage des 1986 publizierten Handbook of Research on Teaching in Verbindung gebracht (vgl. Ashton, 2015; Bromme, 1992). Ein neues Kapitel über „teachers' thought processes" mit einem Abschnitt zu "teachers" theories and beliefs" steht dort stellvertretend für die in diesem Zeitraum vermehrt durchgeführten empirischen Untersuchungen zu den Kognitionen der Lehrpersonen. Entscheidungen und Handlungen von Lehrpersonen wurden dabei - in Anlehnung an Erkenntnisse aus der kognitionspsychologischen Expertiseforschung - fortan als abhängige Variable von (wissensgeleiteter) Wahrnehmung und Interpretation betrachtet (vgl. Bromme, 2008, S. 160; vgl. Krauss \& Bruckmaier, 2014). Ein „Expertenlehrer“ bzw. eine erfolgreiche Lehrperson, so wurde festgestellt, würde Problemsituationen anders wahrnehmen und interpretieren als eine Lehrperson mit geringerer Expertise (vgl. Bromme, 2008, S. 160).

Um die lehrerseitigen Voraussetzungen für „guten“ Unterricht zu identifizieren, wurde in der Folge versucht, die Inhalte und Strukturen des sogenannten professionellen Wissens von (erfolgreichen) Lehrpersonen zu definieren. Eine zentrale Rolle spielten hierbei die Arbeiten von Shulman (1986; 1987), der auf Basis einer theoretischen Anforderungsanalyse eine Taxonomie des Lehrerwissens entwickelte. Dieser später von Bromme (1992) aufgegriffene und erweiterte Vorschlag fand insbesondere mit den Wissenskategorien content knowledge (Fachwissen), pedagogical knowledge (pädagogisches

\footnotetext{
${ }^{10}$ So wurden, wie Ashton (2015) in seinem historischen Überblick über die Lehrerüberzeugungsforschung aufzeigt, Lehrerüberzeugungen bereits in den 1940er und -50er Jahren in Form einzelner Items von Lehrerpersönlichkeitstests erfasst. Später, unter Einfluss des Behaviorismus, wurden kognitive Konstrukte wie Überzeugungen jedoch nur noch marginal untersucht (vgl. ebd.). 
Wissen) und pedagogical content knowledge (fachdidaktische Wissen) Eingang in aktuelle, theoretische Modelle zur professionellen (Handlungs-)Kompetenz von Lehrpersonen (vgl. Baumert \& Kunter, 2006; Blömeke, Kaiser, et al., 2008).

Diesen Modellen liegt - in Anlehnung an Bromme (1992; 1997) und das Kompetenzverständnis von Weinert (2001) - die Auffassung zugrunde, „dass Wissen nur dann eine handlungsleitende Funktion erhält, wenn es in den subjektiven Überzeugungsbestand von Lehrerinnen und Lehrern übernommen wird“ (vgl. Blömeke, Müller, Felbrich \& Kaiser, 2008, S. 219; vgl. auch Baumert \& Kunter, 2011, S. 31). Professionelle Kompetenz von Lehrpersonen wird demnach als ein Zusammenspiel von einerseits professionellem Wissen (Professionswissen), andererseits Überzeugungen/Werthaltungen sowie - zumindest im bislang am meisten aufgegriffenen Kompetenzmodell von Baumert und Kunter - motivationalen Orientierungen und selbstregulativen Fähigkeiten verstanden und modelliert (vgl. Baumert \& Kunter, 2011, S. 32; Kunter \& Trautwein, 2013, S. 144).

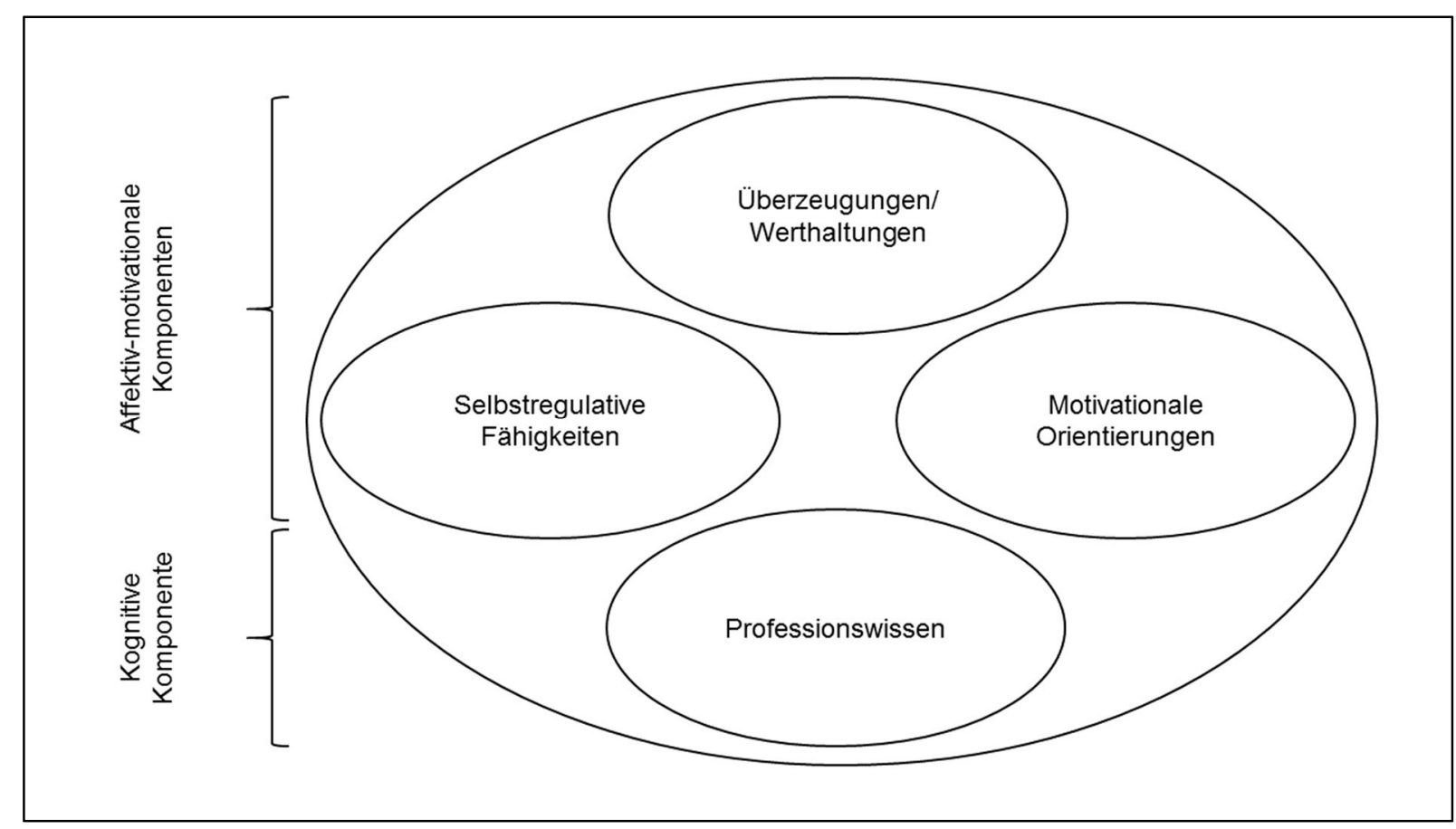

Abbildung 2. Aspekte professioneller Kompetenz von Lehrpersonen. Quelle: Verändert nach Baumert und Kunter (2011) 
Das in Fachwissen, pädagogisches Wissen und fachdidaktisches Wissen aufgegliederte Professionswissen wird dabei meist als kognitive, letztere drei Aspekte (Überzeugungen/Werthaltungen, motivationale Orientierungen, selbstregulative Fähigkeiten) als affektiv-motivationale Komponenten ${ }^{11}$ betrachtet (vgl. Blömeke, Gustafsson \& Shavelson, 2015; Blömeke, Suhl \& Döhrmann, 2012; König, 2016) (vgl. Abb. 2). ${ }^{12}$

\footnotetext{
${ }^{11}$ Die Binnenstrukturen der affektiv-motivationalen Komponenten werden im Gegensatz zu derjenigen des Professionswissens uneinheitlich modelliert. Ursache dafür dürfte der (noch) nicht hinreichend präzisierte Begriff der Überzeugungen sein. So modellieren etwa Blömeke, Müller, et al. (2008) die „motivationalen, volitionalen und sozialen Bereitschaften und Fähigkeiten“ (Weinert, 2001, S. 27-28) „mangels einer präzisen Begrifflichkeit und hinreichend trennscharfer Abgrenzung zu anderen Konstrukten (...) übergreifend als Überzeugung " (S. 219220) und bestimmen damit den Begriff insbesondere in Abgrenzung zu Wissen. Ob vor dem Hintergrund eines bis heute fehlenden Konsens über den Begriff „Überzeugungen“ weitere Abgrenzungen zu motivationalen Orientierung und selbstregulativen Fähigkeiten - denen ihrerseits wiederum Kontroll- und Selbstwirksamkeitsüberzeugungen subsumiert werden (vgl. Baumert \& Kunter, 2011) - sinnvoll sind, bleibt zumindest fraglich.

${ }^{12}$ Wie in Kapitel 2.1.2 dargelegt, ist eine trennscharfe Unterscheidung zwischen affektiv-motivationalen und kognitiven Konstrukten sowohl theoretisch als auch empirisch nicht gerechtfertigt. Vielmehr ist von gegenseitigen mehr oder weniger stark affektiv aufgeladenen Konstrukten auszugehen. Die in Modellen professioneller Kompetenz von Lehrpersonen vorgenommene Unterscheidung hat demnach lediglich einen analytisch-heuristischen Zweck. Ausgehend von der Annahme, dass die dem Handeln zugrundeliegenden Dispositionen (Komponenten) lern- bzw. veränderbar sind, soll die Ausdifferenzierung in einzelne Konstrukte eine gezielte Kompetenzentwicklung ermöglichen (vgl. auch Blömeke et al., 2015).
} 


\subsubsection{Funktionen (Wirkungsweisen) von Lehrerüberzeugungen}

Je nach Anforderungssituation bzw. erfolgreich zu bewältigender Aufgabe sowie in Abhängigkeit der bei einer Lehrperson zur Verfügung stehenden Dispositionen (Komponenten), so die Annahme, ist das „Muster des Zusammenspiels“ (Blömeke et al., 2012, S. 423) der verschiedenen Komponenten unterschiedlich: „Cognition, affect-motivation, and performance are linked as a system, cobbled together in response to task demands, somewhat differently for each person" (Blömeke et al., 2015, S. 6). Wahrnehmung, Interpretation und Entscheidungsbildung stellen dabei die Prozesse dar, die verbindend zwischen den kognitiven und affektiv-motivationalen Dispositionen und dem von aussen beobachtbaren Handeln liegen (ebd., S. 7) (vgl. Abb. 3).

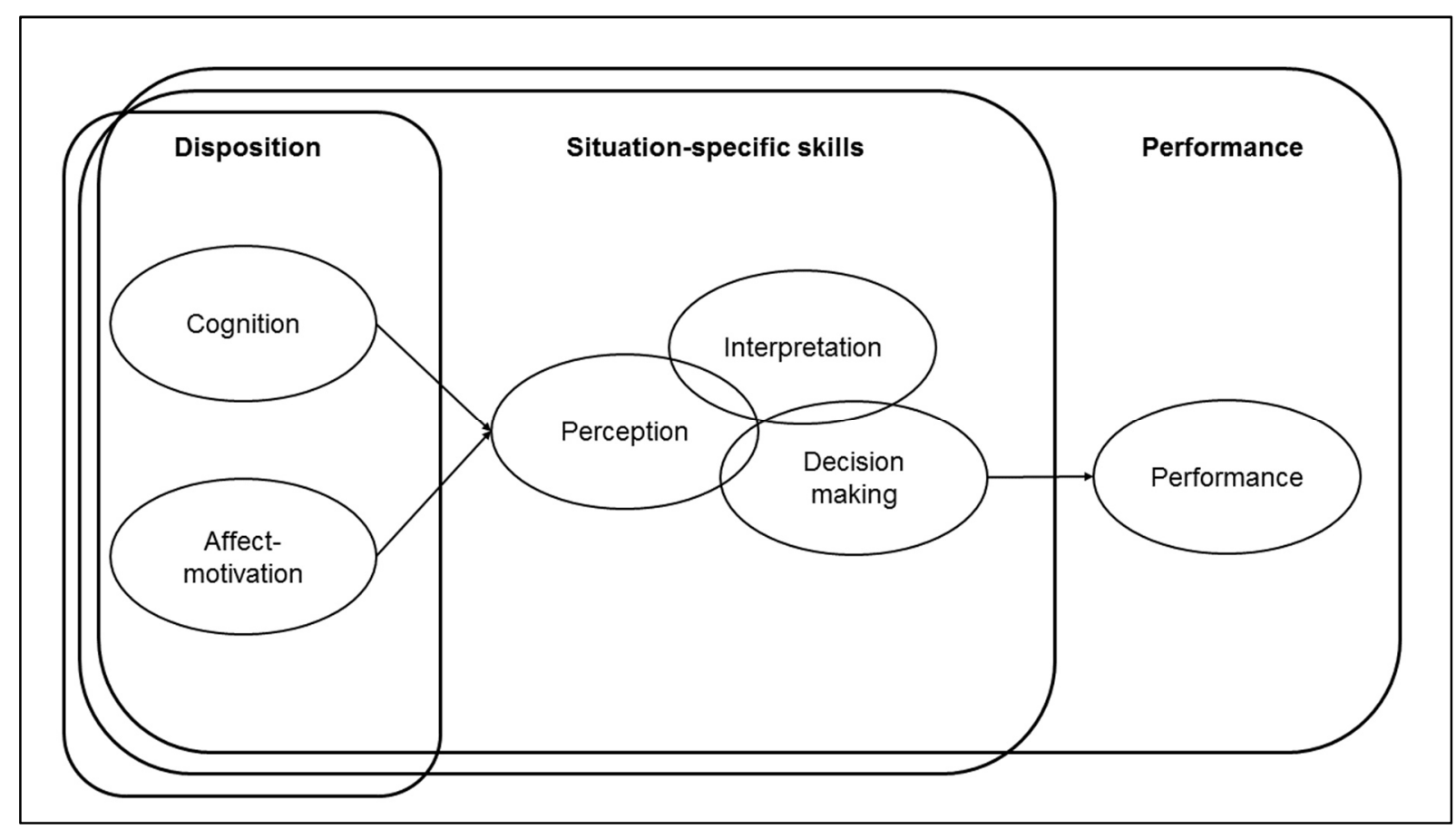

Abbildung 3. Professionelle Kompetenz als Kontinuum zwischen Disposition und Handeln. Quelle: Blömeke et al. (2015, S. 7)

Damit angesprochen sind letztlich die Funktionen, welche die zur (erfolgreichen) Bewältigung einer Berufssituation eingesetzten kognitiven und affektiv-motivationalen Ressourcen haben. Bezogen auf die hier interessierenden affektiv-motivationalen Ressourcen bzw. Überzeugungen der Lehrpersonen stellt sich dabei die Frage, wie sich diese hinsichtlich des Handelns der Lehrpersonen in spezifischen Situationen entfalten bzw. wie sie wirken.

Fives und Buehl (2012) greifen die zentralen in der Literatur besprochenen Wirkungsweisen von Lehrerüberzeugungen auf, indem sie - ähnlich zu den von Blömeke et al. (2015) genannten, zwischen 
Dispositionen und Berufshandeln liegenden Prozessen - zwischen einer Filter-, einer Frame- und einer Guide-Funktion von Lehrerüberzeugungen unterscheiden (vgl. Abb. 5). ${ }^{13}$

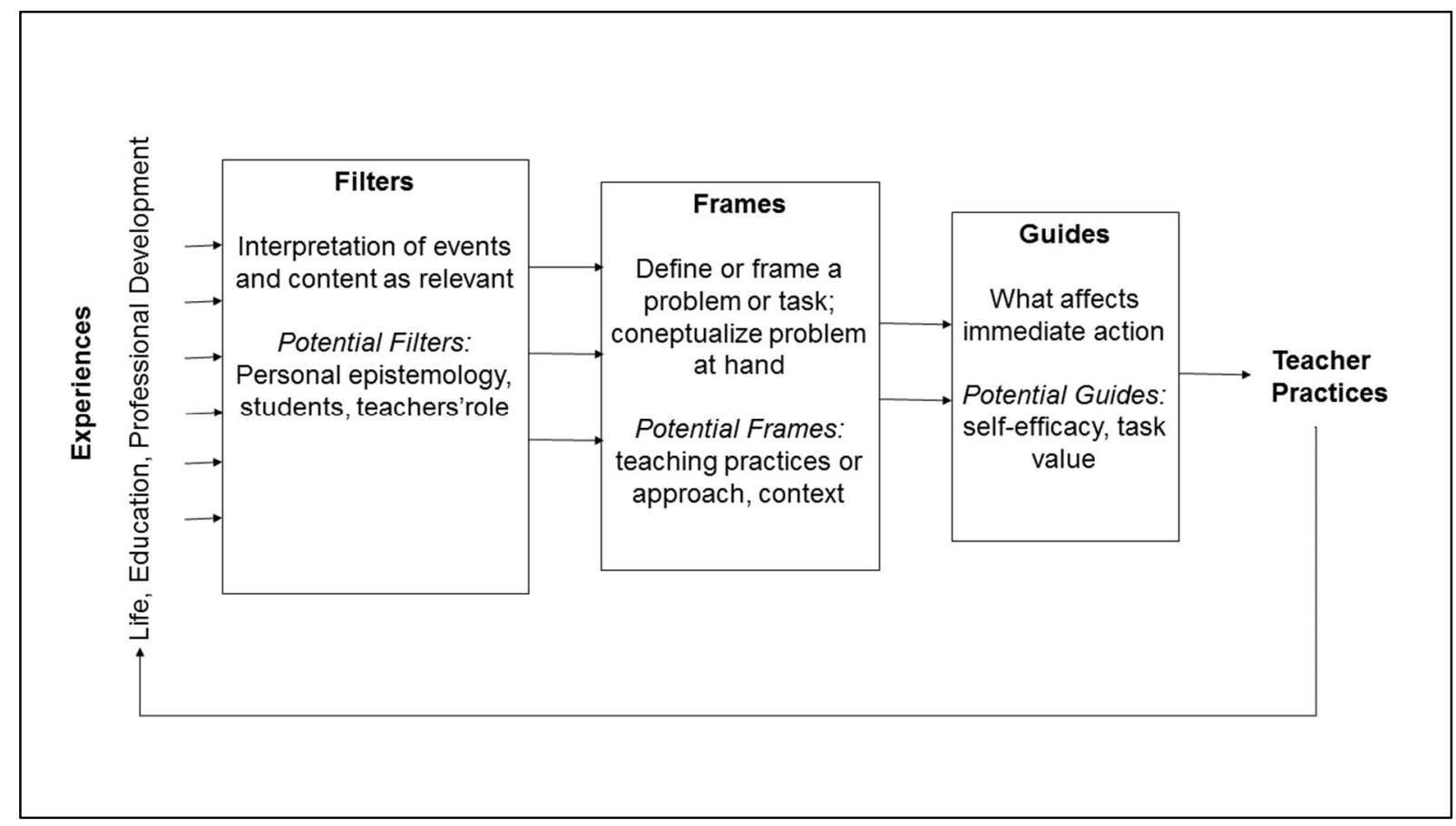

Abbildung 4. Funktionen von Lehrerüberzeugungen. Quelle: Fives und Buehl (2012, S. 478)

Ausgehend von biographischen, ausbildungsbezogenen und berufspraktischen Erfahrungen wirken Überzeugungen zunächst dahingehend als Filter, dass sie die Wahrnehmung und Interpretation von Ereignissen und Informationen beeinflussen. Mit anderen Worten: Eine Lehrperson nimmt die Realität stets durch die Brille ihrer individuellen Überzeugungen (z.B. über Schülerinnen und Schüler oder über ihre Rolle als Lehrperson) wahr (vgl. Fives \& Buehl, 2012, S. 478). Die Unterscheidung zwischen relevanten und irrelevanten Informationen geschieht dabei eher intuitiv, weshalb manche Autoren die Funktion von Überzeugungen auch mit dem auf Bourdieu (1974) zurückgehenden Konzept des Habitus vergleichen. So würde der Praxis von Lehrpersonen ein Habitus als „ein System verinnerlichter Muster“ zugrunde liegen, über das berufstypische Situationen „quasi unbewusst und automatisch“ wahrgenommen und interpretiert werden (vgl. Reusser \& Pauli, 2014, S. 445f.; vgl. auch Gates, 2006).

Ebenso ähnlich zum Habitus-Konzept steht die Frame-Funktion von Überzeugungen. Sind die relevanten Informationen erst einmal gefiltert, so muss ein vorliegendes Problem bzw. eine Aufgabe einge-

\footnotetext{
${ }^{13}$ In ähnlicher Weise unterscheiden Kunter und PohImann (2015) in Anlehnung an Pajares (1992) zwischen einem Filtereffekt, einem Motivierenden Effekt und einem Steuerungseffekt von Überzeugungen. 
ordnet bzw. genauer definiert werden. Da Probleme bzw. Aufgaben, mit denen Lehrpersonen typischerweise konfrontiert sind, überaus komplex und (deshalb) „ill-defined“"14 (Nespor, 1987, S. 324) sind, erlauben Überzeugungen als (im Unterschied zum Professionswissen) nicht zwingend argumentationsfähige Strukturen (vgl. Kap. 2.1.1) eine - u. U. rasche - Bewertung und Einordnung einer solchen Situation. Dies ist nicht zuletzt deshalb von Bedeutung, weil Unterrichten für Lehrpersonen gemeinhin als ein Entscheiden und Handeln unter Zeitdruck betrachtet wird (vgl. Dann, 2008, S. 182).

Schliesslich können Lehrerüberzeugungen auch als Guides betrachtet werden, indem sie motivierend bzw. lenkend hinsichtlich einer unmittelbaren Handlung wirken. Wird beispielsweise eine Unterrichtsmethode als "gut" oder "schlecht" bewertet, so wird sich dies unmittelbar auf die Unterrichtsgestaltung auswirken. Ausserdem können Selbstwirksamkeitsüberzeugungen von Lehrpersonen einen Einfluss auf die Anstrengungsbereitschaft und die Ziele, die (sich) Lehrpersonen setzen, haben (vgl. Fives \& Buehl, 2012, S. 479).

Abbildung 4 suggeriert, dass Lehrerüberzeugungen in Abhängigkeit ihrer Funktion mehr oder weniger nahe beim realisierten Handeln einer Lehrperson liegen. Entsprechend dürfte eine Überzeugung, von der - im Kontext einer spezifisch zu bewältigenden Aufgabe - primär eine Filter-Funktion angenommen wird, gegenüber einer Überzeugung, die im selben Kontext eine unmittelbar lenkende Funktion einnimmt, schwieriger im Unterrichtshandeln einer Lehrperson zu identifizieren sein. Vor diesem Hintergrund bemerken Buehl und Beck (2015, S. 79), dass im Hinblick auf die empirische Erfassung und die Interpretation der Handlungswirksamkeit von Lehrerüberzeugungen stets die Funktion und damit die Stellung, welche die spezifische Überzeugung hinsichtlich des Handelns ausfüllt, mitbedacht werden sollte.

\subsubsection{Effekte von Lehrerüberzeugungen}

Ob und welche Effekte Lehrerüberzeugungen auf das Handeln der Lehrpersonen und darüber hinaus auf die Schülerinnen- und Schülerleistungen haben, gehört zu den zentralen Fragestellungen der Forschung zu Lehrerüberzeugungen (vgl. Reusser \& Pauli, 2014).

Bislang am häufigsten untersucht wurden die Auswirkungen lerntheoretischer Überzeugungen auf die Unterrichtsgestaltung. Ausgehend von der Unterscheidung zwischen Überzeugungen, die tendenziell an konstruktivistischen Lerntheorien orientiert sind, und solchen, die stärker auf transmissiven Lerntheorien basieren, lassen sich die Befunde insgesamt dahingehend interpretieren, dass sich erstere

\footnotetext{
${ }^{14}$ Insbesondere im Zusammenhang mit der Messung der Kompetenzen von Lehrpersonen wird der Lehrerberuf gemeinhin als „schlechtdefinierte Domäne“ beschrieben, weil Lehrpersonen - im Gegensatz zum häufig in diesem Zusammenhang aufgegriffenen Schachspieler - „in der Regel mit komplexen Aufgaben konfrontiert [sind], die aus vielen kleinen Teilproblemen bestehen, für die es oft keine eindeutigen Lösungen gibt" (Krauss \& Bruckmaier, 2014, S. 247).
} 
eher positiv (letztere negativ) auf Qualitätsmerkmale von Unterricht (kognitive Aktivierung, konstruktive Unterstützung, Freiräume für Schülerinnen und Schüler, Strukturiertheit des Unterrichts, Gruppenarbeiten etc.) auswirken (vgl. Dubberke, Kunter, McElvany, Brunner \& Baumert, 2008; Hartinger et al., 2006; Seifried, 2012; Staub \& Stern, 2002; Voss et al., 2011). ${ }^{15}$

Weitere Arbeiten, in denen Effekte von Überzeugungen auf Unterrichtsmerkmale festgestellt werden konnten, betreffen Überzeugungen zum Thema „Sitzenbleiben“ bzw. Wiederholen einer Klasse (retention) (vgl. König, Darge \& Schreiber, 2012), zum Einsatz bestimmter Lehr-Lern-Methoden (vgl. Seifried, 2009), zu Feedback (vgl. Brown, Harris \& Harnett, 2012; zit. n. Buehl \& Beck, 2015), zur Selbstwirksamkeit (vgl. Schwarzer \& Warner, 2014) oder zu Leistungen von Schülerinnen und Schülern (z.B. Jussim \& Harber, 2005; Kronig, 2007; vgl. auch Kap. 3.1.2).

Untersuchungen zu Wirkungen von Lehrerüberzeugungen auf Schülerinnen- und Schülerleistungen erfassten letztere entweder direkt und/oder mediiert über die Unterrichtsgestaltung. Zu den Studien, die die Lehrerüberzeugungen direkt zu ermitteln versucht haben, gehören die Studien von Peterson, Fennema, Carpenter und Loef (1989), Gilleece (2012), Dubberke et al. (2008) sowie Voss et al. (2011). In allen vier Arbeiten konnte festgestellt werden, dass Schülerinnen und Schüler von Lehrpersonen mit konstruktivistischen Überzeugungen höhere (Mathematik-)Leistungen erbringen als Schülerinnen und Schüler von Lehrpersonen mit weniger konstruktivistisch orientierten bzw. eher transmissiven Überzeugungen. In den Studien von Dubberke et al. (2008), Staub und Stern (2002) sowie Voss et al. (2011) konnte darüber hinaus bestätigt werden, dass die mit diesen Überzeugungen zusammenhängenden Unterrichtsmerkmale (vgl. Ausführungen weiter oben) als Mediator hinsichtlich dieser Schülerinnenund Schülerleistungen wirken. Weitere Arbeiten, in welchen die Wirkungskette Lehrerüberzeugungen - Unterrichtsmerkmale - Unterrichtswirkungen untersucht wurde, stellten Zusammenhänge zwischen der Lesemotivation der Schülerinnen und Schüler und den im Unterricht angewandten Leseaktivitäten bzw. den diesen prädiktiv vorgelagerten Lehrerüberzeugungen zur Wichtigkeit des Lesens fest (vgl. Fives \& Buehl, 2012, S. 480).

Trotz empirischer Evidenzen zur Wirkung von Überzeugungen auf den Unterricht finden sich auch Studien, in denen Inkongruenzen zwischen Lehrerüberzeugungen und dem Handeln von Lehrpersonen festgestellt wurden. So berichten etwa Aguirre und Speer (2000) von Lehrpersonen, die der Überzeugung waren, dass im Mathematikunterricht Gruppenarbeiten (collaborative learning beliefs) stattfinden sollten, in ihrem (beobachteten) Unterricht aber dennoch keine Gruppenarbeiten anregten. In einer anderen Studie wurde festgestellt, dass die Mehrheit der Lehrpersonen trotz schülerorientierter

\footnotetext{
${ }^{15}$ Dubberke et al. (2008) und Voss et al. (2011) erfassten die Qualitätsmerkmale des Unterrichts mittels Schülerinnen- und Schülereinschätzungen, Hartinger et al. (2006) sowie Staub und Stern (2002) (zusätzlich) mittels Unterrichtsbeobachtungen und Seifried (2012) mittels Selbsteinschätzung.
} 
Überzeugungen (learner-centered beliefs) ihren Unterricht primär als Frontalunterricht (lecturing) gestalteten (vgl. Liu, 2011).

Buehl und Beck (2015) bringen in ihrem Handbuch-Beitrag zum Zusammenhang zwischen Lehrerüberzeugungen und Lehrerhandeln verschiedene Erklärungen für solche Inkongruenzen an: Erstens scheinen Inkongruenzen insbesondere dann vorzuliegen, wenn die Überzeugungen noch kaum durch Praxiserfahrungen reflektiert wurden. Der Unterricht von unerfahreneren Lehrpersonen, so stellen sie bezugnehmend auf empirische Untersuchungen fest, widerspricht dementsprechend häufiger den Überzeugungen als bei erfahreneren Lehrpersonen. Zweitens sind Überzeugungen, die im Hinblick auf eine bestimmte Unterrichtshandlung weniger eine lenkende, sondern vielmehr eine filternde oder bewertende Funktion einnehmen, im realisierten Unterrichtshandeln durch Beobachtung schwieriger zu identifizieren (vgl. Kap. 2.3.2). Schliesslich und drittens können unterschiedliche internale und externale Faktoren die Umsetzung von Überzeugungen im Unterrichtshandeln erschweren oder gar verhindern. So fassen Buehl und Beck (2015, S. 73f.) verschiedene empirische Studien zusammen, die darauf hinweisen, dass andere Überzeugungen (z.B. Selbstwirksamkeitsüberzeugungen) sowie verschiedene Kontextfaktoren (z.B. Klassengrösse, Motivation der Schülerinnen und Schüler, Unterstützung im Kollegium, curriculare Vorgaben) die Lehrpersonen daran hindern können, entsprechend ihren Überzeugungen zu unterrichten.

\subsection{Zusammenfassung}

Die vorangegangenen Ausführungen haben gezeigt, dass die Untersuchung von Lehrerüberzeugungen auf eine lange Forschungstradition zurückblickt. Denn seitdem Lehrerkognitionen interessieren, stehen neben verschiedenen objektivierten Wissensformen insbesondere auch jene Formen von Kognitionen im Fokus, die vergleichsweise subjektiv geprägt sind. Obwohl in der Forschungspraxis nach wie vor unterschiedliche Begriffe dafür verwendet werden (u.a. Subjektive Theorien, Werthaltungen, Einstellungen), hat sich der Begriff der Lehrerüberzeugungen (teachers' beliefs) als Leitbegriff für solche mentalen Prozesse durchgesetzt. Folgende Merkmale zeichnen sie (abgrenzend zu Lehrerwissen) aus:

- Niedrige oder keine Anforderungen an die Widerspruchsfreiheit, Wahrheitsfähigkeit und Rechtfertigungsfähigkeit

- Hohe affektive Aufladung und evaluativ-normativer Charakter

- Stabile Aneignung durch primär individuelle Sozialisation und lebensgeschichtliche (Schul-)Erfahrungen, aber auch durch Ausbildung und Berufshandeln

- Mentale Verankerung entlang von Episoden, teilweise auch theorieförmig mit „quasi-logischer Struktur", sowie Verwobenheit in Überzeugungssystemen mit zentralen und peripheren Subsystemen 
- Hoher impliziter Anteil (aufgrund informeller Aneignung) und grosse Handlungsnähe im Sinne von theories-in-use

Für die Annahme, dass solche Lehrerüberzeugungen das berufsbezogene Denken und Handeln wesentlich beeinflussen, bestehen heute empirische Evidenzen. In der aktuellen Forschung zur professionellen Kompetenz von Lehrpersonen gelten Lehrerüberzeugungen neben dem Professionswissen (und weiteren Aspekten wie motivationalen Orientierungen und selbstregulativen Fähigkeiten) deshalb als zentrales Antezedens der Qualität des Berufshandelns von Lehrpersonen. Professionelle Kompetenz von Lehrpersonen wird dabei als ein Zusammenspiel der verschiedenen lehrerseitigen Antezedenzien verstanden und modelliert. Je nach (erfolgreich) zu bewältigender Anforderung und in Abhängigkeit der bei einer Lehrperson dafür vorliegenden Ressourcen, so die Annahme, nehmen Lehrerüberzeugungen unterschiedliche Funktionen ein. Unterschieden wird zwischen einer Informationen filternden, einer Situationen bewertenden bzw. beurteilenden und einer unmittelbar Lehrerhandlungen leitenden Funktion. Als Gegenstandsbereiche von Überzeugungen gelten dabei nicht nur der Lehr-LernKontext (Überzeugungen über Lehr-Lernprozesse, über Lernende und Lerninhalte), sondern auch das Selbst der Lehrperson (Überzeugungen zur Lehrerrolle und Selbstwirksamkeit), das Bildungssystem (Überzeugungen über Inhalte der Lehrerbildung und Aufgaben von Lehrpersonen) sowie der gesellschaftliche Kontext (Überzeugungen über die Funktionen der Schule). 


\section{Lehrerüberzeugungen angesichts migrationsbedingter Heterogeni-}

\section{tät}

Dieses Kapitel stellt den aktuellen Stand der empirischen Forschung zu Lehrerüberzeugungen im Zusammenhang mit migrationsbedingt heterogenen Schulen bzw. Klassen dar. Bislang hat sich weder eine einheitliche Terminologie noch ein allgemein akzeptiertes Klassifikationssystem für derartige Überzeugungen durchgesetzt. Zu den verwendeten Begrifflichkeiten gehören u.a. teachers' beliefs about multiculturalism (vgl. Acquah \& Commins, 2013; Moore \& Reeves-Kazelskis, 1992; Petty \& Narayan, 2012), teacher cultural models (vgl. Bryan \& Atwater, 2002), teachers' beliefs about cultural diversity (vgl. Gay, 2015), teachers' views of cultural diversity (vgl. Castro, 2010), Kulturelle Überzeugungen (vgl. Hachfeld, 2013) oder Akkulturationseinstellungen bzw. teachers' acculturation attitudes (vgl. Grimminger, 2012; Makarova \& Herzog, 2013; Van Dick, Wagner, Adams \& Petzel, 1997). Die inhaltliche Ausdifferenzierung bzw. die Klassifizierung der erfassten Überzeugungen wird dabei entweder in Anlehnung an sozial- bzw. kulturpsychologische Modelle (z.B. Van Dick et al., 1997) oder durch Entwicklung induktiver Kategorien (z.B. Bender-Szymanski, 2001) vorgenommen. Hinzu kommen Studien, die sich auf eine allgemeine Beschreibung der erfassten Überzeugungen beschränken (z.B.Marburger et al., 1997).

Untersuchungen aus der (deutschsprachigen) Sportlehrerforschung liegen bislang nur wenige vor. Kleindienst-Cachay (2016) bemerkt dementsprechend: „Der Zweig der sportpädagogischen Forschung, der sich mit Interkulturalität und Migration beschäftigt, hat sich zwar intensiv mit Fragen des pädagogischen Sollens beschäftigt, also mit normativen Fragen, aber bisher kaum - mit wenigen Ausnahmen - mit dem pädagogischen Sein, d.h. mit der alltäglichen Konstruktion migrationsbedingter Differenzen (..)“ (S. 188, Hervorh. i. O.).

Vor diesem Hintergrund wurde entschieden, zunächst empirische Ergebnisse (zum pädagogischen Sein) ausserhalb der Sportlehrerforschung, d. h. Arbeiten mit anderen bzw. - und das betrifft die Mehrheit der Studien - keinen spezifischen Fachperspektiven und hierin wiederum auch Befunde aus dem nord- bzw. US-amerikanischen Raum zu berichten (vgl. Kap. 3.1). Diese sollen einerseits der Identifizierung von Forschungslücken innerhalb der Sportlehrerforschung bzw. der Formulierung konkreter Fragestellungen, andererseits der Interpretation und Diskussion der Ergebnisse dienen. Aus der vergleichsweise grossen Zahl an Forschungsarbeiten liessen sich dabei drei zentrale Gegenstandsbereiche herauskristallisieren, unter die sich die Untersuchungen subsumieren lassen. Es sind dies:

- Akkulturationseinstellung (acculturation attitudes) und Ideologien (ideologies) im Umgang mit migrationsbedingter Heterogenität (vgl. Kap. 3.1.1)

- Überzeugungen über Schülerinnen und Schüler mit Migrationshintergrund (vgl. Kap. 3.1.2) 
- Vorstellungen zur unterrichtlichen Umsetzung und Umsetzbarkeit interkultureller Erziehung (vgl. Kap. 3.1.3)

Die wenigen empirischen Ergebnisse aus der Sportlehrerforschung werden schliesslich erst in Kapitel 3.2 resümiert. Abschliessend stellt Kapitel 3.3 eine im Hinblick auf die Identifikation von Forschungslücken bilanzierende Zusammenfassung des gesamten Forschungsstandes dar.

\subsection{Empirische Ergebnisse ausserhalb der Sportlehrerforschung}

3.1.1 Akkulturationseinstellungen und Ideologien im Umgang mit migrationsbedingter Heterogenität

Die Begriffe Akkulturationseinstellungen (acculturation attitudes) und Ideologien (ideologies) gehen auf sozial- bzw. kulturpsychologische Modelle zu sozialen Prozessen im (nationalstaatlichen) Zusammenleben verschiedener kultureller Gruppen zurück. Dabei wird angenommen, dass „individuelle Einstellungen der Mitglieder der verschiedenen Gruppen zu Art und Zielen der Koexistenz" die Qualität des Zusammenlebens unmittelbar beeinflussen (vgl. Van Dick et al., 1997, S. 83). Übertragen auf die Lehrerforschung wird davon ausgegangen, dass sich Akkulturationseinstellungen und Ideologien von Lehrpersonen auf deren Unterrichtshandeln bzw. deren Bewältigungsstrategien im Umgang mit migrationsbedingter Heterogenität auswirken. In den bislang zur Erforschung solcher Einstellungen und Ideologien von Lehrpersonen herangezogenen Modellen wird zwischen den Akkulturationsvarianten Assimilation, Integration/Multikulturalismus, Segregation/Separation, Marginalisierung und Egalitarismus unterschieden (vgl. Berry, Kim, Power, Young \& Bujaki, 1989; Bourhis, Moïse, Perreault \& Senécal, 1997; Hahn, Judd \& Park, 2010). Assimilation steht dabei allgemein für die Aufgabe der Herkunftskultur zugunsten der Mehrheitskultur, Integration/Multikulturalismus für die Beibehaltung der Herkunftskultur bei gleichzeitiger Annahme (von Aspekten) der Mehrheitskultur, Segregation/Separation für die Beibehaltung der Herkunftskultur und fehlenden Kontakt zur Mehrheitskultur, Marginalisierung für die Aufgabe der Herkunftskultur ohne Annahme einer neuen Kultur und Egalitarismus schliesslich - und im Gegensatz zu den vorangegangenen Varianten - für eine Vorstellung des Zusammenlebens auf Basis universeller bzw. kulturunabhängiger Werte ${ }^{16} .{ }^{17}$

\footnotetext{
${ }^{16}$ An dieser Stelle sei vorweggenommen, dass die Begriffsführung im Zusammenhang mit dieser letzten Akkulturationsvariante nicht einheitlich ist. Genauere Angaben dazu bzw. zum für diese Arbeit herangezogenen Begriffsverständnis finden sich in Kapitel 3.1.1.2.

${ }^{17}$ Für die hier naheliegende Annahme, dass Lehrereinstellungen im Sinne dieser Akkulturationsvarianten nicht losgelöst vom jeweiligen gesellschafts- bzw. bildungspolitischen und historischen Kontext zu betrachten sind (vgl. auch Plaut, 2010), liefert u.a. Sievers (2009) empirische Argumente. So finden sich denn auch offensichtliche Parallelen zwischen Akkulturationseinstellungen und in der interkulturellen Pädagogik - ebenso vor dem Hinter34
} 
Wenngleich der Mehrheit der im Folgenden referierten Untersuchungen eine explizite Anbindung an diese theoretischen Modelle fehlt ${ }^{18}$, so lassen sich die eben skizzierten Akkulturationsvarianten bzw. Ideologien im Umgang mit migrationsbedingter Heterogenität dennoch als konzeptioneller Rahmen zur systematischen Darstellung der zu diesem Gegenstandsbereich vorliegenden Befunde heranziehen. In der Lehrerforschung (hypothesenprüfend) untersucht und auch in explorativen Studien bislang hauptsächlich identifiziert, wurden Überzeugungen von Lehrpersonen im Sinne der Varianten AssimiIation (im Folgenden: assimilative Überzeugungen), Egalitarismus (im Folgenden: egalitäre Überzeugungen) und Integration/Multikulturalismus (im Folgenden: integrativ-multikulturelle Überzeugungen). ${ }^{19}$

\subsubsection{Studien zu assimilativen Überzeugungen}

Assimilative Überzeugungen lassen sich durch eine generelle Orientierung an der Mehrheitskultur kennzeichnen. Lehrpersonen, die assimilative Überzeugungen vertreten, richten ihre Unterrichtsziele und -inhalte dementsprechend an der gesellschaftlichen Mehrheit aus. Daraus resultieren oft defizitorientierte Sichtweisen auf Schülerinnen und Schüler mit Migrationshintergrund und eine allgemeine Aufforderung zur Assimilation. Wird diese nicht erfüllt, können daraus wiederum Überzeugungen im Sinne der Akkulturationsvariante Segregation/Separation resultieren.

Eine ältere Studie, in der solche Überzeugungen zum Ausdruck kamen, ist die Untersuchung von Marburger et al. (1997), die $n=40$ Berliner Grundschul-Lehrpersonen zu deren Sichtweisen und Orientierungen zur Multiethnizität der bundesdeutschen Gesellschaft und den Konsequenzen für Schulen und Unterricht befragten. Als zentrales Ergebnis identifizieren die Autoren eine Sichtweise, die besagt, dass „nichtdeutsche SchülerInnen den hiesigen Normen unterliegen, an hiesigen Anforderungen gemessen und nach ihnen beurteilt werden“ (S. 45) und kommen weiter zum Schluss: „Massgeblich für alle SchülerInnen sind ungeachtet unterschiedlicher herkunftsbedingter Voraussetzungen, Möglichkeiten und Optionen im hiesigen Schulsystem allein Ziel- und Inhaltsvorgaben der Mehrheitsgesellschaft" (S. 45f.).

grund unterschiedlicher gesellschaftspolitischer Positionen bzw. Phasen - thematisierten Konzepten interkultureller Erziehung, wie etwa der Assimilationspädagogik (vgl. Nieke, 2008; Nohl, 2010; Prengel, 2006), dem pädagogischen Universalismus (vgl. Prengel, 2006) oder der multicultural education (vgl. Banks \& McGee Banks, 2004). ${ }^{18}$ Ausnahmen bilden die Untersuchungen von Van Dick et al. (1997), Wagner, Van Dick, Petzel und Auernheimer (2001), Hachfeld (2013), Grimminger (2009) sowie Makarova und Herzog (2013).

${ }^{19}$ Wie Van Dick et al. (1997) im Rahmen der Entwicklung und Evaluation eines Fragebogens zu den Akkulturationseinstellungen von Lehrpersonen aufzeigen konnten, korreliert die Variante Segregation/Separation hoch mit der Variante Assimilation. Die Autorinnen und Autoren erklären diesen Befund damit, dass erstere Variante gewissermassen der Konsequenz einer nicht gerecht gewordenen Aufforderung zur Assimilation entspricht. Die Variante Marginalisierung wurde bislang lediglich in der Studie von Makarova und Herzog (2013) operationalisiert und erfasst. Dabei favorisierte nur eine von $n=225$ befragten Lehrpersonen diese Variante. 
Darüber hinaus dokumentieren die Autorinnen und Autoren eine „infantilisierende Sicht- und Umgangsweise" (S. 52) auf bzw. mit nichtdeutschen Eltern und fassen verschiedene, diesbezüglich einschlägige Aussagen mit einer „Einschätzung von nichtdeutschen Eltern als defizitär, rückständig und unmündig" (S. 51) zusammen.

Ebenso dem assimilativen Überzeugungstyp zuordenbar ist die von Bender-Szymanski (2001, S. 81f., Hervorhebungen i. O.) bei Studienreferendaren in einer qualitativen Längsschnittstudie festgestellte, „ethnoorientierte Verarbeitung kulturbezogener Erfahrungen“. Im Gegensatz zum ebenfalls identifizierten und für die Mehrheit der insgesamt 20 interviewten angehenden Lehrpersonen geltenden „synergieorientierten Verarbeitungsmodus" (vgl. auch Kap. 3.1.1.3) sehen ethnoorientierte Referendare „Defizite in den intellektuellen Fähigkeiten“, eine „unangemessene Planung der eigenen Migration“, „mangelnde Fähigkeiten zur Sozialisation“ sowie „Defizite in der psychischen Verfassung" als Ursachen für Kulturkonflikte in der Schule. Ein schulisches und gesellschaftliches Zusammenleben sei dabei nur dann möglich, „wenn die fremdkulturell orientierten Schüler und ihre Eltern ihr Realitätsmodell änderten, die Normen und Regeln der Residenzkultur achteten und sich an sie anpassten“.

Zu ähnlichen Resultaten gelangt Sievers (2009) in ihrer international vergleichenden Studie zu den Einstellungen deutscher und französischer Lehrkräfte zu Heterogenität im Unterricht. So schreiben manche der von ihr befragten Lehrpersonen die schlechteren Deutschkenntnisse von Schülerinnen und Schülern mit Migrationshintergrund deren „mangelnder Denkfähigkeit“ zu (S. 96).

Eine defizitorientierte Sicht mancher Lehrpersonen zeigte sich auch in der qualitativen Untersuchung von Lanfranchi (2013; vgl. auch Lanfranchi, 2002), der Strategien des Umgangs mit der migrationsbedingten Vielfalt im Kindergarten und in der Schule untersuchte. So finden sich in seiner Typologie von Lehrpersonen im Umgang mit Verschiedenheit zwei Typen von Lehrpersonen bzw. „Interkulturalitätsstrategien“, bei denen es „im Wesentlichen um die Alternativen Assimilation oder Segregation“ mit dem Ziel der Herstellung von „Unterrichtsfähigkeit“ von Schülerinnen und Schülern mit Migrationshintergrund geht (ebd., S. 243). Während für Lehrpersonen des Typs „mit geringer Lebensweltbezogenheit anpassungsfordernd und ausgrenzungswillig" die Maxime: „Integration durch ein vorgängiges Lernen des für die Schule notwendigen Regelsystems sowie des Spracherwerbs“ (S. 241, Hervorhebungen i.O.) lautet, sprechen sich Lehrpersonen des Typs „zwischen Gleichmacherei und Stereotypisierung schwankend“ ebenso pauschal für die Bearbeitung von „,Lücken'“ bzw. „'vorhandenen Mängel[n] ““ bei Schülerinnen und Schülern mit Migrationshintergrund an vor- oder nebengeordneten Einrichtungen aus oder aber tendieren dazu, die Bedeutung kultureller Unterschiede zu leugnen (S. 242, Hervorhebungen i.O.; vgl. auch Kap. 3.1.1.2).

Schliesslich beschreibt auch Edelmann (2008) in ihrer mit $n=40$ Primar- und Sekundarschullehrpersonen der Stadt Zürich durchgeführten Interviewstudie Lehrpersonen, für welche eine defizitorientierte 
Einschätzung von Schülerinnen und Schülern mit Migrationshintergrund typisch ist. Sowohl die Haltung des "abgrenzend-distanzierten" Typs als auch diejenige des "stillschweigend-anerkennenden" Typs interpretiert Edelmann „aufgrund der Nichtbeachtung oder des Stillschweigens der Hintergründe von ihren SchülerInnen mit Migrationshintergrund“ als „Aufforderung zur Assimilation“ (ebd., S. 201, Hervorhebungen i.O.).

Im Gegensatz zu den bisher beschriebenen Studien gingen Wagner et al. (2001) sowie Makarova und Herzog (2013) weniger hypothesengenerierend, sondern vielmehr hypothesenprüfend vor. In beiden Untersuchungen wurden in Anlehnung an die weiter oben skizzierten sozial- bzw. kulturpsychologischen Akkulturationsmodelle (vgl. Berry et al., 1989; Bourhis et al., 1997) zwischen verschiedenen Akkulturationseinstellungen unterschieden und geprüft, wie bzw. ob sich diese Einstellungen auf das Unterrichtshandeln der Lehrpersonen bzw. auf Schülervariablen auswirken.

Wagner et al. (2001) stellten unter Anwendung der von Van Dick et al. (1997) entwickelten 13 Items ${ }^{20}$ umfassenden Skala zur Erfassung der Akkulturationseinstellungen von Lehrpersonen und mittels Mehrebenenanalyse fest, dass „Lehrpersonen mit assimilativ-segregativen Akkulturationseinstellungen eher Aussiedlerschüler mit starker Präferenz für autochthone deutsche Klassenkameraden [unterrichten], wogegen Lehrpersonen mit integrativen Akkulturationseinstellungen eher Aussiedlerschüler unterrichten, die eine geringe Präferenz für autochthone Deutsche aufweisen“ (S. 36). Die Autoren (ebd.) erklären sich die Resultate mit dem in Abhängigkeit der Akkulturationseinstellungen unterschiedlichen Erwartungsdruck der Lehrpersonen, sich als Aussiedlerschülerin bzw. -schüler mehr oder weniger stark zu assimilieren.

Makarova und Herzog (2013) prüften unter Anwendung einer eigens in Anlehnung an das Akkulturationsmodell von Bourhis et al. (1997) entwickelten Skala ${ }^{21}$ u. a. den Zusammenhang zwischen Akkulturationseinstellungen von Schweizer Primarschullehrpersonen $(n=225)$ und verschiedenen - durch Selbsteinschätzung erfassten - weiteren Lehrervariablen (Lehrerreaktionen auf schlechtes Benehmen von Schülerinnen und Schülern, Lehrerwahrnehmung von Unterrichtsstörungen, Diagnostische Kompetenz hinsichtlich sozialer Spannungen in der Klasse). Die Resultate zeigen, dass Lehrperson mit assimilativen Akkulturationseinstellungen ihrer Schülerinnen und Schüler vergleichsweise oft bestrafen

\footnotetext{
${ }^{20}$ Z.B. Item 8: „LehrerInnen sollten darauf achten, dass SchülerInnen anderer ethnischer Herkunft in den Schulpausen unter sich nur deutsch sprechen" oder Item 12: „LehrerInnen sollten den Kontakt zwischen SchülerInnen verschiedener ethnischer Herkunft fördern“ (Van Dick et al., 1997, S. 86f.).

${ }^{21}$ Die vier Akkulturationsvarianten wurden über je fünf Items, stehend für a) „teachers' attitudes towards the maintenance of the culture of ethnic origin " und b) "teachers' attitudes towards the adoption of the culture of the host society" (Makarova \& Herzog, 2013, S. 259), erfasst. Hohe Werte in beiden Dimensionen wurden schliesslich mit der Akkulturationseinstellung Integration assoziiert, tiefe Werte in der Dimension a) und hohe in der Dimension b) mit Assimilation, hohe Werte in der Dimension a) und tiefe in der Dimension b) mit Separation und tiefe Werte in beiden Dimensionen mit Marginalisierung.
} 
und eine geringe diagnostische Kompetenz für soziale Spannungen in der Klasse aufweisen. Die Autorin und der Autor kommen zum Schluss, „(...) that teachers who prefer an assimilative attitude are less likely to be successful in providing order during their lessons and preventing social conflicts among their students" (S. 264).

Weitere Untersuchungen, in denen sich assimilative Überzeugungen von Lehrpersonen als evident erwiesen, finden sich schliesslich auch in der US-amerikanischen Forschung. Ausgehend von der Feststellung, dass multicultural education einem Unterrichtsansatz mit potenziell unterschiedlichen Zielvorstellungen entspricht, befragte Nel (1993) $n=280$ angehende Lehrpersonen, nach welchem Ziel sie in einer migrationsbedingt heterogenen Klasse am ehesten unterrichten würden. Aus der standardisierten Befragung mittels Fragebogen resultierte, dass 67\% der Befragten das (assimilative) Ziel verfolgen würden, Schülerinnen und Schüler mit Migrationshintergrund im Erwerb der Kompetenzen zu unterstützen, die für ein erfolgreiches Bestehen in der Kultur der Mehrheitsgesellschaft notwendig sind. Ähnliche Resultate erhielten Guyton und Wesche (2005) im Rahmen ihrer Entwicklung und Validierung der Multicultural Efficacy Scale (MES) sowie Nadelson et al. (2012), welche dieses Instrument bei $n=88$ angehenden Lehrpersonen einsetzten. Über die Hälfte der 633 bzw. 88 befragten angehenden Lehrpersonen vertreten Konzepte von multicultural education, die der Kategorie assimilation entsprechen.

\subsubsection{Studien zu egalitären Überzeugungen}

Egalitäre Überzeugungen von Lehrpersonen im Umgang mit migrationsbedingter Heterogenität werden hier als Pendant zur im anglo-amerikanischen Sprachraum genannten Colorblindness von Lehrpersonen gefasst. Diese werden von Woolfolk Hoy et al. (2006) folgendermassen beschrieben: „Teachers intentionally try not to recognize and build on students' race and expect success by rejecting the cultural and racial capital that students bring into the learning environment" (S. 719). ${ }^{22}$ Die hinter dieser Absicht stehende Überzeugung: "Teachers choose to believe that to be fair means to not be aware" (Bryan \& Atwater, 2002, S. 830).

Zu den deutschsprachigen qualitativen Studien, welche diese Überzeugungsvariante von Lehrpersonen abbilden, gehören die Untersuchungen von Auernheimer, von Blumenthal, Stübig und Willmann (1996), Marburger et al. (1997), Hallitzky und Schiessleder (2008) und von Sievers (2009).

\footnotetext{
${ }^{22}$ Woolfolk Hoy et al. (2006, S. 722) verweisen diesbezüglich auf eine Studie von Tatum (1997), aus der hervorging, dass Lehrkräfte aus Angst davor, als Rassist bezeichnet zu werden, sich als „colorblind“ charakterisierten. 
In den Schulfallstudien von Auernheimer et al. (1996, S. 231) berichten die Autoren bilanzierend von Egalitarismus, weil befragte Lehrpersonen geltend machten, über die ethnische Zusammensetzung ihrer Klasse wenig zu wissen, diese auch gar nicht wahrzunehmen und ohnehin nicht bemüht zu sein, kulturelle Unterschiede zu thematisieren bzw. die Aufmerksamkeit darauf zu lenken.

In der Berliner Studie von Marburger et al. (1997) wurden die Lehrpersonen (u. a.) mit der Frage „'Meinen Sie, dass die Schule gegenüber deutschen und nichtdeutschen Schülerinnen und Schülern auch unterschiedliche Aufgaben bzw. Verpflichtungen hat?““ (S. 43) konfrontiert. Exemplarisch für die Aussagen der meisten Befragten steht für die Autoren die Antwort „'Nein, es gibt keine Unterschiede. Ich sehe keinen Unterschied. Ich wüsste gar nicht, warum. Ich nehme jeden so, wie er ist. Ich mache da keinen Unterschied, weil Kinder sind Kinder, grundsätzlich““ (S. 43). Das Bekenntnis zur grundsätzlichen Gleichbehandlung, so folgern Marburger und ihre Mitautorinnen und -autoren, erfolgte, „weil man sich als tolerant, verständnisvoll und humanistisch sieht, weil man sich abgrenzen möchte von jenen, die Nichtdeutsche diskriminieren" (S. 43).

Hallitzky und Schiessleder (2008) untersuchten die „pädagogischen Leitbilder“, welche deutsche Lehramtsstudierende in Bezug auf den Umgang mit migrationsbedingter Heterogenität haben. Die qualitative Inhaltsanalyse machte zwei unterschiedliche Absichten der Einbindung von Kindern mit Migrationshintergrund deutlich: eine "förderorientierte" und eine "homogenisierende". Als "homogenisierend" wurden dabei diejenigen Aussagen kodiert, in welchen die Befragten Unterschiede zwischen den Schülerinnen und Schülern ausblendeten bzw. einzuebnen versuchten (ebd., S. 269, Hervorhebungen i. O.), was ebenfalls als egalitäre Überzeugungen aufgefasst werden kann.

In der im selben Zeitraum von Sievers (2009) durchgeführten, ländervergleichenden Studie konnte festgestellt werden, dass (im Gegensatz zu den deutschen Lehrpersonen) die französischen Lehrpersonen kulturelle bzw. ethnische Aspekte kaum beachten bzw. thematisieren. In einer später erschienenen Publikation erklärt Sievers (2012) diese länderspezifische Prägung der Wahrnehmungen und Denkmuster mit dem französischen Selbstverständnis, „,wonach die französische Bevölkerung zu einer Nation gehört, die im öffentlichen Leben und somit auch im Bildungssystem keine Rücksicht auf ethnische, religiöse oder kulturelle Besonderheiten nimmt“ (S. 292). Exemplarisch dafür steht folgende Aussage einer Lehrperson: „'En les traitant comme tout le monde, c'est-à-dire en leur proposant ni plus ni moins que ce qui est proposé aux autres élèves (soutien ou approfondissement basés uniquement sur le volontariat des élèves)““ (ebd., 2009, S. 152).

In einer neueren Forschungsarbeit entwickelten Hachfeld et al. (2011), in Anlehnung an das sozialpsychologische Modell von Hahn et al. (2010), die Teacher Cultural Beliefs Scale (TCBS) zur Erfassung von multicultural beliefs und egalitarian beliefs von Lehrpersonen. Egalitarian beliefs werden hier zwar auch mit dem weiter oben erläuterte Konzept der Colorblindness in Verbindung gebracht, auf Item- 
Ebene schliesslich jedoch so ausformuliert ${ }^{23}$, dass weniger die prinzipielle Nicht-Berücksichtigung kultureller Unterschiede, sondern vielmehr die Suche nach kulturübergreifenden Gemeinsamkeiten letztlich dem pädagogisch angemessensten Umgang mit migrationsbedingter Heterogenität entspricht: „The egalitarianism subscale was designed to measure how strongly participants focus on cultural similarities and endorse the belief that all students should be treated equally, regardless of their cultural background" (Hachfeld et al., 2011, S. 988). Damit liegt der Subskala egalitarian beliefs trotz der begrifflichen Analogie zu den hier berichteten egalitären Überzeugungen ein konzeptuell zumindest teilweise davon abweichendes Verständnis zugrunde.

Unter Anwendung der TCBS konnte Hachfeld (2013) in einer Teilstudie mit $n=305$ angehenden deutschen Mathematiklehrpersonen feststellen, dass Junglehrpersonen der obligatorischen Schule stärkere egalitarian (sowie auch stärkere multicultural) beliefs aufweisen als Junglehrpersonen an Gymnasien (vgl. auch Kap. 3.1.1.3). Aus einer weiteren Studie mit $n=433$ angehenden deutschen Mathematiklehrpersonen konnten Hachfeld, Hahn, Schroeder, Anders und Kunter (2015, S. 51) (u.a.) zudem ableiten, dass egalitarian beliefs negativ mit der über eine 5-Item-Skala erfassten „willingness to adapt teaching to a culturally diverse student body" (= Bereitschaft den Unterricht auf die kulturell heterogene Schülerschaft anzupassen) korrelieren. Sie kommen entsprechend zum Schluss, „(that) the goal to treat all students equally, though noble in its intent, can result in a lack of willingness to prepare one's lessons adequately for the challenges of a diverse classroom" (ebd.).

Schliesslich bringen verschiedene amerikanische Studien zum Ausdruck, dass (angehende) Lehrpersonen ohne Migrationshintergrund stärker dazu neigen, colorblind zu sein als Lehrpersonen mit Migrationshintergrund (vgl. McIntyre, 1997; Valli, 1995; zit. n. Sleeter, 2001).

\subsubsection{Studien zu integrativ-multikulturellen Überzeugungen}

Lehrpersonen mit integrativ-multikulturellen Überzeugungen lassen sich von solchen mit assimilativen oder egalitären Überzeugungen im Wesentlichen durch eine Bereitschaft, den Unterricht den migrationsbedingt heterogenen Bedingungen anzupassen, unterscheiden. Darüber hinaus erachten sie in der Schülerschaft zum Ausdruck kommende kulturelle Unterschiede grundsätzlich als (Lern-)Potenzial. Im Umgang mit migrationsbedingter Heterogenität sehen sie ihre Aufgabe folglich darin, im Unterricht

\footnotetext{
${ }^{23}$ Die Subskala egalitarian beliefs umfasst folgende vier Items: Item $7=$ "Schools should aim to foster and support the similarities between the students from different cultural backgrounds", Item $8=$ "In the classroom, it is important that students of different origins recognize the similarities that exist between them", Item $9=$ "When there are conflicts between students of different origins, they should be encouraged to resolve the argument by finding common ground", Item $10=$ "Children should learn that people of different cultural origins often have a lot in common" (vgl. Hachfeld et al., 2011, S. 989).
} 
aktiv für Bedingungen zu sorgen, in denen kulturelle Unterschiede bzw. unterschiedliche Lebenswelten thematisiert werden können.

Zu den Studien aus dem deutschsprachigen Raum, die Befunde zu derartigen Überzeugungen von Lehrpersonen liefern, gehören die (in Kap. 3.1.1.1 und Kap. 3.1.1.2 bereits erörterten) Arbeiten von BenderSzymanski (2001), Edelmann (2008), Wagner et al. (2001), Hachfeld et al. (2015) sowie Makarova und Herzog (2013).

Neben einem „ethnoorientierten Verarbeitungsmodus“ identifizierte Bender-Szymanski (2001, S. 71f., Hervorhebungen i.O.) bei der Mehrheit der interviewten Referendarinnen und Referendaren $(n=20)$ einen durch das „Bemühen um wechselseitige Verständigung und Annäherung an den Interaktionspartner" gekennzeichneten „synergieorientierten Verarbeitungsmodus“. Dieser kommt dadurch zum Ausdruck, dass Lehrpersonen bestrebt sind, von Schülerinnen und Schülern zu lernen bzw. deren Standpunkte zuzulassen und das eigene - als kulturgebunden erkannte - Denken und Handeln zu reflektieren. Diese „akzeptanz- und verständnisorientierte“ Haltung schlägt sich nach Aussage der Interviewten in einer Neugestaltung der eigenen Zielvorstellungen und einer Veränderung der eigenen Rollendefinition nieder.

Ebenso integrativ-multikulturelle Überzeugungen finden sich in den vier von Edelmann (2008) beschriebenen "individuell-sprachorientierten", „kooperativ-sprachorientierten", „individuell-synergieorientierten" und "kooperativ-synergieorientierten" Typen von Lehrpersonen. So sehen die beiden sprachorientierten Typen sprachliche Differenzen als Lernpotenzial, weshalb sie bewusst Situationen einplanen, in welchen diese Ressource genutzt wird. Der "kooperativ-sprachorientierte" Typ ist ausserdem - und im Gegensatz zum „individuell-sprachorientierten“ Typ - in ein „innovatives Team eingebunden, das sich in einem ständigen fachlichen Austausch mit der sprachlichen Heterogenität auseinandersetzt“ (ebd., S. 161, Hervorhebungen i.O.). Der „individuell-synergieorientierte" Typ versteht nicht nur die sprachliche, sondern die migrationsbedingte Heterogenität an sich als Lernpotenzial und berücksichtigt diese für die Gestaltung eines mehrperspektivischen Unterrichts in allen Fächern. Er sucht vergebens nach Unterstützung im Team und handelt deshalb eigeninitiativ und mit persönlichem Engagement. Im Gegensatz dazu erfährt der „kooperativ-synergieorientierte“ Typ Unterstützung durch das Kollegium (vgl. ebd., S. 152f.).

Wagner et al. (2001) untersuchten mögliche Zusammenhänge zwischen den Akkulturationseinstellungen von Lehrpersonen und deren Umgang mit Interkulturellen Differenzen und Konflikten. Unter Anwendung der Akkulturationsskala von Van Dick et al. (1997) konnten sie aufzeigen, dass integrative Akkulturationseinstellungen negativ mit der Neigung zu Bestrafungen korrelieren: „Je stärker die Befragten Integration präferieren, umso weniger wollen sie mit Strafe auf die Problemsituationen reagieren“ (Wagner et al., 2001, S. 27). Darüber hinaus zeigten die Resultate, dass integrative Akkulturationseinstellungen negativ mit ethnischen Vorurteilen korrelieren (vgl. ebd., S. 25). 
Als integrativ-multikulturelle Überzeugungen lassen sich auch die um die Gruppe von Hachfeld mittels Teacher Cultural Beliefs Scale (TCBS) in verschiedenen Studien (vgl. Hachfeld, Hahn, Schroeder, Anders \& Kunter, 2012; Hachfeld et al., 2015; kumulativ in Hachfeld, 2013) erfassten multicultural beliefs ${ }^{24}$ bezeichnen: „Proponents of multiculturalism believe that (...) intercultural differences should be embraced and viewed as enriching. In the educational context, teachers with multicultural beliefs can be expected to incorporate students' different cultures into everyday school practice when planning their lessons, choosing materials, and interacting with students in class" (Hachfeld et al., 2011, S. 987). Die Untersuchung der Bedeutung von multicultural beliefs und egalitarian beliefs hinsichtlich diverser Variablen professioneller Kompetenz im Kontext migrationsbedingter Heterogenität (Selbstwirksamkeitserwartungen, Enthusiasmus für das Unterrichten von Schülerinnen und Schülern mit Migrationshintergrund, Vorurteile über schulbezogene Lernmotivation von Schülerinnen und Schülern mit Migrationshintergrund, Bereitschaft, den Unterricht auf die kulturell heterogene Schülerschaft anzupassen) zeigte, dass Lehrpersonen mit multicultural beliefs (gegenüber solchen mit egalitarian beliefs) eine höhere Selbstwirksamkeitserwartung und einen höheren Enthusiasmus, Schülerinnen und Schüler mit Migrationshintergrund zu unterrichten, haben. Analog zum Befund von Wagner et al. (2001) korrelieren zudem multicultural beliefs negativ mit (negativen) Vorurteilen über die Lernmotivation von Schülerinnen und Schülern mit Migrationshintergrund und positiv mit der Bereitschaft, den Unterricht auf die kulturell heterogene Schülerschaft anzupassen, wohingegen die egalitarian beliefs diesbezüglich unkorreliert bzw. negativ korreliert ausfielen (vgl. Hachfeld et al., 2015; vgl. auch Kap. 3.1.1.2). Die Autoren schliessen deshalb: „(..) multicultural beliefs are more beneficial for teaching in culturally diverse settings than colorblind beliefs" (S. 51). Ergänzend dazu stellen sie in der Studie von 2012 fest, dass angehende Lehrpersonen mit Migrationshintergrund generell ausgeprägtere multicultural beliefs aufweisen als solche ohne Migrationshintergrund.

Weitere Evidenzen dafür, dass integrativ-multikulturelle Überzeugungen hinsichtlich eines angemessenen Umgangs mit migrationsbedingter Heterogenität vorteilhaft sein können, liefert auch die Studie von Makarova und Herzog (2013; vgl. auch Kap. 3.1.1.1). So zeigen ihre Resultate, dass Lehrpersonen mit integrativen (verglichen mit solchen assimilativer oder segregativer) Akkulturationseinstellungen eine hohe diagnostische Kompetenz für soziale Spannungen in der Klasse haben. Daraus folgern sie: „These teachers have the best prospects for establishing an appropriate academic environment based on social order and prevention of conflict situations in their classrooms" (ebd., S. 264).

\footnotetext{
${ }^{24}$ Die Teilskala multicultural beliefs umfasst folgende sechs Items: Items $1=$, In the classroom, it is important to be responsive to differences between cultures"; Item $2=$ „It is important for children to learn that people from other cultures can have different values“; Item $3=$ ",Respecting other cultures is something that children should learn as early as possible“; Item $4=$ "In counseling parents who have a different cultural background than I do, I try to be considerate of cultural particularities"; Item $5=$ "When meeting with parents of different cultural backgrounds, I spend more time trying to understand and empathize with their perspective"; Item $6=$ "Dealing with cultural diversity should be taught in teacher training courses" (vgl. Hachfeld et al., 2011, S. 989).
} 
Schliesslich wurden integrativ-multikulturelle Überzeugungen auch in den amerikanische Studien von Nel (1993), Guyton und Wesche (2005) sowie Nadelson et al. (2012) erfasst. Die Studie Preservice Teachers' Perceptions of the Goals of Multicultural Education von Nel (1993; vgl. auch Kap. 3.1.1.1) ergab, dass den insgesamt $67 \%$ der Befragten mit einem assimilativen Verständnis von multicultural education 33\% Befragte gegenüberstanden, die multikulturelle Zielvorstellungen, d. h. die Absicht vertreten, Sprache und Kultur von Schülerinnen und Schülern mit Migrationshintergrund aktiv in den Unterricht einzubinden und als Lerngelegenheit zu betrachten. Diesen Befund kommentiert Nel (ebd., S. 124) mit: „Maintaining the status quo spells disaster for the culturally different students in the United States". Ähnliche Resultate lieferten die Untersuchungen von Guyton und Wesche (2005) sowie Nadelson et al. (2012), in welchen weniger als die Hälfte der 633 bzw. 88 befragten angehenden Lehrpersonen pluralistische bzw. multikulturelle Konzepte von multicultural education vertraten.

\subsection{2 Überzeugungen über Schülerinnen und Schüler mit Migrationshintergrund}

Neben Akkulturationseinstellungen bzw. Ideologien im Umgang mit migrationsbedingter Heterogenität findet sich eine Vielzahl von Studien, die sich explizit den Überzeugungen über Schülerinnen und Schüler mit Migrationshintergrund widmen. Ein Grossteil davon lässt sich konzeptionell als Lehrererwartungen (teacher expectations), d. h. als Schlüsse, welche die Lehrpersonen aufgrund bisheriger Erfahrungen mit und Informationen über einzelne Schülerinnen und Schüler (mit Migrationshintergrund) bezüglich schulisch relevanter Aspekte (fachliche Leistung, Sozialverhalten) ziehen, fassen (Schweer \& Thies, 2000, S. 65; Ulich, 2001, S. 86f.). Die lehrerseitige Bedeutung der Lehrererwartungen liegt dabei darin, dass sie als Wahrnehmungsfilter und Beurteilungsraster fungieren und damit eine rasche Eindrucksbildung ermöglichen (vgl. Ulich, 2001, S. 86; vgl. auch Kap. 2.3.2). ${ }^{25}$ Schülerseitig wird die Bedeutsamkeit von Lehrererwartungen insbesondere unter dem Aspekt der Erwartungseffekte (teacher expectation effects) thematisiert. Darunter versteht man, „dass eine Lehrkraft bestimmte Überzeugungen über das Potenzial eines Schülers hat und allein diese Erwartungen dazu beitragen, dass sich der Schüler so verhält oder Leistungen zeigt, wie die Lehrkraft es erwartet hat" (Kunter \& Pohlmann, 2015,

\footnotetext{
${ }^{25}$ Eng mit dem Konzept der Lehrererwartungen verknüpft, sind die ebenso als Filtermechanismus wirkenden impliziten (Persönlichkeits-)Theorien von Lehrpersonen (vgl. Schweer \& Thies, 2000, S. 61f.). Diese lassen sich als Bündel von Eigenschaften (einer Person) verstehen, die subjektiv als zusammengehörig erlebt werden: „Wird eine der als zusammengehörig erlebten Eigenschaften wahrgenommen, wird automatisch [bzw. implizit] auf das Vorliegen weiterer Eigenschaften geschlossen - unabhängig davon, ob diese Eigenschaften nun tatsächlich gehäuft gemeinsam auftreten oder aber ob dies eben nicht der Fall ist" (ebd., S. 61). Entsprechend fassen Ziegler et al. (1998) implizite Theorien von Lehrpersonen als „Vorstellungen von Lehrkräften über (...) Schüler/innen, die freilich auch Vorurteile und Stereotype enthalten können“ (S. 273).
} 
S. 269). ${ }^{26}$ Als dahinterliegender Wirkmechanismus wird ein in Abhängigkeit der Lehrererwartung variierendes Verhalten der Lehrpersonen gegenüber den betreffenden Schülerinnen und Schülern gesehen. Dabei scheinen insbesondere das sozioemotionale Klima und das Lernangebot eine Rolle zu spielen (vgl. Kunter \& Pohlmann, 2015, S. 270). ${ }^{27}$ Da sich Lehrererwartungen nachweislich auch auf die Notengebung und Selektionsentscheide auswirken können (z.B.Trautwein \& Baeriswyl, 2007), wurden Lehrererwartungen im Kontext migrationsbedingter Heterogenität insbesondere als möglicher Erklärungsansatz für den wiederholt konstatierten geringeren Bildungserfolg von Schülerinnen und Schülern mit Migrationshintergrund untersucht.

Da die Erforschung von Lehrererwartungen im Kontext migrationsbedingter Heterogenität insbesondere in den USA auf eine längere Tradition zurückblickt, umfassen die folgenden Ausführungen zunächst einen Überblick über Forschungsbefunde aus dem US-amerikanischen Sprachraum. Daran anschliessend werden in ausführlicherer Version einschlägige Befunde aus dem deutsch- (und holländisch)sprachigen Raum referiert.

Tenenbaum und Ruck (2007) fassen für den US-amerikanischen Sprachraum in einer Meta-Analyse Forschungsergebnisse von insgesamt 39 Untersuchungen zu Lehrererwartungen im Zusammenhang mit ethnischen Minoritäten zusammen. Als zentrales Resultat eruieren sie einen (kleinen) signifikanten Effekt des ethnischen Hintergrunds der Schülerinnen und Schüler auf die Lehrererwartungen bezüglich akademischer Leistung und/oder sozialem Verhalten. Ihren Berechnungen zufolge haben die Lehrpersonen die höchsten Erwartungen gegenüber Schülerinnen und Schüler mit asiatisch-amerikanischem Hintergrund (Asian american children), gefolgt von solchen europäisch-amerikanischer (European American children), lateinamerikanischer (Latino/a children) und schliesslich afro-amerikanischer ( $A f$ rican American children) Herkunft (ebd., S. 262).

Weitere empirische Evidenz für diesen Befund findet sich in der Studie von Arnold und Cross (2003; zit. n. Strasser, 2012, S. 193). Die hier befragten Lehrpersonen schätzten die mathematikbezogenen Kompetenzen und Interessen asiatischer Kinder höher ein als diejenigen weisser Kinder und deren wiederum höher als diejenigen afro-amerikanischer Kinder.

In eine ähnliche Richtung gehen zudem die Resultate zweier qualitativer Studien. Olmedo (1997) kommt aufgrund einer Analyse von Unterrichtsmaterialien und -protokollen von 29 angehenden Lehrpersonen zum Schluss, dass Schülerinnen und Schüler mit Migrationshintergrund aus Sicht der

\footnotetext{
${ }^{26}$ Als Ursprung der Erforschung solcher Erwartungseffekten gilt die Studie von Rosenthal und Jacobson (1968) zum sog. Pygmalioneffekt.

${ }^{27}$ So konnte bereits Hofer (1986, zit. n. Schweer, Thies \& Lachner, 2017, S. 130) aufzeigen, dass von den Lehrpersonen als disziplinlos und wenig begabt kategorisierte Schülerinnen und Schüler häufiger getadelt und weniger gefördert werden als solche, die eher dem Klassenprimus entsprachen. Letztere wurden ausserdem seltener direkt aufgerufen und erhielten qualitativ andere Aufgaben als ihre Mitschülerinnen und Schüler.
} 
Lehrpersonen generell als nicht lernmotiviert eingeschätzt werden. Solomon, Battistich und Hom (1996; zit. n. Bryan \& Atwater, 2002, S. 827) gelangen zum selben Befund und identifizieren darüber hinaus eine autoritärere und enger kontrollierte Unterrichtspraxis der Lehrpersonen gegenüber Schülerinnen und Schülern mit Migrationshintergrund.

Keinen Einfluss der Herkunft der Kinder auf die Leistungserwartungen von Kindergarten-Lehrpersonen stellen hingegen Hauser-Cram, Sirin und Stipek (2003) fest. Wie ein Vergleich zwischen den von den Lehrpersonen erfassten Leistungseinschätzungen und den objektiv erfassten Kompetenzen der Kinder im Bereich von Literalität und Mathematik verdeutlicht, erweisen sich zudem die Einschätzungen der Lehrpersonen als akkurat (vgl. ebd., S. 816). Allerdings konnten Zusammenhänge zwischen den Leistungserwartungen gegenüber Kindern und der Übereinstimmung bzw. Nicht-Übereinstimmung der pädagogischen Wertvorstellungen (teachers'values) mit denjenigen der Eltern dieser Kinder erkannt werden. So fielen die Leistungserwartungen gegenüber Kindern, deren Eltern nicht dieselben erzieherischen Wertvorstellungen wie die Lehrpersonen vertreten, deutlich niedriger aus als bei Kindern von Eltern mit Wertvorstellungen, die mit jenen der Lehrpersonen kongruieren.

Analog zur Arbeit von Hauser-Cram et al. (2003) und im Kontrast zur Mehrheit der US-amerikanischen Studien liegen für den deutsch- (und holländisch)sprachigen Raum vor allem Studien vor, die Leistungserwartungen stets auch unter Berücksichtigung von deren Akkuratesse untersuchten und diskutierten. ${ }^{28}$ Stimmen die Lehrererwartungen mit den tatsächlich realisierten Leistungen der Schülerinnen und Schüler nicht überein, ist gerade im Kontext migrationsbedingter Heterogenität häufig auch von (ethnischen) Stereotypen, verzerrten Erwartungen oder Vorurteilen die Rede. Diesbezügliche Meta-Analysen, wie sie für den US-amerikanischen Sprachraum vorliegen, liessen sich auf Basis der durchgeführten Literaturrecherche für den deutschsprachigen und niederländischen Raum keine finden. Grundsätzlich lassen sich die Studien jedoch danach unterscheiden, ob sie die Akkuratesse der Lehrerurteile hinsichtlich Schulleistungen und/oder Intelligenz der Schülerinnen und Schüler oder aber hinsichtlich formeller Lehrerurteile (Benotung, Selektionsentscheide) fokussierten (vgl. Carigiet Reinhard, 2012). Abgesehen von diesen auf Basis numerischer Daten durchgeführten Studien finden sich zudem verschiedene qualitative Interviewstudien zur Wahrnehmung und Einschätzung von Schülerinnen und Schülern mit Migrationshintergrund.

Zu den Studien mit Fokus auf der Akkuratesse der Lehrerurteile hinsichtlich Schulleistungen und/oder Intelligenz der Schülerinnen und Schüler gehören die Untersuchungen von Jungbluth (1994), Kronig,

\footnotetext{
${ }^{28}$ Solche Untersuchungen fallen oft auch unter den Begriff der diagnostischen Kompetenz von Lehrpersonen (vgl. Carigiet Reinhard, 2012)
} 
Haeberlin und Eckhart (2000), Carigiet Reinhard (2012), Hachfeld (2013) sowie Lorenz, Gentrup, Kristen, Stanat und Kogan (2016).

In seiner vielzitierten Studie untersuchte Jungbluth (1994) die Erwartungen und Zielsetzungen niederländischer Grundschullehrpersonen in Abhängigkeit von Ethnizität und Schichtzugehörigkeit der Schülerinnen und Schüler. Der Vergleich der von den Lehrpersonen $(n=44)$ eingeschätzten Schulleistungen in Mathematik und Lesen mit dem objektiv erfassten nonverbalen Intelligenztest zeigt, dass Schülerinnen und Schüler marokkanischer und türkischer Herkunft von den Lehrpersonen unterschätzt werden (ebd., S. 119). Darüber hinaus machen die Resultate deutlich, dass die Lernzielniveaus trotz gleichen intellektuellen Fähigkeitspotenzials bei marokkanischen und türkischen Schülerinnen und Schülern tiefer angesetzt werden als bei niederländischen Schülerinnen und Schülern. Bei näherer Betrachtung bzw. durch eine Varianzanalyse kommt dabei zum Ausdruck, dass sich weder die seitens der Lehrpersonen geäußerten Fähigkeitseinschätzungen ihrer Schülerinnen und Schüler, noch die anvisierten Lernzielniveaus systematisch mit der Ethnizität der Schülerinnen und Schüler, hingegen aber mit deren Schichtzugehörigkeit erklären lassen. So erklärt die Sozialschicht der Schülerinnen und Schüler 9\% der Varianz bei den wahrgenommenen Schülerfähigkeiten und $4 \%$ bei den gesetzten Lernzielniveaus (vgl. ebd., S. 121).

Kronig et al. (2000) untersuchten die Akkuratesse der von den Lehrpersonen bezüglich Fähigkeiten in der Unterrichtssprache erfassten Leistungserwartungen von Schweizer Lehrpersonen $(n=111)$ der zweiten Primarschulstufe. Die Ergebnisse verweisen insgesamt auf eine verzerrte diagnostische Wahrnehmung der Lehrpersonen zuungunsten der „Immigrantenkinder“. So wurden Immigrantenkinder in ihrer Leistungsfähigkeit signifikant niedriger eingeschätzt als Schweizer Kinder, „die bezüglich der Intelligenz, des Alters und der Leistung zum Zeitpunkt der Urteilsbildung vergleichbar sind und deren Geschlecht jeweils identisch ist" (ebd., S. 193). Die Bedeutsamkeit der Ergebnisse erhöht sich durch den weiteren Befund, dass die Leistungsentwicklung (im Sinne eines Erwartungseffekts) in der Unterrichtssprache bei unterschätzten Immigrantenkindern signifikant geringer ausfällt als bei vergleichbaren Immigrantenkindern, deren Leistung angemessen eingeschätzt oder gar überschätzt wurde (vgl. ebd., S. 144).

Die Studie von Carigiet Reinhard (2012) war vom Ziel geleitet, die über die individuellen Schülerinnenund Schülervoraussetzungen hinausgehenden Bedingungsfaktoren für (ungleiche) Schulleistungen am Ende der dritten Primarschulklasse zu identifizieren. Mit Blick auf Lehrererwartungen und die Herkunft von Schülerinnen und Schülern konnte die Autorin auf Basis einer Stichprobe von 42 Lehrpersonen und 757 Schülerinnen und Schülern aus dem deutschsprachigen Teil des Kantons Bern zunächst feststellen, „dass die Lehrpersonen von Schülerinnen und Schülern aus sozioökonomisch unterprivilegierten, migrierten sowie nicht-deutschsprachigen Familien im Fach Deutsch weniger erwarten" (S. 298). Durch den Vergleich mit objektiv erfassten Testleistungen der Schülerinnen und Schüler im Fach Deutsch 
konnte sie darüber hinaus aufzeigen, dass Schülerinnen und Schüler aus einheimischen Familien „relativ adäquat bzw. leicht überschätzt“ (ebd.), solche aus immigrierten Familien „im Schnitt um einen Drittel bzw. eine halbe Standardabweichung der Fehleinschätzung unterschätzt" (ebd.) werden. Sowohl der Sprachgebrauch zuhause (6.7\% Varianzaufklärung) als auch der Migrationshintergrund (3.2\% Varianzaufklärung) erwiesen sich dabei als bedeutsame Prädiktoren für das Ausmass an Fehleinschätzung. Ähnlich fielen die Resultate in Bezug auf die Lehrerurteile über die Intelligenz der Schülerinnen und Schüler aus. Dabei stellte sich heraus, „dass die Lehrpersonen das intellektuelle Potenzial von Schülerinnen und Schüler aus dem Balkan und der Türkei (bei gleichem sozioökonomischem Status und gleichen objektiv gemessenen kognitiven Grundfähigkeiten) signifikant tiefer einschätzen als die kognitiven Grundfähigkeiten von einheimischen sowie von Schülerinnen und Schülern mit südwesteuropäischer oder anderer Herkunft“ (ebd., S. 299f.). Das Erklärungspotenzial des Sprachgebrauchs zuhause (2\% Varianzaufklärung) und des Migrationshintergrunds (3\% Varianzaufklärung) erwies sich hier jedoch als eher klein. Als bedeutsamer zur Vorhersage des Urteils der Lehrpersonen kristallisierten sich die Leistungen im Fach Deutsch heraus. Carigiet Reinhard (2012) schliesst daraus, „dass Lehrpersonen v.a. (schulische) Leistungsdiagnostiker/-innen sind, welche die intellektuellen Fähigkeiten von Schülerinnen und Schülern nur insofern einzuschätzen vermögen, als diese sich in schulischen Leistungen niederschlagen“ (S. 382).

In der_von Hachfeld (2013, S. 71f.) mit $n=305$ deutschen Mathematiklehrpersonen durchgeführten Studie wurden die von Lehrpersonen eingeschätzten Leistungen ihrer Schülerinnen und Schüler bezüglich zweier sich durch das Sprachniveau voneinander unterscheidenden Mathematikaufgaben mit den tatsächlich realisierten Leistungen der Schülerinnen und Schüler verglichen. Unterschieden wurde dabei zwischen Schülerinnen und Schülern ohne Migrationshintergrund und mit deutscher Muttersprache, solchen mit Migrationshintergrund und nicht-deutscher Muttersprache sowie solchen mit Migrationshintergrund und sowohl deutscher als auch nicht-deutscher Muttersprache (bilinguale Gruppe). In Bezug auf die Akkuratesse der Einschätzungen der Lehrpersonen resultierten insgesamt keine im Zusammenhang mit dem ethnischen und sprachlichen Hintergrund der Schülerinnen und Schüler stehenden Effekte. Allerdings zeigte eine die sprachliche Schwierigkeit der Aufgaben berücksichtigende Auswertung, dass bilinguale Schülerinnen und Schüler hinsichtlich der sprachlich anspruchsvolleren Aufgabe im Vergleich zur sprachlich einfacheren Aufgabe signifikant überschätzt wurden. Die Autorin schliesst daraus, „, [that] teachers are not always aware of bilingual students language difficulties or of the linguistic demands that specific mathematics problems may pose" (ebd., S. 88).

Zu Resultaten, die analog zu den Ergebnissen von Hachfeld (2013) auf (zumindest teilweise) positive Verzerrungen der Lehrererwartungen hinsichtlich der Schulleistungen von Kindern mit Migrationshintergrund hinweisen, gelangen in einer neueren Studie Lorenz et al. (2016). In der mit $n=77$ Grund- 
schullehrpersonen in Nordrhein-Westfalen durchgeführten Untersuchung wurden die Lehrerwartungen dahingehend erfasst, als die Lehrpersonen zu Beginn des Schuljahres einschätzen sollten, „wo das Kind mit seinen Leistungen [in den Fächern Mathematik und Deutsch] am Ende des kommenden Schuljahres im Vergleich zu anderen Kindern aus seiner Klasse voraussichtlich stehen wird" (ebd., S. 97). Der Vergleich mit den objektiv erfassten Leistungen der Schülerinnen und Schüler machte für das Fach Mathematik deutlich, dass türkisch-stämmige Schülerinnen und Schüler akkurat und Schülerinnen und Schüler mit osteuropäischer ${ }^{29}$ Herkunft jedoch überschätzt wurden. Anders stellen sich die Ergebnisse für das Fach Deutsch dar: Während sich die Erwartungen gegenüber Kindern mit einem osteuropäischen Migrationshintergrund als nicht verzerrt bzw. akkurat erwiesen, unterschätzten die Lehrpersonen dagegen Kinder und insbesondere Jungen mit einem türkischen Zuwanderungshintergrund. Die Autorinnen und Autoren fassen zusammen, dass in beiden Fächern „systematische Verzerrungen der Lehrererwartungen nach askriptiven Schülermerkmalen beobachtbar sind" (ebd., S. 105) und schliessen daraus, dass die erfassten Lehrerurteile „zu grossen Teilen die Folge kategorisierender Wahrnehmungsprozesse gewesen sein könnten“ (ebd.).

Ergebnisse zu formellen Lehrerurteilen (Benotung, Selektionsentscheide) unter Berücksichtigung der Herkunft der Schülerinnen und Schüler liefern die Untersuchungen von Moser und Rhyn (1999), Lanfranchi (2007) und Kronig (2007).

In der Schweizer Studie von Moser und Rhyn (1999) wurde der Frage nachgegangen, „welche Merkmale von Schülerinnen und Schülern die Notengebung beeinflussen“"(S. 126). Ihren Resultaten zufolge beeinflussen nach Kontrolle des Schultyps und der mittels standardisiertem Test erfassten tatsächlichen Leistung im Fach Deutsch das Geschlecht, die Muttersprache und die soziale Herkunft der Schülerinnen und Schüler signifikant die Note. Am grössten fällt der Unterschied zwischen einem fremdsprachigen Schüler aus der Unterschicht und einer deutschsprachigen Schülerin aus der Mittel- oder Oberschicht aus. So wird ersterem von den Lehrpersonen $(n=167)$ eine im Schnitt um 0.5 Notenpunkte tiefere Note erteilt als letzterer. Nur unwesentliche Unterschiede zwischen den verschiedenen Schülergruppen errechnen die Autoren hingegen im Fach Mathematik. Die Autoren erklären sich die fachspezifisch unterschiedlich ausgefallenen Befunde insbesondere damit, dass die mündlichen Sprachfähigkeiten der Schülerinnen und Schüler im Fach Deutsch vermutlich stärker in die Benotung miteinfliessen als im Fach Mathematik. Eine „vorurteilsfreie Notengebung“ (ebd., S. 127) sei im Fach Mathematik deshalb einfacher zu erreichen als im Fach Deutsch.

\footnotetext{
${ }^{29}$ Dieser Kategorie wurden Schülerinnen und Schüler aus Staaten der ehemaligen Sowjetunion, Polen, Tschechien, Bulgarien, Rumänien, Moldawien, Ungarn, Slowakei und aus Slowenien zugeordnet (vgl. Lorenz et al., 2016, S. 98).
} 
Vor dem Hintergrund gestiegener Massnahmen sonderpädagogischer Versorgung von Schülerinnen und Schülern untersuchte Lanfranchi (2007), nach welchen Kriterien Schweizer Primarschul-Lehrpersonen $(n=1916)$ in sechs Deutschschweizer Kantonen die Versetzung schulschwacher Kinder in Sonderklassen und Sonderschulen beantragen. Als zentrales Ergebnis konnte festgestellt werden, dass sowohl bei „Unterschichtskindern“ als auch bei „Migrationskindern“ bei gleicher Problemmanifestation $^{30}$ signifikant häufiger separierende Massnahmen (Beratung durch schulpsychologischen Dienst, Individuelle Lernziele, Repetition, Aufgabenhilfe, Logopädie usw.) anvisiert werden als bei „Oberschichtskindern" bzw. Schweizer Kindern.

Kronig (2007) interessierte sich in seiner Längsschnittstudie Systematische Zufälligkeit des Bildungserfolgs sowohl für die Leistungsbewertung als auch für Selektionsentscheide von Schweizer Lehrpersonen ( $n=112$ ) des sechsten Schuljahres. In Übereinstimmung mit den weiter oben referierten Befunden von Moser und Rhyn (1999) weisen seine Resultate aus, dass - auch unter Berücksichtigung des Referenzgruppenfehlers - ein Schweizer Mädchen mit privilegierter sozioökonomischer Herkunft für die gleiche durchschnittliche Leistung im Fach Deutsch mit 0.4 Notenpunkten besser bewertet wird als ein Junge aus einer Zuwandererfamilie mit unterprivilegiertem sozioökonomischem Hintergrund (vgl. ebd., S. 203). Für das Fach Mathematik leisten die erfassten Personenmerkmale (Geschlecht, Nationalität, sozioökonomischer Status) ebenso einen signifikanten, wenn auch etwas geringeren Beitrag zur Vorhersage der Mathematiknote (vgl. ebd., S. 205). Darüber hinaus kann Kronig (im Sinne eines Erwartungseffekts) nachweisen, dass die Notengebung einen Einfluss auf die Leistungsentwicklung der Schülerinnen und Schüler hat. Der Vergleich von in Bezug auf Geschlecht, nationalstaatlicher Herkunft, Einwanderungszeitpunkt, Lernstand zu Beginn des Schuljahres, kognitive Grundfähigkeiten und sozioökonomischen Status komparablen, jedoch notenmässig nicht gleich bewerteten Schülerpaaren zeigt, dass schlechter, d. h. mit Note 4 bewertete Schülerinnen und Schüler signifikant geringere Leistungsfortschritte erzielen als besser, d.h. mit Note 5 bewertete Schülerinnen und Schüler (vgl. ebd., S. 207). Schliesslich lassen die Resultate auch auf eine herkunftsabhängige Selektion schliessen. So zeigen die Kennwerte des Vorhersagemodells, dass Schweizer Schülerinnen und Schüler mit mittlerem oder hohem sozioökonomischen Status (Referenzgruppe) mit einer Wahrscheinlichkeit von 83.3 Prozent, Schülerinnen und Schüler aus Zuwandererfamilien und mit tiefem sozioökonomischem Status (bei gleicher Leistungsfähigkeit) mit einer Wahrscheinlichkeit von 51.7

\footnotetext{
${ }^{30}$ Sämtlichen Lehrpersonen wurden zwei in schriftlicher Form beschriebene Schülerfälle vorgelegt. Der eine Fall beschrieb einen lese- und rechtschreibeschwachen sowie rechenschwachen Schüler mit eher niedriger Intelligenz. Der zweite Fall einen verhaltensauffälligen Schüler. Um Aussagen über das Zuweisungsverhalten der Lehrpersonen in Abhängigkeit von Schichtzugehörigkeit und ethnischem Hintergrund des Schülers machen zu können, wurden die Fallbeschreibungen (in Anlehnung an die Methode des „practice testing“) systematisch im Bereich des Schülernamens (Lukas vs. Bekir) und des Berufes des Vater (Chefarzt vs. Bauarbeiter) variiert (vgl. Lanfranchi, 2007, S. 132-133).
} 
Prozent eine Sekundarschulempfehlung erhalten (vgl. ebd., S. 211f.). ${ }^{31}$ Leistungsgerechte bzw. meritokratische schulische Allokation findet dabei nur an den Extremen der Leistungsverteilung, d. h. nur dort, wo die Leistungen „um mehr als eine Standardabweichung vom Durchschnitt der Stichprobe entfernt" (ebd., S. 214) sind, statt. Kronig folgert entsprechend, „dass über zwei Drittel der Schülerinnen und Schüler nicht mit einer leistungsgerechten Selektion rechnen können“ (ebd.).

Aufschlussreich in Bezug auf potenzielle Erklärungsansätze verzerrter Leistungserwartungen scheint die von Hachfeld et al. (2015; vgl. auch Hachfeld, 2013, S. 133f.) durchgeführte Untersuchung Should teachers be colorblind? How multicultural and egalitarian beliefs differentially relate to aspects of teachers' professional competence for teaching in diverse classrooms. In der Befragung mit $n=433$ angehenden Mathematiklehrpersonen konnte aufgezeigt werden, dass negative Vorurteile über die Lernmotivation ${ }^{32}$ von Schülerinnen und Schülern mit Migrationshintergrund sowie über den Bildungshintergrund ihrer Eltern negativ mit multicultural beliefs korrelieren.

Ergänzend zu den berichteten quantitativen Befunden liefern schliesslich auch qualitative Studien Hinweise für verzerrte Leistungserwartungen. Marburger et al. (1997) eruieren in ihrer Untersuchung vorurteils- und stereotypbehaftete Sichtweisen, die insbesondere die Eltern der Kinder mit Migrationshintergrund bzw. deren Familien- und Geschlechterverhältnisse betreffen. Bender-Szymanski (2001, S. 82; vgl. auch Bender-Szymanski, 2013) sowie Sievers (2009) berichten ausserdem von pauschalisierenden Aussagen von Studienreferendaren und Lehrpersonen. In ihrer Analyse der von Lehrpersonen zur Beschreibung ihrer Schülerschaft verwendeten Begrifflichkeiten stellt Sievers (2009, S. 90) fest, dass deutsche Lehrpersonen stark „nationalitäten- bzw. herkunftsländergebunden“ antworten und französische Lehrpersonen nach Migrantengenerationen oder Migrantengruppen kategorisieren.

\subsubsection{Vorstellungen zur unterrichtlichen Umsetzung und Umsetzbarkeit interkultureller Erzie-} hung

Vorstellungen von Lehrpersonen zur unterrichtlichen Umsetzung und Umsetzbarkeit interkultureller Erziehung im Unterricht betreffen die Fragen nach dem subjektiven Verständnis interkultureller Erziehung (Was) und der (davon abhängigen) Art und Weise, wie diese Zielorientierung im Unterricht umgesetzt wird bzw. werden kann. Hintergrund der wenigen hierzu vorliegenden Untersuchungen ist der

\footnotetext{
${ }^{31}$ Eine mit denselben Daten errechnete logistische Regression, in welcher auch die Variable Geschlecht mitberücksichtigt wurde, bringt ergänzend zum Ausdruck, „dass Schweizer Mädchen zu 83\% einen Sekundarschulentscheid erhalten, Schweizer Jungen zu 70\%, ausländische Mädchen zu 65\% und ausländische Jungen zu 37\%" (Haeberlin, Imdorf \& Kronig, 2004b, S. 50).

32 Itembeispiel für negatives Vorurteil über Lernmotivation: "Immigrant students are less interested in schoolrelated topics". Itembeispiel für negatives Vorurteil über Bildungshintergrund der Eltern: "Immigrant students often come from families that communicate little school-related knowledge" (Hachfeld, 2013, S. 142). 
über die Lehrpläne an Lehrpersonen konkret herangetragene Anspruch der Förderung interkulturellen Lernens bzw. eines konstruktiven Umgangs mit migrationsbedingter Heterogenität.

Strohmeier und Fricker (2007) untersuchten die aus Sicht der Lehrpersonen für die Zielerreichung des interkulturellen Lernens am besten geeigneten Lehrmethoden. Hierzu liessen sie von den 26 österreichischen Mittelschullehrpersonen die Wichtigkeit von acht vorgegebenen Unterrichtsmethoden (Gruppenarbeiten, SchülerInnentandems, offenes Lernen, Diskussionen mit der gesamten Klasse, Diskussionen in der Kleingruppe, Frontalunterricht, Projektunterricht, Teamteaching) hinsichtlich der Zielperspektive interkulturellen Lernens einschätzen. Daraus resultierte folgende, nach Wichtigkeit absteigende Reihenfolge: Teamteaching, Gruppenarbeit, Projektunterricht, Diskussionen in der ganzen Klasse, offenes Lernen, SchülerInnentandems, Diskussionen in Kleingruppen, Frontalunterricht. In der Studie von Edelmann (2008) fungierten u. a. „die Art und Weise, wie die migrationsbedingte Heterogenität im Unterricht als Potenzial verstanden wird“ (S. 133) sowie die „Methodik und Didaktik, die im Kontext der kulturellen Heterogenität als besonders unterstützend eingeschätzt wird“ (ebd.) als Faktoren für die Herausbildung der von ihr identifizierten - sich im Umgang mit migrationsbedingter Heterogenität unterscheidenden - Lehrertypen. Lehrpersonen des Typs „abgrenzend-distanziert“ (S. 134f.) und "stillschweigend-anerkennend" (S. 143f.) messen der migrationsbedingten Heterogenität in ihrer Klasse grundsätzlich kein Lernpotenzial bei. Entsprechend nehmen sie auch keine Anpassungen in ihrem Unterricht vor bzw. planen keine Unterrichtsmomente ein, in denen den verschiedenen Hintergründen der Schülerinnen und Schüler bewusst Raum gegeben wird. Wenn Schülerinnen und Schüler von sich aus etwas einbringen, dann zeigt jedoch der „stillschweigend-anerkennende“ Typ Interesse dafür. Ansonsten bleibt die Thematisierung der migrationsbedingten Heterogenität auf das Fach Religion beschränkt - sofern die Lehrpersonen dieses überhaupt unterrichten. Der „individuellsprachorientierte“ (S. 152) Typ und der "kooperativ-sprachorientierte“ (S. 161f.) Typ schenken vor allem der Sprachenvielfalt Beachtung. So hat die Sprachförderung in allen Fächern ihres Unterrichts einen hohen Stellenwert, da Deutschkenntnisse einen zentralen Faktor für den schulischen Erfolg darstellen. Zudem werden auch die Herkunftssprachen der Schülerinnen und Schüler in den Unterricht durch Sprachvergleiche mit einbezogen. Abgesehen von der Behandlung verschiedener Feiertage (Weihnachten, Ostern usw.) bleiben Religion und Kultur jedoch Unterrichtsthemen des Religionsunterrichts. Um den unterschiedlichen Leistungsniveaus der Schülerinnen und Schüler gerecht zu werden, wird auf individualisierten Unterricht (z.B. durch Wochenpläne oder Arbeit in Kleingruppen) gesetzt und der Wunsch nach nicht zu grossen Klassen geäussert. Bei Lehrpersonen der Typen „individuell-synergieorientiert" (S. 170f.) und „kooperativ-synergieorientiert" (S. 179f.) kommt der kulturelle Hintergrund der Schülerinnen und Schüler im Unterricht in verschiedensten Fächern häufig und deutlich zum Tragen. Lehrpersonen dieser Typen sind der Überzeugung, dass es ihren Schülerinnen und 
Schülern wichtig ist, dass ihre kulturelle Herkunft bzw. ihre Sprachkenntnisse und ihre religiösen Orientierungen thematisiert werden. Entsprechend wird die migrationsbedingte Heterogenität in der Unterrichtsplanung gezielt berücksichtigt und auf Möglichkeiten geachtet, dass die Schülerinnen und Schüler ihre Normen und Wertvorstellungen in den Unterricht einbringen können. Dies geschieht über verschiedene Formen individualisierten und offenen Unterrichts sowie über den Austausch im Klassengespräch. Die „kooperativ-synergieorientierte“ Lehrperson führt zudem klassenübergreifende Projekte zur Sprachförderung durch, wobei beide Typen bewusst sowohl die Verwendung der Standardsprache als auch der Mundartsprache anregen. Letztere, so eine Lehrperson des „individuell-synergieorientierten"Typs, sei wichtig für „das Emotionale“ (S. 174).

Binder und Daryabegi (2002) befragten österreichische Lehrpersonen, „,wie sie persönlich das Unterrichtsprinzip Interkulturelles Lernen verstehen, und wie sie es verankert und verstanden wissen wollen“ (S. 36). Viele der befragten Lehrpersonen sehen interkulturelles Lernen als „Chance, den SchülerInnen die verschiedensten Kulturen näher zu bringen“ (ebd.) und dabei sowohl die Differenzen als auch die Gemeinsamkeiten hervorzuheben. Die Ziele interkulturellen Lernens werden von den Lehrpersonen mit „'(...) voneinander lernen und einander kennenlernen"“ (S. 37), „'Respekt und Achtung vor anderen Kulturen'“ (S. 38), „'Verständnis wecken für verschiedene kulturelle Ansätze'“ (ebd.) oder „(...) möglichst gute Integration (...)'“ (S. 39) beschrieben. Insgesamt schätzen die Lehrpersonen Fremdsprachenunterricht, Deutsch, Geschichte, Sozialkunde, Geografie und Wirtschaftskunde als besonders geeignet für interkulturelles Lernen ein. Naturwissenschaftliche Fächer wie Mathematik, Physik, Chemie oder Biologie werden hingegen als vergleichsweise wenig geeignet betrachtet (vgl. ebd., S. 44). Die erfassten Klassenprotolle zeigen exemplarisch, wie sich die Lehrpersonen eine fächerspezifische Auseinandersetzung mit interkulturellem Lernen vorstellen. Beispielsweise werden im Fremdsprachenunterricht (Schulbuch-)Texte zu Themen wie Migration oder Begräbnisritualen, im Deutschunterricht zu Lebenswelten von Kindern aus anderen Ländern als Anlass zu Dialogen und Diskussion mit dem Ziel interkulturellen Lernens gesehen. Im Deutschunterricht würde sich aus Sicht mancher Lehrpersonen ausserdem das Thema Fremdwörter und Lehnwörter zu Sprachvergleichen und Diskussionen rund um Begriffe anbieten. Im Rahmen des Musikunterrichts sehen die Lehrpersonen insbesondere verschiedene Musikgenres (z. B. Blues, Gospel) als Anknüpfungspunkte, um über unterschiedliche kulturelle Ausdrucksweisen zu sprechen. Im Fach Geografie bieten sich aus Sicht der Lehrpersonen u.a. Referate über das eigene Herkunftsland sowie das Thema Migration als Anknüpfungspunkt für persönliche Erzählungen der Schülerinnen und Schüler an. Hintergründe und Lebenswelten der Kinder liessen sich zudem auch im Fach Geschichte einbeziehen. Allerdings sind viele Lehrpersonen der Meinung, dass Diskussionen über Kriege im ehemaligen Jugoslawien „'zu heiss'“ (S. 52) wären. Schliesslich sehen die Lehrpersonen im Fach Biologie das Thema Fortpflanzung und die damit im Zusammenhang stehenden kulturellen Normen und Werte, im Fach Kochen und Haushaltslehre das 
Thema Essensvorschriften und -gewohnheiten (z. B. Ramadan) als Chancen für interkulturelles Lernen. Die Fächer Mathematik und Physik bieten sich nach Auskunft der Lehrpersonen kaum für interkulturelles Lernen an (vgl. ebd., S. 44f., 56, 58). In Bezug auf sich eignende Unterrichtsformen für interkulturelles Lernen plädieren die Lehrpersonen insbesondere für fächerübergreifendes, projektorientiertes Lernen (z.B. über Migration und Vorurteilsabbau), das zu Gesprächs- und Diskussionskulturen beitragen würde, in denen sich Schülerinnen und Schüler (im Vergleich zu herkömmlichen Unterrichtsmethoden) mehr im Unterricht einbringen könnten (vgl. ebd., S. 84). Als problematisch würde sich hier allerdings der Leistungsdruck erweisen, weil dieser „einen lockeren Umgang mit der zur Verfügung stehenden Zeit kaum zulasse“ (ebd., S. 59). Zusammenfassend kommen die Autoren zum Schluss, dass in der von ihnen untersuchten schulischen Praxis ein „grundsätzliches Denken in Richtung interkulturelles Lernen“ fehlt (ebd., S. 43). Interkulturelles Lernen würde (nur) situativ zur Sprache kommen, was sie an folgender Interviewaussage festmachen: „'Für mich ist Interkulturelles Lernen nicht etwas, was ich aufsetze, sondern was einfliesst und eben einfach kommt. Daher kann ich so viel oder so wenig machen, wie ich in der Situation für notwendig erachte”“ (S. 43). Anknüpfungspunkte würden sich nach Aussage verschiedener Lehrpersonen insbesondere durch die Anwesenheit fremdsprachiger Schülerinnen und Schülern ergeben (vgl. ebd., S. 43f.).

In der Studie von Marburger et al. (1997) kommt zum Ausdruck, dass für einen Grossteil der befragten Berliner Lehrkräfte durch Einbezug von Liedern, Tänzen, Festen oder Kulinarischem „Minderheitenkulturen vermittelt" werden können. Als sich für die Berücksichtigung von Minderheitenkulturen potenziell eignende Fächer nennen die Lehrpersonen Deutsch, bildende Kunst, Erdkunde, Geschichte und Sport. Wie auch in der oben dargestellten Studie von Binder und Daryabegi (2002) sind ausserdem viele Lehrpersonen der Ansicht, dass sich insbesondere auch überfachliche Projekttage dafür eignen . Ähnliche Antworten liefern die von Sievers (2009) interviewten Lehrpersonen: Interkulturelles Lernen komme höchstens im Religionsunterricht - „'(...)wenn es um andere Sitten, andere Gebräuche geht““ (S. 130) - oder aber im Rahmen von Schulfesten und Projektwochen vor. Die Aussagen zeigen ausserdem, dass sich v. a. die befragten französischen Junglehrpersonen noch wenig mit interkulturellem Lernen beschäftigt haben. Diejenigen Lehrpersonen, die sich intensiver mit der interkulturellen Pädagogik bzw. interkulturellem Lernen auseinandergesetzt haben, kommen dagegen zum Schluss, dass die Idee zwar spannend und aktuell, jedoch zu abstrakt und oft aufgrund fehlender Zeitressourcen nicht umsetzbar sei (vgl. ebd., S. 132f.).

Quantitative Untersuchungen zu Lehrervorstellungen, wie der migrationsbedingten Heterogenität methodisch-didaktisch begegnet werden kann, existieren nach derzeitigem Kenntnisstand keine. Der TCBScale von Hachfeld et al. (2011; vgl. auch Kap. 3.1.1) sind jedoch drei Items zu entnehmen, die aufgrund ihrer Formulierung als Vorstellungen zur konkreten Unterrichtspraxis gedeutet werden können. In der 
Subskala multicultural beliefs sind dies die Items 4 und 5, in der Subskala egalitarian beliefs das Item 9 (vgl. ebd., S. 989). ${ }^{33}$

\subsection{Empirische Ergebnisse aus der Sportlehrerforschung}

Wie einführend in das Kapitel 3 erwähnt, liegt bislang nur eine spärliche Anzahl an Untersuchungen zu Überzeugungen von Sport unterrichtenden Lehrpersonen im Kontext migrationsbedingter Heterogenität vor. Im deutschsprachigen Raum bleiben die Befunde auf Ergebnisse verschiedener explorativer Teilstudien von Grimminger $(2009,2012)$, im nordamerikanischen Sprachraum insbesondere auf die Studien von Sparks und Wayman (1993), Stanley (1996; 1997), Chepyator-Thomson, You und Russell (2000) sowie Columna, Foley und Lytle (2010) beschränkt. ${ }^{34}$ Aufgrund des geringen Forschungsvolumens werden die Studien im Folgenden getrennt nach Sprachraum und in chronologischer Reihenfolge präsentiert. Eine mögliche Einordnung der Studien in die im vorangegangenen Kapitel 3.1 vorgenommene Systematisierung von Forschungsarbeiten findet sich in Kapitel 3.3.

Für den deutschsprachigen Raum liefern bislang lediglich zwei Publikationen von Grimminger (2009, 2012) einschlägige Forschungsbefunde.

Als explorative Vorstudie im Hinblick auf die Entwicklung eines Fortbildungskonzepts zur Förderung interkultureller Kompetenz von Sportlehrpersonen eruierte Grimminger (2009) im Rahmen einer Interviewstudie mit $n=11$ deutschen Sportlehrpersonen, welche spezifischen Probleme sich für Sportlehrpersonen im Sportunterricht mit einer kulturell heterogenen Schülerschaft ergeben und wie sie damit umgehen. Als spezifische, von den Lehrpersonen berichtete Schwierigkeiten im Umgang mit Schülerinnen und Schülern mit Migrationshintergrund identifiziert Grimminger schlechtere Deutschkenntnisse, „erschwerte Elternarbeit mit Migranteneltern“, „ethnische Cliquenbildung“, „übermässige Reaktionen“ bzw. „Macho- und Mackerverhalten türkischer Jungen in Konfliktsituationen", "mangelnde Kenntnisse in traditionellen Sportarten“, „geringe oder gar keine Schwimmfähigkeiten“, die

\footnotetext{
33 Item 4: "In counseling parents who have a different cultural background than I do, I try to be considerate of cultural particularities". Item 5: "When meeting with parents of different cultural backgrounds, I spend more time trying to understand and empathize with their perspective". Item 9: "When there are conflicts between students of different origins, they should be encouraged to resolve the argument by finding common ground".

${ }^{34}$ Die in jüngerer Zeit in Deutschland vermehrt durchgeführten Forschungsarbeiten zu Haltungen, Einstellungen oder subjektiven Theorien von Sportlehrpersonen zu Inklusion im Sportunterricht (vgl. u.a. Leineweber, 2015; Meier \& Ruin, 2015; Meier, Ruin \& Leineweber, 2017; Rischke, Heim \& Gröben, 2017) verwenden als normativen Begründungsrahmen zwar einen mit Inklusion verbundenen Begriff der Vielfalt, der sämtliche Dimensionen von Heterogenität - und damit auch die Heterogenitätsdimension Kultur - miteinschliesst (z.B. Meier \& Ruin, 2015, S. 83; Meier et al., 2017, S. 4; Rischke et al., 2017, S. 2), fokussieren empirisch jedoch keine spezifische Heterogenitätsdimension bzw. erfassen Einstellungen und Haltungen der Lehrpersonen hinsichtlich heterogener Lerngruppen im Allgemeinen.
} 
„Nicht-Teilnahme muslimischer Mädchen am Sport- bzw. Schwimmunterricht" sowie die eingeschränkte körperliche Leistungsfähigkeit muslimischer Schülerinnen und Schüler während des Ramadans (S. 31f.). Grimminger bringt in diesem Zusammenhang an, dass - abgesehen von einer Ausnahme - alle Befragten die Verhaltensweisen von Schülerinnen und Schülern mit Migrationshintergrund mit deren kulturellem Hintergrund (weg)erklären bzw. die Schülerinnen und Schüler stereotypisierend wahrnehmen (vgl. ebd., S. 31). Auffällig, so stellt Grimminger weiter fest, sind dabei insbesondere auch geschlechtsstereotype Wahrnehmungen von Mädchen und Jungen mit türkischem Migrationshintergrund. Dass türkische Mädchen je nach Interviewpartner/in als „rebellisch“, als „Sportmuffel“ oder als „,begeisterte Fussballerinnen“ beschrieben werden, sieht Grimminger als Beweis dafür, „dass eine kulturalistische Zuschreibung ebenso wenig gerechtfertigt ist wie eine geschlechtsstereotype" (ebd., S. 31). ${ }^{35}$ Bezüglich des Umgangs mit kultureller Heterogenität stellt die Autorin (ebd., S. 32) in Anlehnung an die Typisierung von Bender-Szymanski (2001; vgl. Kap. 3.1.1) sowohl "ethnoorientierte" als auch „synergieorientierte" Verhaltensweisen der Lehrpersonen fest. Darüber hinaus identifiziert sie Interviewaussagen, die sie den Akkulturationseinstellungen Assimilation/Segregation und Integration zuordnet. Aussagen zur anteilsmässigen Verteilung der befragten Sportlehrpersonen auf die beiden Verhaltensweisen bzw. die unterschiedlichen Akkulturationseinstellungen werden jedoch keine gemacht. In der Publikation von 2012 präsentiert Grimminger zwei explorative Teilstudien mit $n=99$ (Studie 1) bzw. $n=18$ (Studie 2) deutschen Sportlehrpersonen zum Einfluss von Akkulturationseinstellungen (acculturation attitudes) und Überzeugungen über interkulturelles Lernen in der Schule und im Sportunterricht (educational beliefs) einerseits auf die Bereitschaft, interkulturelle Kompetenz bei den Schülerinnen und Schülern im Sportunterricht zu fördern (Studie 1), andererseits auf den tatsächlich realisierten Unterricht (Studie 2). Zur Erfassung der Akkulturationseinstellungen verwendete sie die (in Kap. 3.1.1 beschriebene) von Van Dick et al. (1997) entwickelte und validierte Akkulturationsskala. Die educational beliefs wurden mittels eines um einen dritten Teil „Sport und Interkulturalität“ erweiterten, ursprünglich von Sercu et al. (2005) zur Erfassung der Vorstellungen zu interkultureller Erziehung von Fremdsprachenlehrpersonen entwickelten Fragebogens erfasst (vgl. auch Grimminger, 2009). Eine Faktorenanalyse ergab bei den educational beliefs folgende drei Faktoren bzw. Überzeugungsvarianten: (1) Interkulturelle Erziehung in der Schule bewirkt etwas und ist idealerweise fächerübergreifend zu inszenieren, (2) Interkulturelle Erziehung in der Schule ist nur erforderlich bzw. sinnvoll, wenn die Klasse von Kindern und Jugendlichen mit Migrationshintergrund besucht wird, (3) Sportunterricht hat primär einen sportmotorischen Auftrag und eignet sich nicht zu interkultureller Erziehung (vgl.

\footnotetext{
${ }^{35}$ Weitere diesbezügliche Evidenzen liefert eine in der Schweiz durchgeführte Fallstudie zu Schülerinnen und Schülern mit Migrationshintergrund und deren Verhältnis zum Sportunterricht (vgl. Barker, Barker-Ruchti, Sattler, Gerber \& Pühse, 2011). Daraus geht hervor, dass sich Schülerinnen und Schüler gleicher Herkunft in Bezug auf ihre Motivation und ihr Engagement im Sport(unterricht) durchaus voneinander unterscheiden können.
} 
Grimminger, 2012, S. 9; vgl. auch Grimminger, 2009, S. 70f.). Die Faktorenanalyse der Items der Akkulturationseinstellungen spiegelte inhaltlich die Faktorenstruktur der von Van Dick et al. (1997) identifizierten Faktoren - separatives, assimilatives und pluralistisches Integrationsverständnis - wider (vgl. Grimminger, 2012, S. 9). Die Auswertungen (Korrelations- und Regressionsanalysen) hinsichtlich des Einflusses von educational beliefs und Akkulturationseinstellungen auf die Bereitschaft, interkulturelle Kompetenz der Schülerinnen und Schüler im Sportunterricht zu fördern (Studie 1), zeigten, dass educational beliefs einen signifikanten, Akkulturationseinstellungen hingegen keinen signifikanten Einfluss auf die Bereitschaft zur Förderung interkultureller Kompetenz haben. ${ }^{36}$ (Mögliche) Auswertungen hinsichtlich der anteilsmässigen Verteilung der Sportlehrpersonen auf die unterschiedlichen Faktoren bzw. Überzeugungsvarianten wurden weder für die educational beliefs noch für die Akkulturationseinstellungen vorgenommen.

Bezüglich der Frage, ob sich educational beliefs und Akkulturationseinstellungen auf den (über qualitative Interviews erfassten) tatsächlich realisierten Sportunterricht auswirken (Studie 2), verwiesen die Daten auf keine nennenswerten Zusammenhänge. Grimminger (2012) bemerkt diesbezüglich: "This means that there can be a self-reported willingness to implement intercultural learning in P.E. lessons; the performance indicators [acculturation attitudes, educational beliefs] can have a beneficial level, but from the teachers' point of view, school organizational as well as personal problems prevent the implication" (S. 11). Perspektivisch weist Grimminger deshalb darauf hin, "to integrate educational beliefs in the context of the social and institutional organization" (ebd., S. 12).

Zu den ersten einschlägigen Arbeiten im nordamerikanischen Sprachraum gehört die Fragebogenstudie Multicultural Understanding in Physical Education von Sparks und Wayman (1993). Sie untersuchten (u. a.) die Überzeugungen (attitudes) von in städtischen und ländlichen Regionen unterrichtenden $n=70$ Sportlehrpersonen hinsichtlich der Zielperspektive multicultural education. Als zentrale Ergebnisse stellen sie fest, dass Sportlehrpersonen aus dem städtischen Raum einem die ethnische Diversität berücksichtigenden Lehrplan mehr Bedeutung zusprechen als Sportlehrpersonen aus ländlichen Regionen. In Bezug auf Vorstellungen zum Konzept interkultureller Erziehung weisen die Resultate ausserdem darauf hin, dass Sportlehrpersonen aus ländlichen Regionen interkulturelles Lernen insbesondere als Kennenlernen verschiedener Bräuche und Traditionen verstehen, wohingegen Sportlehrpersonen aus urbanen Regionen vielmehr die Förderung kommunikativer Fähigkeiten für wichtig erachten.

\footnotetext{
${ }^{36}$ Bei den educational beliefs korrelierten die erste und zweite (oben genannte) Überzeugungsvariante signifikant positiv bzw. negativ mit der Bereitschaft zur Förderung interkultureller Kompetenz. Die im Zusammenhang mit den Akkulturationseinstellungen berechneten Regressionsanalysen wiesen keine der drei identifizierten Faktoren (separative Einstellung, assimilative Einstellung, pluralistische Einstellung) als signifikante Prädiktorvariable hinsichtlich der Kriteriumsvariable, interkulturelle Lern- und Bildungsprozesse im Sportunterricht inszenieren zu wollen (,Through my physical education lessons, I would like to promote intercultural competence of children and youth") aus (vgl. Grimminger, 2012, S. 8).
} 
Weiter entwickelte Stanley (1996) einen Fragenbogen zur Erfassung von Überzeugungen von Sportlehrpersonen über kulturelle Vielfalt. Das validierte Instrument, the Pluralism and Diversity Attitude Assessment (PADAA), umfasst insgesamt 19 Items verteilt über vier Subskalen. Die ersten drei Subskalen, (Appreciate Pluralism, Value Pluralism und Implement Pluralism), repräsentieren Art und Intensität der Auseinandersetzung mit kultureller Vielfalt im Sportunterricht - von (An-)Erkennung und Wertschätzung kultureller Vielfalt bis hin zur Absicht, kulturelle Vielfalt im Unterricht als Thema zu implementieren. Die vierte Subskala, Uncomfortable with Diversity, steht für eine Überzeugung, die kulturelle Vielfalt in der Gesellschaft und in der Schule grundsätzlich problematisiert.

In einer Folgestudie wandte Stanley (1997) das entwickelte Instrument bei $n=215$ angehenden Sportlehrpersonen an 15 amerikanischen Universitäten an. Dabei resultierten hohe Scores in der ersten und zweiten Subskala (Appreciate Pluralism, Value Pluralism) und tiefe Scores in der dritten und vierten Subskala (Implement Pluralism, Uncomfortable with Diversity). Stanley kommt zum Schluss: „Preservice teachers may personally respect and value cultural diversity but may not take actions or implement pedagogical practices that are culturally responsive“ (ebd., S. 244). Dieser Befund, so zeigen die Resultate weiter, trifft insbesondere auf die Subgruppe der männlichen Lehrpersonen zu.

Zehn Jahre später wandten auch Columna et al. (2010) den Fragenbogen in leicht modifizierter Form bei $n=433$ amerikanischen Sportlehrpersonen an. Ihre Analysen mit Fokus auf Subgruppenunterschieden (angehende Sportlehrpersonen, praktizierende Sportlehrpersonen, praktizierende Sportlehrpersonen mit spezieller Ausbildung in adapted physical education) machten deutlich, dass sich die Überzeugungen der befragten Lehrpersonen nicht in Abhängigkeit der Ausbildung und Berufserfahrung, sondern - analog zu den Ergebnissen der oben referierten Studie von Stanley (1997) - vielmehr geschlechtsspezifisch unterscheiden. So wiesen weibliche Sportlehrpersonen sowohl im Bereich der Anerkennung und Wertschätzung kultureller Vielfalt als auch im Bereich der Absicht, kulturelle Vielfalt im Unterricht berücksichtigen zu wollen, höhere Werte auf als männliche Sportlehrpersonen. Als mögliche Erklärung für diesen Befund nennen die Autoren eine historisch gewachsene höhere Sensibilität der Frauen hinsichtlich der Gefahr der Diskriminierung von Minderheitengruppen (vgl. ebd., S. 306). Chepyator-Thomson et al. (2000) befragten schliesslich $n=72$ Sportlehrpersonen nach deren Verständnis interkultureller Erziehung („How would you define multicultural education?“ (S. 560)). Eine qualitativ inhaltsanalytische Auswertung der Antworten ergab vier Typen von Lehrpersonen. Dem Typ non-committal wurden Lehrpersonen zugeordnet, die keine (deutliche) Antwort auf die Frage lieferten, dem Typ acquisition of conceptual knowledge solche, die interkulturelle Erziehung mit konzeptuell anzueignendem Wissen assoziieren, sich über ihren Unterricht aber nicht als zuständig dafür verstehen, dem Typ awareness of cultural diversity through teaching solche, die interkulturelle Erziehung grundsätzlich als eine unterrichtliche Angelegenheit interpretieren, und dem Typ inclusive education solche, die darüber hinaus konkretere Massnahmen zur Umsetzung interkultureller Erziehung im 
Sportunterricht (z.B. „include games and activities from other countries“ (ebd. 2000, S. 560f.)) nannten.

\subsection{Zusammenfassung der Forschungsergebnisse und Identifikation von For- schungslücken}

Die vorangegangenen Kapitel haben verdeutlicht, dass das Forschungsvolumen zu Überzeugungen Sport unterrichtender Lehrpersonen im Kontext migrationsbedingter Heterogenität bislang sehr gering geblieben ist. Mit Blick auf den deutschsprachigen Raum beschränken sich die Befunde auf drei explorative Teilstudien aus Deutschland (vgl. Grimminger, 2009, 2012). Untersuchungen aus der Schweiz liegen noch keine vor. Demgegenüber findet sich eine vergleichsweise grosse Zahl an Untersuchungen ausserhalb der Sportlehrerforschung bzw. mit anderen oder - und dies mehrheitlich - keinen spezifischen Fachbezügen. ${ }^{37}$ Aus diesen liessen sich drei zentrale Gegenstandsbereiche identifizieren, unter welche sich die verschiedenen Untersuchungen subsumieren lassen. Es sind dies:

- Akkulturationseinstellungen und Ideologien im Umgang mit migrationsbedingter Heterogenität (vgl. Kap. 3.1.1)

- Lehrerüberzeugungen über Schülerinnen und Schüler mit Migrationshintergrund (vgl. Kap. 3.1.2)

- Vorstellungen zur unterrichtlichen Umsetzung und Umsetzbarkeit interkultureller Erziehung (vgl. Kap. 3.1.3)

Im Folgenden werden zuerst die zentralen Befunde der diesen drei Gegenstandsbereichen zugeordneten Studien (ausserhalb der Sportlehrerforschung) zusammengefasst. Zur Identifikation von Forschungslücken werden daran anschliessend die in Kapitel 3.2 dargestellten Ergebnisse aus der Sportlehrerforschung der allgemeinen Forschungslage gegenübergestellt.

Studien zum Gegenstandsbereich der Akkulturationseinstellungen und Ideologien im Umgang mit migrationsbedingter Heterogenität befassen sich im Wesentlichen mit Einstellungen von Lehrpersonen zu „Art und Zielen der Koexistenz" verschiedener kultureller Gruppen in einem gesellschaftlichen Kontext (vgl. Van Dick et al., 1997, S. 83). Hintergrund dieser Untersuchungen ist die Annahme, dass sich solche

\footnotetext{
${ }^{37}$ In diesem Zusammenhang sei denn auch festgehalten, dass der dieser Arbeit zugrundliegenden allgemeinen Fragestellung bislang in verschiedenen (Teil-)Disziplinen (Pädagogische Psychologie, Sozial- bzw. Kulturpsychologie, Sozialanthropologie) nachgegangen wurde. Nicht zuletzt deshalb findet sich in den dokumentierten Arbeiten sowohl eine Vielzahl an Alias-Konstrukten (Einstellungen, Sichtweisen, Vorstellungen, Erwartungen etc.) für den hier als Leitkonzept verwendeten Begriff der Überzeugungen als auch ein vergleichsweise breites Spektrum an Forschungsdesigns.
} 
Einstellungen auf Strategien der Lehrpersonen im Umgang mit migrationsbedingter Heterogenität und damit auch auf ihr Unterrichtshandeln auswirken. Aus Studien, die sich dieser Fragerichtung angenommen haben, lassen sich dabei insbesondere Lehrerüberzeugungen im Sinne der Akkulturationsvarianten Assimilation (assimilative Überzeugungen), Egalitarismus (egalitäre Überzeugungen) und Integration/Multikulturalismus (integrativ-multikulturelle Überzeugungen) identifizieren.

Lehrpersonen mit assimilativen Überzeugungen vertreten die Haltung, dass sich Schülerinnen und Schüler mit Migrationshintergrund an die Normen und Bedingungen der Schule der Mehrheitsgesellschaft anpassen sollten. Aufgrund ihres dementsprechend monokulturell ausgerichteten Unterrichts nehmen sie Schülerinnen und Schüler mit Migrationshintergrund tendenziell als defizitär wahr und sehen ihre Rolle als Lehrperson gegenüber diesen Schülerinnen und Schülern insbesondere in der „Herstellung von Unterrichtsfähigkeit" (Lanfranchi, 2013, S. 243). Mögliche Folgen assimilativer (im Vergleich zu integrativ-multikulturellen) Überzeugungen können erhöhte Abgrenzungstendenzen innerhalb der Schülerschaft und ein höheres Ausmass an Bestrafungen sowie eine geringere Diagnosekompetenz für soziale Spannungen in der Klasse sein (vgl. Makarova \& Herzog, 2013; Wagner et al., 2001). Egalitäre Überzeugungen im Sinne von Colorblindness entsprechen der Vorstellung, dass Schülerinnen und Schüler ungeachtet ihres kulturellen Hintergrunds gleichbehandelt werden sollten. Kulturelle Unterschiede werden dabei im Hinblick auf einen pädagogisch angemessenen Umgang mit migrationsbedingter Heterogenität als gleichsam irrelevant betrachtet. Entsprechend gering ist die Bereitschaft, den Unterricht auf die migrationsbedingt heterogene Schülerschaft anzupassen. Vertreter egalitärer Überzeugungen scheinen zudem insbesondere Lehrpersonen unterer Schulstufen, aus Ländern mit ohnehin egalitären Selbstverständnissen (z.B. Frankreich) und ohne Migrationshintergrund zu sein (vgl. Hachfeld, 2013; Hachfeld et al., 2015; Sievers, 2012; Sleeter, 2001). Integrativ-multikulturelle Überzeugungen liegen schliesslich dann vor, wenn Lehrpersonen der Überzeugung sind, dass migrationsbedingte Heterogenität in der Schülerschaft grundsätzlich ein (Lern-)Potenzial darstellt. Entsprechend hoch ist die Bereitschaft, andere Standpunkte zuzulassen, diese zu thematisieren und das eigene - als kulturgebunden erkannte - Denken und Handeln zu reflektieren (vgl. Bender-Szymanski, 2001). Studien auf Basis numerischer Daten zeigten dabei, dass integrativ-multikulturelle Überzeugungen negativ mit der Neigung zu Bestrafungen und zu negativen Vorurteilen gegenüber Schülerinnen und Schülern mit Migrationshintergrund korrelieren (vgl. Hachfeld et al., 2015; Wagner et al., 2001). Darüber hinaus weisen Lehrpersonen mit integrativ-multikulturellen Überzeugungen (gegenüber solchen mit egalitären Überzeugungen) eine höhere Selbstwirksamkeitserwartung, einen höheren Enthusiasmus, Schülerinnen und Schüler mit Migrationshintergrund zu unterrichten, und eine höhere Bereitschaft, ihren Unterricht der kulturell heterogenen Schülerschaft anzupassen, auf (vgl. Hachfeld et al., 2015). Insgesamt liegen keine verlässlichen Befunde zur anteilsmässigen Verteilung der Akkulturationseinstellungen und Ideologien im Umgang mit migrationsbedingter Heterogenität vor. 
Dem Gegenstandsbereich Überzeugungen über Schülerinnen und Schüler mit Migrationshintergrund wurden insbesondere Studien mit Fokus auf die Akkuratesse von Lehrerurteilen hinsichtlich Schulleistungen und/oder Intelligenz oder hinsichtlich formeller Lehrerurteile (Benotung, Selektionsentscheide) subsumiert (vgl. Carigiet Reinhard, 2012). Hintergrund dieser Untersuchungen ist der nachgewiesene Effekt von Lehrererwartungen auf das Lehrerverhalten (teacher expectation effects) bzw. auf die Notengebung und Selektionsentscheide. Unter Berücksichtigung objektiv erfasster Schülerleistungen bzw. Intelligenztests lassen sich dabei Aussagen darüber machen, ob (ethnische) Stereotype, verzerrte Erwartungen bzw. Vorurteile seitens der Lehrpersonen vorliegen bzw. ob dem Anspruch einer chancengerechten Schule entsprochen wird. Für den US-amerikanischen Sprachraum liess sich meta-analytisch ein (kleiner) signifikanter Effekt des ethnischen Hintergrunds der Schülerinnen und Schüler auf die Lehrererwartungen bezüglich akademischer Leistung und/oder sozialem Verhalten errechnen (vgl. Tenenbaum \& Ruck, 2007). Dabei scheinen Lehrpersonen die höchsten Erwartungen gegenüber Schülerinnen und Schülern mit asiatisch-amerikanischem Hintergrund (Asian american children), gefolgt von solchen europäisch-amerikanischer (European American children), lateinamerikanischer (Latino/a children) und schliesslich afro-amerikanischer (African American children) Herkunft zu haben. Weitere, auf Basis verbaler Daten durchgeführte Studien gelangten zum Ergebnis, dass Schülerinnen und Schüler mit Migrationshintergrund (im Vergleich zu solchen ohne Migrationshintergrund) von den Lehrpersonen generell als weniger lernmotiviert eingeschätzt werden, und stellen darüber hinaus eine autoritärere und enger kontrollierte Unterrichtspraxis der Lehrpersonen gegenüber Schülerinnen und Schüler mit Migrationshintergrund fest (vgl. Bryan \& Atwater, 2002; Olmedo, 1997). Für den deutschsprachigen Raum resultierten aus mehreren (u. a. in der Schweiz durchgeführten) Studien systematisch verzerrte Erwartungen zuungunsten von Schülerinnen und Schülern mit Migrationshintergrund bzw. mit tieferer Schichtzugehörigkeit (vgl. Bonefeld, Dickhäuser, Janke, Praetorius \& Dresel, 2017; Carigiet Reinhard, 2012; Jungbluth, 1994; Kronig, 2007; Kronig et al., 2000; Lorenz et al., 2016; Moser \& Rhyn, 1999). Vulnerabel scheinen dabei insbesondere Schülerinnen und Schüler aus Südosteuropa in Bezug auf ihre Leistungen im Fach Deutsch zu sein (vgl. Carigiet Reinhard, 2012; Lorenz et al., 2016). Ergänzend zu diesen auf Basis numerischer Daten durchgeführten Studien liefern auch qualitative Interviewstudien Hinweise auf verzerrte Leistungserwartungen bzw. vorurteils- und stereotypbehaftete Sichtweisen von Lehrpersonen (vgl. Bender-Szymanski, 2001, 2013; Marburger et al., 1997; Sievers, 2009).

In Arbeiten zu Vorstellungen zur unterrichtlichen Umsetzung und Umsetzbarkeit interkultureller Erziehung wurde den Fragen nach dem subjektiven Verständnis interkultureller Erziehung (Was) und insbesondere der Art und Weise, wie diese Zielorientierung im Unterricht umgesetzt wird bzw. werden 
kann, nachgegangen. In Bezug auf subjektive Verständnisse interkultureller Erziehung bleiben die Befunde auf floskelhafte Aussagen einzelner Lehrpersonen aus einer Studie von Binder und Daryabegi (2002) beschränkt. Studien zu Vorstellungen über die methodische Umsetzung interkultureller Erziehung bringen übereinstimmend zum Ausdruck, dass Lehrpersonen insbesondere die Anwesenheit fremdsprachiger Schülerinnen und Schüler als Ausgangspunkt für interkulturelle Erziehung sehen. Als förderlich für interkulturelles Lernen werden in verschiedenen Studien zudem Unterrichtsformen wie (fächerübergreifender) Projektunterricht, Austausch in Klassengesprächen oder Referate über das eigene Herkunftsland genannt. Darüber hinaus sehen die Lehrpersonen in Sprachvergleichen, in Liedern und Musik sowie in der Thematisierung verschiedener Feiertage und weiterer "folkloristischer" Aspekte Potenzial für interkulturelles Lernen. Als generell limitierende Faktoren für interkulturelles Lernen werden Leistungsdruck, fehlende Zeitressourcen und/oder zu grosse Klassen genannt (vgl. Binder \& Daryabegi, 2002; Edelmann, 2008; Sievers, 2009). Bezüglich der Eignung von Fächern für interkulturelles Lernen bzw. der Umsetzbarkeit interkultureller Erziehung erweisen sich die Befunde als nicht einhellig.

Mit Blick auf die Identifikation von Forschungslücken innerhalb des dieser Arbeit zugrundeliegenden, noch wenig erforschten (fach-)spezifischen Erkenntnisgegenstands - Überzeugungen Sport unterrichtender Lehrpersonen angesichts migrationsbedingter Heterogenität - stellt sich insbesondere die Frage nach der Übertragbarkeit der eben berichteten Befunde auf den (Lehr-Lern-)Kontext des Sportunterrichts. Angesichts des hohen Anteils an Studien, in denen keine spezifische Fachperspektive eingenommen wurde, erscheint die Übertragbarkeit zumindest auf den ersten Blick unbedenklich.

Für den Bereich der Akkulturationseinstellungen und Ideologien im Umgang mit migrationsbedingter Heterogenität dürfte dies tatsächlich auch unproblematisch sein. So konnte Grimminger sowohl über Interviews (vgl. ebd., 2009) als auch unter Verwendung von Fragebogen (vgl. ebd., 2012) ähnliche Einstellungsmuster und -varianten bei Sportlehrpersonen feststellen, wie sie auch in anderen Studien ohne spezifische (oder mit anderer) Fachperspektive festgestellt wurden. Als allgemeinere, aus der Sozial- bzw. Kulturpsychologie auf den Schulkontext übertragene Einstellungen zur Koexistenz von Mitgliedern verschiedener kultureller Gruppen lassen sich Akkulturationseinstellungen von Lehrpersonen demnach nicht nur aus theoretischer, sondern auch empirischer Sicht grundsätzlich als fachunabhängige Überzeugungen angesichts migrationsbedingter Heterogenität fassen.

Demgegenüber sprechen sowohl theoretische als auch empirische Argumente für eine fachspezifische Betrachtung von Lehrerüberzeugungen über Schülerinnen und Schüler mit Migrationshintergrund. So lassen sich schülerbezogene Überzeugungen konzeptionell als Lehrererwartungen fassen, die sich insbesondere auf die fachliche Leistung und das Sozialverhalten von Schülerinnen und Schülern in einem spezifischen Fachkontext beziehen (vgl. Kap. 3.1.2). Empirische Befunde verweisen dabei insbesondere 
auf unterschiedliche Leistungserwartungen an Schülerinnen und Schüler mit Migrationshintergrund in den Fächern Deutsch und Mathematik (z.B. Carigiet Reinhard, 2012). Darüber hinaus kamen auch in der Studie von Grimminger (2009) offensichtlich auf den Lehr-Lern-Kontext des Sportunterrichts bezogene Lehrererwartungen (z.B. „mangelnde Kenntnisse in traditionellen Sportarten“ (ebd., S. 31)) gegenüber Schülerinnen und Schülern mit Migrationshintergrund zum Ausdruck.

Wie die Datenlage zeigt, ist schliesslich auch von fachspezifisch unterschiedlichen Lehrervorstellungen zur unterrichtlichen Umsetzung und Umsetzbarkeit interkultureller Erziehung auszugehen. Obwohl zwar ähnliche Vorstellungen hinsichtlich der Zielorientierung interkultureller Erziehung und deren Umsetzung zu bestehen scheinen, wird zumindest die Frage nach der Umsetzbarkeit interkultureller Erziehung (in einem bestimmten Fach) von Lehrpersonen uneinheitlich beantwortet: Während Grimminger (2009) Vorstellungen von Sportlehrpersonen eruiert, in denen dem Sportunterricht ein rein sportmotorischer Auftrag und kein Potenzial für interkulturelle Erziehung zugeschrieben wird, schätzen die von Marburger et al. (1997) befragten Lehrpersonen das Fach Sport einhellig als sich für die Berücksichtigung von Minderheitenkulturen eignend ein.

Zusammenfassend ist festzuhalten, dass sich die Forschungslage zu Überzeugungen von Sport unterrichtender Lehrpersonen angesichts migrationsbedingter Heterogenität somit nicht nur aufgrund des geringen sportspezifischen Forschungsvolumens, sondern insbesondere auch deshalb, weil sich ein Grossteil der vergleichsweise umfassenden, jedoch nicht sportspezifischen Forschungsergebnisse nicht auf den Lehr-Lern-Kontext des Sportunterrichts übertragen lassen, als prekär erweist. Hinzu kommt - ergänzend zu den obigen Ausführungen und in Anlehnung an die Resultate der ländervergleichenden Studie von Sievers (2009) -, dass Überzeugungen von Lehrpersonen im Zusammenhang mit migrationsbedingter Heterogenität vor dem Hintergrund unterschiedlicher gesellschafts- bzw. bildungspolitischer Kontexte zu deuten sind. Befunde aus unterschiedlichen nationalen bzw. sprachkulturellen Kontexten oder auch aus unterschiedlichen Phasen demographischer Zustände innerhalb eines Landes sind demnach nur schwer verallgemeinerbar. Mit anderen Worten: Wenngleich empirische Ergebnisse (aus explorativen Teilstudien) zu Überzeugungen von deutschen Sportlehrpersonen angesichts migrationsbedingter Heterogenität vorliegen (vgl. Grimminger, 2009), so lassen sich diese kaum auf die bislang noch unerforscht gebliebenen Überzeugungen von Schweizer Sport unterrichtenden Lehrpersonen übertragen. Wie die damit identifizierte Forschungslücke verringert werden soll, wird im folgenden Kapitel näher erläutert. 


\section{Präzisierung der Fragestellung}

In diesem Kapitel soll die in der Einleitung formulierte, forschungsleitende Fragestellung unter Berücksichtigung des aktuellen Forschungsstandes bzw. der im vorangegangenen Kapitel identifizierten Forschungslücke präzisiert bzw. konkretisiert werden.

Das dieser Arbeit zugrundeliegende Forschungsinteresse ist die Erforschung der Überzeugungen Sport unterrichtender Lehrpersonen angesichts migrationsbedingter Heterogenität im Sportunterricht. Ausgehend davon wurde folgende, forschungsleitende Fragestellung formuliert: Welche Überzeugungen haben Sport unterrichtende Lehrpersonen angesichts der migrationsbedingten Heterogenität im Sportunterricht? (vgl. Kap. 1.1).

Die Erfassung und Analyse des aktuellen Forschungsstandes machte deutlich, dass insgesamt noch kaum Forschungsergebnisse zur Beantwortung dieser Frage vorliegen. Als besonders prekär erweist sich dabei die Forschungslage zu Überzeugungen über Schülerinnen und Schüler mit Migrationshintergrund sowie zu Vorstellungen über die unterrichtliche Umsetzung und Umsetzbarkeit interkultureller Erziehung im Sportunterricht. Um diese Forschungslücke ansatzweise zu schliessen, wurde entschieden, den empirischen Fokus dieser Arbeit auf diese beiden Gegenstandsbereiche zu legen. Im Folgenden werden die pro Gegenstandsbereich formulierten, konkreten Fragestellungen bzw. ihre Ausdifferenzierungen in Teilfragen näher erläutert.

\section{(1) Überzeugungen über Schülerinnen und Schüler mit Migrationshintergrund im Sportunterricht}

Lehrerüberzeugungen über Schülerinnen und Schüler lassen sich konzeptionell als Lehrererwartungen fassen. Diese wiederum bezeichnen Schlüsse, welche die Lehrpersonen aufgrund bisheriger Erfahrungen mit und Informationen über einzelne Schülerinnen und Schüler (mit Migrationshintergrund) bezüglich schulisch relevanter Aspekte (fachliche Leistung, Sozialverhalten) ziehen (vgl. Schweer \& Thies, 2000, S. 65; Ulich, 2001, S. 86f.). Sie fungieren lehrerseitig als Wahrnehmungsfilter und ermöglichen damit eine rasche Eindrucksbildung zu „Persönlichkeitsmerkmalen, Eigenschaften, Einstellungen und Fähigkeiten der Schüler/innen (...)“ (Ziegler et al., 1998, S. 273) (vgl. Kap. 3.1.2). Zur empirischen Untersuchung von Lehrerüberzeugungen über Schülerinnen und Schüler mit Migrationshintergrund im Sportunterricht wurde ausgehend von diesen konzeptionellen Überlegungen folgende erste konkrete Fragestellung formuliert:

Fragestellung 1: Wie nehmen Sport unterrichtende Lehrpersonen Schülerinnen und Schüler mit Migrationshintergrund im Sportunterricht wahr? 
Da gemäss den rezipierten und hier vorgestellten empirischen Studien Schülerinnen und Schüler mit Migrationshintergrund im Unterricht anders wahrgenommen werden als solche ohne Migrationshintergrund, wurde obige Frage in folgende Teilfragestellungen ${ }^{38}$ ausdifferenziert:

a) Unterscheiden sich aus Sicht der Lehrpersonen Schülerinnen und Schüler mit Migrationshintergrund von solchen ohne Migrationshintergrund?

b) Falls ja-wodurch (unterscheiden sich Schülerinnen und Schüler mit Migrationshintergrund von solchen ohne Migrationshintergrund)?

(2) Vorstellungen zur unterrichtlichen Umsetzung und Umsetzbarkeit interkultureller Erziehung im Sportunterricht

Vorstellungen von Lehrpersonen zur unterrichtlichen Umsetzung und Umsetzbarkeit interkultureller Erziehung betreffen die Fragen nach dem subjektiven Verständnis interkultureller Erziehung (Was) und der (davon abhängigen) Art und Weise, wie diese Zielorientierung im Unterricht umgesetzt wird bzw. werden kann (vgl. Kap. 3.1.3). Mit Blick auf das Lernen der Schülerinnen und Schüler geht es dabei um Lehrervorstellungen darüber, ob und inwiefern interkulturelles Lernen im Unterricht gefördert werden kann. Um Aussagen über das diesbezüglich spezifische Potenzial des Sportunterrichts aus Sicht der Lehrpersonen machen zu können, wurde folgende zweite konkrete Fragestellung formuliert:

Fragestellung 2: Welche Vorstellungen zum Potenzial des Sportunterrichts für interkulturelles haben Sport unterrichtende Lehrpersonen?

Da Vorstellungen zum Potenzial des Sportunterrichts für interkulturelles Lernen einerseits Vorstellungen hinsichtlich interkulturellen Lernens generell förderlicher Unterrichtsbedingungen, andererseits Vorstellungen zu den spezifischen Gegebenheiten des Fachs Sport bzw. zum Sportunterricht umfassen, wurde die Fragestellung in folgende zwei Teilfragestellungen weiter ausdifferenziert:

a) Welche unterrichtlichen Bedingungen stellen aus Sicht der Lehrpersonen generell Chancen für interkulturelles Lernen dar?

b) Welche Chancen interkulturellen Lernens treffen aus Sicht der Lehrpersonen spezifisch auf den Sportunterricht zu?

\footnotetext{
${ }^{38}$ Auf die Formulierung von Forschungshypothesen wurde hier aufgrund kaum vorliegender bzw. auf den Sportunterricht kaum übertragbarer Vorarbeiten explizit verzichtet. 


\section{Methode}

Ausgehend von den beiden konkreten Fragestellungen dieser Arbeit (vgl. Kap. 4) beschreibt dieses Kapitel zuerst die wesentlichen Aspekte der in dieser Untersuchung angewandten Repertory Grid-Technik (RGT) (Kap. 5.1). In einem ersten Kapitel wird zunächst begründet, weshalb sich dieses Datenerfassungsverfahren zur Beantwortung der Fragestellungen eignet (Kap. 5.1.1). Aufgrund der geringen Standardisierung des Verfahrens werden sodann die Hauptschritte der Vorbereitung und Durchführung der RGT - mit Berücksichtigung der für diese Arbeit relevanten Variationsmöglichkeiten - etwas ausführlicher erläutert (Kap. 5.1.2).

Kapitel 5.2 beschreibt die Kriterien für die Auswahl der Untersuchungsteilnehmenden sowie den Prozess der Stichprobengewinnung. Die für die beiden Fragestellungen je eigens entwickelten Instrumente werden in Kapitel 5.3, die im Zusammenhang mit der Untersuchungsdurchführung stehenden Aspekte in Kapitel 5.4 geschildert. Kapitel 5.5 bezieht sich schliesslich auf die Auswertungsstrategien und die entsprechenden Analyseverfahren.

\subsection{Die Repertory Grid-Technik}

\subsubsection{Zur Wahl der Repertory Grid-Technik}

Die Erfassung von Lehrerüberzeugungen scheint grundsätzlich an keine verbindliche Methodik gebunden zu sein. Obwohl qualitative Untersuchungen dominieren (vgl. Reusser et al., 2014), umfasst die Erforschung von Lehrerüberzeugungen das gesamte Methodenspektrum quantitativer und qualitativer Sozialforschung. ${ }^{39}$

Zu den bislang angewandten sowie in Übersichtsartikeln (vgl. Calderhead, 1996; Maag Merki \& Werner, 2011; Priemer, 2006) empfohlenen qualitativen Methoden gehören auf Stimuli (z. B. eigene Unterrichtsvideos, fremde Videovignetten) basierende Interviewtechniken (think-aloud commentaries, stimulated recall, teachers' talk, narratives), Strukturlegetechniken (Dialog-Konsens-Verfahren, concept mapping), Repertory Grid-Verfahren, die Auswertung von Dokumenten (Unterrichtsjournale, Portfolios), Beobachtungsformen sowie ethnographische Fallstudien (z.B. Fischler, Schröder, Tonhäuser \& Zedler, 2002; Groeben \& Scheele, 2010; Leuchter, 2009).

\footnotetext{
${ }^{39}$ Grund dafür ist das weite Spektrum der Inhaltsbereiche der Lehrerüberzeugungen. So variiert die methodische Vorgehensweise einerseits in Abhängigkeit des unterschiedlich weit vorangeschrittenen Forschungsstandes, andererseits aber auch aufgrund der unterschiedlichen (theoretischen) Konstrukte, die innerhalb der verschiedenen Inhaltsbereiche untersucht werden.
} 
Quantitative Methoden sind in jüngerer Zeit insbesondere im Rahmen gross angelegter Studien zur professionellen Kompetenz von Lehrpersonen (vgl. Blömeke, Kaiser, et al., 2008; OECD, 2009) eingesetzt worden (vgl. auch Reusser \& Pauli, 2014).

Lehrerüberzeugungen, die sich - wie in dieser Arbeit - auf die migrationsbedingte Heterogenität in der Schule beziehen, wurden bislang ebenfalls sowohl quantitativ als auch qualitativ erfasst.

Im Bereich der quantitativen Methoden scheint die Erfassung der Lehrerüberzeugungen mittels Fragebogen zu dominieren. Letztere sind jedoch häufig kaum theoriegeleitet (z.B. Jungbluth, 1994, 1997; Lanfranchi, 2007; Wagner et al., 2001; Walter, 1999), verfügen über keine zufriedenstellenden psychometrischen Eigenschaften (z.B. Diehm \& Kodron, 1990) oder berichten diese nicht (z.B. Wischmeier, 2012) (vgl. auch Hachfeld, 2013). Ausnahmen bilden diesbezüglich die Teacher Cultural Beliefs Scale (TCBS) von Hachfeld et al. (2011), die Multicultural Efficacy Scale (MES) von Guyton und Wesche (2005) sowie die Akkulturationsskala von Van Dick et al. (1997). Alle drei Skalen umfassen jedoch Items verschiedener Gegenstandbereiche von Lehrerüberzeugungen und bleiben damit sehr allgemein (vgl. Kap. 2.2). Fach- bzw. sportspezifische Überzeugungen - wie sie in dieser Arbeit interessieren - lassen sich mit ihnen nicht erfassen. Schliesslich bleibt fraglich, ob Überzeugungen - verstanden als tendenziell implizite Wissensform (vgl. Kap. 2.1.2) - über die Erfassung mittels Fragebogen überhaupt zugänglich sind.

Standard der qualitativen Erfassung von Überzeugungen angesichts migrationsbedingter Heterogenität scheinen (problemzentrierte) Leitfadeninterviews zu sein (z.B. Bender-Szymanski, 2013; Edelmann, 2008; Hallitzky \& Schiessleder, 2008; Heintze et al., 1997; Sievers, 2009). Ausnahmen bilden die Studien von Olmedo (1997), Auernheimer, Van Dick, Petzel und Wagner (2001) und Merz-Atalik (2001), in welchen die Daten aus Journalen und Essays, aus Gruppendiskussionen sowie mittels Struktur-LegeTechnik gewonnen wurden. Insgesamt bleiben aber auch hier die untersuchten Konstrukte („Erfahrungen“, „Erkenntnisse“, „Strategien“, „Sichtweisen“, „Einschätzungen“, „Orientierungen“, „Einstellungen“, „Interpretationen“) vielfach in einer alltagsprachlichen Verwendung (z.B. Auernheimer et al., 2001; Edelmann, 2008; Heintze et al., 1997; Sievers, 2009). Eine theoretische Auseinandersetzung mit dem zu untersuchenden Konstrukt und eine darauf aufbauende Argumentation für ein valides Erhebungsverfahren bleiben aus.

Im Gegensatz dazu erfolgte die Wahl der Untersuchungsmethode in der vorliegenden Arbeit u. a. aufgrund einer theoretischen Erörterung des zu erfassenden Konstrukts. Die Wahl fiel dabei aus folgenden Gründen auf die RGT:

Erstens lag dem Erkenntnisinteresse dieser Untersuchung ein theoretisches Konstrukt - Lehrerüberzeugungen - zugrunde, von dem angenommen wird, dass es oft in impliziter und damit nur schwer artikulierbarer Form vorliegt (vgl. Herman, 1996, S. 440; Hillier, 1998; Munby, 1982, S. 217; Reusser \& 
Pauli, 2014, S. 646; vgl. auch Kap. 2.1.2). Die RGT gilt aufgrund ihrer speziellen Fragetechnik als ein Datenerhebungsverfahren, das sich besonders zur Erfassung solch „präverbal“ vorliegenden Wissens eignet (vgl. Burr, King \& Butt, 2014, S. 343; Fransella, Bell \& Bannister, 2004, S. 3; Fromm, 1995, S. 203; 2010, S. 527; Scheer, 1993, S. 32). Zweitens erforderte der bislang kaum erforschte Untersuchungsgegenstand eine explorative Orientierung. Die RGT als Interviewform mit offenen Fragen gewährleistet die dafür notwendige Offenheit. Drittens erhöht die RGT als strukturiertes Interview die Vergleichbarkeit der von mehreren Personen erhobenen Daten.

\subsubsection{Hauptschritte der Repertory Grid-Technik}

Die RGT ist eine spezifische Interviewtechnik. Sie wurde von George A. Kelly (1991) zur Erfassung persönlicher Konstrukte einer Person entwickelt. Kurz zusammengefasst, wird die Untersuchungsperson aufgefordert, eine Serie von Unterscheidungen zwischen verschiedenen Elementen (= Personen, Objekte, Situationen) vorzunehmen und zu benennen. Dabei repräsentieren die Elemente das im Interesse einer Erhebung stehende Thema bzw. einen bestimmten Erfahrungsbereich der befragten Person. Das so zustande kommende, in einer Matrix (Grid) dargestellte Repertoire (repertory) an persönlichen Konstrukten entspricht der subjektiven Bedeutung und Struktur, die der Erfahrungsbereich für die befragte Person hat.

Seit der Entwicklung der Urform der $\mathrm{RGT}^{40}$ hat das Verfahren viele weitere Anwendungsgebiete gefunden. Dazu gehören neben der Lehrerforschung u. a. das Marketing und die Marktforschung, die Organisationsentwicklung und das Management, die Politik, die Sportpsychologie oder auch die Berufsberatung (vgl. Fransella et al., 2004). Angesichts dieser Anwendungsbreite ist es wenig verwunderlich, dass heute eine Vielzahl spezifischer Vorgehensweisen der RGT existiert. Ihnen allen ist jedoch gemeinsam, dass sie sich in eine Vorbereitungs-, eine Durchführungs- und eine Auswertungsphase gliedern (vgl. Abb. 5). Im Folgenden werden die einzelnen Schritte der Vorbereitungs- und Durchführungsphase anhand eines fiktiven Beispiels, nämlich der Identifikation möglicher Einflussfaktoren der Studienfachwahl angehender Studierender, erläutert. Auf die Auswertungsmöglichkeiten von Rohgrids wird in Kapitel 5.5 eingegangen.

\footnotetext{
40 Urform aller Varianten der RGT ist der sogenannte Role Construct Repertory Test (Rep Test) (vgl. Kelly, 1991, S. 152 f.). Dieses ursprünglich für die Psychotherapie entwickelte Instrument diente der Identifikation schwieriger Aspekte im (sozialen) Leben von Personen. Als Elemente wurden hier Personen gewählt, die im Leben des/r Befragten eine bestimmte Rolle, d.h. eine spezifische soziale Beziehung zum/r Befragten (z.B. die Mutter; eine Lehrperson, die man mochte; die erfolgreichste Person, die man kennt) haben/hatten. Im gleichen Kapitel forderte Kelly jedoch explizit zur Weiterentwicklung seines Rep Tests auf (vgl. ebd., S. 160).
} 


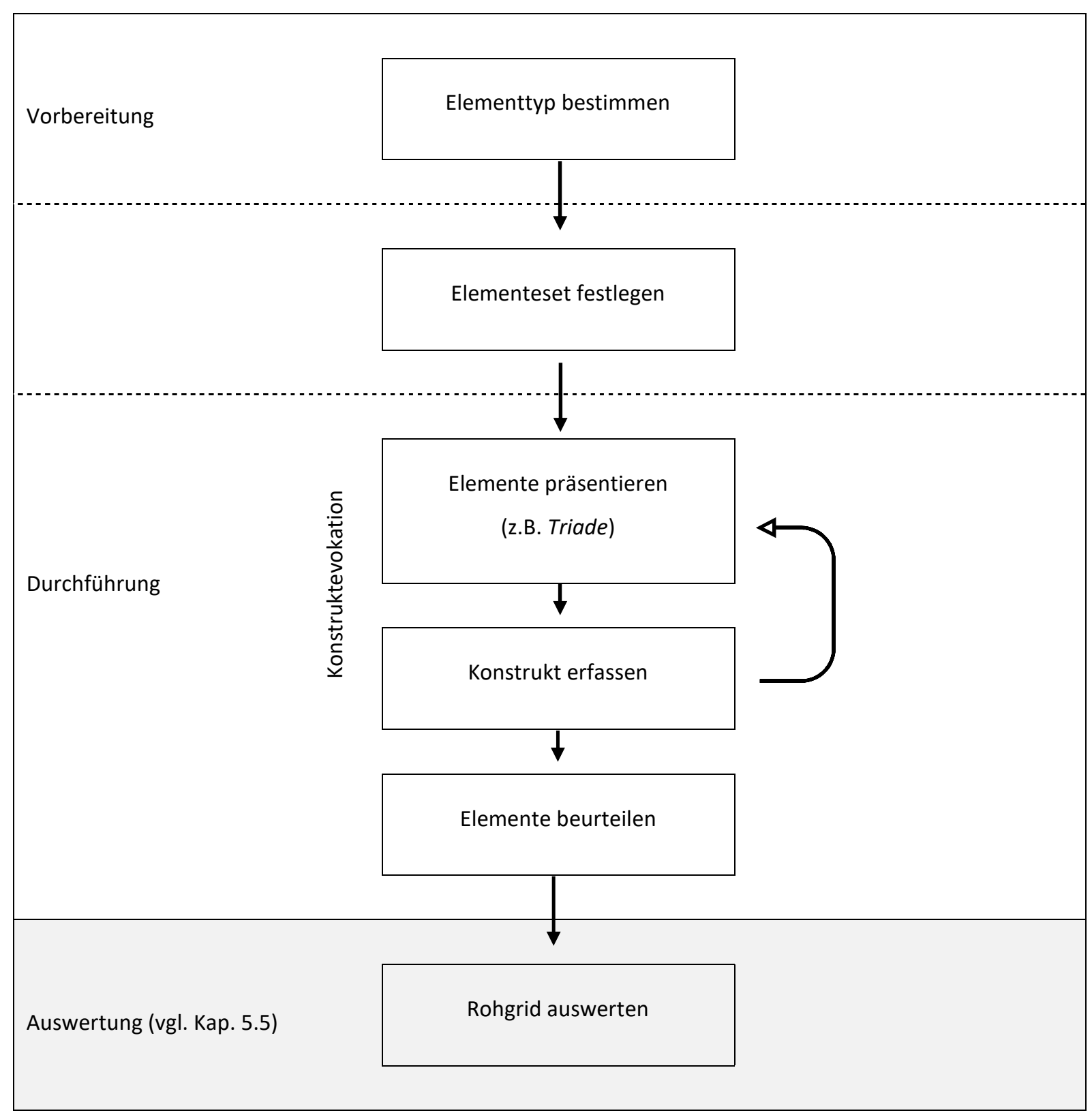

Abbildung 5. Schematische Darstellung der Hauptschritte einer Repertory Grid-Untersuchung. Quelle: Verändert nach Fromm (2010, S. 529); Rosenberger und Freitag (2009, S. 481)

\subsubsection{Schritte der Vorbereitung}

Ziel der RGT ist die Erfassung der wichtigsten (handlungsleitenden) Dimensionen (Konstrukte), mit denen eine Person subjektiv einen bestimmten Erfahrungsbereich wahrnimmt und strukturiert. Im fiktiven Beispiel geht es demzufolge darum, Dimensionen zu erfassen, die angehende Studierende zur Beurteilung von Studienfächern heranziehen. Diese lassen sich schliesslich als mögliche Einflussfaktoren der Studienfachwahl interpretieren. 


\section{Elementtyp bestimmen}

Zentraler, vorbereitender Schritt einer Repertory Grid-Untersuchung ist die Bestimmung des Elementtyps. Gemäss PCP sind Elemente für ein Individuum bedeutsame Dinge oder Ereignisse („things or events" (Kelly, 1991, S. 95)), die durch persönliche Konstrukte abstrahiert werden. Sollen also Konstrukte einer Person zu einem bestimmten Thema (z.B. Studienfächer) erfasst werden, muss dieses in Elemente (z.B. „Physik“, „Sportwissenschaft“, „Archäologie“ usw.) aufgeschlüsselt werden, von denen ausgegangen werden kann, dass sie das Thema exemplarisch repräsentieren. Fransella et al. (2004) erwähnen verschiedene (in Untersuchungen verwendete) Elementtypen wie Autos, Kunstgegenstände, Krankheiten, Angst auslösende Situationen, Länder, Freizeitaktivitäten, Verwandte oder Berufsgruppen und verdeutlichen damit, dass ,elements that are used in repertory grids can be almost anything (...)“(S. 21).

\section{Elementeset festlegen}

Der zweite Schritt einer Repertory Grid-Untersuchung besteht im Festlegen des Elementesets. Hier geht es im Wesentlichen darum, die einzelnen Elemente, die später zur Konstrukterfassung präsentiert werden sollen, festzulegen. Je nachdem, ob dies durch die befragende oder durch die zu befragende Person vorgenommen wird, gehört dieser Schritt noch zur Vorbereitung oder er ist bereits Bestandteil der Durchführung (vgl. gepunktete Linien in Abb. 5). Der Entscheid für die eine oder andere Vorgehensweise - Elemente vorgeben vs. Elemente erheben - hängt primär von der Intention der Untersuchung ab:

Ist die Untersuchung idiographisch-qualitativ orientiert, d.h. liegt das Interesse bei Einzelfällen, werden die Elemente üblicherweise von der befragten Person festgelegt (vgl. Rosenberger \& Freitag, 2009, S. 482). Für das verwendete Beispiel der Studienfachwahl bedeutete dies, dass die angehenden Studierenden je individuell ihr eigenes Set von bspw. sechs Studienfächern festlegen würden.

Soll hingegen eine grössere Anzahl Personen zum selben Thema befragt werden, setzt dies eher ein für alle Befragten standardisiertes Elementeset voraus. Dies kann entweder so geschehen, dass die Elemente vom Befrager/von der Befragerin deduktiv aus der Literatur bzw. aus bestimmten Thesen zum Untersuchungsgegenstand abgeleitet werden oder aber von ausgewählten Befragten (bspw. mittels Gruppendiskussion oder leitfadengestützten Interviews) zunächst erhoben werden, um dann einen einheitlichen Elementsatz für alle Befragten zu verwenden (vgl. Rosenberger \& Freitag, 2009, S. 482). Im Beispiel der Studienfächer könnte bspw. eine Studie vorliegen, welche Aufschluss über die Anzahl der Studierenden pro Studienfach gibt. Nun könnte interessieren, wie Studienfächer mit hohen Studierendenzahlen gegenüber solchen mit niedrigen Studierendenzahlen von angehenden Studierenden wahrgenommen werden. Ausgehend davon könnte der/die Befrager/in ein Set von Studienfächern festlegen, das Fächer mit unterschiedlichen Studierendenzahlen umfasst. 
Unabhängig von den beiden Vorgehensweisen der Festlegung des Elementesets sollte sichergestellt werden, dass die im Elementeset vorkommenden Elemente den Erfahrungsbereich der Befragten abdecken bzw. im Geltungsbereich (range of convenience) der zu erfassenden Konstrukte liegen (vgl. Fransella et al., 2004, S. 18). Mit anderen Worten: Haben die Elemente für die Befragten wenig oder keine Bedeutung, muss mit entsprechend wenig aussagekräftigen Konstrukten bzw. mit wenig validen Daten gerechnet werden. Idiographisch ausgerichtete Untersuchungen sind hier im Gegensatz zu solchen, in denen das Elementeset durch die Befragenden festgelegt wird, kaum gefährdet. Für letztere schlägt Fromm (1995, S. 63) denn auch Probeerhebungen vor. Ferner besteht die Möglichkeit, die Elemente zwar auch vorzugegeben, aber nur soweit, dass sie einer allgemeineren Beschreibung eines Vergleichsobjekts (z. B. ein „naturwissenschaftliches Studienfach“) entsprechen. Die Befragten werden danach aufgefordert, das Element individuell zu konkretisieren (z. B. „Physik“) ${ }^{41}$ (vgl. auch Fromm, 1995, S. 76).

Insgesamt gilt es zu beachten, dass die gewählten Elemente möglichst homogen sind. Besteht das Elementeset z. B. aus fünf naturwissenschaftlichen und nur einem sozialwissenschaftlichen Studienfach, kann dies zu einer Verzerrung der Varianzverteilung führen. Das sozialwissenschaftliche Fach lässt dann die naturwissenschaftlichen Fächer ähnlicher erscheinen, als sie ohne dessen Einbezug ins Elementeset wären (vgl. Scheer, 1993, S. 29). Ausserdem evozieren heterogene Elemente eher abstraktere Konstrukte, die die befragte Person wiederum kognitiv und verbal überfordern können (vgl. Fromm, 1995, S. 79). Dies kann nach Fromm (1995, S. 80) allerdings auch sinnvoll sein, nämlich dann, wenn Konstrukte bzw. Konstruktsysteme erweitert oder gar verändert werden sollen.

Die Anzahl der Elemente ergibt sich grundsätzlich aus der Problemstellung. Empfohlen wird jedoch ein Minimum von sechs Elementen, da weniger keine sinnvolle Anzahl an Vergleichskombinationen ergeben und zu einer künstlichen Vereinfachung der Konstruktsysteme führen würden. Als Maximum werden 15-25 Elemente vorgeschlagen, da zu viele Elemente zu „redundanten Informationen“ bzw. „Fliessbandarbeit“ führen (vgl. Fromm, 1995, S. 80; Scheer, 1993, S. 30). Im verwendeten Beispiel umfasst das Elementeset die Studienfächer "Physik“, „Sportwissenschaft“, „Archäologie“, „Betriebswirtschaft", „Soziologie“ und „Religionswissenschaft“.

\footnotetext{
${ }^{41}$ Dieser auch unter dem Stichwort „Rollenbeschreibung“ (Fromm, 1995, S. 76) explizierten Variante lässt sich auch der Rep Test von Kelly subsumieren. Insgesamt dominiert jedoch sowohl im englisch- wie auch im deutschsprachigen Raum die rein deduktive Festlegung der Elemente (vgl. Rosenberger \& Freitag, 2009, S. 482; Fransella et al., 2004, S. 21, 154). 


\subsubsection{Schritte der Durchführung}

Ist das Elementeset einer Repertory Grid-Untersuchung festgelegt, kann mit dem eigentlichen Interview bzw. der Durchführung der Datenerhebung begonnen werden. Um ein Konstrukt zu erfassen ${ }^{42}$, wird der befragten Person eine zufällige oder zuvor festgelegte Elementkombination vorgelegt (= Elemente präsentieren). Der/die Befragte wird dabei aufgefordert, eine Unterscheidung bzw. Sortierung der Elemente in Bezug auf deren (Un-)Ähnlichkeit vorzunehmen. Im Anschluss daran wird über die getätigte Unterscheidung gesprochen und nach einer dafür passenden Formulierung gesucht ${ }^{43}$, sodass ein zweipoliges Konstrukt notiert werden kann (= Konstrukt erfassen). Nach dieser Konstruktevokation werden schliesslich sämtliche Elemente auf einer Skala, deren Skalenenden durch die beiden Pole des Konstrukts definiert sind, quantitativ bewertet (= Elemente beurteilen).

\section{Elemente präsentieren und Konstrukt erfassen (Konstruktevokation)}

Für dieses (hier vereinfacht beschriebene) Vorgehen finden sich in der Forschungspraxis und Methodenliteratur unzählige Varianten. Für die ersten beiden Schritte, die Konstruktevokation, wird in der aktuellen Forschungspraxis am häufigsten die Triadenmethode eingesetzt. ${ }^{44}$

Als vorbereitender Schritt der Triadenmethode werden zunächst die Elemente des Elementesets in die Kopfspalte eines vorgefertigten Protokollblatts (vgl. Abb. 6) sowie auf einzelne Karten geschrieben. Danach werden drei davon (z.B. „Physik“, „Soziologie“ und „Religionswissenschaft") (= Triade) der befragten Person vorgelegt. ${ }^{45}$ Die Instruktion dazu lautet: „In what important way are two of them alike but different from the third?" (Kelly, 1991, S. 154, Hervorhebung i. O.). Die vom/von der Befragten formulierte Ähnlichkeit zweier der drei Elemente (z. B. „Sozialwissenschaft“), der sog. Konstruktpol, wird von der interviewenden Person im vorgefertigten Protokollblatt notiert. Anschliessend zeigt der/die Interviewer/in auf das dritte Element und fragt: „How is this (...) different?" (ebd.). Der vom/von der Befragten genannte bzw. das dritte Element bezeichnende Unterschied (z.B. „Naturwissenschaft"), der sog. Kontrastpol, wird ebenfalls im Protokollblatt notiert. Schliesslich wird mit dem

\footnotetext{
${ }^{42}$ Die Vorgabe von Konstrukten, wie sie in einer Minderheit von Repertory Grid-Studien zu finden ist (z.B. Kreber \& Klampfleitner, 2013), ist meist mit der Motivation verbunden, die Vergleichbarkeit von Grid-Daten zu maximieren. Solche eher nomothetisch motivierten Anwendungen der RGT stehen jedoch tendenziell im Widerspruch zur Theorie der persönlichen Konstrukte (vgl. dazu Fromm, 1995, S. 86; Neimeyer, Neimeyer, Hagans \& Van Brunt, 2002, S. 166; Scheer, 1993, S. 37).

${ }^{43}$ In dieser Differenzierung zwischen der Unterscheidung als Tun und der Unterscheidung als Formulierung liegt letztlich die Chance der RGT, implizites Wissen durch seine Anwendung explizit zu machen (vgl. Fromm, 2010, S. 527; Hemmecke, 2012, S. 62) (vgl. auch Kap. 5.4.2).

${ }^{44} \mathrm{Ihr}$ Grundprozedere geht auf die von Kelly im Zusammenhang mit seinem Rep Test beschriebene Minimum Context Card Form zurück (vgl. Kelly, 1991, S. 154-155).

45 Theoretischer Hintergrund der Präsentation von jeweils drei Elementen ist Kelly's Postulat, dass eine Unterscheidung von Erfahrungsgegenständen bzw. ein persönliches Konstrukt einer Feststellung von sowohl einer Ähnlichkeit als auch einer Unähnlichkeit entspricht (vgl. Fromm, 1995, S. 81). Das bedeutet, dass ein Konstrukt stets auf mindestens drei Elementen basieren muss.
} 
Prozedere wieder von vorne begonnen, d.h. weitere bipolare Konstrukte (z.B. „interessiert mich - interessiert mich nicht“; „einfacher Berufseinstieg - schwieriger Berufseinstieg“; "langweilig - spannend") werden mit derselben Instruktion, aber anderen Triaden erhoben (vgl. Feedbackschlaufe in Abb. 5).

\begin{tabular}{|c|c|c|c|c|c|c|c|}
\hline Konstruktpol (1) & 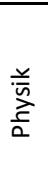 & 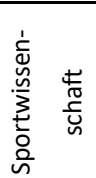 & $\begin{array}{l}\frac{0}{60} \\
\frac{0}{0} \\
: \frac{\pi}{0} \\
\frac{5}{0} \\
\frac{0}{4}\end{array}$ & 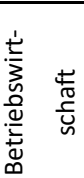 & $\begin{array}{l}\frac{0}{80} \\
\frac{0}{0} \\
\frac{0}{0} \\
0\end{array}$ & 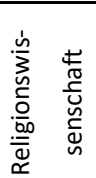 & Kontrastpol (5) \\
\hline Sozialwissenschaft & & & & & & & Naturwissenschaft \\
\hline interessiert mich & & & & & & & interessiert mich nicht \\
\hline einfacher Berufseinstieg & & & & & & & schwieriger Berufseinst. \\
\hline langweilig & & & & & & & spannend \\
\hline$\ldots$ & & & & & & & $\ldots$ \\
\hline & & & & & & & \\
\hline
\end{tabular}

Anmerkung. Grau markierte Zellen entsprechen den zur Unterscheidung vorgegebenen Elementen (Triaden).

Abbildung 6. Protokollblatt einer (fiktiven) Repertory Grid-Untersuchung in der Phase der Konstruktevokation

Weitere, von der Triadenmethode mehr oder weniger abweichende Varianten der Konstruktevokation unterscheiden sich insbesondere a) in der Präsentationsform der Elemente sowie b) bezüglich der Erfassung des Kontrastpols. ${ }^{46}$

a) Variationsmöglichkeiten im Bereich der Präsentation der Elemente betreffen primär die Anzahl bzw. Kombination der zur Unterscheidung bzw. Sortierung vorgelegten Elemente. Neben der Triadenmethode finden sich hier insbesondere die Dyadenmethode sowie die Full Context Form. Diese unterscheiden sich von der Triadenmethode dadurch, dass jeweils nur zwei Elemente (z. B. „Physik“ und „Soziologie“) bzw. alle Elemente (z. B. sechs Studienfächer) des Elementesets präsentiert werden.

Obwohl es aus theoretischer Sicht unproblematisch ist, jeweils nur zwei Elemente vorzulegen ${ }^{47}$, wird die Dyadenmethode vergleichsweise wenig angewandt. In der von verschiedenen Autoren (z.B. Landfield, 1971; Keen \& Bell, 1983; zit. n. Fransella et al., 2004, S. 28f.) vorgeschlagenen Variante wird insbesondere der Vorteil der geringeren Komplexität der Befragung für die Befragten gesehen. Sie wird demnach bei Befragung von Kindern oder Personen mit Lernschwierigkeiten (z.B. Barton, Walton \& Rowe, 1976; zit. n. Fransella et al., 2004, S. 28) eingesetzt.

\footnotetext{
${ }^{46}$ Gröbere Abweichungen vom ursprünglichen Rep Test Kelly's betreffen Varianten wie das Tacit Construing (Sprachfreie Erhebung), Gruppenverfahren oder Computerhebungen (vgl. Fromm, 1995, S. 152f.).

${ }^{47}$ Theoretischer Hintergrund der Präsentation von jeweils drei Elementen ist Kelly's Postulat, dass eine Unterscheidung von Erfahrungsgegenständen bzw. ein persönliches Konstrukt einer Feststellung von sowohl einer Ähnlichkeit als auch einer Unähnlichkeit entspricht (vgl. Kelly, 1991, S. 81). Das bedeutet zwar, dass ein Konstrukt stets auf mindestens drei Elementen basieren muss, determiniert aber nicht, wann, wie viele und in welcher Reihenfolge Elemente vorgelegt werden müssen.
} 
Häufiger in Forschungsarbeiten findet sich hingegen die auf Kelly zurückgehende Variante ${ }^{48}$ der Full Context Form. Das Sortieren des gesamten Elementesets in beliebig grosse Gruppen wird insbesondere als Ergänzung zur Triadenmethode vorgeschlagen (vgl. Fromm, 1995, S. 83) bzw. angewandt (vgl. Müller, 2009; Sulimma, 2012). Ein Wechsel der Erhebungsform kann gemäss Fromm (ebd.) helfen, weitere mittels Triadenmethode nicht evozierte Konstrukte zu erheben.

b) Im Bereich der Erfassung des Kontrastpols geht es vor allem um Variationen in Zusammenhang mit der Formulierung der Unterscheidungsaufgabe (Instruktion). Zu unterscheiden sind hier die difference method, die opposite method und die contrast method (vgl. Neimeyer, Bowman \& Saferstein, 2005).

Die difference method entspricht der von Kelly verwendeten Instruktion und fragt nach dem Unterschied (difference) zwischen den Elementen (vgl. Ausführungen oben).

Bei der von Epting, Suchman und Nickerson (1971) als Weiterentwicklung der difference method eingeführten opposite method interessiert nicht der Unterschied, sondern der (grundsätzliche) Gegensatz (opposite) zur Ähnlichkeit zweier Elemente. Das heisst, dass der Kontrastpol unabhängig von den vorgelegten Elementen erhoben werden soll. ${ }^{49}$ Nach Erhebung des Konstruktpols („How are any two of these alike?“) wird der Kontrastpol entsprechend mit „Nominate the opposite“ erhoben (vgl. Neimeyer et al., 2005, S. 244).

Mit der contrast method nach Neimeyer et al. (2005) wird der Kontrastpol ebenfalls unabhängig von den vorgelegten Elementen erfasst. Im Unterschied zur opposite method wird dafür jedoch der Begriff „Kontrast“ (contrast) verwendet ${ }^{50}$ : „To you, being (...) would contrast with someone being ...” (Neimeyer et al., 2005, S. 244, Hervorhebung i. O.). Zudem werden die Kontrastpole erst dann erhoben, wenn sämtliche Konstruktpole erhoben worden sind.

Neben den Variationsmöglichkeiten in der Präsentation der Elemente sowie der Erfassung des Kontrastpols existieren verschiedene Techniken, die helfen sollen, das von den Befragten Gemeinte systematisch weiter zu verdeutlichen. In der Methodenliteratur werden diese Techniken überschrieben mit "eliciting constructs from constructs“ (Fransella et al., 2004, S. 39) oder „Beziehungen zwischen Konstrukten" (Fromm, 1995, S. 103). Dahinter steht die theoretische Annahme, dass persönliche Konstrukte innerhalb eines Konstruktsystems einander über- bzw. untergeordnet sind. Da die Stellung eines Konstrukts innerhalb des Systems alleine aufgrund der Formulierung jedoch nicht erkennbar ist,

\footnotetext{
${ }^{48}$ Kelly (1991, S. 155-158) beschreibt neben der Minimum Context Card Form und der Full Context Form insgesamt sechs weitere Variationen seines Rep Tests .

${ }^{49}$ Das empirisch nachgewiesene Argument, das Epting et al. (1971) und später auch andere Autoren für diese Variante einbringen, ist die Reduktion sogenannter bent constructs, welche die Auswertung bzw. Interpretation der Grids erschweren. Damit sind Konstrukte gemeint, deren Pole (im Gegensatz zu den eher gewünschten straight constructs) nicht in einem bipolaren, sondern gleichsam orthogonalen Verhältnis zueinander stehen und deshalb eigentlich Konstruktpole zweier verschiedener Konstrukte darstellen (vgl. Neimeyer et al., 2005, S. 240; vgl. auch Yorke, 2001).

${ }^{50}$ Grund dafür ist der empirische Nachweis, dass durch die Verwendung des Begriffs „Kontrast" (contrast) statt des Begriffs „Gegensatz“ (opposite) die Kontrastpole weniger extrem ausfallen und sich deshalb „besser" auf alle Elemente anwenden lassen (vgl. Hagans et al., 2000; Neimeyer et al., 2002).
} 
wurden Techniken entwickelt, diese im Rahmen der Konstrukterfassung zu eruieren (vgl. Fromm, 1995, S. 103f.). Das bekannteste Verfahren ist das sog. laddering („Leiternbildung“).

Das laddering-Verfahren zielt darauf ab, den Ausgangskonstrukten übergeordnete Konstrukte (superordinate constructs) bzw. Implikationen von diesen zu erfassen. In der ursprünglichen Form nach Hinkle (1965; zit. n. Fransella et al., 2004) wird der/die Befragte nach Erfassung eines bipolaren Konstrukts zunächst gebeten, den bevorzugten Pol zu nennen. Danach wird der/die Befragte aufgefordert, zu begründen, weshalb er/sie jenen gegenüber dem anderen Pol präferieren würde ${ }^{51}$, wobei die Antwort darauf als übergeordnetes bipolares Konstrukt interpretiert wird. Da ausgehend von verschiedenen Ausgangskonstrukten potenziell dasselbe übergeordnete Konstrukt genannt wird, entstehen Verknüpfungen zwischen den einzelnen Konstrukten, die sich später in einem Schaubild des Konstruktsystems darstellen lassen (vgl. Fromm, 1995, S. 107).

Der Schritt der Konstruktevokation gilt generell als abgeschlossen, wenn „man ungefähr so viele Konstrukte hat, wie Elemente vorlagen, also vielleicht 10 oder 13 oder 20" (Scheer, 1993, S. 26). Nach Fromm (1995) soll die Beendigung der Erhebung vom Untersuchungszweck abhängen. Ist ein Vergleich der Einzelerhebungen wichtig, wird in der Regel die Anzahl der Konstrukte definiert. Um einerseits die relevanten Konstrukte zu erfassen, andererseits aber auch die Formulierung von „Gefälligkeitskonstrukten“ zu vermeiden, empfiehlt Fromm (ebd., S. 99) einen „Höchstwert von 10 bis 15 Wahlen“.

\section{Elemente beurteilen}

Im letzten Schritt der Datenerhebung (= Elemente beurteilen) werden sämtliche Elemente hinsichtlich der erfassten Konstrukte eingeschätzt. Die zuvor für bestimmte Elementkombinationen formulierten Konstrukte werden somit als Beurteilungsdimensionen für alle Elemente herangezogen. Vorgeschlagen werden hierfür 5- oder 7-stufige Ratingskalen ${ }^{52}$, deren Skalenenden durch die beiden Pole des Konstrukts definiert sind (vgl. Fransella et al., 2004, S. 59; Fromm, 1995, S. 94; Jankowicz, 2004, S. 37; Riemann, 1991, S. 22). Die Ratings lassen sich entweder Zeile für Zeile (construct-by-construct rating: Jedes Konstrukt wird auf alle Elemente hintereinander angewandt) oder Spalte für Spalte (element-byelement rating: Jedes Element wird in Bezug auf alle Konstrukte geratet) durchführen (vgl. Fransella et al., 2004). ${ }^{53}$

\footnotetext{
51 " (...) why you would prefer to be here rather than here ... What are the advantages of this side in contrast to the disadvantages of that side as you see it?" (Hinkle, 1965, S. 32f.; zit. n. Fransella et al., 2004, S. 39).

${ }^{52}$ Im Gegensatz zu Kelly's 2-Punkt Nominalskala des Rep Tests oder an anderen Stellen diskutierten Rangskalierungen (vgl. Scheer \& Catina, 1993) haben stärker differenzierende Ratingskalen den Vorteil, dass sie einerseits den/die Befragte/ $\mathrm{n}$ nicht zu künstlichen Entzerrungen der Elemente zwingen, andererseits sich am besten für rechnerische Auswertungen eignen (Scheer, 1993, S. 33).

${ }^{53}$ Diskutiert wird hier vor allem der Effekt dieser beiden Varianten auf die Differenziertheit des Konstruktssystems (z.B. Fransella et al., 2004, S. 64). Aufgrund inkonsistenter Forschungsergebnisse kommt (Bell, 2011) diesbezüglich zum Schluss: „Should the ratings be collected construct by construct, rating each element in turn, or element by element, rating each construct in turn? Evidence suggests (Bell et al., 2002; Neimeyer and Hagans, 2002) that it does not matter"(S. 501-502).
} 
Ein Repertory Grid-Interview resultiert schliesslich in einer durch Elemente und Konstrukte aufgespannten Matrix mit Zahlen (vgl. Abb. 7). Dieses sog. Rohgrid kann somit sowohl qualitativ als auch quantitativ ausgewertet werden (vgl. Kap. 5.5).

\begin{tabular}{|c|c|c|c|c|c|c|c|}
\hline Konstruktpol (1) & $\frac{\frac{r}{\tilde{n}}}{\frac{a}{a}}$ & 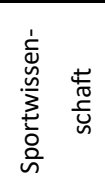 & $\begin{array}{l}\frac{0}{60} \\
\frac{0}{0} \\
: \frac{\pi}{0} \\
\frac{0}{4} \\
\frac{3}{4}\end{array}$ & 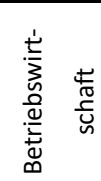 & $\begin{array}{l}\frac{0}{00} \\
\frac{0}{0} \\
\frac{0}{N} \\
0\end{array}$ & 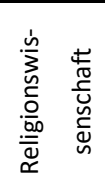 & Kontrastpol (5) \\
\hline Sozialwissenschaft & 1 & 4 & 2 & 2 & 5 & 2 & Naturwissenschaft \\
\hline interessiert mich & 2 & 2 & 4 & 1 & 4 & 5 & interessiert mich nicht \\
\hline éinfacher Berufseinstieg & 4 & 5 & 2 & 1 & 2 & 2 & schwieriger Berufseinst. \\
\hline langweilig & 4 & 4 & 2 & 4 & 1 & 2 & spannend \\
\hline wenig Praxis & 3 & 5 & 2 & 2 & 1 & 1 & Viel Praxis \\
\hline kaum weibl. Studierende & 1 & 5 & 2 & 5 & 5 & 5 & auch weibl. Studierende \\
\hline
\end{tabular}

Anmerkung. Grau markierte Zellen entsprechen den zur Unterscheidung vorgegebenen Elementen (Triaden).

Abbildung 7. Protokollblatt einer (fiktiven) Repertory Grid-Untersuchung nach Abschluss der Datenerfassung

Wie die Ausführungen in diesem Kapitel zu den Hauptschritten der RGT gezeigt haben, wird dem Anwender der RGT viel Spielraum gelassen. Neimeyer et al. (2005) kommen diesbezüglich zum Schluss, dass der gemeinsame Nenner aller Repertory Grid-Untersuchungen so beschrieben werden kann, dass es sich letztlich um "sorting tasks [handelt] which enable the person to tell us something of the way in which he sees and orders the world" (S. 239).

\subsection{Untersuchungsteilnehmende}

Im folgenden Kapitel werden zunächst die Kriterien erläutert, nach denen die Untersuchungsteilnehmenden bestimmt wurden (vgl. Kap. 5.2.1). Im Anschluss daran werden der Prozess der Stichprobengewinnung und die Charakteristika des daraus resultierenden Samples beschrieben (vgl. Kap. 5.2.2).

\subsubsection{Selektionskriterien}

Interessierende Untersuchungsgruppe der vorliegenden Untersuchung waren Sport unterrichtende Lehrpersonen, für die das Unterrichten in migrationsbedingt heterogenen Klassenverhältnissen Berufsalltag bedeutet. Vor dem Hintergrund der beiden Fragestellungen wurden Sport unterrichtende Lehrpersonen gesucht, die sich infolge ihrer Berufsausübung (bewusst oder unbewusst) Gedanken 
über Lernende mit Migrationshintergrund sowie über interkulturelles Lernen (im Sportunterricht) machen.

Ausgehend von diesen Überlegungen ging es zunächst darum, als übergeordnetes Selektionskriterium eine Schulstufe zu identifizieren, in dem Sport unterrichtende Lehrpersonen erstens besonders stark mit migrationsbedingt heterogenen Klassenverhältnissen konfrontiert sind und zweites ein eher fächerspezifisches statt fächerübergreifendes Unterrichtsverständnis vorweisen. Ein fächerspezifisches Unterrichtsverständnis, so die Annahme, würde die Lehrpersonen eher dazu befähigen, den Sportunterricht (in Abgrenzung zu anderen Fächern) in Bezug auf sein interkulturelles Lernpotenzial einzuschätzen.

Da der Anteil fremdsprachiger Schülerinnen und Schüler auf der Primarstufe (ISCED 1) und der Sekundarstufe I (ISCED 2) generell höher ist als auf der Sekundarstufe II (ISCED 34+35), wurde die Grundgesamtheit somit erst einmal auf Sport unterrichtende Lehrpersonen der ersten beiden Schulstufen (ISCED 1+2) eingeschränkt. Aufgrund der beschränkten zur Verfügung stehenden Ressourcen wurde der Untersuchungsraum zudem auf den deutschsprachigen Teil des Kantons Bern eingeengt. Obwohl hier der Anteil fremdsprachiger Schülerinnen und Schüler auf der Primarstufe höher ist als auf der Sekundarstufe I, wurde die Zielpopulation weiter auf Sport unterrichtende Lehrpersonen der Sekundarstufe I eingeschränkt. Diese Entscheidung wurde deshalb getroffen, weil Sport unterrichtende Lehrpersonen der Sekundarstufe I aufgrund ihrer Ausbildung und Berufsausübung viel eher ein fächerspezifisches Unterrichtsverständnis aufweisen dürften als Lehrpersonen der Primarstufe. ${ }^{54}$

Abgesehen von der Schulstufe, auf der die Lehrperson unterrichtet, wurden als weitere Selektionskriterien a) eine untere Grenze an Sportunterrichtserfahrung und b) ein Minimal- und Maximalanteil fremdsprachiger Schülerinnen und Schüler einer potenziell zu kontaktierenden Schule herangezogen.

a) In Bezug auf die Unterrichtserfahrung wurde davon ausgegangen, dass Lehrpersonen mit längerer Berufserfahrung bereits stärker validierte und deshalb stabilere Konstruktionen bzw. klarere Überzeugungen aufweisen als solche mit kürzerer Berufserfahrung. Hinweise dafür boten denn auch sechs Pretests, die u. a. aufzeigten, dass es berufserfahreneren Lehrpersonen generell leichter fällt, eine Formulierung für ihre persönlichen Konstrukte zu finden. Schliesslich wurde angenommen, dass mit zunehmender Berufserfahrung auch das Repertoire an Fächern, die bis dahin unterrichtet wurden, grösser ist. Dies war insofern von Bedeutung, als das im Zusammenhang mit der zweiten Fragestellung behan-

\footnotetext{
${ }^{54}$ Für die Sekundarstufe SI ausgebildete Lehrpersonen absolvieren aktuell eine vier bis fünf Jahre dauernde Lehrbefähigung für drei bis vier Fächer. Ihre Anstellung beschränkt sich (in der Regel) auf das Unterrichten dieser Fächer. Primarlehrpersonen werden hingegen während drei Jahren zur Lehrperson für sieben bis acht Fächer (bzw. für fast alle Fächer) ausgebildet. Aus professionstheoretische Sicht entsprechen sie daher eher Generalisten mit fächerübergreifendem und weniger fächerspezifischem Unterrichtsverständnis. 
delte Thema - Interkulturelles Lernen - einer überfachlichen Zielorientierung von Unterricht entspricht (vgl. Kap. 1.1). Um aufgrund der weiteren Selektionskriterien die Gesamtheit potenzieller Untersuchungsteilnehmender dennoch nicht zu stark zu minimieren, wurde als untere Grenze eine Sportunterrichtserfahrung von minimal fünf Jahren festgelegt.

b) Das im Zusammenhang mit der ersten Fragestellung entwickelte Instrument erforderte einen ungefähr je hälftigen Anteil fremdsprachiger und nicht-fremdsprachiger Schülerinnen und Schüler in der als Gesprächsgrundlage herangezogenen Klasse. Dies deshalb, weil unter den 10 zufällig davon ausgewählten und in der Folge von der Lehrperson zu konstruierenden bzw. zu vergleichenden Schülerinnen und Schülern (vgl. Kap. 5.4.1) fremdsprachige und nicht-fremdsprachige Schülerinnen und Schüler zu möglichst gleichen Anteilen vorkommen sollten (vgl. Kap. 5.3.1). Um die beschriebene Erfordernis bestmöglich zu erfüllen, wurde ein Anteil von 30-80\% fremdsprachigen Schülerinnen und Schülern einer potenziell zu kontaktierenden Schule (mit Sekundarstufe I) vorausgesetzt.

Die Strategie der Stichprobenwahl dieser Untersuchung ist gekennzeichnet durch eine Vorab-Festlegung von (insbesondere theoretischen) Selektionskriterien für potenzielle Untersuchungsteilnehmende. Diese für qualitative Forschung typische Auswahlstrategie wird je nach Autor (mit kleinen Differenzen) als qualitativer Stichprobenplan (vgl. Lamnek, 2005), selektives Sampling (vgl. Kluge \& Kelle, 1999) oder purposive sampling (vgl. Cohen, Manion \& Morrison, 2013) beschrieben. Sie grenzt sich vom schrittweisen bzw. theoretischen Sampling (vgl. Flick, 2016; Lamnek, 2005) ab, bei dem „Entscheidungen über die Auswahl und Zusammensetzung des empirischen Materials (Fällen, Untersuchungsgruppen, Institutionen ...) im Prozess der Datenerhebung und -auswertung gefällt" werden (Flick, 2016, S. 158f., Hervorhebung i. O.). Die Entscheidung für eine Vorab-Festlegung gegenüber einer iterativen Festlegung von Merkmalen potenzieller Untersuchungsteilnehmer ist dabei in der Regel vom (vermeintlichen) Vorwissen der Forscher zum Untersuchungsgegenstand abhängig (vgl. Flick, 2016, S. 155). Da in der vorliegenden Untersuchung theoretische Annahmen zu Charakteristiken typischer Personen („critical cases“ (Cohen et al., 2013, S. 115)) vorlagen, entschied ich mich für eine Vorab-Bestimmung eines Kriterienkatalogs. Hierzu gilt es anzumerken, dass aufgrund dieser Kriterien kein Anspruch an statistische Repräsentativität erhoben wurde. Vielmehr sollte das Sample der für die qualitative Forschung angemessenen Forderung nach inhaltlicher Repräsentativität nachkommen (vgl. Lamnek, 2005, S. 192f.). 


\subsubsection{Stichprobengewinnung und Samples}

Von den insgesamt 295 (deutschsprachigen) Schulen mit Sekundarstufe I im Kanton Bern haben 58 Schulen einen Fremdsprachigenanteil zwischen $30 \%$ und $80 \%{ }^{55}$ (vgl. Tab. 1). Da für die vorliegende Untersuchung keine Kontaktangaben von Sport unterrichtenden Lehrpersonen dieser Schulen zur Verfügung standen, mussten diese zuerst von den ausgewählten Schulen angefordert werden. Hier stellten sich die Schulleitungen als erste entscheidende Hürde in der Gewinnung möglicher Untersuchungsteilnehmender heraus. Nach zunächst schriftlicher, dann mündlicher individueller Kontaktierung der Schulen zur Darlegung des Untersuchungsinteresses und dessen Implikationen ${ }^{56}$ (Forschungsziel, Erhebungsmethode, Zeitaufwand etc., vgl. Anhang A) gewährten 26 der 58 Schulen Zugang zu den ihnen angehörenden Sport unterrichtenden Lehrpersonen (vgl. Tab 1).

Tabelle 1. Prozessdiagramm zur Stichprobengewinnung

Selektionskriterien und Kontaktierungs- Resultierende Anzahl Schulen schritte nach Anwendung der Selektionskriterien bzw. Kontaktierungsschritte
Resultierende Anzahl Lehrpersonen nach Anwendung der Selektionskriterien bzw. Kontaktierungsschritte

\begin{tabular}{l|l}
\hline $\begin{array}{l}\text { Deutschsprachige Schule der Se- } \\
\text { kundarstufe I (Kanton Bern) }\end{array}$ & 295 \\
\hline \hline & $\begin{array}{l}\text { Schriftliche und telefonische An- } \\
\text { frage an die Schulleitungen }\end{array}$ \\
\hline $\begin{array}{l}\text { Telefonische Anfrage an die } \\
\text { Lehrpersonen: } \geq 5 \text { Jahre Sport- } \\
\text { unterrichtserfahrung }\end{array}$
\end{tabular}

Als Hauptargument der Schulen gegen eine Teilnahme wurden primär die fehlenden zeitlichen Ressourcen der Lehrpersonen angebracht. ${ }^{57}$

55 Die Zahlen wurden den online veröffentlichten Statistiken der Erziehungsdirektion des Kantons Bern (http://www.erz.be.ch/erz/de/index/direktion/organisation/generalsekretariat/statistik/schul-_und_gemeindetabellen.html) entnommen und entsprechen den Angaben mit Stichtag 15.09.2014.

${ }^{56} \mathrm{Um}$ etwaige Antwortverzerrungen bestmöglich auszuschliessen, wurden die Schulleitungen darauf hingewiesen, die Informationen nicht an die betreffenden Lehrpersonen weiterzuleiten.

${ }^{57}$ Trotz Bemühungen um Transparenz und Vertrauen (mittels Informationsschreiben und persönlichem Telefongespräch) dürften hier letztendlich aber auch die von Flick (2016, S. 90) beschriebenen, typischen Probleme im Zusammenhang mit dem Zugang zu Institutionen - Forschung als Intervention, Forschung als Störfaktor - mit hineingespielt haben. 
Aus den 26 bereitwilligen Schulen resultierten schliesslich 80 Kontaktangaben potenzieller Untersuchungsteilnehmender (vgl. Tab. 1). Da es nach Bortz und Döring (2006) für explorative Studien „weitgehend unerheblich [ist], wie die Untersuchungsteilnehmer aus der interessierenden Population ausgewählt werden“ (S. 71), wurden die Lehrpersonen in der Reihenfolge der von den Schulleitungen zeitlich versetzt eingegangenen Kontaktangaben per E-Mail und/oder telefonisch kontaktiert und über das Untersuchungsvorhaben informiert. Hervorgehoben wurde hier insbesondere, dass Untersuchungsteilnehmenden die Gelegenheit geboten würde, als erfahrene Lehrpersonen über ihren eigenen Sportunterricht berichten zu können. Der ihnen damit zugesprochene Expertenstatus sowie die Zusicherung einer als Weiterbildung anerkennbaren Teilnahmebestätigung sollte die Bereitschaft der Lehrpersonen erhöhen. Obwohl die Lehrpersonen gemeinhin hohes Interesse bekundeten, zeigte sich - wie auch schon auf Ebene der Schulen - der hohe Aufwand von zwei mit 60 bis 90 Minuten veranschlagten Interviews als die Bereitschaft hauptsächlich limitierender Faktor. Ausserdem erfüllten einige Lehrpersonen das Kriterium von mindestens fünf Jahren Sportunterrichtserfahrung nicht oder blieben über die angegebenen Kontaktangaben unerreichbar.

Unter Berücksichtigung der Selektionskriterien konnten schliesslich 26 Lehrpersonen aus 19 Schulen für die Untersuchung gewonnen werden (vgl. Tab. 1). Die Anzahl rekrutierter Lehrpersonen entspricht somit nicht einer vorab festgelegten, sondern einer nach Anwendung der Selektionskriterien und Kontaktierung der Schulen und Lehrpersonen erzielten Samplegrösse.

In Tabelle 2 sind die 19 teilnehmenden Schulen, ihr Anteil an fremdsprachigen Schülerinnen und Schülern sowie die Anzahl befragter Lehrpersonen pro Schule dargestellt. Die aufgesuchten Schulen verteilen sich über alle fünf Schulverwaltungskreise des Kantons Bern (Bern-Mittelland, Biel/Bienne, Emmental, Oberaargau, Thun) und liegen sowohl in (vor-)städtischen als auch in ländlichen Gemeinden. Der durchschnittliche Anteil an fremdsprachigen Schülerinnen und Schülern pro Schule belief sich zum Zeitpunkt des Monitorings auf $43.6 \%{ }^{58}$ Keine der Schulen fiel dabei aus dem als Selektionskriterium definierten Bereich von 30\%-80\% (vgl. Kap. 5.2.1).

\footnotetext{
${ }^{58}$ Die Angaben zum Anteil fremdsprachiger Schülerinnen und Schüler pro Schule wurden den von der Erziehungsdirektion des Kantons Bern per 15.9.2014 erhobenen und online veröffentlichten Schülerinnen- und Schülerstatistiken entnommen (http://www.erz.be.ch/erz/de/index/direktion/organisation/generalsekretariat/statistik/schul__und_gemeindetabellen.html). Sie entsprechen dem Anteil an fremdsprachigen Schülerinnen und Schülern der von den befragten Lehrpersonen unterrichteten Niveaustufe. In Schulen, in welchen der Sportunterricht integriert, d.h. in nach Niveau gemischten Klassen unterrichtet wird, wurde jeweils der Mittelwert des Anteils zwischen Real- und Sekundarklassen angegeben.
} 
Tabelle 2. Angaben zu den teilnehmenden Schulen

\begin{tabular}{|c|c|c|}
\hline Name der Schule (Gemeinde) & $\begin{array}{c}\text { Anteil fremdsprachiger } \\
\text { Schülerinnen und Schüler } \\
{[\%]}\end{array}$ & $\begin{array}{c}\text { Anzahl befragter } \\
\text { Lehrpersonen }\end{array}$ \\
\hline 1) Schule Liebefeld-Steinhölzli (Köniz) & 66 & 1 \\
\hline 2) Sekundarstufe I Neuenegg (Neuenegg) & 30 & 2 \\
\hline 3) Oberstufenschule Schönau (Steffisburg) & 33 & 2 \\
\hline 4) Oberstufe Herzogenbuchsee (Herzogenbuchsee) & 39 & 3 \\
\hline 5) Schulzentrum Pestalozzi-Gotthelf (Burgdorf) & 33 & 2 \\
\hline 6) Schule Niederbipp (Niederbipp) & 40 & 1 \\
\hline 7) Schule Burgerbeunden (Nidau) & 56 & 1 \\
\hline 8) Schulzentrum Elzmatte (Langenthal) & 56 & 3 \\
\hline 9) Schule Bärau (Langnau i. E.) & 42 & 1 \\
\hline 10) Schule Lengnau (Lengnau) & 31 & 1 \\
\hline 11) Schule Oberburg (Oberburg) & 31 & 1 \\
\hline 12) Schule Moos (Muri b. Bern) & 50 & 1 \\
\hline 13) Schule Bützberg Byfang (Thunstetten) & 34 & 1 \\
\hline 14) Schule Lee 1-4 (Urtenen-Schönbühl) & 46 & 1 \\
\hline 15) Schule Trubschachen (Trubschachen) & 31 & 1 \\
\hline 16) Oberstufenzentrum Ittigen (Ittigen) & 55 & 1 \\
\hline 17) Schule Niederwangen (Köniz) & 44 & 1 \\
\hline 18) Schule Dennigkofen (Ostermundigen) & 79 & 1 \\
\hline 19) Oberstufe Kehrsatz (Kehrsatz) & 32 & 1 \\
\hline Mittelwert & 43.6 & 1.4 \\
\hline
\end{tabular}

Von der Mehrzahl der Schulen konnte nur eine Lehrperson gewonnen werden. Aus drei Schulen konnten je zwei und aus zwei Schulen je drei Lehrpersonen rekrutiert werden, sodass im Mittel 1.4 befragte Lehrpersonen pro Schule resultierten (vgl. Tab. 2).

Tabelle 3 präsentiert neben soziodemographischen Daten die berufsbezogenen Angaben der Lehrpersonen. ${ }^{59}$ Die Analysen zur Fragestellung 1 basieren auf den Interviews sämtlicher 26 Untersuchungsteilnehmender. In die Auswertungen zur Fragestellung 2 wurden aufgrund mangelnder Validität hingegen die Interviews von zwei Teilnehmenden nicht miteinbezogen (vgl. Kap. 5.5.2), sodass hier 24 Interviews als Datengrundlage resultierten. Tabelle 3 stellt deshalb die Angaben differenziert nach Stichprobe $1\left(n_{1}\right)$ und $2\left(n_{2}\right)$ dar.

\footnotetext{
${ }^{59}$ Die Angaben wurden mittels Kurzfragebogen im Anschluss an die beiden Interviews erfasst (vgl. Anhang B).
} 
Der Wegfall von je einer weiblichen und einer männlichen Lehrperson aus $n_{1}(w=10, m=16)$ gegenüber $n_{2}(w=9, m=15)$ wirkt sich kaum auf das Durchschnittsalter $\left(n_{1}=43.8\right.$ Jahre, $n_{2}=43.4$ Jahre $)$ und die durchschnittliche Unterrichtserfahrung als Sport unterrichtende Lehrperson ( $n_{1}=18.5$ Jahre, $n_{2}=$ 18.4 Jahre) aus. Ebenso unwesentlich weichen die beiden Samples in Bezug auf die durchschnittlichen Anteile der pädagogischen Ausbildungsabschlüsse (Lehrerpatent: $n_{1}=53.8 \%, n_{2}=58.3 \%$, Lehrdiplom SI: $n_{1}=42.3 \%, n_{2}=37.5 \%$, Lehrdiplom SII: $n_{1}=3.8 \%, n_{2}=4.2 \%$ ) der befragten Lehrpersonen aus (vgl. Tab. 3).

Tabelle 3. Alter, Sportunterrichtserfahrung (SU-Erfahrung), Ausbildung (Lehrerpatent, Lehrdiplom SI, Lehrdiplom SII) und Unterrichtspensum der befragten Lehrpersonen differenziert nach Stichprobe $1\left(n_{1}\right)$ und Stichprobe $2\left(n_{2}\right)$

\begin{tabular}{|c|c|c|c|c|c|c|c|}
\hline & $n_{1} / n_{2}$ & Alter & $\begin{array}{c}\text { SU- } \\
\text { Erfahrung }\end{array}$ & Lehrerpatent & Lehrdiplom SI & $\begin{array}{l}\text { Lehrdiplom } \\
\text { SII }\end{array}$ & $\begin{array}{l}\text { Unterrichts- } \\
\text { pensum }\end{array}$ \\
\hline & & [Jahre] & [Jahre] & [\%] & [\%] & [\%] & [\%] \\
\hline weiblich & $10 / 9$ & $40.8 / 39.8$ & $13 / 11.7$ & $40 / 44.4$ & $60 / 55.6$ & $0 / 0$ & $69.3 / 73.7$ \\
\hline männlich & $16 / 15$ & $45.6 / 45.5$ & $22 / 22.4$ & $62.5 / 66.7$ & $31.3 / 26.7$ & $6.3 / 6.7$ & $87.4 / 89.8$ \\
\hline Gesamt & $26 / 24$ & 43.8/43.4 & $18.5 / 18.4$ & $53.8 / 58.3$ & $42.3 / 37.5$ & $3.8 / 4.2$ & $80.4 / 83.8$ \\
\hline
\end{tabular}

Betrachtet man die weiblichen und männlichen Anteile der Stichproben getrennt, lässt sich für beide Stichproben ein Altersunterschied von fünf bis sechs Jahren zugunsten der Männer $\left(n_{1}: \mathrm{w}=40.8\right.$ Jahre, $m=45.6$ Jahre, $n_{2}: w=39.8$ Jahre, $m=45.5$ Jahre) konstatieren. Die in beiden Stichproben höheren Anteile bei den männlichen Lehrpersonen mit - in der Schweiz seit 2005 nicht mehr angebotenem Lehrerpatent $\left(n_{1}: \mathrm{m}=62.5 \%, \mathrm{w}=40 \%, n_{2}: \mathrm{m}=66.7 \%, \mathrm{w}=44.4 \%\right)$ und die höheren Anteile bei den weiblichen Lehrpersonen mit Lehrdiplom für die Sekundarstufe I $\left(n_{1}: \mathrm{w}=60 \%, \mathrm{~m}=31.3 \%, n_{2}: \mathrm{w}=\right.$ $55.6 \%, \mathrm{~m}:=26.7 \%)$ dürfte eine Folge davon sein. Ebenso Ausdruck dieses geschlechtsbezogenen Altersunterschieds dürfte die ungefähr doppelt so hohe Unterrichtserfahrung im Fach Sport seitens der Männer sein ( $n_{1}: \mathrm{m}=22$ Jahre, $\mathrm{w}=13$ Jahre, $n_{2}: \mathrm{m}=22.4, \mathrm{w}=11.6$ Jahre).

Schliesslich ist Tabelle 3 ein Geschlechterunterschied in Bezug auf das Unterrichtspensum zu entnehmen. Zum Zeitpunkt der Befragung waren die Lehrpersonen durchschnittlich zu $n_{1}=80.4$ bzw. $n_{2}=83.8$ Stellenprozenten angestellt. In beiden Stichproben betrug der Unterschied zwischen Frauen und Männern ungefähr 20 Stellenprozente ( $n_{1}: \mathrm{w}=69.3 \%, \mathrm{~m}=87.4 \%, n_{2}: \mathrm{w}=73.7 \%, \mathrm{~m}=89.8 \%$ ).

Abschliessend ist festzuhalten, dass mit dem/den gewonnenen Sample(s) das Selektionskriterium von mindestens fünf Jahren Unterrichtserfahrung im Fach Sport (vgl. Kap. 5.2.1) eingehalten werden 
konnte. Das Kriterium, Lehrpersonen mit eher fächerspezifischem Verständnis bzw. mit einem Lehrdiplom SI zu befragen (vgl. Kap. 5.2.1), trifft hingegen - zumindest in Bezug auf die formelle Qualifikation der Lehrpersonen ${ }^{60}$ - nur für $42.3 \%$ bzw. 37.5\% der befragten Lehrpersonen zu. Da - wie in Kap. 1.1 dargelegt - das unterrichtsbezogene Denken der Lehrpersonen im Kontext migrationsbedingter Heterogenität jedoch primär auf subjektiven (Unterrichts-)Erfahrungen der Lehrpersonen und weniger auf deren formellen Qualifikationen zu basieren scheint, wurde hinsichtlich des Kriteriums eines fächerspezifischen Unterrichtsverständnisses dennoch von einem homogenen Sample ausgegangen.

\subsection{Instrumente}

Dieses Kapitel beschreibt die Instrumente, die zur Durchführung der Interviews entwickelt wurden. Kapitel 5.3.1 bezieht sich auf das Instrument zur Erfassung der Überzeugungen über Schülerinnen und Schüler mit Migrationshintergrund, Kapitel 5.3.2 erläutert das Instrument, das zur Erhebung der Überzeugungen über das Fach Sport in Bezug auf interkulturelles Lernen verwendet wurde.

Nach Durchführung der Interviews wurden mittels Kurzfragebogen neben soziodemographischen Daten weitere Angaben zur Schule, Ausbildung und Berufserfahrung der Lehrpersonen erfasst (vgl. Anhang B).

\subsection{1 Überzeugungen über Schülerinnen und Schüler mit Migrationshintergrund im Sportun-} terricht

Ausgehend von der Frage nach der Wahrnehmung Sport unterrichtender Lehrpersonen von Schülerinnen und Schülern mit Migrationshintergrund im Sportunterricht sollten mit diesem Instrument „Vorstellungen über Persönlichkeitsmerkmale, Eigenschaften, Einstellungen und Fähigkeiten der Schüler/innen (...) [mit Migrationshintergrund]“ (Ziegler et al., 1998, S. 273) erfasst werden (vgl. Kap. 4). Migrationshintergrund wurde definiert als das Vorliegen einer Muttersprache ${ }^{61}$ einer Schülerin/eines Schülers, die nicht der Unterrichtssprache (Deutsch) entspricht.

\footnotetext{
${ }^{60}$ Aufgrund des komplexen Prozesses der Stichprobengewinnung wurde die formelle Qualifikation der Lehrpersonen nicht gezielt, sondern nur indirekt durch die Selektion der Schulstufe mitberücksichtigt. Da heute Lehrpersonen mit unterschiedlichen Ausbildungen auf der Sekundarstufe I unterrichten, musste deshalb von einem diesbezüglich heterogenen Sample ausgegangen werden. Der grosse Anteil an Lehrpersonen mit Lehrerpatent dürfte dabei insbesondere damit zu tun haben, dass aufgrund des Selektionskriteriums von mindestens $30 \%$ Fremdsprachigen pro zu kontaktierender Schule (vgl. Kap. 5.2.1) häufiger Schulen angefragt wurden, die nur Realklassen führen, welche wiederum (gemäss telefonischer Auskunft der Erziehungsdirektion des Kantons Bern) primär von Lehrpersonen mit Lehrerpatent unterrichtet werden.

${ }^{61}$ Die Muttersprache entspricht in der Regel der Sprache, die in der Familie gesprochen wird und kann deshalb stellvertretend für das (schulisch relevante) Erziehungsmilieu eines Schülers bzw. einer Schülerin betrachtet werden.
} 


\subsubsection{Elementtyp und Elementeset}

Der Anwendungsbereich (range of convenience) persönlicher Konstrukte Sport unterrichtender Lehrpersonen bezüglich fremdsprachiger Schülerinnen und Schüler im Sportunterricht umfasst sowohl fremdsprachige als auch nicht-fremdsprachige Schülerinnen und Schüler im Sportunterricht. ${ }^{62}$ Dieser Überlegung zufolge - und um Aussagen zu potenziellen Unterschieden zwischen Schülerinnen und Schülern mit und solchen ohne Migrationshintergrund machen zu können - wurde als Elementtyp fremdsprachige sowie nicht-fremdsprachige Schülerinnen und Schüler im Sportunterricht bestimmt. Die Anzahl Elemente wurde aus forschungsökonomischen Gründen auf 10 Schülerinnen und Schüler und damit auf ca. 50\% einer Klasse beschränkt. Ausgewählt wurde dafür jede/r zweite/r Schüler/in einer alphabetisch geordneten Klassenliste einer aktuell von der befragten Lehrperson unterrichteten Sportklasse (vgl. dazu ausführlicher Kap. 5.4.1). Hinter dieser alphabetischen Wahl der Schülerinnen und Schüler stand die Absicht, keine a priori festgelegten Denk- bzw. Schülerkategorien (Schülerinnen und Schüler mit Migrationshintergrund vs. Schülerinnen und Schüler ohne Migrationshintergrund) in die Untersuchung hineinzutragen. ${ }^{63}$ Da nur Lehrpersonen rekrutiert wurden, von denen bekannt war, dass sie hohe Anteile an fremdsprachigen Schülerinnen und Schülern in ihren Klassen aufweisen würden (vgl. Kap. 5.2.1), konnte aufgrund dieser Wahlsystematik davon ausgegangen werden, dass sich in den Elementesets der Lehrpersonen letztendlich sowohl fremdsprachige als auch nicht-fremdsprachige Schülerinnen und Schüler finden würden. Zur Identifikation fremdsprachiger Schüler und Schülerinnen wurden schliesslich im Nachgang an die Interviews die jeweiligen Schulsekretariate kontaktiert. $^{64}$

Zusätzlich zu den 10 realen Schülerinnen und Schülern wurde ein sogenannter „Ideal“-Schüler als 11. Element hinzugefügt (vgl. Abb. 8). ${ }^{65}$ Dieses Element diente allerdings nicht als Stimulus zur Erfassung

\footnotetext{
${ }^{62}$ Fransella et al. (2004) führen in diesem Zusammenhang folgendes Beispiel zur Wahl von Grid-Elementen an: „If you want to find out a homosexual person's views of sex, then you will need both homosexuals and heterosexuals represented in the elements. However, if you want to find out a homosexual person's view of some aspect of homosexuality (e.g. sexual positions), then clearly the elements would need to be other homosexual people only" (S. 18).

${ }^{63}$ Zur Gefahr der Reifizierung in der Heterogenitätsforschung vgl. Budde (2012, S. 525f.; 2013, S. 13) sowie Degele und Schirmer (2004, S. 107). Ihnen zufolge ist die Frage nach möglichen Differenzlinien empirisch (a posteriori) zu beantworten.

${ }^{64}$ Die Schulsekretariate erfassen Muttersprache und Staatsbürgerschaft aller unterrichteten Schülerinnen und Schüler.

65 Die Integration eines „Ideal“-Elements in ein Elementeset geht auf Anwendungen der RGT in der klinischen Psychologie zurück, in der u.a. die „Real-Idealselbst Kongruenz“ erfasst wurde (vgl. Riemann, 1991). Innerhalb der Lehrerkognitionsforschung finden „Ideal“-Elemente in den RGT-Untersuchungen von Boei, Corporaal und van Hunen (1989) und Fischler (2000a) Verwendung. Beide Untersuchungen waren vom Interesse geleitet, „guten" Unterricht aus Sicht der Lehrpersonen zu erforschen. Als Elemente wurden unterschiedliche Lehrerverhaltensweisen (vgl. Boei et al., 1989) bzw. fachdidaktische Intentionen (vgl. Fischler, 2000a) verwendet und mitei-
} 
der Konstrukte, sondern wurde erst nach Abschluss der Konstruktevokation und der Beurteilung der realen Schülerinnen und Schüler eingeführt und beurteilt (vgl. Kap. 5.4.2). Ziel war es, im Hinblick auf die Datenauswertung einen normativ-idealen Bezugspunkt zu den im Zusammenhang mit den realen Schülerinnen und Schülern formulierten Konstrukten zu erhalten.

\subsubsection{Konstruktevokation}

Die Präsentation der Elemente erfolgte mittels Triadenmethode. Von den bei 10 Elementen maximal 120 möglichen Kombinationen wurden in jedem Interview standardisiert dieselben 10 Triaden vorgelegt. Jedes Element kam dabei dreimal in einer Triade vor (vgl. Abb. 8).

Die zusätzlich zur mündlichen Erwähnung auf einem Merkzettel notierte Konstruktevokationsfrage lautete: „Denke an deinen Sportunterricht. ${ }^{66}$ Welche zwei von diesen drei Schülern und/oder Schülerinnen sind sich ähnlich? Wie würdest du diese Ähnlichkeit bezeichnen?".

Um sicherzustellen, dass sämtliche Konstrukte erfasst wurden, wurde im Anschluss an die Triadenmethode (oder auch bei Sättigungsanzeichen) zusätzlich die full context form (vgl. Kap. 5.1.2) zur Erhebung von maximal drei weiteren Konstrukten angewandt.

Um die Kontrastpole möglichst unabhängig von den zur Erfassung der Konstruktpole sortierten Elementen zu erheben, wurden diese erst nach Benennung aller Konstruktpole, d.h. mittels Kontrastmethode erhoben (vgl. Kap. 5.1.2). Die Frage dazu lautete: „Wie lautet für dich der Kontrast zu diesem Merkmal?".

\subsubsection{Beurteilung der Elemente}

Zur Beurteilung der Schülerinnen und Schüler hinsichtlich der erfassten Merkmale wurde in Anlehnung an die aktuelle Lehrmeinung (vgl. Kap. 5.1.2) eine 5-Punkte Skala verwendet (vgl. Abb. 9). Als verbale Marken wurden die Begriffe "trifft zu“ für die Werte 1 und 5 und "trifft eher zu“ für die Werte 2 und 4 verwendet. Mit dem Wert 3 konnte ein Schüler/eine Schülerin mit keiner deutlichen Ausprägung weder des einen noch des anderen Pols beurteilt werden. Die Nicht-Beurteilbarkeit einer Schülerin/eines Schülers in Bezug auf ein bestimmtes Merkmal konnten die Befragten mit einem Strich ( - ) deutlich machen.

nander verglichen. Um die persönliche Position der Befragten zu eruieren, wurden die Elementesets nach Abschluss der Konstruktevokation mit den Elementen „I as an ideal teacher“ (Boei et al., 1989, S. 184) bzw. „guter Physikunterricht" (Fischler, 2000a, S. 35) ergänzt.

${ }^{66} \mathrm{Da}$ die meisten Lehrpersonen die vorgelegten Schüler und Schülerinnen auch in anderen Fächern unterrichteten, sollte dieser der Frage vorgelagerte Satz sicherstellen, dass die Lehrpersonen Konstrukte nannten, welche die Schüler und Schülerinnen im Kontext des Sportunterrichts charakterisierten. 
Als „Richtung des Bewertens“ wurde in Anlehnung an Fransella et al. (2004, S. 64) die horizontale, d.h. die construct-by-construct rating-Vorgehensweise gewählt (vgl. Kap. 5.1.2).

\begin{tabular}{|l|l|l|l|l|l|l|l|l|l|l|l|l|}
\hline Konstruktpol (1) & S1 & S2 & S3 & S4 & S5 & S6 & S7 & S8 & S9 & S10 & "Idea & Kontrastpol (5) \\
\hline & & & & & & & & & & & & \\
\hline & & & & & & & & & & & & \\
\hline & & & & & & & & & & & & \\
\hline & & & & & & & & & & & & \\
\hline & & & & & & & & & & & & \\
\hline & & & & & & & & & & & & \\
\hline & & & & & & & & & & & & \\
\hline & & & & & & & & & & & & \\
\hline & & & & & & & & & & & & \\
\hline & & & & & & & & & & & & \\
\hline & & & & & & & & & & & & \\
\hline & & & & & & & & & & & & \\
\hline
\end{tabular}

Anmerkung. Grau markiert sind die jeweils präsentierten Elemente (10 Triaden, 3x full context form).

Abbildung 8. Verkleinerte Darstellung des Protokollblatts zum Interview über Schülerinnen und Schüler

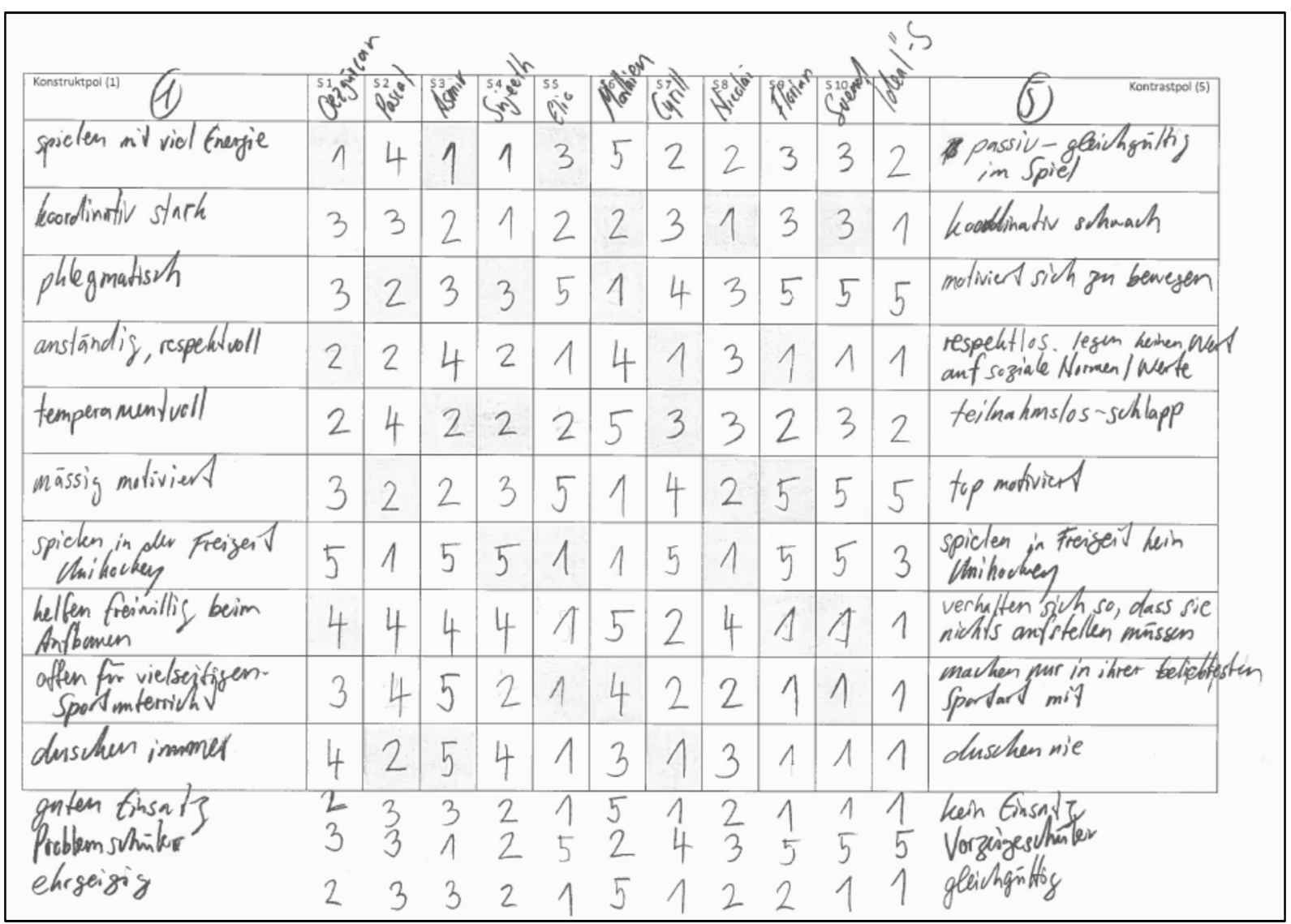

Abbildung 9. Beispiel eines komplettierten Protokollblatts zum Interview über Schülerinnen und Schüler 
5.3.2 Vorstellungen zur unterrichtlichen Umsetzung und Umsetzbarkeit interkultureller Erziehung im Sportunterricht

Ausgangslage der Entwicklung dieses Instruments war die Frage nach den Vorstellungen Sport unterrichtender Lehrpersonen zum Potenzial des Sportunterrichts für interkulturelles Lernen (vgl. Kap. 4). Erfasst werden sollten dabei einerseits unterrichtliche Bedingungen, die aus Sicht der Lehrpersonen generell Chancen für interkulturelles Lernen darstellen (Teilfragestellung 2a). Andererseits sollten die Daten Aussagen zu spezifisch auf den Sportunterricht zutreffenden Chancen interkulturellen Lernens ermöglichen (Teilfragestellung $2 b$ ).

\subsubsection{Elementtyp und Elementeset}

Da interkulturelles Lernen gemeinhin als überfachliche Zielorientierung konzeptualisiert wird (Erziehungsdirektion des Kantons Bern, 1995; 2016b; vgl. auch Kap. 1.1) , können unterschiedliche Schulfächer als Anwendungsbereich (range of convenience) persönlicher Konstrukte von Lehrpersonen zu Chancen interkulturellen Lernens aufgefasst werden. Dieser Überlegung zufolge wurden Schulfächer als Elementtyp dieses Instruments definiert.

Da insbesondere Vorstellungen bzw. Chancen zum interkulturellen Lernen im Sportunterricht interessierten, wurde das Fach Sport als Element vorgegeben. Um sicherzustellen, dass die weiteren Elemente bzw. Schulfächer ebenso im Anwendungsbereich der persönlichen Konstrukte der Befragten zu interkulturellem Lernen lagen, wurden diese vor jedem Interview individuell erhoben (vgl. Kap. 5.4.1). Die Anzahl Elemente wurde aus forschungsökonomischen Gründen auf sechs Fächer (inklusive dem Fach Sport) beschränkt und entspricht damit der empfohlenen Mindestanzahl von Elementen (vgl. Kap. 5.1.2).

\subsubsection{Konstruktevokation}

Die Konstruktevokationsfrage dieses Instruments war bestimmt durch die ihr zugrundeliegenden sehr spezifischen Teilfragestellungen der zweiten konkreten Fragestellung. Diese umfassen aus konstruktivistischer Sicht zwei Konstruktsysteme: zum einen das Konstruktsystem zum Sportunterricht, zum anderen das Konstruktsystem zum Konzept bzw. den Chancen interkulturellen Lernens. Um diese beiden Konstruktsysteme miteinander zu verbinden, wurde in Anlehnung an Fromm (1995, S. 143) eine Frage entwickelte, die nicht nur danach fragt, inwiefern sich Elemente ähnlich sind, sondern spezifischer, inwiefern sich die Elemente unter einem bestimmten Aspekt (hier: in Bezug auf interkulturelles Lernen) 
ähnlich sind ${ }^{67}$ Nach Testung einer zunächst anderen Variante ${ }^{68}$ wurde schliesslich folgende Konstruktevokationsfrage formuliert und den Befragten auf einem Merkzettel vorgelegt:

„Denke an potenzielle Chancen und Hindernisse für interkulturelles Lernen. Welche zwei von diesen drei Fächern ${ }^{69}$ sind sich diesbezüglich ähnlich? Benenne diese Ähnlichkeit!".

Die sechs Fächer wurden in acht unterschiedlichen Triaden vorgelegt. Um die Benennung von Ähnlichkeiten bzw. Unähnlichkeiten für die Befragten zu erleichtern, wurden die Triaden nicht zufällig, sondern in Bezug auf ihr interkulturelles Lernpotenzial gezielt möglichst heterogen gebildet. Die im Protokollblatt festgehaltene Systematik der Fächerkombinationen orientierte sich dabei an der im Rahmen der Elementewahl (vgl. Kap. 5.4.1) bereits erfolgten Grobeinschätzung der Fächer, die durch die Reihenfolge der Fächer in der Kopfzeile des Protokollblattes repräsentiert war. Jedes Element wurde dabei viermal in Kombination mit anderen Fächern präsentiert (vgl. Abb. 10).

Damit die Ähnlichkeiten bzw. Konstruktpole im Sinne von Chancen oder Hindernissen formuliert wurden, sollten die Befragten nach jeder Sortierung der Elemente zunächst bekunden, ob die einander zugeordneten Elemente aufgrund einer gemeinsamen Chance oder aufgrund eines gemeinsamen Hindernisses für interkulturelles Lernen ähnlich sind. Jeder Konstruktpol wurde sodann mit einem "C" (Chance) oder einem „H“ (Hindernis) gekennzeichnet (vgl. Abb. 11).

Nach der Formulierung der Chance bzw. des Hindernisses wurden die Befragten aufgefordert, zu begründen, weshalb der genannte Konstruktpol einer Chance bzw. einem Hindernis für interkulturelles Lernen entspricht („Weshalb entspricht diese Ähnlichkeit einer Chance bzw. einem Hindernis für interkulturelles Lernen?"). Die Antworten darauf bzw. die übergeordneten Konstrukte wurden unter die jeweiligen Ausgangskonstrukte notiert (vgl. Abb. 10). Dieses in Anlehnung an die Technik des laddering bzw. der „Leiternbildung“ (vgl. Kap. 5.1.2) entwickelte Vorgehen sollte im Hinblick auf die Auswertung einerseits die Nachvollziehbarkeit der Chancen und Hindernisse erhöhen, andererseits Anhaltspunkte zu den subjektiven Verständnissen des Konzepts des interkulturellen Lernens bieten.

Wie auch im Interview zu den Überzeugungen über Schülerinnen und Schüler wurde im Anschluss an die acht erhobenen Konstrukte (oder bei Sättigungsanzeichen) die full context form zur Erfassung von maximal drei weiteren Konstrukten angewandt.

\footnotetext{
${ }^{67}$ Hemmecke $(2012$, S. 332) nennt diese elaborierte Vorgehensweise „eine kontextualisierende Wendung in der Konstrukterhebung". Zu den empirischen Studien in der Lehrerkognitionsforschung, in denen mit derart formulierten Konstruktevokationsfragen gearbeitet wurde, gehören u.a. die Arbeiten von Svennberg, Meckbach und Redelius (2014) und Sulimma (2012). In beiden Untersuchungen sollten die Lehrpersonen Schülerinnen und Schüler unterscheiden. Bei Svennberg et al. (ebd.) in Bezug auf „aspects relevant to grading“ (S. 204), bei Sulimma (ebd.) in Bezug auf „Wissen und Lernen“ (S. 82).

${ }^{68}$ Bei der in Probeinterviews verwendeten Konstruktevokationsfrage „Denke an Chancen für interkulturelles Lernen. Welche zwei dieser drei Fächer sind sich diesbezüglich ähnlich?“ stellte sich heraus, dass die Lehrpersonen teilweise auch Ähnlichkeiten im Sinne von Hindernissen für interkulturelles Lernen nennen wollten. Diesen Umstand berücksichtigend, wurde die Konstruktevokationsfrage mit dem Gegenbegriff Hindernis ergänzt.

${ }^{69}$ Der Begriff Fach wurde hier stellvertretend für Fachunterricht verwendet.
} 
Ebenso analog zum ersten Interview erfolgte die Erhebung der Kontrastpole mittels Kontrastmethode. Die Frage dazu lautete: „Wie lautet für dich der Kontrast zu dieser Ähnlichkeit?“.

\begin{tabular}{|l|l|l|l|l|l|l|l|}
\hline Konstruktpol (1) & Fach 1 & Fach 2 & Fach 3 & Fach 4 & Fach 5 & Fach 6 & Kontrastpol (5) \\
\hline & & & & & & & \\
\hline & & & & & & & \\
\hline & & & & & & & \\
\hline & & & & & & & \\
\hline & & & & & & & \\
\hline & & & & & & & \\
\hline & & & & & & & \\
\hline & & & & & & & \\
\hline & & & & & & & \\
\hline & & & & & & & \\
\hline
\end{tabular}

Anmerkung. Grau markiert sind die jeweils präsentierten Elemente (8 Triaden, 3x full context form).

Abbildung 10. Verkleinerte Darstellung des Protokollblatts zum Interview über Fächer und interkulturelles Lernen

\subsubsection{Beurteilung der Elemente}

Die Beurteilung der Fächer hinsichtlich der erfassten Chancen bzw. Hindernisse erfolgte analog zum ersten Interview auf einer 5-Punkte Skala nach der construct-by-construct-Vorgehensweise.

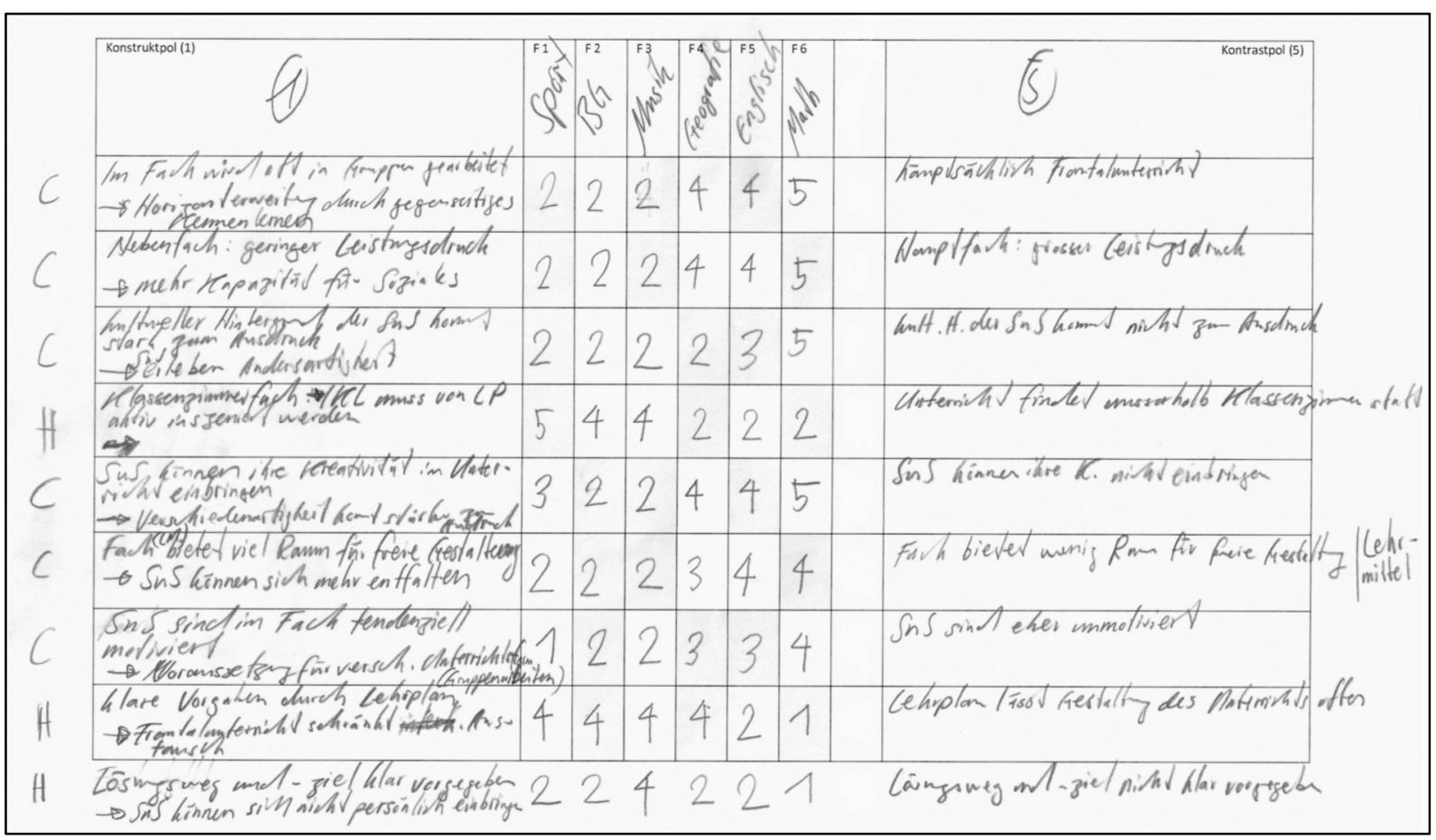

Abbildung 11. Beispiel eines komplettierten Protokollblatts zum Interview über Fächer und interkulturelles Lernen 


\subsection{Durchführung der Interviews}

Dieses Kapitel beschreibt die zentralen Aspekte der Untersuchungsdurchführung. Kapitel 5.4.1 informiert über die Organisation und Vorbereitung der Interviews, Kapitel 5.4 .2 beleuchtet die wesentlichen Aspekte der Gesprächsführung im Zuge der Datenerfassung.

\subsubsection{Organisation und Vorbereitung der Interviews}

Die Interviews fanden im Zeitraum vom 23.3.2015 bis 27.06.2015 in 19 Schulen (vgl. Kap. 5.2.2) statt. Die Interviews zu den Überzeugungen über die Schülerinnen und Schüler dauerten im Schnitt 74 $(S D=20.8)$ Minuten, diejenigen zu den Überzeugungen über das Fach $84(S D=27.7)$ Minuten. Zusätzlich wurden jeweils ca. 15 Minuten in das Briefing und die Vorbereitung der Instrumente investiert. Aufgrund dieser hohen zeitlichen Beanspruchung wurden die Befragten (abgesehen von einer Ausnahme) während zwei Terminen besucht. Während des ersten Termins wurde das Interview zu den Schülerinnen und Schülern, während des zweiten Termins das Interview zu den Fächern sowie die schriftliche Kurzbefragung durchgeführt. Grund für diese Reihenfolge war die Absicht, die Lehrpersonen thematisch möglichst unbefangen zu ihren Schülerinnen und Schülern zu befragen (vgl. Kap. 5.4.1.1).

\subsubsection{Zum Briefing der Befragten}

Vor Beginn des ersten Interviews wurden die Befragten über das Ziel und den Ablauf des Interviews informiert. Als Untersuchungsabsicht wurde die Erfassung der Wahrnehmungen von Schülerinnen und Schülern im Sportunterricht seitens der Lehrpersonen erklärt. Um etwaige Antwortverzerrungen bestmöglich zu kontrollieren, wurde das primäre Interesse - die Wahrnehmung und Einschätzung von Schülerinnen und Schülern mit Migrationshintergrund - nicht erwähnt. Ebenso unerwähnt blieben deshalb auch inhaltliche Angaben zum zweiten Interview, welches erst zum Zeitpunkt dessen Durchführung eingeführt wurde. Auch hier wurde der Hauptfokus der Untersuchung - die Einschätzung des Potenzials des Fachs Sport in Bezug auf interkulturelles Lernen - zunächst nicht erläutert. Die höhere Gewichtung der Angaben zum Fach Sport sollte den Befragten während der Interviews nicht bewusst sein. 
Die vollständige Aufklärung der Befragten über das Hauptinteresse und -ziel der beiden Interviews fand somit jeweils erst im Nachgang an das zweite Interview statt. ${ }^{70}$ Dabei wurde den Befragten offengelassen, ob sie die von ihnen erfassten Daten zurückziehen oder dennoch zur weiteren Verarbeitung zur Verfügung stellen wollen.

Zu den Informationen über den Ablauf der Befragung gehörten der Beschrieb der einzelnen Verfahrensschritte (Elemente auswählen und notieren, Konstrukt- und Kontrastevokationsfrage sowie Rating erläutern), die Erklärung des Protokollblattes, eine Zeitprognose zur Dauer des Interviews sowie der Hinweis auf den Voicerecorder. Die einzelnen Schritte wurden in Anlehnung an die aktuelle Lehrmeinung (vgl. Bell, 2011, S. 500) anhand eines themenunabhängigen Beispiels (mit verschiedenen Früchten als Elementen) erläutert und eingeübt. Die Befragten wurden zudem in Anlehnung an Fromm (1995) über die Rollenverteilung zwischen Befrager und Befragtem/r informiert. Befragten würde die Rolle des/r erfahrenen Experten/in, dem Befrager die des Zuhörers, zuweilen jedoch auch des penetranten Nachfragers zukommen. Auch wurden die Befragten beispielhaft darüber informiert, wie eine Unterhaltung zur Eruierung persönlicher Konstrukte zwischen Befrager und Befragtem verlaufen könnte. Dazu gehörte nicht zuletzt die Information, dass Mehrfachnennungen von Konstrukten durchaus „üblich“ und keineswegs als „mangelhafte Kompetenz" interpretiert würden. ${ }^{71}$

Schliesslich wurden die Befragten darauf hingewiesen, dass ihre Antworten weder richtig noch falsch sein können, sondern dass vielmehr ihre subjektive Wahrnehmung und Einschätzung interessieren würde. Auch wurde innen eine anonyme Darstellung der Ergebnisse zugesichert.

Vor dem zweiten Interview wurden diese Informationen nochmals kurz in Erinnerung gerufen. Als Ziel der Befragung wurde die Erfassung der subjektiven Meinung über interkulturelles Lernen in verschiedenen Schulfächern erklärt. Um Antwortverzerrungen bestmöglich zu vermeiden, blieb auch hier das primäre Untersuchungsinteresse - die Einschätzung des Fachs Sport in Bezug auf interkulturelles Lernen - bis nach Abschluss des Interviews unerwähnt. Der Begriff des interkulturellen Lernens wurde mit der Begründung, dass explizit das subjektive Verständnis der Befragten interessieren würde, nicht erläutert.

\footnotetext{
${ }^{70}$ Nach Bortz und Döring (2006, S. 44) ist eine zunächst unvollständige Aufklärung der Untersuchungsteilnehmenden unter Voraussetzung eines späteren Debriefings legitim.

${ }^{71}$ Dieser Hinweis sollte die Wahrscheinlichkeit der Erfindung von „Gefälligkeitskonstrukten“ minimieren, die wie Fromm (1995, S. 89) anführt - insbesondere dann erhöht ist, wenn die Befragten vom Anspruch ausgehen, für jede Triade eine weitere (neue) Unterscheidung nennen zu müssen.
} 


\subsubsection{Zum Festlegen des Elementesets}

Instrument 1: Zur Bestimmung der Elemente (Schülerinnen und Schüler) wurden die Lehrpersonen gebeten, eine nach Alphabet sortierte Namensliste einer aktuell und mindestens seit Beginn des Schuljahres 2014/15 unterrichteten Sportklasse zum Interview mitzubringen. Der Interviewer markierte darauf jeden zweiten Namen und stellte so ein Elementeset aus 10 „zufällig“ gewählten Schülerinnen und Schülern der eigenen Klasse zusammen. Bei Klassengrössen von unter 20 Lernenden wurde zur Auszählung weiterer Elemente im Alphabet wieder von vorne begonnen. Schliesslich wurden die Vornamen der 10 Schülerinnen und Schüler in die Kopfzeile des Protokollblatts und auf je einen Elementzettel notiert.

Instrument 2: Zur Wahl der Elemente des zweiten Interviews wurde den Befragten ein Blatt mit den 14 aktuell im Lehrplan des Kantons Bern stehenden Schulfächern der Sekundarstufe I vorgelegt. ${ }^{72}$ Die Befragten wurde gebeten, (abgesehen vom bereits vorgegebenen Fach Sport) all diejenigen und mindestens fünf Fächer davon auszuwählen, bei denen sie sich in der Lage fühlten, Aussagen hinsichtlich deren Potenzial für interkulturelles Lernen zu treffen. ${ }^{73}$ Damit sollte sichergestellt werden, dass die gewählten Elemente im Anwendungsbereich (range of convenience) der jeweiligen individuellen Konstrukte zum interkulturellen Lernen lagen (vgl. Kap. 5.1.2; Kap. 5.3.2). Anschliessend wurden die Lehrpersonen aufgefordert, die von ihnen gewählten Fächer und das Fach Sport gemäss einer ersten Grobeinschätzung bezüglich des interkulturellen Lernpotenzials graphisch auf einem Kontinuum (zwischen hohem und tiefem Potenzial) einzuordnen (vgl. Abb. 12). Analog zur Einordnung der Fächer auf diesem Kontinuum wurden die Fächer schliesslich nach abnehmendem interkulturellen Lernpotenzial von links nach rechts in die Kopfzeile des Protokollblatts sowie auf die sechs Element-Zettel übertragen. Bei mehr als sechs Fächern wurden diejenigen Fächer übertragen, die (graphisch) am weitesten auseinanderlagen. Schliesslich enthielt so jedes Protokollblatt sechs Fächer (inkl. des Fachs Sport), von denen davon ausgegangen werden konnte, dass sie nach Meinung der Befragten ein unterschiedlich hohes Potenzial für interkulturelles Lernen aufweisen würden. Dieses Prozedere der Elementewahl hatte den

\footnotetext{
72 Diese Vorlage sollte den Lehrpersonen einerseits die Wahl erleichtern und andererseits eine über die verschiedenen Lehrpersonen hinweg einheitliche Bezeichnung der je nach Schule zuweilen unterschiedlich bezeichneten Fächer garantieren. Im Kanton Bern heissen die 14 Fächer: Mathematik, Deutsch, Französisch, Englisch, Italienisch, Geographie (Natur - Mensch - Raum), Naturkunde (Natur - Mensch - Technik), Geschichte (Mensch - Zeit - Gesellschaft), Bewegung und Sport, Musik, Hauswirtschaft (Mensch - Konsum - Haushalt), Religion/Lebenskunde (Religion - Mensch - Ethik), Textiles und technisches Gestalten und Bildnerisches Gestalten.

${ }^{73}$ Aufgrund von Probeerhebungen wurde davon ausgegangen, dass sich die Befragten in der Lage fühlen würden, nicht nur das Fach Sport, sondern auch etliche weitere Fächer bezüglich interkulturellem Lernpotenzial einzuschätzen. Dies deshalb, weil erfahrenere Lehrpersonen der Stufe SI in der Regel in diversen Schulfächern über Unterrichtserfahrung verfügen bzw. vielfach das Lehrerseminar mit Lehrberechtigung für sämtliche Fächer auf Stufe SI absolvierten.
} 
Zweck, in den Interviews möglichst diskrete, klar voneinander unterscheidbare Elemente zu verwenden und damit die Benennung von Ähnlichkeiten bzw. Unähnlichkeiten für die Befragten zu erleichtern. Ausserdem sollte dadurch die Wahrscheinlichkeit erhöht werden, den Erfahrungsbereich der persönlichen Konstrukte bestmöglich abzudecken und letztlich die Validität der erfassten Daten zu erhöhen (vgl. dazu Kap. 5.1.2).

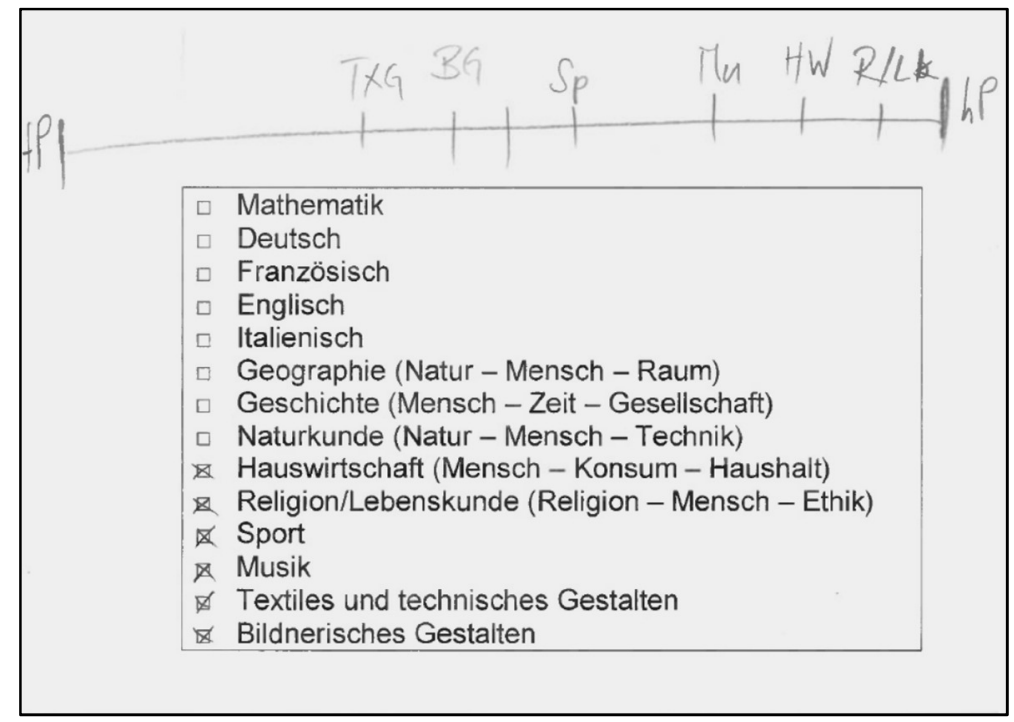

Anmerkung. tP = tiefes Potenzial; hP = hohes Potenzial.

Abbildung 12. Beispiel einer Elementewahl (angekreuzte Fächer) und einer ersten Grobeinschätzung des Potenzials für interkulturelles Lernen

\subsubsection{Gesprächsführung und Prozess der Datenerfassung}

Nach dem Briefing der Befragten und der Bestimmung der Elementesets konnte mit der eigentlichen Datenerfassung bzw. mit dem Interview begonnen werden. Es gliederte sich entsprechend der entwickelten Instrumente (vgl. Kap. 5.3.1; Kap. 5.3.2) in drei Teilschritte: In einem ersten Schritt wurden die Konstruktpole (vgl. Kap. 5.4.2.1), in einem zweiten Schritt die Kontrastpole (vgl. Kap. 5.4.2.2) und in einem dritten Schritt die Beurteilung der Elemente (rating) (vgl. Kap. 5.4.2.3) auf Basis der bipolaren Konstrukte erfasst. Dieses Kapitel beleuchtet zentrale Aspekte der Gesprächsführung und Datenerfassung entlang dieser drei Teilschritte des Interviews.

\subsubsection{Erfassung der Konstruktpole}

Fromm (1995) formuliert zwei allgemeine Anforderungen, die ein Repertory Grid-Interview gewährleisten sollte. Es sind dies a) die Möglichkeit für den/die Interviewte/n, „seine [ihre] Sicht der Dinge möglichst unbeeinträchtigt zu artikulieren“ und b) „Verständnis- und Übersetzungshilfen“ für den/die 
Interviewer/in, „die es ihm erlauben, das vom Befragten Ausgedrückte im Rahmen seines eigenen Konstruktsystems zu rekonstruieren“ (S. 55). Wie mit diesen Anforderungen im Schritt der Erfassung der Konstruktpole umgegangen wurde, soll mit den folgenden Ausführungen verdeutlicht werden.

Während die Unterscheidung bzw. Sortierung der vorgelegten Elemente („Welche zwei dieser drei Elemente sind sich ähnlich?") durch die Befragten vergleichsweise rasch erfolgte, stellte sich die Formulierung der Ähnlichkeit der einander zugeordneten Elemente („Benenne diese Ähnlichkeit!“) für die Befragten als wesentlich schwieriger heraus. ${ }^{74}$ Insbesondere bei den Interviews über Schulfächer und interkulturelles Lernen bekundeten die Lehrpersonen Mühe, ihre persönlichen Konstrukte begrifflich zu fassen. Um die in diesem Zusammenhang oft entstandene Verunsicherung der Befragten abzuschwächen, wurden die Befragten in Anlehnung an Fromm (1995, S. 88) aufgefordert, laut zu denken und sich Zeit zu nehmen. Den Befragten wurde zudem nochmals verdeutlicht, dass Konstruktformulierungen nicht „gut" oder "schlecht" sein könnten, sondern (lediglich) das bezeichnen würden, was aus Sicht der Befragten die Ähnlichkeit zwischen zwei Elementen ausmacht. Weiter wurden die Befragten angeregt, Beispiele zu nennen, anhand welcher sie die (implizite) Ähnlichkeit zweier Elemente verdeutlichen bzw. formulieren könnten. Solche Impulse erwiesen sich im Hinblick auf eine unbeeinträchtigte Formulierung von Konstruktpolen durch die Befragten als sehr hilfreich.

Um sicherzustellen, dass das vom Befragten Ausgedrückte „richtig“ verstanden wurde, blieb der Befrager aber dennoch meist auf "Verständnis- und Übersetzungshilfen“ (Fromm, 1995, S. 55) für genannte Aussagen oder Begriffe angewiesen. Folgender Interviewausschnitt aus dem zweiten Interview mit L9 soll dies beispielhaft verdeutlichen:

I: „Also, dann wäre die erste Kombination Sport, Bildnerisches Gestalten und Mathematik. Denke an potenzielle Chancen und Hindernisse für interkulturelles Lernen in diesen drei Fächern. Wo hast du das Gefühl, gibt es Überschneidungen?"

L: (fügt die Fächer Sport und Bildnerisches Gestalten zusammen)

I: „Sport und Bildnerisches Gestalten.“

L: (überlegt lange)

I: „Denkst du eher an eine Chance oder an ein Hindernis?"

L: „Also ich denke jetzt hier eher an eine Chance. (I: Hm.) Ehm...und zwar...ehm...es werden hier einfach andere...ehm...wie soll ich sagen...ehm...ein bisschen andere Aspekte angesprochen, als einfach kognitive Überlegungen oder Logiken oder so."

\footnotetext{
${ }^{74}$ Diese für Repertory Grid-Interviews typische Schwierigkeit wird auf Basis der theoretischen Annahmen der PCP damit begründet, dass persönliche Konstrukte oft nur in impliziter Form, d.h. als zunächst nicht verbalisierbare Unterscheidung vorliegen.
} 


\section{I: „Was meinst du konkret...?"}

$\mathrm{L}:$ „Beides geht in den musischen Bereich...ehm...und ehm mich dünkt, da ist immer...da ist immer viel Potenzial vorhanden. Ehm...und jetzt sollte ich so einen gemeinsamen Nenner finden, da von diesen zwei?"

I: „Ja. Hm...du meinst der musische Bereich ist für dich eher nicht kognitiv...dann wäre für dich das Musische so ein bisschen das Gegenteil vom Kognitiven, wenn ich dich richtig verstehe."

L: „Ja, also ja auch der emotionale Bereich ist natürlich hier auch stärker drin, als z. B. hier in der Mathematik. (I: ok) Also so emotionale...also wie soll ich dem sagen...emotionale Intelligenz und so Phantasie, Ausdruck...ehm...das kommt ja hier (zeigt auf Fach Sport) auch vor, Phantasie und Ausdruck...zum Teil...je nachdem, was man macht. Und hier (zeigt auf Fach Bildnerisches Gestalten) auch. Da sehe ich eigentlich das Potenzial und da gibt es weniger Barrieren als bei diesem Logischen, Analytischen...ehm...Kognitiven (zeigt auf Fach Mathematik). (I: Hm). Aber ich weiss also nicht, wie ich das formulieren soll, auf was ich das reduzieren könnte."

I: „Du sagst dieses Emotionale als Gegensatz zum Kognitiven...also auch das Kreative und...

L: „...Kreativ-emotional, vielleicht. Kreativ-emotional."

I: „Dann kann ich es so festhalten?"

$\mathrm{L}:$ „Ja."

I: „Also, dass die Inhalte kreativ-emotional sind?"

$\mathrm{L}:$ „Ja."

I: (notiert Konstruktpol: Inhalte sind kreativ-emotional).

Dem Interviewausschnitt ist zu entnehmen, dass die Aussagen und Fragen des Interviewers generell darauf abzielen, die Gedanken bzw. Aussagen des Befragten besser nachvollziehen zu können.

Nach der erfolgten Klärung, ob Chance oder Hindernis („Denkst du eher an eine Chance oder an ein Hindernis?"), versuchte der Interviewer an drei Stellen, das von der Lehrperson Gesagte für sich zu verdeutlichen. An der ersten Stelle wird um eine genauere Erläuterung gebeten (,Was meinst du konkret...?"), an der zweiten Stelle wird gewissermassen zusammengefasst, was gesagt wurde (,...dann wäre für dich das Musische so ein bisschen das Gegenteil vom Kognitiven, wenn ich dich richtig verstehe.") und an der dritten Stelle wird ein eigener Begriff (,...also auch das Kreative...") zur Verdeutlichung eingeführt.

„Übersetzungshilfen“ in Form von Bitten um Erläuterungen oder Paraphrasierungen sollen nach Fromm (1995, S. 58) denn auch „nachhaltig und durchgängig angefordert“ werden. Auf stellvertretende Formulierungen soll zum Schutz der Artikulationsfreiheit der Befragten jedoch verzichtet werden (ebd., S. 56). Wie dieser Interviewausschnitt zeigt, konnte gerade diese letzte Position nicht in 
letzter Konsequenz umgesetzt werden. Die Aussage „....also auch das Kreative...“ entspricht einer stellvertretenden Formulierung bzw. einer begrifflichen Erweiterung des vom Befragten Gesagten und fand schliesslich sogar Eingang in die definitive Formulierung des Konstruktpols.

Formulierungsangebote erwiesen sich insbesondere auch dann als unvermeidbar, wenn Befragte nach längeren Eruierungsversuchen keine für sie befriedigende Formulierung für den Konstruktpol fanden. Obwohl in solchen Fällen dieselben Triaden zu einem späteren Zeitpunkt, mit dem Ziel, die Bezeichnung des Konstruktpols ggf. nochmals zu reformulieren, vorgelegt wurden, forderten manche Lehrpersonen (zumindest implizit) Formulierungshilfe ein. In anderen Situationen blieben die Konstruktpole für den Interviewer trotz mehrfachen Bittens um Erläuterung für den Interviewer zu unklar. Hier bestand die Gefahr, die Befragten durch zu beharrliches Nachfragen zusätzlich zu verunsichern oder gar zu demotivieren. Stellvertretende Formulierungen wirkten hier oft entlastend und begünstigten ein Gespräch, in dem sich die Befragten dennoch als Experten akzeptiert fühlten.

Die Variante des im Rahmen der Erfassung von Chancen und Hindernissen für interkulturelles Lernen vorgesehenen laddering (vgl. Kap. 5.1.2; Kap. 5.3.2) konnte aus oben geschilderten Gründen nicht bei allen Lehrpersonen konsequent angewandt werden. Bei Lehrpersonen, für die sich bereits die Nennung von Chancen bzw. Hindernissen für interkulturelles Lernen als sehr anspruchsvoll erwies, wurde ad hoc entschieden, entweder gänzlich oder zumindest teilweise auf die Erfassung übergeordneter Konstrukte zu verzichten. Auch wurden in manchen Fällen für noch unklar gebliebene Formulierungen keine weiteren Verständnishilfen eingefordert. Dies hatte zwar zur Folge, dass manche Begründungen von Chancen bzw. Hindernissen so formuliert blieben, dass sie letztlich nicht wesentlich zur Explikation der Konstrukte beitrugen. ${ }^{75}$ Wie auch bei der Erfassung der Konstruktpole hatte jedoch auch hier die Aufrechterhaltung einer positiven Befindlichkeit der Befragten oberste Priorität.

Insgesamt ist somit festzuhalten, dass weitestmöglich Übersetzungshilfen durch Nachfragen eingefordert und auf Formulierungshilfen verzichtet wurde. Je nach Befindlichkeit des/r Befragten konnte jedoch mehr oder weniger hartnäckig an diesen Prinzipien der Gesprächsführung festgehalten werden. Fromm (1995, S. 60) bemerkt diesbezüglich zu Recht, dass die Entscheidung, wann die Verständigungsbemühungen abgebrochen bzw. eine hinreichende Übereinkunft über einen Begriff zwischen Interviewer und Interviewtem/r erreicht wurde, „spontan und intuitiv“ bleiben müsse.

\footnotetext{
${ }^{75}$ So wurde beispielsweise die wenig aufschlussreiche Begründung Erschwert interkulturelles Lernen zum Konstruktpol Fach ist text- und sprachlastig (L20: K4) als solche notiert, jedoch später nicht in die Auswertung miteinbezogen (vgl. Kap. 5.5.2).
} 


\subsubsection{Erfassung der Kontrastpole}

In derselben Reihenfolge wie die Konstruktpole erhoben wurden, sollten die Befragten in der zweiten Phase des Gesprächs nun den einschlägigen Kontrast zu jedem Konstruktpol nennen („,Wie lautet für dich der Kontrast zu dieser Ähnlichkeit / zu diesem Merkmal?") (vgl. Kap. 5.3.1.2; Kap. 5.3.2.2). Den Befragten wurde dabei nochmals verdeutlicht, dass der von ihnen subjektiv empfundene Kontrast und nicht das im Wörterbuch stehende Antonym interessieren würde.

Bezüglich der Gesprächsführung gestaltete sich die Erfassung der Kontrastpole deutlich einfacher als die Konstruktpol-Erfassung. Da im Rahmen der Konstruktpol-Erfassung der letztlich beiden Polen inhärente Bedeutungsgehalt von Aussagen und Begriffen diskutiert wurde, waren in diesem Schritt weniger Übersetzungshilfen notwendig. Dies war insbesondere auch dann der Fall, wenn die Befragten (lediglich) Negationen der Konstruktpole (z.B. motiviert - nicht motiviert) nannten.

Nachdem für jeden Konstruktpol ein Kontrastpol formuliert worden war, wurden die Befragten aufgefordert, sämtliche Konstruktformulierungen nochmals zu überblicken und gegebenenfalls zu justieren. Auch hier konnte der Interviewer nochmals gezielt unklar gebliebene Formulierungen ansprechen und vom Befragten erläutern bzw. umformulieren lassen.

\subsubsection{Beurteilung der Elemente}

Die Beurteilung der Elemente bzw. die Erfassung der ratings erforderte zunächst eine Erläuterung der Bedeutung der einzelnen Skalenwerte (vgl. Kap. 5.3). Um etwaige Verzerrungseffekte zu minimieren, wurden die Befragten zudem darauf hingewiesen, dass die Bewertung der Elemente unabhängig von der Wertigkeit der Pole, sondern lediglich auf Basis des Zutreffens zu den einzelnen Polen vorzunehmen sei. Mit der Bitte, laut zu denken, vervollständigten die Befragten schliesslich die Matrix eigenhändig von oben nach unten im Sinne eines construct-by-construct rating (vgl. Kap. 5.1.2). Hier zeigte sich insbesondere bei der Einschätzung der Schülerinnen und Schüler, dass die Lehrpersonen oft bestimmte Elemente bzw. Schülerinnen oder Schüler als Referenz zur Beurteilung der anderen Elemente heranzogen. Die in diesem Zusammenhang diskutierte Gefahr des primacy-recency-effects (vgl. Bortz \& Döring, 2006, S. 184) wurde bewusst in Kauf genommen, da letztlich die subjektive und damit möglicherweise auch verzerrte Wahrnehmung und Einschätzung der Lehrpersonen interessierte. Interveniert wurde jedoch, wenn die Lehrpersonen die mit den beiden Polen assoziierten Ratingwerte versehentlich verwechselten. Da sich die Lehrpersonen in ihrer Beurteilung oft am Pol positiver Wertigkeit orientierten und dieser sowohl links (= Wert 1 ) als auch rechts (= Wert 5$)$ im Protokollblatt stehen konnte, war dies relativ häufig der Fall.

Nach Einschätzung sämtlicher Elemente hinsichtlich aller Konstrukte wurde das Interview über die Fächer und interkulturelles Lernen beendet. 
Im Interview über die Schülerinnen und Schüler hingegen wurde nach Abschluss der Einschätzung der realen Schülerinnen und Schüler der „Ideal“-Schüler in die 11. Element-Spalte eingetragen (vgl. Kap. 5.3.1). Diesen, so die Instruktion, sollten die Lehrpersonen wiederum laut denkend und unabhängig von der Einschätzung ihrer eigenen (realen) Schülerinnen und Schüler einschätzen. Um dies bestmöglich zu gewährleisten, wurden die ratings der realen Schülerinnen und Schüler mittels Schablone abgedeckt.

\subsection{Auswertungsstrategie und Analyseverfahren}

Ausgangspunkt der Datenanalyse von Repertory Grid-Daten ist das mittels RGT erarbeitete Protokollblatt (Rohgrid). Die in ihm enthaltenen Daten werden - zumindest in der Anwendungsform dieser Arbeit - durch ein zweigliedriges Face-to-Face-Gespräch ermittelt. Dies geschieht zum einen durch ein auf Elementvergleichen basierendes Interview mit offenen Fragen, zum anderen durch ein darauf aufbauendes numerisches Urteilsverfahren. Entsprechend resultiert hieraus eine sowohl durch qualitative (Konstrukte) als auch quantitative Daten (rating) ausgedrückte Repräsentation der persönlichen Wahrnehmung und Strukturierung eines Grid-Themas.

Entsprechend den unterschiedlichen Skaleneigenschaften von Grid-Daten bieten sich grundsätzlich unterschiedliche Möglichkeiten für deren Analyse an. In der Literatur zur Repertory Grid-Methodik wird folglich zwischen auf die Konstrukte ausgerichteten, qualitativen und auf die ratings fokussierenden quantitativen Analysezugängen unterschieden (vgl. Bortz \& Döring, 2006; Fromm, 2010; Fromm \& Paschelke, 2010; Green, 2004; Rangosch-Schneck, 2007; Thieme, 2011). Als qualitativer Zugang gilt üblicherweise die Inhaltsanalyse, als quantitative Zugänge gelten die Clusteranalyse und die Hauptkomponentenanalyse (vgl. Fromm \& Paschelke, 2010). Wie stark der Analysefokus schliesslich auf den Text und/oder auf die Zahlen in einem Grid gelegt wird, ist vom jeweiligen Untersuchungsdesign bzw. vom Auswertungsziel abhängig.

Im Folgenden werden die Überlegungen im Hinblick auf die für diese Arbeit gewählten Auswertungsstrategien und Analyseverfahren dargelegt.

\subsubsection{Auswertungsstrategie zur Fragestellung 1}

1) Wie nehmen Sport unterrichtende Lehrpersonen Schülerinnen und Schüler mit Migrationshintergrund im Sportunterricht wahr?

Datengrundlage zur Beantwortung der Fragestellung 1 waren die 26 Protokollblätter zu den Interviews über Schülerinnen und Schüler migrationsbedingt heterogener Sportklassen. Die darin festgehaltenen 
qualitativen Daten entsprechen bipolaren Schülermerkmalen, welche die Lehrpersonen aufgrund von Schülervergleichen zum Ausdruck brachten. Die quantitativen Daten entsprechen den auf Basis dieser Merkmale skalierten Urteilen der Lehrpersonen über ihre Schülerinnen und Schüler (zuzüglich des „Ideal“-Schülers). Schliesslich ist auf den Protokollblättern vermerkt, welche der Schülerinnen und Schüler fremdsprachig sind bzw. Migrationshintergrund haben.

Sämtliche 26 Protokollblätter wurden zunächst mittels der Software Gridsuite (Version 4.0.2, Fromm, 2014) digital gesichert. Eingelesen wurden sämtliche Daten abgesehen von zwei Elementen ${ }^{76}$ und den dazugehörenden Ratingwerten. Konstruktformulierungen, die im Plural notiert waren, wurden in Einzahl umformuliert.

Im Folgenden werden die Auswertungsstrategien und Analysenverfahren zur Beantwortung der Teilfragen a) und b) näher erläutert.

a) Unterscheiden sich aus Sicht der Lehrpersonen Schülerinnen und Schüler mit Migrationshintergrund von solchen ohne Migrationshintergrund?

Um diese Frage beantworten zu können, wurden die beiden Schülergruppen (fremdsprachige Schülerinnen und Schüler vs. nicht-fremdsprachige Schülerinnen und Schüler) in Bezug auf ihre mittlere Distanz zum „Ideal“-Schüler miteinander verglichen. Als Distanzmass wurden matchings scores (vgl. Jankowicz, 2004) berechnet. Diese Masszahl gibt an, wie hoch die Ratingwerte zwischen einzelnen Elementen (auf einer Skala von 0-100\%) übereinstimmen und entspricht der Summe der Differenzen zwischen zwei Elementen als relativer Anteil der maximal möglichen Differenzsumme subtrahiert von 100 (vgl. ebd., S. 101). ${ }^{77}$ Der Vorteil dieses scores liegt im Gegensatz zu herkömmlichen Distanzmassen (wie z. B. der euklidischen Distanz) darin, dass hier die Distanzen - ausgedrückt in Prozentwerten - nicht in Abhängigkeit der Anzahl der zur Berechnung der Distanz berücksichtigten Variablen bzw. Konstrukte variieren (vgl. Brosius, 2013, S. 695). Da die Lehrpersonen unterschiedlich viele, d.h. 10-13 Konstrukte nannten, konnten somit die mittleren matching scores der beiden Schülergruppen nicht nur fallspezifisch ermittelt, sondern auch fallübergreifend miteinander verglichen werden. Die matching scores wurden mittels der Repertory Grid-Analysesoftware Gridsuite (Version 4.0.2, Fromm, 2014), der Mittelwertvergleich mittels SPSS (Version 23) berechnet.

b) Falls ja-wodurch (unterscheiden sich Schülerinnen und Schüler mit Migrationshintergrund von solchen ohne Migrationshintergrund)?

\footnotetext{
${ }^{76}$ Diese zwei Elemente repräsentierten je eine Schülerin bei zwei Lehrpersonen, welche diese (nach eigener Aussage) noch zu wenig gut kannten und deshalb in Bezug auf fünf bzw. sechs Konstrukte nicht beurteilen konnten. ${ }^{77}$ Die Formel dazu lautet: $\left.100-([S D /[L R-1) \times C]] \times 100\right) . S D=$ sum of differences, $L R=$ largest rating, $C=$ number of constructs (vgl. Jankowicz, 2004, S. 101).
} 
Da sich Schülerinnen und Schüler mit Migrationshintergrund von solchen ohne Migrationshintergrund aus Sicht der Lehrpersonen unterschieden (vgl. Kap. 6.1.1), wurde der daran anschliessenden Frage nachgegangen, wodurch sich die beiden Schülergruppen (aus Sicht der Lehrpersonen) unterscheiden. Um hierzu fallübergreifende Aussagen machen zu können, mussten zuerst sämtliche erfassten Konstrukte nach inhaltlichen Kategorien zusammengefasst und strukturiert werden. Hierzu wurde eine qualitative Inhaltsanalyse durchgeführt. ${ }^{78}$ Anschliessend wurden die Anteile der Schülerinnen und Schüler pro Schülergruppe (fremdsprachige vs. nicht-fremdsprachige Schülerinnen und Schüler), auf welche die präferierten Pole (vgl. dazu weiter unten) einer Kategorie angewandt wurden, miteinander verglichen.

Die durchgeführte, qualitative Inhaltsanalyse stützt sich zum einen auf die von Jankowicz (2004, S. 148f.) spezifisch für die aggregierte Auswertung mehrerer Grids beschriebene bootstrapping technique als induktiver Ansatz der content analysis, zum anderen auf die allgemeine Anleitung zur qualitativen Inhaltsanalyse von Kuckartz (2014) bzw. spezifischer auf seine Erläuterungen zur inhaltlich strukturierenden qualitativen Inhaltsanalyse (vgl. ebd., S. 77f.). Um die Inhaltsanalyse computergestützt durchführen zu können, wurden die Grids mittels Gridsuite (Version 4.0.2, Fromm, 2014) zunächst in ExcelDateien konvertiert und anschliessend in Tabellenform in die qualitative Datenanalyse-Software MAXQDA (Version 11) importiert.

Als Codiereinheit wurde das zweipolige Konstrukt definiert. Da Konstrukte per se bereits Abstraktionen (persönlicher Erfahrungen) darstellen (vgl. Kap. 5.1.2) und darüber hinaus in bereits paraphrasierter

\footnotetext{
${ }^{78}$ Die damit verbundene Intention der Zusammenfassung qualitativer Grid-Daten lässt sich grundsätzlich mit verschiedenen Analyseformen verfolgen. Die in Repertory Grid-Studien hierfür am häufigsten angewandten Clusteranalysen und Hauptkomponentenanalysen eignen sich insbesondere dann, wenn kleine Samplegrössen bzw. Fallanalysen vorliegen (z.B. Hewitt, 2005) und/oder den Befragten dieselben Elemente (und/oder Konstrukte) vorgegeben wurden (vgl. Bell, 2011, S. 507; Fransella et al., 2004, S. 98f.; Jankowicz, 2004, S. 145f.; Riemann, 1991, S. 45f.). Da für das Design dieser Untersuchung weder der eine noch der andere Fall zutrifft, wurde als Analyseform die qualitative Inhaltsanalyse gewählt. Inhaltsanalysen gehören im Rahmen der Repertory Grid-Methode zu den vergleichsweise selten angewandten Auswertungstechniken. Im therapeutischen Kontext werden Inhaltsanalysen meist in Bezug auf bestimmte Auffälligkeiten, wie z.B. Sprachniveau, Macht oder Sequenz der persönlichen Konstrukte (vgl. Kelly, 1991, S. 162f.) oder aber anhand vorab festgelegter Kategoriensysteme durchgeführt (vgl. Fransella et al., 2004, S. 49; Jankowicz, 2004, S. 167f.). In Anwendungsbereichen ausserhalb der Beratung geschieht die Kategorienbildung allerdings meist am Material. So etwa in der Organisationspsychologie, wenn es um die Identifikation von Merkmalen "Guter Arbeit“ aus Sicht von Erwerbstätigen unterschiedlicher Institutionen (vgl. Mösken, Dick \& Wehner, 2015) oder in der Pädagogik um die Feststellung von Eigenschaften „guter", „effektiver" oder ",authentischer“ Lehrpersonen aus Sicht von Lernenden, Studierenden oder Dozierenden geht (z.B. Boei et al., 1989; Kreber \& Klampfleitner, 2012, 2013). Abgesehen eines Beitrags von Jankowicz (2004) gibt es allerdings kaum differenziertere Methodenbeiträge zu Inhaltsanalysen im Rahmen von Repertory Grid-Untersuchungen. Autoren und Autorinnen, die Inhaltsanalysen von Grid-Daten durchführten, stützten sich dementsprechend auf Methodenliteratur ausserhalb der Repertory Grid-Methode (vgl. Boei et al., 1989; Mösken et al., 2015; Sulimma, 2012) oder entwickelten im Zuge ihrer Untersuchung eigene, gleichsam als Methodenbeiträge publizierte Vorgehensweisen (vgl. Rangosch-Schneck, 2007).
} 
Form festgehalten wurden, wurde der Abstraktionslevel der Kategorien nicht weiter angehoben. Die Benennung der Kategorien orientierte sich somit terminologisch weitestmöglich an den Formulierungen der einer Kategorie subsumierten Konstrukte. Das Hauptaugenmerk wurde dabei auf die mit dem „Ideal“-Schüler assoziierten Pole bzw. auf diejenigen Pole der Konstrukte gelegt, die (mittels der Ratingwerte 1 und $5=$ „trifft zu“ sowie 2 und 4 = „trifft eher $z u^{\prime \prime}$ ) auf den „Ideal“-Schüler angewandt wurden. Um diese - im Folgenden als „präferierte Pole“ bezeichneten - Pole im Rahmen der Analyse und Dokumentation besser erkennbar zu machen, wurden diejenigen Konstrukte, deren präferierte Pole im Protokollblatt in der Spalte der Kontrastpole (d.h. rechts) notiert waren, umgepolt. ${ }^{79}$ Die Ratingwerte umgepolter Konstrukte wurden entsprechend angepasst (1 zu 5, 2 zu 4 und umgekehrt).

Das Codieren folgte schliesslich dem von Jankowicz (2004, S. 149f.) und Kuckartz (2014, S. 77f.) empfohlenen iterativ-zyklischen Vorgehen. Das heisst, nach Bildung und Beschreibung erster Kategorien aufgrund eines ersten Codierdurchgangs wurden diese unter Berücksichtigung der Konstrukt-Retrievals (vgl. Anhang E) und der noch nicht kategorisierten Konstrukte entweder weiter (in Subkategorien) ausdifferenziert oder aber zusammengefasst und neu definiert.

Konstrukte, die nicht unidirektional formuliert wurden (z.B. motiviert - nicht motiviert) und deren beiden Pole in je eine der definierten Kategorien fielen, wurden doppelt codiert. Mehrfachnennungen von Konstrukten durch dieselbe Lehrperson wurden auch codiert und derselben Kategorie zugeordnet. ${ }^{80} \mathrm{Um}$ die Objektivität der Kategorisierung zu erhöhen, wurde schliesslich in Anlehnung an Gramespacher, Albert, Hunger und Lüsebrink (2009) eine Deutungsabsicherung in Form einer argumentativen bzw. konsensuellen Validierung mit vier weiteren Forschenden vorgenommen.

Zur Berechnung der kategorienspezifischen Unterschiede der beiden Schülergruppen (fremdsprachige vs. nicht-fremdsprachige Schülerinnen und Schüler) wurden zunächst pro Lehrperson die relativen Anteile ${ }^{81}$ der Schülerinnen und Schüler, auf welche die präferierten Pole der Konstrukte der betreffenden Kategorie angewandt wurden ${ }^{82}$, ermittelt. Wandte beispielsweise eine Lehrperson einen präferierten Pol auf drei von vier fremdsprachigen und auf vier von sechs nicht-fremdsprachigen Schülerinnen und Schülern an, so bedeutete dies, dass $75 \%$ der von ihr beurteilten fremdsprachigen und $66.7 \%$ der von

\footnotetext{
79 Diese Art von Reorganisation von Grids findet in Repertory Grid-Analysen insbesondere dann Anwendung, wenn der Fokus der Analyse - wie auch hier - auf einzelne Elemente gelegt wird (vgl. Fransella et al., 2004, S. 91f.).

${ }^{80}$ Zur Überprüfung der inhaltlichen Ähnlichkeit bzw. Übereinstimmung mehrerer von derselben Lehrperson formulierter Konstrukte wurden zusätzlich die Ratingwerte der entsprechenden Konstrukte verglichen.

${ }^{81}$ Da nicht alle Lehrpersonen gleichviele fremdsprachige und nicht-fremdsprachige Schülerinnen und Schüler einschätzten (vgl. Kap. 6.1), wurden zugunsten der interindividuellen Vergleichbarkeit der Einschätzungen die relativen Anteile anstelle der absoluten Anzahlen ermittelt.

${ }^{82}$ Die Differenzierung zwischen den beiden Zutreffensgraden "trifft zu“ (= „1“, ,5“) und "trifft eher zu“ (= „2", "4") wurde dabei im Sinne einer Dichotomisierung der Konstrukte aufgegeben. 
ihr beurteilten nicht-fremdsprachigen Schülerinnen und Schüler mit dem präferierten Pol des entsprechenden Konstrukts übereinstimmen. Bei mehreren von einer Lehrperson derselben Kategorie zugeordneten Konstrukten (Mehrfachnennungen) wurde das arithmetische Mittel der beiden Prozentwerte berechnet. Um schliesslich fallübergreifende Aussagen über die Einschätzungen der Schülerinnen und Schüler pro Kategorie machen zu können, wurde das arithmetische Mittel der pro Lehrperson ermittelten Prozentwerte berechnet.

\subsubsection{Auswertungsstrategie zur Fragestellung 2}

2) Welche Vorstellungen zum Potenzial des Sportunterrichts für interkulturelles Lernen haben Sport unterrichtende Lehrpersonen?

Die Datengrundlage zur Beantwortung der Fragestellung 2 waren die Protokollblätter zu den Fachvorstellungen in Bezug auf interkulturelles Lernen. Die darin festgehaltenen persönlichen Konstrukte entsprechen dem Repertoire an potenziellen Chancen und Hindernissen für interkulturelles Lernen, das von den Lehrpersonen genannt wurde. In ihm zum Ausdruck kommen verschiedene unterrichtsbezogene Aspekte, welche die Lehrpersonen für interkulturelles Lernen als potenziell zuträglich (Chancen) oder nicht zuträglich (Hindernisse) erachteten. Ihre Anwendung auf ein Fach (rating) lässt sich damit als Bewertung eines Faches in Bezug auf dessen Potenzial für interkulturelles Lernen interpretieren. Von den 26 während der Interviews ausgefüllten Protokollblättern wurden $24^{83}$ als Rohgrids mittels Gridsuite (Version 4.0.2, Fromm, 2014) digital gesichert. Da keine fehlenden Ratingwerte zu verzeichnen waren, beschränkte sich die Datenbereinigung auf grammatikalische Korrekturen von in den Protokollblättern notierten Konstruktformulierungen und deren allfälligen, in Anlehnung an die Methode des laddering erfassten Begründungen. Leergebliebene Grid-Zeilen wurden nicht eingelesen, da diese Information im Hinblick auf die Beantwortung der Fragestellung nicht relevant war.

Im Folgenden werden die Auswertungsstrategien und Analyseverfahren zur Beantwortung der Teilfragen a) und b) näher erläutert.

a) Welche unterrichtlichen Bedingungen stellen aus Sicht der Lehrpersonen generell Chancen für interkulturelles Lernen dar?

\footnotetext{
${ }^{83}$ Eine Teilnehmende fühlte sich (aufgrund ihres kleinen Unterrichtspensums von 30 Prozent) mit der Aufgabe überfordert und weigerte sich kurz nach Beginn des Interviews, am Gespräch teilzunehmen. Ein anderer Teilnehmender stellte sich für das Gespräch zwar zur Verfügung, zeigte sich aber wenig anstrengungsbereit und unmotiviert, sodass das Gespräch nach vergleichsweise kurzen 30 Minuten beendet war. Es wurde entschieden, diese Daten aufgrund mangelnder Reliabilität bzw. Validität nicht in die Auswertung miteinzubeziehen.
} 
Um Antworten auf diese Teilfragestellung zu finden, wurde analog zum Analyseverfahren im Zusammenhang mit der Teilfragestellung 1b) (vgl. Kap. 5.5.1) eine qualitative Inhaltsanalyse der Konstrukte durchgeführt. Hierzu wurden sämtliche Pole identifiziert, die fortan als „Chancen-Pol“ interpretiert werden sollten. Dies waren zunächst all diejenigen Konstruktpole, die von den Befragten explizit als Chance für interkulturelles Lernen bezeichnet wurden, andererseits aber auch die Kontrastpole der von den Befragten als Hindernis für interkulturelles Lernen bezeichneten Konstruktpole. Um Analyse und Dokumentation zu vereinfachen, wurden diejenigen Konstrukte, bei denen der Chancen-Pol in der Spalte der Kontrastpole notiert war, umgepolt (vgl. dazu auch Kap. 5.5.1).

Um die Inhaltsanalyse schliesslich computergestützt durchführen zu können, wurden die reorganisierten Grids via Gridsuite (Version 4.0.2, Fromm, 2014) und Microsoft Excel (Microsoft Office 2013) in die Software MAXQDA (Version 11) importiert.

Das Codieren bzw. Kategorisieren der Konstrukte erfolgte analog zur Vorgehensweise im Zusammenhang mit der Teilfragestellung 1b) (vgl. Kap. 5.5.1). Die Benennung der Kategorien orientierte sich terminologisch primär an den "Chancen-Polen“. Da die Mehrheit der Konstrukte unidirektional, d.h. mit lediglich einem Pol und dessen Negation (z.B. Rollenverständnis kommt zum Ausdruck - Rollenverständnis kommt nicht zum Ausdruck (L17: K6)) formuliert wurden, erwies sich die Zuordnung der Konstrukte insgesamt als vergleichsweise einfach. Im Gegensatz zur Inhaltsanalyse der Konstrukte zur Fragestellung 1 wurde folglich auf Mehrfachcodierungen verzichtet.

Nicht codiert wurden die durch in Form eines ladderings erfassten Begründungen einzelner „Chancenbzw. Hindernis-Pole", da diese nicht systematisch erfasst werden konnten (vgl. dazu Kap. 5.4.2). Gleichwohl wurden sie einerseits zur Beschreibung der Kategorien (vgl. Kap. 6.2.1), andererseits zur inhaltlichen Verdichtung bzw. zur Beschreibung der Beziehungen zwischen den Chancen-Kategorien (vgl. Exkurs Kap. 6.2.1) herangezogen. Begründungen, die nicht weiter zur Explikation der Ausgangskonstrukte beitrugen (vgl. dazu Kap. 5.4.2), wurden nicht in die Auswertung miteinbezogen. ${ }^{84}$

b) Welche Chancen interkulturellen Lernens treffen aus Sicht der Lehrpersonen spezifisch auf den Sportunterricht zu?

Zur Eruierung der Chancen für interkulturelles Lernen, die aus Sicht der Lehrpersonen spezifisch auf den Sportunterricht zutreffen, wurden die den Chancen-Kategorien subsumierten Konstrukte hinsichtlich ihres Zutreffens auf den Sportunterricht analysiert. Die den Kategorien subsumierten Konstrukte wurden folglich dahingehend geprüft, ob ihr jeweiliger "Chancen-Pol“ auf das Fach Sport angewandt

\footnotetext{
${ }^{84}$ Folgende Begründungen wurden nicht berücksichtigt: Bedingung für IKL (L12: K4); Kann IkL verhindern (L22: K3); IkL kann kaum zum Thema gemacht werden (L23: K7); Viel Lerngelegenheiten für IkL (L8: K5). 
wurde. ${ }^{85}$ Berücksichtigt wurden dabei nur die Ratingwerte, die inhaltlich klar mit dem „Chancen-Pol“ assoziiert wurden, d. h. die Werte 1 und 2 („trifft zu“, "trifft eher zu“). Der Ratingwert 3 wurde aufgrund seiner nicht eindeutigen Zuordenbarkeit zu einem der beiden Pole nicht berücksichtigt.

85 Ähnlich sind Mösken et al. (2015) in ihrer RGT-Studie zur Ermittlung von Unterschieden zwischen Erwerbsarbeit und frei-gemeinnütziger Tätigkeit vorgegangen. Nach einer aggregierten Auswertung der persönlichen Konstrukte verschiedener Formen von Arbeit (u.a. Hobby, Erwerbsarbeit, Hausarbeit, frei-gemeinnützige Tätigkeit) wurde eine differenzierende Analyse der Elemente frei-gemeinnützige Tätigkeit, Erwerbsarbeit und Ideal vorgenommen. Hierzu wurden all diejenigen Konstrukte in den Blick genommen, die auf die jeweilige Tätigkeit angewandt wurden. Daraus ergab sich für beide Tätigkeiten ein je eigenes Merkmalsprofil mit je unterschiedlich hoher Überlappung mit dem Ideal-Profil. 


\section{ERGEBNISSE}

In diesem Kapitel werden die Ergebnisse mit Bezug zur Fragestellung 1 (vgl. Kap. 6.1) und zur Fragestellung 2 (vgl. Kap. 6.2) dargestellt. Beide Kapitel gliedern sich entlang den Teilfragestellungen der beiden Hauptfragstellungen.

\subsection{Schülerinnen und Schüler mit Migrationshintergrund im Sportunterricht aus Sicht der Lehrpersonen}

Das vorliegende Kapitel referiert die Ergebnisse der empirischen Untersuchung zur Fragestellung 1 (Wie nehmen Sport unterrichtende Lehrpersonen Schülerinnen und Schüler mit Migrationshintergrund im Sportunterricht wahr?). Kapitel 6.1.1 bezieht sich auf die Teilfragestellung 1a, Kapitel 6.1.2 auf die Teilfragestellung 1b. In Kapitel 6.1.3 werden die Ergebnisse zur Fragestellung 1 zusammengefasst. Folgende Ausführungen geben zunächst einen Überblick über die von den Lehrpersonen beurteilten bzw. in die Elementesets gewählten Schülerinnen und Schüler.

Tabelle 4 präsentiert die Anteile fremdsprachiger Schülerinnen und Schüler der im Rahmen der Interviews einbezogenen Klassen sowie die Anzahl fremdsprachiger Schülerinnen und Schüler, die ins Elementeset gewählt wurden. Mit 45.5\% liegt der durchschnittliche Anteil fremdsprachiger Schülerinnen und Schüler pro Klasse leicht über demjenigen der selektierten Schulen (43.6\%, vgl. Kap. 5.2.2). Die mittlere Anzahl von $4.6(S D=1.7$, range: $2-8)$ Fremdsprachigen pro Grid zeigt, dass die Zufallswahl der Schülerinnen und Schüler in etwa den Anteil fremdsprachiger Schülerinnen und Schüler in der Klasse widerspiegelt. ${ }^{86}$

Die Verteilung der Klassen nach Niveaustufe ergab sich erwartungsgemäss: Die Mehrheit $(n=14)$ der für die Interviews einbezogenen Klassen waren Realklassen, 11 entsprachen integrierten Klassen und lediglich eine Klasse war eine Sekundarklasse. Diese Verteilung spiegelt die aktuellen Schülerstatistiken des Kantons Bern wider, die für Realklassen gegenüber Sekundarklassen höhere Anteile an fremdsprachigen Schülerinnen und Schüler verzeichnen (vgl. Allraum, Weissbaum \& Wassmer, 2017).

\footnotetext{
${ }^{86}$ Wie in Kapitel 5.5.1 erwähnt, konnten zwei Elemente aus je einem Elementeset nicht in die Datenauswertung miteinbezogen werden. Zwei Elementesets umfassten somit nur neun (statt 10) Schülerinnen und Schüler. 
Tabelle 4. Anteil fremdsprachiger SuS pro Klasse und pro Grid differenziert nach Niveaustufe der Klasse

\begin{tabular}{lccc} 
& $n$ & Anteil fremdspr. SuS pro Klasse & $\begin{array}{c}\text { Mittlere Anzahl fremdspr. SuS } \\
\text { in Grid }\end{array}$ \\
\hline & & {$[\%]$} & 5.1 \\
Realklassen & 14 & 51.1 & 5 \\
Sekundarklassen & 1 & 43 & 4 \\
Integrierte Klassen & 11 & 38.6 & $\mathbf{4 . 6}$ \\
Gesamt & $\mathbf{2 6}$ & $\mathbf{4 5 . 5}$ & \\
\hline
\end{tabular}

Anmerkung. Die mittlere Anzahl nicht-fremdsprachiger SuS pro Grid entspricht $5.3(S D=1.6$, range: $2-8)$ Schülerinnen und Schülern.

Tabelle 5 zeigt die Verteilung der für die Interviews herangezogenen Schülerinnen und Schüler nach Muttersprache. Nach Bereinigung der Daten verblieben 258 Schülerinnen und Schüler im Datensatz, wovon 120 bzw. $46.5 \%$ fremdsprachig sind.

Insgesamt wurden 20 verschiedene Muttersprachen identifiziert, die nicht der Unterrichtssprache (Deutsch) entsprechen. Mit Abstand am häufigsten von den Lehrpersonen beurteilt wurden Schülerinnen und Schüler, deren Muttersprachen in den Ländern Ex-Jugoslawiens oder in Albanien gesprochen werden (Albanisch: 34, Serbisch: 13, Kroatisch: 2, Bosnisch: 2, Mazedonisch: 1). Ihr Anteil an den fremdsprachigen Schülerinnen und Schülern beträgt aufsummiert 43.7\%. Grössere Anteile kommen zudem Schülerinnen und Schülern mit Türkisch (11.8\%), Tamilisch (10.9\%) oder Italienisch (8.4\%) als Muttersprache zu. Mit kleineren Anteilen vertreten sind schliesslich die Sprachen Französisch (4.2\%), Portugiesisch (3.4\%) und Spanisch (3.4\%). Alle weiteren Sprachen entsprechen den Muttersprachen von jeweils maximal drei der beurteilten Schülerinnen und Schüler. 
Tabelle 5. Muttersprachen der beurteilten Schülerinnen und Schüler

\begin{tabular}{|c|c|c|c|c|c|c|c|}
\hline & Muttersprache & Anzahl SuS & Anteil SuS & & & & \\
\hline & & $n$ & [\%] & & & & \\
\hline 1 & Deutsch & 138 & 53.5 & & & & \\
\hline 2 & $\begin{array}{l}\text { fremdsprachig } \\
\text { davon: }\end{array}$ & 120 & 46.5 & & & & \\
\hline 2.1 & Albanisch & 34 & 28.6 & 2.12 & Kurdisch & 2 & 1.7 \\
\hline 2.2 & Türkisch & 14 & 11.8 & 2.13 & Kroatisch & 2 & 1.7 \\
\hline 2.3 & Tamilisch & 13 & 10.9 & 2.14 & Bosnisch & 2 & 1.7 \\
\hline 2.4 & Serbisch & 13 & 10.9 & 2.15 & Englisch & 2 & 1.7 \\
\hline 2.5 & Italienisch & 10 & 8.4 & 2.16 & Lettisch & 1 & 0.8 \\
\hline 2.6 & Französisch & 5 & 4.2 & 2.17 & Russisch & 1 & 0.8 \\
\hline 2.7 & Portugiesisch & 4 & 3.4 & 2.18 & Ukrainisch & 1 & 0.8 \\
\hline 2.8 & Spanisch & 4 & 3.4 & 2.19 & Mazedonisch & 1 & 0.8 \\
\hline 2.9 & Tygrinisch & 3 & 2.5 & 2.20 & Vietnamesisch & 1 & 0.8 \\
\hline 2.10 & Arabisch & 3 & 2.5 & 2.21 & unbekannt & 2 & 1.7 \\
\hline 2.11 & Persisch & 2 & 1.7 & & & & \\
\hline
\end{tabular}

6.1.1 Unterschied zwischen fremdsprachigen und nicht-fremdsprachigen Schülerinnen und Schülern

Dieses Kapitel referiert die Befunde, die sich auf die Teilfragestellung 1a (Unterscheiden sich aus Sicht der Lehrpersonen Schülerinnen und Schüler mit Migrationshintergrund von solchen ohne Migrationshintergrund?) beziehen. Ziel war es, zu eruieren, ob sich aus Sicht der Lehrpersonen fremdsprachige von nicht-fremdsprachigen Schülerinnen und Schülern unterscheiden (vgl. Kap. 5.5.1).

Tabelle 6 präsentiert die Mittelwerte der mittleren matching scores (vgl. Kap. 5.5.1) fremdsprachiger und nicht-fremdsprachiger Schülerinnen und Schüler mit dem „Ideal“-Schüler. Es zeigt sich, dass die nicht-fremdsprachigen Schülerinnen und Schüler $(M=69.2, S D=7.1)$ durchschnittlich stärker mit dem „Ideal"-Schüler matchen, d.h. aus Sicht der Lehrpersonen näher am „Ideal"-Schüler liegen als die fremdsprachigen Schülerinnen und Schüler $(M=60.4, S D=13.8)$.

Zur Überprüfung der Bedeutung dieses Unterschieds wurde ein „explorativer Signifikanztest“ (Bortz \& Döring, 2006, S. 379) ${ }^{87}$ durchgeführt und auf Basis der daraus resultierenden Teststatistik die Effektstärke nach Cohen (1988) berechnet. Als Signifikanztest wurde ein t-Test für unabhängige Stichproben eingesetzt. Die Berechnung der Effektstärke erfolgte nach Borenstein, Hedges, Higgins und Rothstein

\footnotetext{
${ }^{87}$ Bortz und Döring (2006, S. 379) beschreiben unter dem Titel „Exploratives Signifikanztesten“ das Vornehmen von "Signifikanztests auf Probe“. Damit sind Signifikanztests gemeint, die unabhängig von a-priori-Hypothesen aufgrund vorgefundener, interessanter Effekte durchgeführt werden können.

106
} 
(2009, S. 228f.). Das Ergebnis des t-Tests weist mit $t(37.5)^{88}=-2.9, p=.006$ einen (auf dem .05-Niveau) überzufällig bzw. statistisch signifikanten Unterschied aus. Die Effektstärke von $d=.80$ entspricht in Anlehnung an Rost (2013, S. 244) einem grossen Effekt.

Tabelle 6. Mittelwerte der mittleren matching scores [\%] fremdsprachiger und nicht-fremdsprachiger Schülerinnen und Schüler mit dem „Ideal"-Schüler

\begin{tabular}{|c|c|c|c|c|}
\hline & $n$ & $M$ & $S D$ & range \\
\hline Mittlere matching scores fremdsprachige SuS & 26 & 60.4 & 13.8 & $29.0-87.5$ \\
\hline Mittlere matching scores nicht-fremdsprachige SuS & 26 & 69.2 & 7.1 & $51.3-86.0$ \\
\hline
\end{tabular}

Wie die Streuungsmasse in Tabelle 6 vermuten lassen, trifft dieser auf aggregierter Ebene festgestellte Unterschied nicht auf alle Lehrpersonen zu. Tabelle 7 verdeutlicht dies anhand der mittleren matching scores der beiden Schülergruppen (fremdsprachige vs. nicht-fremdsprachige Schülerinnen und Schüler) differenziert nach Lehrperson. Dabei zeigt sich, dass bei sieben (26.9\%) (grau markiert) der insgesamt 26 Lehrpersonen die mittleren matchings scores der nicht-fremdsprachigen Schülerinnen und Schüler mit dem „Ideal“-Schüler negativ von denjenigen der fremdsprachigen Schülerinnen und Schüler abweichen. Während bei vier (L7, L8, L11, L22) dieser sieben Lehrpersonen die Differenz vergleichsweise moderat ausfällt, stechen insbesondere die matching scores von L17, L18 und L23 hervor. Im Gegensatz zur überwiegenden Mehrheit der Lehrpersonen schätzten diese Lehrpersonen die von innen beurteilten fremdsprachigen Schülerinnen und Schüler deutlich „idealer" ein als die nicht-fremdsprachigen Schülerinnen und Schüler. Eine Augenscheinanalyse der soziodemographischen, berufsbezogenen und klassenbezogenen Angaben dieser Lehrpersonen bot jedoch keine plausible Erklärung für diese vorgefundenen „Ausreisser".

In Bezug auf die Teilfragestellung 1a) bleibt somit - bezogen auf die Gesamtstichprobe - festzuhalten, dass sich in der Wahrnehmung der Lehrpersonen Schülerinnen und Schüler mit Migrationshintergrund im Sportunterricht von solchen ohne Migrationshintergrund unterscheiden. Nach Einschätzung der Lehrpersonen stimmen Letztere signifikant stärker mit dem „Ideal“-Schüler überein als Erstere.

\footnotetext{
${ }^{88}$ Aufgrund vorliegender Varianzheterogenität wurde ein t-Test (für unabhängige Stichproben) mit „Welch-Korrektur" bzw. mit angepassten Freiheitsgraden berechnet.
} 
Tabelle 7. Mittlere matching scores [\%] fremdsprachiger und nicht-fremdsprachiger SuS mit dem „Ideal“-S differenziert nach Lehrperson

\begin{tabular}{|c|c|c|c|}
\hline \multirow{2}{*}{$\begin{array}{l}\text { Lehr- } \\
\text { person }\end{array}$} & \multicolumn{2}{|c|}{ mittlerer matching score mit „Ideal“-S [\%] } & \multirow{2}{*}{$\begin{array}{l}\text { positive/negative Abweichung der } \\
\text { matching scores der nicht-fremd- } \\
\text { sprachigen SuS von den matching } \\
\text { scores der fremdsprachigen SuS [\%] }\end{array}$} \\
\hline & fremdsprachige SuS & nicht-fremdsprachige SuS & \\
\hline L1 & 60.4 & 62.2 & +1.8 \\
\hline L2 & 67.1 & 71.3 & +4.2 \\
\hline L3 & 51.8 & 66.2 & +14.4 \\
\hline L4 & 47.2 & 65.4 & +18.2 \\
\hline L5 & 50.3 & 76.0 & +25.7 \\
\hline L6 & 60.0 & 75.8 & +15.8 \\
\hline L7 & 67.4 & 66.5 & -0.9 \\
\hline L8 & 70.3 & 68.7 & -1.6 \\
\hline L9 & 73.0 & 86.0 & +13.0 \\
\hline L10 & 49.0 & 74.3 & +25.3 \\
\hline L11 & 72.0 & 68.6 & -3.4 \\
\hline L12 & 53.3 & 68.6 & +15.3 \\
\hline L13 & 49.0 & 61.6 & +11.6 \\
\hline L14 & 52.8 & 69.8 & +17.0 \\
\hline L15 & 43.7 & 71.3 & +27.6 \\
\hline L16 & 38.5 & 68.7 & +30.2 \\
\hline L17 & 82.5 & 51.3 & -31.2 \\
\hline L18 & 87.5 & 70.1 & -17.4 \\
\hline L19 & 63.8 & 66.3 & +3.5 \\
\hline L20 & 66.5 & 86.0 & +19.5 \\
\hline L21 & 55.3 & 63.0 & +7.7 \\
\hline L22 & 74.5 & 68.5 & -6.0 \\
\hline L23 & 76.4 & 63.8 & -12.6 \\
\hline L24 & 65.8 & 68.8 & +3.0 \\
\hline L25 & 29.0 & 74.9 & +45.9 \\
\hline L26 & 63.7 & 66.7 & +3.0 \\
\hline Gesamt & 60.4 & 69.2 & +8.8 \\
\hline
\end{tabular}

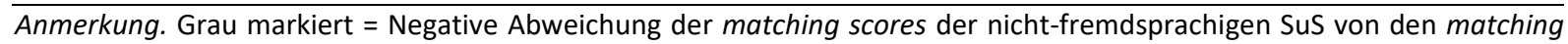
scores der fremdsprachigen SuS 
6.1.2 Kategorienspezifische Unterschiede zwischen fremdsprachigen und nicht-fremdsprachigen Schülerinnen und Schülern

Nachdem im vorangegangenen Kapitel auf den allgemeinen Unterschied zwischen Schülerinnen und Schülern mit und ohne Migrationshintergrund aus Sicht der Lehrpersonen eingegangen wurde, beleuchtet dieses Kapitel zusätzlich die inhaltlichen Dimensionen dieses Unterschieds. Die im Folgenden dargestellten Ergebnisse beziehen sich somit auf die Teilfragestellung $1 \mathrm{~b}$ (Falls ja - wodurch (unterscheiden sich Schülerinnen und Schüler mit Migrationshintergrund von solchen ohne Migrationshintergrund)?).

Die 26 Lehrpersonen nannten insgesamt 315 Konstrukte. Bei einer Vorgabe von minimal 10 und maximal 13 Konstrukten (vgl. Kap. 5.3.1) führten die Lehrpersonen im Schnitt 12.1 (SD=0.9, range = 10-13) Konstrukte an.

Aus der Inhaltsanalyse bzw. nach Kategorisierung der Konstrukte (vgl. Kap. 5.5.1) resultierten 19 induktiv gebildete Kategorien mit Konstrukten, die von minimal fünf (19.2\%) Lehrpersonen genannt wurden. 16 dieser Kategorien liessen sich als Subkategorien zu vier thematischen Hauptkategorien zusammenfassen: 1) Körperlichkeit/Sportliche Leistungsfähigkeit, 2) Engagement/Beteiligung, 3) Motivation/Interesse und 4) Mit Anderen. Eine fünfte übergeordnete Kategorie, 5) Verschiedenes, fasst die restlichen vier Subkategorien, die keiner der vier thematischen Hauptkategorien zugeordnet werden konnten, zusammen (vgl. Tab. 8).

Die Kategorien 1) - 5) umfassen zusammen 283 (89.8\%) Konstrukte ${ }^{89}$ und schliessen damit Aussagen von je minimal 18 (69.2\%) und maximal 25 (96.2\%) der 26 Lehrpersonen ein. Konstrukte, die keiner dieser Kategorien eindeutig zugeordnet werden konnten oder im minimalen Vergleich weniger als vier weiteren Konstrukten von vier Lehrpersonen ähnlich waren ${ }^{90}$, wurden der Kategorie 6) Besondere zugeordnet.

\footnotetext{
${ }^{89}$ Doppelcodierungen wurden nur 1-fach gezählt.

${ }^{90}$ Eine von fünf Lehrpersonen genannte Kategorie wurde unter Berücksichtigung der Samplegrösse $(n=26)$ und der maximalen Nennungshäufigkeit einer Kategorie $(n=21)$ als minimale Kategoriengrösse bestimmt. Auf weniger als fünf Fälle pro Kategorie wurde nicht zuletzt angesichts des Zwecks qualitativer Inhaltsanalysen - die Zusammenfassung und Reduktion von Komplexität (vgl. Kuckartz, 2014) - verzichtet. In der folgenden Darstellung der Ergebnisse wurde die Kategorie 6) deshalb nicht weiter berücksichtigt.
} 
a) Körperliche Voraussetzungen und Fähigkeiten

$17(65.4 \%) / 28$

b) (Spiel-)Sportarten-Können

$12(46.2 \%) / 16$

c) Umgang mit Körper / Körperkontrolle

$11(42.3 \%) / 13$

d) (Sportliche) Leistung generell

$8(30.8 \%) / 9$

2) Engagement / Beteiligung
a) Einsatz / Anstrengungsbereitschaft
b) Fokussiertheit / Selbständigkeit
c) Besonnenheit
d) Mut

25 (96.2\%) / 60

Motivation / Interesse

21 (80.8\%) / 31

$12(46.2 \%) / 18$

$6(23.1 \%) / 6$

$5(19.2 \%) / 5$
a) Offenheit / Begeisterungsfähigkeit
b) Motivation / Freude an Bewegung
c) Ehrgeiz / Siegeswille
d) Persönliche Vorlieben

23 (88.5\%) / 68

19 (71.1\%) / 26

15 (57.7\%) / 23

$11(42.3 \%) / 12$

$5(19.2 \%) / 7$

4) Mit Anderen

23 (88.5\%) / 63

a) Aufrichtigkeit / Loyalität / Fairness

16 (61.5\%) / 22

b) Teamfähigkeit

14 (53.8\%) / 21

c) Geltungs- / Profilierungsbedürfnis

$9(34.6 \%) / 12$

d) Beliebtheit in Klasse

$6(23.1 \%) / 7$

\section{5) Verschiedenes}

$18(69.2 \%) / 33$
a) Selbstvertrauen / Selbsteinschätzung
b) Lernfähigkeit
c) Sportengagement ausserhalb Sportunterricht
d) Elternhaus

$$
\begin{aligned}
& 11(42.3 \%) / 13 \\
& 6(23.1 \%) / 6 \\
& 6(23.1 \%) / 6 \\
& 5(19.2 \%) / 8
\end{aligned}
$$

\section{6) Besondere}

\section{$21(80.8 \%) / 32$}

$\overline{\text { Anmerkungen. Doppelcodierungen innerhalb derselben Hauptkategorie wurden nur 1-fach gezählt. Die Reihenfolge der Ka- }}$ tegorien 1) - 5) und ihrer Subkategorien entspricht von oben nach unten der abnehmenden Nennungshäufigkeit.

In den folgenden fünf Unterkapiteln (vgl. Kap. 6.1.2.1-6.1.2.5) werden die Kategorien 1) - 5) bzw. ihre Subkategorien anhand von Beispielen genannter Konstrukte näher beschrieben. ${ }^{91}$ In Anlehnung an die auf den „Ideal“-Schüler angewandten bzw. von den Lehrpersonen präferierten Pole werden dabei die Idealvorstellungen der Lehrpersonen pro Kategorie zusammengefasst. ${ }^{92}$ Diese dienen schliesslich als

\footnotetext{
${ }^{91}$ Die Konstrukt-Retrievals mit sämtlichen einer Kategorie zugeordneten Konstrukte finden sich im Anhang E. In kursiver Schrift dargestellte Wörter entsprechen den Originalformulierungen in den Rohgrids.

${ }^{92}$ Konstrukte, bei denen in der Spalte des „Ideal“-Schülers ein mittlerer (,3“) Ratingwert zu verzeichnen war bzw. (eindeutige) Angaben zur Präferenz des einen oder anderen Pols fehlen, wurden nicht berücksichtigt. Dies betrifft insgesamt 25 (7.9\%) Konstrukte.
} 
normativ-ideale Bezugspunkte zur Beschreibung der auf Basis der kategorienspezifischen Teilstichproben errechneten Unterschiede zwischen den beiden Schülergruppen (fremdsprachige vs. nicht-fremdsprachige Schülerinnen und Schüler).

Um die vorgefundenen Unterschiede explorativ auf statistische Signifikanz und praktische Bedeutsamkeit zu testen, wurden auf Basis der Teilstichproben der Kategorien 1) - 4) „explorative Signifikanztests“" (Bortz \& Döring, 2006, S. 379; vgl. Kap. 6.1.1) (t-Tests für unabhängige Stichproben) durchgeführt und auf Grundlage der daraus resultierenden Teststatistiken die Teststärken nach Cohen (1988) berechnet.

\subsubsection{Körperlichkeit/Sportliche Leistungsfähigkeit}

Die Kategorie Körperlichkeit/Sportliche Leistungsfähigkeit umfasst sämtliche Konstrukte, die sich auf die körper- bzw. bewegungsbezogene Leistung der Schülerinnen und Schüler beziehen. Sie schliesst insgesamt 65 von 25 (96.2\%) Lehrpersonen genannte Konstrukte ein und ist ausdifferenziert in die Subkategorien a) Körperliche Voraussetzungen und Fähigkeiten, b) (Spiel-)Sportarten-Können, c) Umgang mit Körper/Körperkontrolle und d) (Sportliche) Leistung generell (vgl. Tab. 9).

Tabelle 9 zeigt, dass fast die Hälfte, d.h. 28 aller körper- und bewegungsbezogenen Konstrukte in die Subkategorie a) Körperliche Voraussetzungen und Fähigkeiten fällt. Aus dem Verhältnis zwischen Anzahl Konstrukten (28) und Anzahl Lehrpersonen (17) geht zudem hervor, dass viele Lehrpersonen mehrere dieser Subkategorie zugeordneten Konstrukte nannten. Eingeschlossen wurden dabei all jene Konstrukte, über welche die Schülerinnen und Schüler aufgrund deren konditionellen und koordinativen Fähigkeiten bzw. Voraussetzungen voneinander unterschieden wurden. So wurden beispielsweise die Konstrukte Hohe Schnelligkeit - „Schneckentempo" (L9: K9), Koordinativ stark - Koordinativ schwach (L12: K2) oder Gute physische Voraussetzungen - Übergewichtig (L24: K3) dieser Kategorie zugeordnet.

Deutlich weniger Konstrukte umfasst die 12 Lehrpersonen einschliessende Subkategorie b) (Spiel-) Sportarten-Können. Die dieser Kategorie zugeordneten, insgesamt 16 Konstrukte beziehen sich insbesondere auf das Spielverständnis (z.B. Gutes Spielverständnis - Schlechtes Spielverständnis (L19: K8)), auf die Antizipationsfähigkeit (z.B. Kann Spiel lesen - Eingeschränkte Antizipationsfähigkeit (L23: K6) sowie generell auf das Können der Schülerinnen und Schüler in (einzelnen) (Spiel-)Sportarten (z.B. Überdurchschnittlich gut in Badminton - Unterdurchschnittlich gut in Badminton (L17: K10)). 
1) Körperlichkeit / Sportliche Leistungsfähigkeit (25 Lehrpersonen / 65 Konstrukte)

a) Körperliche Voraussetzungen und Fähigkeiten (17/28)

\begin{tabular}{l|l}
\hline Hohe Schnelligkeit & „Schneckentempo“ (L9: K9) \\
Koordinativ stark & Koordinativ schwach (L12: K2) \\
Gute physische Voraussetzungen & Übergewichtig (L24: K3) \\
\hline
\end{tabular}

b) (Spiel-)Sportarten-Können (12/16)

\begin{tabular}{l|l}
\hline Überdurchschnittlich gut in Badminton & Unterdurchschnittlich gut in Badminton (L17: K10) \\
Gutes Spielverständnis & Schlechtes Spielverständnis (L19: K8) \\
Kann Spiel lesen & \\
\hline
\end{tabular}

c) Umgang mit Körper / Körperkontrolle (11/13)

\begin{tabular}{l|l}
\hline Gutes Körpergefühl & Ungeschickt (L2: K8) \\
Kontrollierte, präzise Bewegungen & Ungeschickt-tollpatschig (L3: K12) \\
Hat gute Körperwahrnehmung & Hat schlechte Körperwahrnehmung (L16: K3) \\
\hline
\end{tabular}

d) (Sportliche) Leistung generell (8/9)

Gute/r bis sehr gute/r Schüler/in

Sportlich leistungsstark

Ungenügende/r Schüler/in (L1: K11)

Leistungsschwach (L2: K9)

5,5er-Turner

4,5er-Turner (L26: K12)

Anmerkungen. Die hinter den Kategorienbezeichnungen in Klammer festgehaltenen Zahlen entsprechen der Anzahl Lehrpersonen bzw. der Anzahl Konstrukte. Die hinter den einzelnen Konstrukten notierten Angaben verweisen auf die Lehrperson und die jeweilige Konstruktzeile im Rohgrid (vgl. Anhang D). In der linken (grau markierten) Spalte finden sich die mit dem „Ideal“-Schüler assoziierten bzw. die von den Lehrpersonen präferierten Pole.

Weiter schliesst die Subkategorie c) Umgang mit Körper/Körperkontrolle 12 Konstrukte ein, mit welchen 10 Lehrpersonen ihre Schülerinnen und Schüler in Bezug auf deren Körperwahrnehmung und Kontrolliertheit der Bewegungen unterschieden. Genannt wurden u.a. die Konstrukte Gutes Körpergefühl-Ungeschickt (L2: K8), Kontrollierte, präzise Bewegungen - Ungeschickt-tollpatschig (L3: K12) oder Hat gute Körperwahrnehmung - Hat schlechte Körperwahrnehmung (L16: K3). 
Schliesslich umfasst die Subkategorie d) (Sportliche) Leistung generell neun Konstrukte, über die sehr allgemein die (sportliche) Leistung der Schülerinnen und Schüler beurteilt wurde. Im Gegensatz zu den vorangegangenen Subkategorien finden sich in den Formulierungen dieser Konstrukte keine Anhaltspunkte hinsichtlich einer spezifischen körper- bzw. bewegungsbezogenen Leistung der Schülerinnen und Schüler (z.B. Gute/r bis sehr gute/r Schüler/in - Ungenügende/r Schüler/in (L1: K11), Sportlich leistungsstark - Leistungsschwach (L2: K9) oder 5,5er-Turner-4,5er-Turner (L26: K12)).

Fasst man die präferierten Pole der beschriebenen Subkategorien zusammen, lassen sich folgende Idealvorstellungen der Lehrpersonen ihrer Schülerinnen und Schüler im Bereich Körperlichkeit/Sportliche Leistungsfähigkeit nachzeichnen:

Ein/e ideale/r Schüler/in ist allgemein sportlich (Kategorie d). Er/sie hat hohe konditionelle und koordinative Fähigkeiten (Kategorie a) und zeigt einen geschickten, feinmotorischen Umgang mit dem eigenen Körper (Kategorie c). Schliesslich beherrscht er/sie verschiedene (Spiel-)Sportarten bzw. erfüllt die dafür notwendigen Anforderungen (z.B. Antizipationsfähigkeit) (Kategorie b).

Der Vergleich der beiden Schülergruppen (fremdsprachige vs. nicht-fremdsprachige Schülerinnen und Schüler) hinsichtlich dieser Idealvorstellungen der Lehrpersonen zeigt, dass nicht-fremdsprachige Schülerinnen und Schüler $(M=60.1, S D=17.4)$ diesen stärker entsprechen als die fremdsprachigen Schülerinnen und Schüler ( $M=45.2, S D=28.4)$ (vgl. Tab. 10). Ein „explorativer Signifikanztest" weist dabei einen signifikanten Unterschied mit mittlerem Effekt aus $\left(t(39.789)^{93}=2.250, p=.030, n=50\right.$, $d=.636)$

Wie die Mittelwerte auf Ebene der Subkategorien zeigen, trifft dieser Unterschied mit mehr oder weniger starker Ausprägung auf alle Subkategorien zu. Am deutlichsten fällt er innerhalb der Subkategorie c) Umgang mit Körper/Körperkontrolle, am schwächsten innerhalb der Kategorie c) (Sportliche) Leistung generell aus (vgl. Tab. 10).

\footnotetext{
${ }^{93}$ Aufgrund vorliegender Varianzheterogenität wurde ein t-Test (für unabhängige Stichproben) mit „,Welch-Korrektur" bzw. mit angepassten Freiheitsgraden berechnet.
} 
Tabelle 10. Mittelwerte, Standardabweichungen und range der mittleren Anteile [\%] fremdsprachiger und nicht-fremdsprachiger Schülerinnen und Schüler, die dem präferierten Pol in der Kategorie "Körperlichkeit/Sportliche Leistungsfähigkeit" entsprechen

\begin{tabular}{|c|c|c|c|c|c|}
\hline Kategorie/Subkategorie & SuS-Gruppe & $n$ & $\begin{array}{l}M \\
{[\%]}\end{array}$ & $S D$ & range \\
\hline \multirow{2}{*}{$\begin{array}{l}\text { 1) Körperlichkeit/Sportli- } \\
\text { che Leistungsfähigkeit }\end{array}$} & fremdsprachige SuS & 25 & 45.2 & 28.4 & $0-100$ \\
\hline & nicht-fremdsprachige SuS & 25 & 60.1 & 17.4 & $33.3-100$ \\
\hline \multirow{2}{*}{$\begin{array}{l}\text { a) Körperliche Vorausset- } \\
\text { zungen und Fähigkeiten }\end{array}$} & fremdsprachige SuS & 17 & 45.6 & 23.1 & $0-83.3$ \\
\hline & nicht-fremdsprachige SuS & 17 & 56.8 & 19.1 & $33.3-100$ \\
\hline \multirow{2}{*}{$\begin{array}{l}\text { b) (Spiel-)Sportarten- } \\
\text { Können }\end{array}$} & fremdsprachige SuS & 11 & 49.2 & 38.8 & $0-100$ \\
\hline & nicht-fremdsprachige SuS & 11 & 59.8 & 20.4 & $33.3-100$ \\
\hline \multirow{2}{*}{$\begin{array}{l}\text { c) Umgang mit Kör- } \\
\text { per/Körperkontrolle }\end{array}$} & fremdsprachige SuS & 11 & 40.8 & 37.0 & $0-100$ \\
\hline & nicht-fremdsprachige SuS & 11 & 58.6 & 15.6 & $33.3-87.5$ \\
\hline \multirow{2}{*}{$\begin{array}{l}\text { d) (Sportliche) Leistung } \\
\text { generell }\end{array}$} & fremdsprachige SuS & 8 & 52.9 & 34.3 & $0-100$ \\
\hline & nicht-fremdsprachige SuS & 8 & 54.4 & 13.6 & 33.3-71.4 \\
\hline
\end{tabular}

Anmerkungen. $n=$ Anzahl Lehrpersonen, die (fremdsprachige und nicht-fremdsprachige) Schülerinnen und Schüler hinsichtlich ihrer den jeweiligen Kategorien bzw. Subkategorien zugeordneten Konstrukten beurteilten. $M=$ Arithmetisches Mittel der pro Lehrperson ermittelten Anteile fremdsprachiger und nicht-fremdsprachiger Schülerinnen und Schüler, auf welche die präferierten Pole angewandt wurden (vgl. Kap. 5.1.5). Konstrukte, bei denen keine (eindeutigen) Angaben zur Präferenz des einen oder anderen Pols gemacht wurden (mittlerer (,,3“) Ratingwert beim „Ideal“-Schüler), wurden nicht berücksichtigt. Die Angaben zur Anzahl Lehrpersonen können deshalb von denjenigen in Tabelle 9 abweichen.

\subsubsection{Engagement/Beteiligung}

Die Kategorie Engagement/Beteiligung enthält Konstrukte, welche die Art und Weise, wie sich die Schülerinnen und Schüler im Sportunterricht engagieren und beteiligen, beschreiben. Im Vordergrund steht dabei die Frage, wie sich die Schülerinnen und Schüler mit der Sache, d. h. den ihnen aufgetragenen Aufgaben im Sportunterricht auseinandersetzen. Damit grenzt sich diese Kategorie von der Kategorie 3) Motivation/Interesse (vgl. Kap. 6.1.2.3) ab, welche Konstrukte umfasst, die vielmehr den inneren Bezug der Schülerinnen und Schüler zur Sache beschreiben.

Die insgesamt 60 von 25 (96.2\%) Lehrpersonen angeführten Konstrukte verteilen sich auf die Subkategorien a) Einsatz/Anstrengungsbereitschaft, b) Fokussiertheit/Selbständigkeit, c) Besonnenheit sowie d) Mut (vgl. Tab 11). 
2) Engagement / Beteiligung (25/60)

a) Einsatz / Anstrengungsbereitschaft (21/31)

\begin{tabular}{l|l}
\hline Hat „Biss" & Wenig Durchhaltewille (L4: K9) \\
Strengt sich an & Faul (L5: K1) \\
Zeigt grossen Einsatz & Zeigt bescheidenen Einsatz (L8: K5) \\
\hline
\end{tabular}

b) Fokussiertheit / Selbständigkeit (12/18)

\begin{tabular}{l|l}
\hline Konzentriert & Unfokussiert (L3: K4) \\
Fokussiert auf Unterricht & Dauernd private Gespräche (L5: K12) \\
Sehr selbständig nach Erteilen des Auftrages & Braucht viel Unterstützung von Seiten Lehrperson \\
& (L22: K11) \\
\hline
\end{tabular}

c) Besonnenheit (6/6)

\begin{tabular}{l|l}
\hline Denkt mit & Ist gleichgültig (L7: K10) \\
Überlegt & Denkt wenig (L8: K10) \\
Kritisch-hinterfragend & Angepasst (L17: K8) \\
\hline
\end{tabular}

d) Mut (5/5)

\begin{tabular}{|c|c|}
\hline Mutig & Ängstlich (L1: K7) \\
\hline Mutig bis übermütig & Eher wenig Mut (L8: K8) \\
\hline Gesunde Risikobereitschaft & Ängstlich (L9: K8) \\
\hline
\end{tabular}

Tabelle 11 ist zu entnehmen, dass 21 Lehrpersonen ihre Schülerinnen und Schüler nach deren generellen Einsatz bzw. Anstrengungsbereitschaft beurteilten. Die von ihnen insgesamt 31 formulierten Konstrukte beziehen sich insbesondere auf die (willentliche) Intensität des Engagements der Schülerinnen und Schüler. Zu den zugeordneten Konstrukten gehören u. a. die Konstrukte Hat „Biss“-Wenig Durchhaltewille (L4: K9), Strengt sich an - Faul (L5: K1) und Zeigt grossen Einsatz - Zeigt bescheidenen Einsatz (L8: K5). 
Die Kategorie Fokussiertheit/Selbständigkeit fasst weiter all jene Konstrukte zusammen, die sich auf den Willen und/oder die Fähigkeit der Schülerinnen und Schüler, sich zu konzentrieren sowie selbständig eine Aufgabe zu bearbeiten, beziehen. Bezeichnend dafür sind Konstrukte wie Konzentriert - Unfokussiert (L3: K4), Fokussiert auf Unterricht - Dauernd private Gespräche (L5: K12) oder Sehr selbständig nach Erteilen des Auftrages - Braucht viel Unterstützung von Seiten Lehrperson (L22: K11).

Weitere sechs Lehrpersonen unterschieden die ihnen vorgelegten Schülerinnen und Schüler hinsichtlich deren Besonnenheit im Unterricht, d. h. danach, ob sie mitdenkend, überlegt, kritisch-hinterfragend oder vielmehr nicht mitdenkend, gleichgültig oder gar draufgängerisch (L4: K13; L7: K10; L8: K10; L16: K5; L17: K8) sind.

Schliesslich umfasst die Subkategorie Mut Konstrukte, über welche fünf Lehrpersonen die Schülerinnen und Schüler nach deren Mut versus Ängstlichkeit sortierten und einschätzten (z.B. Mutig - Ängstlich (L1: K7), Mutig bis übermütig - Eher wenig Mut (L8: K8), Gesunde Risikobereitschaft - Ängstlich (L9: K8)).

Die Idealvorstellungen der Lehrpersonen innerhalb der Kategorie Engagement/Beteiligung lassen sich in Anlehnung an die präferierten Pole wie folgt zusammenfassen:

Ein/e ideale/r Schüler/in im Sportunterricht zeigt allgemein einen hohen Einsatz und eine hohe Anstrengungsbereitschaft (Kategorie a). Er/sie arbeitet fokussiert und selbständig (Kategorie b), beteiligt sich auf eine besonnene Art und Weise (Kategorie c) und zeigt Mut in der Auseinandersetzung mit den ihm/ihr aufgetragenen Aufgaben (Kategorie d).

Aus Tabelle 12 geht hervor, dass die nicht-fremdsprachigen Schülerinnen und Schüler auch im Bereich Engagement/Beteiligung hinsichtlich der Idealvorstellungen der Lehrpersonen insgesamt besser abschneiden als die fremdsprachigen Schülerinnen und Schüler: Erstere stimmen im Mittel zu 63.6\% $(S D=21.7)$, letztere zu 47.1\% $(S D=31.5)$ mit den präferierten Polen überein, was mit $t(39.021)^{94}=2.061, p=.046, d=.608$ ebenso einem statistisch signifikanten Unterschied mit mittlerem Effekt entspricht.

Die auf Ebene der einzelnen Subkategorien berechneten Mittelwerte zeigen, dass sich dieser Gruppenunterschied aus den Einschätzungen in den Bereichen Einsatz/Anstrengungsbereitschaft, Fokussiertheit/Selbständigkeit und Besonnenheit ergibt. In diesen drei Subkategorien schnitten die nicht-fremdsprachigen Schülerinnen und Schüler hinsichtlich der Idealvorstellungen der Lehrpersonen im Mittel besser ab als die fremdsprachigen Schülerinnen und Schüler. Am deutlichsten fiel der Unterschied in

\footnotetext{
${ }^{94}$ Aufgrund vorliegender Varianzheterogenität wurde ein t-Test (für unabhängige Stichproben) mit „,Welch-Korrektur" bzw. mit angepassten Freiheitsgraden berechnet. 
der Subkategorie b) Fokussiertheit/Selbständigkeit aus. Während die nicht-fremdsprachigen Schülerinnen und Schüler hier im Mittel zu 69.6\% (SD = 18.3) dem präferierten Pol entsprechen, sind es bei den fremdsprachigen Schülerinnen und Schülern 26.0\% (SD = 29.8) (vgl. Tab. 12).

Im Gegensatz zum allgemeinen Unterschied stehen die Mittelwerte in der Subkategorie d) Mut. Die fünf Lehrpersonen, die ihre Schülerinnen und Schüler diesbezüglich beurteilten, schätzten die fremdsprachigen Schülerinnen und Schüler $(M=68.0, S D=29.5)$ im Mittel mutiger ein als die nicht fremdsprachigen Schülerinnen und Schüler $(M=54.7, S D=26.3)$.

Tabelle 12. Mittelwerte, Standardabweichungen und range der mittleren Anteile [\%] fremdsprachiger und nicht-fremdsprachiger Schülerinnen und Schüler, die dem präferierten Pol in der Kategorie „Engagement/Beteiligung" entsprechen

\begin{tabular}{llllll}
\hline Kategorie/Subkategorie & SuS-Gruppe & $n$ & $M$ & SD
\end{tabular}

[\%]

\begin{tabular}{|c|c|c|c|c|c|}
\hline \multirow{2}{*}{$\begin{array}{l}\text { 2) Engagement/Beteili- } \\
\text { gung }\end{array}$} & fremdsprachige SuS & 23 & 47.1 & 31.5 & $0-100$ \\
\hline & nicht-fremdsprachige SuS & 23 & 63.6 & 21.7 & $33.3-100$ \\
\hline \multirow{2}{*}{$\begin{array}{l}\text { a) Einsatz/Anstrengungs- } \\
\text { bereitschaft }\end{array}$} & fremdsprachige SuS & 19 & 50.7 & 32.8 & $0-100$ \\
\hline & nicht-fremdsprachige SuS & 19 & 66.8 & 24.1 & $20-100$ \\
\hline \multirow{2}{*}{$\begin{array}{l}\text { b) Fokussiertheit/Selb- } \\
\text { ständigkeit }\end{array}$} & fremdsprachige SuS & 12 & 26.0 & 29.8 & $0-75$ \\
\hline & nicht-fremdsprachige SuS & 12 & 69.6 & 18.3 & $33.3-100$ \\
\hline \multirow[t]{2}{*}{ c) Besonnenheit } & fremdsprachige SuS & 6 & 46.4 & 36.7 & $0-100$ \\
\hline & nicht-fremdsprachige SuS & 6 & 59.0 & 12.2 & $50-80$ \\
\hline \multirow[t]{2}{*}{ d) Mut } & fremdsprachige SuS & 5 & 68.0 & 29.5 & $40-100$ \\
\hline & nicht-fremdsprachige SuS & 5 & 54.7 & 26.3 & $33.3-100$ \\
\hline
\end{tabular}

Anmerkungen. $n$ = Anzahl Lehrpersonen, die (fremdsprachige und nicht-fremdsprachige) Schülerinnen und Schüler hinsichtlich ihrer den jeweiligen Kategorien bzw. Subkategorien zugeordneten Konstrukten beurteilten. $M=$ Arithmetisches Mittel der pro Lehrperson ermittelten Anteile fremdsprachiger und nicht-fremdsprachiger Schülerinnen und Schüler, auf welche die präferierten Pole angewandt wurden (vgl. Kap. 5.1.5). Konstrukte, bei denen keine (eindeutigen) Angaben zur Präferenz des einen oder anderen Pols gemacht wurden (mittlerer (,,3“) Ratingwert beim „Ideal“-Schüler), wurden nicht berücksichtigt. Die Angaben zur Anzahl Lehrpersonen können deshalb von denjenigen in Tabelle 11 abweichen. 


\subsubsection{Motivation/Interesse}

Die Kategorie Motivation/Interesse umfasst Konstrukte, die sich auf die geistige Einstellung bzw. Orientierung der Schülerinnen und Schüler zum Sport(-unterricht) beziehen. Im Zentrum steht dabei die Frage, wie die Schülerinnen und Schüler allgemein zum Sport(-unterricht) stehen bzw. welchen inneren Bezug sie zur Sache aufweisen. Damit grenzt sich diese Kategorie von der Kategorie 2) Engagement/Beteiligung ab (vgl. Kap. 6.1.2.2), der Konstrukte zugeordnet wurden, die sich darauf beziehen, wie sich die Schülerinnen und Schüler (aufgrund ihres inneren Bezugs zur Sache) schliesslich mit der Sache bzw. den innen aufgetragenen Aufgaben im Sportunterricht auseinandersetzen.

Tabelle 13. Beispiele genannter Konstrukte der Kategorie "Motivation / Interesse"

3) Motivation / Interesse (23 Lehrpersonen / 68 Konstrukte)

a) Offenheit / Begeisterungsfähigkeit (19/26)

\begin{tabular}{l|l}
\hline Offen für Neues & Nicht willig, Neues zu lernen (L3: K6) \\
Vielseitig interessiert & Nicht offen für Neues (L17: K1) \\
Begeisterungsfähig & \\
\hline
\end{tabular}

b) Motivation / Freude an Bewegung (15/23)

\begin{tabular}{l|l}
\hline Überdurchschnittlicher Bewegungsdrang & Unterdurchschnittlicher Bewegungsdrang (L11: K9) \\
Viel Eigenmotivation & Wenig Eigenmotivation (L17: K4) \\
Freut sich, sich zu bewegen & Unmotiviert (L23: K3) \\
\hline
\end{tabular}

\section{c) Ehrgeiz / Siegeswille (11/12)}

\begin{tabular}{l|l}
\hline Ehrgeizig & Gleichgültig (L5: K4) \\
Will gewinnen & Gleichgültig zu verlieren (L18: K12) \\
Es zählt nur der Sieg & Resultat zweitrangig (L26: K7) \\
\hline
\end{tabular}

d) Persönliche Vorlieben (5/7)

\begin{tabular}{l|l}
\hline Hat gerne Geräteturnen & Abneigung gegenüber Geräteturnen (L6: K3) \\
Betätigt sich gerne kreativ, tänzerisch & Betätigt sich nicht gerne kreativ, tänzerisch (L14: K9) \\
Fussballbegeistert & Fussball-Hasser (L21: K2)
\end{tabular}

Anmerkungen. Die hinter den Kategorienbezeichnungen in Klammern festgehaltenen Zahlen entsprechen der Anzahl Lehrpersonen bzw. der Anzahl Konstrukte. Die hinter den einzelnen Konstrukten notierten Angaben verweisen auf die Lehrperson und die jeweilige Konstruktzeile im Rohgrid (vgl. Anhang D). In der linken (grau markierten) Spalte finden sich die mit dem „Ideal“-Schüler assoziierten bzw. die von den Lehrpersonen präferierten Pole. 
Insgesamt wurden 68 Konstrukte von 23 (88.5\%) Lehrpersonen dieser Kategorie zugeordnet. Die der Kategorie subsumierten Konstrukte verteilen sich auf die Subkategorien a) Offenheit/Begeisterungsfähigkeit, b) Motivation/Freude an Bewegung, c) Ehrgeiz/Siegeswille und c) Persönliche Vorlieben (vgl. Tab. 13).

Tabelle 13 macht deutlich, dass die meisten Konstrukte der Kategorie Motivation/Interesse in die Subkategorien a) Offenheit/Begeisterungsfähigkeit (26 Konstrukte) und b) Motivation/Freude an Bewegung (23 Konstrukte) fallen. Ersterer wurden Konstrukte zugeordnet, über welche die Schülerinnen und Schüler bezüglich allgemeiner, d.h. vom Unterrichtsinhalt unabhängiger Offenheit, Begeisterungsfähigkeit und Lernwilligkeit unterschieden wurden (z.B. Offen für Neues - Nicht willig, Neues zu lernen (L3: K6), Vielseitig interessiert - Einseitig interessiert (L15: K11), Begeisterungsfähig - Nicht offen für Neues (L17: K1). Letztere grenzt sich hiervon insbesondere durch Konstrukte ab, die mit Formulierungen wie Bewegungsdrang (L11: K9), Eigenmotivation (L4: K9; L17: K4; L20: K8) oder Freude (L23: K3) noch stärker die „innere Bedingung" für das Engagement im Unterricht beschreiben. Insgesamt wird aus dem Verhältnis zwischen Anzahl Konstrukten und Anzahl Lehrpersonen in beiden Kategorien deutlich, dass viele Lehrpersonen mehrere diesen Kategorien zugeordnete Konstrukte nannten.

Deutlich weniger umfassend sind dagegen die je 11 bzw. fünf Lehrpersonen einschliessenden Subkategorien c) Ehrgeiz/Siegeswille (12 Konstrukte) und d) Persönliche Vorlieben (7 Konstrukte). In erstere Kategorie wurden Konstrukte eingeschlossen, die explizit zur Unterscheidung der Schülerinnen und Schüler in Bezug auf ihren Ehrgeiz und ihren Willen zu gewinnen angewandt wurden. Genannt wurden etwa die Konstrukte Ehrgeizig - Gleichgültig (L5: K4), Will gewinnen - Gleichgültig zu verlieren (L18: K12) oder Es zählt nur der Sieg - Resultat zweitrangig (L26: K7). Letztere Kategorie steht schliesslich für Konstrukte, mit denen Schülerinnen und Schüler hinsichtlich ihrer Freude an bzw. Begeisterung für spezifische(n) Sportarten oder Unterrichtsinhalte charakterisiert wurden (z.B. Hat gerne Geräteturnen - Abneigung gegenüber Geräteturnen (L6: K3), Betätigt sich gerne kreativ, tänzerisch - Betätigt sich nicht gerne kreativ, tänzerisch (L14: K9), Fussballbegeistert - Fussball-Hasser (L21: K2)).

Der/die ideale Schüler/in aus Sicht der Lehrpersonen lässt sich auf Grundlage der präferierten Pole im Bereich Motivation/Interesse folgendermassen beschreiben:

Ideal ist ein/e Schüler/in, wenn er/sie einen inneren Bewegungsdrang aufweist bzw. allgemein Freude an Bewegung hat (Kategorie b), wenn er/sie offen und (für vielseitigen Unterricht) begeisterungsfähig ist (Kategorie a) und wenn er/sie ehrgeizig ist bzw. siegen will (Kategorie c). Schliesslich zeigt der/die ideale Schüler/in Freude an bzw. Begeisterung für spezifische(n) Sportarten oder Unterrichtsinhalte(n). 
Analog zu den vorangegangenen Kategorien weist der Vergleich der beiden Schülergruppen hinsichtlich der Idealvorstellungen der Lehrpersonen auch hier auf eine positivere Einschätzung der nichtfremdsprachigen Schülerinnen und Schüler $(M=65.3, S D=20.1)$ gegenüber den fremdsprachigen Schülerinnen und Schülern $(M=54.8, S D=25.8)$ hin (vgl. Tab. 14). Das Ergebnis des T-Tests weist jedoch einen statistisch nicht signifikanten Unterschied aus $(t(44)=1.538, p=.131)$. Die Effektstärke $(d$ $=0.454)$ entspricht einem kleinen Effekt.

Wie die Angaben in Tabelle 14 deutlich machen, trifft die Richtung des Unterschieds denn auch nicht auf alle Subkategorien zu. So zeigt sich, dass nicht-fremdsprachige Schülerinnen und Schüler zwar als vergleichsweise offener bzw. begeisterungsfähiger sowie allgemein motivierter bzw. bewegungsfreudiger eingeschätzt wurden, gleichzeitig aber in den Kategorien Ehrgeiz/Siegeswille sowie Persönliche Vorlieben bezüglich Idealvorstellungen schlechter als die fremdsprachigen Schülerinnen und Schüler abschneiden (vgl. Tab. 14).

Schneiden die nicht-fremdsprachigen Schülerinnen und Schüler in der Kategorie Motivation/Interesse insgesamt besser ab als die fremdsprachigen Schülerinnen und Schüler, dann also nicht, weil sie aus Sicht der Lehrpersonen ehrgeiziger bzw. siegeswilliger oder mehr Interesse an spezifischen Unterrichtsinhalten zeigen, sondern vielmehr deshalb, weil sie eher dem Ideal eines/r offenen und (für vielseitigen Unterricht) begeisterungsfähigen und allgemein motivierten Schülers/in entsprechen. 
Tabelle 14. Mittelwerte, Standardabweichungen und range der mittleren Anteile [\%] fremdsprachiger und nicht-fremdsprachiger Schülerinnen und Schüler, die dem präferierten Pol in der Kategorie "Motivation/Interesse" entsprechen

\begin{tabular}{|c|c|c|c|c|c|}
\hline Kategorie/Subkategorie & SuS-Gruppe & $n$ & $M$ & $S D$ & range \\
\hline & & & {$[\%]$} & & \\
\hline \multirow[t]{2}{*}{ 3) Motivation/Interesse } & fremdsprachige SuS & 23 & 54.8 & 25.8 & $0-100$ \\
\hline & nicht-fremdsprachige SuS & 23 & 65.3 & 20.1 & $26.7-100$ \\
\hline \multirow{2}{*}{$\begin{array}{l}\text { a) Offenheit/Begeiste- } \\
\text { rungsfähigkeit }\end{array}$} & fremdsprachige SuS & 19 & 51.6 & 34.7 & $0-100$ \\
\hline & nicht-fremdsprachige SuS & 19 & 65.2 & 20.3 & $33.3-100$ \\
\hline \multirow{2}{*}{$\begin{array}{l}\text { b) Motivation/Freude an } \\
\text { Bewegung }\end{array}$} & fremdsprachige SuS & 15 & 58.6 & 33.0 & $0-100$ \\
\hline & nicht-fremdsprachige SuS & 15 & 66.6 & 25.4 & $20-100$ \\
\hline \multirow[t]{2}{*}{ c) Ehrgeiz/Siegeswille } & fremdsprachige SuS & 10 & 62.1 & 24.9 & $25-100$ \\
\hline & nicht-fremdsprachige SuS & 10 & 60.4 & 28.8 & $20-100$ \\
\hline \multirow[t]{2}{*}{ d) Persönliche Vorlieben } & fremdsprachige SuS & 5 & 61.4 & 24.8 & $33.3-100$ \\
\hline & nicht-fremdsprachige SuS & 5 & 46.5 & 22.9 & $16.6-66.6$ \\
\hline
\end{tabular}

Anmerkung. $n=$ Anzahl Lehrpersonen, die (fremdsprachige und nicht-fremdsprachige) Schülerinnen und Schüler hinsichtlich ihrer den jeweiligen Kategorien bzw. Subkategorien zugeordneten Konstrukten beurteilten. $M=$ Arithmetisches Mittel der pro Lehrperson ermittelten Anteile fremdsprachiger und nicht-fremdsprachiger Schülerinnen und Schüler, auf welche die präferierten Pole angewandt wurden (vgl. Kap. 5.1.5). Konstrukte, bei denen keine (eindeutigen) Angaben zur Präferenz des einen oder anderen Pols gemacht wurden (mittlerer (,,3“) Ratingwert beim „Ideal“-Schüler), wurden nicht berücksichtigt.

\subsubsection{Mit Anderen}

Die Kategorie „Mit Anderen“ schliesst Konstrukte ein, die von den Lehrpersonen zur Unterscheidung bzw. Beurteilung der Schülerinnen und Schüler in Bezug auf ihr soziales Verhalten und ihre soziale Rolle im Sportunterricht herangezogen wurden.

Die insgesamt 63 von 23 (88.5\%) Lehrpersonen genannten Konstrukte verteilen sich auf die Subkategorien a) Aufrichtigkeit/Loyalität/Fairness, b) Teamfähigkeit, c) Geltungs-/Profilierungsbedürfnis, d) Beliebtheit in Klasse (vgl. Tab. 15). 
4) Mit Anderen (23/63)

a) Aufrichtigkeit / Loyalität / Fairness (16/22)

\begin{tabular}{l|l}
\hline Sehr korrekt & "Falsch“ (L6: K4) \\
Fair & Unfair-unehrlich (L21: K5) \\
Anerkennt Lehrperson & Stellt Lehrperson halblaut in Frage (L25: K4) \\
\hline
\end{tabular}

b) Teamfähigkeit $(14 / 21)$

\begin{tabular}{l|l}
\hline Motiviert andere & Demotiviert andere durch Zurechtweisung (L7: K6) \\
Gruppenkonstellation spielt keine Rolle & Gruppenkonstellation hat hohe Bedeutung (L11: K7) \\
Teamplayer & Hat Mühe, den Teamgedanken zu leben (L14: K8) \\
\hline
\end{tabular}

c) Geltungs- /Profilierungsbedürfnis (9/12)

\begin{tabular}{l|l}
\hline Eigene Leistung wird „innerlich“ gefeiert & Übertriebenes Feiern der eigenen Leistung (L2: K11) \\
Stille Macher & Wird gerne in der Klasse wahrgenommen (L14: K5) \\
Braucht Rampenlicht nicht & \\
\hline
\end{tabular}

d) Beliebtheit in Klasse (6/7)

\begin{tabular}{l|l}
\hline Beliebt in der Klasse & Nicht gut in Klassenverband integriert (L1: K10) \\
Sozial gut integriert & Sozial schlecht integriert (L15: K9) \\
Leader & Wird von anderen SuS gerne links liegen gelassen \\
\hline Anmerkungen. Die hinter den Kategorienbezeichnungen in Klammern festgehaltenen Zahlen entsprechen der Anzahl Lehr- \\
personen bzw. der Anzahl Konstrukte. Die hinter den einzelnen Konstrukten notierten Angaben verweisen auf die Lehrperson \\
und die jeweilige Konstruktzeile im Rohgrid (vgl. Anhang D). In der linken (grau markierten) Spalte finden sich die mit dem \\
„Ideal“-Schüler assoziierten bzw. die von den Lehrpersonen präferierten Pole.
\end{tabular}

Tabelle 15 zeigt, dass je ungefähr ein Drittel aller dieser Kategorie subsumierten Konstrukte in die ersten beiden Subkategorien a) Aufrichtigkeit/Loyalität/Fairness (22 Konstrukte) und b) Teamfähigkeit (21 Konstrukte) fallen.

Die Subkategorie a) Aufrichtigkeit/Loyalität/Fairness umfasst Konstrukte, die sich allgemein auf die „moralische Korrektheit“ des Verhaltens der Schülerinnen und Schüler gegenüber anderen Schülerinnen und Schülern aber auch gegenüber der Lehrperson beziehen. Zu den dieser Kategorie zugeordneten Konstrukten gehören u.a. Sehr korrekt - „Falsch“ (L6: K4), Fair - Unfair-unehrlich (L21: K5) sowie Anerkennt Lehrperson - Stellt Lehrperson halblaut in Frage (L21: K5). 
Die Subkategorie b) Teamfähigkeit bezieht sich auf das soziale Verhalten der Schülerinnen und Schüler spezifisch im Kontext von Spielsituationen oder sonstigen, in Gruppen bzw. Teams zu bewältigenden Aufgaben. So wurden die Schülerinnen und Schüler u. a. danach beurteilt, ob sie andere (schwächere) Mitschülerinnen und -schüler tolerieren und in die Gruppe integrieren können (z.B. Gruppenkonstellation spielt keine Rolle - Gruppenkonstellation hat hohe Bedeutung (L11: K7)) und ob sie andere ermutigen können (z.B. Motiviert andere - Demotiviert andere durch Zurechtweisung (L7: K6)).

Die Subkategorie c) Geltungs-/Profilierungsbedürfnis schliesst weitere 12 Konstrukte ein, über welche neun Lehrpersonen ihre Schülerinnen und Schüler hinsichtlich deren Umgang mit der eigenen Leistung und der eigenen Person im sozialen Kontext charakterisierten. Genannt wurden u. a. die Konstrukte Eigene Leistung wird „innerlich“ gefeiert - Übertriebenes Feiern der eigenen Leistung (L2: K11), Stille Macher - Bluffer (L4: K3) oder Braucht Rampenlicht nicht - Wird gerne in der Klasse wahrgenommen (L14: K5).

In der kleinsten der vier Subkategorien, Beliebtheit in Klasse (6/7), finden sich schliesslich Konstrukte zur sozialen Rolle bzw. Stellung der Schülerinnen und Schüler im Klassenverband. Hier unterschieden die Lehrpersonen ihre Schülerinnen und Schüler danach, wie gut bzw. ob sie in der Klasse beliebt, integriert oder auch Leader sind (L1: K10; L7: K12; L10: K5; L15: K9; L21: K6; L24: K11).

Gemäss den präferierten Polen in den beschriebenen Subkategorien lassen sich „ideale“ Schülerinnen und Schüler aus Sicht der Lehrpersonen folgendermassen charakterisieren:

Schülerinnen und Schüler sollten gegenüber ihren Mitschülerinnen und -schülern sowie gegenüber der Lehrperson aufrichtig, loyal und fair sein (Kategorie a). In Spielsituationen bzw. in Teams sollten sie sich auf andere Schülerinnen und Schüler einlassen bzw. diese akzeptieren und unterstützen können (Kategorie b). Weiter sollten sie einen angemessenen Umgang mit ihrer Person und ihrer Leistung pflegen, d. h. z. B. eigene Schwächen zugeben können, nicht bluffen, sich aber auch nicht schämen (Kategorie c). Schliesslich präferieren die Lehrpersonen Schülerinnen und Schüler, die gut in die Klasse integriert sind (Kategorie d).

Die Berechnung der durchschnittlichen Anteile der Schülerinnen und Schüler, die diesen Idealvorstellungen entsprechen, zeigt - analog zu den vorangegangenen Kategorien - eine insgesamt positivere Einschätzung der nicht-fremdsprachigen Schülerinnen und Schüler im Vergleich zu den fremdsprachigen Schülerinnen und Schülern. Während im Mittel 66.5\% (SD = 19.5) der nicht-fremdsprachigen Schülerinnen und Schüler mit dem präferierten Pol übereinstimmen, sind es bei den fremdsprachigen Schülerinnen und Schülern durchschnittlich 53.1\% (SD = 20.7) (vgl. Tab. 16). Dieser Unterschied fällt mit $t(44)=2.270, p=.028$ statistisch signifikant aus, wobei die Effektstärke von $d=.669$ auf einen mittleren Effekt hinweist. 
Die auf Ebene der einzelnen Subkategorien berechneten Unterschiede zeigen, dass der Gesamtunterschied insbesondere auf den Unterschied in der Kategorie a) Aufrichtigkeit/Loyalität/Fairness zurückzuführen ist (vgl. Tab. 16). Gemäss ratings dieser 16 Lehrpersonen trifft bei 78.2\% (SD = 16.9) der nichtfremdsprachigen Schülerinnen und Schüler und bei 43.4\% $(S D=26.9)$ der fremdsprachigen Schülerinnen und Schüler der präferierte Pol zu. Ebenso vergleichsweise häufiger wurde der präferierte Pol auf die nicht-fremdsprachigen Schülerinnen und Schüler in der Kategorie c) Geltungs- und Profilierungsbedürfnis angewandt (Nicht-Fremdsprachige: $M=63.6, S D=28.5$, Fremdsprachige: $M=54.9, S D=$ 20.4). Fast identisch fielen hingegen die Mittelwerte in der Kategorie c) Teamfähigkeit aus (Fremdsprachige: $M=54.9, S D=31.4$, Nicht-Fremdsprachige: $M=54.7, S D=23.4$ ). Die Kategorie d) Beliebtheit in Klasse ist schliesslich die einzige Kategorie, in welcher fremdsprachige Schülerinnen und Schüler ( $M=$ $54.4, S D=15.9)$ häufiger mit dem präferierten Pol assoziiert wurden als die nicht-fremdsprachigen Schülerinnen und Schüler $(M=39.9, S D=7.0)$.

Tabelle 16. Mittelwerte, Standardabweichungen und range der mittleren Anteile [\%] fremdsprachiger und nicht-fremdsprachiger Schülerinnen und Schüler, die dem präferierten Pol in der Kategorie „Mit Anderen“ entsprechen

\begin{tabular}{|c|c|c|c|c|c|}
\hline Kategorie/Subkategorie & SuS-Gruppe & $n$ & $M$ & $S D$ & range \\
\hline & & & [\%] & & \\
\hline \multirow[t]{2}{*}{ 4) Mit Anderen } & fremdsprachige SuS & 23 & 53.1 & 20.7 & $20.8-100$ \\
\hline & nicht-fremdsprachige SuS & 23 & 66.5 & 19.5 & $38.2-100$ \\
\hline \multirow{2}{*}{$\begin{array}{l}\text { a) Aufrichtigkeit/Loyali- } \\
\text { tät/Fairness }\end{array}$} & fremdsprachige SuS & 16 & 43.4 & 26.9 & $0-83.3$ \\
\hline & nicht-fremdsprachige SuS & 16 & 78.2 & 16.9 & $58.3-100$ \\
\hline \multirow[t]{2}{*}{ b) Teamfähigkeit } & fremdsprachige SuS & 13 & 54.9 & 31.4 & $12.5-100$ \\
\hline & nicht-fremdsprachige SuS & 13 & 54.7 & 23.4 & $37.5-100$ \\
\hline \multirow{2}{*}{$\begin{array}{l}\text { c) Geltungs- } \\
\text { rungsbedürfnis }\end{array}$} & fremdsprachige SuS & 8 & 54.9 & 20.4 & $33.3-100$ \\
\hline & nicht-fremdsprachige SuS & 8 & 63.6 & 28.5 & $18.8-100$ \\
\hline \multirow[t]{2}{*}{ d) Beliebtheit in Klasse } & fremdsprachige SuS & 5 & 54.4 & 15.9 & $28.6-66.6$ \\
\hline & nicht-fremdsprachige SuS & 5 & 39.9 & 7.0 & $33.3-50$ \\
\hline
\end{tabular}

Anmerkungen. $n$ = Anzahl Lehrpersonen, die (fremdsprachige und nicht-fremdsprachige) Schülerinnen und Schüler hinsichtlich ihrer den jeweiligen Kategorien bzw. Subkategorien zugeordneten Konstrukten beurteilten. $M=$ Arithmetisches Mittel der pro Lehrperson ermittelten Anteile fremdsprachiger und nicht-fremdsprachiger Schülerinnen und Schüler, auf welche die präferierten Pole angewandt wurden (vgl. Kap. 5.1.5). Konstrukte, bei denen keine (eindeutigen) Angaben zur Präferenz des einen oder anderen Pols gemacht wurden (mittlerer (, $\left.3^{\prime \prime}\right)$ Ratingwert beim „Ideal“-Schüler), wurden nicht berücksichtigt. 


\subsubsection{Verschiedenes}

Die Kategorie 5) Verschiedenes schliesst die den anderen Hauptkategorien thematisch nicht zuordenbaren Subkategorien a) Selbstvertrauen/Selbsteinschätzung, b) Lernfähigkeit und c) Sportengagement ausserhalb Sportunterricht und d) Elternhaus ein. Darin enthalten sind insgesamt 33 von 18 Lehrpersonen genannte Konstrukte (vgl. Tab. 17).

Tabelle 17. Beispiele genannter Konstrukte in der Kategorie "Verschiedenes"

5) Verschiedenes (18/33)

a) Selbstvertrauen / Selbsteinschätzung (11/13)

\begin{tabular}{l|l}
\hline Selbstsicher & Zweifelt an sich (L4: K4) \\
Hohes Selbstvertrauen & Tiefes Selbstvertrauen (L16: K4) \\
Kann eigene Leistung realistisch einschätzen & Kann eigene Leistung nicht realistisch einschätzen \\
\hline
\end{tabular}

b) Lernfähigkeit (6/6)

\begin{tabular}{l|l}
\hline Rasche Auffassungsgabe & Langsame Auffassungsgabe (L2: K2) \\
Lernfähig & Wachig lernfähig (L3: K9) \\
Lernt ring & \\
\hline
\end{tabular}

c) Sportengagement ausserhalb Sportunterricht (6/6)

\begin{tabular}{l|l}
\hline Treibt in Freizeit regelmässig Sport & Hat keinen Bezug zum Sport (L7: K11) \\
Ist in einem Sportverein & Ist nicht in Sportverein (L13: K9) \\
Ist auch ausserhalb Sportunterricht aktiv & Nicht aktiv ausserhalb Sportunterricht (L19: K7) \\
\hline
\end{tabular}

d) Elternhaus (5/8)

\section{Bringt grossen Erfahrungsschatz mit \\ Hat Migrationshintergrund \\ Eltern informieren und fordern nicht}

Anmerkungen. Die hinter den Kategorienbezeichnungen in Klammern festgehaltenen Zahlen entsprechen der Anzahl Lehrpersonen bzw. der Anzahl Konstrukte. Die hinter den einzelnen Konstrukten notierten Angaben verweisen auf die Lehrperson und die jeweilige Konstruktzeile im Rohgrid (vgl. Anhang D). In der linken (grau markierten) Spalte finden sich die mit dem „Ideal“-Schüler assoziierten bzw. die von den Lehrpersonen präferierten Pole. 
Die Subkategorie a) Selbstvertrauen/Selbsteinschätzung fasst 13 Konstrukte zusammen, über welche 11 Lehrpersonen die Bewertung, welche die Schülerinnen und Schüler von sich selbst haben, einschätzten. Der Kategorie zugeordnet wurden u.a. die Konstrukte Selbstsicher-Zweifelt an sich L4: K4), Hohes Selbstvertrauen - Tiefes Selbstvertrauen (L16: K4) und Kann eigene Leistung realistisch einschätzen Kann eigene Leistung nicht realistisch einschätzen (L18: K13).

Weiter beurteilten sechs Lehrpersonen die Lernfähigkeit der Schülerinnen und Schüler (Kategorie 5b). Konstrukte, die dazu genannt wurden, betreffen die Auffassungsgabe (L2: K2; L10: K11; L24: K7) bzW. die Lerngeschwindigkeit und -fähigkeit (L2: K2; L3: K9; L21: K8; L22: K2) der Schülerinnen und Schüler. Die Kategorie c) Sportengagement ausserhalb Sportunterricht umfasst weitere sechs Konstrukte, die sich auf die Sportaktivität der Schülerinnen und Schüler ausserhalb der Schule beziehen. Genannt wurden etwa die Konstrukte Treibt in der Freizeit regelmässig Sport - Hat keinen Bezug zum Sport (L7: K11), Ist in einem Sportverein - Ist nicht in Sportverein (L13: K9), Ist auch ausserhalb Sportunterricht aktiv - Nicht aktiv ausserhalb Sportunterricht (L19: K7).

Schliesslich nannten fünf Lehrpersonen Konstrukte, die sich auf das Elternhaus der Schülerinnen und Schüler beziehen (Kategorie 5c). Angesprochen wurden dabei insbesondere die Sportaffinität der Familie (L1: K12; L19: K4) sowie die Herkunft der Schülerinnen und Schüler bzw. ihrer Eltern (L4: K13; L13: K10; L25: K9).

Die Idealvorstellungen der Lehrpersonen in dieser gemischten Kategorie lassen sich in Anlehnung an die präferierten Pole wie folgt zusammenfassen:

Präferiert werden Schülerinnen und Schüler, die ein angemessenes Vertrauen in die eigenen Fähigkeiten haben und ihre Leistung realistisch einschätzen können (Kategorie a), die schnell und ring lernen (Kategorie b), die auch ausserhalb des Sportunterrichts sportaktiv sind (Kategorie c) und die aus einem sportaffinen Elternhaus stammen (Kategorie d).

Die Berechnung der mittleren Anteile der Schülerinnen und Schüler, die den Idealvorstellungen der Lehrpersonen in den verschiedenen Subkategorien entsprechen, zeigt, dass mit Ausnahme der Subkategorie c) Sportengagement ausserhalb Sportunterricht, in welcher die nicht-fremdsprachigen Schülerinnen und Schüler $(M=46.8, S D=19.9)$ deutlich „idealer" eingeschätzt wurden als die fremdsprachigen Schülerinnen und Schüler $(M=34.0, S D=26.7)$, sich keine wesentlichen bzw. vergleichsweise nur kleine Mittelwertunterschiede zwischen den fremdsprachigen und nicht-fremdsprachigen Schülerinnen und Schülern finden (vgl. Tab. 18). Auf „explorative Signifikanztests“ wurde aufgrund der kleinen - und deshalb in Bezug auf die Gesamtstichprobe nur wenig aussagekräftigen - Teilstichproben verzichtet. 
Tabelle 18. Mittelwerte, Standardabweichungen und range der mittleren Anteile [\%] fremdsprachiger und nicht-fremdsprachiger Schülerinnen und Schüler, die dem präferierten Pol in den Subkategorien der Kategorie "Verschiedenes" entsprechen

\begin{tabular}{|c|c|c|c|c|c|}
\hline Kategorie/Subkategorie & SuS-Gruppe & $n$ & $\begin{array}{l}M \\
{[\%]}\end{array}$ & $S D$ & range \\
\hline \multirow{2}{*}{$\begin{array}{l}\text { 5a) Selbstver- } \\
\text { trauen/Selbsteinschät- } \\
\text { zung }\end{array}$} & fremdsprachige SuS & 10 & 56.5 & 27.4 & $25-100$ \\
\hline & nicht-fremdsprachige SuS & 10 & 58.1 & 21.3 & $33.3-100$ \\
\hline \multirow[t]{2}{*}{ 5b) Lernfähigkeit } & fremdsprachige SuS & 6 & 51.2 & 30.5 & $25-100$ \\
\hline & nicht-fremdsprachige SuS & 6 & 48.6 & 27.1 & $25-100$ \\
\hline \multirow{2}{*}{$\begin{array}{l}\text { 5c) Sportengagement } \\
\text { ausserhalb Sportunter- } \\
\text { richt }\end{array}$} & fremdsprachige SuS & 6 & 34.0 & 26.7 & $0-75$ \\
\hline & nicht-fremdsprachige SuS & 6 & 46.8 & 19.9 & $20-75$ \\
\hline \multirow[t]{2}{*}{ 5d) Elternhaus } & fremdsprachige SuS & 4 & 60.0 & 49.0 & $0-100$ \\
\hline & nicht-fremdsprachige SuS & 4 & 56.8 & 21.8 & $40-87.5$ \\
\hline $\begin{array}{l}\text { Inmerkungen. } n=\text { Anzahl Lehr } \\
\text { ch ihrer den jeweiligen Subkat } \\
\text { rmittelten Anteile fremdsprac } \\
\text { ngewandt wurden (vgl. Kap. } 5 \\
\text { en Pols gemacht wurden (mitt }\end{array}$ & $\begin{array}{l}\text { oersonen, die (fremdsprachige } \\
\text { egorien zugeordneten Konstruk } \\
\text { higer und nicht-fremdsprachig } \\
\text { 1.5). Konstrukte, bei denen kei } \\
\text { lerer („,3“) Ratingwert beim „Id }\end{array}$ & cht-fr & $\begin{array}{l}\text { chige) S } \\
\text { Arithme } \\
\text { Schüler, } \\
\text { saben zu }\end{array}$ & $\begin{array}{l}\text { anen un } \\
\text { Mittel c } \\
\text { elche die } \\
\text { renz de }\end{array}$ & $\begin{array}{l}\text { uler hinsicl } \\
\text { Lehrpers } \\
\text { erierten Pc } \\
\text { n oder anc }\end{array}$ \\
\hline
\end{tabular}

\subsubsection{Zusammenfassung der Ergebnisse}

Der Vergleich der Ratingwerte der beiden Schülergruppen (fremdsprachige vs. nicht-fremdsprachige Schülerinnen und Schüler) hat gezeigt, dass sich fremdsprachige und nicht-fremdsprachige Schülerinnen und Schüler aus Sicht der Lehrpersonen im Sportunterricht unterscheiden. Erstere liegen im Mittel signifikant $(p=.006)$ weiter von den Idealvorstellungen der Lehrpersonen entfernt als letztere. Die Berechnung der Effektstärke $(d=.80)$ verweist dabei auf einen Unterschied mit hoher praktischer Bedeutsamkeit.

Um inhaltliche Aussagen zu diesem festgestellten Unterschied machen zu können (vgl. Teilfragestellung 1b), wurde zunächst eine qualitative Inhaltsanalyse der erfassten Konstrukte durchgeführt. Diese machte deutlich, dass die Schülerinnen und Schüler von je 25 (96.2\%) bzw. 23 (88.8\%) Lehrpersonen in Bezug auf ihre körper- und bewegungsbezogene Leistung (Kategorie 1: Körperlichkeit/Sportliche Leistungsfähigkeit), ihre Art und Weise, wie sie sich im Sportunterricht engagieren und beteiligen (Kategorie 2: Engagement/Beteiligung), ihre Einstellung bzw. ihren inneren Bezug zur Sache (Kategorie 3: 
Motivation/Interesse) sowie hinsichtlich ihres sozialen Verhaltens und ihrer sozialen Rolle im Sportunterricht (Kategorie 4: Mit Anderen) unterschieden und eingeschätzt wurden. Weitere, von kleineren Teilstichproben (min. $n=5$ ) genannte Aspekte wurden in der Kategorie 5: Verschiedenes zusammengefasst. Pro Kategorie resultierten vier Subkategorien.

Auf Grundlage der von den Lehrpersonen je Kategorie präferierten Konstrukt-Pole wurden sodann kategorienspezifische Idealvorstellungen von Schülerinnen und Schülern im Sportunterricht formuliert. Ein „Ideal“-Schüler ist demnach:

1) Allgemein sportlich, (d. h.) hat hohe konditionelle und koordinative Fähigkeiten, zeigt einen geschickten, feinmotorischen Umgang mit dem eigenen Körper, beherrscht verschiedene (Spiel-) Sportarten bzw. erfüllt die dafür notwendigen Anforderungen (z.B. Antizipationsfähigkeit),

2) zeigt einen hohen Einsatz und eine hohe Anstrengungsbereitschaft, arbeitet fokussiert und selbständig, beteiligt sich auf eine besonnene Art und Weise, zeigt Mut in der Auseinandersetzung mit den ihm aufgetragenen Aufgaben,

3) weist einen inneren Bewegungsdrang bzw. allgemein Freude an Bewegung auf, ist offen und (für vielseitigen Unterricht) begeisterungsfähig, ist ehrgeizig bzw. siegeswillig, zeigt Freude an bzw. Begeisterung für spezifische(n) Sportarten oder Unterrichtsinhalte(n),

4) ist gegenüber seinen Mitschülerinnen und -schülern sowie gegenüber der Lehrperson aufrichtig, loyal und fair, kann sich in Spielsituationen bzw. in Teams auf andere Schülerinnen und Schüler einlassen bzw. diese akzeptieren und unterstützen, pflegt einen angemessenen Umgang mit seiner Person und seiner Leistung (d. h. kann z. B. eigene Schwächen zugeben und blufft nicht), ist gut in die Klasse integriert,

5) hat ein angemessenes Vertrauen in die eigenen Fähigkeiten und kann seine Leistung realistisch einschätzen, lernt ring, ist auch ausserhalb des Sportunterrichts sportaktiv und hat ein sportaffines Elternhaus.

Um kategorienspezifische Unterschiede zwischen den beiden Schülergruppen (fremdsprachige vs. nicht-fremdsprachige Schülerinnen und Schüler) zu eruieren, wurden schliesslich pro Kategorie und Subkategorie die prozentualen Anteile der Schülerinnen und Schüler errechnet, auf welche diese Idealvorstellungen aus Sicht der Lehrpersonen zutreffen. Die Mittelwertvergleiche machten dabei deutlich, dass die fremdsprachigen Schülerinnen und Schüler in manchen, von kleineren Teilstichproben ( $n$ = 5-10) genannten Subkategorien (2d: Mut, 3c: Ehrgeiz/Siegeswille, 3d: Persönliche Vorlieben, 4d: Beliebtheit in Klasse, 5b: Lernfähigkeit, 5d: Elternhaus) im Vergleich zu den nicht-fremdsprachigen Schülerinnen und Schülern zwar häufiger den Idealvorstellungen der Lehrpersonen entsprechen, auf Ebene der vier thematischen Hauptkategorien 1) - 4), d. h. auf Basis der aggregierten Teilstichproben $(n=23-25)$ jedoch deutlich weiter von den Idealvorstellungen entfernt liegen als die nicht-fremdsprachigen Schülerinnen und Schüler. „Explorative Signifikanztests“ der Mittelwertunterschiede in den 
Hauptkategorien verwiesen dabei auf statistisch signifikante Unterschiede in den Kategorien 1) Körperlichkeit/Sportliche Leistungsfähigkeit, 2) Engagement/Beteiligung und 4) Mit Anderen. Als statistisch nicht signifikant fiel hingegen der Unterschiede in der Kategorie 3) Motivation/Interesse aus (vgl. Abb. 13).

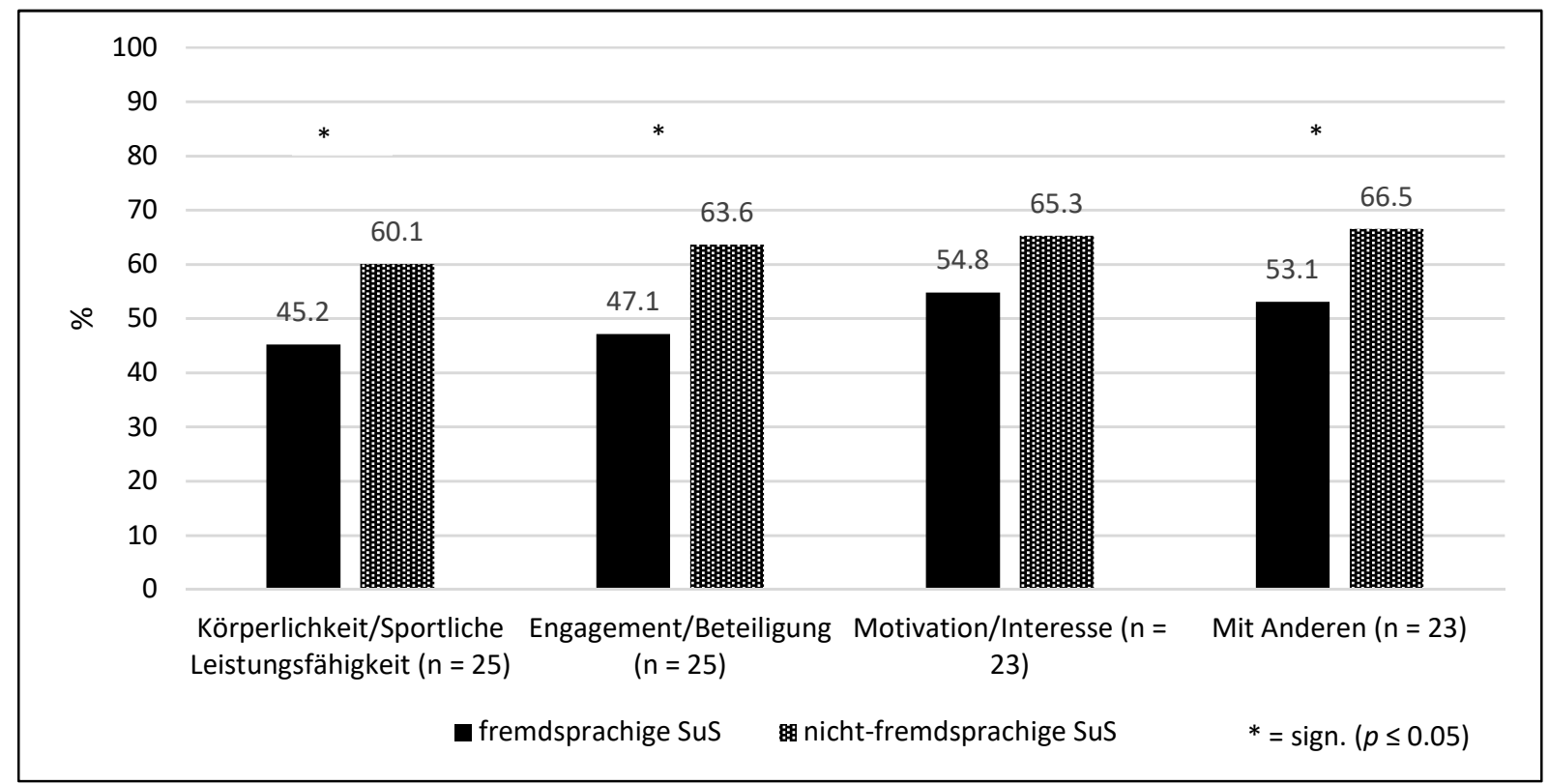

Abbildung 13. Anteil Schülerinnen und Schüler, die den Idealvorstellungen der Lehrpersonen entsprechen 


\subsection{Sportunterricht und interkulturelles Lernen aus Sicht der Lehrpersonen}

Dieses Kapitel referiert die Ergebnisse der empirischen Untersuchung zu Fragestellung 1 (Welche Vorstellungen zum Potenzial des Sportunterrichts für interkulturelles Lernen haben Sport unterrichtende Lehrpersonen?). Es ist gegliedert in je ein Kapitel zur Teilfragestellung 2a (vgl. Kap. 6.2.1) und 2b (vgl. Kap. 6.2.2) und schliesst ab mit einer Zusammenfassung der Ergebnisse (vgl. Kap. 6.2.3).

\subsubsection{Allgemeine Chancen interkulturellen Lernens aus Sicht der Lehrpersonen}

In diesem Kapitel werden die Ergebnisse zur Teilfragestellung 2a) (Welche unterrichtlichen Bedingungen stellen aus Sicht der Lehrpersonen generell Chancen für interkulturelles Lernen dar?) dargestellt. Präsentiert werden folglich die aus der qualitativen Inhaltsanalyse der Konstrukte hervorgegangen Kategorien an von den Lehrpersonen genannten allgemeinen Chancen für interkulturelles Lernen.

Insgesamt nannten die 24 Lehrpersonen 171 Konstrukte, was durchschnittlich 7.3 (SD = 1.4, range: 410) Konstrukten pro Lehrperson entspricht. Aus der Inhaltsanalyse resultierten 17 induktiv gewonnene Chancen-Kategorien, die Konstrukte von jeweils mindestens vier (16.7\%) ${ }^{95}$ und maximal $13(54.2 \%)$ der 24 Lehrpersonen zusammenfassen. Sie repräsentieren damit die - zumindest gemessen an der Nennungshäufigkeit - zentralen Gelingensbedingungen für interkulturelles Lernen aus Sicht der Lehrpersonen. Konstrukte bzw. Chancen, die keiner dieser Kategorien zugeordnet werden konnten, fielen in die Residualkategorie "Sonstige Chancen“. Pro Lehrperson wurden durchschnittlich 5.7 (SD = 1.7, range: 2-9) Chancen genannt. ${ }^{96}$

Tabelle 19 fasst die 17 Chancen-Kategorien, geordnet nach der Nennungshäufigkeit der Chance, in absteigender Reihenfolge zusammen.

\footnotetext{
${ }^{95}$ Eine von vier Lehrpersonen gemeinsam genannte Chance wurde - relativ zur Samplegrösse $(n=24)$ und zur maximalen Nennungshäufigkeit einer Chance $(n=13)$ - als ausreichend bedeutsam erachtet, um daraus eine Chancen-Kategorie zu formulieren. Auf weniger als vier Fälle umfassende Kategorien wurde nicht zuletzt angesichts des Zwecks qualitativer Inhaltsanalysen - die Zusammenfassung und Reduktion von Komplexität (vgl. Kuckartz, 2014) - verzichtet.

${ }^{96}$ Etwaige Zusammenhänge zwischen der Anzahl Chancen und der Anzahl Jahre an Unterrichtserfahrung sowie zwischen der Anzahl Chancen und dem pädagogischen Ausbildungsabschluss konnten nicht gefunden werden. 130
} 
(2) Geringe Sprachgebundenheit

(3) Kulturbezogene Fachthemen

(5) Offene Lernwege, variierende Ergebnisse

(6) Pers. Hintergründe und Haltungen der SuS kommen zum Ausdruck

(7) Homogenes Vorwissen der Schülerinnen und Schüler

(8) Kulturneutrale Fachinhalte

(9) Arbeiten in Gruppen

(10) Emotions- und erlebnisreicher Unterricht

(11) Physische Bewegung, wechselnde Sitzordnungen

(12) Austauschen, Diskutieren, Kommunizieren

(13) Motivierte, offene Schülerinnen und Schüler

(14) Fach mit Alltags- und Lebensbezug

(15) Interdisziplinäre Fachthemen

$4(16.7 \%) / 5$

(16) Hoher Praxisanteil

$4(16.7 \%) / 4$

(17) Pers. Hintergründe und Haltungen der SuS kommen nicht zum Ausdruck

$4(16.7 \%) / 4$

Im Folgenden werden die 17 Chancen-Kategorien unter Bezugnahme auf die jeweiligen Konstrukt-Retrievals (vgl. Anhang G) und zwei bzw. drei ausgewählte Konstrukt-Beispiele näher beschrieben. ${ }^{97}$ Da die Kategorienbezeichnungen entlang der Formulierungen der Chancen-Pole vorgenommen wurden (vgl. Kap. 5.5.2), fokussieren auch die Beschreibungen der Kategorien primär die Chancen-Pole. Formulierungen der Hindernis-Pole wurden nur dann aufgegriffen, wenn sie durch Nennung von Gegenbegriffen zum Verständnis der Chancen beitragen.

\footnotetext{
${ }^{97}$ Die kursiven Textteile entsprechen dabei den Originalformulierungen in den Rohgrids (vgl. Anhang F).
} 
Ebenso in die Beschreibung der Kategorien miteinbezogen wurden die in Anlehnung an das laddering erhobenen Begründungen der Chancen und Hindernisse. Obwohl diese nicht systematisch erhoben werden konnten (vgl. Kap. 5.4.4), tragen sie dennoch zum Verständnis der Chancen-Kategorien bzw. dem diesen zugrundeliegenden Verständnis interkulturellen Lernens bei. Die Ergebnisse einer systematischeren Analyse dieser Begründungen finden sich in Form einer Heuristik interkulturellen Lernens aus Sicht der Lehrpersonen im diesem Kapitel nachfolgenden Exkurs.

(1) Einbezug persönlicher Hintergründe der Schülerinnen und Schüler

Aus 13 (54.2\%) Interviews ging je ein Konstrukt hervor, über welches Fächer hinsichtlich ihrer Möglichkeiten, die persönlichen Hintergründe der Schülerinnen und Schüler einzubeziehen, unterschieden wurden. Alle 13 Lehrpersonen schätzten dies als Chance für interkulturelles Lernen ein. Bezeichnend für die der Kategorie subsumierten Chancen-Pole sind Formulierungen, in denen das Einbeziehen bzw. Einbinden von Erzählungen von SuS aus ihren Ländern (L2: K6), von persönlichen Schul- oder Vorschulbiographien (L8: K2) oder gar von kulturspezifischen Fähigkeiten/Fertigkeiten von Schülerinnen und Schülern (L13: K4) angesprochen wurde (vgl. Tab. 20).

Die Lehrpersonen beurteilten das Einbeziehen persönlicher Hintergründe der Schülerinnen und Schüler deshalb als Chance, weil dadurch Akzeptanz und Verständnis (L7: K1, L24: K7, L26: K1), Empathie gefördert (L23: K1) und Interesse aneinander geweckt würde (L21: K3), weil authentische Erzählungen bereichernd wären (L20: K2) oder überhaupt, weil interkulturelles Lernen so explizit stattfinden bzw. von den SuS ausgehen würde (L1: K5, L5: K3).

Zwei Hindernis-Pole dieser Kategorie bringen zum Ausdruck, unter welchen Bedingungen interkulturelles Lernen durch den Einbezug persönlicher Hintergründe der Schülerinnen und Schüler potenziell erschwert sein kann. So stellen für L20 lehrerzentrierter Sachunterricht (K2), für L23 Mathematik: Spezialfach, das wenig Schülerbezüge anbietet (K1) potenzielle Hindernisse für interkulturelles Lernen dar.

(2) Geringe Sprachgebundenheit

Ein von 12 (50\%) Lehrpersonen verwendetes Unterscheidungskriterium von Schulfächern war der Stellenwert der Sprache in einem Fach. Als Bezugspunkte der in dieser Kategorie zusammengefassten 19 Konstrukte lassen sich die allgemeine Sprachlastigkeit eines Faches (L2: K7, L12: K5, L18: K3, L23: K9, L26: K4), die Sprachlastigkeit von in Fächern angewandten Vermittlungsformen (L2: K2, L14: K12, L18: K4, L18: K5, L20: K4, L26: K8) sowie die Relevanz der (Deutsch-)Sprachkenntnisse fremdsprachiger Schülerinnen und Schüler (L1: K3, L7: K7, L9: K3, L9: K7, L12: K1, L19: K6, L21: K1, L21: K6) identifizieren. Beispiele einschlägiger Chancen-Pole sind Sprache spielt eine untergeordnete Rolle (L18: K3), SuS mit 
wenig Deutsch-Sprachkenntnissen sind nicht benachteiligt (L19: K6) oder Instruktion/Lernen ist auch durch Vor- und Nachmachen möglich (L26: K8) (vgl. Tab. 20).

Insgesamt waren die Lehrpersonen der Meinung, dass wenig sprachgebundene Fächer (im Gegensatz zu sprachlastigen Fächern) eine Chance für interkulturelles Lernen sein können. Dies wurde einerseits damit begründet, dass Schülerinnen und Schüler mit weniger guten Deutschkenntnissen in weniger sprachgebundenen Fächern in Bezug auf fachliches Lernen und Leisten nicht benachteiligt sind und dadurch besser in der Klasse integriert (L1: K3) werden bzw. mehr Anerkennung, Akzeptanz und Eingebundenheit in der Klasse erfahren (L2: K2, L7: K7, L12: K5, L20: K4, L21: K6, L26: K4). Andererseits sehen die Lehrpersonen in sprachunabhängigeren Fächern auch den Vorteil, dass Schülerinnen und Schüler unabhängig von ihren Deutschkenntnissen dennoch zusammenarbeiten sich austauschen und (voneinander) lernen können (L2: K7, L12: K1, L14: K2, L18: K4, L21: K1, L26: K8).

(3) Kulturbezogene Fachthemen

11 (45.8\%) Lehrpersonen nannten insgesamt 24 Konstrukte, über welche die Fächer hinsichtlich ihres „Kulturgehalts" bzw. darüber, ob in den Fächern kulturbezogene Themen vorkommen, unterschieden wurden. So unterschieden die Lehrpersonen zwischen Fächern, in denen Kultur stark enthalten (L23: K2) ist, und solchen, die kulturlos (L3: K3) sind. Darüber hinaus wurde aber auch zwischen Fächern differenziert, deren Inhalte nebst der eigenen Kultur auch Aspekte anderer Kulturen - wie Religion (L17: K5), „fremdkulturelle“ Instrumente (L10: K7), Personen (L10: K1), Rituale an Feiertagen (L4: K7) oder Traditionen (L16: K5) - betreffen.

Insgesamt betrachteten die Lehrpersonen Fächer mit kulturbezogenen Fachthemen als Chance für interkulturelles Lernen. Zu den hierzu formulierten Chancen-Polen gehören u.a. Im Fach lassen sich verschiedene Kulturen thematisieren (L2: K4), Fachinhalt ist kulturell gehaltvoll (L3: K3), Im Fach kann man kulturelle Anlässe anderer Kulturen thematisieren (L8: K4), Fach bietet Möglichkeit, kulturelle Aspekte (geistig oder handfest) in Unterricht einzubauen (L13: K5) (vgl. auch Tab. 20).

Begründet wird diese Chance damit, dass andere Kulturen bzw. Lebensweisen kennengelernt (L3: K1, L13: K3) und unterschieden werden können (L3: K3) und dass diese das gegenseitige Verständnis fördern (L23: K2) und den Horizont erweitern würden (L26: K5).

(4) Geringer Leistungs- und Stoffdruck

10 (41.7\%) Lehrpersonen nannten zusammen 14 Konstrukte, die der Kategorie (4) Geringer Leistungsund Stoffdruck zugeordnet wurden. Die Schulfächer wurden insbesondere nach dem Verhältnis zwischen der für ein Fach zur Verfügung stehenden Zeit und dem durch den Lehrplan und die jeweiligen Lehrmittel vorgegebenen Stoffvolumen voneinander unterschieden. Beispiele hierzu formulierter 
Chancen-Pole sind Freiheit in der Wahl des Lehrmittels (L1: K2), Lehrplan lässt Gestaltung des Unterrichts offen (L11: K8) oder Frei wählbarer Stoffplan (L22: K3). Drei der genannten Konstrukte rekurrieren ausserdem auf den Unterschied zwischen Haupt- und Nebenfächern (L11: K2, L23: K7) bzw. auf deren Promotionsrelevanz (L25: K4).

Die Lehrpersonen waren der Meinung, dass Leistungs- und Stoffdruck für interkulturelles Lernen ein Hindernis darstellen. Freiheit in der Gestaltung des Unterrichts durch wenige Lehrplanvorgaben und durch geringeres Stoffvolumen würde interkulturelles Lernen hingegen begünstigen, weil dadurch Raum zur Behandlung von Themen anderer Kulturen (L1: K2) sowie zur Berücksichtigung der Interessen und Stärken der Schülerinnen und Schüler frei würde (L1: K1, L1: K7, L5: K5, L14: K5). L11 begründete die Chance zudem mit Mehr Kapazität für Soziales (K2), dass sich die SuS mehr entfalten können würden (K6), und damit, dass zu klare Vorgaben durch den Lehrplan mehr Frontalunterricht implizieren und damit wiederum den Austausch einschränken würden (K8). Weiter bestand die Chance für L19 darin, Dinge praktisch ausprobieren zu können (K3). 


\section{(1) Einbezug persönlicher Hintergründe der Schülerinnen und Schüler}

\begin{tabular}{l:l}
\hline Chancen-Pol & Hindernis-Pol \\
\hline Möglichkeit, Erzählungen von SuS aus ihren Ländern & SuS haben keine Möglichkeit, ihre Erzählungen einzu- \\
einzubeziehen & bringen (L2: K6) \\
Im Fach lassen sich persönliche Schul- oder Vorschulbi- Persönliche Biographien lassen sich nicht einbinden \\
ographien einbinden & (L8: K2) \\
Im Fach kann persönlicher Hintergrund einzelner SuS & Im Fach können keine persönlichen Hintergründe der \\
einbezogen werden & SuS einbezogen werden (L21: K3) \\
\hline
\end{tabular}

\section{(2) Geringe Sprachgebundenheit}

\begin{tabular}{l|l}
\hline Sprache spielt eine untergeordnete Rolle & Fach ist sprachlastig (L18: K3) \\
SuS mit wenig Deutsch-Sprachkenntnissen sind nicht & SuS mit wenig Deutsch-Sprachkenntnissen sind be- \\
benachteiligt & nachteiligt (L19: K6) \\
Instruktion/Lernen ist auch durch Vor- und Nachma- & Fach ist sprachlastig (L26: K8) \\
chen möglich &
\end{tabular}

\section{(3) Kulturbezogene Fachthemen}

\begin{tabular}{l|l}
\hline Fachinhalt ist kulturell gehaltvoll & Fachinhalt ist kulturlos (L3: K3) \\
Fachinhalte bieten Möglichkeit zu Vergleichen ver- & Inhalte sind kulturunabhängig (L4: K3) \\
schiedener Kulturen & \\
Im Unterricht lernt man andere Nationen kennen & Im Unterricht lernt man keine anderen Nationen ken- \\
\end{tabular}

(4) Geringer Leistungs- und Stoffdruck

Fach ist offen für unterschiedliche Themen, viel Spiel- Starres Programm, keine Freiheit in Stoffwahl (L1: K1)
raum
Hoher Freiraum in der Unterrichtsgestaltung
$\begin{array}{ll}\text { Nebenfach: Geringer Leistungsdruck } & \text { Hauptfach: Grosser Leistungsdruck (L11: K2) }\end{array}$

Anmerkung. Die hinter den Hindernis-Polen in Klammern festgehaltenen Angaben verweisen auf die Lehrperson und die jeweilige Konstruktzeile im Rohgrid (vgl. Anhang F). 
(5) Offene Lernwege, variierende Ergebnisse

Acht (33.3\%) Lehrpersonen nannten 10 Konstrukte, die der Kategorie (5) Offene Lernwege, variierende Ergebnisse zugeordnet wurden. Die Kategorie enthält Konstrukte, die sich auf die Offenheit des Unterrichts und konkreter auf die Offenheit von Lernwegen und von Resultaten beziehen. Unterschieden wurde demnach zwischen Fächern, in denen der Lernweg und die erbrachten Lösungen bzw. Produkte variieren können, und solchen, in denen der Lernweg klar vorgegeben und nur eine Lösung bzw. ein Produkt richtig ist (L1: K10, L7: K5, L11: K5, L24: K2). Weitere Konstrukte beschreiben das Thema über den Begriff der Kreativität und differenzieren zwischen Fächern, die Raum für Kreativität seitens der Schülerinnen und Schüler bieten, und solchen, die stark reguliert (L23: K5) sind bzw. in denen starres Üben/Ausführen (L24: K2) im Vordergrund steht. Schliesslich führte L20 den Unterschied zwischen Entdeckendem Lernen und Sachvermittlung (K7) an (vgl. auch Tab. 21).

Alle acht Lehrpersonen waren der Ansicht, dass offene Lernwege und die Möglichkeit variierender Ergebnisse als Chance für interkulturelles Lernen aufzufassen sind, weil durch die Möglichkeit unterschiedlicher Lernwege und Ergebnisse sich die Schülerinnen und Schüler persönlich einbringen (L11: K9) und Produkte mit interkulturellem Lerninhalt (L23: K5) realisiert werden könnten, Verschiedenartigkeit zwischen den Schülerinnen und Schüler eher zum Ausdruck kommen würde (L11: K5), dies auch Anlass zu Interaktion, Austausch und Reflexion (L1: K10, L24: K2, L24: K4, L24: K5) sowie Horizonterweiterung (L7: K5) und gegenseitigem voneinander Lernen (L14: K1) sein könne.

(6) Persönliche Hintergründe und Haltungen der Schülerinnen und Schüler kommen zum Ausdruck

Acht (33.3\%) Lehrpersonen nannten zusammen 9 Konstrukte, die sie zur Unterscheidung der Fächer hinsichtlich der Chance, dass persönliche Hintergründe und Haltungen der Schülerinnen und Schüler zum Ausdruck kommen können, heranzogen. Zu den hierzu formulierten Chancen-Polen gehören Kultureller Hintergrund der SuS kommt stark zum Ausdruck (L11: K3), Familiäre, kulturelle Hintergründe manifestieren sich stark (L24: K6) oder Haltungen/Wertvorstellungen kommen zum Ausdruck (L17: K2) (vgl. Tab. 21). Weitere Formulierungen beziehen sich auf die Sicht- bzw. Wahrnehmbarkeit (kultureller) Unterschiede (L5: K7; L12: K4) zwischen den Schülerinnen und Schülern.

Die Manifestation persönlicher Hintergründe und Haltungen der Schülerinnen und Schüler stellt aus Sicht dieser Lehrpersonen deshalb eine Chance für interkulturelles Lernen dar, weil die SuS (dadurch) Andersartigkeit erleben würden (L11: K3), dies zur Horizonterweiterung beitragen (L16: K1) und gegenseitigen Respekt fördern würde (L18: K2) oder auch Anlass zu Diskussionen (L24: K6) sein könne. 
(7) Homogenes Vorwissen der Schülerinnen und Schüler

Acht (33.3\%) Lehrpersonen nannten insgesamt neun Konstrukte, mit welchen sie zwischen Fächern unterschieden, in welchen das Vorwissen von Schülerinnen und Schüler eher homogen oder eher heterogen ist.

Homogenes Vorwissen der Schülerinnen und Schüler in einem Fach beurteilten sie als Chance für interkulturelles Lernen (z.B. Wenig Leistungsunterschiede bezüglich Vorwissen der SuS (L9: K4), SuS kommen mit gleichem Vorwissen ins Fach (L18: K6)). Zwei Lehrperson sprachen dabei explizit potenzielle Unterschiede zwischen Schülerinnen und Schülern mit und solchen ohne Migrationshintergrund an (z.B. Fach, in dem SuS mit MH [Migrationshintergrund] gleiches oder besseres Fachwissen haben (L25: K5)).

Homogenes oder ähnliches Vorwissen würde die Schülerinnen und Schüler verbinden (L18: K6) und wäre Grundlage dafür, dass sich die Schülerinnen und Schüler füreinander interessieren und attraktiv sind (Angebot, von dem profitiert werden kann (L25: K5), Fördert gegenseitige Neugier (L26: K3)), mit einander in Kontakt treten und sich austauschen (L19: K2, L25: K1). Einseitiges oder wenig Vorwissen von Schülerinnen und Schülern würde sich hingegen hinderlich für interkulturelles Lernen auswirken, da die Schülerinnen und Schüler in diesem Fall zu stark mit ihrer eigenen Leistung im Fach beschäftigt wären (L16: K3) bzw. mehr Aufmerksamkeit durch die Lehrperson bräuchten (L23: K3).

(8) Kulturneutrale Fachinhalte

Sieben (29.2\%) Lehrpersonen beurteilten die Schulfächer bezüglich der Kulturunabhängigkeit bzw. Universalität der Fachinhalte. Sind Fachinhalte bzw. Regeln, Gesetze oder Definitionen in einem Fach international (L2: K1), kulturneutral (L5: K5, L9: K5, L14: K6), kulturunabhängig (L16: K4) bzw. fix (L26: K7), dann wird darin eine Chance für interkulturelles Lernen gesehen. Gemäss L16 trifft dies deshalb zu, weil die Schülerinnen und Schüler realisieren würden, dass sie trotz verschiedener Hintergründe zusammenarbeiten können (K4) oder gemäss L26 alle vom Selben reden würden (K7). Kulturell geprägte Fachinhalte könnten laut L5 hingegen Fronten zwischen SuS verstärken (K1). 
Tabelle 21. Beispiele genannter Konstrukte der Chancen-Kategorien (5) - (8)

\section{(5) Offene Lernwege, variierende Ergebnisse}

\begin{tabular}{l|l}
\hline Chancen-Pol & Hindernis-Pol \\
\hline Fach mit offenen Lösungswegen, Zugängen & Exakte Wissenschaften: Kein Spielraum bezgl. Resul- \\
Entdeckendes Lernen steht im Vordergrund & Sachvermittlung steht im Vordergrund (L20: K6) \\
Kreatives Erarbeiten von Inhalten & Starres Üben/Ausführen (L24: K2) \\
\hline
\end{tabular}

(6) Persönliche Hintergründe und Haltungen der Schülerinnen und Schüler kommen zum Ausdruck

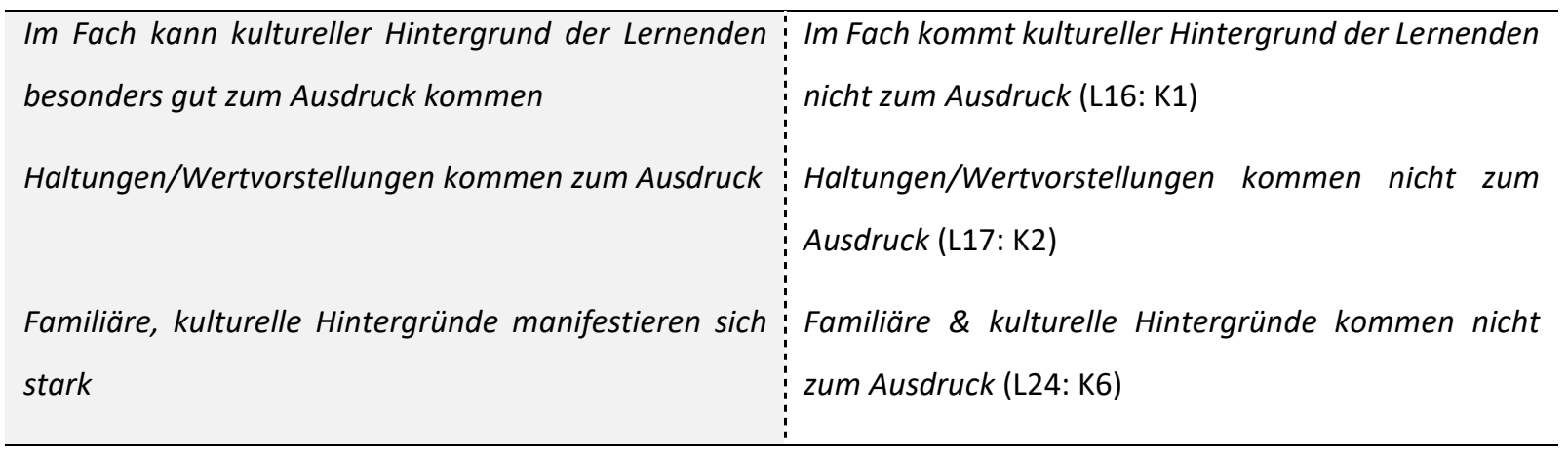

\section{(7) Homogenes Vorwissen der Schülerinnen und Schüler}

Wenig Leistungsunterschiede bezüglich Vorwissen der
SuS

\section{(8) Kulturneutrale Fachinhalte}

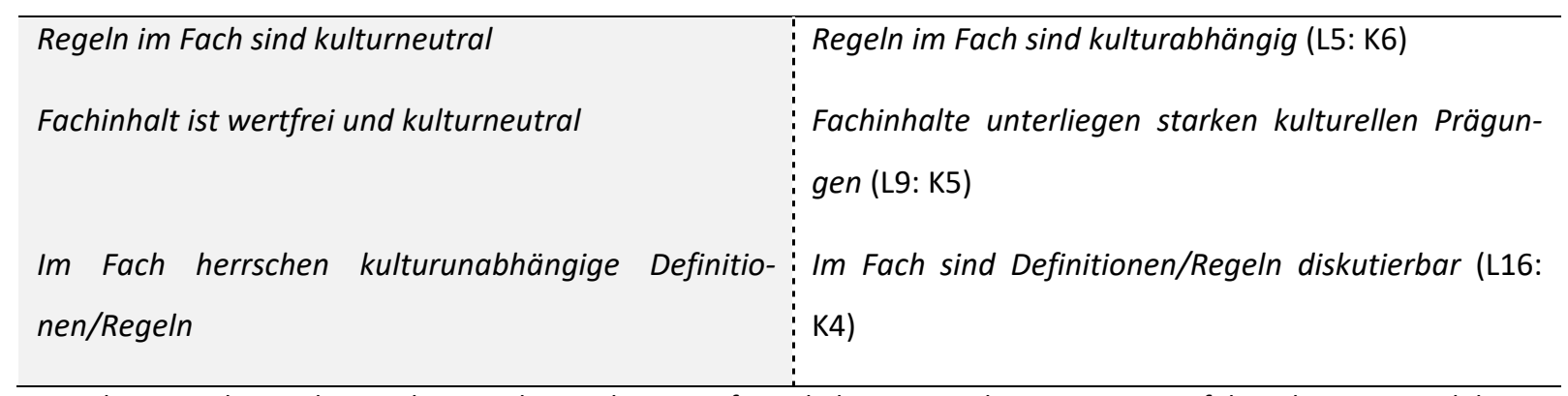

Anmerkung. Die hinter den Hindernis-Polen in Klammern festgehaltenen Angaben verweisen auf die Lehrperson und die jeweilige Konstruktzeile im Rohgrid (vgl. Anhang F). 
(9) Arbeiten in Gruppen

Sieben (29.2\%) der befragten Lehrpersonen nannten Konstrukte, die sie zur Differenzierung der Fächer hinsichtlich der Interaktionsstruktur anwandten. Die Lehrpersonen unterschieden dabei zwischen Fächern, in denen hauptsächlich frontal unterrichtet (L1: K4; L11: K1; L23: K4) und viel Einzelarbeit (L1: K4; L4: K9; L20: K5) geleistet wird, und solchen, die sich potenziell für das Arbeiten in der Gruppe bzw. im Team eignen.

Fächern, die sich für Gruppenarbeiten eignen, massen die Lehrpersonen ein höheres Potenzial für interkulturelles Lernen bei. Dies, weil Gruppenarbeit den Horizont erweitern (L11: K1), die Kooperationsfähigkeit und Akzeptanz (L12: K2) das gegenseitige Verständnis für unterschiedliche Gedankengänge (L20: K5) fördern, das Klassenteam stärken (L23: K4), die SuS untereinander verbinden (L26: K6) und sie zum Austausch anregen würde (L1: K4).

(10) Emotions- und erlebnisreicher Unterricht

Die dieser Kategorie zugeordneten Konstrukte beschreiben Fächer und ihre Themen hinsichtlich ihres Vermögens, Emotionen auszulösen oder in Bezug auf ihren Erlebnisreichtum. Bezeichnend für die von sechs (25\%) Lehrpersonen genannten Konstrukte sind Formulierungen wie Inhalte sind kreativ-emotional - Inhalte sind logisch-analytisch (L9: K1), Fachinhalt weckt Emotionen - Fachinhalt ist kognitiv (L18: K1) oder Fach ist erlebnisreich - Fach bietet wenig Gelegenheit zum Entdecken und Erleben (L9: K9) (vgl. auch Tab. 14). Emotions- und erlebnisreiche Fächer wurden dabei von den Lehrpersonen als Chance, trockene (L7: K2), logisch-analytische (L9: K1) oder kognitive (L18: K1) Fächer hingegen als Hindernis für interkulturelles Lernen eingeschätzt.

Die Chance sehen die Lehrpersonen darin, dass sich die Schülerinnen und Schüler durch gemeinsame (emotionale) Erlebnisse direkter und unmittelbarer kennenlernen (L7: K2) bzw. emotionale Reaktionen anderer mit den eigenen vergleichen können (L20: K3). L18 war zudem der Überzeugung, dass interkulturelles Lernen durch emotionale Abstützung generell besser gelingt (K1).

(11) Physische Bewegung, wechselnde Sitzordnung

Diese Kategorie repräsentiert sechs Konstrukte von sechs (25\%) Lehrpersonen, mit denen Fächer hinsichtlich der Frage, ob Schülerinnen und Schüler nur sitzen oder sich auch physisch bewegen können bzw. ob der Unterricht innerhalb oder ausserhalb eines Schulzimmers stattfindet, unterschieden wurden. Physische Bewegung (L4: K8), wechselnde Sitzordnungen (L1: K6) und Unterricht ausserhalb des Klassen- bzw. Schulzimmers (L11: K4, L24: K3) wurde als Chance für interkulturelles Lernen bezeichnet. Lernen ausserhalb des Schulzimmers würde Gelegenheiten bieten, sich besser kennenzulernen (L24: K3) bzw. ein faires Miteinander fördern (L26: K2). Demgegenüber müsste in einem Klassenzimmerfach 
das interkulturelle Lernen aktiv inszeniert werden (L11: K4). Fixe Sitzordnungen würden zudem mit wenig Austausch zwischen SuS einhergehen (L1: K6).

(12) Austauschen, Diskutieren, Kommunizieren

Sechs (25\%) Lehrpersonen unterschieden die vorgelegten Fächer hinsichtlich des Aspekts des Austauschens, Diskutierens und Kommunizierens (z. B. Im Fach finden offene Diskussionen statt - Im Fach finden wenige offene Diskussionen statt (L7: K8), Fach fördert in besonderem Masse die Kommunikation zwischen SuS - Fach fördert Kommunikation zwischen SuS nicht (L19: K5), vgl. auch Tab. 22).

Nach Überzeugung der Lehrpersonen ist Austauschen, Diskutieren und Kommunizieren eine Chance für interkulturelles Lernen, weil SuS-Themen aufgenommen werden können (L5: K5), andere Sichtweisen kennengelernt und verstanden (L7: K8), zur eigenen Meinungsbildung und zu Toleranz beigetragen (L16: K6) und persönlichere Kommunikation ermöglicht werden würde/n (L21: K2). 


\section{(9) Arbeiten in Gruppen}

\begin{tabular}{l|l}
\hline Chancen-Pol & Hindernis-Pol \\
\hline Fach eignet sich für Gruppenarbeiten & Fach wird v. a. frontal unterrichtet, viel Einzelarbeit \\
Viel Interaktion/Gruppenarbeit & Viel Einzelarbeit (L4: K9) \\
Projektarbeit gut möglich & Lehrerzentriertes, einseitiges, frontal unterrichtetes \\
& Fach (L23: K4) \\
\hline
\end{tabular}

(10) Emotions- und erlebnisreicher Unterricht

\begin{tabular}{l|l}
\hline Im Fach wird gemeinsam erlebt & Fach ist "trocken" (L7: K2) \\
Inhalte sind kreativ-emotional & Inhalte sind logisch-analytisch (L9: K1) \\
Fachinhalt weckt Emotionen & Fachinhalt ist kognitiv (L18: K1) \\
\hline
\end{tabular}

(11) Physische Bewegung, wechselnde Sitzordnungen

Viel Raum, wechselnde Sitzordnungen
Zugang zueinander über (physische) Bewegung mög-
lich

(12) Austauschen, Diskutieren, Kommunizieren

\begin{tabular}{|c|c|}
\hline Im Fach finden offene Diskussionen statt & $\begin{array}{l}\text { Im Fach finden wenige offene Diskussionen statt (L7: } \\
\text { K8) }\end{array}$ \\
\hline Im Fach kann viel diskutiert und ausgetauscht werden & $\begin{array}{l}\text { Im Fach arbeitet jeder für sich, kein Austausch (L16: } \\
\text { K6) }\end{array}$ \\
\hline Kommunikativer Aspekt ist zentral & Kommunikativer Aspekt ist nicht relevant (L17: K3) \\
\hline
\end{tabular}


(13) Motivierte, offene Schülerinnen und Schüler

Fünf (20.8\%) Lehrpersonen sortierten die vorgelegten Fächer hinsichtlich der Motivation und Offenheit der Schülerinnen und Schüler in den jeweiligen Fächern. Verwendet wurden dabei Begriffe wie Offenheit (L9: K6, L23: K6), Motivation (L11: K7, L25: K6) oder Leistungsbereitschaft (L12: K3), wobei drei der fünf Lehrpersonen insbesondere an die Motivation und Offenheit von Schülerinnen und Schülern mit Migrationshintergrund dachten (L9: K6, L12: K3, L25: K6).

Motivierte und offene Schülerinnen und Schüler wurden deshalb als Chance für interkulturelles Lernen bezeichnet, weil dies Voraussetzung für Unterrichtsformen wie Gruppenarbeit sei (L11: K7) oder weil ähnliche Einstellungen zum Fach generell einen Vorteil für interkulturelles Lernen darstellen würden (L12: K3). Gehemmte Schülerinnen und Schüler in einem Fach würden hingegen den gegenseitigen Austausch erschweren (L23: K6).

(14) Fach mit Alltags- und Lebensbezug

Fünf (20.8\%) Lehrpersonen nannten je ein Konstrukt, mit dem sie die Schulfächer in Bezug auf deren Bedeutung im Alltag bzw. im Leben der Schülerinnen und Schüler beurteilten. Dabei erachteten die Lehrpersonen die Möglichkeit, auf alltägliche und/oder lebensnahe Themen im Unterricht eingehen zu können, als Chance für interkulturelles Lernen. Genannt wurden etwa die Chancen-Pole Fach als Lebensschule: Fach ermöglicht Thematisierung lebensnaher Themen (L7: K4) oder Über Fachinhalt lassen sich Bezüge zum alltäglichen Leben herstellen (L8: K7) (vgl. auch Tab. 23).

Die Schülerinnen und Schüler würden dadurch angeregt, über eigenes Handeln nachzudenken (L7: K4) oder würden über „ausserschulische Themen“ Zugang zueinander finden (L19: K7).

(15) Interdisziplinärer Fachthemen

Vier (16.7\%) Lehrpersonen formulierten zusammen fünf Konstrukte, mit denen sie Fächer in Bezug auf die Möglichkeit, interdisziplinär zu unterrichten, beurteilten. Die Schulfächer wurden folglich danach beurteilt, ob Transfers (L5: K4), inhaltliche Verbindungen (L14: K7) oder Parallelen (L21: K7) zu anderen Fächern möglich sind. L22 unterschied ausserdem zwischen Fächern, in denen Gelerntes aus anderen Fächern angewandt werden kann, und solchen, in denen dies nicht möglich ist (K2) (vgl. auch Tab. 14). Die Möglichkeit interdisziplinären Unterrichts ist für L22 zudem nicht nur ein Fachspezifikum, sondern auch davon abhängig, ob ein Fach eher von Klassenlehrpersonen oder von Fachlehrpersonen unterrichtet wird, da erstere eher fächerverbindend unterrichten könnten (K4).

Insgesamt sehen die Lehrpersonen interdisziplinären Unterricht als Chance für interkulturelles Lernen, weil er das vernetzte Denken (L22: K2) und das Sprachenlernen fördern (L21: K7) sowie die Notwendigkeit der Zusammenarbeit zwischen den Schülerinnen und Schülern verstärken kann (L14: K7). 
(16) Hoher Praxisanteil

Vier (16.7\%) Lehrpersonen äusserten sich über je ein Konstrukt zum Thema Theorie-Praxis-Verhältnis im Fach. Über drei der vier Konstrukte wurde explizit zwischen praktischen Fächern, in denen praktische Erfahrungen gemacht werden, und theoretischen bzw. theorielastigen Fächern, in denen theoretisches Wissen gelernt wird, unterschieden (L4: K5, L9: K2, L17: K7). ${ }^{98}$

Für alle vier Lehrpersonen bedeutete ein hoher Praxisanteil im Fach eine Chance für interkulturelles Lernen. L1 begründete diese Ansicht damit, dass in praktischen Fächern die Differenzen zwischen Kulturen stärker zum Ausdruck kommen würden (K9).

(17) Persönliche Hintergründe und Haltungen der Schülerinnen und Schüler kommen nicht zum Ausdruck

Im Gegensatz zu anderen (acht) Lehrpersonen (vgl. Chance 6) plädierten vier (16.7\%) Lehrpersonen für die Chance, dass persönliche Hintergründe und Haltungen der Schülerinnen und Schüler nicht zum Ausdruck kommen. Drei der insgesamt vier Chancen-Pole rekurrieren dabei spezifisch auf das Nichtzum-Ausdruck kommen von religiösen und kulturellen Hintergründen bzw. Haltungen der Schülerinnen und Schüler (z. B. Kein religionsbedingtes, andersartiges Verhalten von SuS (L3: K5); Im Fach kommen kulturbedingte Rollenbilder nicht zum Ausdruck (L7: K3)). Auffällig ist ausserdem, dass zwei Lehrpersonen (L5, L7) zum Ausdruck kommende Rollenbilder als potenzielles Hindernis für interkulturelles Lernen sehen.

Würden persönliche Hintergründe und Haltungen der SuS zum Ausdruck kommen - so die Begründungen - könnte dies Vorurteile erzeugen (L21: K4), einen negativen Einfluss auf die Gruppendynamik haben (L7: K3) oder gar andere SuS abschrecken (L3: K5).

\footnotetext{
${ }^{98}$ K9 von L1 (Handlungsorientiertes Fach - Kognitives Fach) wurde trotz anderer Begrifflichkeiten ebenfalls in die Kategorie aufgenommen. Nach Konsultation des einschlägigen Audioabschnitts (47:12) lässt sich handlungsorientiert im Verständnis dieser Lehrperson als „Tätigkeit“ und kognitiv als „Denkarbeit“ übersetzen.
} 
(13) Motivierte, offene Schülerinnen und Schüler

\begin{tabular}{l|l}
\hline Chancen-Pol & Hindernis-Pol \\
\hline Offenheit aller SuS gegenüber Fachinhalt & Negative Vorurteile seitens fremdsprachiger SuS ge- \\
SuS sind im Fach tendenziell motiviert & SuS sind eher unmotiviert (L11: K7) \\
Gelöste, offene, extrovertierte SuS im Fach & Oft gehemmte SuS im Fach (L23: K6) \\
\hline
\end{tabular}

(14) Fach mit Alltags- und Lebensbezug

Fach als Lebensschule: Fach ermöglicht Thematisie- Fachinhalt ist weniger lebensnah (L7: K4)

rung lebensnaher Themen

Über Fachinhalt lassen sich Bezüge zum alltäglichen Leben herstellen

Fachinhalt bietet keine Bezüge zum alltäglichen Leben (L8: K7)

Fach hat viele alltagsbezogene Themen

Viele Themen im Fach sind nur schulbezogen (L19: K7)

\section{(15) Interdisziplinäre Fachthemen}

Im Fach lassen sich Transfers zu anderen Fächern herstellen

Im Fach sind keine Transfers zu anderen Fächern möglich (L5: K4)

Im Fach besteht Möglichkeit, Gelerntes aus anderen Im Fach besteht keine Möglichkeit, Gelerntes aus anFächern im Fach anzuwenden deren Fächern anzuwenden (L22: K2)

(16) Hoher Praxisanteil

Handlungsorientiertes Fach Kognitiv orientiertes Fach (L1: K9)

Fach ist körperlich/praktisch Fach ist theoretisch (L17: K7)

(17) Persönliche Hintergründe und Haltungen der Schülerinnen und Schüler kommen nicht zum Ausdruck Kulturbedingte Rollenverständnisse können nicht zum Ausdruck kommen zum Ausdruck kommen (z.B. Macho-Verhalten) (L5: K8)

Im Fach kommen kulturbedingte Rollenbilder nicht Im Fach können kulturbedingte Rollenbilder stark zum zum Ausdruck Ausdruck kommen (L7: K3)

Anmerkung. Die hinter den Hindernis-Polen in Klammern festgehaltenen Angaben verweisen auf die Lehrperson und die jeweilige Konstruktzeile im Rohgrid (vgl. Anhang F). 
Sonstige Chancen (Residualkategorie)

Die Residualkategorie umfasst 16 Konstrukte. Die darin enthaltenen Chancen wurden zwar nur von einzelnen (bzw. maximal drei) Lehrpersonen genannt und gehören deshalb - gemessen an der Nennungshäufigkeit - nicht zu den zentralen Gelingensbedingungen für interkulturelles Lernen aus Sicht der Lehrpersonen. Dennoch bedürfen einige davon der Erwähnung, da sie gleichsam im Widerspruch zu manchen der oben beschriebenen Chancen-Kategorien stehen. Zu diesen zählt etwa der ChancenPol Austausch über Sprache/Texte (L4: K1) im Gegensatz zur Kategorie Geringe Sprachgebundenheit, der Chancen-Pol Geschlossener Unterricht (L5: K2) im Gegensatz zur Kategorie Offene Lernwege/variierende Ergebnisse sowie der Chancen-Pol Fachinhalt ist emotionslos (L25: K2) in Opposition zur Kategorie Emotions- und erlebnisreicher Unterricht. Geschlossener Unterricht würde keine Separation in der Klasse fördern, emotionsloser Fachinhalt Fronten zwischen SuS verhindern (L5: K2; L25: K2).

Exkurs: Interkulturelles Lernen aus Sicht der Lehrpersonen - eine Heuristik

Wie in Kapitel 5.3.2 erläutert, wurden in Anlehnung an die Technik des ladderings (vgl. Kap. 5.1.2) sog. übergeordnete Konstrukte in Form von Begründungen für genannte Chancen und Hindernisse („Weshalb entspricht diese Ähnlichkeit einer Chance bzw. einem Hindernis für interkulturelles Lernen?") erfasst. Die Erhebung übergeordneter Konstrukte ermöglicht die Eruierung inhaltlich-hierarchischer Verbindungen zwischen den Ausgangskonstrukten und damit die Erhellung der Stellung eines Konstrukts innerhalb eines Konstruktsystems (vgl. Kap. 5.1.2). Wie die vorangegangene Darstellung der ChancenKategorien offenbarte, stehen die verschiedenen Chancen über ihre Begründungen denn auch explizit miteinander in Verbindung. So stellt etwa die für die Chance (9) Arbeiten in Gruppen genannte Begründung Austausch zwischen SuS findet statt (L1: K4) eine argumentative Verbindung zur Chance (12) Austauschen, Diskutieren, Kommunizieren dar.

In den nachfolgenden Ausführungen werden daher die Ergebnisse einer systematischen Analyse solcher Verbindungen beschrieben. Ziel war es, die im vorangegangenen Kapitel unabhängig voneinander dargestellten Chancen-Kategorien anhand ihrer inhaltlichen Verflechtung weiter zu verdeutlichen und davon eine Heuristik interkulturellen Lernens aus Sicht der Lehrpersonen abzuleiten. Da sich diese Ergebnisse nicht explizit auf die Teilfragestellung 2a beziehen, sind sie im Sinne weiterführender Ergebnisse bzw. eines Exkurses zu lesen.

Abbildung 14 visualisiert die Verknüpfungen der 17 ermittelten Chancen interkulturellen Lernens auf Basis erfasster übergeordneter Konstrukte. Ein von einer Chance ausgehender Pfeil markiert dabei mindestens eine erhobene Begründung eines Konstruktpols, die eine inhaltliche Verbindung zu einer 
anderen (übergeordneten) Chance aufzeigt. Damit stellt Abbildung 14 ein aggregiertes Schaubild ${ }^{99}$ der Über- bzw. Unterordnungsverhältnisse der Konstruktsysteme der Lehrpersonen dar. Die Tabellen 24, 25 und 26 fassen die einschlägigen Konstruktpol-Begründungen bzw. die empirische Basis der Visualisierung in Abbildung 14 zusammen.

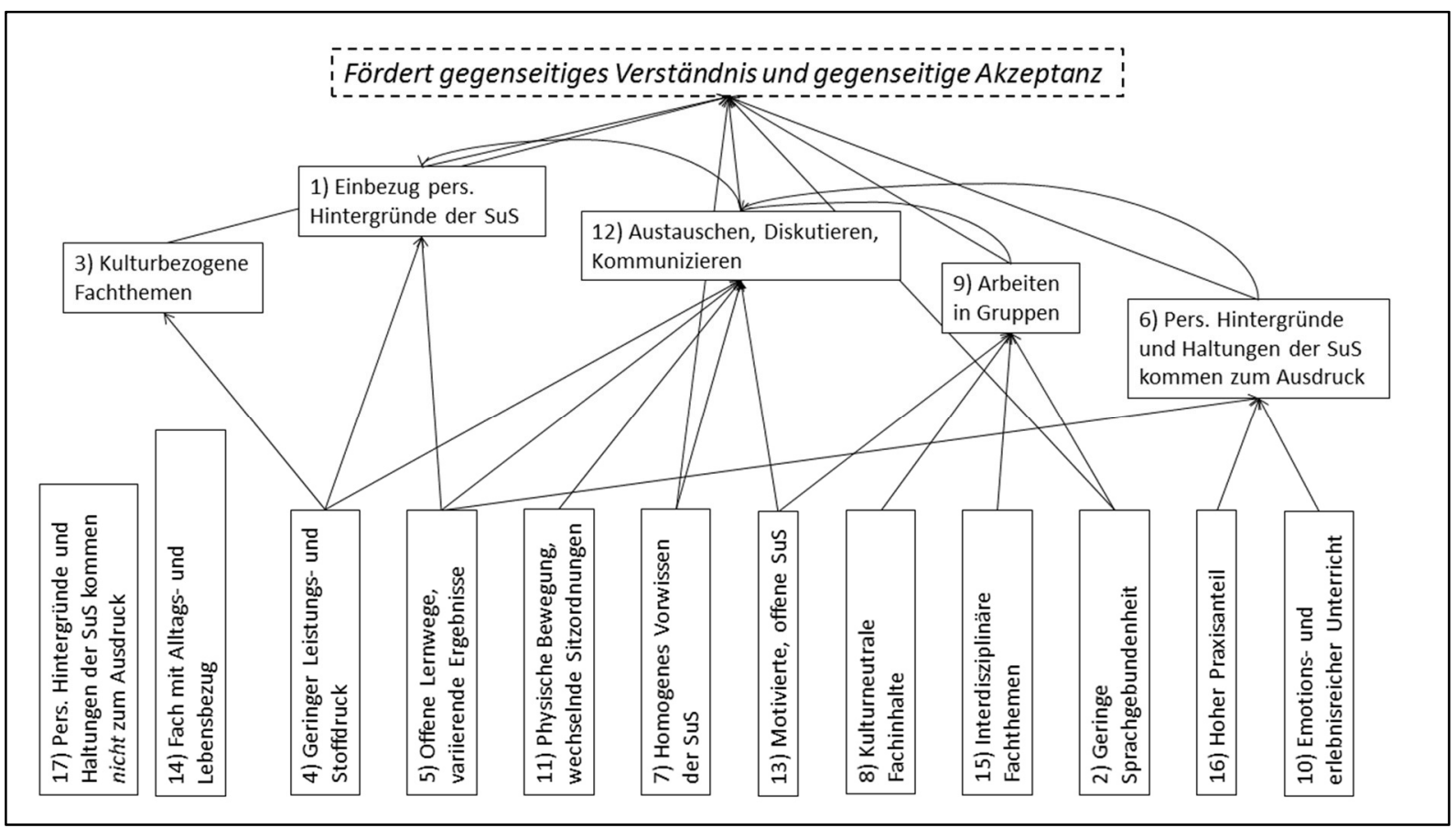

Anmerkung. Die Nummerierung der Chancen entspricht der im vorangegangenen Kapitel in Abhängigkeit der Nennungshäufigkeit vorgenommenen Sortierung der Chancen.

Abbildung 14. Inhaltliche Verknüpfungen der Chancen-Kategorien mit gemeinsamem übergeordnetem Konstrukt

Abbildung 14 verdeutlicht zunächst, dass mit Ausnahme von zwei Chancen (17 und 14) sämtliche Chancen über die erfassten Begründungen inhaltlich-hierarchisch miteinander in Verbindung stehen. Die fehlende Anbindung der Chance (17) Pers. Hintergründe und Haltungen der SuS kommen nicht zum Ausdruck ist inhaltlich (vgl. dazu Ausführungen weiter unten), diejenige der Chance (14) Fach mit Alltags- und Lebensbezug mit der Tatsache, dass hierzu keine erfassten Begründungen vorliegen, zu erklären ${ }^{100}$. Weiter lässt sich Abbildung 14 folgendermassen lesen:

Auf der unteren Ebene der Hierarchie finden sich Chancen (4, 5, 11, 7, 13, 8, 15, 2, 16, 10), die aufgrund ihrer Begründungen anderen Chancen-Kategorien (der mittleren Ebene) untergeordnet sind bzw. nicht

\footnotetext{
${ }^{99}$ Aggregiert ist das Schaubild in dem Sinne, als es die Konstruktsysteme der 24 Lehrpersonen gewissermassen in übereinander gelagerter Form abbildet. Das bedeutet, dass nicht nur die Chancen-Kategorien, sondern auch die Verbindungen zwischen diesen unterschiedlich stark empirisch erhärtet sind.

${ }^{100}$ Wie in Kapitel 5.4.2 beschrieben, konnte aufgrund teilweiser Überforderung befragter Lehrpersonen nicht für jeden Konstruktpol eine Begründung erfasst werden. Die in den Tabellen 24 und 25 pro Chance aufgelisteten Begründungen stehen deshalb nicht repräsentativ für die Anzahl der den Chancen zugeordneten Konstrukte, sondern variieren in ihrer Anzahl in Abhängigkeit der zu den Chancen erfassten Begründungen.
} 
gleichzeitig auch eine Begründung anderer Chancen-Kategorien darstellen. Auf der mittleren Hierarchieebene finden sich fünf Chancen-Kategorien $(3,1,12,9,6)$, die gleichzeitig Begründungen anderer Chancen (der unteren Ebene sowie auch der mittleren Ebene) und damit gewissermassen übergeordnete Chancen darstellen. Auf der obersten Hierarchieebene findet sich schliesslich ein gemeinsames übergeordnetes Konstrukt - Fördert gegenseitiges Verständnis und gegenseitige Akzeptanz - aller Chancen der mittleren und einzelner Chancen der unteren Hierarchieebene. In ihm kommt zum Ausdruck, woran sich die Lehrpersonen im Prozess der Unterscheidung von Fächern hinsichtlich deren Chancen und Hindernisse für interkulturelles Lernen implizit orientierten. Damit stellt das Konstrukt gewissermassen die Zielkategorie interkulturellen Lernens aus Sicht der Lehrpersonen dar.

Ausgehend von dieser Lesart lässt sich in Anlehnung an Abbildung 14 das von den Lehrpersonen Gemeinte bzw. ihre persönlichen Konstrukte in aggregierter Form rekonstruieren und reformulieren. Eine solche Reformulierung könnte folgendermassen lauten:

Interkulturelles Lernen soll aus Sicht der Lehrpersonen insbesondere darin resultieren, dass sich die Schülerinnen und Schüler in ihrer Unterschiedlichkeit (besser) verstehen und (dadurch) akzeptieren lernen. Neben kulturbezogenen Fachthemen, die zunächst eine allgemeine Horizonterweiterung der Schülerinnen und Schüler in Bezug auf andere Kulturen und Lebensweisen ermöglichen können, werden dabei primär die Schülerinnen und Schüler bzw. deren persönlichen Hintergründe und Haltungen als Ausgangspunkt dieses Lernens gesehen.

Interkulturelles Lernen kann aus Sicht der Lehrpersonen demnach dann potenziell stattfinden, wenn Unterricht dazu beiträgt, dass Persönliches der Schülerinnen und Schüler zum Ausdruck kommen kann - sei dies, indem die persönlichen Hintergründe der Schülerinnen und Schüler (von der Lehrperson) aufgegriffen und einbezogen werden, die Schülerinnen und Schüler in Gruppen arbeiten, oder indem ausgetauscht, diskutiert und kommuniziert werden kann. Ein solcher Unterricht wiederum wird potenziell dadurch begünstigt, dass geringer Leistungs- und Stoffdruck vorherrscht, offene Lernwege und variierende Ergebnisse möglich sind, es physische Bewegung bzw. wechselnde Sitzordnungen gibt, alle Schülerinnen und Schüler Vorwissen mitbringen sowie motiviert und offen sind, die Fachinhalte bzw. -themen kulturneutral und interdisziplinär sind, das Fach wenig sprachgebunden ist, einen hohen Praxisanteil aufweist und schliesslich emotions- und erlebnisreich ist.

Interkulturelles Lernen basiert entsprechend der Argumentation dieser Heuristik insbesondere auf einem Unterricht, in dem Differenzerfahrungen möglich sind. Konträr zu dieser Vorstellung - und das suggeriert auch Abbildung 14 - steht hier allerdings die von insgesamt vier Lehrpersonen genannte Chance (17) Persönliche Hintergründe und Haltungen der SuS kommen nicht zum Ausdruck. Diffe- 
renzerfahrungen würden gemäss den erfassten Begründungen dieser Lehrpersonen andere SuS abschrecken, einen negativen Einfluss auf die Gruppendynamik haben oder Vorurteile erzeugen (vgl. Kap. 6.2.1). Dass diese Lehrpersonen gleichzeitig aber auch andere, auf Differenzerfahrungen ausgerichtete Chancen nannten, weist insgesamt auf ambivalente Vorstellungen zur unterrichtlichen Umsetzung interkultureller Erziehung dieser Lehrpersonen hin. 


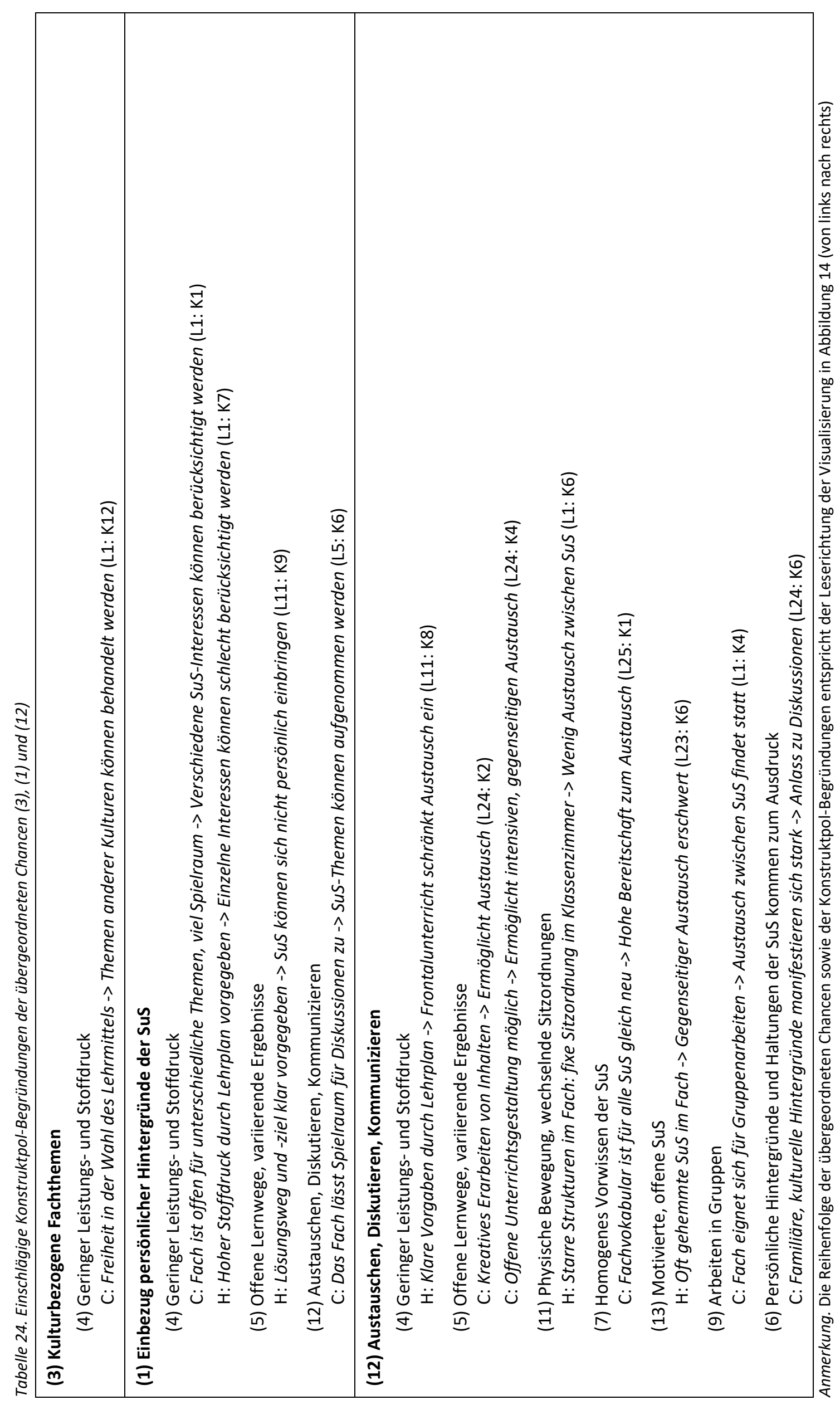




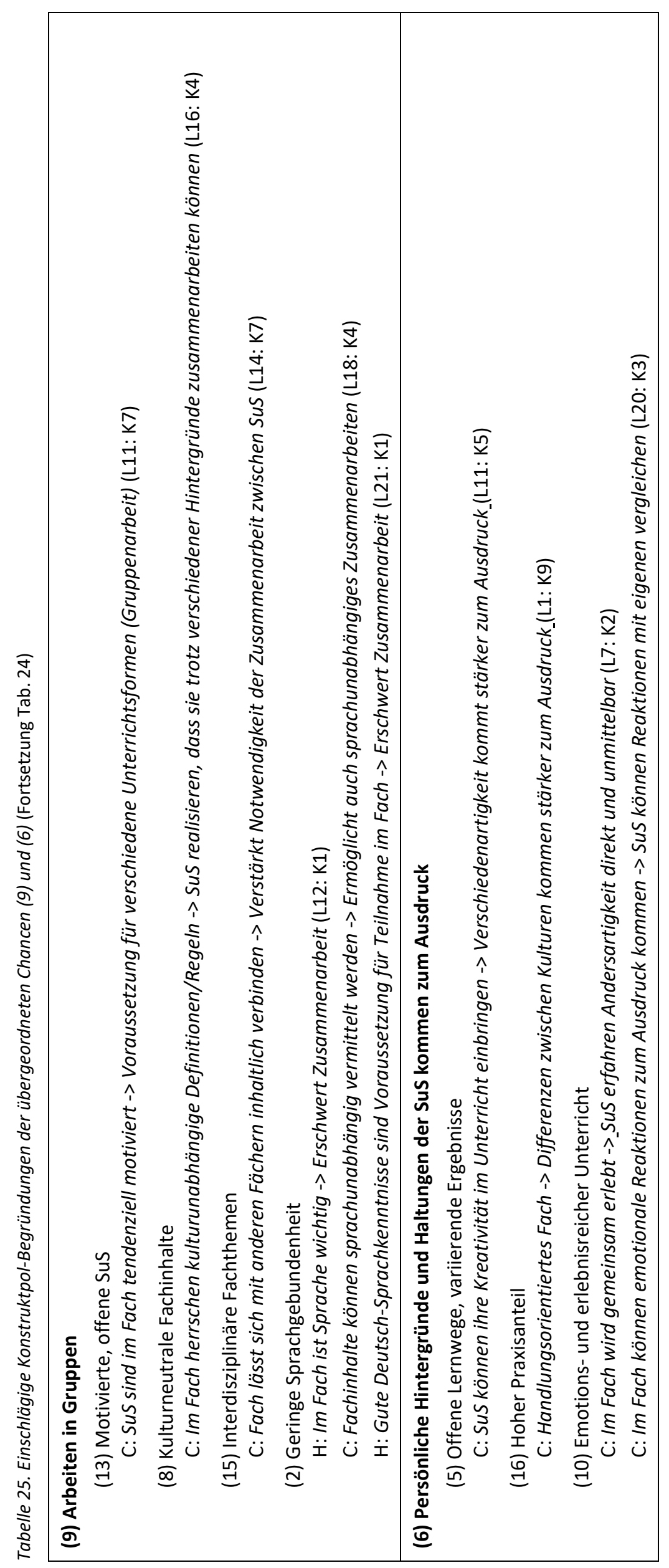




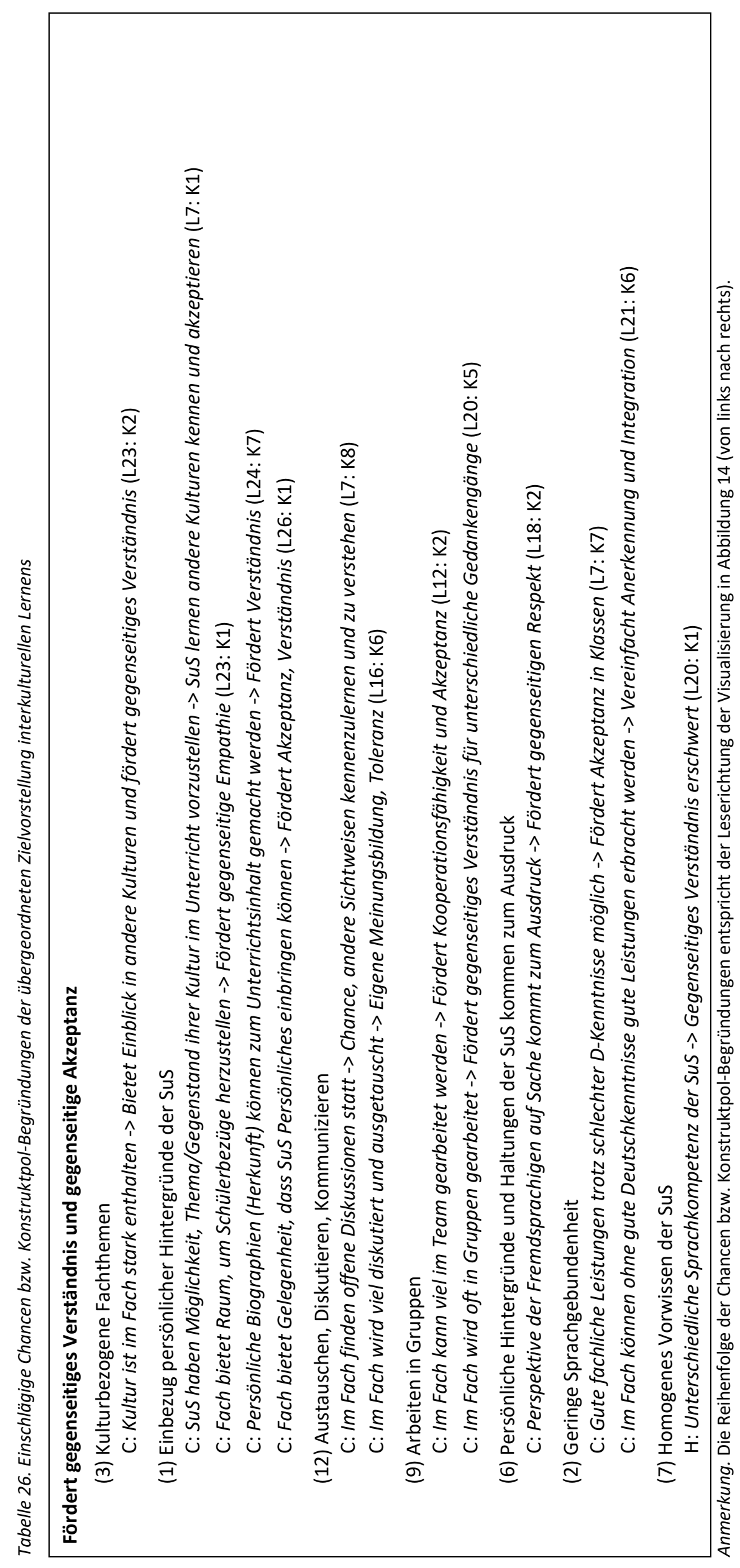




\subsubsection{Chancen interkulturellen Lernens im Sportunterricht aus Sicht der Lehrpersonen}

Nachdem im vorangegangenen Kapitel geäusserte allgemeine Chancen für interkulturelles Lernen und deren Verbindungen aus Sicht der Lehrpersonen dargestellt wurden, werden im Folgenden die Einschätzungen der Lehrpersonen des Fachs Sport hinsichtlich dieser Chancen präsentiert. Die nachfolgenden Ausführungen beziehen sich demnach auf die Teilfragestellung 2b) (Welche Chancen interkulturellen Lernens treffen aus Sicht der Lehrpersonen spezifisch auf den Sportunterricht zu?).

Grundlage der Beschreibung der Einschätzung des Sportunterrichts durch die Lehrpersonen bildet Abbildung 15. In ihr sind für jede der 17 Chancen-Kategorien (vgl. Kap. 6.2.1) die Anzahl Lehrpersonen, welche die jeweilige Chance in Bezug auf das Fach Sport positiv bzw. als zutreffend ${ }^{101}$ einschätzten (gepunktete Säulen), im Verhältnis zur Anzahl Lehrpersonen, welche die Chance überhaupt nannten (schwarze Säulen), dargestellt. Damit visualisiert die Graphik pro Chance den Grad der Zustimmung der Lehrpersonen, dass diese Chance auf das Fach Sport bzw. den entsprechenden Unterricht zutrifft: Je näher die Höhen der beiden Säulen beieinanderliegen, umso höher ist der Grad der Zustimmung der Lehrpersonen in Bezug auf diese Chance. Differenzen zwischen den beiden Säulenhöhen repräsentieren entsprechend die (Anzahl) Lehrpersonen, welche die Chance in Bezug auf das Fach Sport nicht eindeutig oder als nicht zutreffend ${ }^{102}$ einschätzten.

\footnotetext{
101 "Zutreffend“ bedeutet, dass die Lehrperson das Fach Sport mit dem Ratingwert „1“ (= Chancen-Pol „trifft zu“) oder "2" (= Chancen-Pol "trifft eher zu“) beurteilte (vgl. Kap. 3.5.2).

102 "Nicht eindeutig“ bzw. „nicht zutreffend“ bedeutet, dass die Lehrpersonen die Chance in Bezug auf das Fach Sport mit einem Ratingwert von "3“ bzw. "4“ (= Hindernis-Pol "trifft eher zu“) oder "5“ (= Hindernis-Pol "trifft zu“) einschätzte.
} 


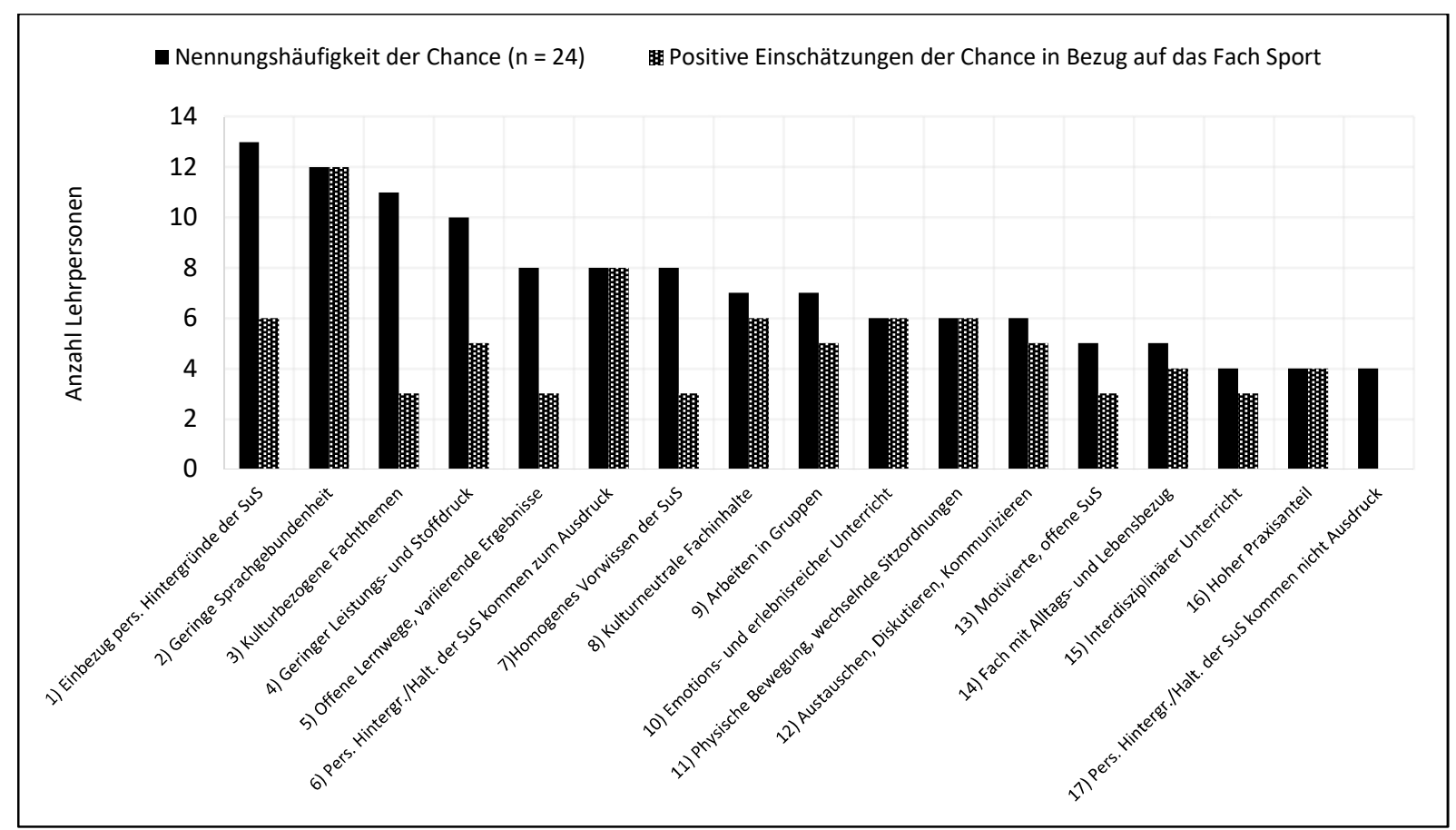

Abbildung 15. Einschätzung des Fachs Sport hinsichtlich genannter Chancen interkulturellen Lernens

Abbildung 16 ist analog zu Abbildung 15 zu lesen. Im Unterschied zu Abbildung 15 stellt sie allerdings zusätzlich die (positiven) Einschätzungen der Chancen in Bezug auf das Fach Mathematik dar (schraffierte Säulen). Dieses war neben dem Fach Sport das am zweithäufigsten, d.h. bei $n=20$ Lehrpersonen in den Elementesets vertretene Fach (vgl. Anhang C). Um weitere Anhaltspunkte bzw. Referenzwerte hinsichtlich der nachfolgenden Interpretation der in Abbildung 15 visualisierten Daten zu erhalten, wurde somit eine zusätzliche, im obigen Sinne beschriebene Analyse der Rating-Werte der Fächer Sport und Mathematik auf Basis einer Teilstichprobe von 20 Lehrpersonen vorgenommen. ${ }^{103}$

\footnotetext{
${ }^{103}$ Den nachfolgenden Ausführungen zu den Abbildungen 15 und 16 ist vorwegzunehmen, dass der empirische Erhärtungsgrad von Aussagen zu den Einschätzungen der verschiedenen Chancen mit abnehmender Nennungshäufigkeit der Chancen abnimmt. Mit anderen Worten: Wurde beispielsweise eine Chance bei 10 Nennungen von fünf Lehrpersonen als zutreffend für ein Fach eingeschätzt, so sind Aussagen über die Einschätzung dieser Chance empirisch „stabiler" als solche zu einer zwar ebenso zu 50\% in Bezug auf ein Fach als zutreffend, aber insgesamt von nur sechs Lehrpersonen genannten Chance.
} 


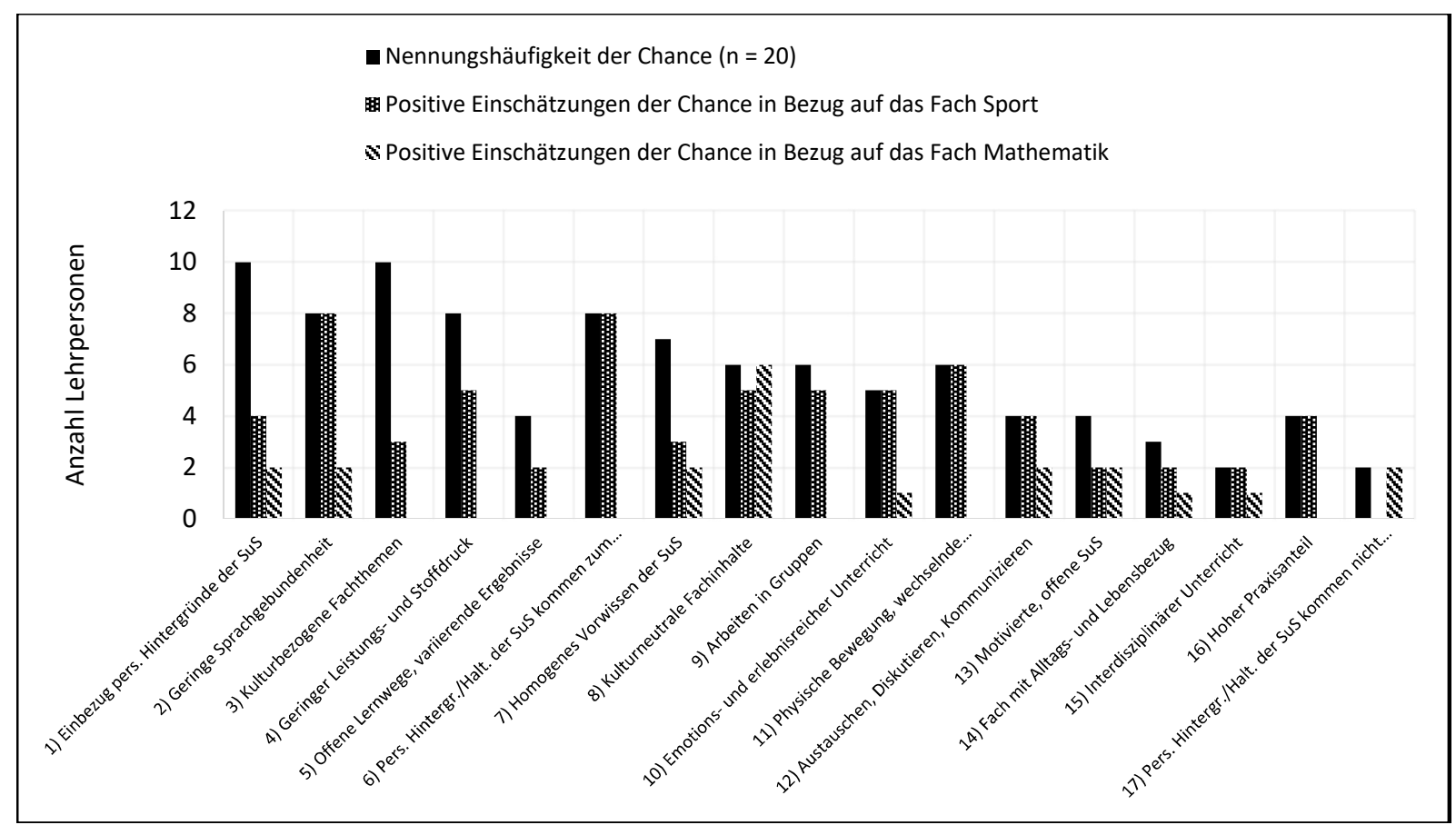

Abbildung 16. Einschätzung des Fachs Sport im Vergleich zum Fach Mathematik hinsichtlich genannter Chancen interkulturellen Lernens

Entsprechend der geschilderten Lesart der Abbildungen 15 und 16 lässt sich zunächst konstatieren, dass jede der 17 kategorisierten Chancen (vgl. Kap. 6.2.1) von mindestens einem Teil der Lehrpersonen, die diese Chancen nannten, in Bezug auf den Sportunterricht als zutreffend eingeschätzt wurde. ${ }^{104}$ Aus Sicht der Lehrpersonen bedeutet dies, dass keine der genannten Chancen dem Sportunterricht grundsätzlich abgesprochen werden kann. Im Gegensatz zum Mathematikunterricht, dem bei insgesamt sieben Chancen positive Einschätzungen gänzlich fehlen, scheint der Sportunterricht aus Sicht der Lehrpersonen demnach einen Lehr-Lern-Kontext darzustellen, der nicht per se Hindernisse in Bezug auf interkulturelles Lernen aufweist.

Dennoch, so ist den Abbildungen zu entnehmen, treffen aus Sicht der Lehrpersonen nicht alle Chancen gleichermassen auf den Sportunterricht zu. Ausgehend von den unterschiedlichen Zustimmungsraten lassen sich die Chancen in eine Gruppe der fachimmanenten Chancen des Sportunterrichts (vgl. Kap. 6.2.2.1), in eine Gruppe der typischen Chancen des Sportunterrichts (vgl. Kap. 6.2.2.2) und in eine Gruppe der eher untypischen Chancen des Sportunterrichts (vgl. Kap. 6.2.2.3) zusammenfassen.

\footnotetext{
${ }^{104}$ Chance 17 wurde von keiner Lehrperson als zutreffend für das Fach Sport eingeschätzt, da sie den gegenteiligen und von allen Lehrpersonen auf das Fach Sport zutreffenden Aspekt von Chance 6 (Pers. Hintergründe und Haltungen der SuS kommen zum Ausdruck) betrifft (vgl. Ausführungen weiter unten). 


\subsubsection{Fachimmanente Chancen des Sportunterrichts}

Zu den fachimmanenten Chancen des Sportunterrichts lassen sich all diejenigen Chancen zählen, die von allen Lehrpersonen, die diese nannten, einhellig bzw. zu 100\% als (eher) zutreffend für das Fach Sport eingeschätzt wurden. Sie können als fachimmanent bezeichnet werden, da sie vergleichsweise unabhängig von (der Einschätzung) der Lehrperson zu existieren scheinen. Darunter fallen die Chancen:

(2) Geringe Sprachgebundenheit,

(6) Persönliche Hintergründe und Haltungen der SuS kommen zum Ausdruck,

(10) Emotions- und erlebnisreicher Unterricht,

(11) Physische Bewegung, wechselnde Sitzordnungen,

(16) Hoher Praxisanteil (vgl. Abb. 15).

Die befragten Lehrpersonen teilten demnach die Meinung, dass die Sprache im Sportunterricht einen geringen Stellenwert hat, dass kulturelle Unterschiede zwischen den Schülerinnen und Schülern gut erkennbar sind und dass Sportunterricht emotions- und erlebnisreich ist. Schliesslich ist aus Sicht der befragten Lehrpersonen auch unstrittig, dass im Sportunterricht physische Bewegung sowie Praxis (im Sinne einer tätigen Auseinandersetzung mit der Sache, vgl. Kap. 6.2.1) zentral sind.

Der Vergleich mit dem Fach Mathematik, dem aus Sicht der Lehrpersonen lediglich zwei Chancen tendenziell fachimmanent sein dürften ((8) Kulturneutrale Fachinhalte, (17) Persönliche Hintergründe und Haltungen der SuS kommen nicht zum Ausdruck) (vgl. Abb. 16), macht deutlich, dass der Sportunterricht per se vergleichsweise viele Aspekte aufweist, die aus Sicht der Lehrpersonen eine Chance für interkulturelles Lernen darstellen.

\subsubsection{Typische Chancen des Sportunterrichts}

Als typische Chancen des Sportunterrichts können Chancen bezeichnet werden, die zwar nicht von allen Lehrpersonen bzw. zu 100\%, aber dennoch mehrheitlich positiv in Bezug auf das Fach Sport eingeschätzt wurden. Hierzu gehören die Chancen: 
(8) Kulturneutrale Fachinhalte,

(9) Arbeiten in Gruppen,

(12) Austauschen, Diskutieren, Kommunizieren,

(13) Motivierte, offene SuS,

(14) Fach mit Alltags- und Lebensbezug,

(15) Interdisziplinäre Fachthemen (vgl. Abb. 15).

Aus Sicht der Lehrpersonen, die diese Chancen anführten, zeichnet sich das Fach Sport also typischerweise durch kulturunabhängige Inhalte, durch Unterricht, in dem eher Gruppen- statt Einzelarbeit geleistet wird und in dem sich die Schülerinnen und Schüler vergleichsweise häufig austauschen bzw. miteinander kommunizieren, aus. Ebenso typisch für das Fach Sport scheinen nach Meinung der Lehrpersonen sein Alltags- bzw. Lebensbezug sowie seine Interdisziplinarität zu sein. Knapp in der Mehrheit sind zudem die Lehrpersonen, die dem Fach tendenziell motivierte, offene Schülerinnen und Schüler zusprechen.

Verglichen mit dem Fach Mathematik hebt sich in dieser Chancen-Gruppe der Sportunterricht insbesondere durch die vergleichsweise hohe Zustimmungsrate der Chance (9) Arbeiten in Gruppen ab. Im Mathematikunterricht, so das Urteil der Lehrpersonen, scheint vielmehr Einzelarbeit geleistet zu werden (vgl. Abb. 16).

\subsubsection{Untypische Chancen des Sportunterrichts}

Zu den untypischen Chancen des Sportunterrichts können diejenigen Chancen gezählt werden, die von mindestens 50\% der Lehrpersonen, die die Chancen nannten, dem Sportunterricht abgesprochen wurden. Es sind dies die Chancen:

(1) Einbezug persönlicher Hintergründe der Schülerinnen und Schüler,

(3) Kulturbezogene Fachthemen,

(4) Geringer Leistungs- und Stoffdruck,

(5) Offene Lernwege, variierende Ergebnisse,

(7) Homogenes Vorwissen der SuS,

(17) Persönliche Hintergründe und Haltungen der SuS kommen nicht zum Ausdruck (vgl. Abb. 15).

Aus Sicht der Lehrpersonen, welche diese Chancen nannten, ist Sportunterricht demnach ein Fach, in dem persönliche Hintergründe der Schülerinnen und Schüler in den Unterricht nicht einbezogen werden bzw. sich nicht einbeziehen lassen. Ebenso eher untypisch für das Fach Sport scheinen für die 
Lehrpersonen unterschiedliche Lernwege und Ergebnisse zu sein sowie die Tatsache, dass das sportbezogene Vorwissen bzw. die Fertigkeiten der Schülerinnen und Schüler homogen sind. Schliesslich schätzen die Lehrpersonen den Sportunterricht als einen Lehr-Lern-Kontext mit wenig oder keinen kulturbezogenen Fachthemen ein.

Aus dem Vergleich mit dem Mathematikunterricht fällt insbesondere auf, dass in diesem im Gegensatz zum Sportunterricht persönliche Hintergründe und Haltungen der Schülerinnen und Schüler nicht zum Ausdruck kommen (vgl. Abb. 16).

Sämtliche 17 Chancen überblickend ist schliesslich festzustellen, dass fünf der sieben insgesamt am häufigsten genannten Chancen gleichzeitig zu den eher untypischen Chancen des Sportunterrichts aus Sicht der Lehrpersonen gehören (vgl. Abb. 15). Dies legt den Schluss nahe, dass der Sportunterricht aus Sicht der Lehrpersonen einen Lehr-Lern-Kontext darzustellen scheint, in dem interkulturelles Lernen vergleichsweise untypisch stattzufinden scheint. 


\subsubsection{Zusammenfassung der Ergebnisse}

Zusammenfassend und in Bezug auf die erste Teilfragestellung (Welche unterrichtlichen Bedingungen stellen aus Sicht der Lehrpersonen generell Chancen für interkulturelles Lernen dar?) lässt sich festhalten, dass insgesamt ein weites Spektrum an Chancen für interkulturelles Lernen genannt wurde. Chancen für interkulturelles Lernen können sich aus Sicht der Lehrpersonen demnach sowohl im Inhalt bzw. den Themen eines Faches (z.B. Kulturbezogene Fachthemen) als auch in der Art und Weise wie Lehren und Lernen inszeniert wird (z.B. Arbeiten in Gruppen) finden. Darüber hinaus äusserten die Lehrpersonen aber auch Chancen im Bereich curricularer Vorgaben (z.B. Geringer Leistungs- und Stoffdruck) sowie hinsichtlich der Voraussetzungen der Schülerinnen und Schüler (z.B. Motivierte, offene Schülerinnen und Schüler). Schliesslich wurden Chancen genannt, die sich gleichsam als Implikationen anderer Chancen fassen lassen (z.B. Austauschen, Diskutieren, Kommunizieren als Implikation der Chance Arbeiten in Gruppen) und/oder deren Begründungen explizit anderen Chancen entsprechen. Die vier am häufigsten genannten Chancen sind Einbezug persönlicher Hintergründe der SuS (13 bzw. 54.2\% Lehrpersonen), Geringe Sprachgebundenheit (12 bzw. 50\% Lehrpersonen), Kulturbezogene Fachthemen (11 bzw. 45.8\% Lehrpersonen), Geringer Leistungs- und Stoffdruck (10 bzw. 41.7\% Lehrpersonen). Wie aus einer Analyse der Begründungen der Chancen hervorging, orientierten sich die Lehrpersonen im Prozess der Unterscheidung der Fächer an einem Verständnis interkulturellen Lernens, das primär im Unterricht anwesende Schülerinnen und Schüler bzw. deren persönlichen, unterschiedlichen Hintergründe und Haltungen als Ausgangspunkt für dieses Lernen ansieht. Es zeigte sich jedoch auch, dass die Vorstellungen mancher Lehrpersonen in Bezug auf Differenzerfahrungen ambivalent sind.

Die Ergebnisse zur zweiten Teilfragestellung (Welche Chancen interkulturellen Lernens treffen aus Sicht der Lehrpersonen spezifisch auf den Sportunterricht zu?) machen zunächst deutlich, dass der Sportunterricht aus Sicht der Lehrpersonen einen Lehr-Lern-Kontext darstellt, der nicht per se Hindernisse hinsichtlich interkulturellen Lernens aufweist. So wurden sämtliche 17 genannten Chancen von mindestens einer der befragten Lehrpersonen als zutreffend für das Fach Sport eingeschätzt. Durch die Berechnung der Verhältnisse zwischen der Anzahl an Lehrpersonen, welche die Chance nannten, und der Anzahl an Lehrpersonen, welche die Chance positiv bzw. als zutreffend für das Fach Sport einschätzten, konnten darüber hinaus drei nach Höhe der Zustimmungsraten abgestufte Chancen-Gruppen gebildet werden:

a) Fachimmanente Chancen des Sportunterrichts (Zustimmungsrate $=100 \%$ ): Geringe Sprachgebundenheit; Persönliche Hintergründe und Haltungen der SuS kommen zum Ausdruck; Emotions- und erlebnisreicher Unterricht; Physische Bewegung, wechselnde Sitzordnungen; Hoher Praxisanteil. 
b) Typische Chancen des Sportunterrichts (Zustimmungsrate > 50\%): Kulturneutrale Fachinhalte; Arbeiten in Gruppen; Austauschen, Diskutieren, Kommunizieren; Motivierte, offene SuS; Fach mit Alltags- und Lebensbezug; Interdisziplinäre Fachthemen.

c) Untypische Chancen des Sportunterrichts (Zustimmungsrate $\leq 50 \%$ ): Einbezug persönlicher Hintergründe der Schülerinnen und Schüler; Kulturbezogene Fachthemen; Geringer Leistungsund Stoffdruck; Offene Lernwege, variierende Ergebnisse; Homogenes Vorwissen der SuS; Persönliche Hintergründe und Haltungen der SuS kommen nicht zum Ausdruck.

Da die überwiegende Mehrheit aller von den Lehrpersonen genannten Chancen in die Gruppe der untypischen Chancen des Sportunterrichts fallen, lässt sich schliesslich folgern, dass interkulturelles Lernen im Sportunterricht aus Sicht der Lehrpersonen vergleichsweise untypisch bzw. fachspezifisch stattzufinden scheint. 


\section{ZUSAMMENFASSUNG UND DISKUSSION DER ERGEBNISSE}

In diesem Kapitel werden die Fragestellungen der Untersuchung unter Berücksichtigung der datenbasierten Ergebnisse beantwortet und vor dem Hintergrund vorliegender Forschungsbefunde zusammengefasst und diskutiert (vgl. Kap. 7.1; Kap. 7.2). Danach werden methodische Aspekte reflektiert (vgl. Kap. 7.3) sowie ein übergreifendes Fazit und Implikationen für Wissenschaft und Praxis formuliert (vgl. Kap. 7.4).

Ausgehend von empirischen Befunden zur Professionalisierung und zum Umgang von Lehrpersonen mit migrationsbedingter Heterogenität wurde in dieser Untersuchung davon ausgegangen, dass das Unterrichtshandeln von (Sport-)Lehrpersonen in migrationsbedingt heterogenen Klassenverhältnissen zu grossen Teilen durch deren subjektive Überzeugungen bestimmt ist. Die Erforschung solcher Überzeugungen war vom Ziel geleitet, Orientierungswissen zu generieren, das u. a. für die (Konzeption der) Aus- und Weiterbildung von (Sport-)Lehrpersonen genutzt werden kann (vgl. Kap. 1.1).

Wie die Erfassung und Analyse des aktuellen Forschungsstandes deutlich machte (vgl. Kap. 3), lassen sich Lehrerüberzeugungen angesichts migrationsbedingter Heterogenität den folgenden drei Gegenstandsbereichen zuordnen:

- Akkulturationseinstellungen und Ideologien im Umgang mit migrationsbedingter Heterogenität als Einstellungen von Lehrpersonen zu „Art und Zielen der Koexistenz“ verschiedener kultureller Gruppen in einem gesellschaftlichen Kontext (vgl. Van Dick et al., 1997, S. 83).

- Überzeugungen über Schülerinnen und Schüler mit Migrationshintergrund als Lehrererwartungen an bzw. -wahrnehmungen von Schülerinnen und Schülern mit Migrationshintergrund.

- Vorstellungen zur unterrichtlichen Umsetzung und Umsetzbarkeit interkultureller Erziehung als Auffassungen von Lehrpersonen zum Konzept interkultureller Erziehung und der (davon abhängigen) Art und Weise, wie diese Zielorientierung im Unterricht umgesetzt wird bzw. werden kann.

Mit Blick auf die Forschungslage zu Überzeugungen Sport unterrichtender Lehrpersonen erwiesen sich insbesondere letztere beiden Gegenstandsbereiche als weitgehend unerforscht. Die damit identifizierten Forschungslücken waren schliesslich Anlass, einerseits die Überzeugungen Sport unterrichtender Lehrpersonen über Schülerinnen und Schüler mit Migrationshintergrund im Sportunterricht, andererseits die Vorstellungen Sport unterrichtender Lehrpersonen zur unterrichtlichen Umsetzung und Umsetzbarkeit interkultureller Erziehung im Sportunterricht empirisch zu untersuchen. 


\subsection{Zu den Lehrerüberzeugungen über Schülerinnen und Schüler mit Migrations- hintergrund im Sportunterricht}

Für die empirische Untersuchung der Überzeugungen Sport unterrichtender Lehrpersonen über Schülerinnen und Schüler mit Migrationshintergrund im Sportunterricht wurde folgende Fragestellung formuliert: Wie nehmen Sport unterrichtende Lehrpersonen Schülerinnen und Schüler mit Migrationshintergrund im Sportunterricht wahr?

Die Ergebnisse zur ersten Teilfragestellung (Unterscheiden sich aus Sicht der Lehrpersonen Schülerinnen und Schüler mit Migrationshintergrund von solchen ohne Migrationshintergrund?) machten deutlich, dass sich Schülerinnen und Schüler mit Migrationshintergrund von solchen ohne Migrationshintergrund in der Wahrnehmung der befragten Lehrpersonen unterscheiden. Es zeigte sich, dass die mittlere Distanz der eingeschätzten fremdsprachigen Schülerinnen und Schüler zu einem in Bezug auf dieselben Dimensionen eingeschätzten „Ideal“-Schüler grösser war als diejenige der beurteilten nichtfremdsprachigen Schülerinnen und Schüler. Ein „Signifikanztest auf Probe“ (Bortz \& Döring, 2006, S. 379) sowie die Berechnung der Effektstärke liess dabei einen hochsignifikanten Unterschied von hoher praktischer Bedeutsamkeit erkennen.

Die Ergebnisse zur zweiten Teilfragestellung (Falls ja - wodurch (unterscheiden sich Schülerinnen und Schüler mit Migrationshintergrund von solchen ohne Migrationshintergrund)?) wiesen darauf hin, dass die befragten Lehrpersonen ihre Schülerinnen und Schüler insbesondere in Bezug auf ihre körper- und bewegungsbezogene Leistung (Kategorie 1), ihre Art und Weise, wie sie sich im Sportunterricht engagieren und beteiligen (Kategorie 2), ihre Einstellung bzw. ihren inneren Bezug zur Sache (Kategorie 3) sowie hinsichtlich ihres sozialen Verhaltens und ihrer sozialen Rolle im Sportunterricht (Kategorie 4) unterschieden und einschätzten. Auf Grundlage der von den Lehrpersonen pro Kategorie präferierten Konstrukt-Pole liessen sich sodann die Idealvorstellungen der Lehrpersonen von Schülerinnen und Schülern im Sportunterricht zusammenfassend beschreiben. Ein „Ideal“-Schüler im Sportunterricht ist demnach:

- Sportlich, d. h. hat hohe konditionelle und koordinative Fähigkeiten, zeigt einen geschickten, feinmotorischen Umgang mit dem eigenen Körper, beherrscht verschiedene (Spiel-)Sportarten bzw. erfüllt die dafür notwendigen Anforderungen (z.B. Antizipationsfähigkeit) (Kategorie 1),

- zeigt einen hohen Einsatz und eine hohe Anstrengungsbereitschaft, arbeitet fokussiert und selbständig, beteiligt sich auf eine besonnene Art und Weise, zeigt Mut in der Auseinandersetzung mit den ihm aufgetragenen Aufgaben (Kategorie 2),

- $\quad$ weist einen inneren Bewegungsdrang bzw. allgemein Freude an Bewegung auf, ist offen und (für vielseitigen Unterricht) begeisterungsfähig, ist ehrgeizig bzw. siegeswillig, zeigt Freude an bzw. Begeisterung für spezifische(n) Sportarten oder Unterrichtsinhalte(n) (Kategorie 3), 
- $\quad$ ist gegenüber seinen Mitschülerinnen und -schülern sowie gegenüber der Lehrperson aufrichtig, loyal und fair, kann sich in Spielsituationen bzw. in Teams auf andere Schülerinnen und Schüler einlassen bzw. diese akzeptieren und unterstützen, pflegt einen angemessenen Umgang mit seiner Person und seiner Leistung (d. h. kann z. B. eigene Schwächen zugeben und blufft nicht) und ist gut in die Klasse integriert (Kategorie 4).

Ein Vergleich der Anteile der Schülerinnen und Schüler pro Schülergruppe (mit Migrationshintergrund vs. ohne Migrationshintergrund), auf welche die präferierten Pole pro Kategorie angewandt wurden, machte deutlich, dass Schülerinnen und Schüler mit Migrationshintergrund in allen vier Hauptkategorien seltener den Idealvorstellungen der Lehrpersonen entsprechen als jene ohne Migrationshintergrund. In den Kategorien 1 (Körperlichkeit/Sportliche Leistungsfähigkeit), 2 (Engagement/Beteiligung) und 4 (Mit Anderen) erwies sich dieser Unterschied gar als signifikant.

Insgesamt weisen die Ergebnisse zur Fragestellung 1 darauf hin, dass Schülerinnen und Schüler mit Migrationshintergrund im Sportunterricht bezogen auf die Idealvorstellungen ihrer Lehrpersonen in allen zur Beurteilung herangezogenen Haupturteilsdimensionen als schlechter wahrgenommen und eingeschätzt werden als Schülerinnen und Schüler ohne Migrationshintergrund.

Dieser Befund deckt sich mit sowohI US-amerikanischen als auch deutschsprachigen Forschungsergebnissen zu Lehrerurteilen hinsichtlich Schulleistungen und/oder Intelligenz von Schülerinnen und Schülern mit Migrationshintergrund. Vergleichbar mit den Resultaten der vorliegenden Untersuchung dokumentierten die Autorinnen und Autoren dieser Publikationen wiederholt niedrigere Leistungs- bzw. Intelligenzerwartungen von Lehrpersonen an Schülerinnen und Schüler mit Migrationshintergrund im Vergleich zu solchen ohne Migrationshintergrund (vgl. Kap. 3.1.2).

Ob die in der vorliegenden Studie erfassten Einschätzungen ebenso - wie dies insbesondere in deutschsprachigen Arbeiten ausserhalb der Sportlehrerforschung festgestellt werden konnte (vgl. Kap. 3.1.2) - von verzerrten Wahrnehmungen zuungunsten von Schülerinnen und Schülern mit Migrationshintergrund bzw. niedrigerer Schichtzugehörigkeit unterlaufen werden, lässt sich aufgrund nicht erfasster Angaben zu den tatsächlichen Leistungen bzw. zum Verhalten der Schülerinnen und Schüler nicht beurteilen. Angesichts der Feststellung von Grimminger (2009, S. 31), wonach Sportlehrpersonen die Verhaltensweisen von Schülerinnen und Schülern mit Migrationshintergrund fast ausnahmslos mit deren kulturellem Hintergrund (weg)erklären bzw. die Schülerinnen und Schüler stereotypisierend wahrnehmen sowie aufgrund des aus der vorliegenden Arbeit hervorgegangenen Befunds, dass Schülerinnen und Schüler mit Migrationshintergrund über alle Urteilskategorien hinweg schlechter einge- 
schätzt wurden als solche ohne Migrationshintergrund, lassen sich kategorisierende Wahrnehmungsprozesse entlang des askriptiven Schülermerkmals „Migrationshintergrund“ jedoch zumindest vermuten.

Für diese Vermutung, also die Annahme einer wenig differenzierten und möglicherweise nicht akkuraten Sichtweise der Lehrpersonen auf ihre Schülerinnen und Schüler im Sportunterricht, würden denn auch theoretische Argumente sprechen: Lehrerüberzeugungen wird hinsichtlich ihrer Wirkungsweise insofern eine Filter- und eine Frame-Funktion zugesprochen, als sie (auf Basis biographischer, ausbildungsbezogener und berufspraktischer Vorerfahrungen) die Wahrnehmung von Ereignissen und Informationen filtern und dadurch rascher einordnen bzw. interpretieren helfen (vgl. Fives \& Buehl, 2012, S. 478; vgl. auch Kap. 2.3.2). Bezogen auf Schülerinnen und Schüler bzw. als implizite Persönlichkeitstheorien ermöglichen Überzeugungen damit eine rasche Eindrucksbildung zu „Persönlichkeitsmerkmalen, Eigenschaften, Einstellungen und Fähigkeiten der Schüler/innen (...)“ (Ziegler et al., 1998, S. 273). Wenngleich schülerbezogene Überzeugungen damit durchaus funktional sein können, ist mit innen gleichzeitig aber auch die Gefahr verbunden, dass bei Wahrnehmung einer von mehreren als zusammengehörig erlebten Eigenschaften automatisch, d. h. implizit auf das Vorliegen der weiteren Eigenschaften geschlossen wird - unabhängig davon, ob diese Eigenschaften tatsächlich gehäuft gemeinsam vorliegen oder nicht (vgl. Schweer \& Thies, 2000, S. 61). Vor diesem Hintergrund liessen sich die vorliegenden Befunde (zumindest theoriebasiert) auch dahingehend interpretieren, dass Sport unterrichtende Lehrpersonen durch die Feststellung eines Migrationshintergrunds bei Schülerinnen und Schülern implizit auf vergleichsweise weit von ihren Idealvorstellungen entfernt liegende Schülereigenschaften schliessen, die unter Umständen nicht auf die betreffenden Schülerinnen und Schüler zutreffen. Die empirische Prüfung solcher Hypothesen könnte Gegenstand weiterführender Untersuchungen sein.

\subsection{Zu den Lehrervorstellungen zur unterrichtlichen Umsetzung und Umsetzbar- keit interkultureller Erziehung im Sportunterricht}

Vorstellungen von Lehrpersonen zur unterrichtlichen Umsetzung und Umsetzbarkeit interkultureller Erziehung betreffen die Fragen nach dem subjektiven Verständnis interkultureller Erziehung und der (davon abhängigen) Art und Weise, wie diese Zielorientierung im Unterricht umgesetzt wird bzw. werden kann (vgl. Kap. 3.1.3). In der vorliegenden empirischen Untersuchung interessierten dabei insbesondere die Auffassungen Sport unterrichtender Lehrpersonen darüber, ob und inwiefern interkulturelles Lernen im Sportunterricht gefördert werden kann. Vor diesem Hintergrund wurde folgender Frage nachgegangen: Welche Vorstellungen zum Potenzial des Sportunterrichts für interkulturelles Lernen haben Sport unterrichtende Lehrpersonen? 
Die Ergebnisse hinsichtlich der ersten Teilfragestellung (Welche unterrichtlichen Bedingungen stellen aus Sicht der Lehrpersonen generell Chancen für interkulturelles Lernen dar?) zeigten zunächst, dass insgesamt ein breites Spektrum an unterrichtlichen Bedingungen als Chancen für interkulturelles Lernen genannt wurde. Chancen für interkulturelles Lernen im Unterricht können sich aus Sicht der Lehrpersonen demnach sowohl in den Inhalten bzw. Themen eines Faches (Kulturbezogene Fachthemen; Kulturneutrale Fachinhalte; Fach mit Alltags- und Lebensbezug; Interdisziplinäre Fachthemen) als auch in der Art und Weise, wie Lehren und Lernen inszeniert wird (Arbeiten in Gruppen; Austauschen, Diskutieren, Kommunizieren; Offene Lernwege, variierende Ergebnisse) finden. Darüber hinaus äusserten die Lehrpersonen aber auch Chancen im Bereich curricularer Vorgaben (Geringer Leistungs- und Stoffdruck) sowie hinsichtlich der Voraussetzungen der Schülerinnen und Schüler (Homogenes Vorwissen der Schülerinnen und Schüler; Motivierte, offene Schülerinnen und Schüler). Als weitere Gelingensbedingungen für interkulturelles Lernen wurden Einbezug persönlicher Hintergründe der SuS, Geringe Sprachgebundenheit, Pers. Hintergründe und Haltungen der SuS kommen zum Ausdruck, Emotions- und erlebnisreicher Unterricht, Physische Bewegung/Wechselnde Sitzordnung sowie Hoher Praxisanteil genannt. Von den insgesamt 17 inhaltsanalytisch kategorisierten Chancen wurden die Chancen Einbezug persönlicher Hintergründe der SuS (13 bzw. 54.2\% Lehrpersonen), Geringe Sprachgebundenheit (12 bzw. 50\% Lehrpersonen), Kulturbezogene Fachthemen (11 bzw. 45.8\% Lehrpersonen) und Geringer Leistungs- und Stoffdruck (10 bzw. 41.7\% Lehrpersonen) am häufigsten genannt.

Als sämtlichen Chancen übergeordnete Kategorie liess sich - als weiterführendes Ergebnis auf Basis einer systematischen Analyse erfasster Chancen-Begründungen (vgl. Kap. 6.2.1) - das Konstrukt Fördert gegenseitiges Verständnis und gegenseitige Akzeptanz identifizieren. Es bringt zum Ausdruck, woran sich die Lehrpersonen im Prozess der Unterscheidung von Fächern hinsichtlich deren Chancen und Hindernisse für interkulturelles Lernen implizit orientierten und stellt damit gewissermassen die Zielkategorie interkulturellen Lernens aus Sicht der Lehrpersonen dar. Neben kulturbezogenen Fachthemen scheinen dabei primär die Schülerinnen und Schüler bzw. deren persönlichen, unterschiedlichen Hintergründe den Ausgangspunkt interkulturellen Lernens zu bilden. Diesem auf Differenzerfahrungen ausgerichteten Verständnis interkulturellen Lernens steht allerdings die von vier Lehrpersonen genannte Chance Persönliche Hintergründe und Haltungen der SuS kommen nicht zum Ausdruck konträr gegenüber. Dies ist ein Befund, der darauf hinweist, dass zumindest manche Lehrpersonen ambivalente Vorstellungen zur unterrichtlichen Umsetzung interkultureller Erziehung haben.

Der Vergleich dieser Befunde mit vorliegenden empirischen Ergebnissen zeigt zunächst, dass die Vorstellungen der befragten Lehrpersonen zu grossen Teilen mit denjenigen, die in anderen Interviewstudien eruiert wurden (z.B. Binder \& Daryabegi, 2002; Edelmann, 2008; Marburger et al., 1997; Sievers, 
2009; Strohmeier \& Fricker, 2007), übereinstimmen. Deutliche Parallelen finden sich dabei insbesondere zu den Resultaten von Edelmann (2008) und Binder und Daryabegi (2002). So erklärten die von Edelmann (2008) befragten Zürcher Lehrpersonen etwa auch die Behandlung verschiedener Feiertage (Ostern, Weihnachten etc.) - in der vorliegenden Studie ein mehrfach genanntes, der Chancen-Kategorie Kulturbezogene Fachthemen zugeordnetes Konstrukt (vgl. Kap. 6.2.1) - , individualisierte, offene Unterrichtsformen, Gruppenarbeiten, Klassengespräche, die Thematisierung individuell-religiöser Orientierungen, geringer Leistungsdruck sowie „das Emotionale“ als potenzielle Gelingensbedingungen für interkulturelles Lernen. In der Studie von Binder und Daryabegi (2002) wurden zudem - analog zu den eigenen Ergebnissen - u. a. die Inszenierung fächerübergreifenden Lernens, die Förderung von Gesprächs- und Diskussionskulturen sowie auch das aktive Einbeziehen persönlicher Hintergründe der Schülerinnen und Schüler als Möglichkeiten zur Förderung interkulturellen Lernens genannt. Unterschiede zwischen den eigenen Befunden und den in diesen beiden Studien berichteten Lehrervorstellungen finden sich dagegen im Bereich der Einschätzung sprachbezogener Aspekte. Während die befragten Lehrpersonen stark sprachgebundenen Unterricht eher als Hindernis für interkulturelles Lernen einschätzten, berichten die anderen beiden Studien vielmehr von Lehrervorstellungen, in denen die Sprache explizit als interkulturelles Lernfeld angesehen wird. Die genauere Betrachtung der Ergebnisse macht dabei deutlich, dass diesem Unterschied eine in Bezug auf Sprache im einen Fall eher defizitorientierte, im anderen Fall eher ressourcenorientierte Sichtweise zugrunde liegt. So fokussieren die in der vorliegenden Studie befragten Lehrpersonen insbesondere die Defizite in der deutschen Sprache und die damit einhergehende Problematik von Anerkennung und Integration von Schülerinnen und Schülern mit Migrationshintergrund, wohingegen die Lehrpersonen der anderen Studien ihre Aufmerksamkeit vielmehr auf die Herkunftssprachen jener Schülerinnen und Schüler als potenzielle Ressource (z. B. durch Förderung von Sprachvergleichen) für interkulturelles Lernen richten.

Eine mögliche Erklärung für diese unterschiedlichen Sichtweisen könnte der von den befragten Lehrpersonen (implizit) eingenommene Fokus auf das Fach Sport und dessen spezifisches Potenzial für interkulturelles Lernen - die geringe Sprachgebundenheit (vgl. nachfolgende Ausführungen zur zweiten Teilfragestellung) - sein. Obwohl die Lehrpersonen vor dem Interview bewusst nicht über den Hauptfokus der Befragung - die Einschätzung des Potenzials des Fachs Sport in Bezug auf interkulturelles Lernen - informiert wurden (vgl. Kap. 5.4.1), dürften die institutionelle Anbindung des Interviewers sowie das im Vorfeld durchgeführte Interview zu Schülerinnen und Schülern im Sportunterricht dazu beigetragen haben.

Unabhängig davon scheint für die von Binder und Daryabegi (2002) befragten Lehrpersonen analog zu den in der vorliegenden Studie befragten Lehrpersonen die Anwesenheit fremdsprachiger Schülerinnen und Schüler gewissermassen die Grundbedingung für interkulturelles Lernen darzustellen. 
Zur Beantwortung der zweiten Teilfragestellung (Welche Chancen interkulturellen Lernens treffen aus Sicht der Lehrpersonen spezifisch auf den Sportunterricht zu?) wurde auf Basis der numerischen Werte bzw. der ratings eruiert, welche der erfassten Chancen für interkulturelles Lernen auf das Fach Sport angewandt wurden. Dabei ist zunächst vorwegzunehmen, dass jede der 17 kategorisierten Chancen von mindestens einem Teil der Lehrpersonen, die diese Chancen nannten, in Bezug auf den Sportunterricht als zutreffend eingeschätzt wurde. Aus Sicht der Lehrpersonen bedeutet dies, dass keine der genannten Chancen dem Sportunterricht grundsätzlich abgesprochen werden kann.

Dieser Befund verdeutlicht, dass sich die Mehrheit der erfassten Chancen nicht per se bestimmten Fächern zuschreiben bzw. absprechen lässt. Insbesondere Chancen im Bereich des Lehrens und Lernens beziehen sich auf Inszenierungsformen von Unterricht, die grundsätzlich in jedem Fach anwendbar sind. Ebenso wenig in Bezug auf ein spezifisches Fach generalisierbar sind die Schülervoraussetzungen, die stark in Abhängigkeit der Schule und/oder der Klassenzusammensetzung variieren können. Oder anders ausgedrückt: Ob ein Fach Alltags- und Lebensbezüge aufweist, ob seine Inhalte kulturneutral sind, ob geringer Leistungs- und Stoffdruck vorherrscht oder ob ein Fach bzw. sein Unterricht emotional und erlebnisreich ist, sind Fragen, deren Antworten letztlich von den subjektiven Vorstellungen zu den Bedingungen und Praktiken eines Faches - wie sie etwa auch im Rahmen der Fachkulturforschung untersucht werden (z.B. Schierz, 2013) - abhängen.

Um Lehrpersonen übergreifende Aussagen zu den Vorstellungen über das Fach Sport machen zu können, wurden vor diesem Hintergrund aus den pro Chance errechneten Verhältnissen zwischen der Anzahl Lehrpersonen, welche die Chance nannten, und der Anzahl Lehrpersonen, welche die Chance positiv bzw. als zutreffend auf das Fach Sport einschätzten, drei nach Höhe der Zustimmungsraten abgestufte Chancen-Gruppen gebildet:

- Fachimmanente Chancen des Sportunterrichts (Zustimmungsrate $=100 \%$ ): Geringe Sprachgebundenheit; Persönliche Hintergründe und Haltungen der SuS kommen zum Ausdruck; Emotions- und erlebnisreicher Unterricht; Physische Bewegung, wechselnde Sitzordnungen; Hoher Praxisanteil.

- Typische Chancen des Sportunterrichts (Zustimmungsrate > 50\%): Kulturneutrale Fachinhalte; Arbeiten in Gruppen; Austauschen, Diskutieren, Kommunizieren; Motivierte, offene SuS; Fach mit Alltags- und Lebensbezug; Interdisziplinäre Fachthemen.

- Untypische Chancen des Sportunterrichts (Zustimmungsrate $\leq 50 \%$ ): Einbezug persönlicher Hintergründe der Schülerinnen und Schüler; Kulturbezogene Fachthemen; Geringer Leistungsund Stoffdruck; Offene Lernwege, variierende Ergebnisse; Homogenes Vorwissen der SuS; Persönliche Hintergründe und Haltungen der SuS kommen nicht zum Ausdruck. 
Der Gruppe der fachimmanenten Chancen des Sportunterrichts wurden all diejenigen Chancen zugeordnet, die von allen Lehrpersonen, welche diese Chancen nannten, als zutreffend auf den Sportunterricht eingeschätzt wurden. Angesichts der hohen Zustimmungsrate von 100\% scheinen der geringe Stellenwert der Sprache, die Emotionalität und Erlebnishaftigkeit, die Manifestation persönlicher Aspekte der Schülerinnen und Schüler, die physische Bewegung sowie der hohe Praxisanteil (im Sinne einer tätigen vs. kognitiven Auseinandersetzung mit der Sache) Unterrichtsmerkmale darzustellen, die vergleichsweise unabhängig (von der Einschätzung) der Lehrperson und damit per se auf den Sportunterricht zuzutreffen scheinen. Im Gegensatz dazu umfasst die Gruppe der typischen Chancen des Sportunterrichts (dem interkulturellen Lernen zuträgliche) Merkmale von Unterricht, die zwar von der Mehrheit, aber nicht mehr von allen Lehrpersonen, welche die Chancen nannten, dem Sportunterricht zugesprochen wurden. Offensichtlich finden sich darunter denn auch Aspekte von Unterricht - etwa zur Inszenierung des Unterrichts (Arbeiten in Gruppen) oder zu den Schülervoraussetzungen (Motivierte, offene SuS) -, die sich wohl tendenziell, aber nicht generell dem Sportunterricht zuschreiben lassen. Untypische Chancen des Sportunterrichts sind schliesslich Chancen, die nach Einschätzung von mindestens der Hälfte der Lehrpersonen nicht auf den Sportunterricht zutreffen. So würden im Sportunterricht aus Sicht der Mehrheit der Lehrpersonen persönliche Hintergründe der Schülerinnen und Schüler nicht einbezogen werden (können). Ebenso eher untypisch für den Sportunterricht scheinen unterschiedliche Lernwege und Ergebnisse zu sein sowie die Tatsache, dass alle Schülerinnen und Schüler (in gleichem Ausmass) sportbezogenes Vorwissen bzw. Fertigkeiten mitbringen. Schliesslich schätzte mindestens die Hälfte der befragten Lehrpersonen den Kulturgehalt sowie den Leistungs- und Stoffdruck des Fachs Sport als gering ein.

Um weitere Anhaltspunkte hinsichtlich der Interpretation dieser Ergebnisse zu erhalten, wurden zusätzlich die Zustimmungsraten der Chancen für das (neben dem Fach Sport am zweithäufigsten in die Elementesets gewählte) Fach Mathematik errechnet. Dabei zeigte sich, dass in Bezug auf den Mathematikunterricht bei insgesamt sieben der 17 Chancen Zustimmungsraten von $0 \%$ und bei nur gerade zwei Chancen solche von $100 \%$ vorliegen. Der Vergleich des sportunterrichtsspezifischen Chancen-Profils mit dem Gesamtrepertoire der genannten bzw. auf andere Fächer angewandten Chancen macht ausserdem deutlich, dass der Sportunterricht aus Sicht der Lehrpersonen vergleichsweise untypische bzw. eher fachspezifische Chancen für interkulturelles Lernen aufweist.

Damit lässt sich Fragestellung 2 folgendermassen beantworten: Sportunterricht stellt aus Sicht der Lehrpersonen einen Lehr-Lern-Kontext dar, der (im Vergleich zu anderen Fächern) nicht per se Hindernisse in Bezug auf interkulturelles Lernen - verstanden als Zuwachs an gegenseitigem Verständnis und gegenseitiger Akzeptanz - aufweist. Neben Chancen im Bereich von Fachinhalten und -themen, im 
Bereich der Unterrichtsinszenierung sowie hinsichtlich Schülervoraussetzungen sehen die Lehrpersonen das Potenzial des Sportunterrichts für interkulturelles Lernen primär in der geringen Sprachgebundenheit, in der Emotionalität und Erlebnishaftigkeit, in der physischen Bewegung, im hohen Praxisanteil sowie in der Tatsache, dass im Sportunterricht persönliche Hintergründe und Haltungen der Schülerinnen und Schüler zum Ausdruck kommen. Dabei weisen die Ergebnisse auf ein aus Sicht der Lehrpersonen vergleichsweise fachspezifisches Potenzial des Sportunterrichts für interkulturelles Lernen hin.

Insgesamt stützen die Resultate den Befund der Interviewstudie von Marburger et al. (1997), wonach Lehrpersonen (u. a.) das Fach Sport als potenziell eignendes Fach für die Berücksichtigung von Minderheitenkulturen nannten. Auch korrespondiert es mit den Ergebnissen von Grimminger (2012), die auf Basis einer Fragebogenstudie mit Sportlehrpersonen vergleichsweise niedrige Zustimmungswerte für die Vorstellung, Sportunterricht habe einen rein sportmotorischen Auftrag und könne nichts zu interkulturellem Lernen beitragen, eruierte.

\subsection{Methodenreflexion}

Die Erfassung von Lehrerüberzeugungen scheint grundsätzlich an keine verbindliche Methodik gebunden zu sein. Lehrerüberzeugungen im Zusammenhang mit migrationsbedingter Heterogenität wurden bislang sowohl quantitativ (primär mittels paper-pencil-Fragebogen) als auch qualitativ (primär mittels problemzentrierter Interviews) erfasst. Da bislang keine Instrumente vorliegen, die sich zur Erfassung der hier interessierenden (fachspezifischen) Lehrerüberzeugungen eignen (vgl. Kap. 5.1.1), wurden zwei eigens für die vorliegende Untersuchung entwickelte Instrumente verwendet.

Übergeordnetes, methodologisches Ziel dieser Untersuchung war dabei eine möglichst adäquate Rekonstruktion der interessierenden Überzeugungen der Lehrpersonen. Vor diesem Hintergrund wurde mit der Wahl der RGT auf ein Erhebungsverfahren gesetzt, das insbesondere dem Anspruch der Erfassung teilweise implizit repräsentierter Wissensformen bzw. Überzeugungen gerecht wird. Darüber hinaus, so die Überlegung, sollte die RGT in der Anwendungsform eines strukturierten Interviews sowohl die für die vorliegende Untersuchung notwendige explorative Orientierung als auch die Vergleichbarkeit der von mehreren Personen erhobenen Daten gewährleisten (vgl. Kap. 5.1.1).

Um Aussagen über die Adäquanz der Rekonstruktion der Überzeugungen und damit auch über die diesbezügliche Eignung der RGT machen zu können, ist insbesondere zu prüfen, ob die zum Untersuchungsthema getroffenen Aussagen als ausreichend gültig und die Art und Weise der Erfassung als ausreichend verlässlich sowie unabhängig von der forschenden Person betrachtet werden können. So 
gesehen, hebt sich diese Untersuchung bezüglich des Qualitätsanspruchs kaum von anderen empirischen Untersuchungen ab, die sich an den klassischen Gütekriterien wissenschaftlicher Forschung (Objektivität, Reliabilität, Validität) orientieren. Allerdings wird die Anwendung traditioneller Reliabilitätsund Validitätskonzeptionen zur Bewertung der wissenschaftlichen Güte einer Repertory Grid-Untersuchung aufgrund der „methodologischen Sonderstellung" der Methode als eher ungeeignet betrachtet (vgl. Fromm, 1995, S. 7; Riemann, 1991). Vor diesem Hintergrund und ganz im Sinne von Flick (2010), der im Zusammenhang mit der Problematik der Übertragung klassischer Gütekriterien auf qualitative Forschung für die „Formulierung alternativer, methodenangemessener Kriterien“ (S. 400) plädiert, formulieren Fromm (1995) sowie Riemann (1991) in Anlehnung an Kelly (1991) Kriterien, wonach Repertory Grid-Studien (in methodenangemessener Weise) geprüft werden können. Diese sollen im Folgenden zur Reflexion der Datenerfassung, -auswertung und -interpretation herangezogen werden.

Mit Blick auf die Datenerfassung steht insbesondere die Prüfung der Konstruktion und Anwendung des Instruments und damit gewissermassen die Inhaltsvalidität im Vordergrund. Diese lässt sich einerseits anhand der Auswahl der Elemente und der Vergleiche der Elemente, andererseits über die Angemessenheit des spezifischen Erhebungsverfahrens beurteilen (vgl. Fromm, 1995, S. 203; Riemann, 1991, S. 58f.).

In Bezug auf die Auswahl und Vergleiche der Elemente ist - in Analogie zur Repräsentativität der Auswahl von Testitems - zu prüfen, ob einerseits die gewählten Elemente repräsentativ für das untersuchte Thema (hier: Schülerinnen und Schüler mit Migrationshintergrund im Sportunterricht sowie Interkulturelles Lernen im Sportunterricht), andererseits die zur Erhebung von Konstrukten schliesslich herangezogenen Elemente repräsentativ in Bezug auf die spezifische Fragestellung sind.

Die Repräsentativität einer Auswahl von Elementen lässt sich dann ermessen, wenn alle möglichen Elemente eines Themas benannt werden können (vgl. Riemann, 1991, S. 58). Da dies im Fall von Schülerinnen und Schülern mit Migrationshintergrund im Sportunterricht - unter der Prämisse, dass jede/r Schüler/in mit Migrationshintergrund im Sportunterricht individuell ist - nicht möglich war, wurde in pragmatischer Absicht als Grundgesamtheit potenzieller Elemente eine von den Befragten aktuell unterrichtete, migrationsbedingt heterogene Klasse und hiervon wiederum eine zufällig gezogene und damit relativ repräsentative Stichprobe von 10 Schülerinnen und Schülern gewählt (vgl. Kap. 5.3.1; Kap. 5.4.1). Im Zusammenhang mit der Erfassung von Konstrukten zum interkulturellen Lernen liessen sich hingegen alle möglichen Elemente in Form der 14 im Kanton Bern auf Stufe SI unterrichteten Schulfächer benennen. Hiervon wurden wiederum all diejenigen Schulfächer als besonders repräsentativ bzw. angemessen im Hinblick auf die Erfassung valider Konstrukte betrachtet, von denen sichergestellt werden konnte, dass sich die Lehrpersonen in der Lage fühlen würden, Aussagen hinsichtlich deren Potenzials für interkulturelles Lernen treffen zu können. Waren dies (inklusive des Fachs Sport) 
mehr als sechs Schulfächer, so wurde das Elementeset aus forschungsökonomischen Gründen weiter auf die nach Angabe der Lehrpersonen bezüglich des interkulturellen Lernpotenzials am weitesten auseinanderliegenden Fächer beschränkt (vgl. Kap. 5.3.2; Kap. 5.4.1). Die Repräsentativität der Elemente hinsichtlich der untersuchten Themen darf somit als zufriedenstellend beurteilt werden.

In Bezug auf die Repräsentativität der zur Konstruktevokation herangezogenen Elementvergleiche existiert zwar kein absolutes Kriterium, jedoch besteht zumindest der Anspruch, etwaige im Zusammenhang mit der Fragestellung spezifische Priorisierungen von Elementkombinationen zu begründen (vgl. Riemann, 1991, S. 58). Da mit beiden Instrumenten insbesondere die Absicht verfolgt wurde, bestmöglich gesättigte Konstruktsysteme zu den beiden Themen generell zu erfassen, wurde keiner Elementkombination eine besondere Bedeutung beigemessen. Vielmehr wurde darauf geachtet, dass alle Elemente gleichmässig in den - aus forschungsökonomischen Gründen auf 10 bzw. acht beschränkten - vorgelegten Triaden vorkamen und etwaige mittels Triadenmethode noch nicht erfasste Konstrukte über die full context form erfasst werden konnten.

Zur Prüfung der Angemessenheit des spezifischen Erhebungsverfahrens schlägt Riemann (ebd.) die Prüfung der Zugänglichkeit der Aufgaben für die Befragten, des Gültigkeitsbereichs der Konstrukte sowie der Gefahr der Erfassung von Artefakten vor. Vor dem Hintergrund der Theorie der persönlichen Konstrukte, nach der Konstrukte häufig präverbal vorliegen und deshalb zunächst schwierig zu formulieren sind, fordert Fromm (1995, S. 203f.) ergänzend dazu ein Vorgehen, das einer eiligen Abarbeitung der vorgelegten Unterscheidungsaufgaben keinen Vorschub leistet. Angemessen bzw. inhaltsvalide ist ein Erhebungsverfahren demnach dann, wenn der vorgesehene Vergleich der Elemente oder weitere damit zusammenhängende Aufgaben die befragten Personen nicht überfordern, den Probanden im Rahmen des ratings die Gelegenheit gegeben wird, nicht einschätzbare Elemente auszulassen, wenn die interviewende Person nicht auf der Bearbeitung von durch Befragte als nicht sinnvoll erachtete Vergleiche oder Aufgaben beharrt und wenn den Befragten allgemein genügend Zeit zum Reflektieren und Formulieren eingeräumt wird.

Zur Gewährleistung der Zugänglichkeit der Aufgaben für die Befragten wurden im Hinblick auf die Optimierung der Instrumente Probeinterviews und im Rahmen der Briefings der Befragten (vgl. Kap. 5.4.1) die einzelnen Schritte des Datenerfassungsverfahrens anhand eines themenunabhängigen Beispiels eingeübt. Dennoch, so wurde mehrfach darauf hingewiesen (vgl. Kap. 5.4.2; Kap. 6.2), konnte die Variante des im Rahmen der Erfassung von Chancen und Hindernissen für interkulturelles Lernen vorgesehenen laddering (vgl. Kap. 5.1.2; Kap. 5.3.2) aufgrund von Überforderung nicht bei allen Lehrpersonen konsequent angewandt werden. Um Artefakte bestmöglich zu vermeiden - und auch die positive Befindlichkeit der Befragten aufrechtzuerhalten -, wurde hier ad hoc entschieden, entweder gänzlich oder zumindest teilweise auf die Erfassung übergeordneter Konstrukte zu verzichten. Dies war insbesondere auch deshalb möglich, weil die übergeordneten Konstrukte nicht den Kern der Daten zur 
Beantwortung der Fragestellung 2 ausmachten, sondern vielmehr ergänzend dazu erfasst wurden (vgl. Exkurs Kap. 6.2.1). Um in einer zukünftigen Untersuchung übergeordnete Konstrukte systematischer erfassen zu können, müssten die Befragten noch intensiver darauf vorbereitet bzw. darüber informiert werden, mit welcher Absicht die befragende Person dermassen penetrant die dem laddering zugrundeliegende „Warum-Frage“ stellt.

Zur Vermeidung von Gefälligkeitseinschätzungen von Elementen wurde den Befragten in beiden Instrumenten die Option eingeräumt, bei Nicht-Beurteilbarkeit einer Schülerin/ eines Schülers bzw. eines Faches hinsichtlich eines bestimmten Konstrukts dies mit einem Strich (-) deutlich zu machen (vgl. Kap. 5.3.1; Kap. 5.3.2). Wie sich zeigte, machten sich diese Option nur zwei Lehrpersonen im Rahmen der Beurteilung je einer Schülerin zunutze, die sie nach eigener Aussage noch zu wenig gut kannten und deshalb in Bezug auf fünf bzw. sechs Konstrukte nicht einzuschätzen vermochten. Diese geringe Zahl mag aufgrund der Tatsache, dass eigene und seit mindestens 3/4 Jahren unterrichtete Schülerinnen und Schüler zu beurteilen waren - so die Vorgabe (vgl. Kap. 5.4.1) -, realistisch sein. Erstaunlich ist hingegen, dass im Rahmen der Beurteilung der Fächer keines der erfassten Konstrukte als nicht anwendbar auf ein Fach empfunden wurde. Insbesondere mit Blick auf Konstrukte geringeren Abstraktionsniveaus ist anzunehmen, dass diese von den Befragten implizit in Bezug auf andere Fächer übersetzt wurden oder aber anstelle der Angabe der Nicht-Beurteilbarkeit der mittlere Ratingwert „3“ angegeben wurde. Um die Validität der ratings zu erhöhen, müsste perspektivisch darauf geachtet werden, das Abstraktionsniveau und damit einhergehend die Formulierung der Konstrukte so anzupassen, dass sie potenziell auf alle Elemente anwendbar sind. Damit würde gleichzeitig gewährleistet, dass die Angabe eines mittleren Ratingwerts tatsächlich einer inhaltlichen Angabe entspricht.

Um schliesslich einer eiligen Abarbeitung der Unterscheidungsaufgaben entgegenzuwirken und damit nicht nur das zu erfassen, was leicht formulierbar ist, wurde einerseits darauf geachtet, dass die beiden pro Lehrperson durchgeführten Interviews an zwei unterschiedlichen Terminen stattfanden und andererseits eine Gesprächssituation etabliert werden konnte, in der etwaige Schwierigkeiten in Zusammenhang mit der Formulierung von Konstrukten als „üblich“ und nicht als „mangelhafte Kompetenz" interpretiert würden. Mit der Begründung, dass derartige Interviews nicht nur sehr zeitintensiv, sondern von den Befragten auch als fordernd und anstrengend erlebt würden, konnten denn auch bis auf eine Lehrperson alle Befragten von zwei Interviewterminen überzeugt werden. Unsicherheiten im Zusammenhang mit der Konstruktformulierung liessen sich zudem einerseits durch vor den Interviews durchgeführte, ausführliche Briefings (zum Verlauf, der Gesprächsführung und Prozess der Konstrukterfassung), andererseits durch die während den Interviews wiederholten Aufforderungen, laut zu denken und sich Zeit zu nehmen, abschwächen bzw. verhindern (vgl. Kap. 5.4). 
Abgesehen von den Herausforderungen im Zusammenhang mit der Konstruktion und Anwendung eines Repertory Grid-Instruments bringt die RGT als Methode zur Erfassung subjektiver Sichtweisen weiter die Schwierigkeit mit sich, dass subjektiv möglichst bedeutungsvolle und relevante Daten in Form individueller (und in kurzer Form formulierter) Bezeichnungen erfasst werden sollen, diese jedoch in aller Regel der Übersetzung in die Terminologie der Interpreten bedürfen. Für die Gesprächsführung bedeutet dies, dass einerseits dem/der Interviewten die Möglichkeit gegeben werden sollte, „seine [ihre] Sicht der Dinge möglichst unbeeinträchtigt zu artikulieren“, andererseits aber auch „Verständnisund Übersetzungshilfen“ für den/die Interviewende/n notwendig sind, „,die es ihm erlauben, das vom Befragten Ausgedrückte im Rahmen seines eigenen Konstruktsystems zu rekonstruieren“ (Fromm, 1995, S. 55; vgl. auch Kap. 5.4.2). Dies hat zur Folge, dass mit jedem Versuch, die Bedeutung eines Konstrukts für den/die Befragende/n klar zu machen - etwa indem stellvertretende Formulierungen bzw. begriffliche Erweiterungen des von der interviewten Person Gesagten eingebracht werden - die inhaltliche Einflussnahme seitens des/der Interviewers/in zunimmt und damit die Qualität des Verfahrens (hinsichtlich des Anspruchs freier Artikulation seitens der Befragten) abnimmt. Wie in Kapitel 5.4.2 ausführlich erläutert wurde, ist vor allem im Zusammenhang mit der Erfassung von Chancen und Hindernissen für interkulturelles Lernen von solchen „Qualitätseinbussen“ auszugehen. Da es jedoch insbesondere darum ging, die subjektive Sicht der Lehrpersonen zu verstehen und kennenzulernen, erschien hier eine primäre Orientierung an einer möglichst objektiven Erfassung nicht angemessen (vgl. auch Fromm, 1995, S. 206).

Mit Blick auf die Datenauswertung und -interpretation finden sich in der Literatur zur RGT keine methodeneigenen Gütekriterien. Ob und inwieweit herkömmliche oder andere Gütekriterien sinnvoll sind, soll vielmehr „vom jeweiligen Untersuchungszweck und den verwendeten Varianten der GridMethodik abhängig gemacht werden“ (Fromm, 1995, S. 205f.).

In dieser Untersuchung getroffene empirische Aussagen basieren auf Ergebnissen von Analyseverfahren, in denen entweder nur die numerischen (Teilfragestellung 1a), nur die verbalen (Teilfragestellung 2a) oder auch beide (Teilfragestellungen $1 \mathrm{~b}$ und $2 \mathrm{~b}$ ) in den Rohgrids erfassten Datenformen als Datenbasis berücksichtigt wurden. Die Frage nach der wissenschaftlichen Güte der Analysen stellt sich dabei insbesondere dort, wo Rohdaten zugunsten der Vergleichbarkeit transformiert bzw. rationalisiert wurden. Konkret betrifft dies einerseits die zur Beantwortung der Teilfragestellungen $1 \mathrm{~b}$ und 2a durchgeführten qualitativen Inhaltsanalysen der Konstrukte, andererseits die im Zusammenhang mit den AnaIysen zu den Teilfragestellungen $1 \mathrm{~b}$ und $2 \mathrm{~b}$ vorgenommenen Dichotomisierungen der Konstrukte bzw. der Ratingskalen.

Um sicherzustellen, dass die aus den Inhaltsanalysen resultierenden Ergebnisse möglichst unabhängig vom Forschenden bzw. objektiv zustande kamen, wurde in Anlehnung an Gramespacher et al. (2009) 
eine Deutungsabsicherung in Form einer argumentativen bzw. konsensuellen Validierung mit vier weiteren Forschenden vorgenommen. Darüber hinaus und zur Erhöhung der intersubjektiven Nachvollziehbarkeit wurden klare Regeln der Konstruktanalyse definiert (vgl. Kap. 5.5).

Die Dichotomisierung der Ratingskalen bzw. das Zusammenführen einzelner Ratingwerte zu einem Ratingwert ist unweigerlich mit einem Verlust an Differenziertheit der Aussagen, welche sich aufgrund der ratings über die einzelnen Elemente machen lassen, verbunden. In der vorliegenden Arbeit wurde der Ratingwert „2“ (= trifft eher zu) analog zum Ratingwert „1“ (= trifft zu) interpretiert. Da mit diesem Entscheid die zentralen empirischen Verhältnisse nicht verändert wurden, darf dieses Vorgehen zwecks einfacherer interindividueller Vergleichbarkeit der Daten - als zulässig betrachtet werden (vgl. Hussy, Schreier \& Echterhoff, 2013).

Zusammenfassend lässt sich festhalten, dass sich das sehr aufwändige Verfahren der RGT angesichts bislang fehlender standardisierter Instrumente zur Erfassung schüler- und fachbezogener Überzeugungen angesichts migrationsbedingter Heterogenität bewährt hat. Wie in den vorangegangenen Ausführungen dargelegt wurde, kann sowohl mit Blick auf die Datenerfassung als auch -auswertung von einer zufriedenstellenden Güte der Untersuchung ausgegangen werden.

\subsection{Fazit und Ausblick}

Die vorliegende Arbeit hat ihren Ausgang beim Problem einer Diskrepanz zwischen bildungspolitischen Forderungen und dem professionellen Handeln von (Sport unterrichtenden) Lehrpersonen angesichts migrationsbedingter Heterogenität genommen. Ausgehend von der Annahme, dass das Unterrichtshandeln von (Sport-)Lehrpersonen in migrationsbedingt heterogenen Klassenverhältnissen zu grossen Teilen durch deren subjektive Überzeugungen bestimmt ist, bestand das Erkenntnisinteresse in der Eruierung von Überzeugungen Sport unterrichtender Lehrpersonen angesichts migrationsbedingter Heterogenität. Nach einer theoretischen Auseinandersetzung mit dem Konstrukt der Lehrerüberzeugungen sowie einer Aufarbeitung des empirischen Forschungsstandes zu Lehrerüberzeugungen angesichts migrationsbedingter Heterogenität wurde spezifisch den Fragen nach der lehrerseitigen Wahrnehmung von Schülerinnen und Schülern mit Migrationshintergrund im Sportunterricht sowie nach den Lehrervorstellungen zum Potenzial des Sportunterrichts für interkulturelles Lernen nachgegangen. Ziel war es, (erstes) Orientierungswissen zu generieren, das u. a. für die (Konzeption der) Aus- und Weiterbildung Sport unterrichtender Lehrpersonen hinsichtlich des Umgangs mit migrationsbedingter Heterogenität genutzt werden kann. Darüber hinaus bestand die Absicht der vorliegenden empirischen Untersuchung darin, einen - zumindest in Bezug auf den Lehr-Lern-Kontext des Sportunterrichts 
- noch wenig strukturierten Gegenstandsbereich möglichst methodenangemessen zu erkunden, Impulse für die hinsichtlich Interkulturalität noch sehr überschaubare, sportdidaktische Theoriebildung zu liefern und die Thematik damit letztlich auch für hypothesengeleitete Untersuchungen zugänglich zu machen.

Mit Blick auf die schülerbezogenen Überzeugungen Sport unterrichtender Lehrpersonen lässt sich aufgrund der Ergebnisse festhalten, dass Schülerinnen und Schüler mit Migrationshintergrund im Sportunterricht weniger den Idealvorstellungen der Lehrpersonen entsprechen als solche ohne Migrationshintergrund. Damit bieten die Ergebnisse zunächst eine mögliche Erklärung für die - als wesentlicher Ausgangspunkt dieser Arbeit beschriebenen - Schwierigkeiten von (Sport unterrichtenden) Lehrpersonen im Umgang mit migrationsbedingter Heterogenität: Je mehr Schülerinnen und Schüler mit Migrationshintergrund im Sportunterricht, desto höher der Anteil an Schülerinnen und Schülern, die (in der Wahrnehmung der Lehrpersonen) ein (sportlich) weniger hohes Leistungsniveau haben, die weniger motiviert und engagiert sind und die sich im Umgang mit anderen weniger angemessen verhalten. Diese vergleichsweise schlechteren Lernvoraussetzungen von Schülerinnen und Schülern mit Migrationshintergrund, so eine mögliche davon abzuleitende Hypothese, erschweren das Unterrichten für (Sport unterrichtende) Lehrpersonen.

Da gemäss den weiteren Ergebnissen Sport unterrichtende Lehrpersonen Schülerinnen und Schüler mit Migrationshintergrund in allen von ihnen zur Beurteilung herangezogenen, zentralen Dimensionen schlechter einschätzen als Schülerinnen und Schüler ohne Migrationshintergrund, sind ausserdem kategorisierende Wahrnehmungsprozesse entlang des askriptiven Schülermerkmals „Migrationshintergrund“ zu vermuten. Die daran anknüpfende, weiterführende Hypothese, dass verzerrte Leistungserwartungen seitens Lehrpersonen zuungunsten von Schülerinnen und Schülern mit Migrationshintergrund vorliegen, lässt sich aufgrund der erfassten Daten nicht eindeutig ableiten. Wenngleich aus anderen Studien (ausserhalb der Sportlehrerforschung) diesbezügliche datenbasierte Erkenntnisse vorliegen, lassen sich diese auch nur beschränkt auf den Lehr-Lern-Kontext des Sportunterrichts übertragen. Die Untersuchung schülerbezogener Überzeugungen unter Berücksichtigung von deren Akkuratesse stellt deshalb - und insbesondere auch vor dem Hintergrund der bildungspolitischen Forderung nach einem chancengerechten Unterricht - ein Forschungsdesiderat innerhalb der Sportlehrerforschung dar. Wünschenswert und womöglich aufschlussreich wären in diesem Zusammenhang zudem Untersuchungen mit Fokus auf potenziellen herkunfts- bzw. ethniespezifischen Unterschieden.

Bezüglich der fachbezogenen Lehrerüberzeugungen machen die Ergebnisse deutlich, dass Sport unterrichtende Lehrpersonen die geringe Sprachgebundenheit, die Emotionalität und Erlebnishaftigkeit, die physische Bewegung und den hohen Praxisanteil sowie das (daraus resultierende) zum Ausdruckkom- 
men persönlicher Hintergründe und Haltungen der Schülerinnen und Schüler als fachimmanente Chancen für interkulturelles Lernen im Sportunterricht sehen. Weitere, typische und dem interkulturellen Lernen zuträgliche Merkmale des Sportunterrichts sind aus Sicht der Lehrpersonen die Kulturneutralität der Fachinhalte, das Arbeiten in Gruppen, das Austauschen/Diskutieren/Kommunizieren, motivierte und offene Schülerinnen und Schüler, der Alltags- und Lebensbezug sowie die Interdisziplinarität des Faches. Fächervergleichende Analysen weisen ausserdem darauf hin, dass dem Sportunterricht seitens der ihn erteilenden Lehrpersonen insgesamt ein hohes Potenzial für interkulturelles Lernen zugesprochen wird. Schliesslich machen die Resultate deutlich, dass interkulturelles Lernen im Sportunterricht aus Sicht der Lehrpersonen aufgrund unterrichtlicher Bedingungen zustande kommt bzw. kommen kann, die für die Mehrheit anderer Schulfächer vergleichsweise untypisch sind.

Insgesamt finden sich in den Vorstellungen und Einschätzungen der Lehrpersonen offenkundige Verbindungen zu verschiedenen sportpädagogischen und -didaktischen Konzepten und Theorien interkulturellen Lernens im Sportunterricht (vgl. im Überblick Giess-Stüber \& Grimminger, 2008). Perspektivisch bzw. ergänzend zu den vorliegenden Befunden liessen sich deshalb (z.B. durch eine fallorientierter Sekundäranalyse der erfassten Lehrervorstellungen) auch Aussagen über Tendenzen der Verteilung der Lehrvorstellungen auf verschiedene Positionen und Konzepte interkulturellen Lernens im Sportunterricht machen.

Vor dem Hintergrund der in der Literatur beschriebenen Annahmen und empirischen Evidenzen über die Herausbildung und Wirkung von Überzeugungen (im Zusammenhang mit migrationsbedingter Heterogenität) (vgl. Kap. 2; Kap. 3) sowie des in dieser Untersuchung erarbeiteten Wissens lassen sich abschliessend folgende erste Überlegungen hinsichtlich (der Konzeption) einer auf einen angemessenen Umgang mit migrationsbedingter Heterogenität ausgerichteten Aus- und Weiterbildung Sport unterrichtender Lehrpersonen skizzieren:

Nicht nur aufgrund der - speziell im Kontext migrationsbedingt heterogener Klassenverhältnisse stark wahrnehmungs- und handlungssteuernden Wirkung von Lehrerüberzeugungen, sondern insbesondere auch zur wirksamen Sensibilisierung für mögliche Schwierigkeiten im Umgang mit migrationsbedingter Heterogenität erscheint es sinnvoll, dass Aus- und Weiterbildungskonzeptionen mit Fokus auf Migration und Interkulturalität ihr Hauptaugenmerk primär auf die Reflexion der Überzeugungen bzw. Selbstverständnisse der (angehenden) Lehrpersonen legen.

Da Lehrerüberzeugungen für ihre Trägerinnen und Träger zu hohen Anteilen nur beschränkt bewusstseinsfähig sind, bedarf es hierfür in einem ersten Schritt geeigneter Instrumente, welche der Evokation implizit vorliegender mentaler Strukturen dienlich sind. Als erfolgreich erwiesen sich zu diesem Zweck bislang Formate mit Rückgriff auf die Conceptual-Change-Literatur (z.B. Möller, Hardy, Jonen, Kleickmann \& Blumberg, 2006) oder auch das fachspezifisch-pädagogische Coaching (vgl. Staub, 2001; 
West \& Staub, 2003) (vgl. auch Kunter \& PohImann, 2015; Reusser \& Pauli, 2014). Ausserdem stützen die Erfahrungen und Ergebnisse der vorliegenden Studie die noch - zumindest innerhalb der Lehrer(bildungs)forschung - wenig vorliegenden Evidenzen (z.B. Bruder, Lengnink \& Prediger, 2003; Fischler, 2000a, 2000b), wonach auch die RGT als ein äusserst erfolgversprechendes Instrument zur Bewusstmachung von Überzeugungen bzw. Denkstrukturen (angehender) Lehrpersonen verwendet werden kann.

Sind die in den persönlichen Denk- und Handlungsroutinen verankerten Überzeugungen erst einmal explizit, sollten die (angehenden) Sport unterrichtenden Lehrpersonen in einem zweiten Schritt in einer kritischen Auseinandersetzung mit den eigenen Annahmen und Vorstellungen unterstützt werden. Hierzu erscheinen insbesondere fachwissenschaftlich-fachdidaktische Inputs - etwa zu Akkulturationseinstellungen, zur impliziten Kategorisierung und Stereotypisierung von Schülerinnen und Schülern im Sportunterricht, zu kulturell geprägten Normalitäts- bzw. Idealvorstellungen oder zu Chancen interkulturellen Lernens im Sportunterricht - von zentraler Bedeutung. So soll die Vermittlung wissenschaftlich fundierten Wissens den (angehenden) Lehrpersonen eine potenzielle Defizitdiagnose hinsichtlich ihrer eigenen Überzeugungen ermöglichen und sie folglich motivieren, ihre Denk- und Handlungsroutinen zu verändern.

Wenngleich die damit erzeugte Betroffenheit eine wichtige Gelingensbedingung zur Veränderung von Überzeugungen darstellt, so dürfte der dritte Schritt, also eine gegebenenfalls notwendige Justierung subjektiver Überzeugungen hin zu sachlich angemesseneren bzw. professionellen Überzeugungen, nicht zuletzt auch aufgrund der generellen Veränderungsresistenz von Lehrerüberzeugungen - eine der Hauptherausforderungen einer auf Lehrerüberzeugungen ausgerichteten Aus- oder Weiterbildungsmassnahme sein. Zwar existieren empirisch fundierte Hinweise, dass (zeit-)aufwändige, an konstruktivistischen Prinzipien orientierte und tutoriell unterstützte Aus- und Weiterbildungsmodelle Veränderungen in auf das Lehren und Lernen von naturwissenschaftlichen Fächern bezogenen Vorstellungen der Lehrpersonen bewirken können (vgl. Möller et al., 2006), jedoch lassen sich damit keine belastbaren Aussagen darüber machen, ob sich auf diese Weise auch angesichts migrationsbedingter Heterogenität bestehende Überzeugungen von (angehenden) Sport unterrichtenden Lehrpersonen umbzw. neustrukturieren lassen. Um im Rahmen der Sportlehrerbildung schüler- und fachbezogene Überzeugungen im Zusammenhang mit migrationsbedingter Heterogenität im Sportunterricht nachhaltig weiterentwickeln - und damit die diagnostische und interkulturelle Kompetenz (angehender) Sport unterrichtender Lehrpersonen fördern zu können -, bedarf es somit weiterführender, spezifisch darauf ausgerichteter Forschungsarbeiten. 


\section{LITERATUR}

Abelson, R. P. (1979). Differences Between Belief and Knowledge Systems. Cognitive Science, 3(4), 355366. doi: 10.1207/s15516709cog0304_4

Acquah, E. O., \& Commins, N. L. (2013). Pre-service teachers' beliefs and knowledge about multiculturalism. European Journal of Teacher Education, 1-19. doi: 10.1080/02619768.2013.787593

Aguirre, J., \& Speer, N. M. (2000). Examining the Relationship Between Beliefs and Goals in Teacher Practice. Journal of Mathematical Behaviour, 18(3), 327-356.

Alexander, P. A., Schallert, D. L., \& Hare, V. C. (1991). How Researchers in Learning and Literacy Talk about Knowledge. Review of educational Research, 61(3), 315-343.

Allemann-Ghionda, C. (2006). Standards und Kompetenzen in der Lehrerinnen- und Lehrerbildung unter besonderer Berücksichtigung der Multikulturalität. In A. Tanner, H. Badertscher, R. Holzer, A. Schindler \& U. Streckeisen (Hrsg.), Heterogenität und Integration. Umgang mit Ungleichheit und Differenz in Schule und Kindergarten (S. 78-90). Zürich: Seismo Verlag.

Allraum, J., Weissbaum, M., \& Wassmer, C. (2017). Bildungsstatistik Kanton Bern: Basisdaten 2016. Bern: Erziehungsdirektion des Kantons Bern.

Argyris, C., \& Schön, D. (1974). Theory in practice: Increasing professional effectiveness. San Francisco: Jossey-Bass.

Artelt, C., Baumert, J., Klieme, E., Neubrand, M., Prenzel, M., Schiefele, U., ... Weiss, M. (Hrsg.). (2001). PISA 2000. Zusammenfassung zentraler Befunde. Berlin: Max-Planck-Institut für Bildungsforschung.

Ashton, P. T. (2015). Historical Overview and Theoretical Perspectives of Research on Teachers' Beliefs. In H. Fives \& M. G. Gill (Hrsg.), International Handbook of Research on Teachers' Beliefs (S. 3147). New York: Routledge.

Auernheimer, G., Van Dick, R., Petzel, T., \& Wagner, U. (Hrsg.). (2001). Interkulturalität im Arbeitsfeld Schule. Empirische Untersuchungen über Lehrer und Schüler. Opladen: Leske + Budrich.

Auernheimer, G., von Blumenthal, V., Stübig, H., \& Willmann, B. (1996). Interkulturelle Erziehung im Schulalltag. Fallstudien zum Umgang von Schulen mit der multikulturellen Situation. Münster: Waxmann.

Banks, J., \& McGee Banks, C. (Hrsg.). (2004). Handbook of research on multicultural education. San Francisco: John Wiley \& Sons.

Barker, D., Barker-Ruchti, N., Sattler, S., Gerber, M., \& Pühse, U. (2011). Understanding youths with migration backgrounds and their relations to physical education. Sportunterricht, 60(8), 239242.

Baumert, J., \& Kunter, M. (2006). Stichwort: Professionelle Kompetenz von Lehrkräften. Zeitschrift für Erziehungswissenschaft, 9(4), 469-520. 
Baumert, J., \& Kunter, M. (2011). Das Kompetenzmodell von COACTIV. In M. Kunter, J. Baumert, W. Blum, U. Klusmann, S. Krauss \& M. Neubrand (Hrsg.), Professionelle Kompetenz von Lehrkräften. Ergebnisse des Forschungsprogramms COACTIV (S. 29-53). Münster: Waxmann.

Baumert, J., \& Kunter, M. (2013). Professionelle Kompetenz von Lehrkräften. In I. Gogolin, H. Kuper, H.-H. Krüger \& J. Baumert (Hrsg.), Stichwort: Zeitschrift für Erziehungswissenschaft (S. 277-337). Wiesbaden: Springer Fachmedien.

Bell, R. (2011). Personal constructs. In L. Cohen, L. Manion \& K. Morrison (Hrsg.), Research Methods in Education (7. Aufl., S. 496-509). London: Routledge.

Bender-Szymanski, D. (2001). Kulturkonflikt als Chance für Entwicklung? In G. Auernheimer, R. van Dick, T. Petzel \& U. Wagner (Hrsg.), Interkulturalität im Arbeitsfeld Schule. Empirische Untersuchungen über Lehrer und Schüler (S. 63-97). Opladen: Leske + Budrich.

Bender-Szymanski, D. (2013). Interkulturelle Kompetenz bei Lehrerinnen und Lehrern aus der Sicht der empirischen Bildungsforschung. In G. Auernheimer (Hrsg.), Interkulturelle Kompetenz und pädagogische Professionalität (2. Aufl., S. 201-227). Wiesbaden: VS Verlag.

Berry, J. W., Kim, U., Power, S., Young, M., \& Bujaki, M. (1989). Acculturation Attitudes in Plural Societies. Applied Psychology, 38(2), 185-206. doi: 10.1111/j.1464-0597.1989.tb01208.x

Binder, S., \& Daryabegi, A. (2002). Interkulturelles Lernen - Bespiele aus der Praxis. In T. Fillitz (Hrsg.), Interkulturelles Lernen. Zwischen institutionellem Rahmen, schulischer Praxis und gesellschaftlichem Kommunikationsprinzip (S. 33-95). Innsbruck: Studien Verlag.

Blömeke, S. (2012). Does Greater Teacher Knowledge Lead to Student Orientation? The Relationship between Teacher Knowledge and Teacher Beliefs. In J. König (Hrsg.), Teachers' Pedagogical Beliefs. Definition and Operationalisation - Connections to Knowledge and Performance Development and Change (S. 15-35). Münster: Waxmann.

Blömeke, S., Gustafsson, H., \& Shavelson, R. (2015). Beyond Dichotomies. Competence Viewed as a Continuum. Zeitschrift für Psychologie, 223(1), 3-13.

Blömeke, S., Kaiser, G., \& Lehmann, R. (Hrsg.). (2008). Professionelle Kompetenz angehender Lehrerinnen und Lehrer. Wissen, Überzeugungen und Lerngelegenheiten deutscher Mathmatikstudierender und -referendare. Erste Ergebnisse zur Wirksamkeit der Lehrerausbildung. Münster: Waxmann.

Blömeke, S., Müller, C., Felbrich, A., \& Kaiser, G. (2008). Epistemologische Überzeugungen zur Mathematik. In S. Blömeke, G. Kaiser \& R. Lehmann (Hrsg.), Professionelle Kompetenz angehender Lehrerinnen und Lehrer. Wissen, Überzeugungen und Lerngelegenheiten deutscher Mathematikstudierender und -referendare. Erste Ergebnisse zur Wirksamkeit der Lehrerausbildung (S. 219-246). Münster: Waxmann.

Blömeke, S., Suhl, U., \& Döhrmann, M. (2012). Zusammenfügen was zusammengehört. Kompetenzprofile am Ende der Lehrerausbildung im internationalen Vergleich. Zeitschrift für Pädagogik, 58(4), 422-438. 
Boei, F., Corporaal, A. H., \& van Hunen, W. H. (1989). Describing Teacher Cognitions with the Repgrid. Some methodological reflections and research findings. In J. Lowyck \& C. M. Clark (Hrsg.), Teacher Thinking and Professional Action (S. 175-192). Leuven: Leuven University Press.

Bohnsack, R. (2013). Gruppendiskussionsverfahren und dokumentarische Methode. In B. Friebertshäuser, A. Langer \& A. Prengel (Hrsg.), Handbuch Qualitative Forschungsmethoden in der Erziehungswissenschaft (4., durchgesehende Aufl.). Weinheim: Beltz Juventa.

Bonefeld, M., Dickhäuser, O., Janke, S., Praetorius, A.-K., \& Dresel, M. (2017). Migrationsbedingte Disparitäten in der Notenvergabe nach dem Übergang auf das Gymnasium. Zeitschrift für Entwicklungspsychologie und Pädagogische Psychologie, 49(1), 11-23.

Borenstein, M., Hedges, L. V., Higgins, J. P. T., \& Rothstein, H. R. (2009). Introduction to Meta-Analysis. Chichester: John Wiley \& Sons.

Bortz, J., \& Döring, N. (2006). Forschungsmethoden und Evaluation für Human- und Sozialwissenschaftler (4. Aufl.). Heidelberg: Springer Medizin Verlag.

Bourdieu, P. (1974). Zur Soziologie der symbolischen Formen. Frankfurt a. M.: Suhrkamp.

Bourhis, R. Y., Moïse, L. C., Perreault, S., \& Senécal, S. (1997). Towards an Interactive Acculturation Model: A Social Psychological Approach. International Journal of Psychology, 32(6), 369-386.

Bromme, R. (1992). Der Lehrer als Experte. Zur Psychologie des professionellen Wissens. Bern: Hans Huber.

Bromme, R. (1997). Kompetenzen, Funktionen und unterrichtliches Handelns des Lehrers. In F. E. Weinert (Hrsg.), Psychologie des Unterrichts und der Schule (S. 177-212). Göttingen: Verlag für Psychologie.

Bromme, R. (2008). Lehrerexpertise. In W. Schneider \& M. Hasselhorn (Hrsg.), Handbuch der Pädagogischen Psychologie (S. 159-167). Göttingen: Hogrefe.

Brosius, F. (2013). SPSS 21. Heidelberg: mitp.

Bruder, R., Lengnink, K., \& Prediger, S. (2003). Wie denken Lehramtsstudierende über Mathematikaufgaben? Ein methodischer Ansatz zur Erfassung subjektiver Theorien mittels Repertory-Grid-Technik. Mathematica Didactica, 26(1), 63-85.

Bryan, L. A., \& Atwater, M. M. (2002). Teacher beliefs and cultural models: A challenge for science teacher preparation programs. Science Education, 86(6), 821-839. doi: 10.1002/sce.10043

Budde, J. (2012). Problematisierende Perspektiven auf Heterogenität als ambivalentes Thema der Schul- und Unterrichtsforschung. Zeitschrift für Pädagogik, 58(4), 522-540.

Budde, J. (2013). Einleitung. In J. Budde (Hrsg.), Unscharfe Einsätze: (Re-)Produktion von Heterogenität im schulischen Feld (S. 7-26). Wiesbaden: Springer Fachmedien.

Buehl, M., \& Beck, J. (2015). The Relationship between Teachers' Beliefs and Teachers' Practices. In H. Fives \& M. G. Gill (Hrsg.), International Handbook of Research on Teachers' Beliefs (S. 66-84). New York: Routledge.

Buholzer, A., Zulliger, S., \& Zutavern, M. (2015). Lehrerinnen- und Lehrerbildung in der Schweiz. Einblicke zum Thema Heterogenität in der Lehrpersonenausbildung. In C. Fischer, M. Veber, C. 
Fischer-Ontrup \& R. Buschmann (Hrsg.), Umgang mit Vielfalt. Aufgaben und Herausforderungen für die Lehrerinnen- und Lehrerbildung. Münster: Waxmann.

Büker, P., \& Rendtorff, B. (2015). Sichtweisen von Lehrkräften auf Ethnizität und Geschlecht. Eine Problemanzeige. Zeitschrift für Pädagogik, 61(1-117).

Bundesamt für Statistik. (2016). Obligatorische Schule. Retrieved 15. 12., 2016, from https://www.bfs.admin.ch/bfs/de/home/statistiken/bildung-wissenschaft/personenausbildung/obligatorische-schule.html

Bundesamt für Statistik. (2017a). Ausländische Bevölkerung. Retrieved 5. 1., 2017, from https://www.bfs.admin.ch/bfs/de/home/statistiken/bevoelkerung/migrationintegration/auslaendische-bevoelkerung.html

Bundesamt für Statistik. (2017b). Bevölkerung nach Migrationsstatus. Retrieved 5. 1., 2017, from https://www.bfs.admin.ch/bfs/de/home/statistiken/bevoelkerung/migrationintegration/nach-migrationsstatuts.html

Burr, V., King, N., \& Butt, T. (2014). Personal construct psychology methods for qualitative research. international Journal of Social Research Methodolgy, 17(4), 341-355.

Calderhead, J. (1996). Teachers: Beliefs and knowledge. In D. C. Berliner \& R. C. Calfee (Hrsg.), Handbook of Educational Psychology (S. 709-725). New York: Macmillan.

Carigiet Reinhard, T. (2012). Schulleistungen und Heterogenität. Eine mehrebenenanalytische Untersuchung der Bedingungsfaktoren der Schulleistungen am Ende der dritten Primarschulklasse. Bern: Haupt.

Castro, A. J. (2010). Themes in the Research on Preservice Teachers' Views of Cultural Diversity: Implications for Researching Millenial Preservice Teachers. Educational Researcher, 39(3), 198210.

Chepyator-Thomson, R., You, J., \& Russell, J. (2000). In-service physical education teachers: background and understanding of multicultural education. Journal of In-Service Education, 26(3), 557-568.

Chisholm, R. (1989). Theory of Knowledge (3. Aufl.). New Jersey: Prentice-Hall International.

Cohen, J. (1988). Statistical power analysis for the behavioral sciences (2. Aufl.). Hillsdale: Erlbaum.

Cohen, L., Manion, L., \& Morrison, K. (2013). Research Methods in Education (7. Aufl.). Oxon: Routledge.

Columna, L., Foley, J. T., \& Lytle, R. K. (2010). Physical Education Teachers' and Candidates' Attitudes Toward Cultural Pluralism. Journal of Teaching in Physical Education, 29(3), 295-311.

Coradi Vellacott, M., \& Wolter, S. C. (2005). Equity in the Swiss Education System: Dimensions, Causes and Policy Responses. National Report from Switzerland contributing to the OECD's Review of "Equity in Education": Swiss Coordination Centre for Research in Education.

Dann, H.-D. (2008). Lehrerkognitionen und Handlungsentscheidungen. In M. K. W. Schweer (Hrsg.), Lehrer-Schüler-Interaktion. Inhaltsfelder, Forschungsperspektiven und methodische Zugänge (2., vollst. überarb. Aufl., S. 177-208). Wiesbaden: VS Verlag. 
Degele, N., \& Schirmer, D. (2004). Selbstverständlich heteronormativ: zum Problem der Reifizierung in der Geschlechterforschung. In S. Buchen, C. Herlfferich \& M. S. Maier (Hrsg.), Gender methodologisch. Empirische Forschung in der Informationsgesellschaft vor neuen Herausforderungen (S. 107-122). Wiesbaden: VS Verlag.

Diehm, I., \& Kodron, C. (1990). Unterricht und Erziehung für eine multikulturelle Gesellschaft. Bericht über eine Pilotstudie zur Erstellung eines Fragebogens in vier europäischen Ländern unter besonderer Berücksichtigung der hessischen Studie. Frankfurt a. M.: Deutsches Institut für Internationale Pädagogische Forschung.

Dirks, U. (2000). Wie werden Englischlehrerlnnen professionell? Eine berufsbiographische Untersuchung in den neuen Bundes/ändern. Münster: Waxmann.

Dresel, M., Steuer, G., \& Berner, V.-D. (2010). Zum Zusammenhang von Geschlecht, kultureller Herkunft und sozialer Herkunft mit Lernen und Leistung im Kontext von Schule und Unterricht. In J. Hagedorn, V. Schurt, C. Steber \& W. Waburg (Hrsg.), Ethnitzität, Geschlecht, Familie und Schule. Heterogenität als erziehungswissenschaftliche Herausforderung (S. 333-349). Wiesbaden: VS Verlag.

Dubberke, T., Kunter, M., McElvany, N., Brunner, M., \& Baumert, J. (2008). Lerntheoretische Überzeugungen von Mathematiklehrkräften. Einflüsse auf die Unterrichtsgestaltung und den Lernerfolg von Schülerinnen und Schülern. Zeitschrift für Pädagogische Psychologie, 22(3-4), 193-206.

Edelmann, D. (2006). Pädagogische Professionalität im transnationalen sozialen Raum. Eine Studie über Sichtweisen und Erfahrungen von Primarlehrpersonen in Bezug auf die kulturelle Heterogenität ihrer Schulklassen. In C. Allemann-Ghionda \& E. Terhart (Hrsg.), Kompetenzen und Kompetenzentwicklung von Lehrerinnen und Lehrern (S. 235-249). Weinheim: Beltz.

Edelmann, D. (2008). Pädagogische Professionalität im transnationalen sozialen Raum. Eine qualitative Untersuchung über den Umgang von Lehrpersonen mit der migrationsbedingten Heterogenität ihrer Klassen (2. Aufl.). Zürich: LIT Verlag.

Epting, F. R., Suchman, D., \& Nickerson, K. J. (1971). An evaluation of elicitation procedures for personal constructs. British Journal of Psychology, 62, 513-517.

Erziehungsdirektion des Kantons Bern (ERZ) (1995). Lehrplan Volksschule Retrieved 15. 8., 2017

Erziehungsdirektion des Kantons Bern (ERZ) (2016a). Integration und besondere Massnahmen in der Volksschule des Kantons Bern (ERZ) (IBEM). Leitfaden zur Umsetzung von Artikel 17 VSG für Lehrpersonen, Schulleitungen und Schulbehörden Retrieved 2. 10., 2017, from http://www.erz.be.ch/erz/de/index/kindergarten_volksschule/kindergarten_volksschule/integ ration_und_besonderemassnahmen.assetref/dam/documents/ERZ/AKVB/de/01_Besondere\% 20Massnahmen/bes_massnahmen_leitfaden_IBEM_d.pdf

Erziehungsdirektion des Kantons Bern (ERZ) (2016b). Lehrplan 21. Grundlagen. Retrieved 23. 10., 2017 Fend, H. (2008). Schule gestalten. Systemsteuerung, Schulentwicklung und Unterrichtsqualität. Wiesbaden: VS Verlag. 
Fenstermacher, G. D. (1994). The Knower and the Known: The Nature of Knowledge in Research on Teaching. Review of Research in Education, 20(1), 3-56.

Fischer, C., Veber, M., Fischer-Ontrup, C., \& Buschmann, R. (2015). Vorwort. In C. Fischer, M. Veber, C. Fischer-Ontrup \& R. Buschmann (Hrsg.), Umgang mit Vielfalt. Aufgaben und Herausforderungen für die Lehrerinnen- und Lehrerbildung (S. 7-9). Münster: Waxmann.

Fischler, H. (2000a). Über den Einfluss von Unterrichtserfahrungen auf die Vorstellungen vom Lehren und Lernen bei Lehrerstudenten der Physik. Teil 1: Stand der Forschung sowie Ziele und Methoden einer Untersuchung. Zeitschrift für Didaktik der Naturwissenschaften, 6, 27-36.

Fischler, H. (2000b). Über den Einfluss von Unterrichtserfahrungen auf die Vorstellungen vom Lehren und Lernen bei Lehrerstudenten der Physik. Teil 2: Ergebnisse der Untersuchung. Zeitschrift für Didaktik der Naturwissenschaften, 6, 79-95.

Fischler, H., Schröder, H.-J., Tonhäuser, C., \& Zedler, P. (2002). Unterrichtsskripts und Lehrerexpertise: Bedingungen ihrer Modifikation. Zeitschrift für Pädagogik, 45 (Beiheft), 157-172.

Fives, H., \& Buehl, M. (2012). Spring cleaning for the "messy" construct of teachers' beliefs: What are they? Which have been examined? What can they tell us? In K. R. Harris, S. Graham \& T. Udan (Hrsg.), Educational Psychology Handbook: (Bd. 2, S. 471-499). Washington, DC: American Psychological Association.

Flick, U. (2010). Gütekriterien qualitativer Forschung. In G. Mey \& K. Mruck (Hrsg.), Handbuch Qualitative Forschung in der Psychologie (S. 395-407). Wiesbaden: VS Verlag.

Flick, U. (2016). Qualitative Sozialforschung. Eine Einführung (7., vollst. überarb. Aufl.). Reinbek: Rowohlt Taschenbuch.

Fransella, F., Bell, R., \& Bannister, D. (2004). A Manual for Repertory Grid Technique (2. Aufl.). Chichester: John Wiley \& Sons

Fromm, M. (1995). Repertory Grid Methodik. Ein Lehrbuch. Weinheim: Deutscher Studienverlag.

Fromm, M. (2010). Grid-Methodik. In G. Mey \& K. Mruck (Hrsg.), Handbuch Qualitative Forschung in der Psychologie (S. 524-537). Wiesbaden: VS Verlag.

Fromm, M. (2014). Gridsuite [Computer Software]. Stuttgart: TGZ constructiv.

Fromm, M., \& Paschelke, S. (2010). GridPractice. Anleitung zur Durchführung und Auswertung von GridInterviews. Noderstedt: Books on Demand.

Gates, P. (2006). Going beyond belief systems: Exploring a model for the social influence on mathematics teacher beliefs. Educational Studies in Mathematics, 63, 347-369.

Gay, G. (2015). Teachers' Beliefs about Cultural Diversity. Problems and Perspectives. In H. Fives \& M. G. Gill (Hrsg.), International Handbook of Research on Teachers' Beliefs (S. 436-452). New York: Routledge.

Giess-Stüber, P., \& Grimminger, E. (2008). Kultur und Fremdheit als sportdidaktische Perspektive. In H. Lange \& S. Sinning (Hrsg.), Handbuch Sportdidaktik (S. 223-244). Balingen: Spitta.

Gilleece, L. (2012). Teachers' Pedagogical Beliefs: Findings from the first OECD Teaching and Learning International Survey. In J. König (Hrsg.), Teachers' Pedagogical Beliefs. Definition and 
Operationalisation - Connections to Knowledge and Performance - Development and Change (S. 109-129). Münster: Waxmann.

Goldin, G. A. (2002). Beliefs, meta-affect, and mathematical belief structures. In G. C. Leder, E. Pehkonen \& G. Törner (Hrsg.), Beliefs: A hidden variable in mathematics education? (S. 59-72). Dordrecht: Kluwer.

Gramespacher, E., Albert, K., Hunger, I., \& Lüsebrink, I. (2009). Forschungswerkstätten - Basis qualitativer Forschung. Leipziger Sportwissenschaftliche Beiträge, 50(1), 98-119.

Green, B. (2004). Personal Construct Psychology and content analysis. Personal Construct Theory \& Practice, 1(3).

Green, T. F. (1971). The Activities of Teaching. New York: McGraw-Hill.

Grimminger, E. (2009). Interkulturelle Kompetenz im Schulsport. Evaluation eines Fortbildungskonzepts. Baltmannsweiler: Schneider Verlag.

Grimminger, E. (2012). An Empirical Study about the Importance of Teachers' Educational Beliefs and Acculturation Attitudes for the Implementation of Intercultural Education in Physical Education. Journal of Physical Education \& Health, 1(1), 5-14.

Groeben, N., \& Scheele, B. (2010). Das Forschungsprogramm Subjektive Theorien. In G. Mey \& K. Mruck (Hrsg.), Handbuch Qualitative Forschung in der Psychologie (S. 151-165). Wiesbaden: VS Verlag.

Groeben, N., Wahl, D., Schlee, J., \& Scheele, B. (1988). Das Forschungsprogramm Subjektive Theorien. Tübingen: Francke.

Guyton, E. M., \& Wesche, M. V. (2005). The Multicultural Efficacy Scale: Development, Item Selection, and Reliability. Multicultural Perspectives, 7(4), 21-29.

Hachfeld, A. (2013). Kulturelle Überzeugungen und professionelle Kompetenz von Lehrenden im Umgang mit kultureller Heterogenität im Klassenzimmer Retrieved 28. 5. , 2014, from http://www.diss.fu-

berlin.de/diss/servlets/MCRFileNodeServlet/FUDISS_derivate_000000012951/e_Hachfeld_Diss ertation_Kulturelle_Ueberzeugungen_und_professionelle_Kompetenz.pdf?hosts=

Hachfeld, A., Hahn, A., Schroeder, S., Anders, Y., \& Kunter, M. (2012). Multikulturelle Überzeugungen. Herkunft oder Überzeugung? Welche Rolle spielen der Migrationshintergrund und multikulturelle Überzeugungen für das Unterrichten von Kindern mit Migrationshintergrund? Zeitschrift für Pädagogische Psychologie, 26(2), 101-120.

Hachfeld, A., Hahn, A., Schroeder, S., Anders, Y., \& Kunter, M. (2015). Should teachers be colorblind? How multicultural and egalitarian beliefs differentially relate to aspects of teachers' professional competence for teaching in diverse classrooms. Teaching and Teacher Education, 48, 44-55.

Hachfeld, A., Hahn, A., Schroeder, S., Anders, Y., Stanat, P., \& Kunter, M. (2011). Assessing teachers' multicultural and egalitarian beliefs: The Teacher Cultural Beliefs Scale. Teaching and Teacher Education, 27, 986-996.

Haeberlin, U., Imdorf, C., \& Kronig, W. (2004a). Chancengleichheit bei der Lehrstellensuche. Der Einfluss von Schule, Herkunft und Geschlecht. In SKBF (Hrsg.). Bern. 
Haeberlin, U., Imdorf, C., \& Kronig, W. (2004b). Von der Schule in die Berufslehre. Untersuchungen zur Benachteiligung von ausländischen und von weiblichen Jugendlichen bei der Lehrstellensuche. Bern: Haupt.

Hagans, C. L., Neimeyer, G. J., \& Goodholm, C. R. (2000). The Effect of Elicitation Methods on Personal Construct Differentiation and Valence. Journal of Constructivist Psychology, 13, 155-173.

Hahn, A., Judd, C. M., \& Park, B. (2010). Thinking About Group Differences: Ideologies and National Identities. Psychological Inquiry, 21(2), 120-126.

Hallitzky, M., \& Schiessleder, M. (2008). Welche pädagogischen Leitbilder haben Lehramtsstudierende in Bezug auf den Umgang mit migrationsbedingter Heterogenität. In J. Ramseger \& M. Wagener (Hrsg.), Chancenungleichheit in der Grundschule. Ursachen und Wege aus der Krise. Wiesbaden: VS Verlag.

Hartinger, A., Kleickmann, T., \& Hawelka, B. (2006). Der Einfluss von Lehrervorstellungen zum Lernen und Lehren auf die Gestaltung des Unterrichts und auf motivationale Schülervariablen. Zeitschrift für Erziehungswissenschaft, 9(1), 110-126.

Hascher, T., \& Krapp, A. (2014). Forschung zu Emotionen von Lehrerinnen und Lehrern. In E. Terhart, H. Bennewitz \& M. Rothland (Hrsg.), Handbuch der Forschung zum Lehrerberuf (S. 679-697). Waxmann: Münster.

Hauser-Cram, P., Sirin, S. R., \& Stipek, D. (2003). When Teachers' and Parents' Values Differ: Teachers' Ratings of Academic Competence in Children From Low-Income Families. Journal of Educational Psychology, 95(4), 813-820.

Heintze, A., Helbig, G., Jungbluth, P., Kienast, E., \& Marburger, H. (1997). Schule und multiethnische Schülerschaft. Sichtweisen, Orientierungen und Handlungsmuster von Lehrerinnen und Lehrern. Frankfurt: IKO-Verlag für Interkulturelle Kommunikation.

Hemmecke, J. (2012). Repertory Grids als Methode zum Explizieren impliziten Wissens in Organisationen: Ein Beitrag zur Methodenentwicklung im Wissensmanagement Retrieved 11.12., 2016, from http://othes.univie.ac.at/27576/1/2012-10-23_9957490.pdf

Herman, R. L. (1996). The repertory grid technique: path to teacher description or teacher potential? Instructional Science, 24(6), 439-459.

Hewitt, A. (2005). Teachers' Personal Construct models of pupil individuality and their influence in the music classroom. Music Education Research, 7(3), 305-330.

Hillier, Y. (1998). Informal practitioner theory: eliciting the implicit. Studies in the Education of Adults, 30(1), 35-52.

Hussy, W., Schreier, M., \& Echterhoff, G. (2013). Forschungsmethoden in Psychologie und Sozialwissenschaften für Bachelor (2. überarb. Aufl.). Heidelberg: Springer.

Jankowicz, D. (2004). The Easy Guide to Repertory Grids. Chichester, UK: John Wiley \& Sons Ltd.

Jungbluth, P. (1994). Lehrererwartungen und Ethnizität. Innerschulische Chancendeterminanten bei Migrantenschülern in den Niederlanden. Zeitschrift für Pädagogik, 40(1), 113-125.

Jungbluth, P. (1997). Lehrererwartungen und die Reproduktion schicht- und ethnienspezifischer Chancenungleichheit. In A. Heintze, G. Helbig, P. Jungbluth, E. Kienast \& H. Marburger (Hrsg.), 
Schule und multiethnische Schülerschaft. Sichtweisen, Orientierungen und Handlungsmuster von Lehrerinnen und Lehrern. Frankfurt: IKO-Verlag für Interkulturelle Kommunikation.

Jurinak, I. (2017). Musliminnen müssen in Schwimmunterricht Tagesanzeiger. Zürich: Tamedia.

Jussim, L., \& Harber, K. D. (2005). Teacher Expectations and Self-Fulfilling Prophecies: Knowns and Unknowns, Resolved and Unresolved Controversies. Personality and Social Psychology Review, 9(2), 131-155. doi: 10.1207/s15327957pspr0902_3

Kagan, D. M. (1992). Implications of Research on Teacher Belief. Educational Psychologist, 27(1), 6590.

Kane, R., Sandretto, S., \& Heath, C. (2002). Telling Half the Story: A Critical Review of Research on the Teaching Beliefs and Practices of University Academics. Review of Educational Research, 72(2), 177-228.

Kelly, G. A. (1991). The psychology of personal constructs (Bd. 1, A theory of personality). London: Routledge. (Reprinted from: 1955).

Kember, D. (1997). A reconceptualisation of the research into university academics' conceptions of teaching. Learning and Instruction, 7(3), 255-275.

Kirchner, V. (2016). Wirtschaftsunterricht aus der Sicht von Lehrpersonen. Eine qualitative Studie zu fachdidaktischen teachers' beliefs in der ökonomischen Bildung. Wiesbaden: Springer VS Verlag.

Kleindienst-Cachay, C. (2016). Zum Umgang mit 'migrationsbedingten' Differenzen im Sportunterricht. In D. Wiesche, M. Fahlenbock \& N. Gissel (Hrsg.), Sportpädagogische Praxis - Ansatzpunkt und Prüfstein von Theorie. 28. Jahrestagung der dvs-Sektion Sportpädagogik vom 30. April-2. Mai 2015 in Bochum (S. 181-190). Hamburg: Czwalina.

Kluge, S., \& Kelle, U. (1999). Vom Einzelfall zum Typus: Fallvergleiche und Fallkontrastierung in der qualitativen Sozialforschung. Opladen: Leske + Budrich.

König, J. (2016). Lehrerexpertise und Lehrerkompetenz. In M. Rothland (Hrsg.), Beruf Lehrer / Lehrerin. Ein Studienbuch (S. 127-148). Stuttgart: UTB.

König, J., Darge, K., \& Schreiber, M. (2012). Teachers' Beliefs about Retention: Effects on Teaching Quality. In J. König (Hrsg.), Teachers' Pedagogical Beliefs. Definition and Operationalisation Connections to Knowledge and Performance - Development and Change (S. 191-204). Münster: Waxmann.

Kratzmann, J., \& Pohlmann-Rother, S. (2012). Ethnische Stereotype im Kindergarten? Erzieherinnenhaltungen gegenüber Zuwanderern aus der Türkei. Zeitschrift für Pädagogik, 58(6), 855-876.

Krauss, S., \& Bruckmaier, G. (2014). Das Experten-Paradigma in der Forschung zum Lehrerberuf. In E. Terhart, H. Bennewitz \& M. Rothland (Hrsg.), Handbuch der Forschung zum Lehrerberuf (S. 241261). Münster: Waxmann.

Kreber, C., \& Klampfleitner, M. (2012). Construing the meaning of authenticity in university teaching: comparing explicit to implicit theories. Journal of Constructivist Psychology, 25(1), 34-69. 
Kreber, C., \& Klampfleitner, M. (2013). Lecturers' and students' conceptions of authenticity in teaching and actual teacher actions and attributes students perceive as helpful. Higher Education, 66(4), 463-487.

Kronig, W. (2007). Die systematische Zufälligkeit des Bildungserfolgs. Theoretische Erklärungen und empirische Untersuchungen zur Lernentwicklung und zur Leistungsbewertung in unterschiedlichen Schulklassen. Bern: Haupt.

Kronig, W., Haeberlin, U., \& Eckhart, M. (2000). Immigrantenkinder und schulische Selektion. Pädagogische Visionen, theoretische Erklärungen und empirische Untersuchungen zur Wirkung integrierender und separierender Schulformen in den Grundschuljahren. Bern: Haupt.

Kuckartz, U. (2014). Qualitative Inhaltsanalyse. Methoden, Praxis, Computerunterstüzung (2. durchgesehene Aufl.). Weinheim: Beltz Juventa.

Kunter, M., \& Pohlmann, B. (2015). Lehrer. In R. Möller \& E. Wild (Hrsg.), Einführung in die Pädagogische Psychologie (S. 261-281). Berlin: Springer.

Kunter, M., \& Trautwein, U. (2013). Psychologie des Unterrichts. Paderborn: Ferdinand Schöningh.

Lamnek, S. (2005). Qualitative Sozialforschung (4., vollst. überarb. Aufl.). Weinheim: Beltz.

Lanfranchi, A. (2002). Schulerfolg von Migrationskindern. Die Bedeutung familienergänzender Betreuung im Vorschulalter. Opladen: Leske + Budrich.

Lanfranchi, A. (2007). Sonderklassenversetzung oder integrative Förderung: Denken und handeln Lehrpersonen kulturell neutral? Vierteljahresheft für Heilpädagogik und ihre Nachbargebiete (VHN), 76(2), 128-141.

Lanfranchi, A. (2013). Interkulturelle Kompetenz als Element pädagogischer Professionalität Schlussfolgerungen für die Lehrerausbildung. In G. Auernheimer (Hrsg.), Interkulturelle Kompetenz und pädagogische Professionalität (4. durchgesehene Aufl., S. 231-260). Wiesbaden: vs Verlag.

Lanfranchi, A., Perregaux, C., \& Thommen, B. (2001). Interkulturelle Pädagogik in der Lehrerinnen- und Lehrerbildung. Zentrale Lernbereiche - Thesen - Literaturhinweise. Schlussbericht. Bern: EDK.

Leineweber, H. (2015). "Ich unterrichte die Kids und nehme sie so, wie sie sind" - Zur Bedeutung subjektiver Theorien von Lehrkräften für die Entwicklung inklusiven Sportunterrichts. In S. Meier \& S. Ruin (Hrsg.), Inklusion als Herausforderung, Aufgabe und Chance für den Schulsport (S. 163183). Berlin: Logos.

Leuchter, M. (2009). Die Rolle der Lehrperson bei der Aufgabenbearbeitung. Unterrichtsbezogene Kognitionen von Lehrpersonen. Münster: Waxmann.

Liu, S.-H. (2011). Factors related to pedagogical beliefs of teachers and technology integration. Computers and Education, 56(4), 1012-1022.

Lorenz, G., Gentrup, S., Kristen, C., Stanat, P., \& Kogan, I. (2016). Stereotype bei Lehrkräften? Eine Untersuchung systematisch verzerrter Lehrerwartungen. Kölner Zeitschrift für Soziologie und Sozialpsychologie, 68, 89-111.

Lortie, D. C. (1975). Schoolteacher. A Sociological Study. Chicago: University of Chicago Press. 
Maag Merki, K., \& Werner, S. (2011). Erfassung und Bewertung professioneller Kompetenz von Lehrpersonen. In E. Terhart, H. Bennewitz \& M. Rothland (Hrsg.), Handbuch der Forschung zum Lehrerberuf (S. 573-591). Münster: Waxmann.

Makarova, E., \& Herzog, W. (2013). Teachers' Acculturation Attitudes and their Classroom Management: an empirical study among fifth-grade primary school teachers in Switzerland. European Educational Research Journal, 12(2), 256-269.

Marburger, H., Helbig, G., \& Kienast, E. (1997). Sichtweisen und Orientierungen Berliner Grundschullehrerinnen und -lehrer zur Multiethnizität der bundesdeutschen Gesellschaft und den Konsequenzen für Schule und Unterricht. In A. Heintze, G. Helbig, P. Jungbluth, E. Kienast \& H. Marburger (Hrsg.), Schule und multiethnische Schülerschaft. Sichtweisen, Orientierungen und Handlungsmuster von Lehrerinnen und Lehrern (S. 4-62). Frankfurt: IKO-Verlag für Interkulturelle Kommunikation.

McLoed, D. B. (1992). Research on Affect in Mathmatics Education: A Reconceptualization. In D. A. Grouws (Hrsg.), Handbook of Research on Mathmatics Teaching and Learning. Of Project of the National Council of TEachers of Mathematics. New York: Macmillan.

Meier, S., \& Ruin, S. (Hrsg.). (2015). Inklusion als Herausforderung, Aufgabe und Chance für den Schulsport. Berlin: Logos.

Meier, S., Ruin, S., \& Leineweber, H. (2017). HainSL - ein Instrument zur Erfassung von Haltungen zu inklusivem Sportunterricht bei (angehenden) Lehrkräften. [journal article]. German Journal of Exercise and Sport Research, 1-10. doi: 10.1007/s12662-016-0429-9

Merz-Atalik, K. (2001). Interkulturelle Pädagogik in Integrationsklassen. Subjektive Theorien von Lehrern im gemeinsamen Unterricht von Kinder mit und ohne Behinderung. Opladen: Leske + Budrich.

Möller, K., Hardy, I., Jonen, A., Kleickmann, T., \& Blumberg, E. (2006). Naturwissenschaften in der Primarstufe. Zur Förderung konzeptionellen Verständnisses durch Unterricht und zur Wirksamkeit von Lehrerfortbildungen. In M. Prenzel \& L. Allolio-Näcke (Hrsg.), Untersuchungen zur Bildungsqualität von Schule. Abschlussbericht des DFG-Schwerpunktprogramms (S. 161-193). Münster: Waxmann.

Moore, T. L., \& Reeves-Kazelskis, C. (1992). Effects of Formal Instruction on Preservice Teachers' Beliefs about Multicultural Education. In J. R. Petry \& G. E. Kenney (Hrsg.), Proceedings of the TwentyFirst Annual Meeting of the Mid-South Educational Research Association (S. 77). Knoxville, TN: ERIC.

Moser, U., \& Rhyn, H. (1999). Schulmodell im Vergleich. Eine Evaluation der Leistungen in zwei Schulmodellen der Sekundarstufe I. Aarau: Verlag Sauerländer.

Mösken, G., Dick, M., \& Wehner, T. (2015). "Gute Arbeit": Welche persönlichen Konstrukte unterscheiden Erwerbsarbeit von frei-gemeinnütziger Tätigkeit? In T. Wehner \& S. T. Güntert (Hrsg.), Psychologie der Freiwilligenarbeit. Motivation, Gestaltung, Organisation (S. 39-56). Heidelberg: Springer. 
Müller, C., Felbrich, A., \& Blömeke, S. (2008). Schul- und professionstheoretische Überzeugungen. In S. Blömeke, G. Kaiser \& R. Lehmann (Hrsg.), Professionelle Kompetenz angehender Lehrerinnen und Lehrer. Wissen, Überzeugungen und Lerngelegenheiten deutscher Mathematikstudierender und -referendare. Erste Ergebnisse zur Wirksamkeit der Lehrerausbildung (S. 277-302). Münster: Waxmann.

Müller, S. (2009). Methoden zur Erfassung epistemologischer Überzeugungen von Handelslehramtsstudierenden - eine empirische Vergleichsstudie. München: Rainer Hampp Verlag.

Munby, H. (1982). The Place of Teachers' Beliefs in Research on Teacher Thinking and Decision Making, and an Alternative Methodology. Instructional Science, 11, 201-225.

Munby, H., Russell, T., \& Martin, A. K. (2001). Teachers' Knowledge and How It Develops. In V. Richardson (Hrsg.), Handbook of Research on Teaching (4. Aufl., S. 877-904). Washington D.C.: American Educational Research Association.

Nadelson, L. S., Boham, M. D., Conlon-Khan, L., Fuentealba, M. J., Hall, C. J., Hoetker, G. A., . . Zenkert, A. J. (2012). A Shifting Paradigm: Preservice Teachers' Multicultural Attitudes and Efficacy. Urban Education, 47(6), 1183-1208.

Neimeyer, G. J., Bowman, J. Z., \& Saferstein, J. (2005). The Effects of Elicitation Techniques on Repertory Grid Outcomes: Difference, Opposite, and Contrast Methods. Journal of Constructivist Psychology, 18, 237-252.

Neimeyer, G. J., Neimeyer, R. A., Hagans, C. L., \& Van Brunt, D. L. (2002). Is There Madness in Our Method? The Effects of Repertory Grid Variations on Measures of Construct System Structure. In G. J. Neimeyer \& R. A. Neimeyer (Hrsg.), Advances in Personal Construct Psychology (Bd. 5, S. 161-200). Westport, CT: Praeger

Nel, J. (1993). Preservice Teachers' Perceptions of the Goals of Multicultural Education: Implications for the Empowerment of Minority Students. Educational Horizons, 71(3), 120-125.

Nespor, J. (1987). The role of beliefs in the practice of teaching. Journal of Curriculum Studies, 19(4), 317-328.

Neuweg, G. H. (2014). Das Wissen der Wissensvermittler. Problemstellungen, Befunde und Perspektiven der Forschung zum Lehrerwissen. In E. Terhart, H. Bennewitz \& M. Rothland (Hrsg.), Handbuch der Forschung zum Lehrerberuf (S. 583-614). Münster: Waxmann.

Nieke, W. (2008). Interkulturelle Erziehung und Bildung. Wertorientierungen im Alltag (3., aktual. Aufl.). Wiesbaden: VS Verlag.

Nohl, A.-M. (2010). Konzepte Interkultureller Pädagogik. Eine systematische Einführung (2., erw. Aufl.). Bad Heilbrunn: Klinkhardt.

OECD. (2009). Creating Effective Teaching and Learning Environments. First Results from TALIS: OECD Publishing.

Olmedo, I. M. (1997). Challenging old assumptions: preparing teachers for inner city schools. Teaching and Teacher Education, 13(3), 245-258. 
Oser, F., Biedermann, H., Brühwiler, C., Kopp, M., Krattenmacher, S., \& Steinmann, S. (2010). Deutschschweizer Lehrerbildung auf dem Prüfstand. Wie gut werden unsere angehenden Lehrpersonen ausgebildet? Ein internationaler Vergleich. Retrieved 17. 8., 2017, from http://www.teds-m.ch/download/Erste_Ergebnisse_110222.pdf

Oser, F., \& Blömeke, S. (2012). Überzeugungen von Lehrpersonen. Einführung in den Thementeil. Zeitschrift für Pädagogik, 58(4), 415-421.

Pajares, M. F. (1992). Teachers' Beliefs and Educational Research: Cleaning up a Messy Construct. Review of Educational Research, 62(3), 307-332.

Peterson, P. L., Fennema, E., Carpenter, T. P., \& Loef, M. (1989). Teachers' Pedagogical Content Beliefs in Mathematics. Cognition and Instruction, 6(1), 1-40.

Petty, L. L., \& Narayan, R. (2012). Investigating Secondary Science Teachers' Beliefs About Multiculturalism and its Implementation in the Classroom. Multicultural Perspectives, 14(4), 212-219. doi: 10.1080/15210960.2012.725327

Philipp, R. A. (2007). Mathmatics Teachers' Beliefs and Affect. In F. K. Lester (Hrsg.), Second Handbook of Research on Mathmatics Teaching and Learning (S. 257-315). Charlotte: Information Age Publishing.

Piguet, E. (2006). Einwanderungsland Schweiz. Fünf Jahrzehnte halb geöffnete Grenzen. Bern: Haupt.

Plaut, V. C. (2010). Diversity Science: Why and How Difference Makes a Difference. Psychological Inquiry, 21(2), 77-99.

Prengel, A. (2006). Pädagogik der Vielfalt. Verschiedenheit und Gleichberechtigung in Interkultureller, Feministischer und Integrativer Pädagogik (3. Aufl.). Wiesbaden: VS Verlag.

Priemer, B. (2006). Deutschsprachige Verfahren der Erfassung von epistemologischen Überzeugungen. Zeitschrift für Didaktik der Naturwissenschaften, 12, 159-175.

Rangosch-Schneck, E. (2007). "Da ist meistens so viel ungesprochen im Hinterkopf drin ..." Erfahrungen mit der Repertory-Grid-Technik als qualitativ-heuristischem Forschungskonzept am Beispiel der Explizierung von "Elternbildern" bei Lehrpersonen. Forum: Qualitative Sozialforschung, 8(1).

Reusser, K., \& Pauli, C. (2014). Berufsbezogene Überzeugungen von Lehrerinnen und Lehrern. In E. Terhart, H. Bennewitz \& M. Rothland (Hrsg.), Handbuch der Forschung zum Lehrerberuf (S. 642661). Münster: Waxmann.

Richardson, V. (1996). The Role of Attitudes and Beliefs in Learning to Teach. In J. Sikula (Hrsg.), Handbook of research on teacher education (2 Aufl., S. 102-119). New York: Macmillan.

Riemann, R. (1991). Repertory Grid Technik. Handanweisung. Göttingen: Hogrefe Verlag für Psychologie.

Rischke, A., Heim, C., \& Gröben, B. (2017). Nur eine Frage der Haltung? Eine empirische Analyse von personen- und institionenbezogenen Einflussgrössen auf die Einstellungen von Sportlehrkräften der Sekundarstufe I zur schulischen Inlusion German Journal of Exercise and Sport Research, 112. 
Rokeach, M. (1989). Beliefs, attitudes and values : a theory of organization and change. San Francisco, Calif.: Jossey-Bass.

Rosenberger, M., \& Freitag, M. (2009). Repertory Grid. In S. Kühl, P. Strodtholz \& A. Taffertshofer (Hrsg.), Handbuch Methoden der Organisationsforschung (S. 477-496). Wiesbaden: VS Verlag.

Rosenthal, R., \& Jacobson, L. (1968). Pygmalion in the classroom. New York: Holt, Rinehart \& Winston. Rost, D. H. (2013). Interpretation und Bewertung pädagogisch-psychologischer Studien. Eine Einführung (3., vollst. überarb. und erw. Aufl.). Bad Heilbrunn: Julius Klinkhardt.

Scheer, J. W. (1993). Planung und Durchführung von Repertory Grid-Untersuchungen. In J. W. Scheer \& A. Catina (Hrsg.), Einführung in die Repertory Grid-Technik. (Bd. 1: Grundlagen und Methoden, S. 24-40). Bern: Verlag Hans Huber.

Scheer, J. W., \& Catina, A. (Hrsg.). (1993). Einführung in die Repertory Grid-Technik. (Bd. 1: Grundlagen und Methoden). Bern: Verlag Hans Huber.

Schierz, M. (2013). Bildungspolitische Reformvorgaben und fachkulturelle Reproduktion Beobachtungen am Beispiel des Schulfachs Sport. Spectrum für Sportwissenschaften, 25(1), 6479.

Schlichter, N. (2012). Lehrerüberzeugungen zum Lehren und Lernen. Dissertation, Georg-AugustUniversität Göttingen, Göttingen.

Schwarzer, R., \& Warner, L. M. (2014). Forschung zur Selbstwirksamkeit bei Lehrerinnen und Lehrern. In E. Terhart, H. Bennewitz \& M. Rothland (Hrsg.), Handbuch der Forschung zum Lehrerberuf (2., überarb. und erw. Aufl., S. 662-676). Münster Waxmann.

Schweer, M. K. W., \& Thies, B. (2000). Situationswahrnehmung und interpersonales Verhalten im Klassenzimmer. In M. K. W. Schweer (Hrsg.), Lehrer-Schüler-Interaktion. Pädagogischpsychologische Aspekte des Lehrens und Lernens in der Schule (S. 59-78). Opladen: Leske + Budrich.

Schweer, M. K. W., Thies, B., \& Lachner, R. P. (2017). Soziale Wahrnehmungsprozesse und unterrichtliches Handeln. Eine dynamisch-transaktionale Perspektive. In M. K. W. Schweer (Hrsg.), Lehrer-Schüler-Interaktion. Inhaltsfelder, Forschungsperspektiven und methodische Zugänge (3., überarb. und aktual. Aufl., S. 121-145). Wiesbaden: VS Verlag.

Schweizerische Konferenz der kantonalen Erziehungsdirektoren (EDK) (1995). Empfehlungen und Beschlüsse. Bern: EDK.

Seifried, J. (2009). Unterricht aus der Sicht von Handelslehrern. Franfurt am Main: Peter Lang.

Seifried, J. (2012). The Role of Teachers' Pedagogical Beliefs - An Analysis from the Perspective of Vocational Education and Training. In J. König (Hrsg.), Teachers' Pedagogical Beliefs. Definition and Operationalisation - Connections to Knowledge and Performance - Development and Change (S. 131-147). Münster: Waxmann.

Sembill, D., \& Seifried, J. (2009). Konzeptionen, Funktionen und intentionale Veränderungen von Sichtweisen. In O. Zlatkin-Troitschanskaia, K. Beck, D. Sembill, R. Nickolaus \& R. Mulder (Hrsg.), Lehrprofessionalität. Bedingungen, Genese, Wirkungen und ihre Messung (S. 345-354). Weinheim: Beltz. 
Sercu, L., Bandura, E., Castro, P., Davcheva, L., Laskaridou, C., Lundgren, U., . . Ryan, P. (2005). Foreign Language Teachers and Intercultural Competence. An International Investigation. Clevedon: Multilingual Matters Ltd.

Shulman, L. S. (1986). Those Who Unterstand: Knowledge Growth in Teaching. Educational Researcher, 15(2), 4-14.

Shulman, L. S. (1987). Knowledge and teaching: Foundations of the new reform. Harvard Educational Review, 57(1), 1-22.

Sieber, P. (2009). Interkulturelle Pädagogik in der Lehrerinnen und Lehrerbildung. vpod-bildungspolitik, 164, 11-14.

Sievers, I. (2012). Zum Umgang von Lehrkräften mit migrationsbedingter Vielfalt und Differenz. In M. Matzner (Hrsg.), Handbuch Migration und Bildung (S. 288-300). Weinheim: Beltz.

Sievers, I. (Hrsg.). (2009). Individuelle Wahrnehmung, nationale Denkmuster. Einstellungen deutscher und französischer Lehrkräfte zu Heterogenität im Unterricht. Frankfurt am Main: Brandes \& Apsel

Skott, J. (2015). The Promises, Problems, and Prospects of Research on Teachers' Beliefs. In H. Fives \& M. G. Gill (Hrsg.), International Handbook of Research on Teachers' Beliefs (S. 13-30). New York: Routledge.

Sleeter, C. E. (2001). Preparing Teachers for Culturally Diverse Schools: Research and the Overwhelming Presence of Whiteness. Journal of Teacher Education, 52(2), 94-106.

Sparks, W., \& Wayman, L. (1993). Multicultural Understanding In Physical Education: A Comparison of Urban and Rural Perspectives. Physical Educator, 50(2), 58-68.

Staatssekretariat für Migration (SEM) (2017). Ausländerstatistik Dezember 2016. Retrieved 6. 3., 2017, from

https://www.sem.admin.ch/sem/de/home/publiservice/statistik/auslaenderstatistik/archiv/20 16/12.html

Stanley, L. S. (1996). The Development and Validation of an Instrument to Assess Attitudes toward Cultural Diversity and Pluralism among Preservice Physical Educators. Educational and psychological measurement, 56(5), 891-897.

Stanley, L. S. (1997). Preservice Physical Educators' Attitudes Toward Cultural Pluralism: A Preliminary Analysis. Journal of Teaching in Physical Education, 16(2), 241-249.

Staub, F. C. (2001). Fachspezifisch-pädagogisches Coaching: Theoriebezogene Unterrichtsentwicklung zur Förderung von Unterrichtsexpertise. Beiträge zur Lehrerbildung, 19(2), 175-198.

Staub, F. C., \& Stern, E. (2002). The Nature of Teachers' Pedagogical Content Beliefs matters for Students' Achievement Gains: Quasi-Experimental Evidence From Elementary Mathematics. Journal of Educational Psychology, 94(2), 344-355.

Steinmann, S., \& Oser, F. (2012). Prägen Lehrerausbildende die Beliefs der angehenden Primarlehrpersonen? Shared Beliefs als Wirkungsgrösse in der Lehrerausbildung. Zeitschrift für Pädagogik, 58(4), 441-459. 
Strasser, J. (2012). Kulturelle Stereotype und ihre Bedeutung für das Verstehen in Schule und Unterricht. In W. Wiater \& D. Manschke (Hrsg.), Verstehen und Kultur. Mentale Modelle und kulturelle Prägungen (S. 191-215). Wiesbaden: VS Verlag.

Strohmeier, D., \& Fricker, A. (2007). Interkulturelles Lernen: Unbekanntes Unterrichtsprinzip oder gelebte schulische Praxis. Erziehung und Unterricht, 157(1+2), 115-128.

Sulimma, M. (2012). Die Entwicklung epistemologischer Überzeugungen von (angehenden) Handelslehrer(inne)n. München: Rainer Hampp Verlag.

Svennberg, L., Meckbach, J., \& Redelius, K. (2014). Exploring PE teachers' 'gut feelings': An attempt to verbalise and discuss teachers' internalised grading criteria. European Physical Education Review, 20(2), 199-214.

Tatum, B. D. (1997). "Where are all the Black kids sitting together in the cafeteria?" and other conversations about race. New York: Basic Books.

Tenenbaum, H. R., \& Ruck, M. D. (2007). Are Teachers' Expectations Different for Racial Minority Than for European American Students? A Meta-Analysis. Journal of Educational Psychology, 99(2), 253-273.

Thieme, N. (2011). Repertory Grid Methodik. In G. Oelerich \& H.-U. Otto (Hrsg.), Empirische Forschung und Soziale Arbeit (S. 281-291). Wiesbaden: VS Verlag.

Trautmann, M., \& Wischer, B. (2011). Heterogenität in der Schule. Eine kritische Einführung. Wiesbaden: VS Verlag.

Trautwein, C. (2013). Lehrebezogene Überzeugungen und Konzeptionen - eine konzeptuelle Landkarte. Zeitschrift für Hochschulentwicklung, 8(3), 1-14.

Trautwein, U., \& Baeriswyl, F. (2007). Wenn leistungsstarke Klassenkameraden ein Nachteil sind. Referenzgruppeneffekte bei Übertrittsentscheidungen. Zeitschrift für Pädagogische Psychologie, 21, 119-133.

Tschannen-Moran, M., \& Hoy, A. W. (2001). Teacher efficacy: Capturing an elusive construct. Teaching and Teacher Education, 17, 783-805.

Ulich, K. (2001). Einführung in die Sozialpsychologie der Schule. Weinheim: Beltz.

Van Dick, R., Wagner, U., Adams, C., \& Petzel, T. (1997). Einstellungen zur Akkulturation: Erste Evaluation eines Fragebogens an sechs deutschen Stichproben. Gruppendynamik. Zeitschrift für angewandte Sozialpsychologie, 28(1), 83-92.

Volksschulgesetz (VSG) des Kantons Bern vom 19. März 1992 (BSG432.210).

Voss, T., Kleickmann, T., Kunter, M., \& Hachfeld, A. (2011). Überzeugungen von Mathematiklehrkräften. In M. Kunter, J. Baumert, W. Blum, U. Klusmann, S. Krauss \& M. Neubrand (Hrsg.), Professionelle Kompetenz von Lehrkräften. Ergebnisse des Forschungsprogramms COACTIV. Münster: Waxmann.

Wagner, U., Van Dick, R., Petzel, T., \& Auernheimer, G. (2001). Der Umgang von Lehrerinnen und Lehrern mit interkulturellen Konflikten. In G. Auernheimer, R. Van Dick, T. Petzel \& U. Wagner (Hrsg.), Interkulturalität im Arbeitsfeld Schule. Empirische Untersuchungen über Lehrer und Schüler (S. 17-40). Opladen: Leske + Budrich. 
Walter, P. (1999). Nichts als ethnozentrische Vorurteile? Kognitionen von Lehrkräften über interkulturelle Erziehung. In R. Dollase, T. Kliche \& H. Moser (Hrsg.), Politische Psycholgie der Fremdenfeindlichkeit (S. 241-255). Weinheim: Juventa Verlag.

Weinert, F. E. (2001). Concepts of Competence: A conceptual clarification. In D. S. Rychen \& L. H. Saganik (Hrsg.), Defining and Selecting Key Competencies (S. 45-65). Seattle: Hogrefe \& Huber.

West, L., \& Staub, F. C. (2003). Content-Focused Coaching: Transforming mathematics lessons. NH: Heinemann.

Wilde, A., \& Kunter, M. (2016). Überzeugungen von Lehrerinnen und Lehrern. In M. Rothland (Hrsg.), Beruf Lehrer / Lehrerin. Ein Studienbuch (S. 299-315). Stuttgart: UTB.

Wischer, B. (2007). Heterogenität als komplexe Anforderung an das Lehrerhandeln. In S. Boller, E. Rosowski \& T. Stroot (Hrsg.), Heterogenität in Schule und Unterricht (S. 32-41). Weinheim: Beltz.

Wischmeier, I. (2012). "Teacher' Beliefs": Überzeugungen von (Grundschul-)Lehrkräften über Schüler und Schülerinnen mit Migrationshintergrund - Theoretische Konzeption und empirische Überprüfung. In W. Wiater \& D. Manschke (Hrsg.), Verstehen und Kultur. Mentale Modelle und kulturelle Prägungen (S. 167-189). Wiesbaden: VS Verlag.

Woolfolk Hoy, A., Davis, H., \& Pape, S. J. (2006). Teacher Knowledge and Beliefs. In P. A. Alexander \& P. H. Winne (Hrsg.), Handbook of Educational Psychology (2. Aufl., S. 715-737). Mahwah: Erlbaum.

Yorke, M. (2001). Bipolarity ... or not? Some Conceptual Problems Relating to Bipolar Rating Scales. British Educational Research Journal, 27(2), 171-186.

Zick, A., \& Six, B. (1997). Autoritarismus, Vorurteile und Einstellungen zur Akkulturation. Gruppendynamik, 28, 305-320.

Ziegler, A., Kuhn, C., \& Heller, A. (1998). Implizite Theorien von gymnasialen Mathematik- und Physiklehrkräften zu geschlechtsspezifischer Begabung und Motivation. Psychologische Beiträge, 40(1), 271-287. 


\section{Anhang}

\section{A Anfrage Schulen}

Schweizerische Eidgenossenschaf Conféderation suisse Confederazione Svizzera Confederaziun svizra
Bundesamt für Sport BASPO

Eidgenössische Hochschule für Sport Magglingen EHSM

\section{Anfrage zur Befragung von Sportlehrpersonen zum Thema „Kulturelle Heterogenität}

im Sportunterricht"

Sehr geehrte Damen und Herren

Lehrpersonen in Schulen mit hohem Anteil an Schülerinnen und Schülern mit Migrationshintergrund stehen heute vielfältigen Herausforderungen gegenüber. Besonders herausfordemd - zumindest in der öffentlichen Wahmehmung - scheint dabei der Sportunterricht zu sein. Die Eidgenössische Hochschule für Sport Magglingen (EHSM) führt vor diesem Hintergrund eine empirische Studie durch, die die Überzeugungen von Sportlehrpersonen zur kulturelIen Heterogenität im Sportunterricht untersucht. Wir möchten herausfinden, was und wie erfahrene Sportlehrpersonen über die kulturelle Heterogenität in Schulen und im Sportunterricht denken. Für diese Untersuchung bitten wir Sie um Ihre Unterstützung.

Befragt werden sollen Sportlehrpersonen der Sekundarstufe I des Kantons Bem, die mindestens 5 Jahre Unterrichtserfahrung haben. Die voraussichtliche Inanspruchnahme einer Lehrperson umfasst zwei mündliche Befragungen von je 60-90 Minuten. Sämtliche Daten werden vollständig anonymisiert, so dass aus den Resultaten keinerlei Rückschlüsse auf befragte Personen gemacht werden können. Teilnehmende erhalten eine Teilnahmebestätigung, die ggf. als Weiterbildungsbestätigung verwendet werden kann.

Als angewandtes Forschungsprojekt sollen die Erkenntnisse der Studie einerseits in die Ausund Weiterbildung von Sportlehrpersonen einfliessen und damit wichtige Anregungen und Hilfen für die Bewältigung ihrer Aufgaben geben. Andererseits sollen die beteiligten Schulen die erzielten Resultate für ihre eigene Schul(sport)entwicklung weiter verwenden können.

Wir würden uns sehr freuen, wenn wir Sportlehrpersonen Ihrer Schule als Teilnehmende dieser Studie gewinnen könnten. Damit wir angehende und tätige Sportlehrpersonen optimal auf die Berufspraxis vorbereiten können, sind wir auf Ihre Unterstütung angewiesen.

Mit freundlichen Grüssen

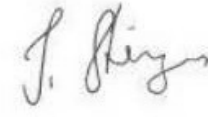

Jonas Steiger

Untersuchungsleiter

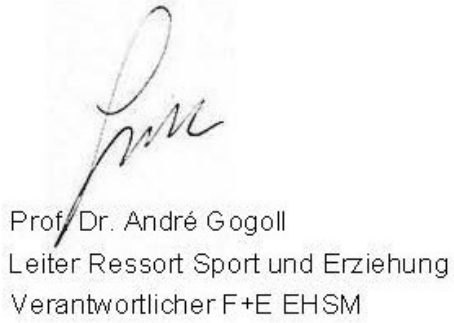

Verantwortlicher F+E EHSM 


\section{B Kurzfragebogen}

Schweizerische Eidgenossenschaft

Confédération suisse

Confederazione Svizzera

Confederaziun svizra
Bundesamt für Sport BASPO

Eidgenössische Hochschule für Sport Magglingen EHSM

\section{Kurzfragebogen}

Vorname / Name:

Geschlecht: $\square$ W $\square \mathrm{m}$

Geburtsdatum:

Name der Schule

Klasseniveau: Schulmodell:

$\square$ Realklasse

$\square$ Sekundarklasse

$\square$ integriert

Unterrichtserfahrung als Sportlehrperson: ...... Jahre

Jahr des pädagogischen Ausbildungsabschlusses:

Diplom:

$\square$ Lehrerpatent (Seminar); Stufe(n):

$\square$ Lehrdiplom SI (Sekundarlehramt / PH / UNI)

$\square$ Lehrdiplom SII (Höheres Lehramt / PH / UNI)

$\square$ Sportlehrdiplom I $\square$ Sportlehrdiplom II

$\square$ Diplom Sonderpädagogik (PH/ UNI)

$\square$ sonstiges Lehrdiplom:

Lehrbefähigung für folgende Fächer

Bislang und gegenwärtig unterrichtete Fächer

$\square$ Mathematik

$\square$ Geschichte (MZG)

$\square$ Deutsch

$\square$ Bewegung und Sport

$\square$ Musik

$\square$ Französisch

$\square$ Hauswirtschaft $(\mathrm{MKH})$

Italieni

$\square$ Religion/Lebenskunde (RME)

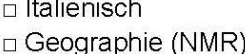

$\square$ Naturkunde (NMT)

$\square$ Bildnerisches Gestalten

Aus- oder Weiterbildungen im Bereich interkulturelle Pädagogik: $\quad$ ja $\quad \square$ nein

Aktuelles Jahrespensum: ......\%

Persönliche Hauptsportart(en)

Befragungstermine: ......... 


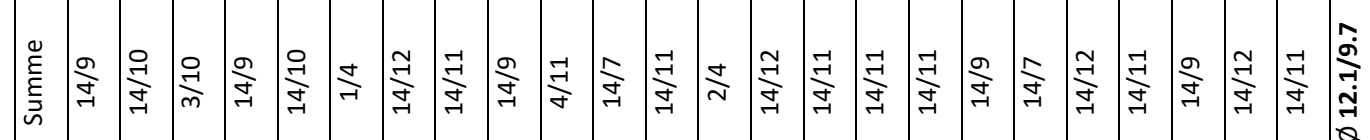

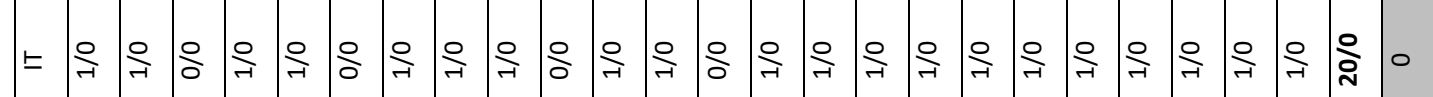

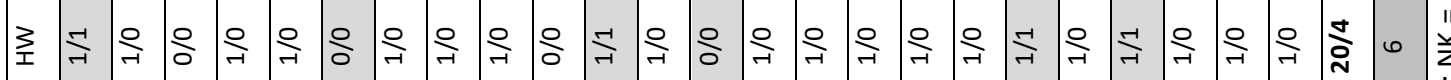

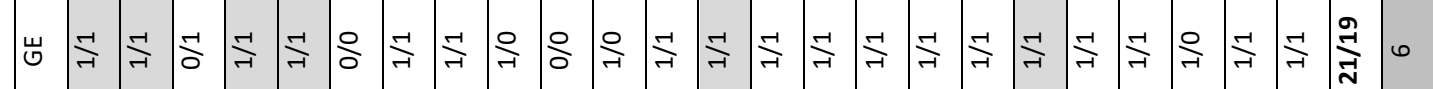

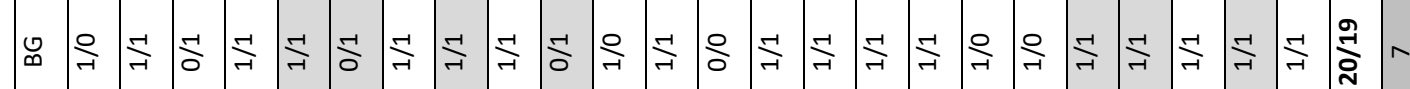

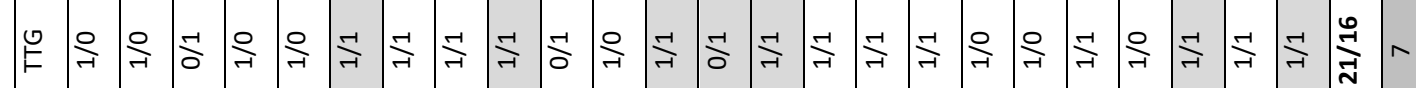

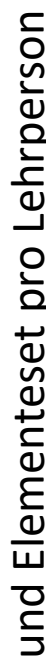

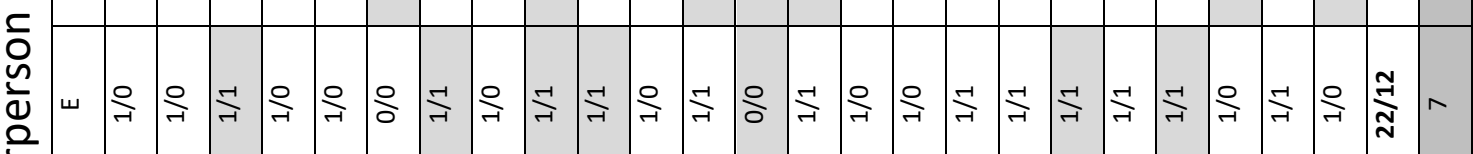

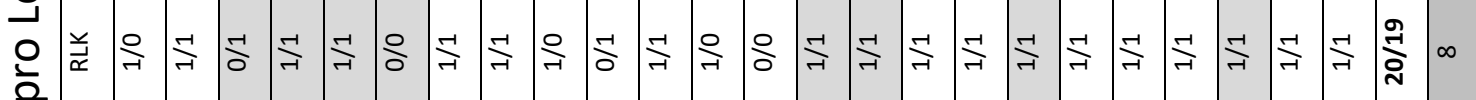

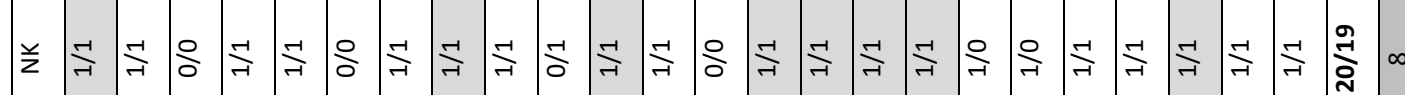

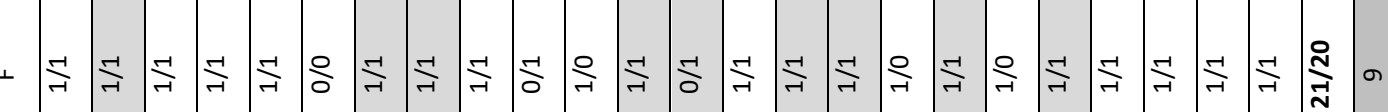
岁

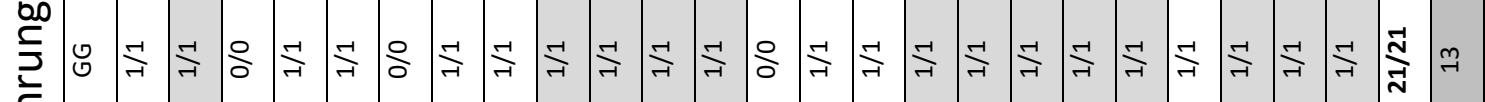

西

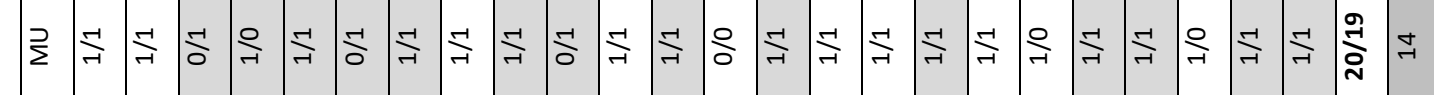

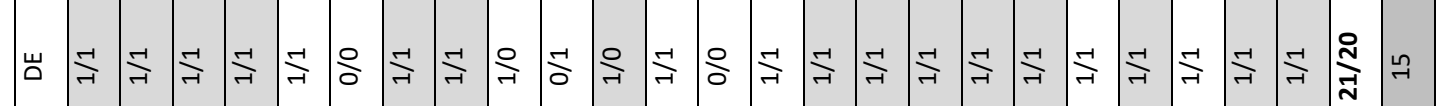
II $\stackrel{0}{\Sigma}$

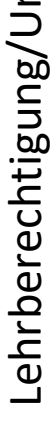

$\cup$ U 


\begin{tabular}{|c|c|c|c|c|c|c|c|c|c|c|c|c|}
\hline in & 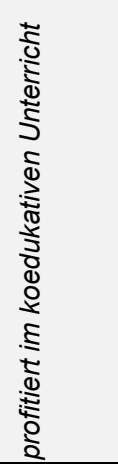 & 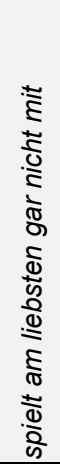 & 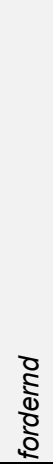 & 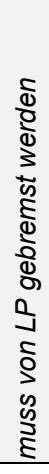 & 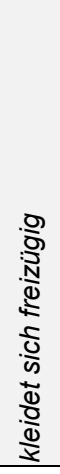 & 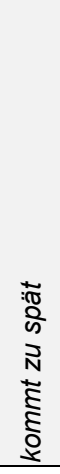 & 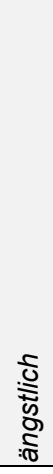 & 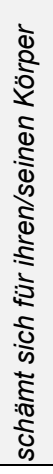 & 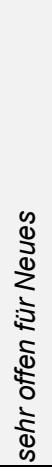 & 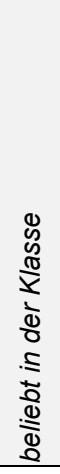 & $\begin{array}{l}\infty \\
\bar{d} \\
\delta \\
\Phi \\
\Phi \\
: \\
\Phi \\
\Phi \\
5 \\
5\end{array}$ & 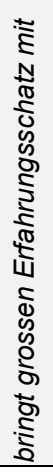 \\
\hline S-.„eəp I. & in & - & 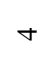 & 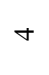 & $n$ & N & - & - & in & 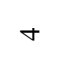 & - & - \\
\hline (๖) E|OX!N & ナ & - & $ナ$ & ナ & $m$ & $\nabla$ & - & 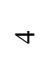 & in & $n$ & - & + \\
\hline 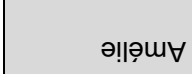 & n & - & + & + & $m$ & - & - & $N$ & in & $\nabla$ & - & n \\
\hline eonา & 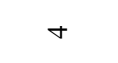 & - & + & ナ & m & N & - & + & 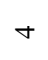 & $m$ & - & in \\
\hline (†) & $N$ & + & - & $N$ & + & in & + & $N$ & $N$ & $n$ & + & $m$ \\
\hline (๖) оा!्u!W & + & v & in & m & m & in & n & in & - & in & $\sim$ & + \\
\hline еגe & - & in & - & - & $m$ & + & in & $m$ & - & $\sim$ & $\nabla$ & - \\
\hline (Ł) чәшәңе & 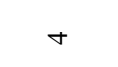 & $\sim$ & - & $m$ & $N$ & - & $\sim$ & $m$ & ナ & N & $\sim$ & $m$ \\
\hline (t) uәsu $\forall$ & m & $m$ & - & n & N & N & $\nabla$ & m & ナ & N & n & $N$ \\
\hline oue!!n!๐ & m & $m$ & - & 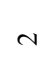 & m & - & n & $\sim$ & ナ & - & n & m \\
\hline Y!lü & 4 & 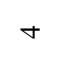 & N & N & in & 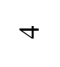 & 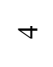 & a & $m$ & $n$ & + & $N$ \\
\hline- & 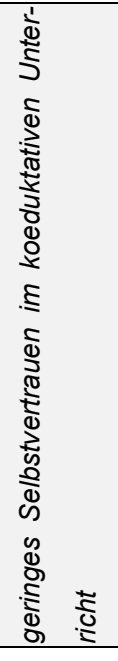 & 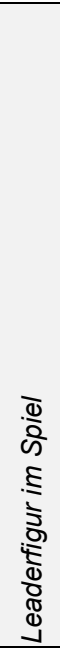 & 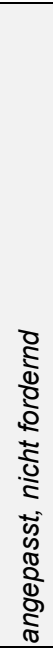 & 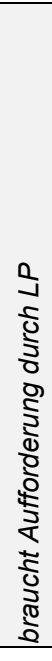 & 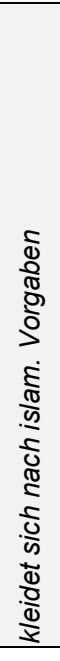 & 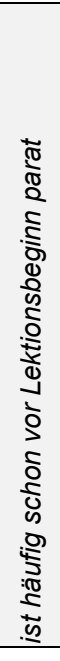 & $\begin{array}{l}\text { D } \\
\text { है }\end{array}$ & 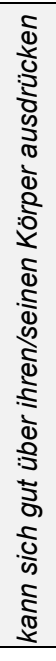 & 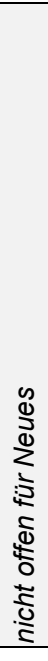 & 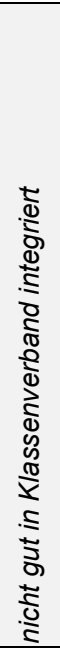 & 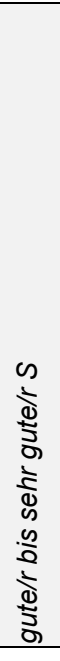 & 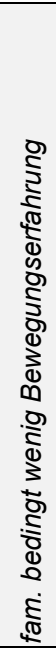 \\
\hline$\exists$ & $\bar{x}$ & $\underline{\mathfrak{Y}}$ & $\underline{m}$ & $\underset{\mathbf{Z}}{\mathbf{Z}}$ & $\underline{\underline{0}}$ & $\underline{\dot{Q}}$ & $\underline{x}$ & $\underline{\infty}$ & $\stackrel{9}{\underline{x}}$ & $\stackrel{\text { ㅁ }}{\underline{y}}$ & $\overline{\bar{\Sigma}}$ & $\frac{\mathfrak{N}}{\underline{x}}$ \\
\hline
\end{tabular}




\begin{tabular}{|c|c|c|c|c|c|c|c|c|c|c|c|}
\hline n & 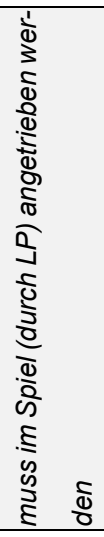 & 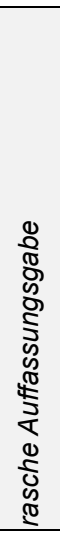 & 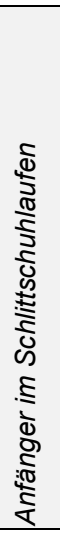 & 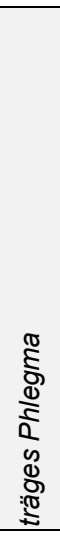 & $\begin{array}{l}\bar{d} \\
\frac{0}{2} \\
\text { D } \\
.5 \\
: \frac{1}{\pi} \\
\end{array}$ & 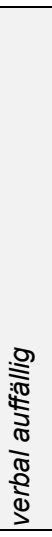 & 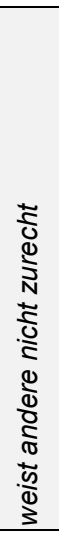 & 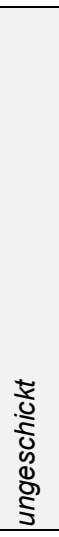 & 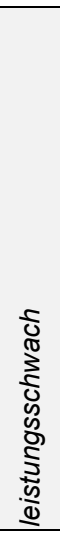 & 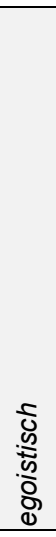 & 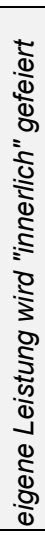 \\
\hline S-|еәр| & m & $n$ & - & - & $n$ & $\sigma$ & m & - & - & - & ナ \\
\hline es!? & + & 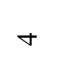 & $\sim$ & - & in & - & $n$ & - & - & $N$ & in \\
\hline 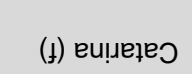 & $\checkmark$ & $\nabla$ & $\nabla$ & $m$ & $n$ & - & $n$ & - & $N$ & - & ナ \\
\hline (Ł) e!|əON & $m$ & $N$ & $N$ & $m$ & + & + & $\nabla$ & $n$ & $m$ & $N$ & 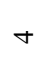 \\
\hline еәา & m & $m$ & $\sim$ & - & in & N & in & $N$ & - & - & $\nabla$ \\
\hline (๖) esnW & $N$ & - & $N$ & - & + & $\nabla$ & $N$ & - & - & $m$ & $N$ \\
\hline (๖) sol!W & - & 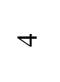 & - & - & $m$ & in & - & - & - & + & - \\
\hline (Ł) żıə০ & $N$ & $m$ & - & $m$ & $N$ & 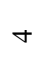 & m & 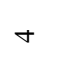 & $m$ & $N$ & m \\
\hline pәшшечоw & - & $m$ & $\sim$ & $\sim$ & - & $n$ & - & $m$ & $\sim$ & $n$ & - \\
\hline ичог & $\nabla$ & $N$ & - & $m$ & $\nabla$ & - & $n$ & $\nabla$ & $\nabla$ & $N$ & in \\
\hline (Ł) uotər & $m$ & N & $\sim$ & - & $n$ & N & n & m & N & - & t \\
\hline- & 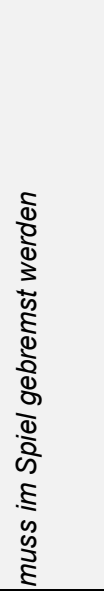 & 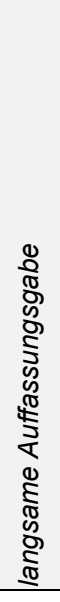 & 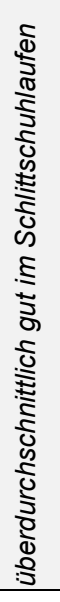 & 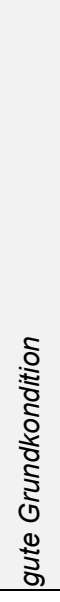 & 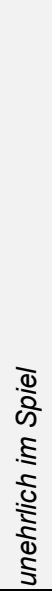 & 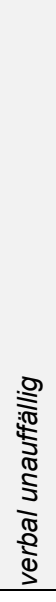 & 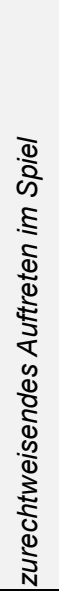 & 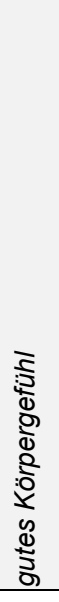 & 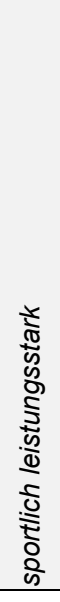 & 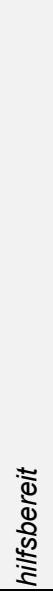 & 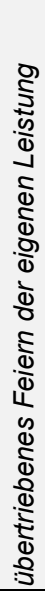 \\
\hline ב & $\underline{\Sigma}$ & $\underline{\widetilde{Y}}$ & $\underline{\tilde{m}}$ & $\mathbb{\pm}$ & $\underline{\underline{n}}$ & $\underline{\varphi}$ & $\underline{\underline{x}}$ & $\stackrel{\infty}{\underline{\infty}}$ & $\underline{\mathscr{9}}$ & $\frac{O}{\underline{y}}$ & $\underline{\Sigma}$ \\
\hline
\end{tabular}




\begin{tabular}{|c|c|c|c|c|c|c|c|c|c|c|c|c|c|}
\hline in & 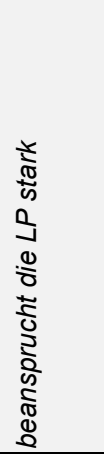 & 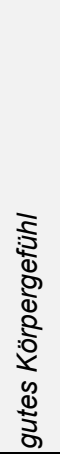 & 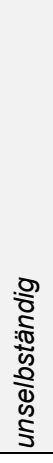 & 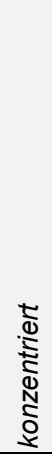 & 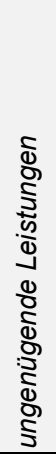 & 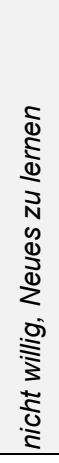 & 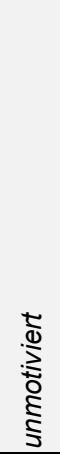 & 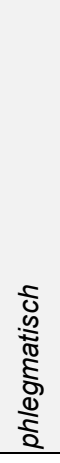 & 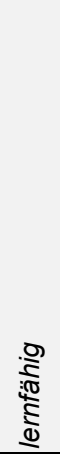 & 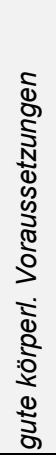 & 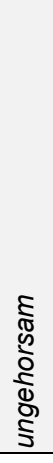 & 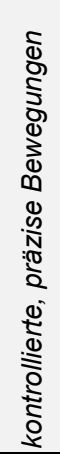 & 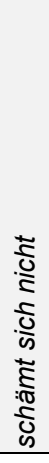 \\
\hline S-ןеәр| & - & n & - & $n$ & - & - & - & N & $n$ & $n$ & - & in & in \\
\hline ұиәэи!^ & $m$ & - & in & $m$ & 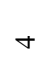 & $m$ & $\nabla$ & $n$ & - & $N$ & m & 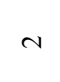 & + \\
\hline (๖) (๖ & $\nabla$ & $\nabla$ & $\nabla$ & $\sim$ & $\sim$ & $\sim$ & $n$ & - & m & in & $\sim$ & $\sigma$ & in \\
\hline |ənuew & $\nabla$ & - & $\sim$ & $\nabla$ & n & $m$ & $\sim$ & $n$ & $\sim$ & - & - & - & $m$ \\
\hline semou」 & in & $\nabla$ & in & - & $\sim$ & 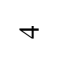 & $n$ & - & n & in & in & 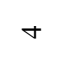 & in \\
\hline (๖) иечұеиог & $N$ & $N$ & $N$ & $m$ & $m$ & $\sim$ & - & $m$ & $N$ & $n$ & - & - & in \\
\hline 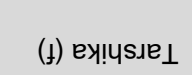 & $m$ & $m$ & $m$ & 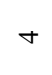 & $m$ & $\sim$ & $\sim$ & $\sim$ & 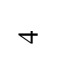 & in & $\sim$ & $\nabla$ & $\sim$ \\
\hline ә!ро|ə઼W & - & + & - & in & $N$ & - & - & $N$ & $n$ & $n$ & - & in & + \\
\hline 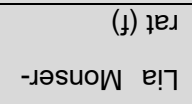 & $\nabla$ & - & in & $m$ & n & in & in & n & - & - & $\theta$ & $N$ & - \\
\hline Еมе!чว & $\sim$ & $n$ & $\sim$ & in & - & - & - & $\sim$ & $n$ & in & - & in & $n$ \\
\hline әnb!̣ụuoo & 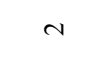 & in & $\sim$ & + & N & - & - & - & in & in & - & in & $n$ \\
\hline- & 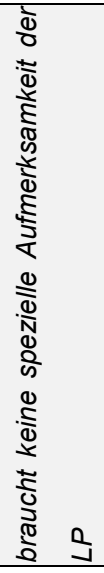 & 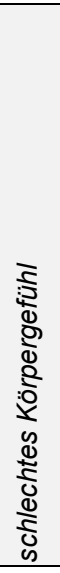 & 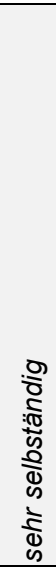 & 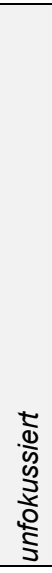 & 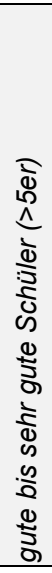 & 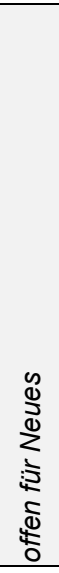 & 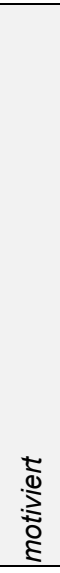 & 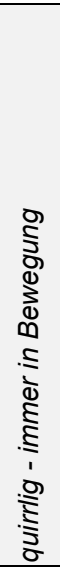 & 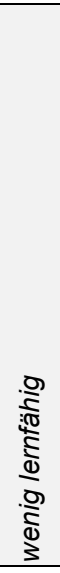 & 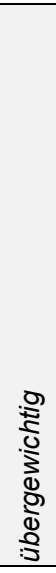 & $\begin{array}{l}0 \\
\frac{1}{d} \\
0 \\
\frac{1}{0} \\
\frac{0}{d} \\
\text { d }\end{array}$ & 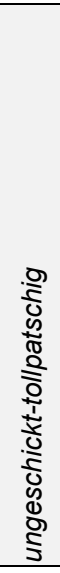 & 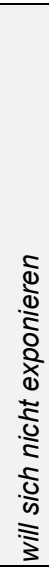 \\
\hline 3 & $\bar{x}$ & $\underline{\tilde{y}}$ & m & 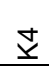 & $\underline{\underline{2}}$ & $\stackrel{\mathscr{q}}{\underline{y}}$ & $\underline{\underline{x}}$ & $\stackrel{\infty}{\underline{x}}$ & $\stackrel{9}{x}$ & 올 & $\bar{\Sigma}$ & $\stackrel{\mathfrak{N}}{\underline{I}}$ & $\stackrel{m}{\underline{x}}$ \\
\hline
\end{tabular}




\begin{tabular}{|c|c|c|c|c|c|c|c|c|c|c|c|c|c|}
\hline n & 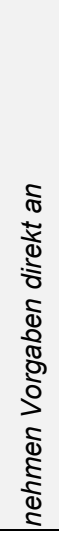 & 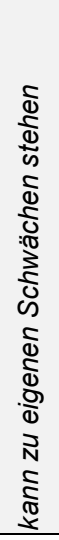 & $\frac{\bar{\Phi}}{\stackrel{\Phi}{\infty}}$ & 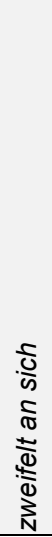 & $\underset{\mathbb{N}}{\mathbb{S}}$ & 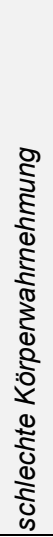 & 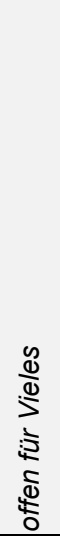 & 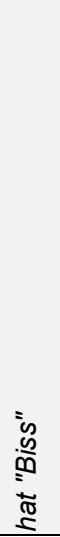 & 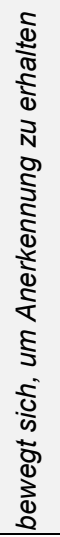 & 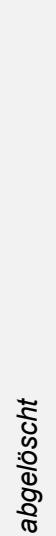 & 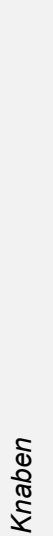 & 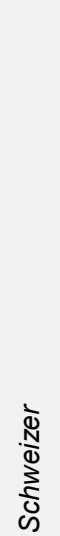 & 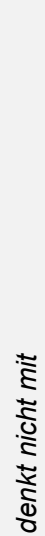 \\
\hline s-|еәр| & in & $n$ & - & - & - & - & $n$ & in & - & - & $\sim$ & ' & - \\
\hline (4) eגe!!! & $m$ & - & $m$ & + & + & + & $\sigma$ & $\sim$ & $\nabla$ & $\sigma$ & - & - & $m$ \\
\hline (๖) unsoy & n & 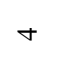 & m & - & n & $m$ & $N$ & 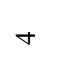 & $\sim$ & $m$ & $n$ & - & N \\
\hline (๖) ue!̣uəə & $N$ & - & 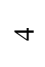 & N & - & a & - & + & $\sim$ & m & n & - & + \\
\hline e!pey & 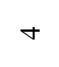 & n & N & - & N & a & n & + & $\sim$ & u & - & n & $\sim$ \\
\hline suər & in & 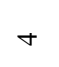 & - & $\nabla$ & N & $m$ & $n$ & $m$ & - & $\sigma$ & $n$ & $n$ & m \\
\hline se!ynew & - & n & N & 4 & a & + & + & m & - & 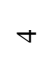 & n & $n$ & - \\
\hline 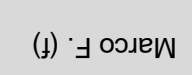 & $N$ & $\sigma$ & m & - & - & - & $N$ & $n$ & $\sim$ & m & in & - & m \\
\hline r osew & - & a & + & m & - & m & N & + & + & m & n & $n$ & - \\
\hline oue!̣ue & n & N & - & $N$ & - & - & $n$ & + & - & - & $n$ & - & N \\
\hline (๖) еə!sser & N & $\nabla$ & $\mathrm{N}$ & in & in & n & 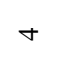 & - & + & in & - & - & n \\
\hline- & 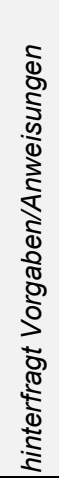 & 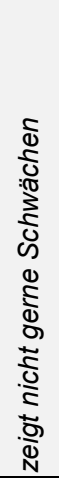 & 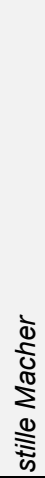 & 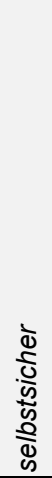 & 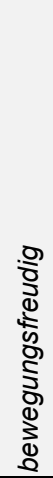 & 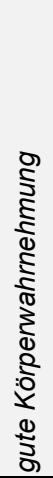 & 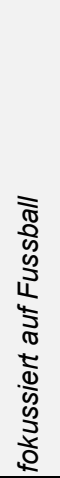 & 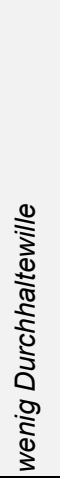 & 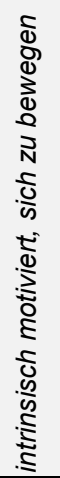 & 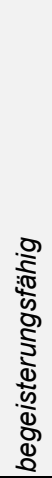 & 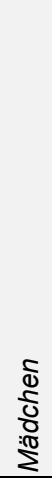 & $\begin{array}{l}\bar{\Phi} \\
\frac{\Phi}{2} \\
\frac{\pi}{0} \\
\frac{3}{\alpha}\end{array}$ & $\begin{array}{l}\text { है } \\
\text { tे } \\
\text { d्ञ }\end{array}$ \\
\hline I & $\bar{x}$ & $\widetilde{\widetilde{x}}$ & $\underline{\tilde{s}}$ & 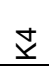 & $\stackrel{\mathscr{p}}{\underline{x}}$ & $\underline{q}$ & $\hat{x}$ & $\underline{\infty}$ & $\stackrel{\mathscr{P}}{\underline{y}}$ & 올 & $\overline{\bar{x}}$ & $\stackrel{\bar{I}}{\bar{I}}$ & $\frac{m}{\underline{x}}$ \\
\hline
\end{tabular}




\begin{tabular}{|c|c|c|c|c|c|c|c|c|c|c|c|c|c|}
\hline n & 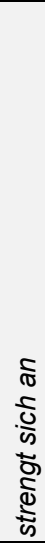 & $\frac{\overrightarrow{3}}{\pi}$ & 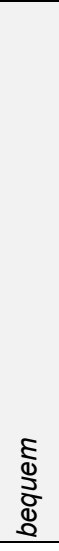 & $\begin{array}{l}0 \\
: 5 \\
: 5 \\
\frac{0}{00} \\
\frac{0}{0} \\
\end{array}$ & 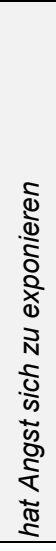 & 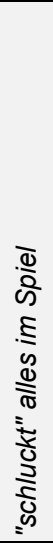 & 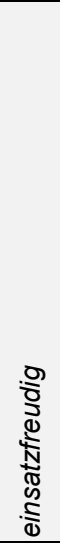 & 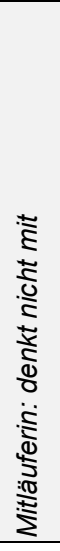 & 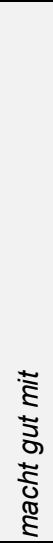 & 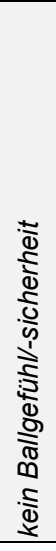 & 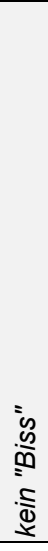 & 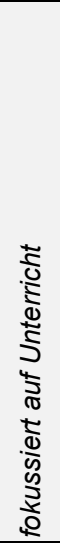 & 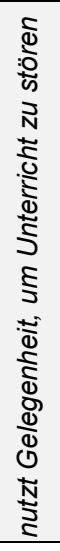 \\
\hline S-ןеәp| & n & $n$ & - & N & - & n & $n$ & $\sim$ & in & a & - & $n$ & - \\
\hline (Ł) еиоәя & m & m & m & N & N & - & $m$ & $n$ & $n$ & N & n & $\sim$ & $n$ \\
\hline вu!ฺәg & + & $n$ & - & - & - & - & in & $\sim$ & in & - & - & 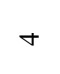 & - \\
\hline (๖) eun7 & - & - & $n$ & in & - & $n$ & - & in & - & + & in & - & $n$ \\
\hline (๖) nu!^ & - & - & $n$ & in & - & N & - & n & - & $N$ & in & - & $n$ \\
\hline (๖) !чәхЕ|әС & + & + & a & $\sim$ & in & n & + & $n$ & $N$ & in & N & $\sim$ & - \\
\hline ess!ue & r & 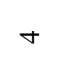 & $\sim$ & $N$ & $ナ$ & $n$ & + & $n$ & $\sim$ & $ナ$ & N & $\sim$ & $\sim$ \\
\hline (๖) ецәઘ & m & n & m & n & - & N & m & n & n & a & m & m & $\sim$ \\
\hline (๖) esury & m & n & $\nabla$ & N & n & N & N & n & n & 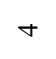 & n & $\sim$ & $\nabla$ \\
\hline (ł) вuәләS & + & in & - & - & n & - & in & - & ナ & - & - & 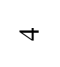 & + \\
\hline eluy & n & in & - & - & + & $n$ & in & - & in & - & - & + & - \\
\hline- & $\underset{\varpi}{\Phi}$ & 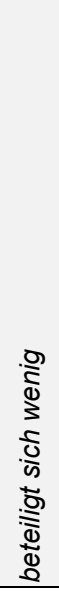 & 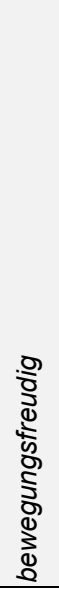 & $\begin{array}{l}\frac{D}{N} \\
\mathbb{D} \\
\stackrel{D}{0}\end{array}$ & 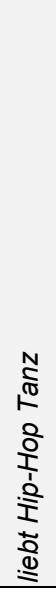 & 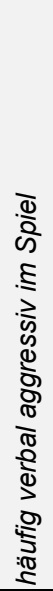 & 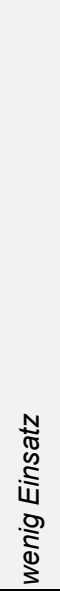 & 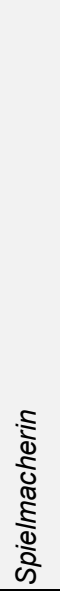 & 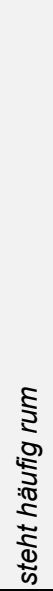 & 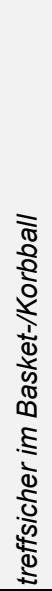 & 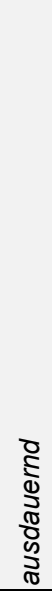 & 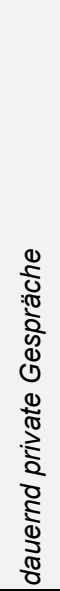 & 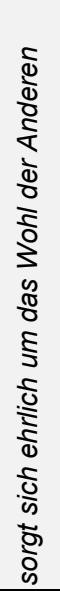 \\
\hline 3 & $\bar{x}$ & $\underline{\widetilde{Y}}$ & $\underline{\tilde{y}}$ & 吉 & $\stackrel{0}{\underline{n}}$ & $\underline{\mathscr{Q}}$ & $\underline{x}$ & 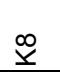 & $\underline{9}$ & $\stackrel{ }{\dot{x}}$ & $\overline{\bar{x}}$ & $\stackrel{\mathfrak{N}}{\underline{I}}$ & $\stackrel{m}{\underline{I}}$ \\
\hline
\end{tabular}




\begin{tabular}{|c|c|c|c|c|c|c|c|c|c|c|c|c|c|}
\hline in & 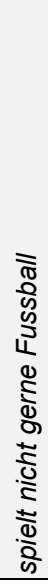 & 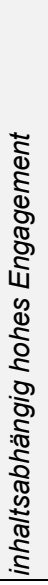 & 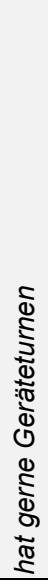 & 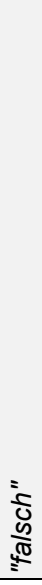 & 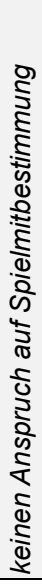 & 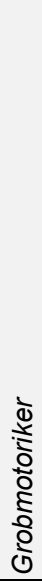 & 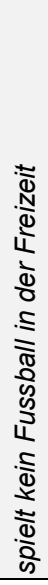 & 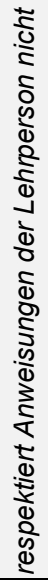 & 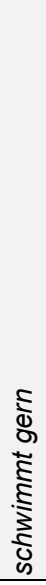 & 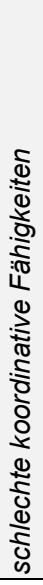 & 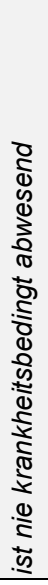 & 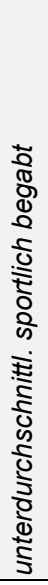 & 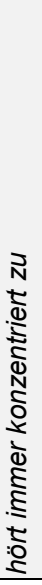 \\
\hline S-ן|eәp| & - & - & $n$ & - & $N$ & - & - & - & $n$ & - & $\nabla$ & $N$ & in \\
\hline (๖) ss!up I & - & $\sim$ & - & $m$ & $m$ & $\nabla$ & $m$ & N & - & $\theta$ & $\theta$ & t & $N$ \\
\hline eu!̣u & - & - & in & - & $m$ & - & $\nabla$ & - & $\nabla$ & - & $\sigma$ & - & + \\
\hline (๖) еب!ә|е $\wedge$ & $N$ & - & $N$ & $N$ & n & n & $\nabla$ & N & $m$ & $m$ & $\sigma$ & $m$ & $n$ \\
\hline ọpne|כ & - & - & $N$ & - & $\sim$ & m & $N$ & - & $m$ & $N$ & + & $m$ & $m$ \\
\hline ןeג!eW & - & - & in & - & m & - & $m$ & - & $m$ & - & $\nabla$ & - & $\nabla$ \\
\hline (๖) ! ! $\forall$ & - & - & - & $m$ & $N$ & $m$ & - & $N$ & - & $m$ & $\nabla$ & $m$ & $N$ \\
\hline (๖) |әપગ̣யәZ & - & - & - & $m$ & - & $m$ & - & $N$ & - & $m$ & $\nabla$ & $m$ & $N$ \\
\hline eu!əдәS & $n$ & - & $m$ & - & $n$ & $m$ & $n$ & - & $m$ & $m$ & $\nabla$ & $m$ & $\theta$ \\
\hline edneך & $m$ & - & $m$ & N & $\nabla$ & $\sim$ & in & - & $m$ & m & $\theta$ & $m$ & 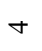 \\
\hline- & 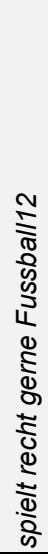 & 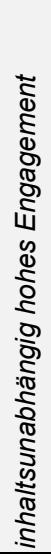 & 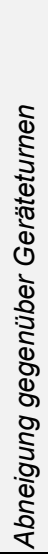 & 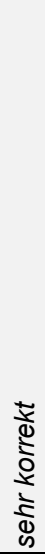 & 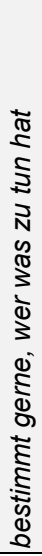 & 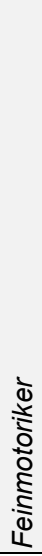 & 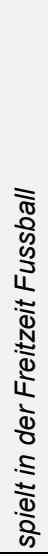 & 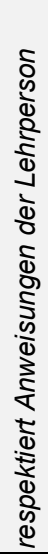 & 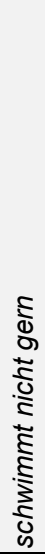 & 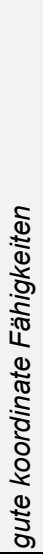 & 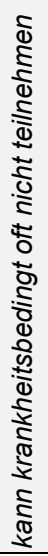 & 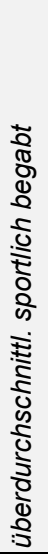 & 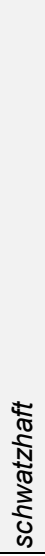 \\
\hline$\underbrace{0}$ & $\bar{z}$ & $\widetilde{\widetilde{V}}$ & $\stackrel{\tilde{m}}{\underline{y}}$ & 志 & $\underline{\underline{u}}$ & $\underline{\underline{z}}$ & $\underline{x}$ & $\underline{\infty}$ & $\underline{0}$ & $\stackrel{ }{\underline{x}}$ & $\underline{\bar{\Sigma}}$ & $\stackrel{\stackrel{N}{\check{I}}}{\underline{T}}$ & $\stackrel{m}{\check{r}}$ \\
\hline
\end{tabular}




\begin{tabular}{|c|c|c|c|c|c|c|c|c|c|c|c|c|c|}
\hline n & 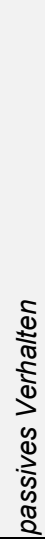 & 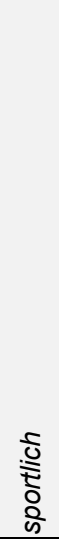 & 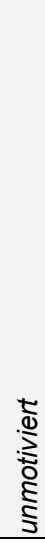 & 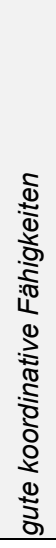 & 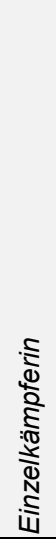 & 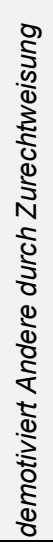 & 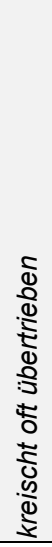 & 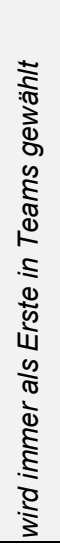 & 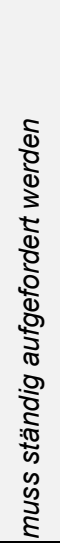 & 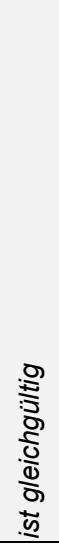 & 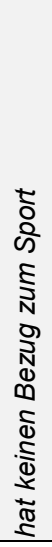 & 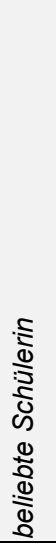 & 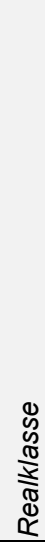 \\
\hline S-|еәp| & $\sim$ & in & - & ナ & n & - & u & $m$ & - & - & - & n & $\mathrm{N}$ \\
\hline 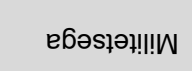 & r & $N$ & + & $N$ & $m$ & $m$ & n & $N$ & + & 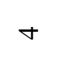 & $n$ & $\sim$ & $n$ \\
\hline e!pneןว & $m$ & m & $N$ & $N$ & $\nabla$ & + & m & - & m & $m$ & + & - & $n$ \\
\hline (ł) ə[!!ques & m & N & - & N & N & u & + & $m$ & $N$ & - & $m$ & + & $n$ \\
\hline (๖) e!!!lejeN & n & 4 & $n$ & a & m & $m$ & m & - & $n$ & $\nabla$ & in & - & in \\
\hline (๖) „nueuəs & $N$ & $\nabla$ & - & $\checkmark$ & $N$ & $ナ$ & $ナ$ & $m$ & - & - & $m$ & $\nabla$ & $n$ \\
\hline (ł) eue!a & N & ナ & N & t & - & - & - & ナ & a & - & $m$ & $\nabla$ & - \\
\hline (๖) ex!ysəy & $\sim$ & $\nabla$ & N & in & - & - & $N$ & 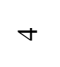 & - & - & $m$ & $n$ & - \\
\hline (Ł) eu|əs & - & $n$ & - & in & - & N & - & $\nabla$ & - & - & - & in & - \\
\hline (๖) дnu!swəs & N & in & N & n & - & 4 & 4 & $n$ & $N$ & - & m & in & - \\
\hline eu!nqes & - & in & - & n & - & - & - & n & - & - & - & in & - \\
\hline- & 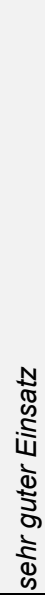 & 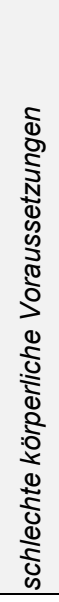 & 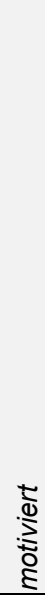 & 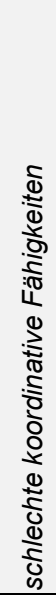 & 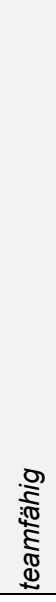 & 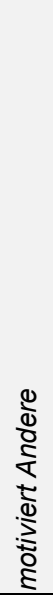 & 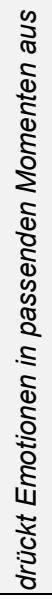 & 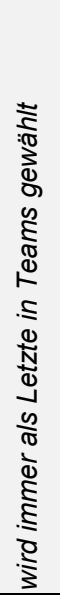 & 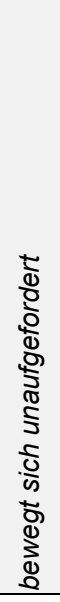 & 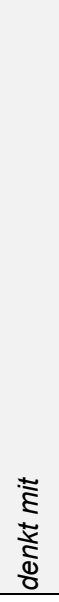 & 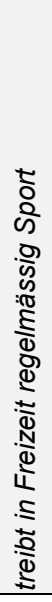 & 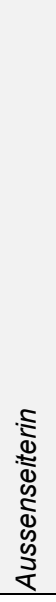 & 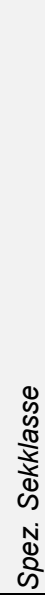 \\
\hline$\Xi$ & $\underline{\bar{x}}$ & $\underline{\widetilde{T}}$ & $\stackrel{\tilde{m}}{\underline{x}}$ & $\mathbb{\pm}$ & $\stackrel{\underline{0}}{\underline{9}}$ & $\underline{\underline{0}}$ & $\underline{\underline{x}}$ & $\stackrel{\infty}{\underline{\infty}}$ & $\underline{\mathscr{g}}$ & 올 & $\underline{\bar{\Sigma}}$ & $\stackrel{\stackrel{N}{\Sigma}}{\underline{Y}}$ & $\stackrel{m}{\underline{m}}$ \\
\hline
\end{tabular}




\begin{tabular}{|c|c|c|c|c|c|c|c|c|c|c|c|}
\hline in & 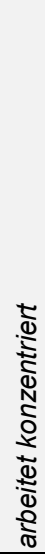 & 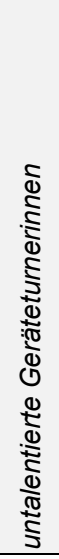 & 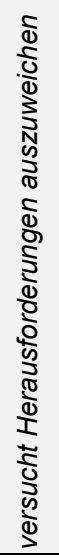 & 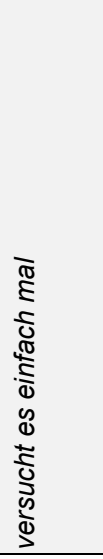 & 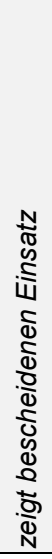 & 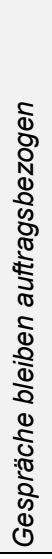 & 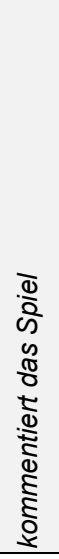 & 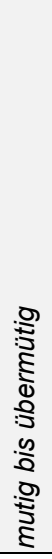 & 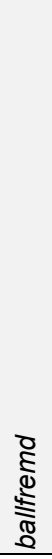 & 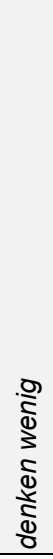 & 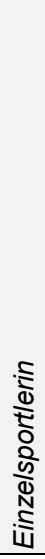 \\
\hline s-|еәр| & $\theta$ & - & - & + & - & $\nabla$ & $\sim$ & + & - & - & $\sim$ \\
\hline (১) ez|ə!a & n & 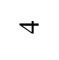 & $\sim$ & ナ & $m$ & $\nabla$ & $\sim$ & - & in & m & ナ \\
\hline !щəоN & ナ & n & $\nabla$ & - & a & m & a & - & 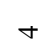 & - & n \\
\hline expues & n & - & - & in & - & in & - & n & - & - & N \\
\hline e!!nर & + & N & - & n & - & $\nabla$ & $\nabla$ & in & N & - & ナ \\
\hline (๖) eue!̣p $\forall$ & ナ & - & a & in & N & 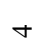 & - & $\nabla$ & - & $N$ & - \\
\hline 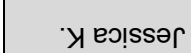 & $\sim$ & $m$ & $m$ & in & $m$ & $\sim$ & $n$ & N & 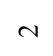 & n & a \\
\hline (๖) еuе!pлt & $\nabla$ & 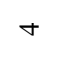 & $\nabla$ & + & $m$ & n & $\nabla$ & N & ナ & $n$ & N \\
\hline yejes & 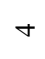 & n & N & 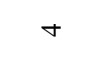 & - & $\nabla$ & - & n & - & - & - \\
\hline uuk & N & 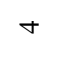 & $n$ & ナ & 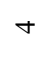 & - & $\nabla$ & - & $m$ & 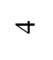 & N \\
\hline (ঊ) eग!sser & $m$ & - & - & in & - & N & - & in & - & - & N \\
\hline- & 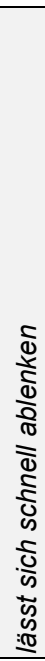 & 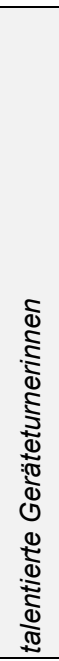 & 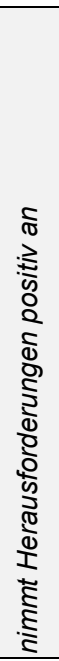 & 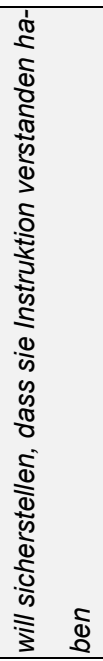 & 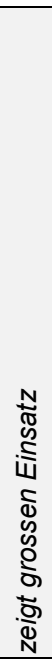 & 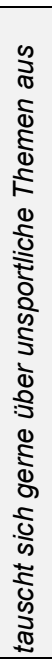 & 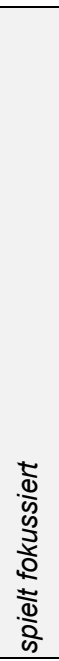 & 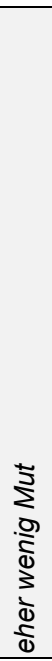 & 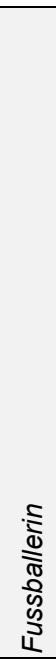 & 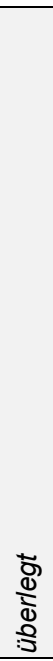 & 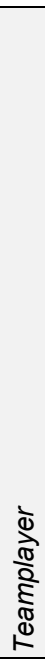 \\
\hline$\stackrel{\infty}{\Theta}$ & $\bar{x}$ & $\underline{\underline{Y}}$ & $\underline{\tilde{m}}$ & $\underline{ \pm}$ & $\underline{\underline{p}}$ & $\underline{\varphi}$ & $\underline{x}$ & $\underline{\infty}$ & $\underline{q}$ & $\stackrel{\circ}{\underline{x}}$ & $\underline{\Sigma}$ \\
\hline
\end{tabular}




\begin{tabular}{|c|c|c|c|c|c|c|c|c|c|c|c|c|}
\hline in & 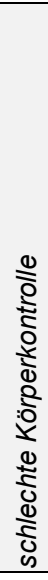 & 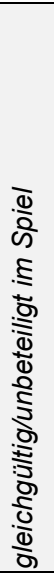 & 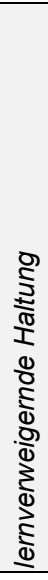 & \begin{tabular}{l}
$\varepsilon$ \\
$\Phi$ \\
\multirow{2}{\sigma}{} \\
$\mathbb{d}$ \\
\end{tabular} & 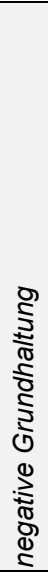 & 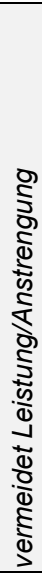 & 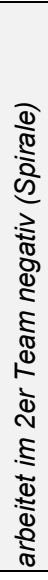 & 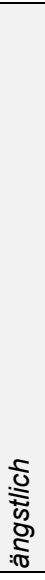 & 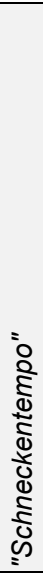 & 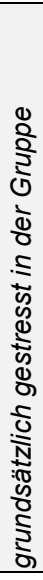 & 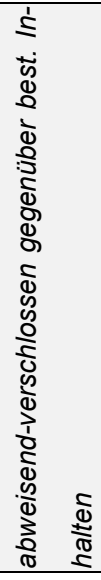 & 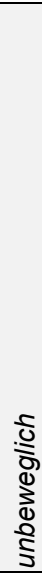 \\
\hline S-ןеәр| & - & - & - & - & - & - & - & - & - & - & - & - \\
\hline иәлS & - & - & $N$ & N & $N$ & $N$ & - & - & - & - & - & - \\
\hline ไ!..pu & - & $N$ & $N$ & $N$ & - & - & - & - & - & - & - & - \\
\hline InN ezאəg & $m$ & $m$ & $m$ & $N$ & $N$ & $N$ & $N$ & $N$ & $m$ & $N$ & $N$ & $m$ \\
\hline елpueəך & $\nabla$ & $m$ & $m$ & $N$ & $N$ & $N$ & - & $N$ & $\nabla$ & - & $N$ & $m$ \\
\hline (t) soxлew & $N$ & - & - & - & - & - & - & - & $N$ & - & - & $N$ \\
\hline$m ! \perp$ & - & - & - & $\sim$ & - & - & $N$ & - & - & - & $N$ & - \\
\hline (Ł) u이 & $m$ & $m$ & $m$ & $m$ & $m$ & $m$ & - & N & $\nabla$ & - & N & $m$ \\
\hline eu!!|əS & $N$ & $N$ & $N$ & $N$ & $N$ & $\sim$ & $N$ & - & $m$ & - & - & $N$ \\
\hline (๖) Ueı!əs & $m$ & $N$ & $m$ & $\sim$ & $N$ & - & - & N & $n$ & - & N & $m$ \\
\hline ә્ર!W & $\alpha$ & - & $N$ & - & - & - & $\alpha$ & - & $\sim$ & - & - & - \\
\hline- & 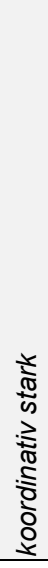 & $\begin{array}{l}0 \\
3 \\
\frac{0}{2} \\
\frac{2}{0} \\
\frac{0}{2}\end{array}$ & 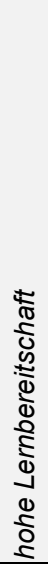 & 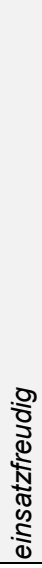 & 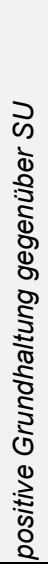 & $\begin{array}{l}\frac{\pi}{0} \\
\frac{0}{0} \\
\frac{0}{5} \\
0 \\
\frac{1}{0} \\
\frac{D}{0}\end{array}$ & 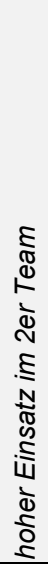 & 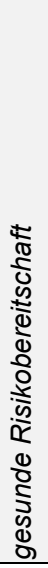 & 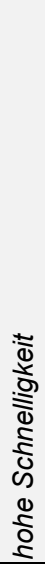 & 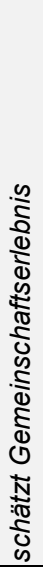 & 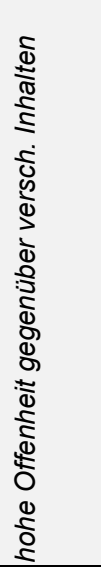 & 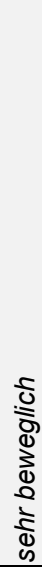 \\
\hline 9 & $\bar{x}$ & $\underline{\widetilde{Y}}$ & $\underline{m}$ & $\underset{ \pm}{ \pm}$ & $\underline{\underline{2}}$ & $\underline{\underline{v}}$ & $\underline{x}$ & $\underline{\infty}$ & $\underline{9}$ & 움 & $\bar{\Sigma}$ & $\stackrel{\mathfrak{N}}{\underline{x}}$ \\
\hline
\end{tabular}




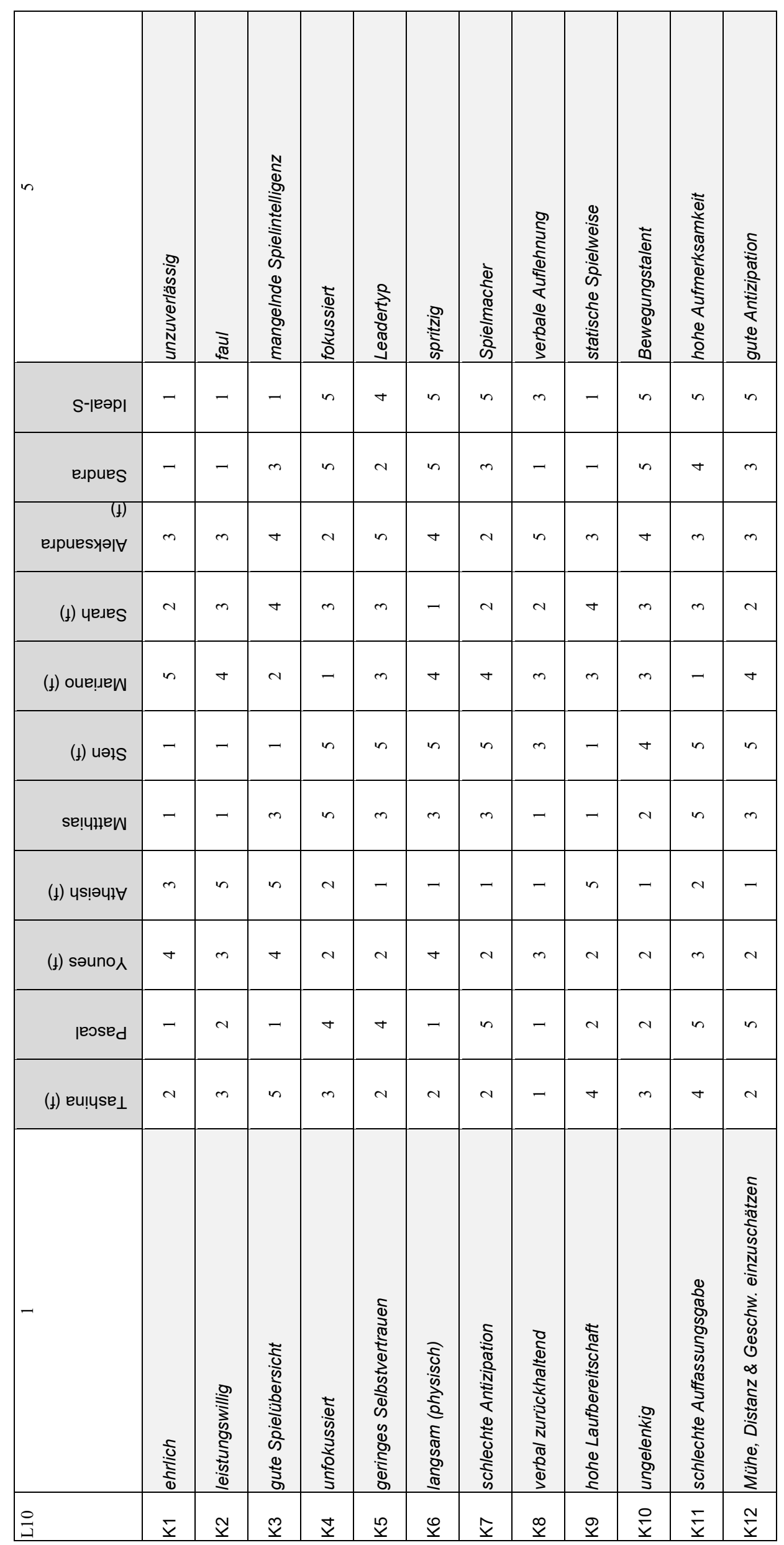




\begin{tabular}{|c|c|c|c|c|c|c|c|c|c|c|c|c|c|}
\hline in & 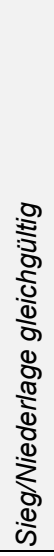 & 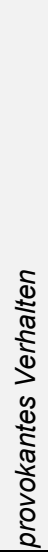 & 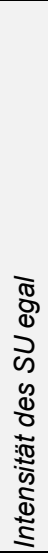 & 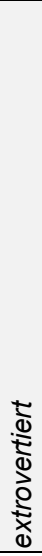 & 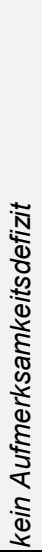 & 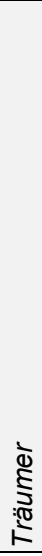 & 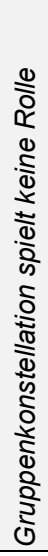 & 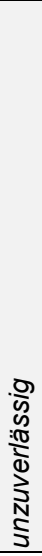 & 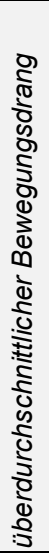 & 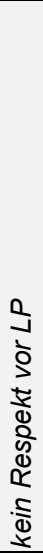 & 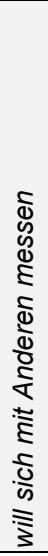 & 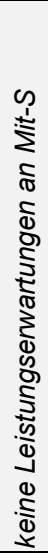 & 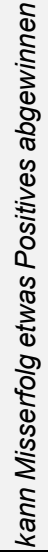 \\
\hline S-ןеәр| & - & $\sim$ & - & $\nabla$ & $\nabla$ & - & 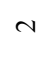 & - & $\nabla$ & - & $\nabla$ & $\sim$ & $n$ \\
\hline seuor & $\nabla$ & $m$ & in & - & $m$ & $n$ & $m$ & 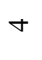 & - & $m$ & $m$ & $\sim$ & $\nabla$ \\
\hline uoy & N & $m$ & $N$ & $\sim$ & $m$ & $\sim$ & $m$ & $\sim$ & $m$ & $m$ & $m$ & $N$ & $\nabla$ \\
\hline onา & - & $\sim$ & $N$ & + & N & $N$ & $N$ & - & $\nabla$ & - & $\nabla$ & $N$ & $n$ \\
\hline uer & $m$ & $m$ & $\nabla$ & $\sim$ & $m$ & $\nabla$ & $n$ & - & $N$ & - & - & $m$ & $n$ \\
\hline x!|ə」 & - & - & - & in & $N$ & - & - & - & in & - & $\nabla$ & - & $\nabla$ \\
\hline seəıpu & $m$ & $m$ & $n$ & $m$ & $m$ & $m$ & $\forall$ & - & - & $N$ & - & $m$ & $\nabla$ \\
\hline (๖) иошлән & - & - & - & $n$ & $N$ & - & - & $N$ & $n$ & $m$ & $n$ & - & - \\
\hline u!ı|^|ә & $N$ & $N$ & $N$ & $n$ & $N$ & $N$ & - & - & $m$ & - & $\nabla$ & $N$ & $N$ \\
\hline se!чમાеW & $m$ & $m$ & $\nabla$ & - & $m$ & in & in & - & $\sim$ & $m$ & - & $m$ & 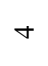 \\
\hline (๖) !ues & - & - & - & $n$ & $N$ & - & - & - & in & N & $n$ & - & $\sim$ \\
\hline- & 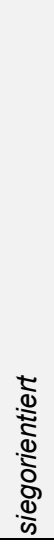 & 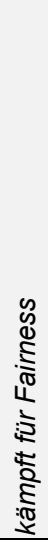 & 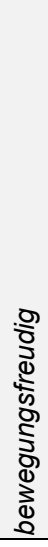 & $\begin{array}{l}\frac{d}{d} \\
\frac{d}{d} \\
\frac{d}{d} \\
: \frac{1}{5} \\
N\end{array}$ & $\begin{array}{l}\bar{d} \\
\frac{2}{0} \\
\frac{1}{0} \\
\frac{1}{N} \\
0 \\
0 \\
0 \\
0\end{array}$ & 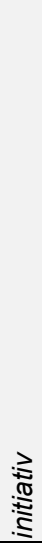 & 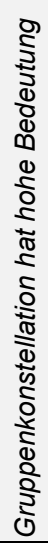 & 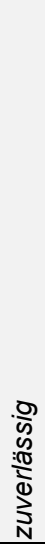 & 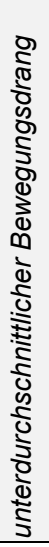 & 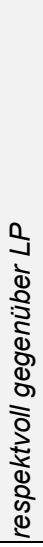 & 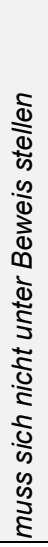 & 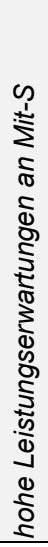 & 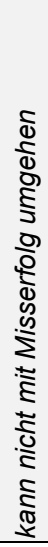 \\
\hline$\Xi$ & $\bar{x}$ & $\underline{\widetilde{Y}}$ & $\underline{m}$ & $\underset{ \pm}{ \pm}$ & $\underline{\ddot{z}}$ & $\underline{x}$ & $\underline{x}$ & $\stackrel{\infty}{\underline{x}}$ & $\underline{9}$ & 음 & $\overline{\bar{x}}$ & $\underset{\mathbb{Y}}{\mathbb{Y}}$ & $\stackrel{m}{\dot{x}}$ \\
\hline
\end{tabular}




\begin{tabular}{|c|c|c|c|c|c|c|c|c|c|c|c|c|c|}
\hline in & 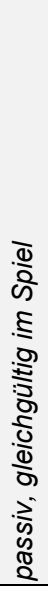 & 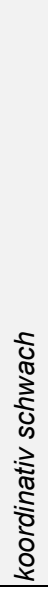 & 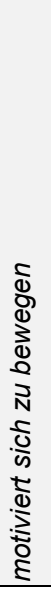 & 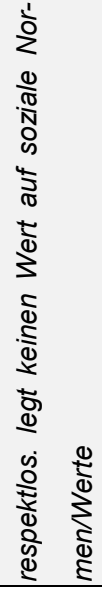 & 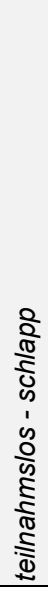 & 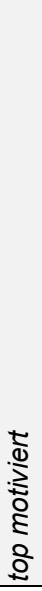 & 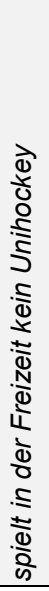 & 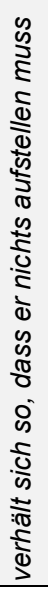 & 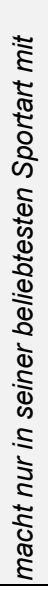 & 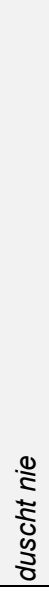 & 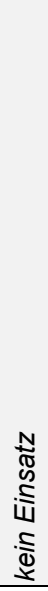 & 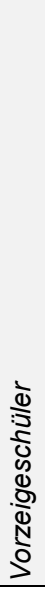 & $\begin{array}{l}\frac{9}{2} \\
\frac{0}{0} \\
\frac{0}{2} \\
\frac{0}{2}\end{array}$ \\
\hline S-|еәрI & $N$ & - & n & - & $\sim$ & $n$ & $\sim$ & - & - & - & - & $n$ & - \\
\hline рuә^S & $m$ & $m$ & n & - & $m$ & n & in & - & - & - & - & $n$ & - \\
\hline ue!̣이 & $m$ & $m$ & in & - & $\sim$ & $n$ & $n$ & - & - & - & - & $n$ & $N$ \\
\hline !e|Oग!N & $\sim$ & - & $n$ & $m$ & $m$ & $N$ & - & $\nabla$ & $N$ & $m$ & $N$ & $n$ & N \\
\hline כ כאו!ו & $\sim$ & $m$ & + & - & $m$ & $\theta$ & n & $\sim$ & $N$ & - & - & $\theta$ & - \\
\hline nə!પાеw & $n$ & $N$ & - & † & in & - & - & in & $\nabla$ & $m$ & $n$ & $\sim$ & $n$ \\
\hline !ㅣ & $m$ & $N$ & $n$ & - & $N$ & $n$ & - & - & - & - & - & in & - \\
\hline (๖) પાәә!̣s & - & - & $n$ & $N$ & $\sim$ & $m$ & $n$ & + & $N$ & $\nabla$ & $N$ & $N$ & $N$ \\
\hline (ı) $)$ l!ms $\forall$ & - & N & $m$ & + & $\sim$ & $N$ & $n$ & 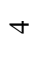 & $n$ & in & $m$ & - & $m$ \\
\hline jeosed & $\nabla$ & $m$ & $\sim$ & $\sim$ & $\nabla$ & $N$ & - & $\nabla$ & $\nabla$ & $N$ & $m$ & $m$ & $m$ \\
\hline ueगגnnbzəo & - & $m$ & $m$ & N & $N$ & $m$ & in & $\nabla$ & $m$ & $\nabla$ & $N$ & $m$ & $N$ \\
\hline- & 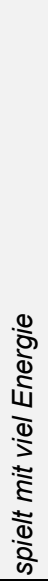 & 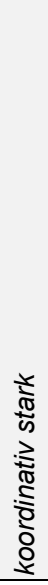 & 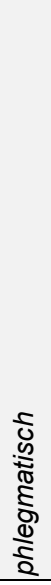 & 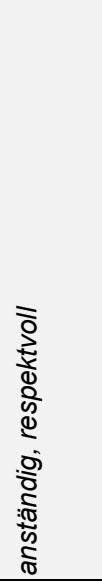 & 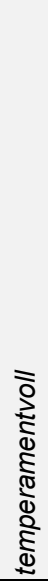 & 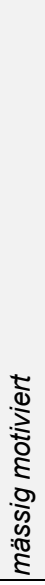 & 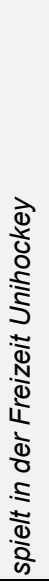 & 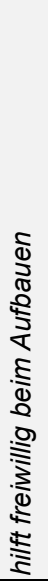 & 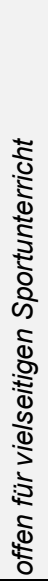 & 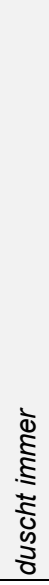 & 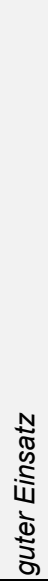 & $\begin{array}{l}\bar{d} \\
: 0 \\
\bar{d} \\
0 \\
\text { ह } \\
\frac{0}{0} \\
0 \\
0\end{array}$ & $\begin{array}{l}\frac{D}{d} \\
\frac{N}{d} \\
\frac{d}{d}\end{array}$ \\
\hline$\Xi$ & $\bar{\Sigma}$ & $\underline{\widetilde{Y}}$ & $\underline{\tilde{O}}$ & $\underline{\mathbf{Z}}$ & $\underline{10}$ & $\underline{\theta}$ & 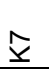 & $\begin{array}{l}\infty \\
\underline{x}\end{array}$ & $\underline{9}$ & 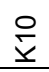 & $\underset{\bar{x}}{\bar{x}}$ & $\underset{\mathbb{Y}}{\stackrel{N}{V}}$ & $\frac{m}{\bar{x}}$ \\
\hline
\end{tabular}




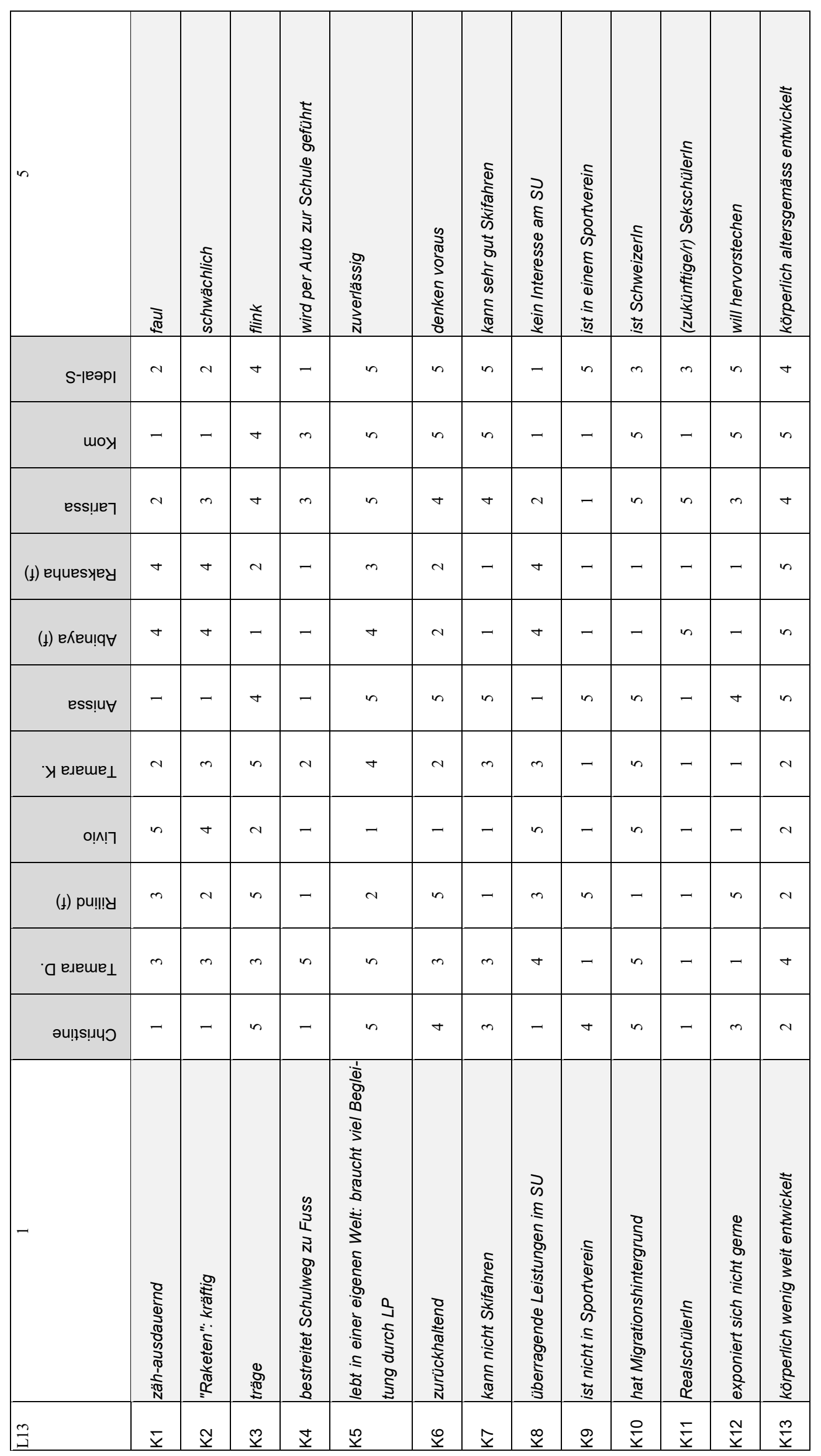




\begin{tabular}{|c|c|c|c|c|c|c|c|c|c|c|c|c|}
\hline in & 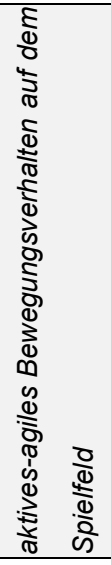 & 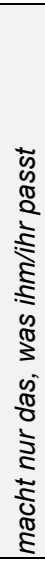 & 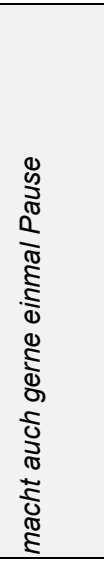 & 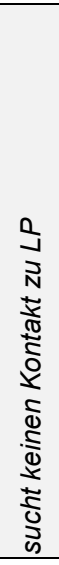 & 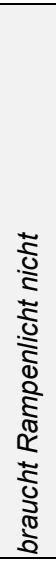 & 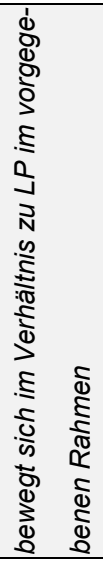 & 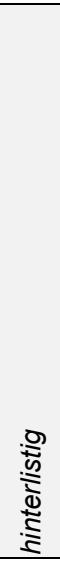 & 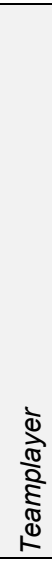 & 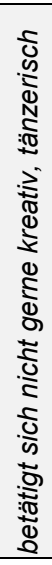 & 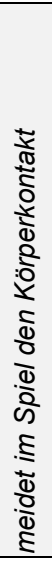 & 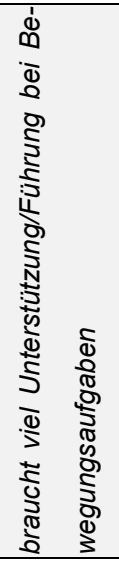 & 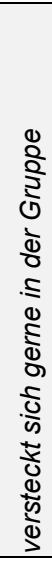 \\
\hline S-|еәр| & $n$ & N & - & N & + & - & - & + & $\sim$ & - & - & - \\
\hline (Ł) и!ฺәəg & N & $m$ & in & n & 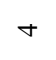 & $\sigma$ & $m$ & N & - & + & in & in \\
\hline (๖) eluuese $\forall$ & 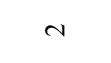 & $m$ & in & n & - & $\alpha$ & m & $\sim$ & - & - & 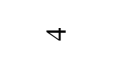 & $m$ \\
\hline 'Id uejəłs & n & N & - & in & in & ナ & $\sigma$ & $m$ & in & - & $N$ & $N$ \\
\hline (๖) челог & ナ & N & N & N & - & - & 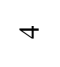 & t & m & m & $m$ & t \\
\hline əગ૦ગ! & in & - & ナ & n & in & n & - & $n$ & n & - & - & - \\
\hline |әEגZ & - & $m$ & $m$ & - & $\sigma$ & $\nabla$ & - & $m$ & in & $\sigma$ & $ナ$ & $\sim$ \\
\hline '시 Uejəłs & in & - & - & in & in & in & - & in & ナ & - & - & - \\
\hline Kuuәr & m & $m$ & in & n & - & m & - & $m$ & - & - & m & $m$ \\
\hline и!цеш & in & N & - & N & 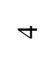 & - & 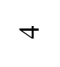 & t & in & - & - & - \\
\hline (๖) pe!!W & $\lambda$ & 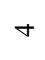 & 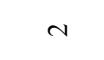 & - & 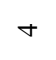 & N & + & $\sim$ & + & N & in & in \\
\hline- & 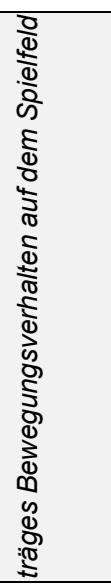 & 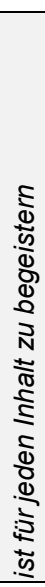 & 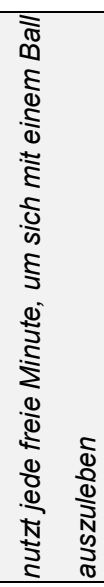 & 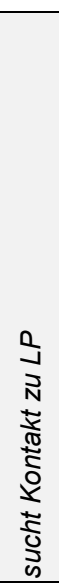 & 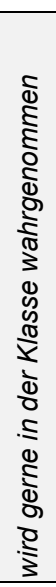 & 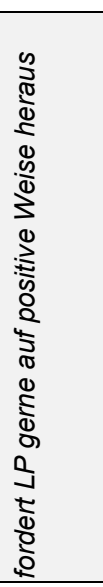 & 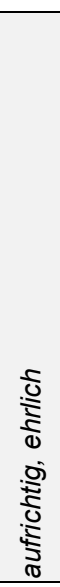 & 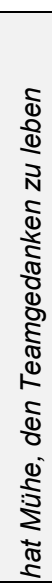 & 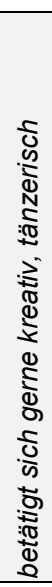 & 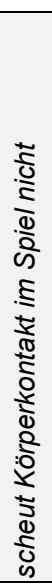 & 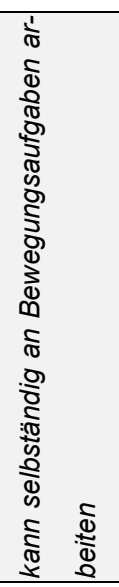 & 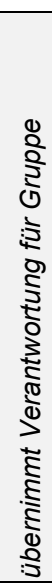 \\
\hline$\Xi$ & $\bar{x}$ & $\widetilde{\widetilde{Y}}$ & $\underline{\underline{y}}$ & 吾 & $\stackrel{\mathscr{L}}{\underline{y}}$ & $\underline{q}$ & $\underline{x}$ & $\stackrel{\infty}{\underline{\perp}}$ & $\stackrel{\mathscr{2}}{\underline{y}}$ & 옴 & $\bar{\Sigma}$ & $\stackrel{\tilde{Y}}{\underline{I}}$ \\
\hline
\end{tabular}




\begin{tabular}{|c|c|c|c|c|c|c|c|c|c|c|c|c|c|}
\hline in & 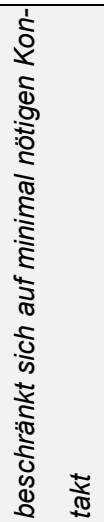 & 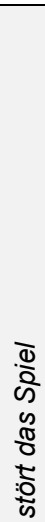 & 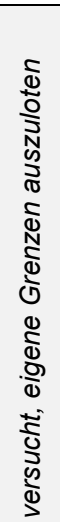 & 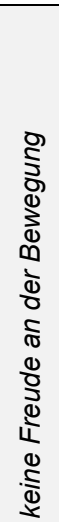 & 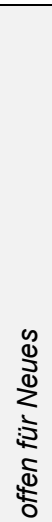 & 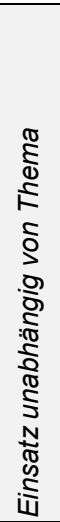 & 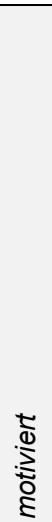 & 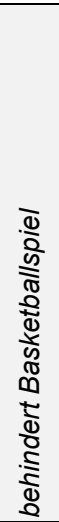 & 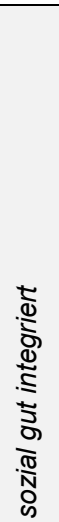 & 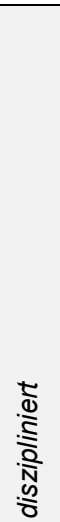 & 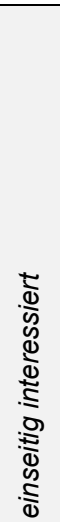 & 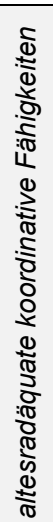 & 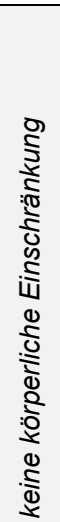 \\
\hline s-ןеәр| & - & $\sim$ & in & - & in & in & n & - & in & in & - & in & $n$ \\
\hline (†) KE!!N & in & - & m & m & N & - & m & - & - & n & in & in & in \\
\hline eə!ssar & $m$ & m & + & N & m & n & + & N & in & m & N & m & - \\
\hline espuesey & m & - & 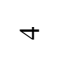 & N & ナ & a & in & - & n & + & N & $n$ & in \\
\hline yejes & - & $\sim$ & $n$ & - & in & $n$ & in & - & $n$ & $n$ & - & in & $n$ \\
\hline ẹu! & + & m & $m$ & $m$ & 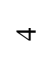 & $m$ & 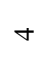 & $\mathrm{N}$ & N & 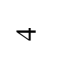 & $m$ & $n$ & in \\
\hline әાә્પગ! & - & $\sim$ & $ナ$ & - & + & + & n & - & in & + & - & $n$ & in \\
\hline me!!! & - & $m$ & $\sim$ & + & $m$ & a & + & m & in & 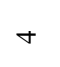 & N & - & N \\
\hline (Ł) ешщ & N & + & N & + & - & N & m & 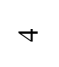 & in & - & m & n & n \\
\hline es! & $m$ & ナ & a & ナ & N & $m$ & $m$ & $\nabla$ & in & - & $m$ & 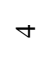 & in \\
\hline (๖) еұәәнэ & $N$ & in & - & in & - & - & - & - & $n$ & - & $m$ & - & $n$ \\
\hline- & 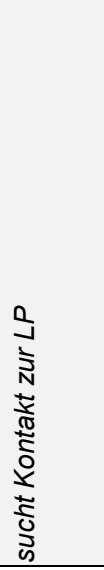 & 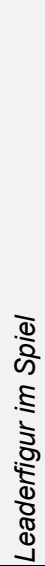 & 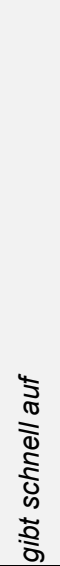 & 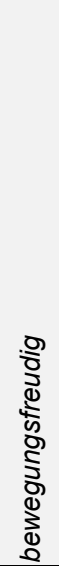 & 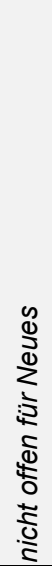 & 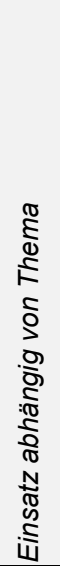 & 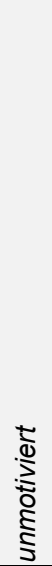 & 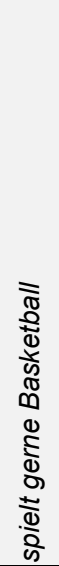 & 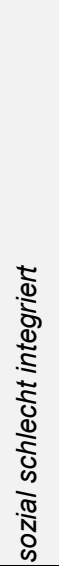 & 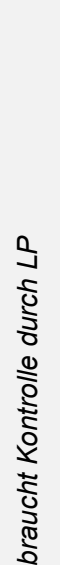 & 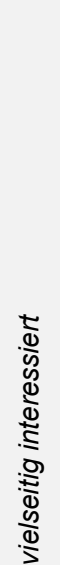 & 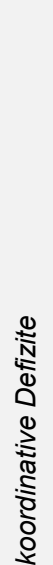 & 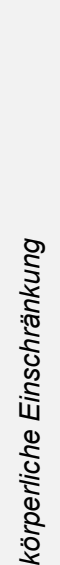 \\
\hline$\dddot{\ddots n}$ & $\underline{\bar{\Sigma}}$ & $\widetilde{\widetilde{x}}$ & $\underline{\tilde{m}}$ & $\underset{\mathbf{I}}{\mathbf{I}}$ & $\underline{\mathscr{R}}$ & $\underline{\varphi}$ & $\underline{\underline{y}}$ & $\stackrel{\infty}{\underline{x}}$ & $\underline{9}$ & 옴 & $\overline{\bar{\Sigma}}$ & $\stackrel{\mathfrak{N}}{\underline{Y}}$ & $\stackrel{m}{\underline{I}}$ \\
\hline
\end{tabular}




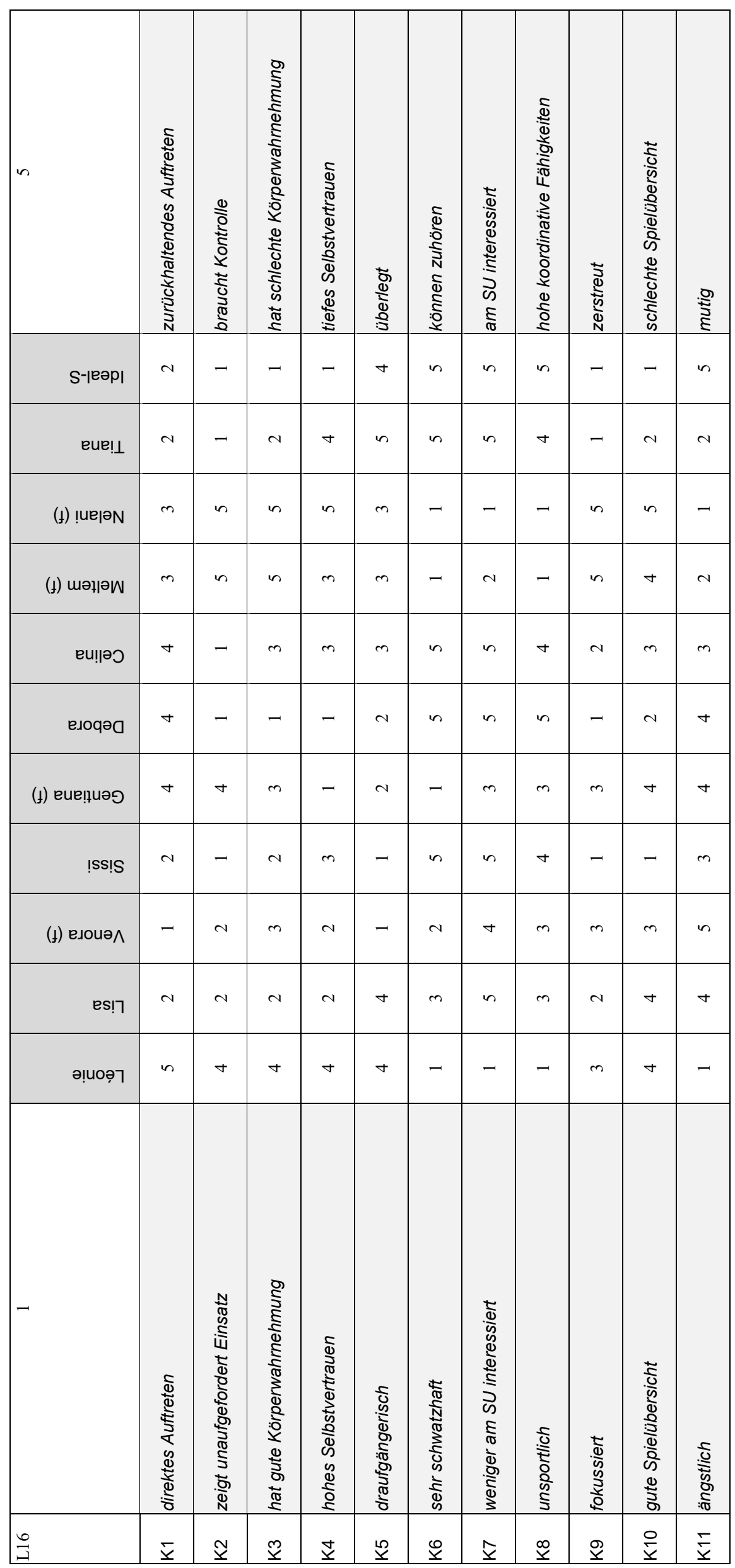

$\stackrel{\sim}{\sim}$ 


\begin{tabular}{|c|c|c|c|c|c|c|c|c|c|c|c|}
\hline n & 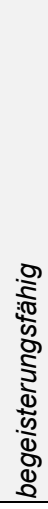 & 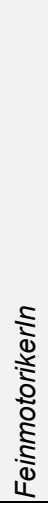 & 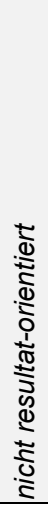 & 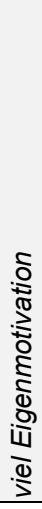 & 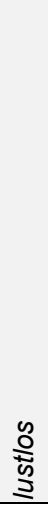 & $\begin{array}{l}\text { P) } \\
\text { है }\end{array}$ & 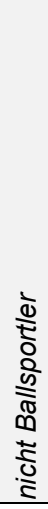 & 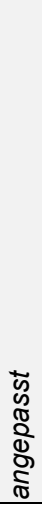 & 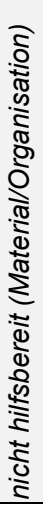 & 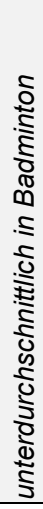 & 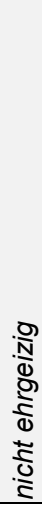 \\
\hline S-ןеәр| & $n$ & $n$ & N & $n$ & - & $n$ & - & - & - & - & - \\
\hline ןənues & + & - & $m$ & $N$ & $\nabla$ & in & $N$ & - & $N$ & - & † \\
\hline p!ıео & $N$ & $\nabla$ & $N$ & - & $n$ & $\nabla$ & $N$ & - & in & 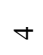 & $n$ \\
\hline (^) деsəg & in & $N$ & $\nabla$ & $n$ & - & in & - & $N$ & $N$ & $\nabla$ & † \\
\hline eגne 7 & $\sim$ & $m$ & - & - & $n$ & - & $n$ & $n$ & $N$ & - & $n$ \\
\hline ןənuew & + & - & in & $\nabla$ & $N$ & $n$ & $N$ & - & - & $N$ & $N$ \\
\hline (๖) $\operatorname{sep} \wedge \wedge \circ 0$ & in & $n$ & $n$ & $n$ & - & $n$ & - & $N$ & - & - & - \\
\hline ( & $n$ & $\nabla$ & in & $n$ & - & in & - & - & in & $N$ & - \\
\hline euuy & - & $\nabla$ & $N$ & $\nabla$ & $N$ & $N$ & - & $\nabla$ & $m$ & $N$ & 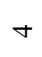 \\
\hline essəue $\wedge$ & - & $N$ & - & $\nabla$ & - & $\neg$ & - & in & - & $N$ & $n$ \\
\hline (๖) es!l & - & $n$ & in & $n$ & - & in & - & $N$ & $N$ & - & - \\
\hline- & 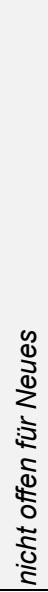 & 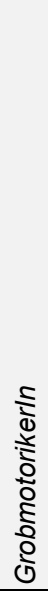 & 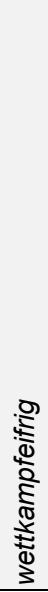 & 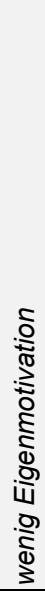 & :D & 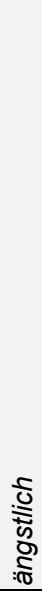 & 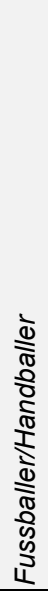 & 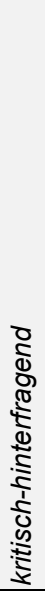 & 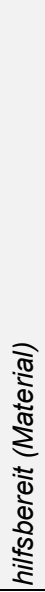 & 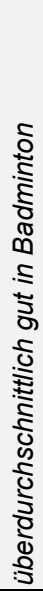 & $\begin{array}{l}\frac{0}{N} \\
\frac{N}{0} \\
\frac{0}{0} \\
\end{array}$ \\
\hline$\exists$ & $\bar{x}$ & $\underline{\widetilde{T}}$ & $\stackrel{\mathscr{q}}{\underline{x}}$ & 妾 & $\stackrel{0}{\underline{n}}$ & $\underline{x}$ & $\underline{x}$ & $\stackrel{\infty}{x}$ & $\stackrel{9}{x}$ & 움 & $\bar{\Sigma}$ \\
\hline
\end{tabular}




\begin{tabular}{|c|c|c|c|c|c|c|c|c|c|c|c|c|c|}
\hline in & 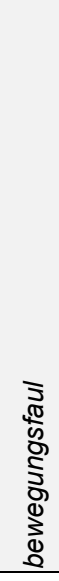 & 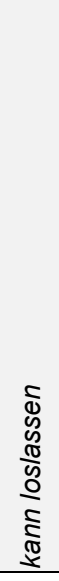 & 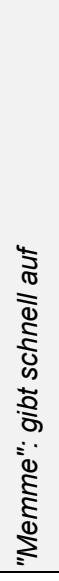 & 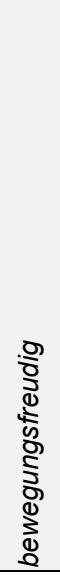 & 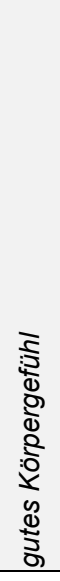 & 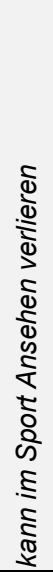 & 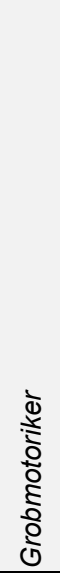 & 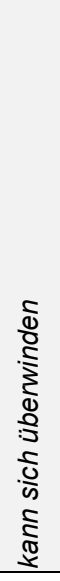 & 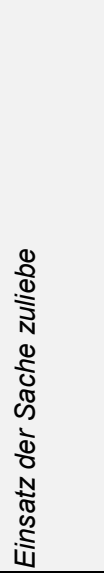 & 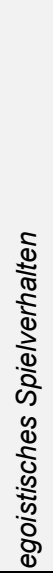 & 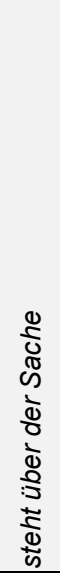 & 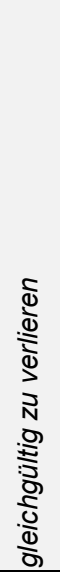 & 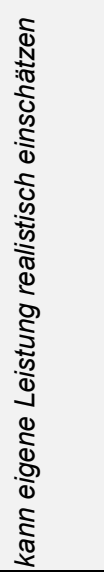 \\
\hline S-|еәp| & - & $n$ & - & $n$ & $n$ & - & $m$ & $n$ & in & - & in & $N$ & $\sigma$ \\
\hline елеуеw & $n$ & - & n & $m$ & $m$ & $m$ & $m$ & N & $N$ & $\sigma$ & - & $m$ & $N$ \\
\hline ue!qe日 & - & in & - & in & $\sigma$ & a & $ナ$ & $n$ & n & $\nabla$ & $n$ & - & N \\
\hline рәчеу & - & 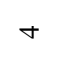 & - & in & $n$ & - & v & $n$ & in & $N$ & $N$ & - & $\sigma$ \\
\hline ордеоэ!у & + & 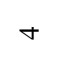 & n & $\nabla$ & $m$ & m & 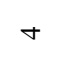 & $m$ & m & $m$ & $m$ & - & N \\
\hline (t) uole & 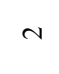 & $ナ$ & $N$ & 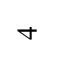 & $n$ & - & n & 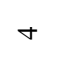 & $\nabla$ & n & $\sim$ & - & 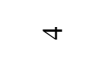 \\
\hline uอ!!ฺ!̣૫ว & N & $ナ$ & - & in & N & - & in & $\nabla$ & 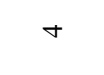 & $\nabla$ & $ナ$ & - & ナ \\
\hline oxew & - & in & - & $n$ & $n$ & - & 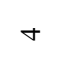 & in & n & N & in & N & in \\
\hline 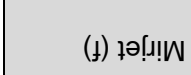 & - & in & - & in & $n$ & - & $n$ & in & in & - & in & N & in \\
\hline u!qoy & in & $n$ & $\nabla$ & - & $\nabla$ & $N$ & $\nabla$ & $N$ & $\nabla$ & - & in & $\sigma$ & in \\
\hline- & 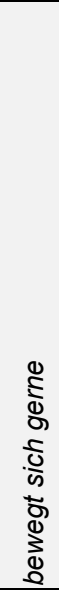 & 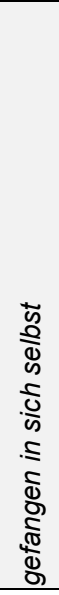 & 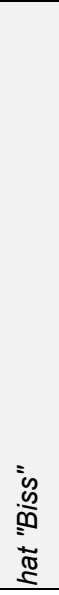 & 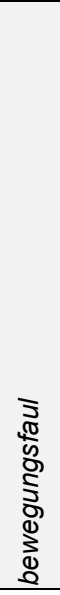 & 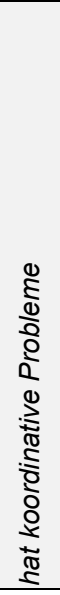 & 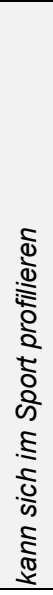 & 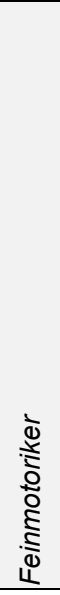 & 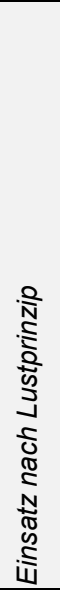 & 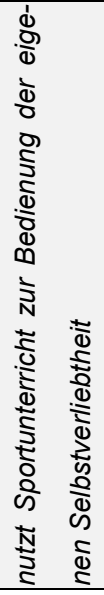 & 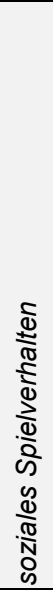 & 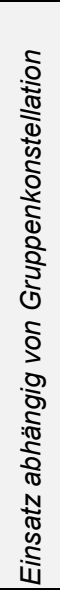 & 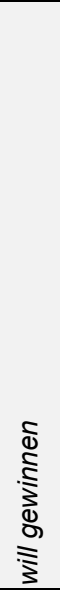 & 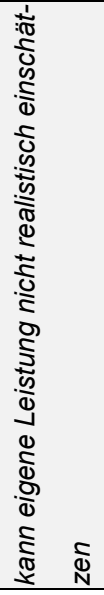 \\
\hline$\stackrel{\infty}{\exists}$ & $\underline{\bar{x}}$ & $\underline{\widetilde{Y}}$ & $\underline{\tilde{x}}$ & $\underset{\Sigma}{ \pm}$ & $\underline{\underline{p}}$ & $\underline{\underline{q}}$ & $\underline{\underline{x}}$ & $\underline{\infty}$ & $\underline{\mathscr{g}}$ & 옴 & $\overline{\bar{\Sigma}}$ & $\stackrel{\mathfrak{I}}{\underline{I}}$ & $\stackrel{m}{\underline{m}}$ \\
\hline
\end{tabular}




\begin{tabular}{|c|c|c|c|c|c|c|c|c|c|c|c|c|}
\hline in & 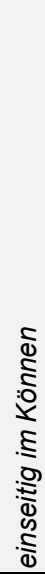 & 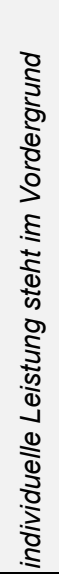 & 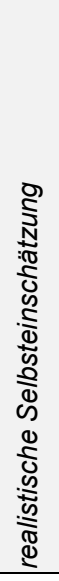 & 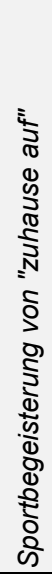 & 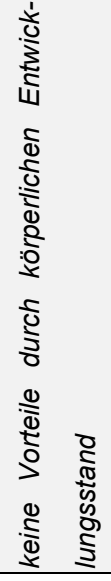 & 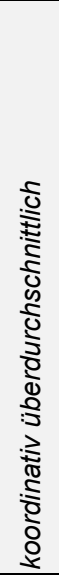 & 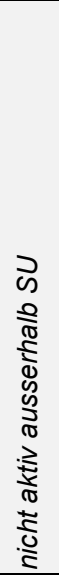 & 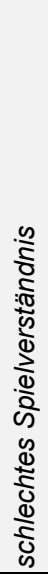 & 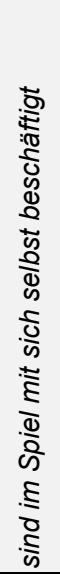 & 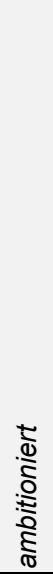 & 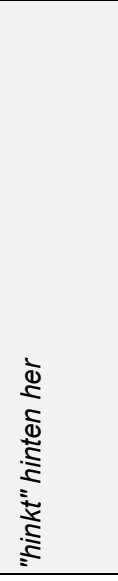 & 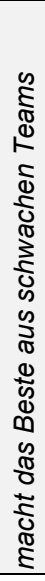 \\
\hline S-|еәр| & - & $m$ & $n$ & + & a & in & - & - & - & 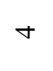 & m & in \\
\hline euer & N & N & n & t & t & ナ & a & a & - & 4 & m & $m$ \\
\hline (๖) jeq!ueH & - & in & $N$ & $n$ & in & in & $N$ & - & - & + & - & N \\
\hline (Ł) & t & a & $n$ & 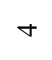 & 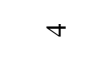 & - & $n$ & N & $n$ & - & in & n \\
\hline ue!̣p $\forall$ & $m$ & u & $n$ & a & ナ & n & m & ナ & 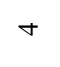 & 4 & ナ & in \\
\hline (๖) nopeurw & $\sim$ & + & - & $\theta$ & m & in & - & N & m & $\nabla$ & N & n \\
\hline әuा़e्र & - & m & ナ & in & m & 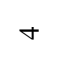 & - & N & N & + & a & in \\
\hline (๖) $e K_{I I I}$ & N & 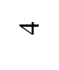 & - & ナ & - & + & - & $\sim$ & m & in & a & N \\
\hline 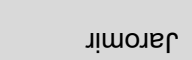 & - & in & $n$ & r & - & $n$ & - & - & - & in & - & in \\
\hline ןәנеW & $m$ & s & n & a & in & - & $\nabla$ & 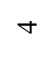 & 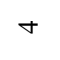 & - & ナ & n \\
\hline x!|ઇ & ナ & $m$ & 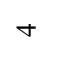 & - & in & a & 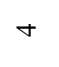 & n & in & - & $\checkmark$ & m \\
\hline- & 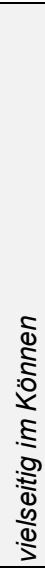 & 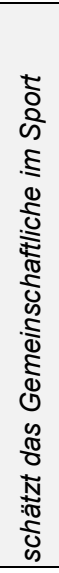 & 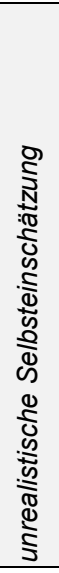 & 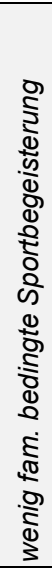 & 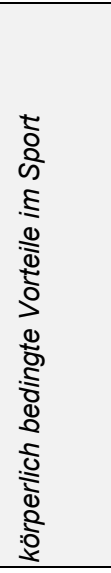 & 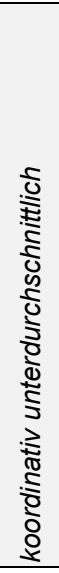 & 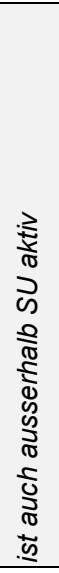 & 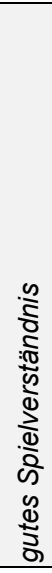 & 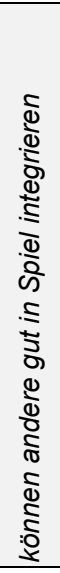 & 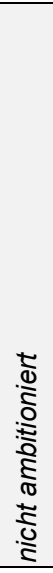 & 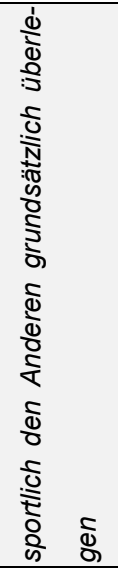 & 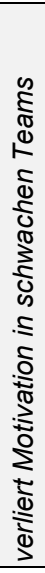 \\
\hline tr & $\underline{\Sigma}$ & $\underline{\widetilde{Y}}$ & $\underline{\tilde{m}}$ & $\underline{ \pm}$ & $\stackrel{\mathscr{b}}{\underline{x}}$ & $\underline{q}$ & $\underline{\underline{x}}$ & $\stackrel{\infty}{\underline{\infty}}$ & $\underline{9}$ & $\stackrel{\circ}{\underline{Y}}$ & $\bar{\Sigma}$ & $\underline{\underline{Y}}$ \\
\hline
\end{tabular}




\begin{tabular}{|c|c|c|c|c|c|c|c|c|c|c|}
\hline in & 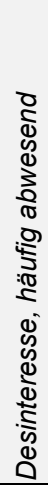 & 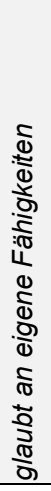 & 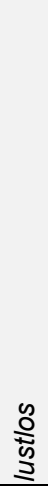 & $\begin{array}{l}\stackrel{0}{1} \\
\frac{0}{0} \\
\frac{0}{0} \\
\frac{0}{0}\end{array}$ & 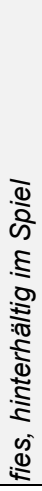 & $\begin{array}{l}\frac{1}{0} \\
\text { s } \\
\frac{1}{0} \\
0 \\
0\end{array}$ & 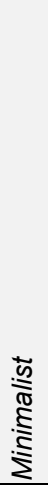 & 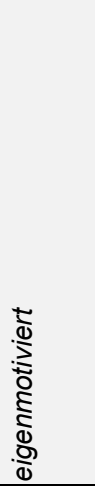 & 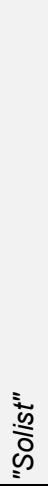 & 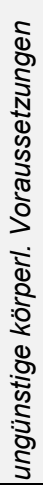 \\
\hline S-|еәр| & - & $n$ & - & $N$ & - & $n$ & - & $\theta$ & $m$ & - \\
\hline (๖) łəu! 시 & $m$ & $\sim$ & N & $\sim$ & N & $\sim$ & $m$ & $\sim$ & N & - \\
\hline (Ł) о!!! $\omega_{\exists}$ & $m$ & n & N & $m$ & + & $N$ & $\forall$ & $N$ & $m$ & $\nabla$ \\
\hline (๖) еu!ı & ナ & $\sim$ & $m$ & $\nabla$ & $\sim$ & $N$ & $m$ & $N$ & $N$ & $m$ \\
\hline eग̣ssər & - & $n$ & - & - & N & $n$ & - & in & $\nabla$ & - \\
\hline (๖) ue!!Z & $\nabla$ & $\sim$ & $\nabla$ & $\nabla$ & - & - & $\nabla$ & - & - & $m$ \\
\hline (๖) ןејиечว & - & $\nabla$ & $N$ & N & $N$ & $\nabla$ & - & 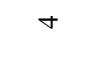 & $N$ & - \\
\hline แ!^әך & $N$ & $m$ & - & N & - & $\nabla$ & $\sim$ & $\checkmark$ & N & $\sim$ \\
\hline (๖) s!̣uəa & - & $\nabla$ & - & N & - & $m$ & $N$ & 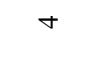 & $\nabla$ & - \\
\hline (๖) uezo & $\sim$ & $\nabla$ & - & - & $m$ & + & - & N & $m$ & in \\
\hline ^ & - & $\nabla$ & - & - & $m$ & $n$ & - & 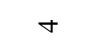 & $m$ & N \\
\hline- & 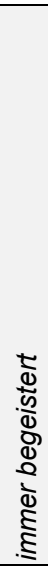 & 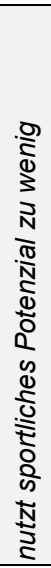 & 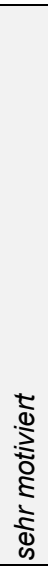 & $\begin{array}{l}\frac{D}{d} \\
\frac{N}{d} \\
\frac{d}{d}\end{array}$ & 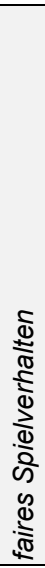 & 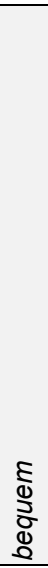 & 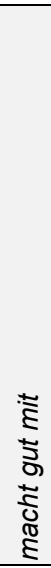 & 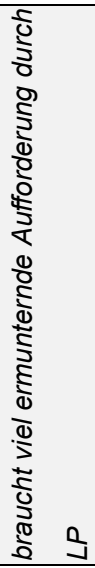 & 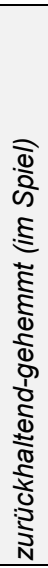 & 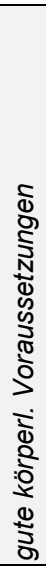 \\
\hline స్త & $\bar{z}$ & $\underline{\pi}$ & $\underline{m}$ & $\underline{ \pm}$ & $\underline{x}$ & $\underline{\mathscr{Q}}$ & $\underline{x}$ & $\stackrel{\infty}{\underline{\infty}}$ & $\underline{9}$ & 음 \\
\hline
\end{tabular}




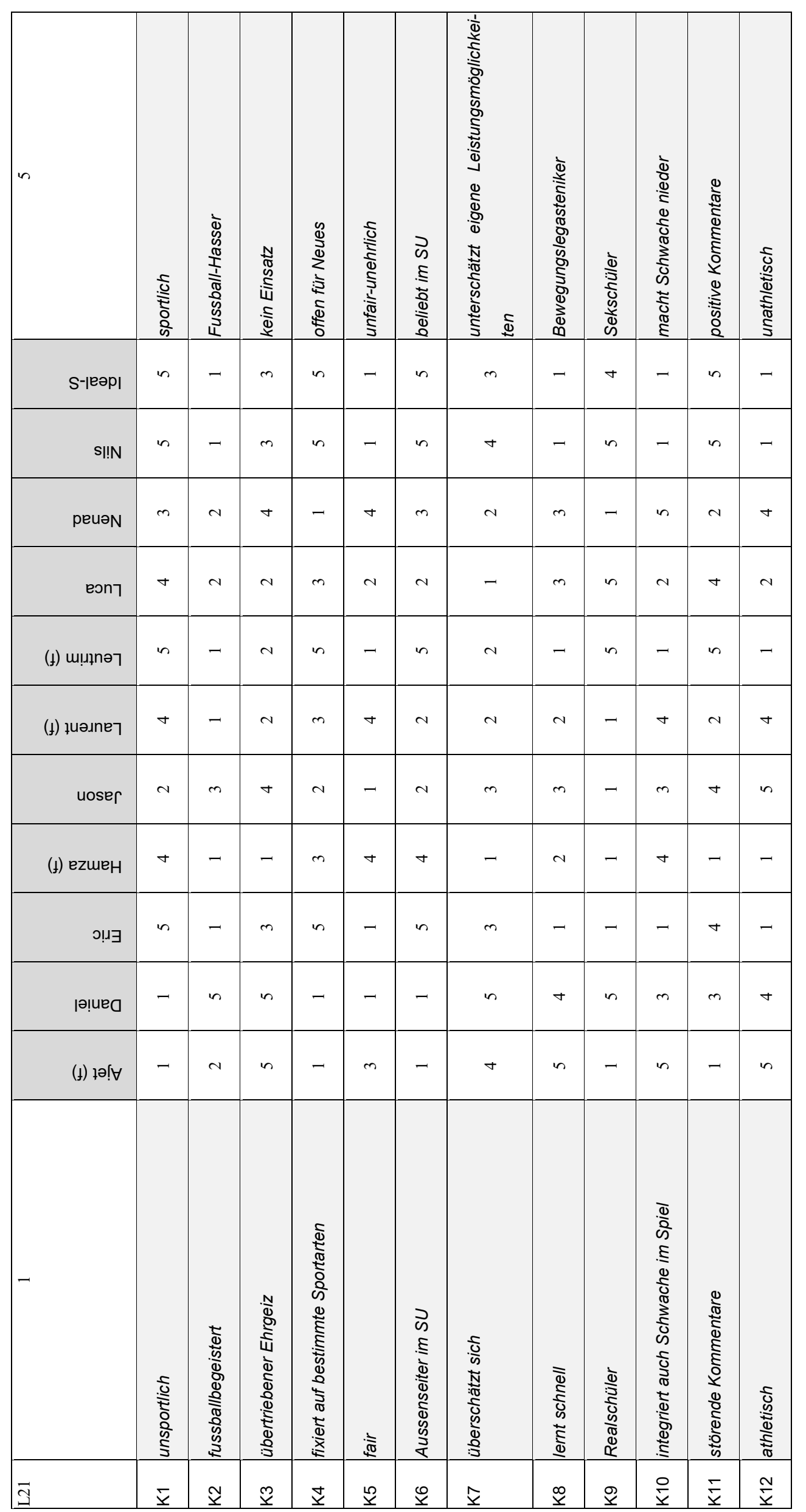




\begin{tabular}{|c|c|c|c|c|c|c|c|c|c|c|c|}
\hline in & 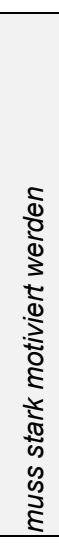 & 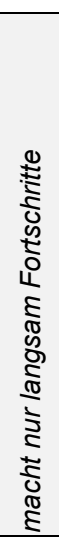 & 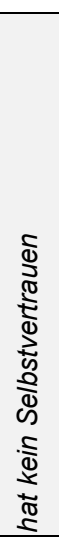 & 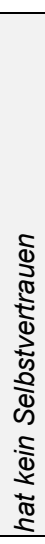 & 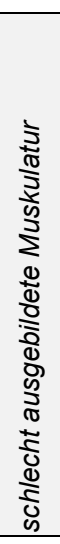 & 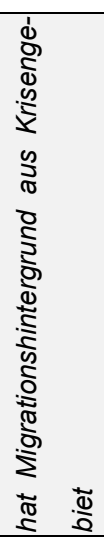 & 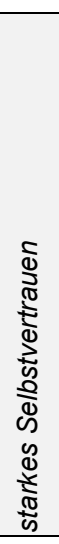 & $\begin{array}{l}\text { : } \\
\text { है } \\
\text { है }\end{array}$ & 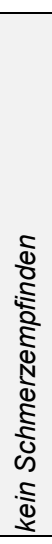 & 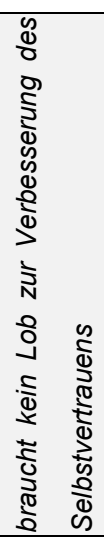 & 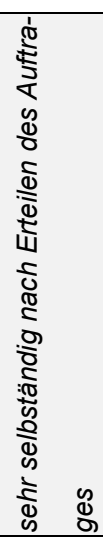 \\
\hline s-ןeәp| & - & - & $m$ & - & - & ' & $n$ & in & $m$ & $m$ & in \\
\hline (ł) us!̣uey & - & - & n & - & - & in & in & in & + & ナ & in \\
\hline (๖) uedı!Yy $\forall$ & m & N & m & - & n & in & m & n & $m$ & $m$ & in \\
\hline u씨이커 & - & - & m & - & - & - & $n$ & in & $\checkmark$ & + & in \\
\hline әи!|ә઼ & $m$ & $N$ & $\sim$ & t & $N$ & $N$ & + & + & $m$ & - & $m$ \\
\hline (Һ) иoəㅅ & $m$ & $m$ & $N$ & - & $\sigma$ & n & $m$ & n & - & - & $\nabla$ \\
\hline essəue $\wedge$ & - & $m$ & $n$ & $\checkmark$ & - & $\alpha$ & in & in & $N$ & - & $m$ \\
\hline exlues & N & in & - & 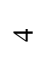 & $\sigma$ & $m$ & in & in & N & - & - \\
\hline (†) o!̣sely & $N$ & $m$ & n & - & $m$ & $m$ & $n$ & $m$ & $m$ & $m$ & $m$ \\
\hline es!n & - & - & n & - & - & - & $\nabla$ & 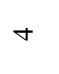 & 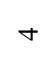 & m & $\nabla$ \\
\hline әม!พ & in & + & a & $\sim$ & in & m & a & - & n & - & $N$ \\
\hline- & 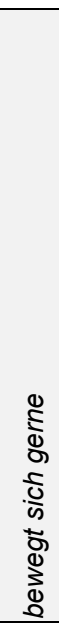 & 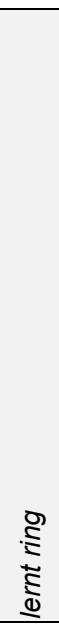 & 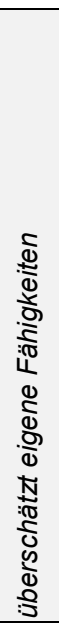 & 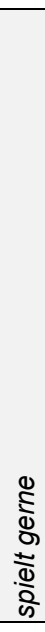 & 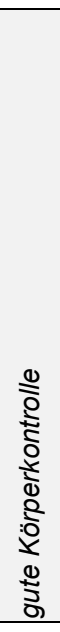 & 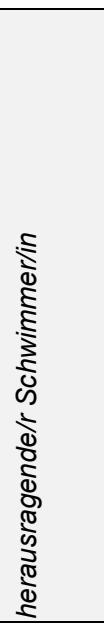 & 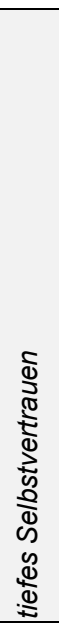 & 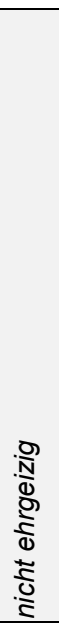 & 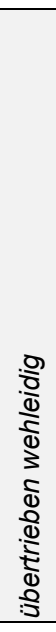 & 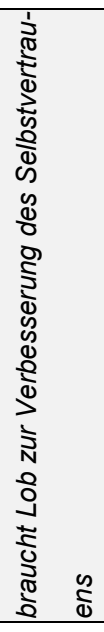 & 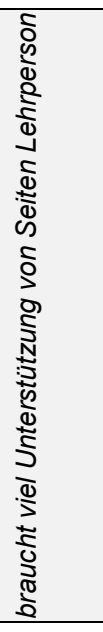 \\
\hline İ & $\underline{\bar{x}}$ & $\underline{\underline{Y}}$ & $\underline{\tilde{x}}$ & $\underline{\underline{x}}$ & $\underline{\underline{a}}$ & $\underline{q}$ & $\underline{x}$ & $\stackrel{\infty}{\underline{\infty}}$ & $\underline{\rho}$ & 옴 & $\overline{\bar{\Sigma}}$ \\
\hline
\end{tabular}




\begin{tabular}{|c|c|c|c|c|c|c|c|c|c|c|c|c|}
\hline n & 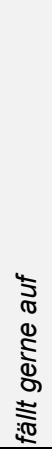 & 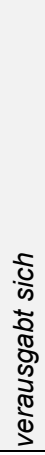 & 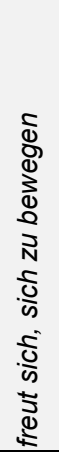 & 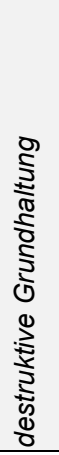 & 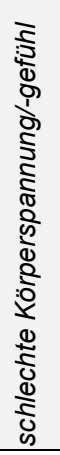 & 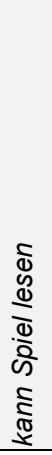 & 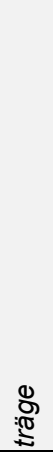 & 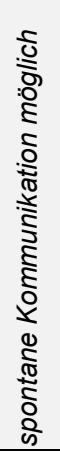 & 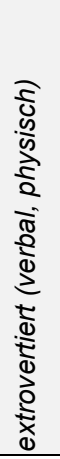 & 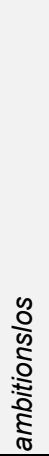 & 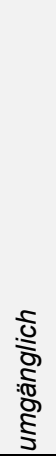 & 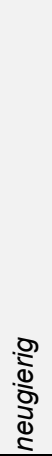 \\
\hline S-|eәp| & $m$ & in & $\nabla$ & - & $\sim$ & in & $\sim$ & in & $n$ & $N$ & $n$ & $\nabla$ \\
\hline 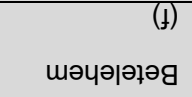 & $N$ & ナ & $\nabla$ & - & $\sim$ & $n$ & $\sim$ & - & n & N & $n$ & $\nabla$ \\
\hline әu!peN & $m$ & N & n & n & $\sim$ & + & n & n & + & m & + & $\sigma$ \\
\hline әңәમәઢฺd & N & - & N & $ナ$ & n & - & $n$ & $n$ & n & $\checkmark$ & 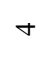 & $\sim$ \\
\hline (๖) & $n$ & m & $\sigma$ & $\sim$ & $\sim$ & + & t & $n$ & n & m & m & in \\
\hline enuew & $\nabla$ & $N$ & $N$ & n & + & - & $\nabla$ & $n$ & $\nabla$ & 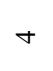 & - & $\nabla$ \\
\hline (ఫ) Koụno & - & n & n & - & - & n & - & n & - & - & n & t \\
\hline uode $\forall$ & in & N & 4 & 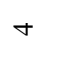 & 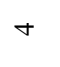 & 4 & in & n & in & 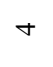 & - & m \\
\hline (Ł) одриеә & in & + & + & $\sim$ & - & $n$ & n & n & n & m & - & in \\
\hline әฺщег & $\sim$ & in & $n$ & - & - & $n$ & $\sim$ & $n$ & $n$ & $\sim$ & in & ナ \\
\hline (Ł) 이어 d $_{d}$ & - & m & $\forall$ & - & $\sim$ & $n$ & + & - & - & m & in & N \\
\hline- & 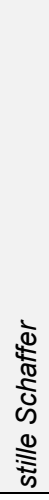 & 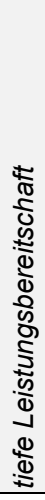 & 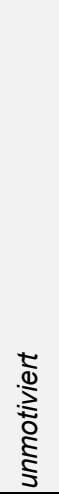 & 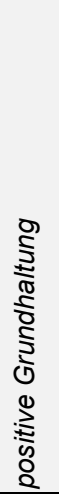 & 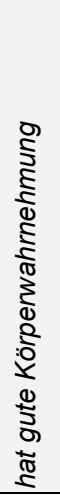 & 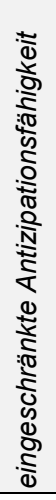 & 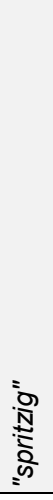 & 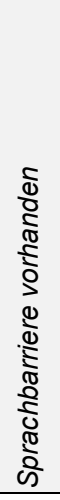 & 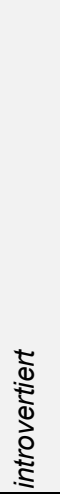 & 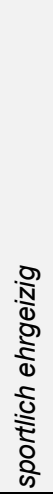 & 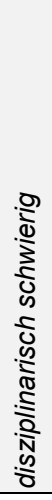 & 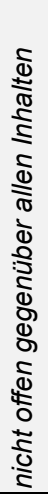 \\
\hline$\tilde{3}$ & $\underline{\bar{x}}$ & $\underline{\tilde{v}}$ & $\underline{\tilde{x}}$ & 吉 & $\underline{\underline{p}}$ & $\underline{\underline{0}}$ & $\underline{x}$ & $\underline{\infty}$ & $\underline{\mathscr{g}}$ & $\stackrel{ }{\underline{x}}$ & $\underline{\Sigma}$ & $\underline{\underline{Y}}$ \\
\hline
\end{tabular}




\begin{tabular}{|c|c|c|c|c|c|c|c|c|c|c|c|}
\hline in & 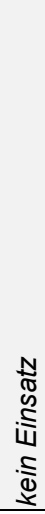 & 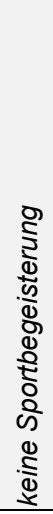 & 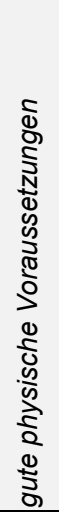 & $\begin{array}{l}3 \\
\text { के } \\
\frac{0}{2} \\
\text { के }\end{array}$ & 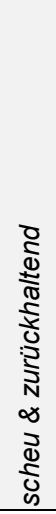 & 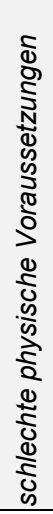 & 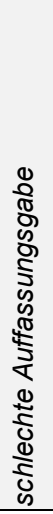 & 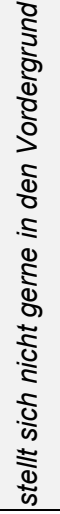 & 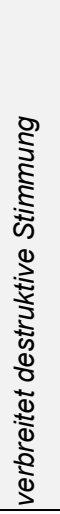 & 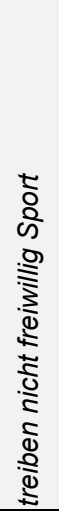 & $\begin{array}{l}\bar{\Phi} \\
\Phi \\
\Phi \\
\unlhd\end{array}$ \\
\hline s-|еәр| & - & v & $\nabla$ & in & $\sim$ & - & - & - & - & - & $\sigma$ \\
\hline (๖) eग!ssər & - & $m$ & $\sigma$ & ナ & N & N & - & - & $\sim$ & $\sigma$ & ナ \\
\hline (ł) euı!ej & - & $m$ & $\nabla$ & ナ & $\nabla$ & $m$ & - & $\nabla$ & n & $\nabla$ & m \\
\hline soJ!n & - & - & $n$ & N & - & - & n & - & - & - & in \\
\hline (ł) uem|əS & $\sim$ & $N$ & - & - & $N$ & m & - & - & - & - & in \\
\hline әશણ઼પગ!พ & $m$ & $\nabla$ & $N$ & $\checkmark$ & $n$ & in & - & n & - & in & - \\
\hline $\mathrm{OO!N}$ & - & - & in & in & - & - & ナ & - & - & - & n \\
\hline (ł) eppoy & n & in & $\sim$ & $ナ$ & in & in & $\sim$ & $\nabla$ & in & $n$ & - \\
\hline (ঊ) pגenp $\exists$ & $m$ & 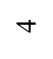 & $n$ & $\sim$ & N & m & $\sim$ & - & - & 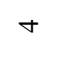 & $\sigma$ \\
\hline (๖) 티이 $\forall$ & - & 4 & $n$ & N & - & - & $\sim$ & $N$ & $\nabla$ & N & ナ \\
\hline 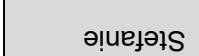 & - & N & - & ナ & N & N & $n$ & + & - & N & $m$ \\
\hline- & 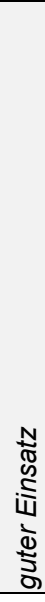 & 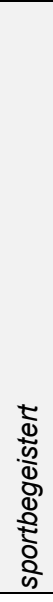 & 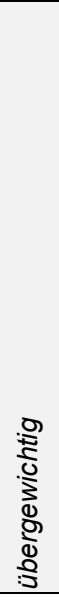 & 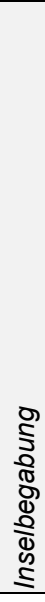 & 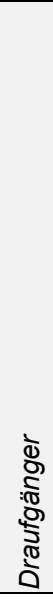 & 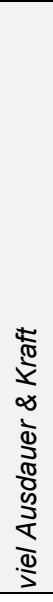 & 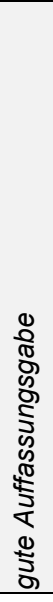 & 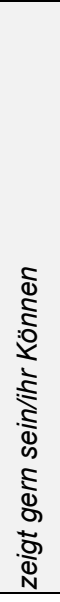 & 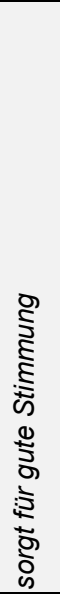 & 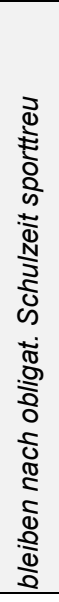 & 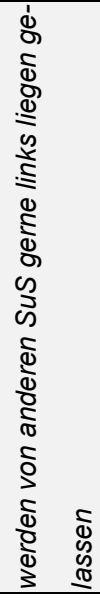 \\
\hline İ & $\bar{x}$ & $\tilde{\underline{x}}$ & $\tilde{\underline{x}}$ & 吉 & $\stackrel{b}{\underline{p}}$ & $\ddot{q}$ & $\underline{x}$ & $\stackrel{\infty}{\check{x}}$ & $\stackrel{\mathscr{P}}{\underline{y}}$ & $\stackrel{\circ}{\underline{Y}}$ & $\bar{\Sigma}$ \\
\hline
\end{tabular}




\begin{tabular}{|c|c|c|c|c|c|c|c|c|c|c|c|c|}
\hline in & 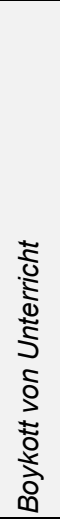 & 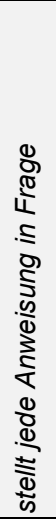 & 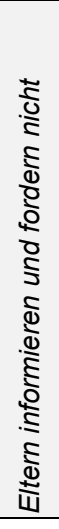 & 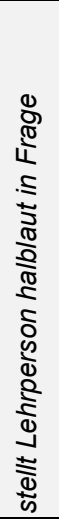 & 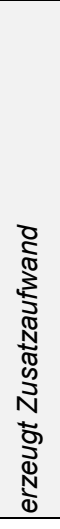 & 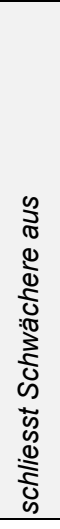 & 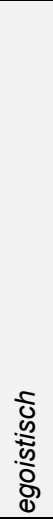 & 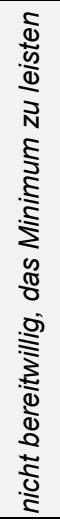 & 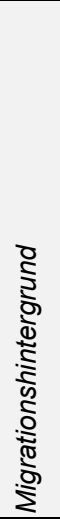 & 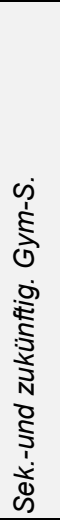 & 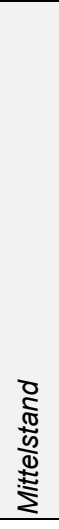 & 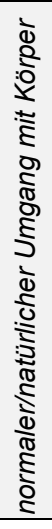 \\
\hline s-|eәp| & $\sim$ & N & a & $\sim$ & $\sim$ & a & n & $a$ & - & in & m & in \\
\hline exes & - & - & - & - & - & - & - & - & - & in & $n$ & $n$ \\
\hline 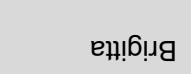 & - & - & - & - & - & - & - & - & $\sim$ & in & $n$ & $n$ \\
\hline Едә^ & $N$ & $\sim$ & $N$ & $\sim$ & - & - & - & $N$ & - & in & $n$ & $n$ \\
\hline EגKY & a & a & - & $\sim$ & $\sim$ & - & N & m & - & n & 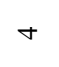 & + \\
\hline elue $\perp$ & - & - & - & - & - & - & - & $m$ & - & in & 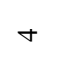 & $n$ \\
\hline es!7 & - & - & a & $\sim$ & $\sim$ & - & - & - & - & in & $n$ & n \\
\hline e!n!7 & n & m & n & in & in & - & in & in & - & - & 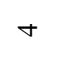 & a \\
\hline eu!|әw & N & $\sim$ & $\mathrm{u}$ & $m$ & $\sim$ & 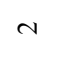 & u & - & - & - & 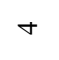 & in \\
\hline (๖) nus!^ & n & n & $n$ & in & in & - & $n$ & $n$ & in & - & - & - \\
\hline (๖) елоunəך & in & in & $n$ & in & $n$ & - & n & $n$ & $n$ & - & - & - \\
\hline- & 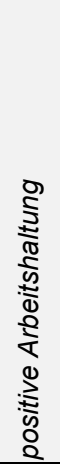 & 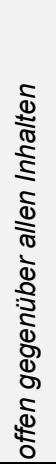 & 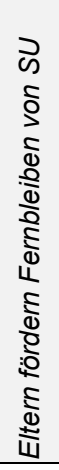 & 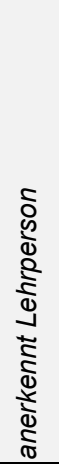 & 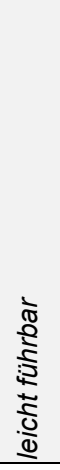 & 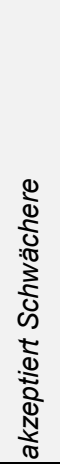 & 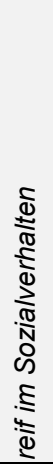 & 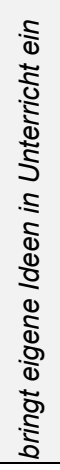 & 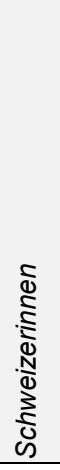 & 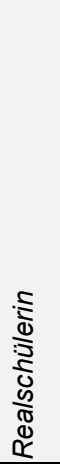 & 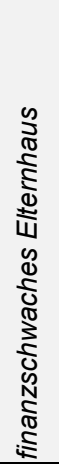 & 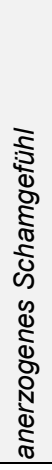 \\
\hline స్త్ & $\bar{x}$ & $\underline{\underline{Y}}$ & $\underline{m}$ & 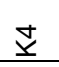 & $\underline{\underline{n}}$ & $\underline{\underline{v}}$ & $\underline{x}$ & $\stackrel{\infty}{\underline{x}}$ & $\underline{\mathscr{g}}$ & $\stackrel{\circ}{\underline{x}}$ & $\bar{\Sigma}$ & $\underline{\underline{Y}}$ \\
\hline
\end{tabular}




\begin{tabular}{|c|c|c|c|c|c|c|c|c|c|c|c|c|c|}
\hline in & 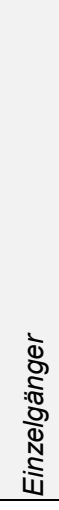 & 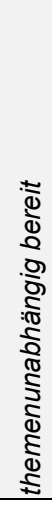 & 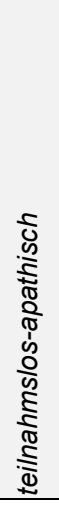 & 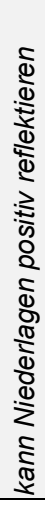 & 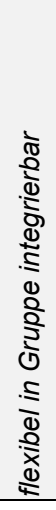 & 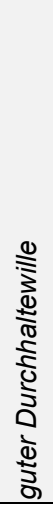 & 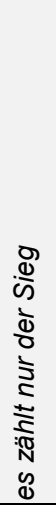 & 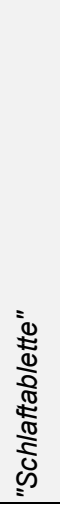 & 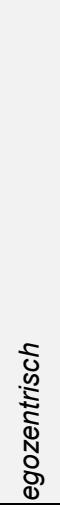 & 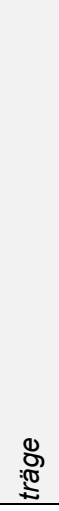 & 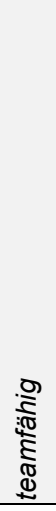 & 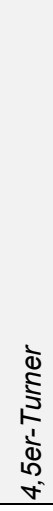 & 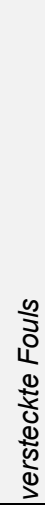 \\
\hline s-|еәp| & $\alpha$ & n & - & $\sigma$ & in & $n$ & $\nabla$ & $m$ & $N$ & - & $n$ & - & - \\
\hline en!eपs & + & $N$ & + & $m$ & - & N & $\sim$ & + & $n$ & + & $N$ & in & $m$ \\
\hline (ఫ) eunda & r & N & $\sigma$ & n & 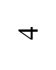 & N & 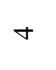 & $\sigma$ & 4 & $n$ & $\nabla$ & in & - \\
\hline (๖)! $6 \mathrm{zz}$ & + & $N$ & + & $m$ & + & $N$ & $m$ & + & $m$ & $n$ & + & $n$ & - \\
\hline (†) pגenpz & $m$ & $n$ & - & - & $\nabla$ & $n$ & $n$ & $\sim$ & $n$ & - & - & $\alpha$ & t \\
\hline euıew & $\nabla$ & + & n & $\checkmark$ & $n$ & $m$ & $m$ & $\nabla$ & - & $N$ & $m$ & $m$ & - \\
\hline om!। & - & $n$ & - & $m$ & in & in & $\nabla$ & $m$ & - & - & in & - & - \\
\hline 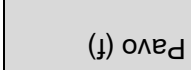 & $\sim$ & $m$ & m & m & in & m & $\sigma$ & m & $N$ & 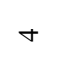 & $\nabla$ & n & $N$ \\
\hline (†) u이 & $n$ & $N$ & $N$ & $\sim$ & n & 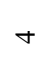 & + & $m$ & + & $N$ & $\nabla$ & $\sim$ & t \\
\hline 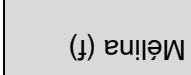 & $\nabla$ & $m$ & - & - & $ナ$ & $\nabla$ & $n$ & $N$ & $\nabla$ & $N$ & m & - & $N$ \\
\hline (Ł) оा!eu!W & n & + & - & - & 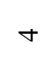 & $\sigma$ & in & N & - & $\sim$ & $m$ & N & N \\
\hline- & 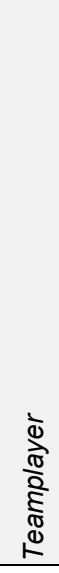 & 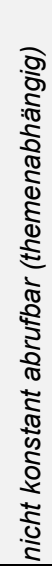 & 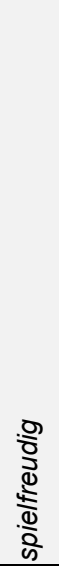 & 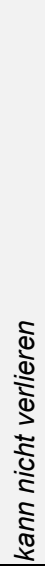 & 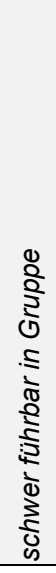 & 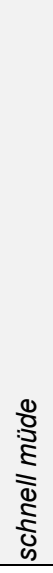 & 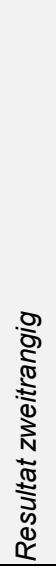 & 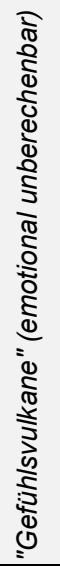 & 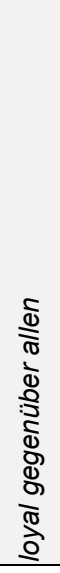 & 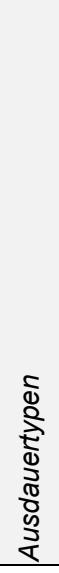 & 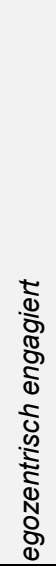 & $\begin{array}{l}\Phi \\
\frac{1}{5} \\
\frac{1}{\Phi} \\
\infty \\
0 \\
0\end{array}$ & $\begin{array}{l}\frac{\sqrt{\frac{2}{2}}}{\frac{2}{\pi}} \\
\end{array}$ \\
\hline 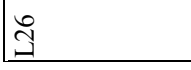 & $\bar{x}$ & $\underline{\tilde{x}}$ & $\underline{\tilde{m}}$ & $\underline{\underline{y}}$ & 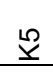 & $\underline{\underline{Q}}$ & $\underline{x}$ & $\stackrel{\infty}{\underline{x}}$ & $\underline{9}$ & 옴 & $\underline{\bar{\Sigma}}$ & $\stackrel{\mathfrak{N}}{\underline{x}}$ & $\stackrel{m}{\underline{m}}$ \\
\hline
\end{tabular}




\section{E Konstrukt-Retrievals Kategorien Fragestellung 1}

1a) Körperliche Voraussetzungen und Fähigkeiten (17/28)

\begin{tabular}{|c|c|c|}
\hline Präferierter Pol & Gegenpol & Dc \\
\hline Gute Grundkondition & Träges Phlegma (L2: K4) & \\
\hline Gute körperl. Voraussetzungen & Übergewichtig (L3: K10) & \\
\hline Gute koordinative Fähigkeiten & Schlechte koordinative Fähigkeiten (L6: K10) & \\
\hline Überdurchschnittl. sportlich begabt & Unterdurchschnittl. sportlich begabt (L6: K12) & \\
\hline Sportlich & Schlechte körperliche Voraussetzungen (L7: K2) & \\
\hline Gute koordinative Fähigkeiten & Schlechte koordinative Fähigkeiten (L7: K4) & \\
\hline Koordinativ stark & Schlechte Körperkontrolle (L9: K1) & \\
\hline Hohe Schnelligkeit & „Schneckentempo" (L9: K9) & \\
\hline Sehr beweglich & Unbeweglich (L9: K12) & \\
\hline Spritzig & Langsam (L10: K6) & \\
\hline Bewegungstalent & Ungelenkig (L10: K10) & \\
\hline Gute Antizipation & $\begin{array}{l}\text { Mühe, Distanz \& Geschw. einzuschätzen (L10: } \\
\text { K12) }\end{array}$ & \\
\hline Koordinativ stark & Koordinativ schwach (L12: K2) & \\
\hline „Raketen“: Kräftig & Schwächlich (L13: K2) & \\
\hline Flink & Träge (L13: K3) & \\
\hline Körperlich altersgemäss entwickelt & Körperlich wenig weit entwickelt (L13: K13) & \\
\hline Altersadäquate koordinative Fähigkeiten & Koordinative Defizite (L15: K12) & \\
\hline Keine körperliche Einschränkung & Körperliche Einschränkung (L15: K13) & \\
\hline Hohe koordinative Fähigkeiten & Unsportlich (L16: K8) & \\
\hline Körperlich bedingte Vorteile im Sport & $\begin{array}{l}\text { Keine Vorteile durch körperlichen Entwicklungs- } \\
\text { stand (L19: K5) }\end{array}$ & \\
\hline Koordinativ überdurchschnittlich & Koordinativ unterdurchschnittlich (L19: K6) & \\
\hline Gute körperliche Voraussetzungen & $\begin{array}{l}\text { Ungünstige körperliche Voraussetzungen (L20: } \\
\text { K10) }\end{array}$ & \\
\hline Athletisch & Unathletisch (L21: K12) & \\
\hline Gute Körperkontrolle & Schlecht ausgebildete Muskulatur (L22: K5) & 1c \\
\hline „Spritzig" & Träge (L23: K7) & \\
\hline Gute physische Voraussetzungen & Übergewichtig (L24: K3) & \\
\hline Viel Ausdauer \& Kraft & Schlechte physische Voraussetzungen (L24: K6) & \\
\hline Ausdauertypen & Träge (L26: K10) & \\
\hline
\end{tabular}

Anmerkung. Dc = Doppelcodierung

1b) (Spiel-)Sportarten-Können (12/16)

\begin{tabular}{l:l|l}
\hline Präferierter Pol & Gegenpol & Dc \\
\hline Überdurchschnittlich gut im Schlittschuhlaufen & Anfänger im Schlittschuhlaufen (L2: K3) & \\
Spielmacherin & Mitläuferin: Denkt nicht mit (L5: K8) \\
Treffsicher im Basket-/Korbball & Kein(e) Ballgefühl/-sicherheit (L5: K10) \\
Talentierte Geräteturnerinnen & Untalentierte Geräteturnerinnen (L8: K2) \\
Fussballerin & Ballfremd (L8: K9) &
\end{tabular}


Gute Spielübersicht

Spielmacher

Kann sehr gut Skifahren

Aktives-agiles Bewegungsverhalten auf dem

Spielfeld

Gute Spielübersicht

Fussballer/Handballer

Überdurchschnittlich gut in Badminton

Gutes Spielverständnis

Herausragende/r Schwimmer/in

Kann Spiel lesen

Polysportiv
Mangelnde Spielintelligenz (L10: K3)

Schlechte Antizipation (L10: K7)

Kann nicht Skifahren (L13: K7)

Träges Bewegungsverhalten auf dem Spielfeld (L14: K1)

Schlechte Spielübersicht (L16: K10)

Nicht Ballsportler (L17: K7)

Unterdurchschnittlich gut in Badminton (L17: K10)

Schlechtes Spielverständnis (L19: K8)

Hat Migrationshintergrund aus Krisengebiet (L22: K6)

Eingeschränkte Antizipationsfähigkeit (L23: K6)

Inselbegabung (L24: K4)

1c) Umgang mit Körper / Körperkontrolle (11/13)

\begin{tabular}{l|l|l}
\hline Präferierter Pol & Gegenpol & Dc \\
\hline $\begin{array}{l}\text { Kann sich gut über ihren/seinen Körper ausdrü- } \\
\text { cken }\end{array}$ & Schämt sich für ihren/seinen Körper (L1: K8) & \\
Gutes Körpergefühl & Ungeschickt (L2: K8) \\
Gutes Körpergefühl & Schlechtes Körpergefühl (L3: K2) & \\
Kontrollierte, präzise Bewegungen & Ungeschickt-tollpatschig (L3: K12) \\
Gute Körperwahrnehmung & Schlechte Körperwahrnehmung (L4: K6) & \\
Feinmotoriker & Grobmotoriker (L6: K6) \\
Hat gute Körperwahrnehmung & Hat schlechte Körperwahrnehmung (L16: K3) & \\
Feinmotorikerln & Grobmotorikerln (L17: K2) \\
Gutes Körpergefühl & Hat koordinative Probleme (L18: K5) & \\
Feinmotoriker & Grobmotoriker (L18: K7) \\
Gute Körperkontrolle & Schlecht ausgebildete Muskulatur (L22: K5) & 1a \\
Hat gute Körperwahrnehmung & Schlechte Körperspannung/-gefühl (L23: K5) & \\
Normaler/natürlicher Umgang mit Körper & Anerzogenes Schamgefühl (L25: K12) & \\
\hline
\end{tabular}

1d) (Sportliche) Leistung generell (8/9)

\begin{tabular}{l|l|l}
\hline Präferierter Pol & Gegenpol & Dc \\
\hline Gute/r bis sehr gute/rS & Ungenügende/r S (L1: K11) & \\
Sportlich leistungsstark & Leistungsschwach (L2: K9) & \\
Gute bis sehr gute Schüler ( $25 e r)$ & Ungenügende Leistungen (L3: K5) & \\
Überragende Leistungen im SU & Kein Interesse am SU (L13: K8) \\
Kann sich im Sport profilieren & Kann im Sport Ansehen verlieren (L18: K6) & \\
Vielseitig im Können & Einseitig im Können (L19: K1) & \\
Sportlich den Anderen grundsätzlich überlegen & „Hinkt“hinten her (L19: K11) & \\
Sportlich & Unsportlich (L21: K1) & \\
5,5er-Turner & 4,5er-Turner (L26: K12) & \\
\hline
\end{tabular}


2a) Einsatz/Anstrengungsbereitschaft (21/31)

\begin{tabular}{|c|c|c|}
\hline Präferierter Pol & Gegenpol & $\begin{array}{l}\mathrm{D} \\
\mathrm{C}\end{array}$ \\
\hline Muss von LP gebremst werden & Braucht Aufforderung durch LP (L1: K4) & \\
\hline Muss im Spiel gebremst werden & $\begin{array}{l}\text { Muss im Spiel (durch LP) angetrieben werden (L2: } \\
\text { K1) }\end{array}$ & \\
\hline Hat „Biss" & Wenig Durchhaltewille (L4: K9) & \\
\hline Strengt sich an & Faul (L5: K1) & \\
\hline Aktiv & Beteiligt sich wenig (L5: K2) & \\
\hline Einsatzfreudig & Wenig Einsatz (L5: K7) & \\
\hline Macht gut mit & Steht häufig herum (L5: K9) & \\
\hline Ausdauernd & Kein „Biss" (L5: K11) & \\
\hline Inhaltsunabhängig hohes Engagement & Inhaltsabhängig hohes Engagement (L6: K2) & 3a \\
\hline Sehr guter Einsatz & Passives Verhalten (L7: K1) & \\
\hline Zeigt grossen Einsatz & Zeigt bescheidenen Einsatz (L8: K5) & \\
\hline Einsatzfreudig & Bequem (L9: K4) & \\
\hline Leistungsbereit & Vermeidet Leistung/Anstrengung (L9: K6) & \\
\hline Leistungswillig & Faul (L10: K2) & \\
\hline Hohe Laufbereitschaft & Statische Spielweise (L10: K9) & \\
\hline Initiativ & Träumer (L11: K6) & \\
\hline Spielt mit viel Energie & Passiv, gleichgültig im Spiel (L12: K1) & \\
\hline Guter Einsatz & Kein Einsatz (L12: K11) & \\
\hline Hilft freiwillig beim Aufbauen & $\begin{array}{l}\text { Verhält sich so, dass er nichts aufstellen muss } \\
\text { (L12: K8) }\end{array}$ & \\
\hline Zäh-ausdauernd & Faul (L13: K1) & \\
\hline Versucht, eigene Grenzen auszuloten & Gibt schnell auf (L15: K3) & \\
\hline Hilfsbereit (Material) & $\begin{array}{l}\text { Nicht hilfsbereit (Material/Organisation) (L17: } \\
\text { K9) }\end{array}$ & \\
\hline Hat „Biss" & „Memme“: Gibt schnell auf (L18: K3) & \\
\hline Kann sich überwinden & Einsatz nach Lustprinzip (L18: K8) & $3 a$ \\
\hline Fordert sich & Bequem (L2O: K6) & \\
\hline Macht gut mit & Minimalist (L20: K7) & \\
\hline Übertriebener Ehrgeiz & Kein Einsatz (L21: K3) & $3 c$ \\
\hline Verausgabt sich & Tiefe Leistungsbereitschaft (L23: K2) & \\
\hline Guter Einsatz & Kein Einsatz (L24: K1) & \\
\hline Bringt eigene Ideen in Unterricht ein & $\begin{array}{l}\text { Nicht bereitwillig, das Minimum zu leisten (L25: } \\
\text { K8) }\end{array}$ & \\
\hline Guter Durchhaltewille & Schnell müde (L26: K6) & \\
\hline
\end{tabular}

2b) Fokussiertheit/Selbständigkeit (12/18)

\begin{tabular}{l|l|l}
\hline Präferierter Pol & Gegenpol & Dc \\
\hline Braucht keine spezielle Aufmerksamkeit der & Beansprucht die LP stark (L3: K1) & \\
Lehrperson & Unselbständig (L3: K3) & \\
Sehr selbständig & Unfokussiert (L3: K4) & \\
Konzentriert & &
\end{tabular}


Fokussiert auf Unterricht

Hört immer konzentriert zu

Bewegt sich unaufgefordert

Arbeitet konzentriert

Gespräche bleiben auftragsbezogen

Spielt fokussiert

Fokussiert

Zuverlässig

Kann selbständig an Bewegungsaufgaben arbeiten

Diszipliniert

Zeigt unaufgefordert Einsatz

Können zuhören

Fokussiert

Sehr selbständig nach Erteilen des Auftrages

Leicht führbar
Dauernd private Gespräche (L5: K12)

Schwatzhaft (L6: K13)

Muss ständig aufgefordert werden (L7: K9)

Lässt sich schnell ablenken (L8: K1)

Tauscht sich gerne über unsportliche Themen aus (L8: K6)

Kommentiert das Spiel (L8: K7)

Unfokussiert (L10: K4)

Lebt in einer eigenen Welt: Braucht viel Begleitung durch Lehrperson (L13: K5)

Braucht viel Unterstützung/Führung bei Bewegungsaufgaben (L14: K11)

Braucht Kontrolle durch LP (L15: K10)

Braucht Kontrolle (L16: K2)

Sehr schwatzhaft (L16: K6)

Zerstreut (L16: K9)

Braucht viel Unterstützung von Seiten Lehrperson (L22: K11)

Erzeugt Zusatzaufwand (L25: K5)

\begin{tabular}{l|l|l}
\hline 2c) Besonnenheit (6/6) & \multicolumn{2}{l}{} \\
\hline Präferierter Pol & Gegenpol & Dc \\
\hline Denkt mit & Denkt nicht mit (L4: K13) & \\
Denkt mit & Ist gleichgültig (L7: K10) & \\
Überlegt & Denken wenig (L8: K10) & \\
Denken voraus & Zurückhaltend (L13: K6) \\
Überlegt & DraufgängerisCh (L16: K5) & \\
Kritisch-hinterfragend & Angepasst (L17: K8) & \\
\hline
\end{tabular}

2d) Mut (5/5)

\begin{tabular}{l|l|l}
\hline Präferierter Pol & Gegenpol & Dc \\
\hline Mutig & Ängstlich (L1: K7) & \\
Mutig bis übermütig & Eher wenig Mut (L8: K8) & \\
Gesunde Risikobereitschaft & Ängstlich (L9: K8) & \\
Mutig & Ängstlich (L16: K11) & \\
Mutig & Ängstlich (L17: K6) & \\
\hline
\end{tabular}

3a) Offenheit/Begeisterungsfähigkeit (26/19)

\begin{tabular}{l|l|l}
\hline Präferierter Pol & Gegenpol & Dc \\
\hline Sehr offen für Neues & Nicht offen für Neues (L1: K9) & \\
Offen für Neues & Nicht willig, Neues zu lernen (L3: K6) & \\
Offen für Vieles & Fokussiert auf Fussball (L4: K7) & \\
Begeisterungsfähig & Abgelöscht (L4: K11) &
\end{tabular}




\begin{tabular}{|c|c|}
\hline $\begin{array}{l}\text { Inhaltsunabhängig hohes Engagement } \\
\text { Nimmt Herausforderungen positiv an }\end{array}$ & $\begin{array}{l}\text { Inhaltsabhängig hohes Engagement (L6: K2) } \\
\text { Versucht Herausforderungen auszuweichen (L8: } \\
\text { K3) }\end{array}$ \\
\hline Hohe Lernbereitschaft & Lernverweigernde Haltung (L9: K3) \\
\hline Positive Grundhaltung gegenüber SU & Negative Grundhaltung (L9: K5) \\
\hline Hohe Offenheit gegenüber versch. Inhalten & $\begin{array}{l}\text { Abweisend-verschlossen gegenüber best. Inhal- } \\
\text { ten (L9: K11) }\end{array}$ \\
\hline Offen für vielseitigen Sportunterricht & $\begin{array}{l}\text { Macht nur in seiner beliebtesten Sportart mit } \\
(\text { L12: K9) }\end{array}$ \\
\hline Ist für jeden Inhalt zu begeistern & Macht nur das, was ihm/ihr passt (L14: K2) \\
\hline Offen für Neues & Nicht offen für Neues (L15: K5) \\
\hline Einsatz unabhängig von Thema & Einsatz abhängig von Thema (L15: K6) \\
\hline Vielseitig interessiert & Einseitig interessiert (L15: K11) \\
\hline Am SU interessiert & Weniger am SU interessiert (L16: K7) \\
\hline Begeisterungsfähig & Nicht offen für Neues (L17: K1) \\
\hline Lernwillig & Lustlos (L17: K5) \\
\hline Kann sich überwinden & Einsatz nach Lustprinzip (L18: K8) \\
\hline Sportbegeisterung von „zuhause auf“ & $\begin{array}{l}\text { Wenig fam. bedingte Sportbegeisterung (L19: } \\
\text { K4) }\end{array}$ \\
\hline Immer begeistert & Desinteresse, häufig abwesend (L2O: K1) \\
\hline Offen für Neues & Fixiert auf bestimmte Sportarten (L21: K4) \\
\hline Positive Grundhaltung & Destruktive Grundhaltung (L23: K4) \\
\hline Neugierig & Nicht offen gegenüber allen Inhalten (L23: K12) \\
\hline Sportbegeistert & Keine Sportbegeisterung (L24: K2) \\
\hline Offen gegenüber allen Inhalten & Stellt jede Anweisung in Frage (L25: K2) \\
\hline Themenunabhängig bereit & $\begin{array}{l}\text { Nicht konstant abrufbar (themenabhängig) (L26: } \\
\text { K2) }\end{array}$ \\
\hline
\end{tabular}

3b) Motivation / Freude an Bewegung (15/23)

\begin{tabular}{l|l|l}
\hline Präferierter Pol & Gegenpol & Dc \\
\hline Motiviert & Unmotiviert (L3: K7) \\
Quirrlig - immer in Bewegung & Phlegmatisch (L3: K8) \\
Bewegungsfreudig & Bëge (L4: K5) \\
Intrinsisch motiviert, sich zu bewegen & K9) \\
& Bequem (L5: K3) \\
Bewegungsfreudig & Unmotiviert (L7: K3) \\
Motiviert & Intensität des SU egal (L11: K3) \\
Spielfreudig & Unterdurchschnittl. Bewegungsdrang (L11: K9) \\
Bewegungsfreudig & Mässig motiviert (L12: K6) \\
Überdurchschnittl. Bewegungsdrang & Macht auch gerne einmal Pause (L14: K3) \\
Motiviert, sich zu bewegen & \\
Top motiviert & Keine Freude an der Bewegung (L15: K4) \\
Nutzt jede freie Minute, um sich mit dem Ballen (L4: \\
auszuleben \\
$\begin{array}{l}\text { Bewegungsfreudig } \\
\text { Motiviert }\end{array}$
\end{tabular}


Viel Eigenmotivation

Bewegt sich gerne

Bewegungsfreudig

Sehr motiviert

Eigenmotiviert

Bewegt sich gerne

Motiviert

Freut sich, sich zu bewegen

Spielfreudig
Wenig Eigenmotivation (L17: K4)

Bewegungsfaul (L18: K1)

Bewegungsfaul (L18: K4)

Lustlos (L20: K3)

Braucht viel ermunternde Aufforderung durch $L P$ (L20: K8)

Muss stark motiviert werden (L22: K1)

Nicht ehrgeizig (L22: K8)

$3 c$

Unmotiviert (L23: K3)

Teilnahmslos-apathisch (L26: K3)

3c) Ehrgeiz / Siegeswille (11/12)

\begin{tabular}{l|l|l}
\hline Präferierter Pol & Gegenpol & Dc \\
\hline Ehrgeizig & Gleichgültig (L5: K4) & \\
Siegorientiert & Sieg/Niederlage gleichgültig (L11: K1) & \\
Ehrgeizig & Gleichgültig (L12: K13) \\
Wettkampforientiert & Nicht resultat-orientiert (L17: K3) & \\
Ehrgeizig & Nicht ehrgeizig (L17: K11) & \\
Will gewinnen & Nicht ambïltig zu verlieren (L18: K12) \\
Ambitioniert & Gleichgültig (L20: K4) & \\
Ehrgeizig & Kein Einsatz (L21: K3) & \\
Übertriebener Ehrgeiz & Nicht ehrgeizig (L22: K8) & \\
Motiviert & Ambitionslos (L23: K10) & \\
Sportlich ehrgeizig & Resultat zweitrangig (L26: K7) & \\
Es zählt nur der Sieg &
\end{tabular}

3d) Persönliche Vorlieben (5/7)

\begin{tabular}{l|l|l}
\hline Präferierter Pol & Gegenpol & Dc \\
\hline Liebt Hip-Hop Tanz & Hat Angst, sich zu exponieren (L5: K5) & \\
Spielt recht gerne Fussball & Spielt nicht gerne Fussball (L6: K1) \\
Hat gerne Geräteturnen & Abneigung gegenüber Geräteturnen (L6: K3) & \\
Schwimmt gerne & Schwimmt nicht gerne (L6: K9) \\
Betätigt sich gerne kreativ, tänzerisch & Betätigt sich nicht gerne kreativ, tänzerisch (L14: & \\
& K9) & \\
Spielt gerne Basketball & Behindert Basketballspieler (L15: K8) \\
Fussballbegeistert & Fussball-Hasser (L21: K2) & \\
\hline
\end{tabular}

4a) Aufrichtigkeit/Loyalität/Fairness (16/22)

\begin{tabular}{l|l|l}
\hline Präferierter Pol & Gegenpol & Dc \\
\hline Ist schon häufig vor Lektionsbeginn parat & Kommt zu spät (L1: K6) & \\
Fair im Spiel & Unehrlich im Spiel (L2: K5) & \\
Gehorcht der Lehrperson & Ungehorsam (L3: K11) & \\
Nimmt Vorgaben direkt an & Hinterfragt Vorgaben/Anweisungen (L4: K1) &
\end{tabular}




\begin{tabular}{l|l|} 
Sorgt sich ehrlich um das Wohl der Anderen & Nutzt Gelegenheit, um Unterricht zu stören (L5: \\
Sehr korrekt & K13) \\
Respektiert Anweisungen der Lehrperson & „Falsch“ (L6: K4) \\
& Respektiert Anweisungen der Lehrperson nicht \\
Ehrlich & (L6: K8) \\
Zuverlässig & Unzuverlässig (L10: K1) \\
Kämpft für Fairness & Unzuverlässig (L11: K8) \\
Respektvoll gegenüber LP & Provokantes Verhalten (L11: K2) \\
Anständig, respektvoll & Kein Respekt von LP (L11: K10) \\
& Respektlos. Legt keinen Wert auf soziale Nor- \\
Zuverlässig & men/Werte (L12: K4) \\
& Lebt in einer eigenen Welt: Braucht viel Beglei- \\
Aufrichtig, ehrlich & tung durch Lehrperson (L13: K5) \\
Faires Spielverhalten & Hinterlistig (L14: K7) \\
Fair & Fies, hinterhältig im Spiel (L20: K5) \\
Positive Kommentare & Unfair-unehrlich (L21: K5) \\
Umgänglich & Störende Kommentare (L21: K11) \\
Positive Arbeitshaltung & Disziplinarisch schwierig (L23: K11) \\
Anerkennt Lehrperson & Boykott von Unterricht (L25: K1) \\
Loyal gegenüber allen & Stellt Lehrperson halblaut in Frage (L25: K4) \\
Fairplay & Egozentrisch (L26: K9) \\
\hline
\end{tabular}

4b) Teamfähigkeit (14/21)

\begin{tabular}{l|l|l}
\hline Präferierter Pol & Gegenpol & Dc \\
\hline $\begin{array}{l}\text { Leaderfigur im Spiel } \\
\text { Zurechtweisendes Auftreten im Spiel }\end{array}$ & Spielt am liebsten gar nicht mit (L1: K2) & \\
Bestimmt gerne, wer was zu tun hat & Keinen Anspruch auf Spielmitbestimmung (L6: \\
Teamfähig & K5) & Einzelkämpferln (L7: K5) \\
Motiviert Andere & Demotiviert Andere durch Zurechtweisung (L7: \\
& K6) \\
Teamplayer & EinzelsportlerIn (L8: K11) \\
Hoher Einsatz im 2er Team & Arbeitet im 2er Team negativ (Spirale) (L9: K7) \\
Schätzt Gemeinschaftserlebnis & Grundsätzlich gestresst in der Gruppe (L9: K10) \\
Gruppenkonstellation spielt keine Rolle & Gruppenkonstellation hat hohe Bedeutung (L11: \\
& K7) \\
Teamplayer & Hat Mühe, den Teamgedanken zu leben (L14: K8) \\
Übernimmt Verantwortung für Gruppe & Versteckt sich gerne in der Gruppe (L14: K12) & \\
Leaderfigur im Spiel & Stört das Spiel (L15: K2) \\
Soziales Spielverhalten & Egoistisches Spielverhalten (L18: K10) \\
Steht über der Sache & Einsatz abhängig von Gruppenkonstellation & \\
Kann Andere gut ins Spiel integrieren & (L18: K11) \\
Macht das Beste aus schwachen Teams & Ist im Spiel mit sich selbst beschäftigt (L19: K9) & \\
& Verliert Motivation in schwachen Teams (L19: & \\
& K12)
\end{tabular}


Integriert auch Schwache im Spiel

Akzeptiert Schwächere

Teamplayer

Flexibel in Gruppen integrierbar

Teamfähig
Macht Schwache nieder (L21: K10)

Schliesst Schwächere aus (L25: K6)

Einzelgänger (L26: K1)

Schwer führbar in Gruppe (L26: K:5)

Egozentrisch engagiert (L26: K11)

4c) Geltungs-/Profilierungsdrang (9/12)

\begin{tabular}{|c|c|c|}
\hline Präferierter Pol & Gegenpol & Dc \\
\hline Eigene Leistung wird „innerlich“ gefeiert & $\begin{array}{l}\text { Übertriebenes Feiern der eigenen Leistung (L2: } \\
\text { K11) }\end{array}$ & \\
\hline Schämt sich nicht & Will sich nicht exponieren (L3: K13) & \\
\hline Kann zu eigenen Schwächen stehen & Zeigt nicht gerne Schwächen (L4: K2) & \\
\hline Stille Macher & Bluffer (L4: K3) & \\
\hline Intrinsisch motiviert, sich zu bewegen & $\begin{array}{l}\text { Bewegt sich, um Anerkennung zu erhalten (L4: } \\
\text { K10) }\end{array}$ & $3 b$ \\
\hline Kein Aufmerksamkeitsdefizit & Provoziert verbal (L11: K5) & \\
\hline Will sich mit Anderen messen & Muss sich nicht unter Beweis stellen (L11: K11) & \\
\hline Will hervorstechen & Exponiert sich nicht gerne (L13: K12) & \\
\hline Braucht Rampenlicht nicht & $\begin{array}{l}\text { Wird gerne in der Klasse wahrgenommen (L14: } \\
\text { K5) }\end{array}$ & \\
\hline Einsatz der Sache zuliebe & $\begin{array}{l}\text { Nutzt Sportunterricht zur Bedienung der eigenen } \\
\text { Selbstverliebtheit (L18: K9) }\end{array}$ & \\
\hline Stille Schaffer & Fällt gerne auf (L23: K1) & \\
\hline Zeigt gerne sein/ihr Können & $\begin{array}{l}\text { Stellt sich nicht gerne in den Vordergrund (L24: } \\
\text { K8) }\end{array}$ & \\
\hline
\end{tabular}

4d) Beliebtheit in Klasse (6/7)

\begin{tabular}{l|l|l}
\hline Präferierter Pol & Gegenpol & Dc \\
\hline Beliebt in der Klasse & Nicht gut in Klassenverband integriert (L1: K10) & \\
Wird immer als Erste/r in Teams gewählt & Wird immer als Letzte/r in Teams gewählt (L7: & \\
& K8) & \\
Beliebte Schülerin & Aussenseiterin (L7: K12) & \\
Leadertyp & Geringes Selbstvertrauen (L10: K5) & 5 \\
Sozial gut integriert & Sozial schlecht integriert (L15: K9) \\
Beliebt im SU & Aussenseiter im SU (L21: K6) \\
Leader & Wird von anderen SUS gerne links liegen gelassen & \\
& (L24: K11) & \\
\hline
\end{tabular}

5a) Selbstbild/Selbsteinschätzung (11/13)

\begin{tabular}{l|l|l}
\hline Präferierter Pol & Gegenpol & Dc \\
\hline Profitiert im koedukativen Unterricht & Geringes Selbstvertrauen im koedukativen Un- & \\
& terricht (L1: K1) \\
Selbstsicher & Zweifelt an sich (L4: K4) & \\
\hline 230 &
\end{tabular}




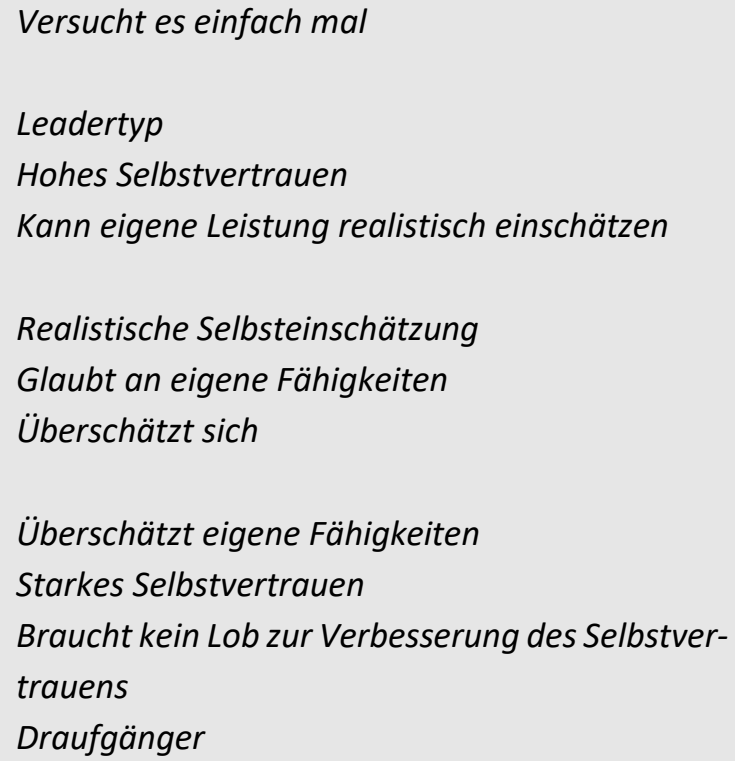

Will sicherstellen, dass er/sie Instruktion verstanden hat (L8: K4)

Geringes Selbstvertrauen (L10: K5)

Tiefes Selbstvertrauen (L16: K4)

Kann eigene Leistung nicht realistisch einschätzen (L18: K13)

Unrealistische Selbsteinschätzung (L19: K3)

Nutzt sportliches Potenzial zu wenig (L20: K2)

Unterschätzt eigene Leistungsmöglichkeiten (L21: K7)

Hat kein Selbstvertrauen (L22: K3)

Tiefes Selbstvertrauen (L22: K7)

Braucht Lob zur Verbesserung des Selbstvertrauens (L22: K10)

Scheu und zurückhaltend (L24: K5)

5b) Lernfähigkeit (6/6)

\begin{tabular}{l|l|l}
\hline Präferierter Pol & Gegenpol & Dc \\
\hline Rasche Auffassungsgabe & Langsame Auffassungsgabe (L2: K2) & \\
Lernfähig & Wenig lernfähig (L3: K9) \\
Hohe Aufmerksamkeit & Schlechte Auffassungsgabe (L10: K11) & \\
Lernt schnell & Bewegungslegasteniker (L21: K8) \\
Lernt ring & Macht nur langsame Fortschritte (L22: K2) & \\
Gute Auffassungsgabe & Schlechte Auffassungsgabe (L24: K7) & \\
\hline
\end{tabular}

5c) Sportengagement ausserhalb SU (6/6)

\begin{tabular}{l|l|l}
\hline Präferierter Pol & Gegenpol & Dc \\
\hline Spielt in der Freizeit Fussball & Spielt keinen Fussball in der Freizeit (L6: K7) & \\
Treibt in Freizeit regelmässig Sport & Sat keinen Bezug zum Sport (L7: K11) \\
Spielt in der Freizeit Unihockey & St nicht in Sportverein (L13: K9) \\
Ist in einem Sportverein & Nicht aktiv ausserhalb SU (L19: K7) \\
Ist auch ausserhalb SU aktiv & Treibt nicht freiwillig Sport (L24: K10) \\
Bleibt nach obligat. Schulzeit sporttreu & & \\
\hline
\end{tabular}

5d) Elternhaus (5/8)

\begin{tabular}{l|l|l}
\hline Präferierter Pol & Gegenpol & Dc \\
\hline Bringt grossen Erfahrungsschatz mit & Fam. bedingt wenig Bewegungserfahrung (L1: & \\
Ausländer & S12) & \\
Bestreitet Schulweg zu Fuss & Wird per Auto zur Schule geführt (L13: K4) \\
Hat Migrationshintergrund & Ist Schweizerln (L13: K10) & \\
Sportbegeisterung von „zuhause auf“ & Wenig fam. bedingte Sportbegeisterung (L19: & 3a
\end{tabular}


Eltern informieren und fordern nicht Migrationshintergrund

Finanzschwaches Elternhaus
Eltern fördern Fernbleiben von SU (L25: K3)

Schweizerinnen (L25: K9)

Mittelstand (L25: K11)

6) Besondere (21/32)

\begin{tabular}{|c|c|c|}
\hline Präferierter Pol & Kontrastpol & $\mathrm{Dc}$ \\
\hline Fordernd & Angepasst, nicht fordernd (L1: K3) & \\
\hline Kleidet sich nach islamischen Vorgaben & Kleidet sich freizügig (L1: K5) & \\
\hline Verbal auffällig & Verbal unauffällig (L2: K6) & \\
\hline Mädchen & Knaben (L4: K12) & \\
\hline Häufig verbal aggressiv im Spiel & „Schluckt" alles im Spiel (L5: K6) & \\
\hline Ist nie krankheitsbedingt abwesend & $\begin{array}{l}\text { Kann krankheitsbedingt oft nicht teilnehmen (L6: } \\
\text { K11) }\end{array}$ & \\
\hline Drückt Emotionen in passenden Momenten aus & Kreischt oft übertrieben (L7: K 7 ) & \\
\hline Spez. Sekklasse & Realklasse (L7: K13) & \\
\hline Verbal zurückhaltend & Verbale Auflehnung (L10: K8) & \\
\hline Extrovertiert & Zurückhaltend (L11: K4) & \\
\hline Hohe Leistungserwartungen an Mit-S & Keine Leistungserwartungen an Mit-S (L11: K12) & \\
\hline Kann Misserfolg etwas Positives abgewinnen & Kann nicht mit Misserfolg umgehen (L11: K13) & \\
\hline Temperamentvoll & Teilnahmslos-schlapp (L12: K5) & \\
\hline Duscht immer & Duscht nie (L12: K10) & \\
\hline Vorzeigeschüler & Problemschüler (L12: K12) & \\
\hline (Zukünftige/r) Sekschülerln & Realschülerln (L13: K11) & \\
\hline Sucht Kontakt zu LP & Sucht keinen Kontakt zu LP (L14: K4) & \\
\hline Fordert LP gerne auf positive Weise heraus & $\begin{array}{l}\text { Bewegt sich im Verhältnis zu LP im vorgegebe- } \\
\text { nen Rahmen (L14: K6) }\end{array}$ & \\
\hline Scheut Körperkontakt im Spiel nicht & Meidet im Spiel den Körperkontakt (L14: K10) & \\
\hline Sucht Kontakt zu LP & $\begin{array}{l}\text { Beschränkt sich auf minimal nötigen Kontakt } \\
\text { (L15: K7) }\end{array}$ & \\
\hline Direktes Auftreten & Zurückhaltendes Auftreten (L16: K1) & \\
\hline Kann loslassen & Gefangen in sich selbst (L18: K16) & \\
\hline Schätzt das Gemeinschaftliche im Sport & $\begin{array}{l}\text { Individuelle Leistung steht im Vordergrund (L19: } \\
\text { K2) }\end{array}$ & \\
\hline Zurückhaltend-gehemmt (Spiel) & „Solist“ (L20: K9) & \\
\hline Sekschüler & Realschüler (L21: K9) & \\
\hline Spielt gerne & Hat kein Selbstvertrauen (L22: K4) & \\
\hline Übertrieben wehleidig & Kein Schmerzempfinden (L22: K9) & \\
\hline Spontane Kommunikation möglich & Sprachbarriere vorhanden (L23: K8) & \\
\hline Extrovertiert & Introvertiert (physisch, verbal) (L23: K9) & \\
\hline Sek- und zukünftig. Gym-S & Realschülerin (L25: K10) & \\
\hline Kann Niederlagen positiv reflektieren & Kann nicht verlieren (L26: K4) & \\
\hline "Gefühlsvulkane" (emotional unberechenbar) & „Schlaftablette“ (L26: K8) & \\
\hline
\end{tabular}




\begin{tabular}{|c|c|c|}
\hline \multicolumn{2}{|c|}{$\begin{array}{l}\text { Zusammenfassung der Konstrukte ohne (eindeutige) Angabe der Präferenz des einen } \\
\text { oder anderen Pols }\end{array}$} & \multirow{2}{*}{$\begin{array}{l}\text { Kat. } \\
6\end{array}$} \\
\hline Kleidet sich nach islamischen Vorgaben & Kleidet sich freizügig (L1: K5) & \\
\hline Muss im Spiel gebremst werden & $\begin{array}{l}\text { Muss im Spiel (durch LP) angetrieben werden } \\
\text { (L2: K1) }\end{array}$ & $2 a$ \\
\hline Zurechtweisendes Auftreten im Spiel & Weist andere nicht zurecht (L2: K7) & $4 b$ \\
\hline Ausländer & Schweizer (L4: K12) & $5 c$ \\
\hline Häufig verbal aggressiv im Spiel & „Schluckt alles im Spiel (L5: K6) & 6 \\
\hline Teamfähig & EinzelkämpferIn (L7: K5) & $4 b$ \\
\hline Wird immer als Erste/r in Teams gewählt & $\begin{array}{l}\text { Wird immer als Letzte/r in Teams gewählt (L7: } \\
\text { K8) }\end{array}$ & $4 d$ \\
\hline Beliebte/r Schüler/in & Aussenseiter/in (L7: K12) & $4 d$ \\
\hline Verbal zurückhaltend & Verbale Auflehnung (L10: K6) & 6 \\
\hline Hat Migrationshintergrund & Schweizer/in (L13: K10) & $5 c$ \\
\hline (Zukünftige/r) Sekschüler/in & Realschüler/in (L13: K11) & 6 \\
\hline Feinmotoriker/in & Grobmotoriker/in (L18: K7) & $1 \mathrm{c}$ \\
\hline Schätzt das Gemeinschaftliche am Sport & $\begin{array}{l}\text { Individuelle Leistung steht im Vordergrund } \\
\text { (L19: K2) }\end{array}$ & 6 \\
\hline Sportlich den Anderen grundsätzlich überlegen & „Hinkt“ hinten her (L19: K11) & $1 d$ \\
\hline Zurückhaltend-gehemmt (im Spiel) & „Solist" (L20: K9) & 6 \\
\hline Übertriebener Ehrgeiz & Kein Einsatz (L21: K3) & $2 a / 3 c$ \\
\hline Überschätzt sich & $\begin{array}{l}\text { Unterschätzt eigene Leistungsmöglichkeiten } \\
\text { (L21: K7) }\end{array}$ & $5 a$ \\
\hline Überschätzt eigene Fähigkeiten & Hat kein Selbstvertrauen (L22: K3) & $5 a$ \\
\hline Herausragende/r Schwimmer/in & $\begin{array}{l}\text { Hat Migrationshintergrund aus Krisengebiet } \\
\text { (L22: K6) }\end{array}$ & $1 b / 5 c$ \\
\hline Übertrieben wehleidig & Kein Schmerzempfinden (L22: K9) & 6 \\
\hline $\begin{array}{l}\text { Braucht kein Lob zur Verbesserung des Selbst- } \\
\text { vertrauens }\end{array}$ & $\begin{array}{l}\text { Braucht Lob zur Verbesserung des Selbstver- } \\
\text { trauens (L22: K10) }\end{array}$ & $5 a$ \\
\hline Stille/r Schaffer & Fällt gerne auf (L23: K1) & $4 c$ \\
\hline Introvertiert & Extrovertiert (L23: K9) & 6 \\
\hline Finanzschwaches Elternhaus & Mittelstand (L25: K11) & $5 c$ \\
\hline „Gefühlsvulkane" & "Schlaftabletten“ (L26: K8) & 6 \\
\hline
\end{tabular}




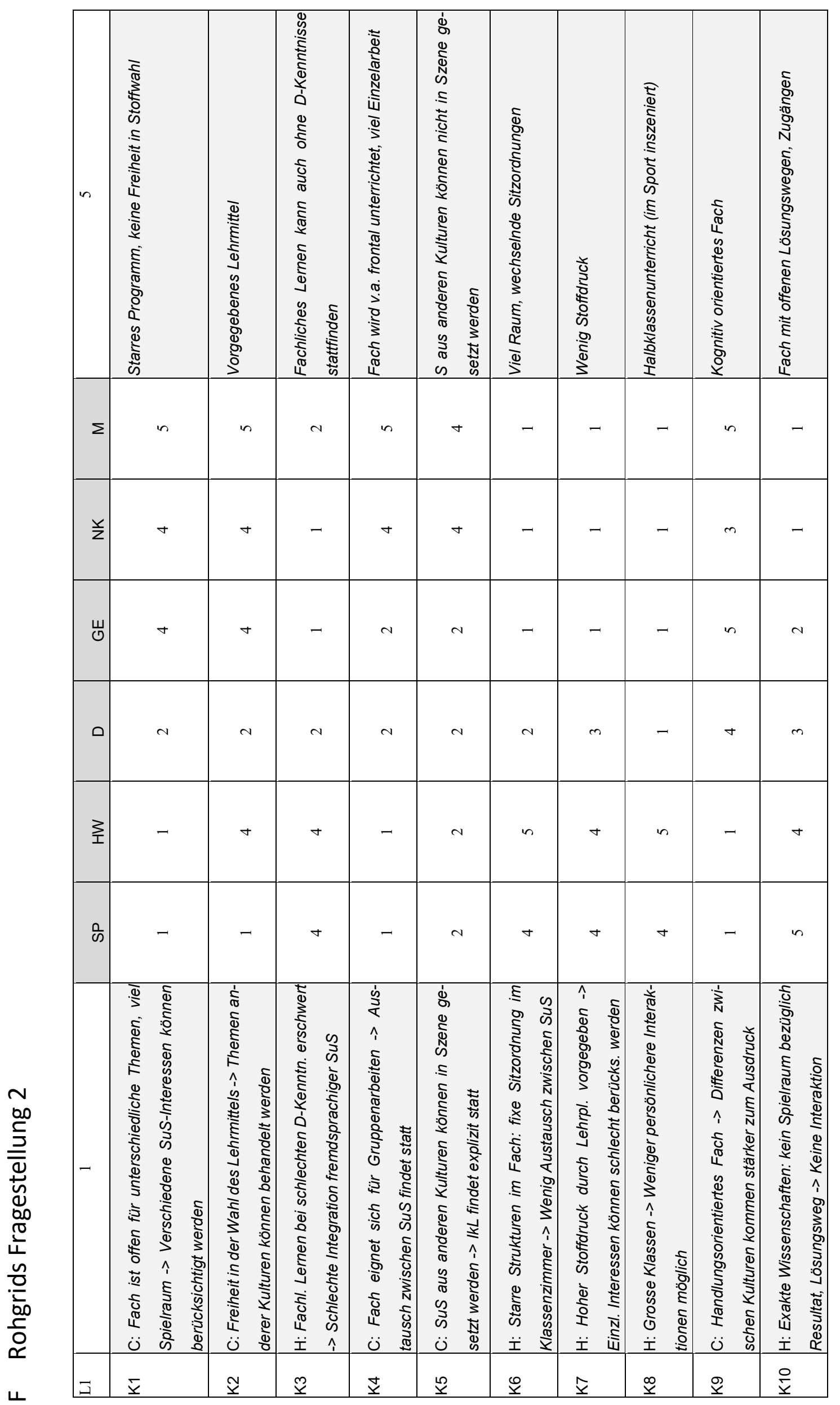




\begin{tabular}{|c|c|c|c|c|c|c|c|}
\hline in & 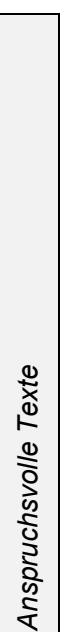 & 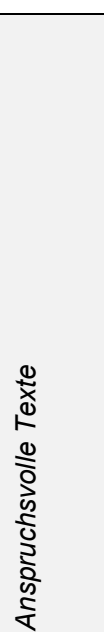 & 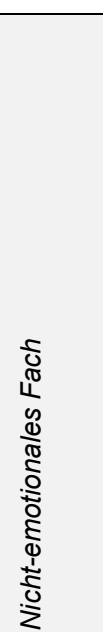 & 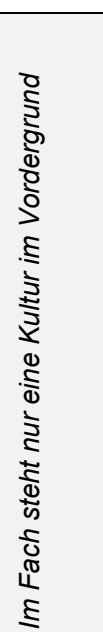 & 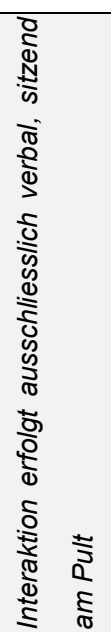 & 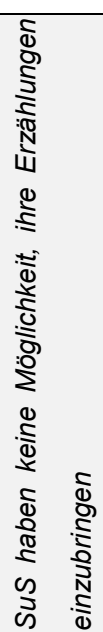 & 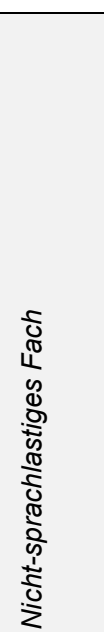 \\
\hline 山् & $\nabla$ & $N$ & $N$ & - & t & $N$ & $N$ \\
\hline 山् & + & $m$ & N & 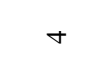 & $N$ & $N$ & - \\
\hline ᄂ & $\sim$ & N & $N$ & in & $\sigma$ & $\nabla$ & $\mathrm{N}$ \\
\hline$\Sigma$ & $\sim$ & $N$ & in & $m$ & $\sigma$ & $\nabla$ & $\sigma$ \\
\hline के & $\sim$ & N & - & $m$ & - & in & in \\
\hline D) & $\sigma$ & $N$ & $\sigma$ & - & $\sigma$ & - & $N$ \\
\hline- & 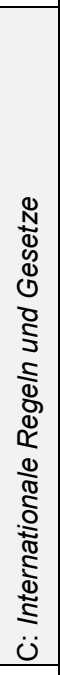 & 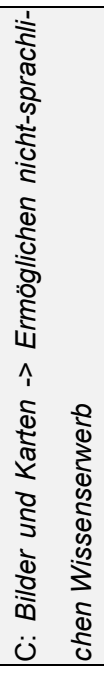 & 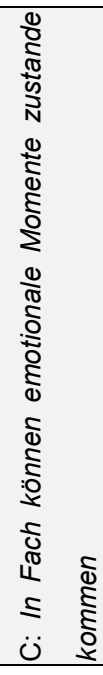 & 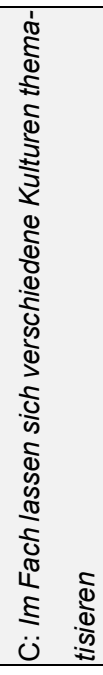 & 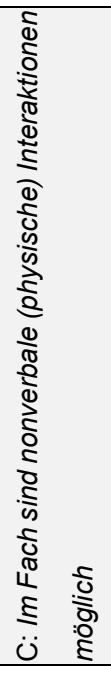 & 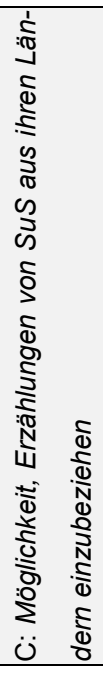 & 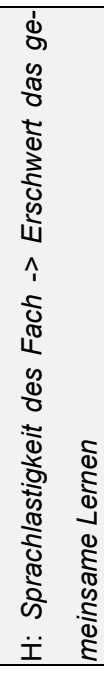 \\
\hline $\mathcal{I}$ & $\bar{x}$ & $\underline{\underline{Y}}$ & $\stackrel{\mathscr{m}}{\underline{x}}$ & $\underset{\Sigma}{\mathbb{I}}$ & $\stackrel{\mathscr{p}}{\underline{p}}$ & $\underline{q}$ & $\hat{x}$ \\
\hline
\end{tabular}




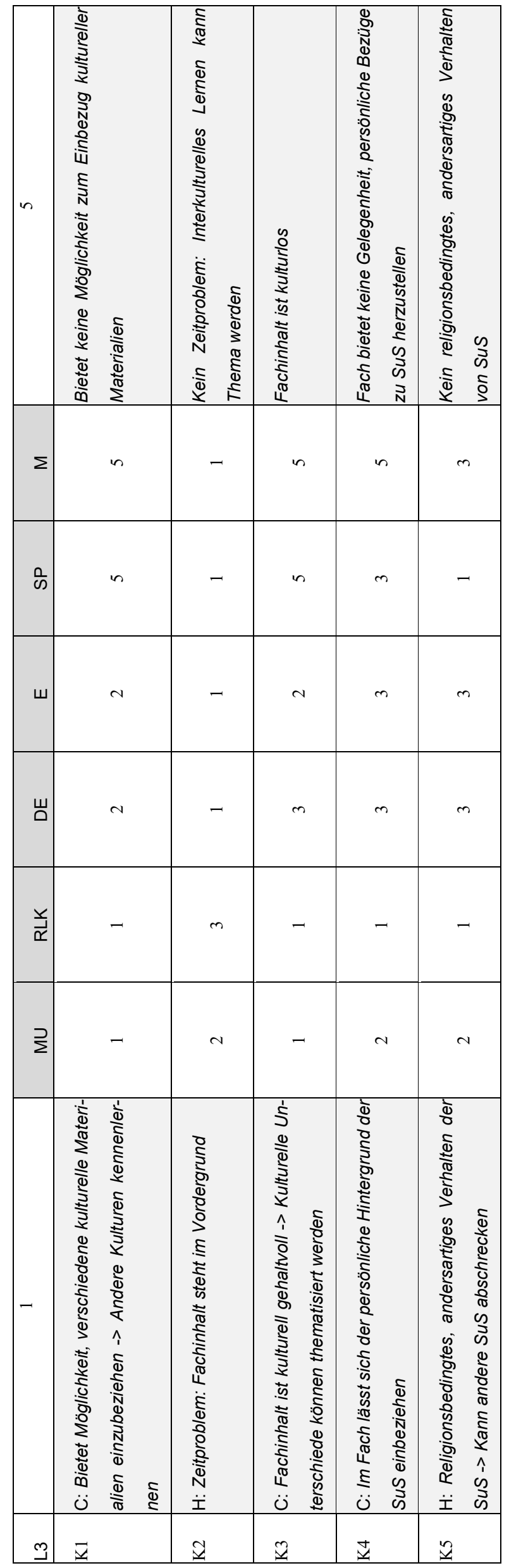




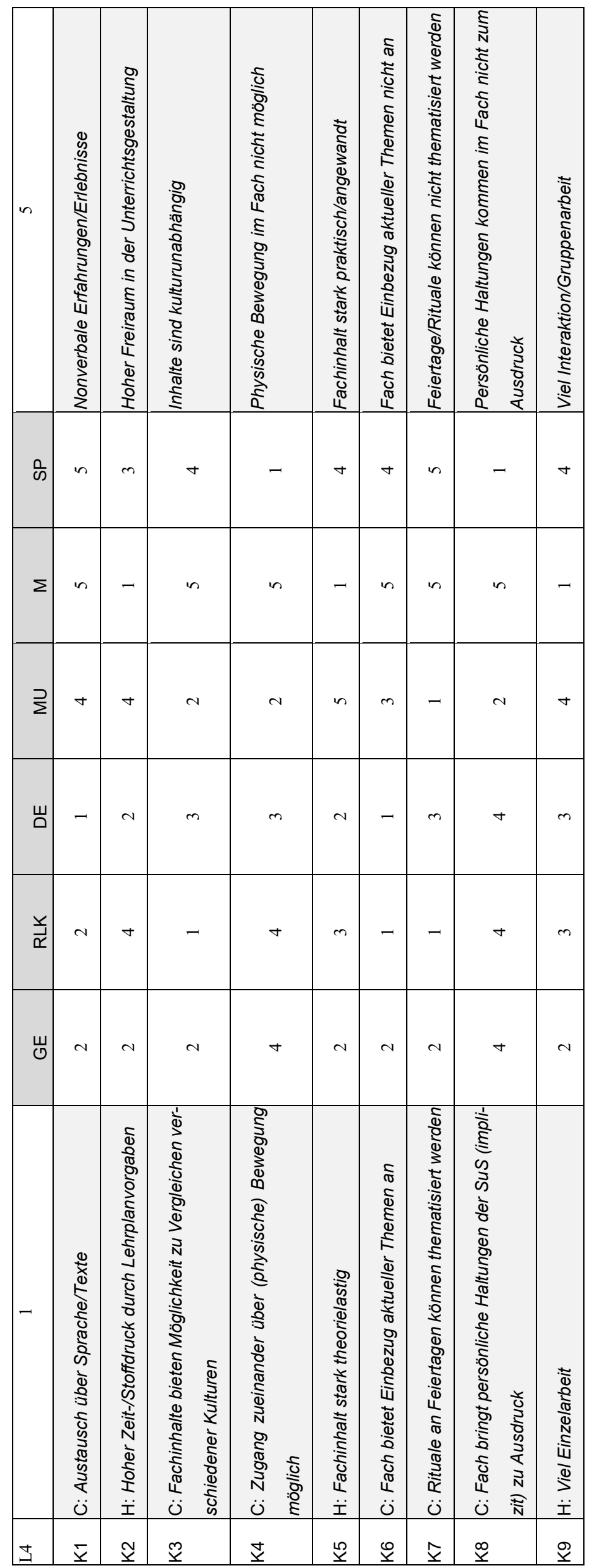

๙ 


\begin{tabular}{|c|c|c|c|c|c|c|c|c|c|}
\hline in & 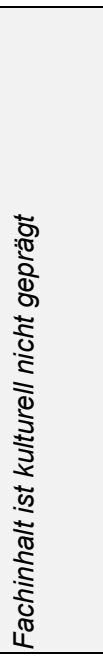 & 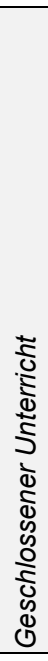 & 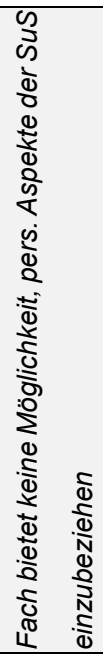 & 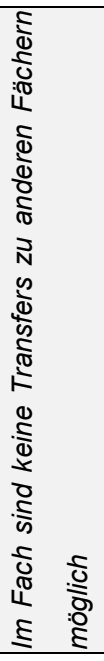 & 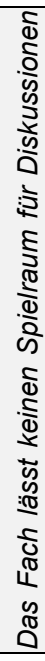 & जे & 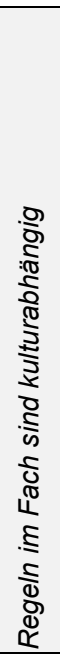 & 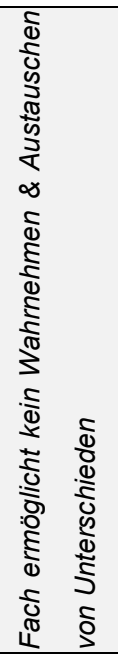 & 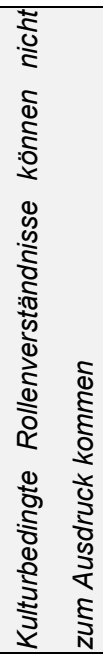 \\
\hline$\Sigma$ & $\nabla$ & ナ & in & - & & + & - & $\nabla$ & 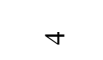 \\
\hline O্ & $m$ & $\sim$ & $N$ & - & & v & - & $N$ & $N$ \\
\hline के & + & 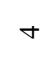 & $N$ & $N$ & & N & - & $N$ & $N$ \\
\hline 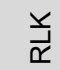 & $N$ & $m$ & - & - & & - & $\nabla$ & - & - \\
\hline$\stackrel{2}{\Sigma}$ & 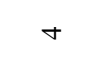 & $n$ & - & - & & - & $\sigma$ & $N$ & - \\
\hline 岁 & - & $\nabla$ & - & - & & - & $\sigma$ & - & - \\
\hline- & 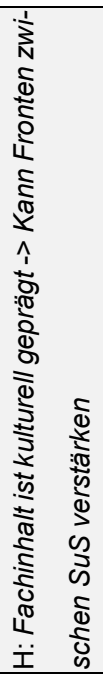 & 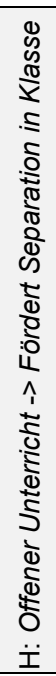 & 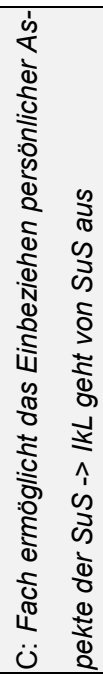 & 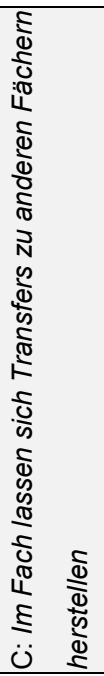 & 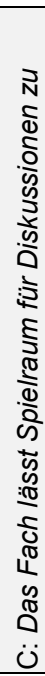 & 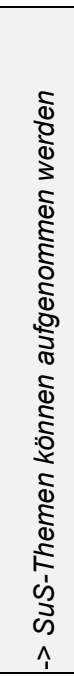 & 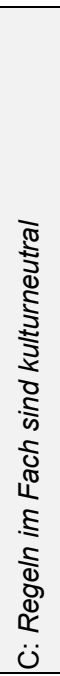 & 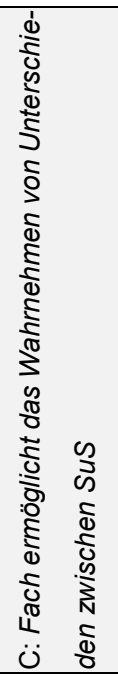 & 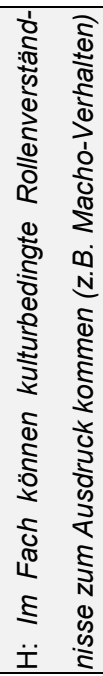 \\
\hline r & $\bar{\Sigma}$ & $\underline{\widetilde{V}}$ & $\underline{\tilde{p}}$ & $\underline{\Sigma}$ & $\underline{\underline{v}}$ & & $\underline{\underline{x}}$ & $\underline{x}$ & $\underline{\infty}$ \\
\hline
\end{tabular}




\begin{tabular}{|c|c|c|c|c|c|c|c|c|c|c|c|}
\hline in & 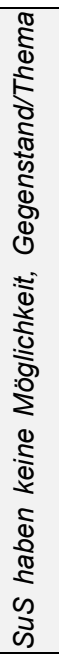 & 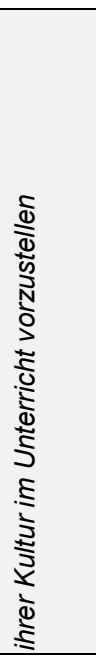 & 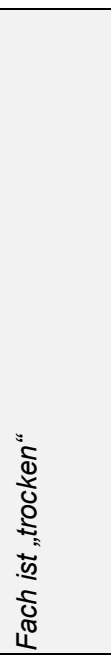 & 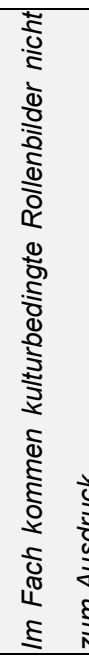 & 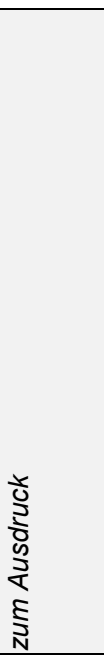 & 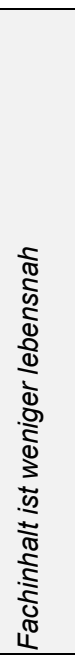 & & 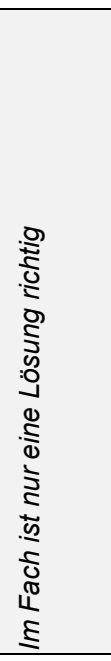 & 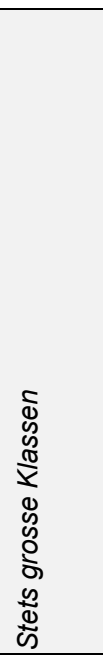 & 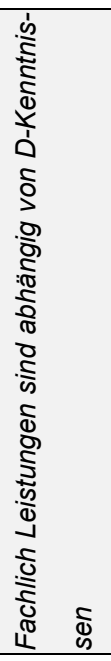 & 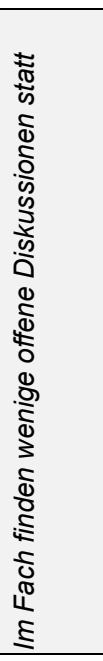 \\
\hline$\stackrel{O}{E}$ & & - & - & & $N$ & & - & $m$ & - & $m$ & $N$ \\
\hline O) & & $\mathrm{N}$ & - & & in & & $m$ & $m$ & in & $N$ & $m$ \\
\hline क & & $N$ & - & & $m$ & & 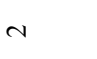 & + & in & - & in \\
\hline 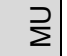 & & $\mathrm{N}$ & - & & in & & $N$ & $m$ & in & N & in \\
\hline 文 & & - & - & & $m$ & & - & - & - & $N$ & N \\
\hline 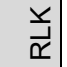 & & - & $N$ & & - & & - & - & in & $\nabla$ & - \\
\hline- & 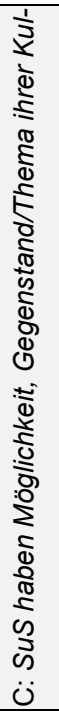 & 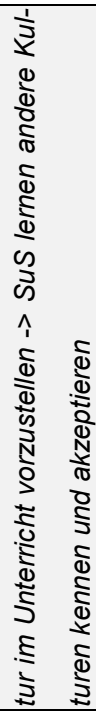 & 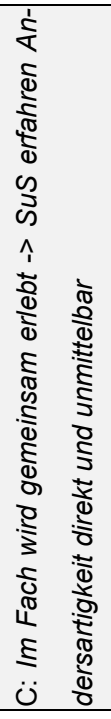 & 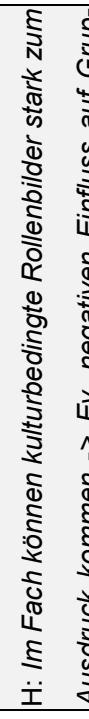 & 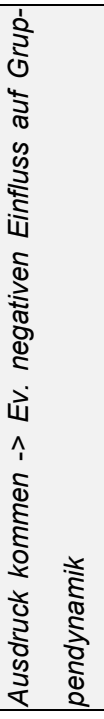 & 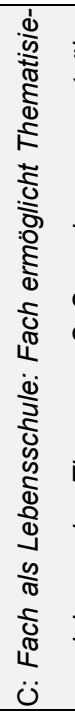 & 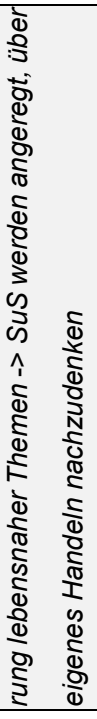 & 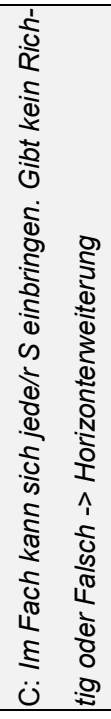 & 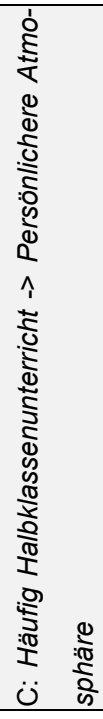 & 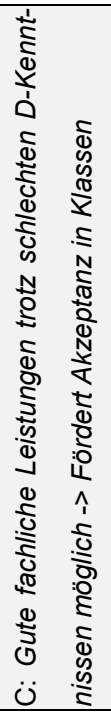 & 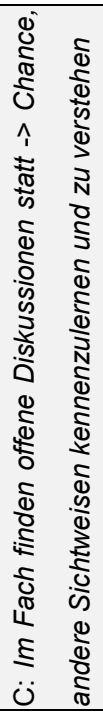 \\
\hline $\bar{\Xi}$ & $\underline{\bar{\Sigma}}$ & & $\underline{\widetilde{v}}$ & $\underline{\tilde{q}}$ & & $\underline{\Psi}$ & & $\underline{\underline{R}}$ & $\underline{\bullet}$ & $\underline{\underline{x}}$ & $\stackrel{\infty}{\underline{x}}$ \\
\hline
\end{tabular}

$\stackrel{\circledast N}{N}$ 


\begin{tabular}{|c|c|c|c|c|c|c|c|c|}
\hline in & 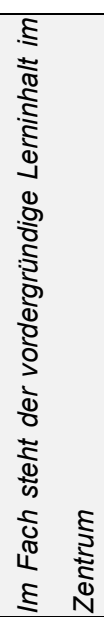 & 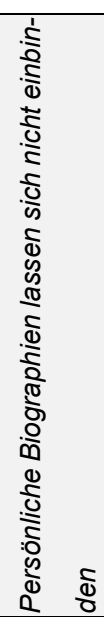 & 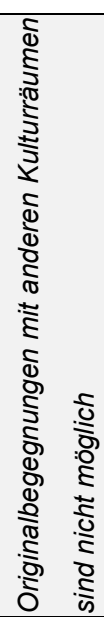 & 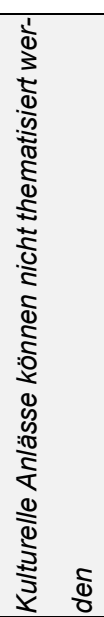 & 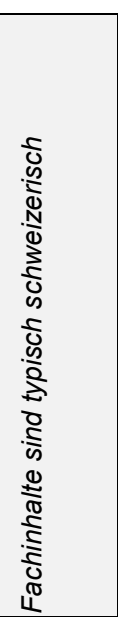 & 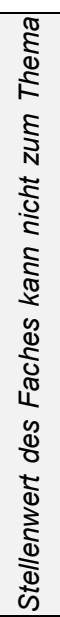 & 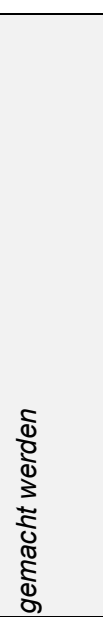 & 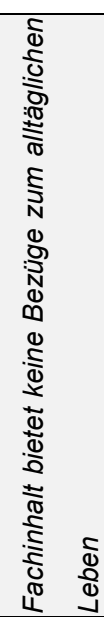 \\
\hline$\Sigma$ & in & - & in & in & $m$ & & $\sim$ & $N$ \\
\hline ш & - & $m$ & - & - & - & & $m$ & 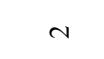 \\
\hline ч & ナ & $m$ & - & - & $m$ & & $m$ & ナ \\
\hline$\vec{z}$ & - & - & $N$ & - & $m$ & & - & - \\
\hline$\frac{0}{\omega}$ & $N$ & $N$ & $N$ & $N$ & $\sim$ & & $\sim$ & $N$ \\
\hline 㟔 & 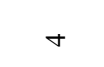 & - & - & - & $\sigma$ & & $m$ & - \\
\hline- & 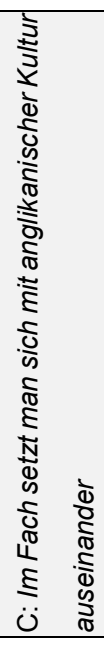 & 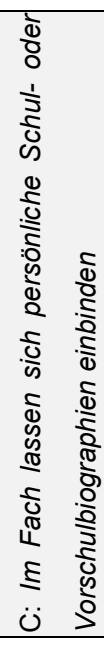 & 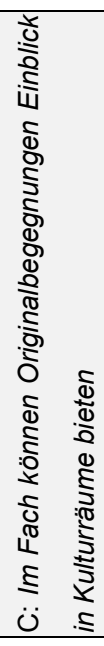 & 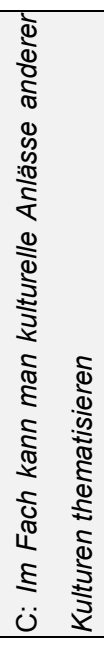 & 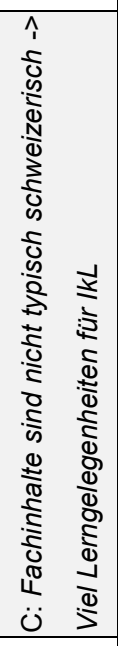 & 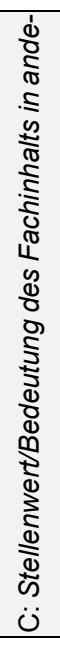 & 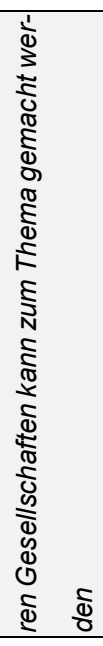 & 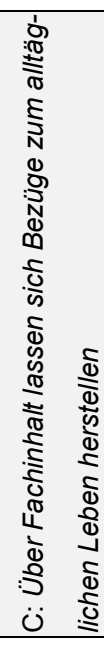 \\
\hline$\stackrel{\infty}{\Theta}$ & $\bar{x}$ & $\underline{\widetilde{r}}$ & $\underline{\tilde{m}}$ & 妾 & $\stackrel{10}{\underline{1}}$ & $\underline{q}$ & & $\underline{x}$ \\
\hline
\end{tabular}




\begin{tabular}{|c|c|c|c|c|c|c|c|c|c|}
\hline in & 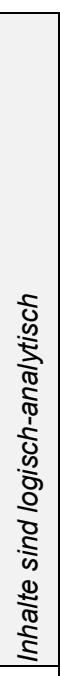 & 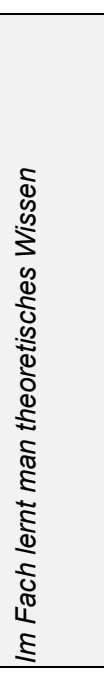 & 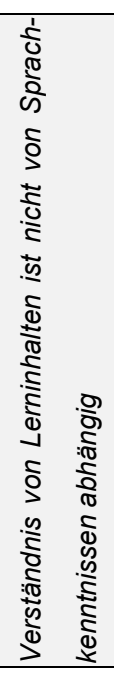 & 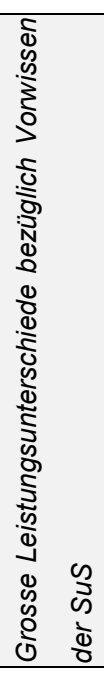 & 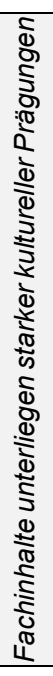 & 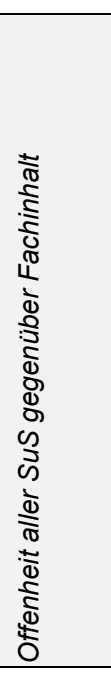 & 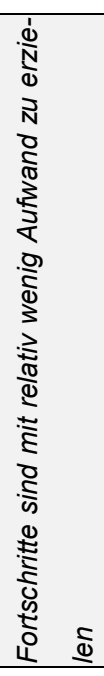 & 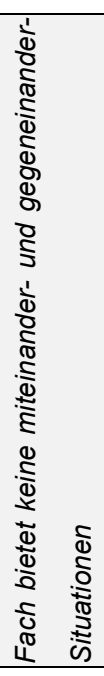 & 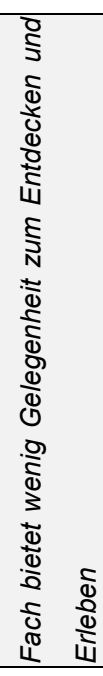 \\
\hline ४ & $m$ & $m$ & - & in & - & $N$ & - & in & in \\
\hline$\Sigma$ & in & $\sigma$ & $N$ & in & - & in & $N$ & in & 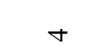 \\
\hline 㟔 & $m$ & $m$ & - & in & - & in & - & in & $\nabla$ \\
\hline ర్ల & - & - & in & $\nabla$ & - & $\nabla$ & in & in & $m$ \\
\hline$\frac{y}{z}$ & $\sim$ & $N$ & $N$ & 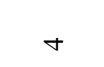 & - & in & $\nabla$ & in & - \\
\hline के & - & - & in & in & - & in & $n$ & - & - \\
\hline- & 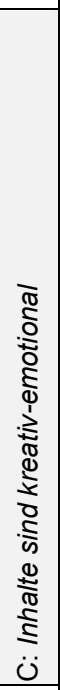 & 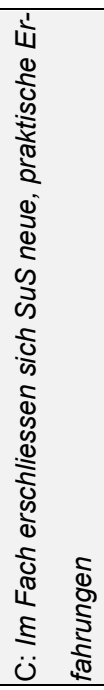 & 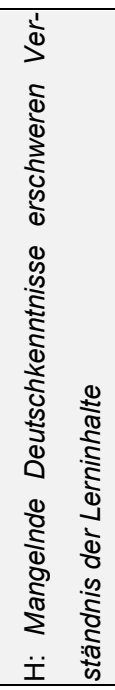 & 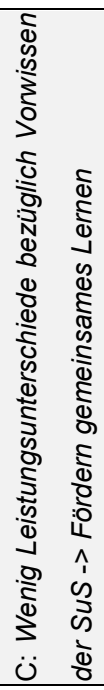 & 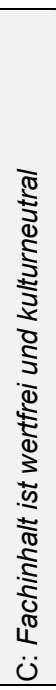 & 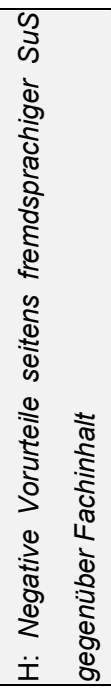 & 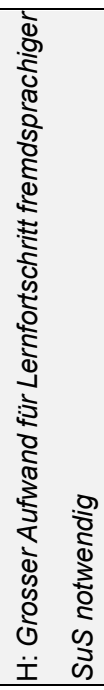 & 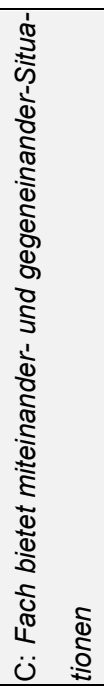 & 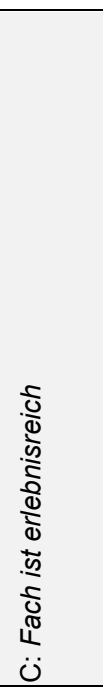 \\
\hline 3 & $\bar{x}$ & $\widetilde{\underline{x}}$ & $\tilde{x}$ & $\underset{\mathbf{z}}{\mathbb{Z}}$ & 岂 & 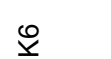 & $\hat{x}$ & $\stackrel{\infty}{\underline{x}}$ & $\stackrel{\mathscr{P}}{\underline{x}}$ \\
\hline
\end{tabular}




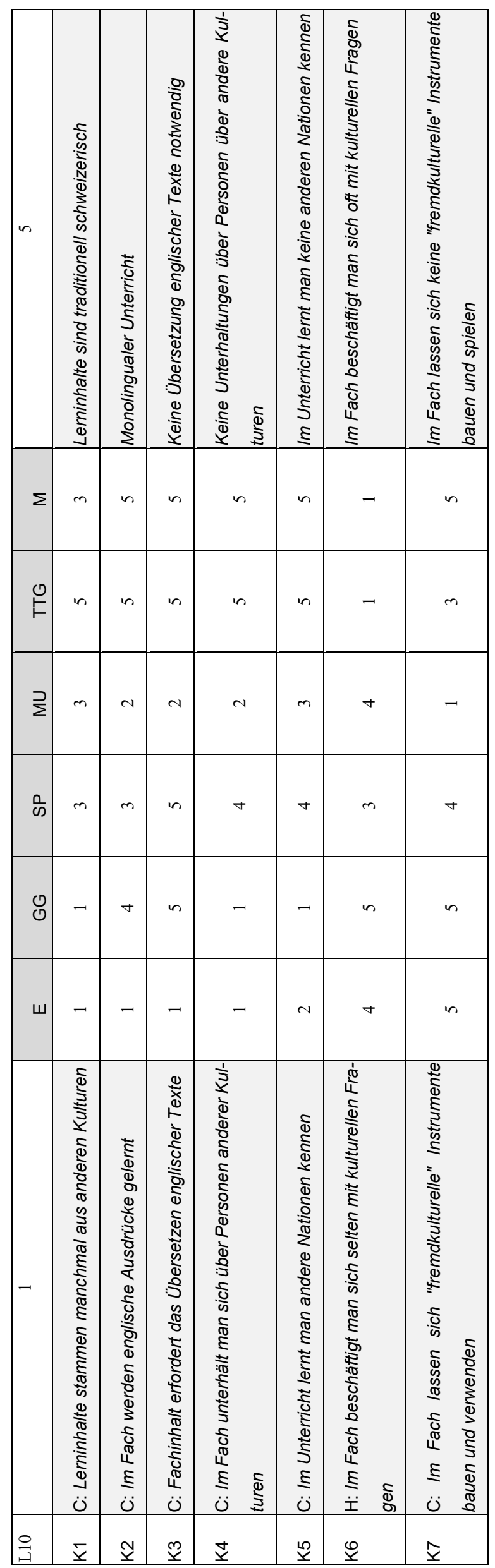




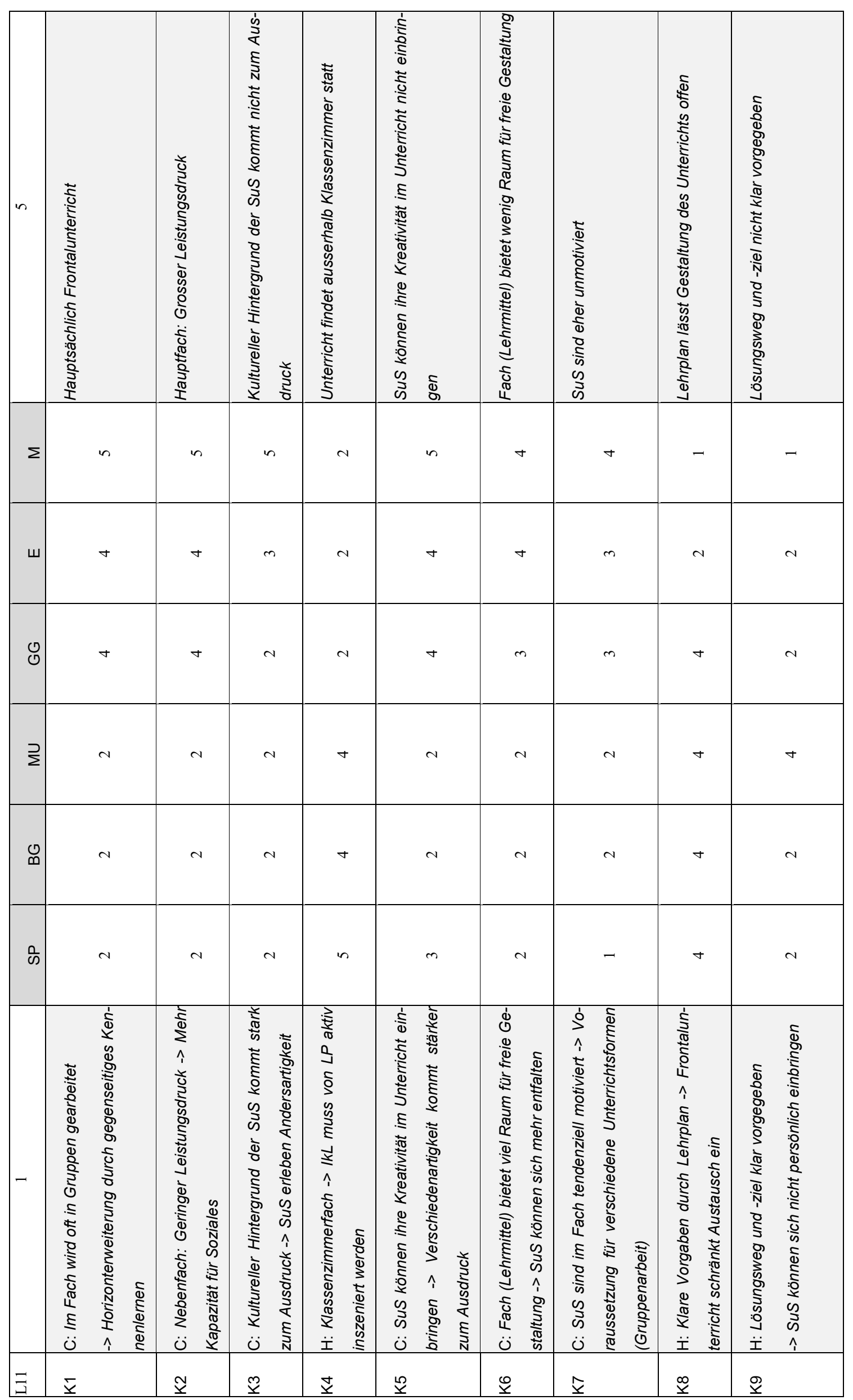

$\stackrel{m}{\sim}$ 


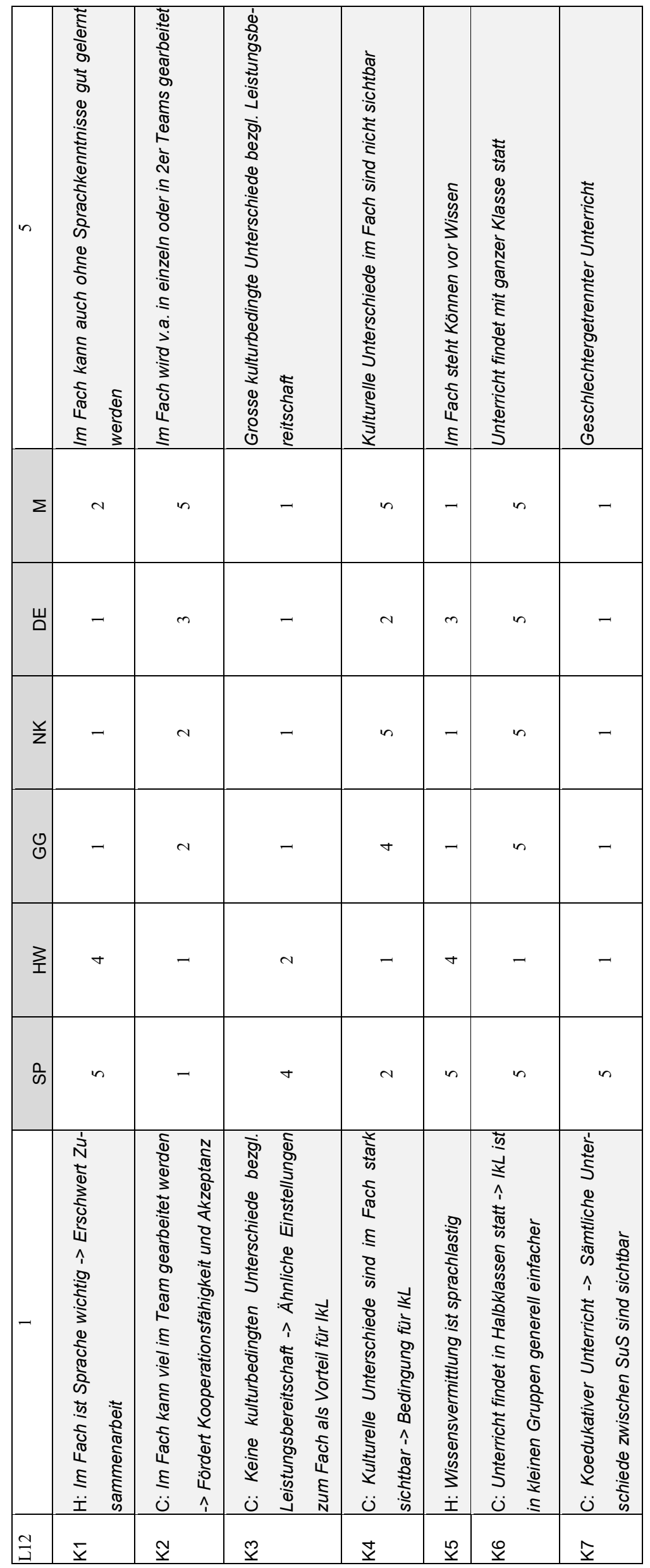




\begin{tabular}{|c|c|c|c|c|c|}
\hline$n$ & 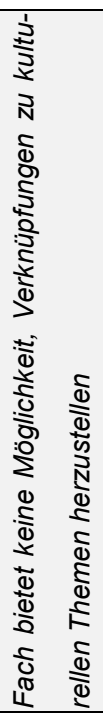 & 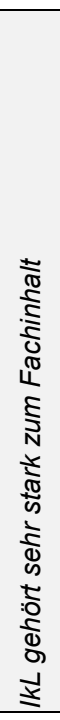 & 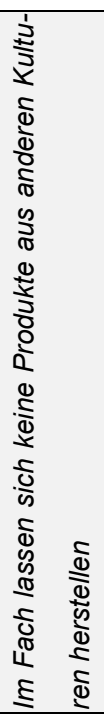 & 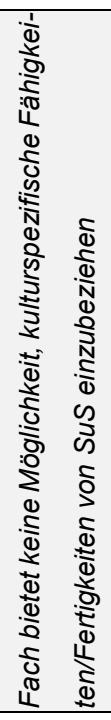 & 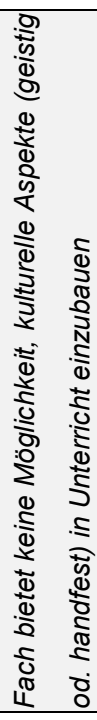 \\
\hline क & $m$ & $\sim$ & in & $m$ & $m$ \\
\hline$\Sigma$ & in & - & in & in & $\nabla$ \\
\hline$\stackrel{O}{E}$ & $m$ & $N$ & - & $m$ & - \\
\hline\llcorner & - & $n$ & - & $m$ & - \\
\hline$\stackrel{2}{\Sigma}$ & - & $m$ & in & - & - \\
\hline স্ত & - & $n$ & $N$ & - & - \\
\hline- & 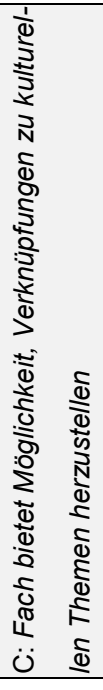 & 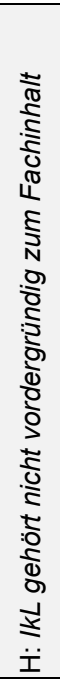 & 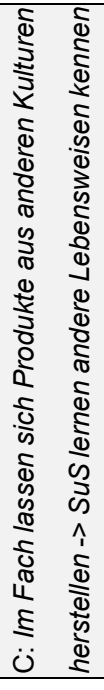 & 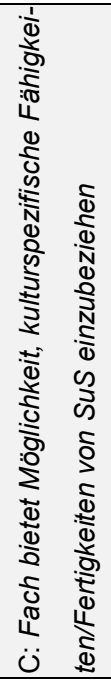 & 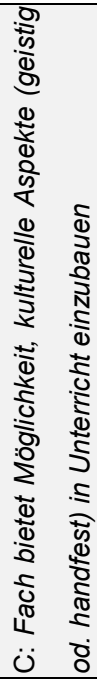 \\
\hline & $\underline{\bar{x}}$ & $x$ & $\underline{\underline{y}}$ & $\underline{\square}$ & $\underline{\underline{p}}$ \\
\hline
\end{tabular}




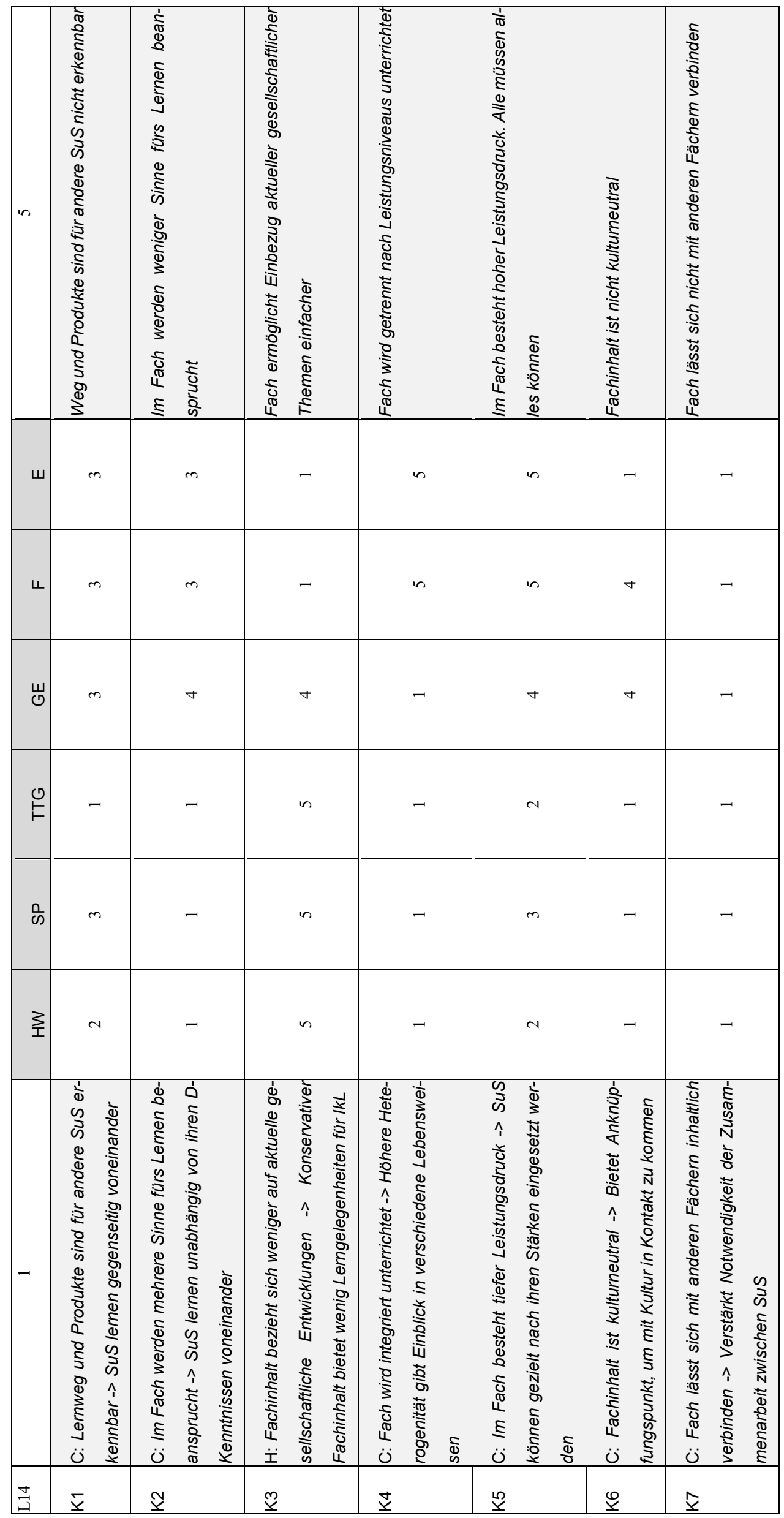




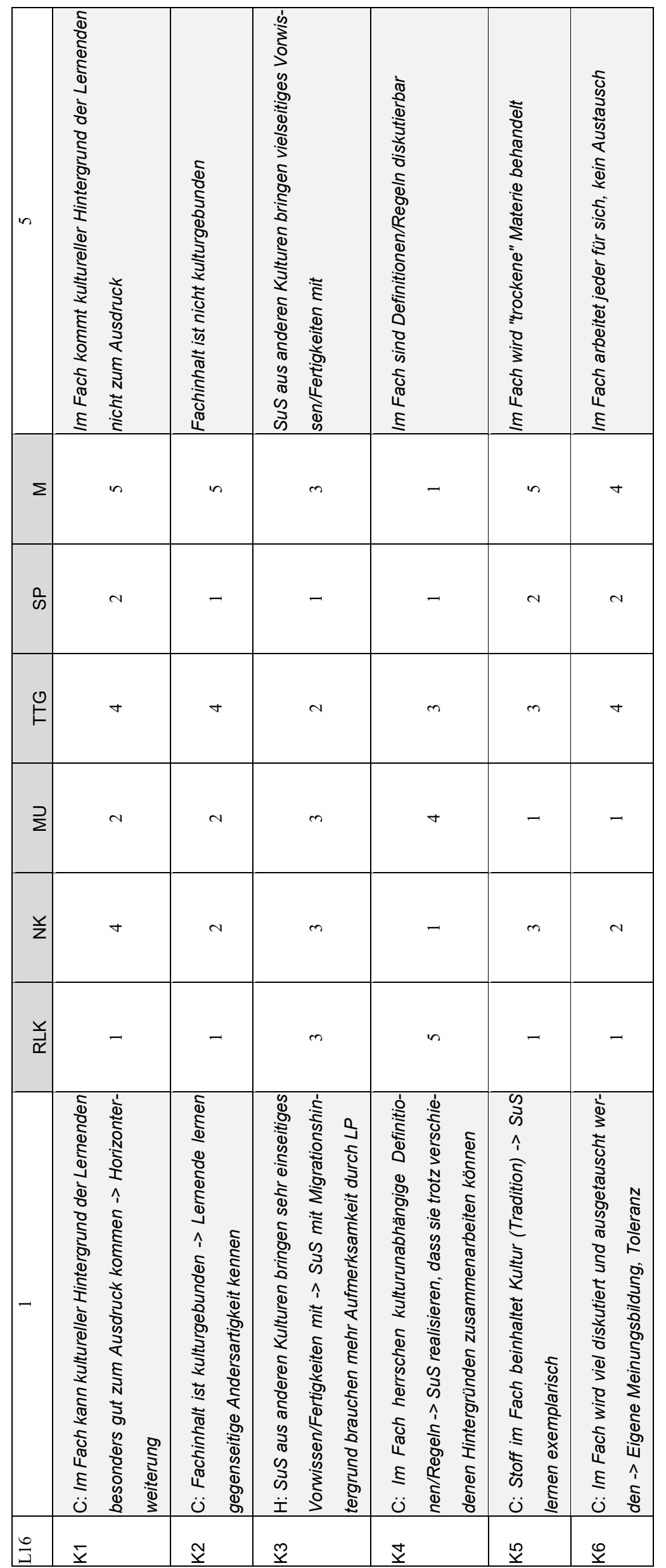




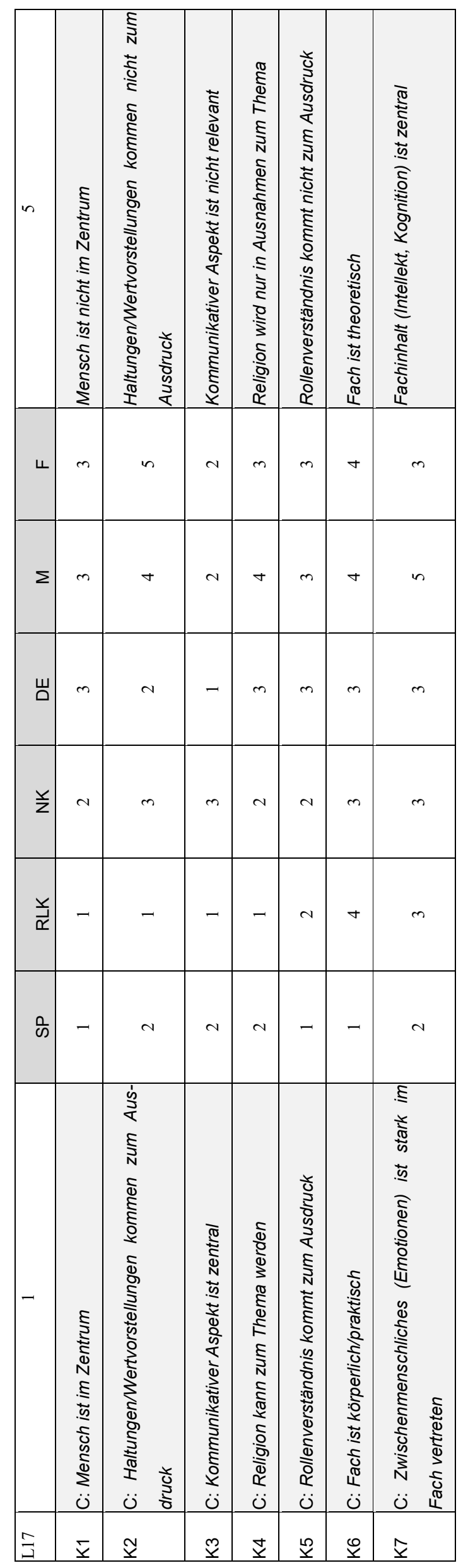




\begin{tabular}{|c|c|c|c|c|c|c|c|}
\hline in & 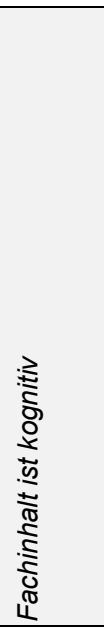 & 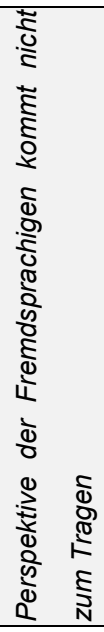 & 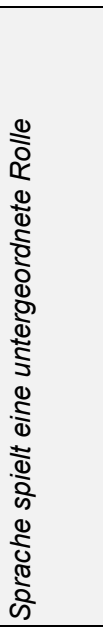 & 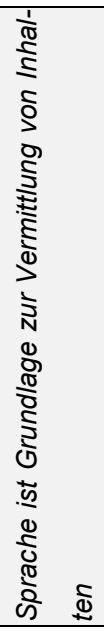 & & 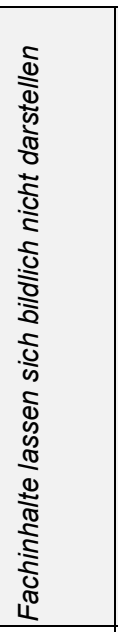 & 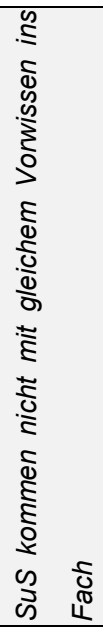 \\
\hline щu & $m$ & $\sim$ & - & in & & $\sigma$ & $m$ \\
\hline ᄂ & $\nabla$ & $N$ & 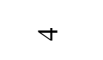 & $m$ & & $\sigma$ & $N$ \\
\hline z & $N$ & $m$ & $m$ & $m$ & & $N$ & $m$ \\
\hline O) & $N$ & $m$ & $N$ & 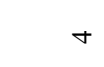 & & $N$ & $m$ \\
\hline$\Sigma$ & $N$ & $\nabla$ & $m$ & $m$ & & $m$ & in \\
\hline$\frac{1}{\omega}$ & - & $N$ & in & $\sim$ & & - & in \\
\hline- & 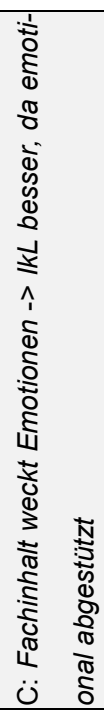 & 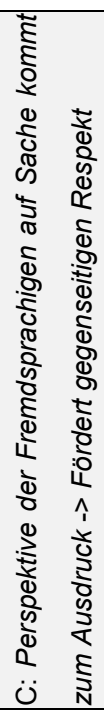 & 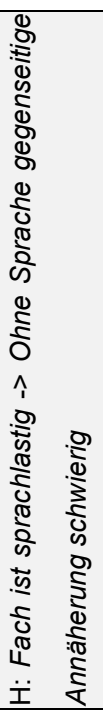 & 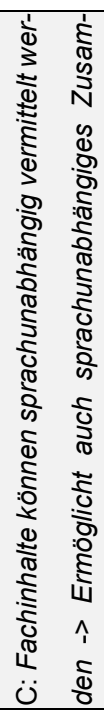 & 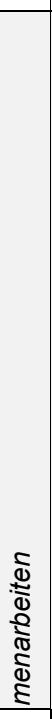 & 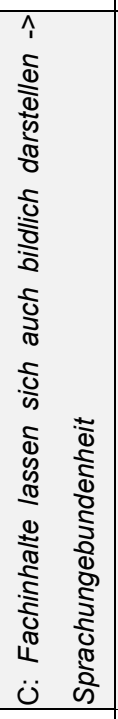 & 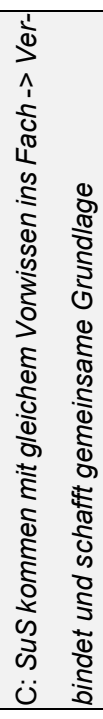 \\
\hline$\infty$ & $\bar{z}$ & $\widetilde{\widetilde{x}}$ & $\underline{\tilde{x}}$ & 年 & & $\underline{\underline{v}}$ & $\underline{\text { qu }}$ \\
\hline
\end{tabular}




\begin{tabular}{|c|c|c|c|c|c|c|c|}
\hline in & 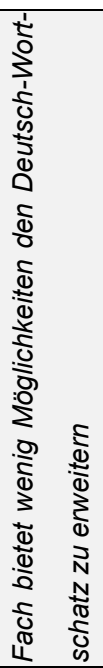 & 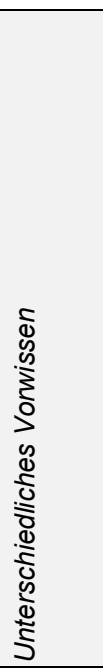 & 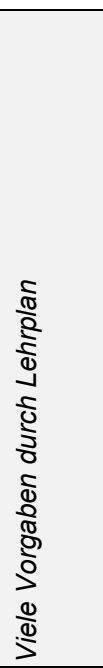 & 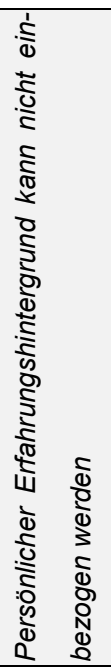 & 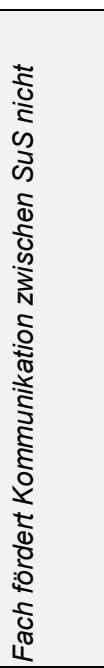 & 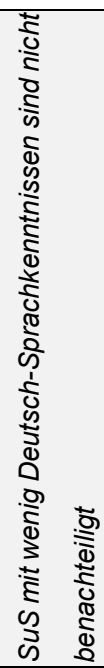 & 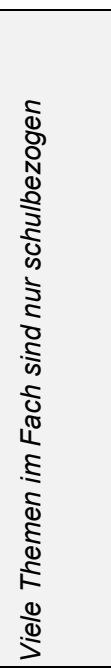 \\
\hline$\Sigma$ & N & $\sim$ & $\nabla$ & N & 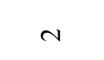 & N & $m$ \\
\hline$\frac{y}{z}$ & $N$ & $\sim$ & $m$ & - & $\sim$ & $N$ & - \\
\hline $\begin{array}{l}\text { D } \\
\text { D. }\end{array}$ & $N$ & - & $m$ & - & $N$ & $N$ & - \\
\hline 㟔 & - & $\sim$ & $\nabla$ & $\sim$ & $\sim$ & - & - \\
\hline के & - & $m$ & - & - & - & $\sigma$ & - \\
\hline$\vec{z}$ & $m$ & $\sim$ & - & - & $m$ & in & $N$ \\
\hline- & 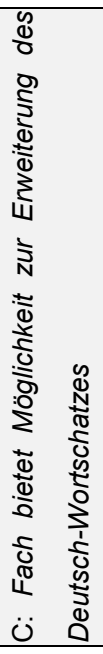 & 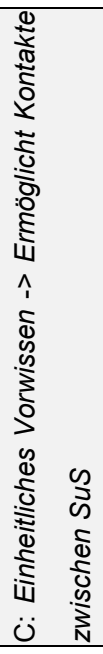 & 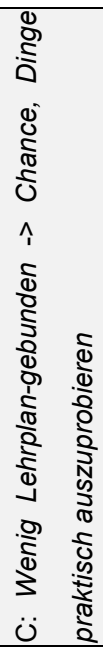 & 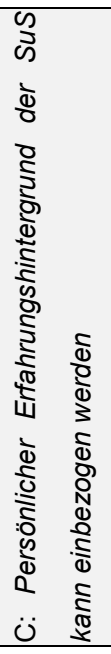 & 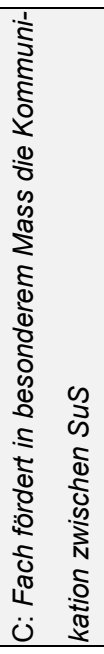 & 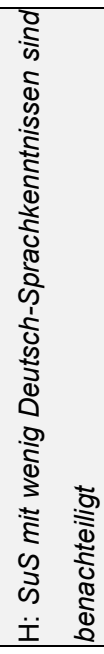 & 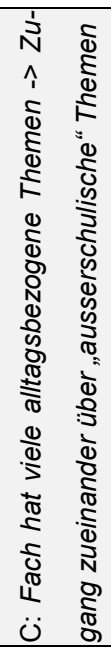 \\
\hline 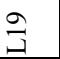 & $\bar{Y}$ & $\widetilde{\widetilde{Y}}$ & $\stackrel{\tilde{y}}{\underline{y}}$ & $\underset{\underline{\Sigma}}{\mathbf{z}}$ & $\underline{\underline{q}}$ & $\underline{q}$ & $\hat{x}$ \\
\hline
\end{tabular}




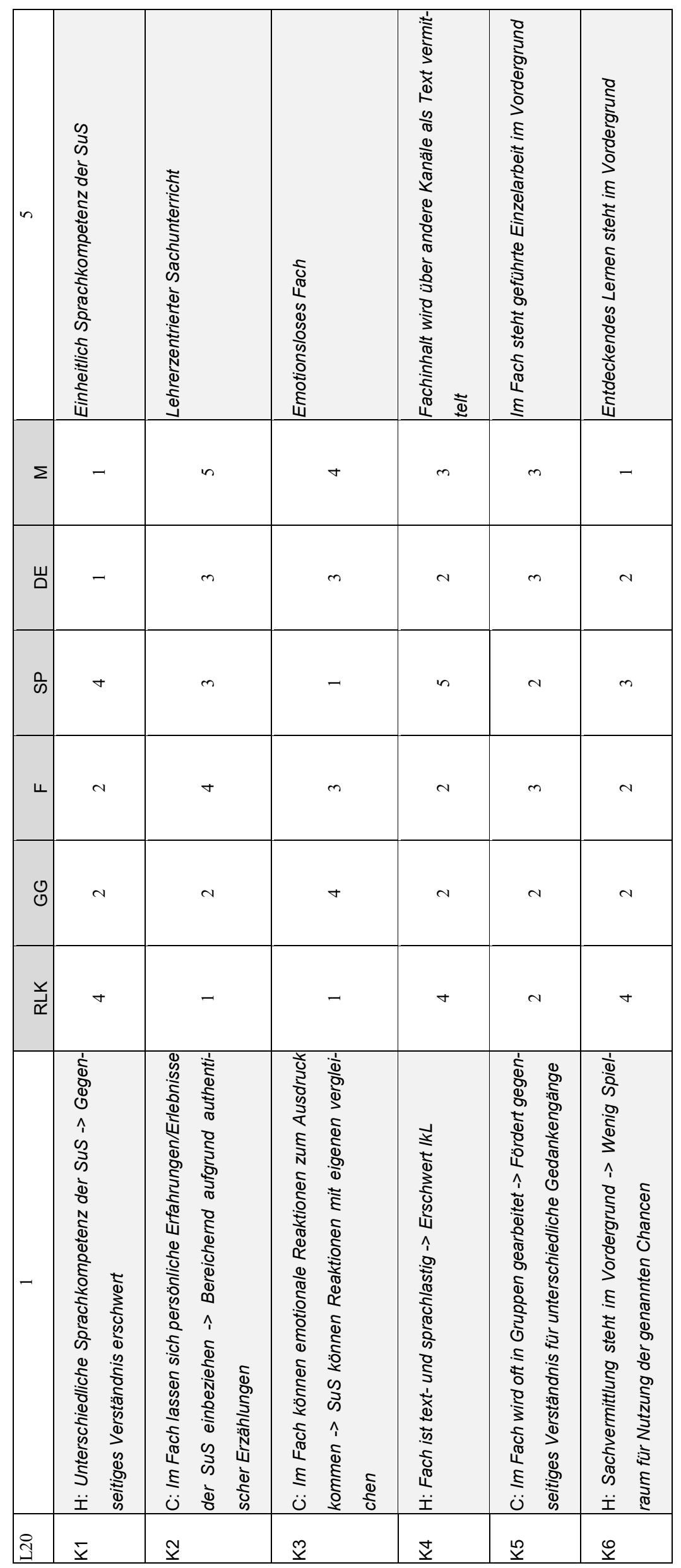




\begin{tabular}{|c|c|c|c|c|c|c|c|c|c|c|c|c|c|c|}
\hline in & 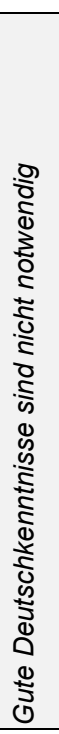 & & 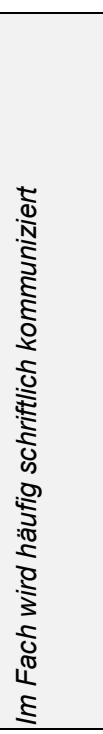 & 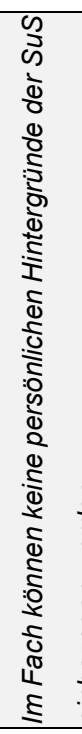 & 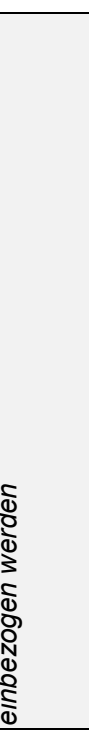 & 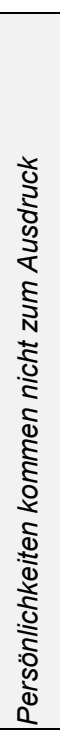 & & 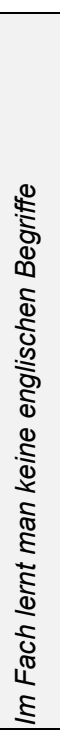 & & & 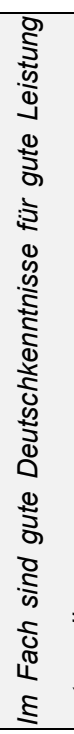 & & & 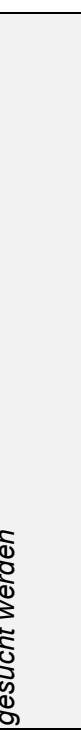 \\
\hline ш & & $N$ & $m$ & & $n$ & $\nabla$ & & & - & & & & & - \\
\hline 㟔 & & - & $m$ & & $n$ & $\nabla$ & & & in & & & & & - \\
\hline$\frac{n}{\omega}$ & & in & - & & - & - & & & N & & & & & $n$ \\
\hline U & & - & $\nabla$ & & $\tau$ & $\nabla$ & & & in & & & & & $n$ \\
\hline O) & & - & $m$ & & - & $\nabla$ & & & $\sim$ & & & & & r \\
\hline$\underset{\text { 竎 }}{ }$ & & 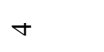 & - & & - & o & & & in & & & & & $n$ \\
\hline- & 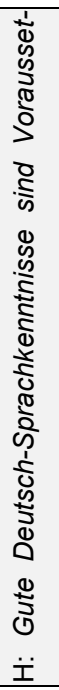 & 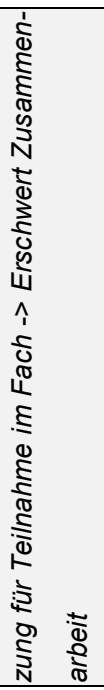 & 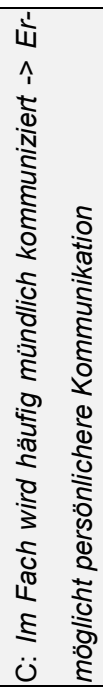 & 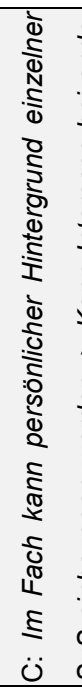 & 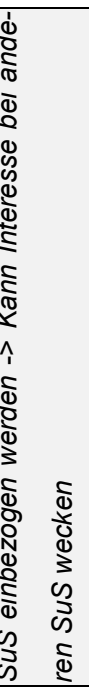 & 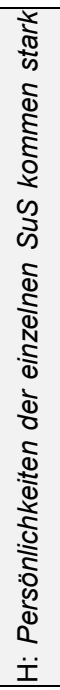 & 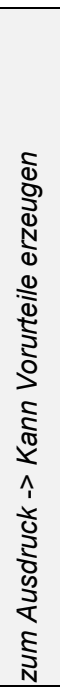 & 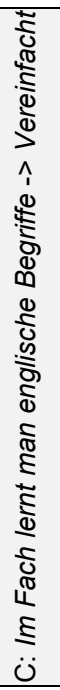 & 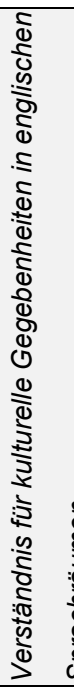 & 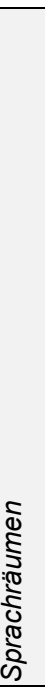 & 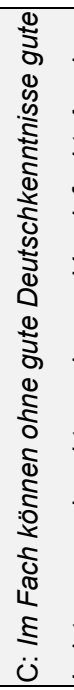 & 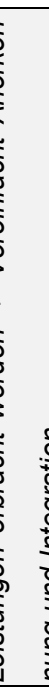 & & 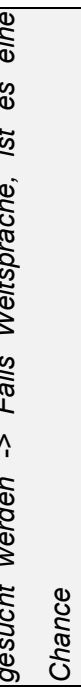 \\
\hline $\overrightarrow{\mathcal{I}}$ & \multicolumn{2}{|l|}{$\bar{x}$} & $\underline{\tilde{z}}$ & $\underline{\tilde{q}}$ & & 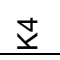 & & $\underline{\underline{R}}$ & & & $\underline{\stackrel{\varphi}{2}}$ & & & \\
\hline
\end{tabular}




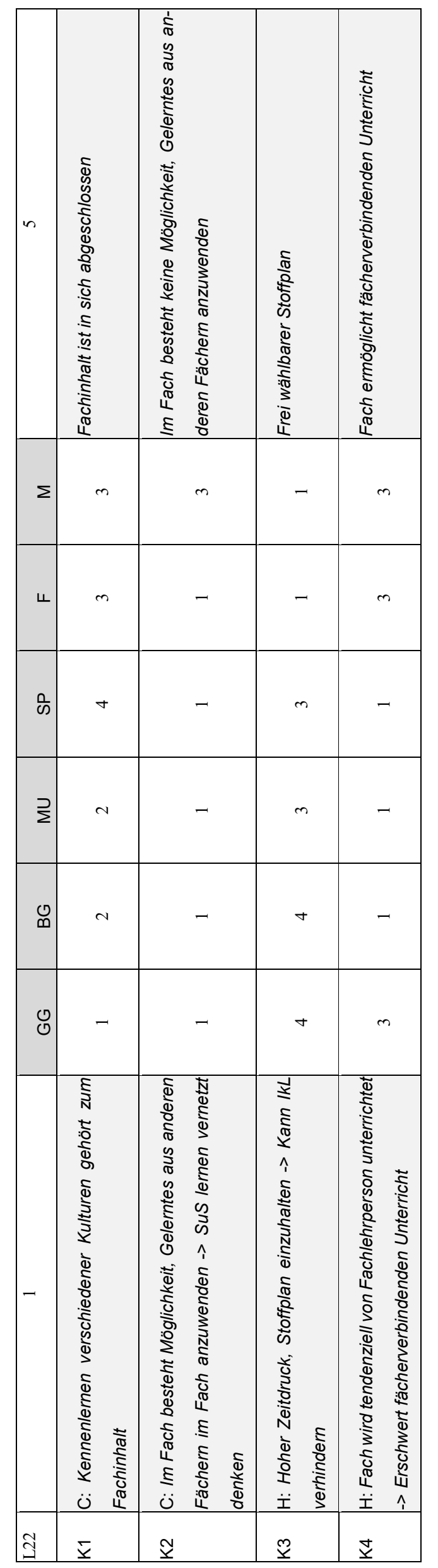

ํํำ 


\begin{tabular}{|c|c|c|c|c|c|c|c|c|c|c|}
\hline in & 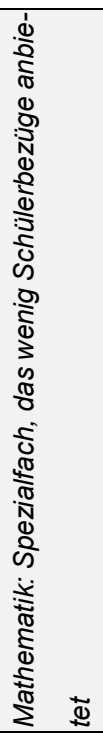 & 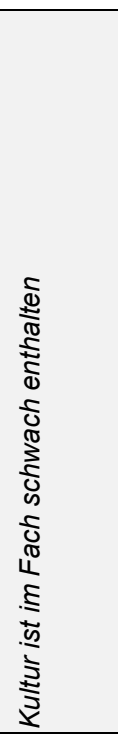 & 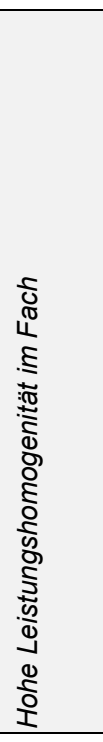 & 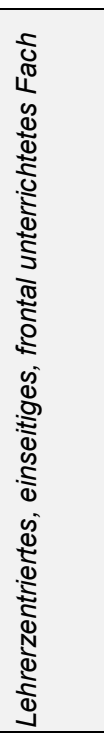 & 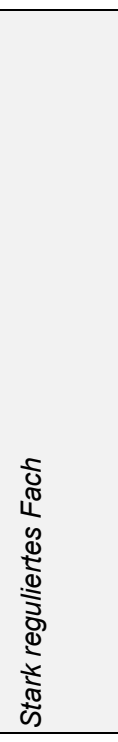 & 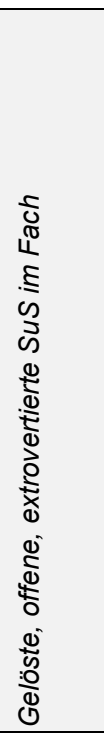 & 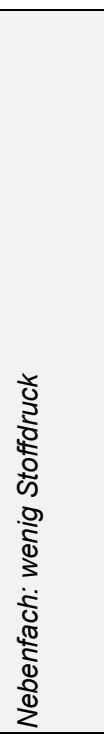 & 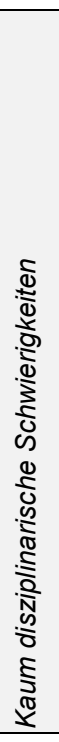 & & 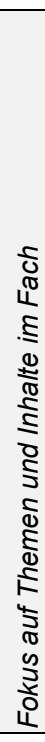 \\
\hline 㟔 & $\nabla$ & $m$ & $m$ & - & $m$ & $m$ & - & & in & - \\
\hline ш & $N$ & - & $N$ & - & $N$ & $N$ & $N$ & & in & $N$ \\
\hline$\frac{0}{\text { के }}$ & $m$ & + & $m$ & $m$ & $\sigma$ & $\sigma$ & $m$ & & $m$ & in \\
\hline স্ৰ & $m$ & N & - & - & - & $\nabla$ & in & & N & in \\
\hline$\stackrel{2}{\Sigma}$ & N & N & - & - & N & $N$ & in & & - & in \\
\hline$\underset{1}{3}$ & $N$ & $N$ & $\nabla$ & - & $N$ & in & $\nabla$ & & - & $n$ \\
\hline- & 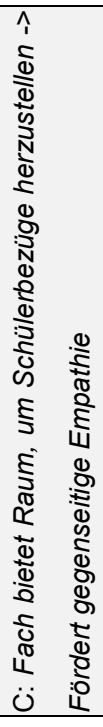 & 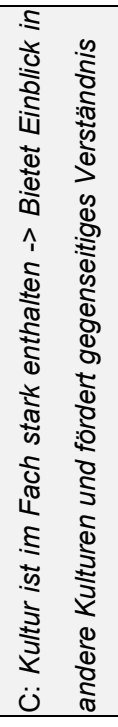 & 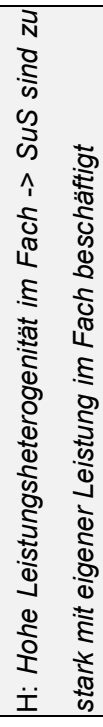 & 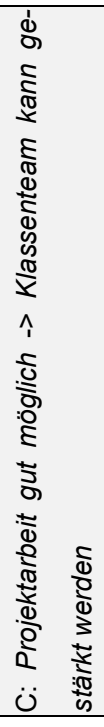 & 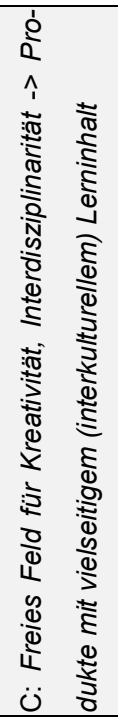 & 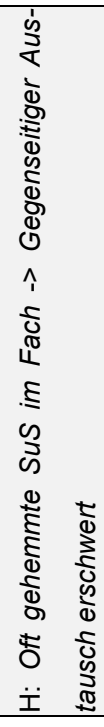 & 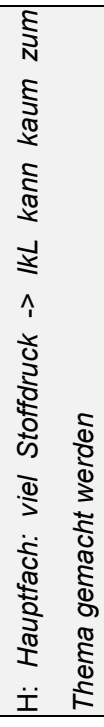 & 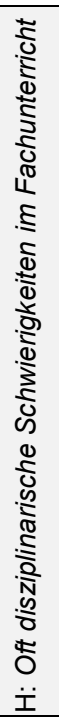 & 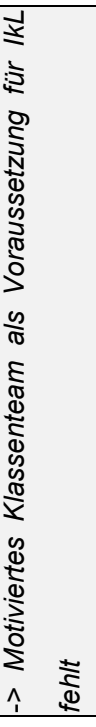 & 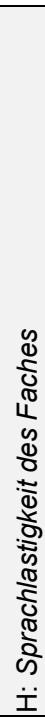 \\
\hline$\widetilde{\Xi}$ & $\overline{\mathbf{z}}$ & $\widetilde{\underline{x}}$ & $\stackrel{m}{\underline{x}}$ & $\underset{\underline{Y}}{ \pm}$ & $\underline{10}$ & $\underline{q}$ & $\hat{x}$ & $\begin{array}{l}\infty \\
\stackrel{\infty}{y}\end{array}$ & & $\underline{\underline{8}}$ \\
\hline
\end{tabular}




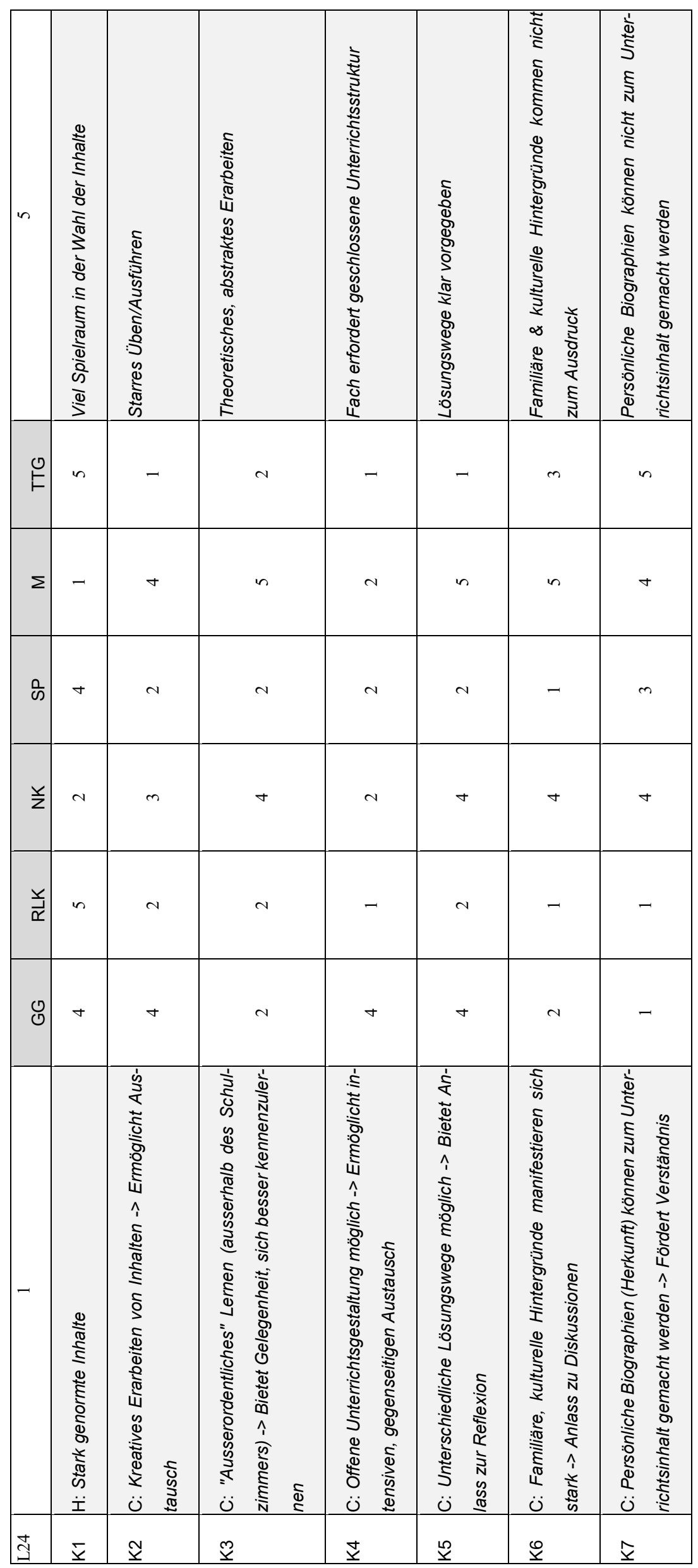




\begin{tabular}{|c|c|c|c|c|c|c|c|}
\hline in & 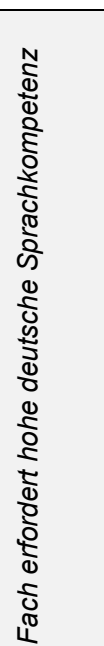 & 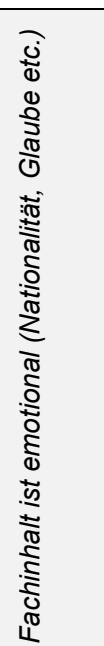 & 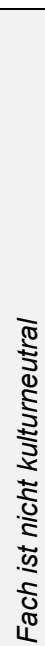 & 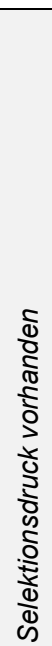 & 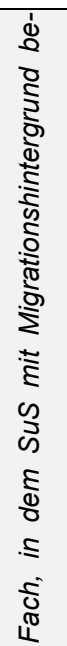 & 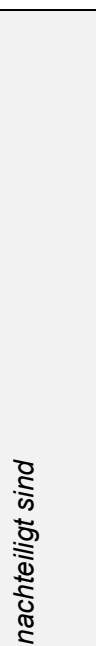 & 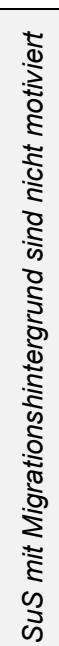 \\
\hline$\stackrel{P}{\Sigma}$ & $\sim$ & in & t & - & & - & 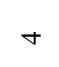 \\
\hline 山् & in & - & - & in & & in & - \\
\hline$\frac{0}{\omega}$ & N & in & 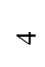 & - & & - & 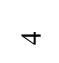 \\
\hline D্ & - & - & + & $n$ & & $\sim$ & $\sigma$ \\
\hline O্ & 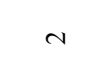 & - & in & - & & - & in \\
\hline$\Sigma$ & - & - & - & in & & $N$ & $m$ \\
\hline- & 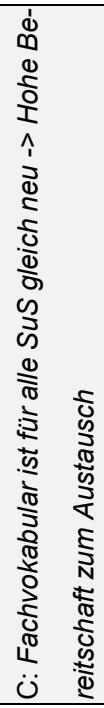 & 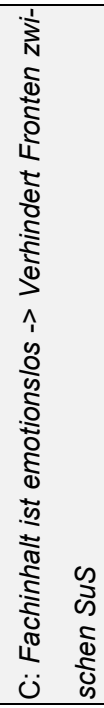 & 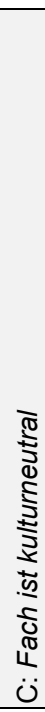 & 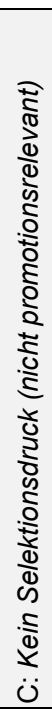 & 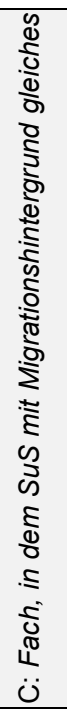 & 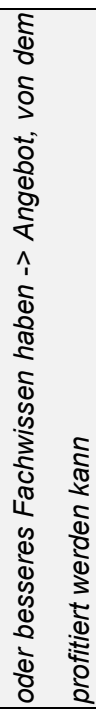 & 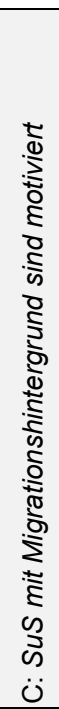 \\
\hline త & $\overline{\mathbf{Y}}$ & $\widetilde{\widetilde{x}}$ & $\tilde{\tilde{x}}$ & $\underset{\Sigma}{ \pm}$ & $\underline{\underline{L}}$ & & $\stackrel{\varphi}{\underline{x}}$ \\
\hline
\end{tabular}




\begin{tabular}{|c|c|c|c|c|c|c|c|c|}
\hline in & 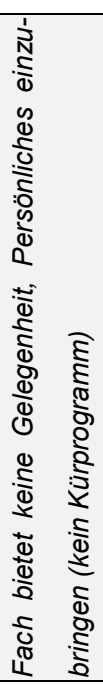 & 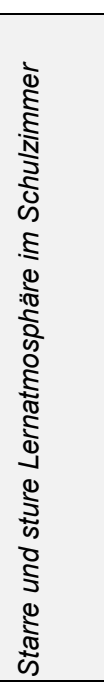 & 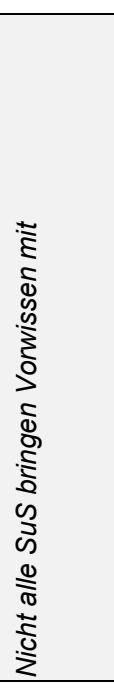 & 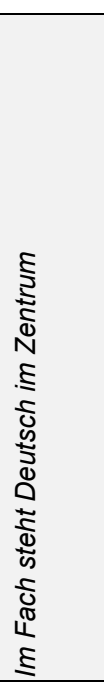 & 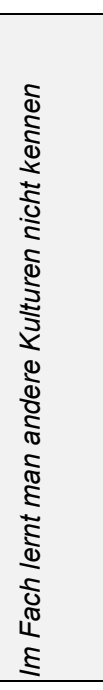 & 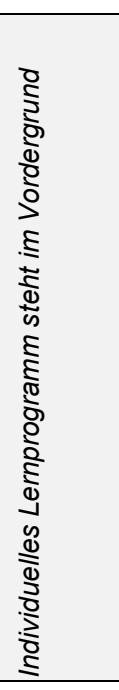 & 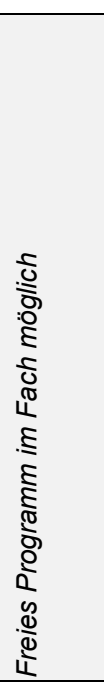 & 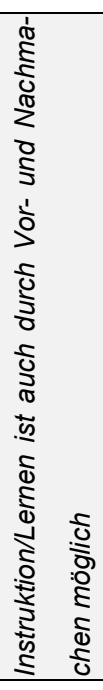 \\
\hline щu & $n$ & $\nabla$ & $\nabla$ & n & t & $\sigma$ & - & - \\
\hline O) & $\alpha$ & $m$ & $m$ & t & 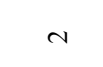 & $m$ & $N$ & $N$ \\
\hline$\Sigma$ & 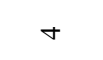 & $\nabla$ & + & $\nabla$ & in & $\nabla$ & - & $\alpha$ \\
\hline 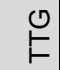 & $m$ & $N$ & $\sigma$ & $N$ & T & $m$ & $N$ & $\nabla$ \\
\hline$\stackrel{P}{\Sigma}$ & $m$ & N & $m$ & 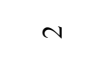 & $\alpha$ & $m$ & $N$ & $\nabla$ \\
\hline$\frac{0}{\omega}$ & ナ & - & - & - & $m$ & $m$ & - & in \\
\hline- & 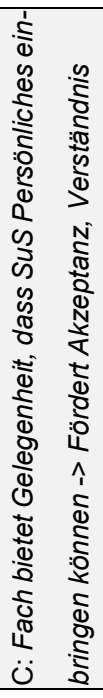 & 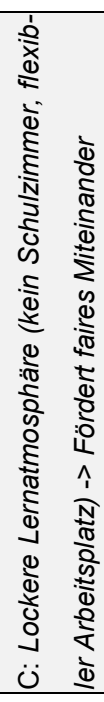 & 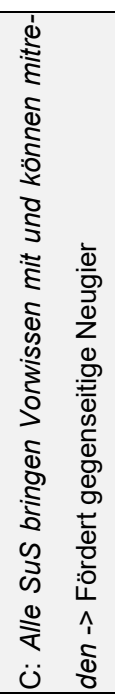 & 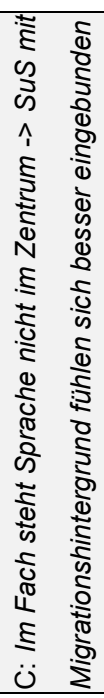 & 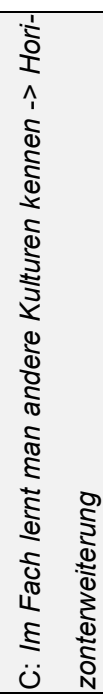 & 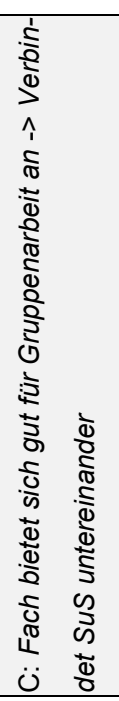 & 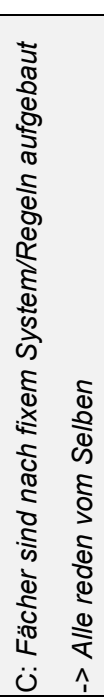 & 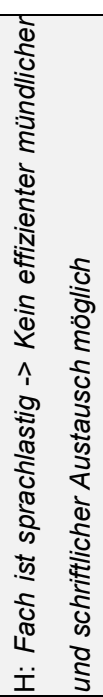 \\
\hline త్ర & $\bar{x}$ & $\widetilde{\Sigma}$ & $\stackrel{m}{\underline{x}}$ & 企 & $\ddot{\varphi}$ & $\mathscr{\mathscr { x }}$ & $\hat{x}$ & $\stackrel{\infty}{\check{\Sigma}}$ \\
\hline
\end{tabular}

$\stackrel{n}{n}$ 


\section{G Konstrukt-Retrievals Kategorien Fragestellung 2}

1) Einbeziehen persönlicher Hintergründe der Schülerinnen und Schüler

Anzahl (Anteil) Lehrpersonen: 13 (54.2\%)

Anzahl Konstrukte: 13

\begin{tabular}{|c|c|}
\hline Chancen-Pol & Hindernis-Pol \\
\hline $\begin{array}{l}\text { SuS aus anderen Kulturen können in Szene gesetzt } \\
\text { werden }->I k L \text { findet explizit statt }\end{array}$ & $\begin{array}{l}\text { SuS aus anderen Kulturen können nicht in Szene ge- } \\
\text { setzt werden (L1: K5) }\end{array}$ \\
\hline $\begin{array}{l}\text { Möglichkeit, Erzählungen von SuS aus ihren Län- } \\
\text { dern einzubeziehen }\end{array}$ & $\begin{array}{l}\text { SuS haben keine Möglichkeit, ihre Erzählungen ein- } \\
\text { zubringen (L2: K6) }\end{array}$ \\
\hline $\begin{array}{l}\text { Im Fach lässt sich der persönliche Hintergrund der } \\
\text { SuS einbeziehen }\end{array}$ & $\begin{array}{l}\text { Fach bietet keine Gelegenheit, persönliche Bezüge } \\
\text { zu SuS herzustellen (L3: K4) }\end{array}$ \\
\hline $\begin{array}{l}\text { Fach ermöglicht Einbeziehen persönlicher Aspekte } \\
\text { der SuS -> IkL geht von SuS aus }\end{array}$ & $\begin{array}{l}\text { Fach bietet keine Möglichkeit, persönliche Aspekte } \\
\text { der SuS einzubeziehen (L5: K3) }\end{array}$ \\
\hline $\begin{array}{l}\text { SuS haben Möglichkeit, Gegenstand/Thema ihrer } \\
\text { Kultur im Unterricht vorzustellen -> SuS lernen an- } \\
\text { dere Kulturen kennen und akzeptieren }\end{array}$ & $\begin{array}{l}\text { SuS haben keine Möglichkeit, Gegenstand/Thema } \\
\text { ihrer Kultur im Unterricht vorzustellen (L7: K1) }\end{array}$ \\
\hline $\begin{array}{l}\text { Im Fach lassen sich persönliche Schul- oder Vor- } \\
\text { schulbiographien einbinden }\end{array}$ & $\begin{array}{l}\text { Persönliche Biographien lassen sich nicht einbinden } \\
\text { (L8: K2) }\end{array}$ \\
\hline $\begin{array}{l}\text { Fach bietet Möglichkeit, kulturspezifische Fähigkei- } \\
\text { ten/Fertigkeiten von SuS einzubeziehen }\end{array}$ & $\begin{array}{l}\text { Fach bietet keine Möglichkeit, kulturspezifische Fä- } \\
\text { higkeiten/Fertigkeiten von SuS einzubeziehen (L13: } \\
\text { K4) }\end{array}$ \\
\hline $\begin{array}{l}\text { Persönlicher Erfahrungshintergrund der SuS kann } \\
\text { einbezogen werden }\end{array}$ & $\begin{array}{l}\text { Persönlicher Erfahrungshintergrund kann nicht ein- } \\
\text { bezogen werden (L19: K4) }\end{array}$ \\
\hline $\begin{array}{l}\text { Im Fach lassen sich persönliche Erfahrungen/Erleb- } \\
\text { nisse der SuS einbeziehen -> Bereichernd aufgrund } \\
\text { authentischer Erzählungen }\end{array}$ & Lehrerzentrierter Sachunterricht (L20: K2) \\
\hline $\begin{array}{l}\text { Im Fach kann persönlicher Hintergrund einzelner } \\
\text { SuS einbezogen werden -> Kann Interesse bei ande- } \\
\text { ren SuS wecken }\end{array}$ & $\begin{array}{l}\text { Im Fach können keine persönlichen Hintergründe } \\
\text { der SuS einbezogen werden (L21: K3) }\end{array}$ \\
\hline $\begin{array}{l}\text { Fach bietet Raum, um Schülerbezüge herzustellen - } \\
>\text { Fördert gegenseitige Empathie }\end{array}$ & $\begin{array}{l}\text { Mathematik: Spezialfach, das wenig Schülerbezüge } \\
\text { anbietet (L23: K1) }\end{array}$ \\
\hline $\begin{array}{l}\text { Persönliche Biographien (Herkunft) können zum } \\
\text { Unterrichtsinhalt gemacht werden -> Fördert Ver- } \\
\text { ständnis }\end{array}$ & $\begin{array}{l}\text { Persönliche Biographien können nicht zum Unter- } \\
\text { richtsinhalt gemacht werden (L24: K7) }\end{array}$ \\
\hline $\begin{array}{l}\text { Fach bietet Gelegenheit, dass SuS Persönliches ein- } \\
\text { bringen können -> Fördert Akzeptanz, Verständnis }\end{array}$ & $\begin{array}{l}\text { Fach bietet keine Gelegenheit, Persönliches einzu- } \\
\text { bringen (kein Kürprogramm) (L26: K1) }\end{array}$ \\
\hline
\end{tabular}

2) Geringe Sprachgebundenheit

Anzahl (Anteil) Lehrpersonen: 12 (50\%)

Anzahl Konstrukte: 19

\begin{tabular}{l|l}
\hline Chancen-Pol & Hindernis-Pol \\
\hline
\end{tabular}




\begin{tabular}{|c|c|}
\hline $\begin{array}{l}\text { Fachliches Lernen kann auch ohne Deutsch-Kennt- } \\
\text { nisse stattfinden }\end{array}$ & $\begin{array}{l}\text { Fachliches Lernen durch schlechte Deutsch-Kennt- } \\
\text { nisse erschwert -> Schlechte Integration fremdspra- } \\
\text { chiger SUS (L1: K3) }\end{array}$ \\
\hline $\begin{array}{l}\text { Bilder und Karten -> Ermöglichen nicht-sprachli- } \\
\text { chen Wissenserwerb }\end{array}$ & Anspruchsvolle Texte (L2: K2) \\
\hline Nicht-sprachlastiges Fach & $\begin{array}{l}\text { Sprachlastigkeit des Faches -> Erschwert das ge- } \\
\text { meinsame Lernen (L2: K7) }\end{array}$ \\
\hline $\begin{array}{l}\text { Gute fachliche Leistungen trotz schlechten } \\
\text { Deutsch-Kenntnissen möglich -> Fördert Akzeptanz } \\
\text { in Klasse }\end{array}$ & $\begin{array}{l}\text { Fachliche Leistungen sind abhängig von Deutsch- } \\
\text { Kenntnissen (L7: K7) }\end{array}$ \\
\hline $\begin{array}{l}\text { Verständnis von Lerninhalten ist nicht von Sprach- } \\
\text { kenntnissen abhängig }\end{array}$ & $\begin{array}{l}\text { Mangelnde Deutsch-Kenntnisse erschweren Ver- } \\
\text { ständnis der Lerninhalte (L9: K3) }\end{array}$ \\
\hline $\begin{array}{l}\text { Fortschritte sind aufgrund geringer Sprachlastig- } \\
\text { keit mit relativ wenig Aufwand zu erzielen }\end{array}$ & $\begin{array}{l}\text { Grosser Aufwand für Lernfortschritt fremdsprachi- } \\
\text { ger SuS notwendig (L9: K7) }\end{array}$ \\
\hline $\begin{array}{l}\text { Im Fach kann auch ohne Sprachkenntnisse gut ge- } \\
\text { lernt werden }\end{array}$ & $\begin{array}{l}\text { Im Fach ist Sprache wichtig } \rightarrow \text { Erschwert Zusam- } \\
\text { menarbeit (L12: K1) }\end{array}$ \\
\hline Im Fach steht Können vor Wissen & Wissensvermittlung ist sprachlastig (L12: K5) \\
\hline $\begin{array}{l}\text { Im Fach werden mehrere Sinne fürs Lernen bean- } \\
\text { sprucht -> SuS lernen unabhängig von ihren } \\
\text { Deutsch-Kenntnissen voneinander }\end{array}$ & $\begin{array}{l}\text { Im Fach werden weniger Sinne fürs Lernen bean- } \\
\text { sprucht (L14: K2) }\end{array}$ \\
\hline Sprache spielt eine untergeordnete Rolle & $\begin{array}{l}\text { Fach ist sprachlastig -> Ohne Sprache gegenseitige } \\
\text { Annäherung schwierig (L18: K3) }\end{array}$ \\
\hline $\begin{array}{l}\text { Fachinhalte können sprachunabhängig vermittelt } \\
\text { werden -> Ermöglicht sprachunabhängiges Zusam- } \\
\text { menarbeiten }\end{array}$ & $\begin{array}{l}\text { Sprache ist Grundlage zur Vermittlung von Inhalten } \\
\text { (L18: K4) }\end{array}$ \\
\hline $\begin{array}{l}\text { Fachinhalte lassen sich auch bildlich darstellen -> } \\
\text { Sprachungebundenheit }\end{array}$ & $\begin{array}{l}\text { Fachinhalte lassen sich bildlich nicht darstellen } \\
\text { (L18: K5) }\end{array}$ \\
\hline $\begin{array}{l}\text { SuS mit wenig Deutsch-Sprachkenntnissen sind } \\
\text { nicht benachteiligt }\end{array}$ & $\begin{array}{l}\text { SuS mit wenig Deutsch-Sprachkenntnissen sind be- } \\
\text { nachteiligt (L19: K6) }\end{array}$ \\
\hline $\begin{array}{l}\text { Fachinhalt wird über andere Kanäle als Text vermit- } \\
\text { telt }\end{array}$ & $\begin{array}{l}\text { Fach ist text- und sprachlastig }->\text { Erschwert IkL (L20: } \\
\text { K4) }\end{array}$ \\
\hline Gute Deutschkenntnisse sind nicht notwendig & $\begin{array}{l}\text { Gute Deutsch-Sprachkenntnisse sind Vorausset- } \\
\text { zung für Teilnahme im Fach -> Erschwert Zusam- } \\
\text { menarbeit (L21: K1) }\end{array}$ \\
\hline $\begin{array}{l}\text { Im Fach können ohne gute Deutschkenntnisse gute } \\
\text { Leistungen erbracht werden -> Vereinfacht Aner- } \\
\text { kennung und Integration }\end{array}$ & $\begin{array}{l}\text { Im Fach sind gute Deutschkenntnisse für gute Leis- } \\
\text { tung notwendig (L21: } \mathrm{K} 6)\end{array}$ \\
\hline Fokus auf Themen und Inhalte im Fach & Sprachlastigkeit des Faches (L23: K9) \\
\hline $\begin{array}{l}\text { Im Fach steht Sprache nicht im Zentrum -> SuS mit } \\
\text { Migrationshintergrund fühlen sich besser einge- } \\
\text { bunden }\end{array}$ & Im Fach steht Deutsch im Zentrum (L26: K4) \\
\hline $\begin{array}{l}\text { Instruktion/Lernen ist auch durch Vor- und Nach- } \\
\text { machen möglich }\end{array}$ & $\begin{array}{l}\text { Fach ist sprachlastig }>>\text { Kein effizienter mündlicher } \\
\text { und schriftlicher Austausch möglich (L26: K8) }\end{array}$ \\
\hline
\end{tabular}




\begin{tabular}{|c|c|}
\hline \multicolumn{2}{|l|}{ 3) Kulturbezogene } \\
\hline Anzahl (Anteil) Lehrpersonen: 11 (45.8\%) & \\
\hline \multicolumn{2}{|l|}{ Anzahl Konstrukte: 23} \\
\hline Chancen-Pol & Hindernis-Pol \\
\hline $\begin{array}{l}\text { Im Fach lassen sich verschiedene Kulturen themati- } \\
\text { sieren }\end{array}$ & $\begin{array}{l}\text { Im Fach steht nur eine Kultur im Vordergrund (L2: } \\
\text { K4) }\end{array}$ \\
\hline $\begin{array}{l}\text { Bietet Möglichkeit, verschiedene kulturelle Materi- } \\
\text { alien einzubeziehen -> Andere Kulturen kennenler- } \\
\text { nen }\end{array}$ & $\begin{array}{l}\text { Bietet keine Möglichkeit zum Einbezug kultureller } \\
\text { Materialien (L3: K1) }\end{array}$ \\
\hline $\begin{array}{l}\text { Fachinhalt ist kulturell gehaltvoll -> Kulturelle Un- } \\
\text { terschiede können thematisiert werden }\end{array}$ & Fachinhalt ist kulturlos (L3: K3) \\
\hline $\begin{array}{l}\text { Fachinhalte bieten Möglichkeit zu Vergleichen ver- } \\
\text { schiedener Kulturen }\end{array}$ & Inhalte sind kulturunabhängig (L4: K3) \\
\hline Rituale an Feiertagen können thematisiert werden & $\begin{array}{l}\text { Feiertage/Rituale können nicht thematisiert wer- } \\
\text { den (L4: K7) }\end{array}$ \\
\hline $\begin{array}{l}\text { Im Fach setzt man sich mit anglikanischer Kultur } \\
\text { auseinander }\end{array}$ & $\begin{array}{l}\text { Im Fach steht der vordergründige Lerninhalt im } \\
\text { Zentrum (L8: K1) }\end{array}$ \\
\hline $\begin{array}{l}\text { Im Fach können Originalbegegnungen Einblick in } \\
\text { Kulturräume bieten }\end{array}$ & $\begin{array}{l}\text { Originalbegegnungen mit anderen Kulturräumen } \\
\text { sind nicht möglich (L8: K3) }\end{array}$ \\
\hline $\begin{array}{l}\text { Im Fach kann man kulturelle Anlässe anderer Kul- } \\
\text { turen thematisieren }\end{array}$ & $\begin{array}{l}\text { Kulturelle Anlässe können nicht thematisiert wer- } \\
\text { den (L8: K4) }\end{array}$ \\
\hline $\begin{array}{l}\text { Fachinhalte sind nicht typisch schweizerisch }->\text { Viel } \\
\text { Lerngelegenheiten für IkL }\end{array}$ & Fachinhalte sind typisch schweizerisch (L8: K5) \\
\hline $\begin{array}{l}\text { Lerninhalte stammen manchmal aus anderen Kul- } \\
\text { turen }\end{array}$ & Lerninhalte sind traditionell schweizerisch (L10: K1) \\
\hline $\begin{array}{l}\text { Im Fach unterhält man sich über Personen anderer } \\
\text { Kulturen }\end{array}$ & $\begin{array}{l}\text { Keine Unterhaltungen über Personen über andere } \\
\text { Kulturen (L10: K4) }\end{array}$ \\
\hline Im Unterricht lernt man andere Nationen kennen & $\begin{array}{l}\text { Im Unterricht lernt man keine anderen Nationen } \\
\text { kennen (L10: K5) }\end{array}$ \\
\hline $\begin{array}{l}\text { Im Fach beschäftigt man sich oft mit kulturellen } \\
\text { Fragen }\end{array}$ & $\begin{array}{l}\text { Im Fach beschäftigt man sich selten mit kulturellen } \\
\text { Fragen (L10: K6) }\end{array}$ \\
\hline $\begin{array}{l}\text { Im Fach lassen sich "fremdkulturelle" Instrumente } \\
\text { bauen und verwenden }\end{array}$ & $\begin{array}{l}\text { Im Fach lassen sich keine "fremdkulturelle" Instru- } \\
\text { mente bauen und spielen (L10: K7) }\end{array}$ \\
\hline $\begin{array}{l}\text { Fach bietet Möglichkeit, Verknüpfungen zu kultu- } \\
\text { rellen Themen herzustellen }\end{array}$ & $\begin{array}{l}\text { Fach bietet keine Möglichkeit, Verknüpfungen zu } \\
\text { kulturellen Themen herzustellen (L13: K1) }\end{array}$ \\
\hline IkL gehört sehr stark zum Fachinhalt & $\begin{array}{l}\text { IkL gehört nicht vordergründig zum Fachinhalt } \\
\text { (L13: K2) }\end{array}$ \\
\hline $\begin{array}{l}\text { Im Fach lassen sich Produkte aus anderen Kulturen } \\
\text { herstellen -> SuS lernen andere Lebensweisen ken- } \\
\text { nen }\end{array}$ & $\begin{array}{l}\text { Im Fach lassen sich keine Produkte aus anderen Kul- } \\
\text { turen herstellen (L13: K3) }\end{array}$ \\
\hline $\begin{array}{l}\text { Fach bietet Möglichkeit, kulturelle Aspekte (geistig } \\
\text { oder handfest) in Unterricht einzubauen }\end{array}$ & $\begin{array}{l}\text { Fach bietet keine Möglichkeit, kulturelle Aspekte } \\
\text { (geistig oder handfest) in Unterricht einzubauen } \\
\text { (L13: K5) }\end{array}$ \\
\hline
\end{tabular}

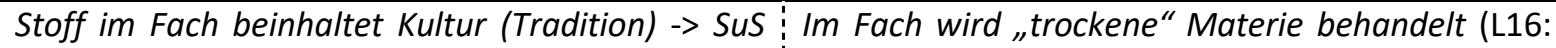
lernen exemplarisch 


\begin{tabular}{l|l}
\hline Religion kann zum Thema werden & Religion wird nur in Ausnahmen zum Thema (L17: \\
& K5) \\
\hline Kennenlernen verschiedener Kulturen gehört zum & Fachinhalt ist in sich abgeschlossen (L22: K1) \\
Fachinhalt & \\
\hline Kultur ist im Fach stark enthalten -> Bietet Einblick & Kultur ist im Fach schwach enthalten (L23: K2) \\
in andere Kulturen und fördert gegenseitiges Ver- & \\
ständnis & \\
\hline Im Fach lernt man andere Kulturen kennen -> Hori- & Im Fach lernt man andere Kulturen nicht kennen \\
zonterweiterung & (L26: K5) \\
\hline
\end{tabular}

4) Geringer Leistungs- und Stoffdruck

Anzahl (Anteil) Lehrpersonen: 10 (41.7\%)

Anzahl Konstrukte: 14

\begin{tabular}{|c|c|}
\hline Chancen-Pol & Hindernis-Pol \\
\hline $\begin{array}{l}\text { Fach ist offen für unterschiedliche Themen, viel } \\
\text { Spielraum }->\text { Verschiedene SuS-Interessen können } \\
\text { berücksichtigt werden }\end{array}$ & $\begin{array}{l}\text { Starres Programm, keine Freiheit in Stoffwahl (L1: } \\
\text { K1) }\end{array}$ \\
\hline $\begin{array}{l}\text { Freiheit in der Wahl des Lehrmittels }->\text { Themen an- } \\
\text { derer Kulturen können behandelt werden }\end{array}$ & Vorgegebenes Lehrmittel (L1: K2) \\
\hline Wenig Stoffdruck & $\begin{array}{l}\text { Hoher Stoffdruck durch Lehrplan vorgegeben -> } \\
\text { Einzelne Interessen können schlecht berücksichtigt } \\
\text { werden (L1: K7) }\end{array}$ \\
\hline $\begin{array}{l}\text { Kein Zeitproblem: Interkulturelles Lernen kann zum } \\
\text { Thema werden }\end{array}$ & $\begin{array}{l}\text { Zeitproblem: Fachinhalt steht im Vordergrund (L3: } \\
\text { K2) }\end{array}$ \\
\hline Hoher Freiraum in der Unterrichtsgestaltung & $\begin{array}{l}\text { Hoher Zeit-/Stoffdruck durch Lehrplanvorgaben } \\
\text { (L4: K2) }\end{array}$ \\
\hline $\begin{array}{l}\text { Nebenfach: Geringer Leistungsdruck -> Mehr Kapa- } \\
\text { zität für Soziales }\end{array}$ & Hauptfach: Grosser Leistungsdruck (L11: K2) \\
\hline $\begin{array}{l}\text { Fach (Lehrmittel) bietet viel Raum für freie Gestal- } \\
\text { tung } \rightarrow \text { SuS können sich mehr entfalten }\end{array}$ & $\begin{array}{l}\text { Fach (Lehrmittel) bietet wenig Raum für freie Ge- } \\
\text { staltung (L11: K6) }\end{array}$ \\
\hline Lehrplan lässt Gestaltung des Unterrichts offen & $\begin{array}{l}\text { Klare Vorgaben durch Lehrplan -> Frontalunterricht } \\
\text { schränkt Austausch ein (L11: K8) }\end{array}$ \\
\hline $\begin{array}{l}\text { Im Fach besteht tiefer Leistungsdruck } \rightarrow \text { SuS kön- } \\
\text { nen gezielt nach Stärken eingesetzt werden }\end{array}$ & $\begin{array}{l}\text { Im Fach besteht hoher Leistungsdruck. Alle müssen } \\
\text { alles können (L14: K5) }\end{array}$ \\
\hline $\begin{array}{l}\text { Wenig Lehrplan-gebunden -> Chance, Dinge prak- } \\
\text { tisch auszuprobieren }\end{array}$ & Viele Vorgaben durch Lehrplan (L19: K3) \\
\hline Frei wählbarer Stoffplan & $\begin{array}{l}\text { Hoher Zeitdruck, Stoffplan einzuhalten -> Kann IkL } \\
\text { verhindern (L22: K3) }\end{array}$ \\
\hline Nebenfach: Wenig Stoffdruck & $\begin{array}{l}\text { Hauptfach: Viel Stoffdruck }>\text { IkL kann kaum zum } \\
\text { Thema gemacht werden (L23: K7) }\end{array}$ \\
\hline Viel Spielraum in der Wahl der Inhalte & Stark genormte Inhalte (L24: K1) \\
\hline Kein Selektionsdruck (nicht promotionsrelevant) & Selektionsdruck vorhanden (L25: K4) \\
\hline
\end{tabular}


5) Offene Lernwege und variierende Ergebnisse

Anzahl (Anteil) Lehrpersonen: 8 (33.3\%)

Anzahl Konstrukte: 10

\begin{tabular}{l|l}
\hline Chancen-Pol & Hindernis-Pol \\
\hline Fach mit offenen Lösungswegen, Zugängen & $\begin{array}{l}\text { Exakte Wissenschaften: Kein Spielraum bezgl. Re- } \\
\text { sultat, Lösungsweg -> Keine Interaktion (L1: K10) }\end{array}$ \\
\hline Im Fach kann sich jede/r S einbringen. Gibt kein & Im Fach ist nur eine Lösung richtig (L7: K5) \\
Richtig oder Falsch -> Horizonterweiterung & \\
\hline SuS können ihre Kreativität im Unterricht einbrin- & SuS können ihre Kreativität im Unterricht nicht ein- \\
gen -> Verschiedenartigkeit kommt stärker zum & bringen (L11: K5) \\
Ausdruck & \\
\hline Lösungsweg und -ziel nicht klar vorgegeben & Lösungsweg und -ziel klar vorgegeben -> SuS kön- \\
& nen sich nicht persönlich einbringen (L11: K9) \\
\hline Lernweg und Produkte sind unterschiedlich -> SuS & Weg und Produkte können variieren (L14: K1) \\
lernen gegenseitig voneinander & \\
\hline Entdeckendes Lernen steht im Vordergrund & Sachvermittlung steht im Vordergrund -> Wenig \\
& Spielraum für Nutzung der genannten Chancen \\
\hline Freies Feld für Kreativität, Interdisziplinarität $->$ & Stark reguliertes Fach (L23: K5) \\
Produkte mit vielseitigem (interkulturellen) Lernin- & \\
halt & \\
\hline Kreatives Erarbeiten von Inhalten -> Ermöglicht & Starres Üben/Ausführen (L24: K2) \\
Austausch & \\
\hline Offene Unterrichtsgestaltung möglich -> Ermög- \\
licht intensiven, gegenseitigen Austausch
\end{tabular}

6) Persönliche Hintergründe und Haltungen der SuS kommen zum Ausdruck

Anzahl (Anteil) Lehrpersonen: 8 (33.3\%)

Anzahl Konstrukte: 9

\begin{tabular}{l|l}
\hline Chancen-Pol & Hindernis-Pol \\
\hline Fach bringt persönliche Haltungen der SuS (implizit) & Persönliche Haltungen kommen im Fach nicht zum \\
zum Ausdruck & Ausdruck (L4: K8) \\
\hline Fach ermöglicht Wahrnehmen von Unterschieden & Fach ermöglicht kein Wahrnehmen \& Austauschen \\
zwischen SuS & von Unterschieden (L5: K7) \\
\hline Kultureller Hintergrund der SuS kommt stark zum & Kultureller Hintergrund der SuS kommt nicht zum \\
Ausdruck -> SuS erleben Andersartigkeit & Ausdruck (L11: K3) \\
\hline Kulturelle Unterschiede sind im Fach stark sichtbar & Kulturelle Unterschiede im Fach sind nicht sichtbar \\
-> Bedingung für IkL & (L12: K4) \\
\hline Im Fach kann kultureller Hintergrund der Lernen- & Im Fach kommt kultureller Hintergrund der Lernen- \\
den besonders gut zum Ausdruck kommen -> Hori- & den nicht zum Ausdruck (L16: K1) \\
zonterweiterung & \\
\hline 262 &
\end{tabular}




\begin{tabular}{l:l}
\hline $\begin{array}{l}\text { Haltungen/Wertvorstellungen kommen zum Aus- } \\
\text { druck }\end{array}$ & $\begin{array}{l}\text { Haltungen/Wertvorstellungen kommen nicht zum } \\
\text { Rollenverständnis kommt zum Ausdruck }\end{array}$ \\
& Rollenverständnis kommt nicht zum Ausdruck (L17: \\
\hline Perspektive der Fremdsprachigen auf Sache kommt & Perspektive der Fremdsprachigen kommt nicht zum \\
zum Ausdruck -> Fördert gegenseitigen Respekt & Tragen (L18: K2) \\
\hline Familiäre, kulturelle Hintergründe manifestieren & Fam. \& kult. Hintergründe kommen nicht zum Aus- \\
sich stark -> Anlass zu Diskussionen & druck (L24: K6) \\
\hline
\end{tabular}

7) Homogenes Vorwissen der SuS

Anzahl (Anteil) Lehrpersonen: 8 (33.3\%)

Anzahl Konstrukte: 9

\begin{tabular}{|c|c|}
\hline Chancen-Pol & Hindernis-Pol \\
\hline $\begin{array}{l}\text { Wenig Leistungsunterschiede bezüglich Vorwissen } \\
\text { der SuS }\end{array}$ & $\begin{array}{l}\text { Grosse Leistungsunterschiede bezüglich Vorwissen } \\
\text { der SuS (L9: K4) }\end{array}$ \\
\hline $\begin{array}{l}\text { SuS aus anderen Kulturen bringen vielseitiges Vor- } \\
\text { wissen/Fertigkeiten mit }\end{array}$ & $\begin{array}{l}\text { SuS aus anderen Kulturen bringen sehr einseitiges } \\
\text { Vorwissen/Fertigkeiten mit -> SUS mit Migrations- } \\
\text { hintergrund brauchen mehr Aufmerksamkeit durch } \\
\text { LP (L16: K3) }\end{array}$ \\
\hline $\begin{array}{l}\text { SuS kommen mit gleichem Vorwissen ins Fach -> } \\
\text { Verbindet und schafft gemeinsame Grundlage }\end{array}$ & $\begin{array}{l}\text { SuS kommen nicht mit gleichem Vorwissen ins Fach } \\
\text { (L18: K6) }\end{array}$ \\
\hline $\begin{array}{l}\text { Einheitliches Vorwissen -> Ermöglicht Kontakte zwi- } \\
\text { schen SuS }\end{array}$ & $\begin{array}{l}\text { Unterschiedliches Vorwissen (Fachkenntnisse) } \\
\text { (L19: K2) }\end{array}$ \\
\hline Einheitlich Sprachkompetenz der SuS & $\begin{array}{l}\text { Unterschiedliche Sprachkompetenz der SuS -> Ge- } \\
\text { genseitiges Verständnis erschwert (20: K1) }\end{array}$ \\
\hline Hohe Leistungshomogenität im Fach & $\begin{array}{l}\text { Hohe Leistungsheterogenität im Fach -> SuS sind zu } \\
\text { stark mit eigener Leistung im Fach beschäftigt (L23: } \\
\text { K3) }\end{array}$ \\
\hline $\begin{array}{l}\text { Fachvokabular ist für alle SuS gleich neu }->\text { Hohe } \\
\text { Bereitschaft zum Austausch }\end{array}$ & $\begin{array}{l}\text { Fach erfordert hohe deutsche Sprachkompetenz } \\
\text { (L25: K1) }\end{array}$ \\
\hline $\begin{array}{l}\text { Fach, in dem SuS mit MH gleiches oder besseres } \\
\text { Fachwissen haben -> Angebot, von dem profitiert } \\
\text { werden kann }\end{array}$ & $\begin{array}{l}\text { Fach, in dem SuS mit MH benachteiligt sind (L25: } \\
\text { K5) }\end{array}$ \\
\hline $\begin{array}{l}\text { Alle SuS bringen Vorwissen mit und können mitre- } \\
\text { den -> Fördert gegenseitige Neugier }\end{array}$ & Nicht alle SuS bringen Vorwissen mit (L26: K3) \\
\hline
\end{tabular}

8) Kulturneutrale Fachinhalte

Anzahl (Anteil) Lehrpersonen: 7 (33.3\%)

Anzahl Konstrukte: 8

\begin{tabular}{l|l}
\hline Chancen-Pol & Hindernis-Pol \\
\hline Internationale Regeln und Gesetze & Anspruchsvolle Texte (L2: K1) \\
\hline
\end{tabular}




\begin{tabular}{l|l}
\hline Fachinhalt ist kulturell nicht geprägt & Fachinhalt ist kulturell geprägt -> Kann Fronten \\
& zwischen SuS verstärken (L5: K1) \\
\hline Regeln im Fach sind kulturneutral & Regeln im Fach sind kulturabhängig (L5: K6) \\
\hline Fachinhalt ist wertfrei und kulturneutral & Fachinhalte unterliegen starker kultureller Prägun- \\
\hline Fachinhalt ist kulturneutral & gen (L9: K5) \\
\hline Im Fach herrschen kulturunabhängige Definitio- & Im Fach sind Definitionen/Regeln diskutierbar (L16: \\
nen/Regeln -> SuS realisieren, dass sie trotz ver- & K4) \\
schiedenen Hintergründen zusammenarbeiten kön- & \\
nen & \\
\hline Fach ist kulturneutral & Fach ist nicht kulturneutral (L25: K3) \\
\hline Fächer sind nach fixem System/Regeln aufgebaut - & Freies Programm im Fach möglich (L26: K7) \\
$>$ Alle reden vom Selben & \\
\hline
\end{tabular}

9) Arbeiten in Gruppen

Anzahl (Anteil) Lehrpersonen: 7 (33.3\%)

Anzahl Konstrukte: 7

\begin{tabular}{l|l}
\hline Chancen-Pol & Hindernis-Pol \\
\hline $\begin{array}{l}\text { Fach eignet sich für Gruppenarbeiten -> Austausch } \\
\text { zwischen SuS findet statt }\end{array}$ & Fach wird v.a. frontal unterrichtet, viel Einzelarbeit \\
\hline Viel Interaktion/Gruppenarbeit & Viel K4) \\
\hline Im Fach wird oft in Gruppen gearbeitet -> Horizon (L4: K9) & Hauptsächlich Frontalunterricht (L11: K1) \\
terweiterung durch gegenseitiges Kennenlernen & \\
\hline Im Fach kann viel im Team gearbeitet werden -> & Im Fach wird v.a. einzeln oder in 2er Teams gear- \\
Fördert Kooperationsfähigkeit und Akzeptanz & beitet (L12: K2) \\
\hline Im Fach wird oft in Gruppen gearbeitet -> Fördert & Im Fach steht geführte Einzelarbeit im Vordergrund \\
gegenseitiges Verständnis für unterschiedliche Ge- & (L20: K5) \\
dankengänge & \\
\hline Projektarbeit gut möglich -> Klassenteam kann ge- & Lehrerzentriertes, einseitiges, frontal unterrichte- \\
stärkt werden & tes Fach (L23: K4) \\
\hline Fach bietet sich gut für Gruppenarbeit an -> Verbin- & Individuelles Lernprogramm steht im Vordergrund \\
det SuS untereinander & (L26: K6) \\
\hline
\end{tabular}

10) Emotions- und erlebnisreicher Unterricht

Anzahl (Anteil) Lehrpersonen: 6 (25\%)

Anzahl Konstrukte: 7

\begin{tabular}{l:l}
\hline Chancen-Pol & Hindernis-Pol \\
\hline Im Fach können emotionale Momente zustande & Nicht-emotionales Fach (L2: K3) \\
kommen & \\
\hline
\end{tabular}




\begin{tabular}{l:l}
\hline $\begin{array}{l}\text { Im Fach wird gemeinsam erlebt -> SuS erfahren An- } \\
\text { dersartigkeit direkt und unmittelbar }\end{array}$ & Fach ist "trocken" (L7: K2) \\
\hline Inhalte sind kreativ-emotional & Inhalte sind logisch-analytisch (L9: K1) \\
\hline Fach ist erlebnisreich & Fach bietet wenig Gelegenheit zum Entdecken und \\
\hline Zwischenmenschliches (Emotionen) ist stark im & Fachinhalt (Intellekt, Kognition) ist zentral (L17: K8) \\
Fach vertreten & \\
\hline Fachinhalt weckt Emotionen -> IkL besser, da emo- & Fachinhalt ist kognitiv (L18: K1) \\
tional abgestützt & \\
\hline Im Fach können emotionale Reaktionen zum Aus- & Emotionsloses Fach (L20: K3) \\
druck kommen -> SuS können Reaktionen mit eige- & \\
nen vergleichen & \\
\hline
\end{tabular}

11) Physische Bewegung, wechselnde Sitzordnung

Anzahl (Anteil) Lehrpersonen: 6 (25\%)

Anzahl Konstrukte: 6

\begin{tabular}{l|l}
\hline Chancen-Pol & Hindernis-Pol \\
\hline Viel Raum, wechselnde Sitzordnungen & Starre Strukturen im Fach: Fixe Sitzordnung im Klas- \\
& senzimmer -> Wenig Austausch zwischen SUS (L1: \\
& K6) \\
\hline Im Fach sind nonverbale (physische) Interaktionen & Interaktion erfolgt ausschliesslich verbal, sitzend \\
möglich & am Pult (L2: K5) \\
\hline Zugang zueinander über (physische) Bewegung & Physische Bewegung im Fach nicht möglich (L4: K4) \\
möglich & \\
\hline Unterricht findet ausserhalb Klassenzimmer statt & Klassenzimmerfach-> IkL muss von LP aktiv insze- \\
& niert werden (L11: K4) \\
\hline "Ausserordentliches" Lernen (ausserhalb des Schul- & Theoretisches, abstraktes Erarbeiten (L24: K3) \\
zimmers) -> Bietet Gelegenheit, sich besser kennen- & \\
zulernen & \\
\hline Lockere Lernatmosphäre (kein Schulzimmer, flexib- & Starre und sture Lernatmosphäre im Schulzimmer \\
ler Arbeitsplatz) -> Fördert faires Miteinander & (L26: K2) \\
\hline
\end{tabular}

12) Motivierte, offene Schülerinnen und Schüler

Anzahl (Anteil) Lehrpersonen: 5 (20.8\%)

Anzahl Konstrukte: 5

\begin{tabular}{l|l}
\hline Chancen-Pol & Hindernis-Pol \\
\hline Offenheit aller SuS gegenüber Fachinhalt & Negative Vorurteile seitens fremdsprachiger SuS \\
& gegenüber Fachinhalt (L9: K6) \\
\hline $\begin{array}{l}\text { SuS sind im Fach tendenziell motiviert -> Vorausset- } \\
\text { zung für verschiedene Unterrichtsformen (Grup- } \\
\text { penarbeit) }\end{array}$ & SuS sind eher unmotiviert (L11: K7) \\
\hline
\end{tabular}




\begin{tabular}{l|l}
\hline $\begin{array}{l}\text { Keine kulturbedingte Unterschiede bezüglich Leis- } \\
\text { tungsbereitschaft } \rightarrow \text { Ähnliche Einstellungen zum } \\
\text { Fach als Vorteil für IkL }\end{array}$ & tungsbereitschaft (L12: K3) \\
\hline Gelöste, offene, extrovertierte SuS im Fach & Oft gehemmte SUS im Fach -> Gegenseitiger Aus- \\
& tausch erschwert (L23: K6) \\
\hline SuS mit Migrationshintergrund sind motiviert & SuS mit Migrationshintergrund sind nicht motiviert \\
& (L25: K6) \\
\hline
\end{tabular}

13) Austauschen, Diskutieren, Kommunizieren

Anzahl (Anteil) Lehrpersonen: 6 (25\%)

Anzahl Konstrukte: 6

\begin{tabular}{l|l}
\hline Chancen-Pol & Hindernis-Pol \\
\hline $\begin{array}{l}\text { Das Fach lässt Diskussionen zu -> SuS-Themen kön- } \\
\text { nen aufgenommen werden }\end{array}$ & Das Fach lässt keine Diskussionen zu (L5: K5) \\
\hline Im Fach finden offene Diskussionen statt -> Chance, & Im Fach finden wenige offene Diskussionen statt \\
andere Sichtweisen kennenzulernen und zu verste- & $(\mathrm{L7:} \mathrm{K8)}$ \\
hen & \\
\hline Im Fach kann viel diskutiert und ausgetauscht wer- & Im Fach arbeitet jeder für sich, kein Austausch (L16: \\
den -> Eigene Meinungsbildung, Toleranz & K6) \\
\hline Kommunikativer Aspekt ist zentral & Kommunikativer Aspekt ist nicht relevant (L17: K3) \\
\hline $\begin{array}{l}\text { Fach fördert in besonderem Mass die Kommunika- } \\
\text { tion zwischen SuS }\end{array}$ & $\begin{array}{l}\text { Fach fördert Kommunikation zwischen SuS nicht } \\
\text { Im Fach wird häufig mündlich kommuniziert -> Er- }\end{array}$ \\
möglicht persönlichere Kommunikation & Im Fach wird häufig schriftlich kommuniziert (L21: \\
\hline
\end{tabular}

14) Fach mit Alltags- und Lebensbezug

Anzahl (Anteil) Lehrpersonen: 5 (20.8\%)

Anzahl Konstrukte: 5

\begin{tabular}{l|l}
\hline Chancen-Pol & Hindernis-Pol \\
\hline Fach bietet Einbezug aktueller Themen an & Fach bietet Einbezug aktueller Themen nicht an (L4: \\
& K6) \\
\hline $\begin{array}{l}\text { Fach als Lebensschule: Fach ermöglicht Thematisie- } \\
\text { rung lebensnaher Themen -> SuS werden angeregt }\end{array}$ & Fachinhalt ist weniger lebensnah (L7: K4) \\
über eigenes Handeln nachzudenken & \\
\hline $\begin{array}{l}\text { Über Fachinhalt lassen sich Bezüge zum alltägli- } \\
\text { chen Leben herstellen }\end{array}$ & Fachinhalt bietet keine Bezüge zum alltäglichen Le- \\
\hline Fach ermöglicht Einbezug aktueller gesellschaftli- & Fachinhalt bezieht sich weniger auf aktuelle gesell- \\
cher Themen einfacher & schaftliche Entwicklungen -> Konservativer Fachin- \\
& halt bietet wenig Lerngelegenheiten für IkL (L14: \\
& K3) \\
\hline
\end{tabular}




\begin{tabular}{l|l}
\hline $\begin{array}{l}\text { Fach hat viele alltagsbezogene Themen -> Zugang } \\
\text { zueinander über ",ausserschulische“" Themen }\end{array}$ & K7) Themen im Fach sind nur schulbezogen (L19: \\
\hline
\end{tabular}

15) Interdisziplinäre Fachthemen

Anzahl (Anteil) Lehrpersonen: 4 (16.7\%)

Anzahl Konstrukte: 5

\begin{tabular}{l:l}
\hline Chancen-Pol & Hindernis-Pol \\
\hline $\begin{array}{l}\text { Im Fach lassen sich Transfers zu anderen Fächern } \\
\text { herstellen }\end{array}$ & Im Fach sind keine Transfers zu anderen Fächern \\
\hline Fach lässt sich mit anderen Fächern inhaltlich ver- & Fach lässt sich nicht mit anderen Fächern verbinden \\
binden -> Verstärkt Notwendigkeit der Zusammen- & $(\mathrm{L14:}$ K7) \\
arbeit zwischen SuS & \\
\hline Im Fach kann nach Parallelen gesucht werden -> & Im Fach kann nicht nach Parallelen zu anderen Fä- \\
Falls Weltsprache, ist es eine Chance & chern gesucht werden (L21: K7) \\
\hline Im Fach besteht Möglichkeit, Gelerntes aus ande- & Im Fach besteht keine Möglichkeit, Gelerntes aus \\
ren Fächern im Fach anzuwenden -> SuS lernen ver- & anderen Fächern anzuwenden (L22: K2) \\
netzt denken & \\
\hline Fach ermöglicht fächerverbindenden Unterricht & Fach wird tendenziell von Fachlehrperson unter- \\
& richtet -> Erschwert fächerverbindenden Unterricht \\
\hline
\end{tabular}

16) Hoher Praxisanteil

Anzahl (Anteil) Lehrpersonen: 4 (16.7\%)

Anzahl Konstrukte: 4

\begin{tabular}{l|l}
\hline Chancen-Pol & Hindernis-Pol \\
\hline $\begin{array}{l}\text { Handlungsorientiertes Fach -> Differenzen zwi- } \\
\text { schen Kulturen kommen stärker zum Ausdruck }\end{array}$ & Kognitiv orientiertes Fach (L1: K9) \\
\hline Fachinhalt stark praktisch/angewandt & Fachinhalt stark theorielastig (L4: K5) \\
\hline Im Fach erschliessen sich SuS neue praktische Er- & Im Fach lernt man theoretisches Wissen (L9: K2) \\
fahrungen & \\
\hline Fach ist körperlich/praktisch & Fach ist theoretisch (L17: K7) \\
\hline
\end{tabular}

17) Persönliche Hintergründe und Haltungen der SuS kommen nicht zum Ausdruck

Anzahl (Anteil) Lehrpersonen: 4 (16.7\%)

Anzahl Konstrukte: 4

\begin{tabular}{l|l}
\hline Chancen-Pol & Hindernis-Pol \\
\hline $\begin{array}{l}\text { Kein religionsbedingtes, andersartiges Verhalten } \\
\text { von SuS }\end{array}$ & Religionsbedingtes, andersartiges Verhalten der \\
\hline
\end{tabular}




\begin{tabular}{l|l}
\hline $\begin{array}{l}\text { Kulturbedingte Rollenverständnisse können nicht } \\
\text { zum Ausdruck kommen }\end{array}$ & $\begin{array}{l}\text { Im Fach können kulturbedingte Rollenverständ- } \\
\text { nisse zum Ausdruck kommen (z.B. Macho-Verhal- } \\
\text { ten) (L5: K8) }\end{array}$ \\
\hline $\begin{array}{l}\text { Im Fach kommen kulturbedingte Rollenbilder nicht } \\
\text { zum Ausdruck }\end{array}$ & $\begin{array}{l}\text { Im Fach können kulturbedingte Rollenbilder stark } \\
\end{array}$ \\
& Gum Ausdruck kommen -> Ev. negativen Einfluss auf \\
\hline Persönlichkeiten kommen nicht zum Ausdruck & Persönlichkeiten der einzelnen SuS kommen stark \\
& zum Ausdruck-> Kann Vorurteile erzeugen (L21: K4) \\
\hline
\end{tabular}

18) Sonstige Chancen

Anzahl Konstrukte: 16

\begin{tabular}{|c|c|}
\hline Chancen-Pol & Hindernis-Pol \\
\hline Halbklassenunterricht (im Sport inszeniert) & $\begin{array}{l}\text { Grosse Klassen -> Weniger persönlichere Interakti- } \\
\text { onen möglich (L1: K8) }\end{array}$ \\
\hline Austausch über Sprache/Texte & Nonverbale Erfahrungen/Erlebnisse (L4: K1) \\
\hline Geschlossener Unterricht & $\begin{array}{l}\text { Offener Unterricht -> Fördert Separation in Klasse } \\
\text { (L5: K2) }\end{array}$ \\
\hline $\begin{array}{l}\text { Häufig Halbklassenunterricht -> Persönlichere At- } \\
\text { mosphäre }\end{array}$ & Stets grosse Klassen (L7: K6) \\
\hline $\begin{array}{l}\text { Stellenwert/Bedeutung des Fachinhalts in anderen } \\
\text { Gesellschaften kann zum Thema gemacht werden }\end{array}$ & $\begin{array}{l}\text { Stellenwert des Faches kann nicht zum Thema ge- } \\
\text { macht werden (L8: K6) }\end{array}$ \\
\hline $\begin{array}{l}\text { Fach bietet miteinander- und gegeneinander-Situa- } \\
\text { tionen }\end{array}$ & $\begin{array}{l}\text { Fach bietet keine miteinander- und gegeneinander- } \\
\text { Situationen (L9: K8) }\end{array}$ \\
\hline Im Fach werden englische Ausdrücke gelernt & Monolingualer Unterricht (L10: K2) \\
\hline $\begin{array}{l}\text { Fachinhalt erfordert das Übersetzen englischer } \\
\text { Texte }\end{array}$ & $\begin{array}{l}\text { Keine Übersetzung englischer Texte notwendig } \\
\text { (L10: K3) }\end{array}$ \\
\hline $\begin{array}{l}\text { Unterricht findet in Halbklassen statt }->I k L \text { ist in } \\
\text { kleinen Gruppen generell einfacher }\end{array}$ & Unterricht findet mit ganzer Klasse statt (L12: K6) \\
\hline $\begin{array}{l}\text { Koedukativer Unterricht -> Sämtliche Unterschiede } \\
\text { zwischen SuS sind sichtbar }\end{array}$ & Geschlechtergetrennter Unterricht (L12: K7) \\
\hline $\begin{array}{l}\text { Fach wird integriert unterrichtet }->\text { Höhere Hetero- } \\
\text { genität gibt Einblick in verschiedene Lebensweisen }\end{array}$ & $\begin{array}{l}\text { Fach wird getrennt nach Leistungsniveaus unter- } \\
\text { richtet (L14: K4) }\end{array}$ \\
\hline Mensch ist im Zentrum & Mensch ist nicht im Zentrum (L17: K1) \\
\hline $\begin{array}{l}\text { Fach bietet Möglichkeit zur Erweiterung des } \\
\text { Deutsch-Wortschatzes }\end{array}$ & $\begin{array}{l}\text { Fach bietet wenig Möglichkeiten den Deutsch- } \\
\text { Wortschatz zu erweitern (L19: K1) }\end{array}$ \\
\hline Im Fach lernt man englische Begriffe & $\begin{array}{l}\text { Im Fach lernt man keine englischen Begriffe (L21: } \\
\text { K5) }\end{array}$ \\
\hline $\begin{array}{l}\text { Oft disziplinarische Schwierigkeiten im Fachunter- } \\
\text { richt }\end{array}$ & Kaum disziplinarische Schwierigkeiten (L23: K8) \\
\hline
\end{tabular}




\begin{tabular}{l|l}
\hline $\begin{array}{l}\text { Fachinhalt ist emotionslos -> verhindert Fronten } \\
\text { zwischen SuS }\end{array}$ & $\begin{array}{l}\text { Fachinhalt ist emotional (Nationalität, Glaube etc.) } \\
(\mathrm{L} 25: \mathrm{K} 2)\end{array}$ \\
\hline
\end{tabular}

